\title{
HYDROGEN FROM AMMONIA BY CATALYTIC SPILLOVER MEMBRANE
}

\author{
BY \\ JONATHAN MARK TAILBY
}

A thesis submitted to the Victoria University of Wellington in fulfilment of the requirements for the degree of Doctor of Philosophy

Victoria University of Wellington 2018 
Jonathan Tailby 


\section{Hydrogen from Ammonia by Catalytic Spillover Membrane}

\section{Abstract}

One of the major challenges to be overcome before hydrogen fuelled vehicles can become commonplace is to store hydrogen with sufficient storage density to be practical. One approach to overcoming this challenge involves converting the hydrogen into a secondary fuel that can be stored more easily, such as ammonia. This introduces the challenge of efficiently retrieving the hydrogen from the secondary fuel with sufficient purity to be used in a polymer electrolyte membrane fuel cell.

Putting the hydrogen producing reaction inside a membrane which is capable of filtering out hydrogen creates a membrane reactor which can increase hydrogen purity and can accelerate the reaction both kinetically and thermodynamically. The most effective materials currently known for hydrogen membranes are high palladium alloys of copper and silver. These are able to absorb hydrogen on the side with high hydrogen partial pressure and desorb that hydrogen on the side with low hydrogen pressure.

Palladium metal is also able to interact with some catalysts by hydrogen spillover. Hydrogen is transported from the surface of the catalyst to the palladium surface more quickly than the hydrogen can desorb from the catalyst, this potentially accelerates both the catalysis and the hydrogen filtration.

This research aimed to create a catalytic spillover membrane to extend the possibility of ammonia as a secondary fuel for hydrogen transport. In this research, several methods to produce a nickel catalyst on the surface of the palladium were explored: electrodeposition with and without a lithographic template; spray coating with nanoparticles; and preshaped nickel mesh and nickel foam. These potential catalysts were tested for ammonia decomposition. Templated electrodeposition created the most effective catalyst, but the nickel foam was most easily applied to the next stage of the research. The nickel foam catalyst was subsequently retested for ammonia decomposition in three scenarios: in contact with palladium foil; in a reactor with a palladium 
Jonathan Tailby

membrane; and in contact with a palladium membrane. The presence of a palladium membrane improved decomposition more than spillover contact between nickel foam catalyst and palladium, however, the combination of spillover contact with a palladium membrane increased the ammonia decomposition further.

The rate of hydrogen flux through the palladium membranes was calculated for the experimental results. These were compared to flux values predicted by a model equation. The results showed that spillover contact between nickel catalyst and palladium membrane increased the hydrogen flux through the membrane..

The research outcomes have generated new knowledge and improved understanding of the morphology and role of nickel catalysts in accelerating ammonia decomposition. The research highlights the complex relationship between reactor design, gas flow paths, catalyst presentation and catalysis chemistry, suggesting promising areas for future research. 


\section{Acknowledgements}

I would like to thank all the people who have helped me through these hard years.

Thank you to both of my supervisors Ian Brown at Industrial Research Limited/Callaghan Innovation/ the MacDiarmid Institute and John Spencer at Victoria University of Wellington/ the MacDiarmid Institute.

For technical help, I would like to thank: Martin Ryan for XRD training and miscellaneous expertise; David Flynn, Ruth Knibbe and Sarah Spencer for SEM training and assistance; Bruce Hamilton for GC advice; Jeremy Wu and Geoff Smith for mass spectrometry and electrodeposition training and assistance; and Andrew Best for microfabrication of electrodeposition templates. I would also like to thank Diego Del Puerto and the Industrial Research Limited/ Callaghan Innovation workshop for so much practical assistance in reactor development. Also, thanks to Alison Speakman of the Industrial Research Limited/ Callaghan Innovation Information Services.

Thanks to Industrial Research Limited/ Callaghan Innovation for lab and office space throughout the project.

For financial support, I would like to thank the MacDiarmid Institute and the Religious Society of Friends Aotearoa/New Zealand.

Big thanks to all the Advanced Materials Team of Industrial Research Limited/ Callaghan Innovation for support, ideas, general assistance and camaraderie.

Thank you to my wife and father for their proof-reading assistance.

The most important people to thank are my wife, my parents and wider family for their longstanding support of me and this research. 
Jonathan Tailby

\section{Table of Contents}

Abstract 3

Acknowledgements $\quad 5$

Table of Contents $\quad 6$

Table of Figures $\quad 9$

List of Tables 23

List of Equations $\quad 29$

Definition of Terms 31

List of Abbreviations 31

1. Introduction 33

1.1. Background: 33

1.2. Literature Review 37

1.2.1. Gas Reformation 37

1.2.2. Ammonia as a Hydrogen Carrier 40

1.2.3. Other Hydrogen Carrier Reactions 49

1.2.4. Membrane Reactors for Gas Reformation 51

1.2.5. Electrochemistry 59

1.2.6. Spillover 66

1.2.7. Palladium-Hydrogen 75

1.2.8. The Palladium-Hydrogen System and Ammonia Decomposition 80

1.3. Research Plan 82

1.3.1. Catalyst Preparation $\quad 82$

1.3.2. Catalyst Characterisation 83

1.3.3. Reaction Testing with the Catalyst 83

1.4. Catalysis with Spillover + Hydrogen Transport + Porous Anodic $\begin{array}{lr}\text { Alumina } & 85\end{array}$

2. Experimental 87

2.1. Experimental Techniques $\quad 87$

$\begin{array}{ll}\text { 2.1.1. Electrodeposition } & 87\end{array}$

2.1.2. Lithography 88

2.1.3. Optical Microscopy 88

2.1.4. X-Ray Diffraction $\quad 89$

2.1.5. Scanning Electron Microscopy 90

2.1.6. Mass Spectrometry 90

2.1.7. Gas Chromatography 91 
2.2. Experimental Method 94

2.2.1. Chemicals and Materials Used 94

2.2.2. Electrodeposition 95

$\begin{array}{ll}2.2 .3 \text {. Spray Coating } & 100\end{array}$

$\begin{array}{ll}\text { 2.2.4. Lithography } & 101\end{array}$

2.2.5. Preshaped Catalysts 106

$\begin{array}{ll}\text { 2.2.6. Optical Microscopy } & 107\end{array}$

$\begin{array}{lll}\text { 2.2.7. X-Ray Diffraction } & 108\end{array}$

2.2.8. Scanning Electron Microscopy 108

2.2.9. Gas Reactor Development 110

2.2.10. Catalyst Testing in the Secondary and Tertiary Inserts 123

2.2.11. Mass Spectrometry for Reaction Analysis 126

2.2.12. Gas Chromatography for Reaction Analysis 127

2.2.12.1. Summary of GC details 130

2.2.13. Electronic Gas Pressure Control 144

3. Results and Discussion 147

$\begin{array}{ll}\text { 3.1. Catalyst Preparation } & 147\end{array}$

3.1.1. Electrodeposition Results 147

$\begin{array}{ll}\text { 3.1.2. Spray Coating } & 174\end{array}$

3.1.3. Electrodeposition with Lithographic Templates 182

$\begin{array}{lll}\text { 3.1.4. } & \text { Preshaped Nickel } & 198\end{array}$

3.2. Reaction Testing with Catalysts 204

3.2.1. Mass Spectrometry Results 204

3.2.2. Reactor Development 208

3.3. Reaction Testing with Enhanced Catalysts 228

3.3.1. Catalyst with Permeation but no Spillover 228

3.3.2. Catalyst with Spillover but no Permeation 232

3.3.3. Catalyst with Spillover and Hydrogen Permeation 236

4. Final Summary 257

4.1. Future Work 259

5. Appendices 261

5.1. Method 261

5.1.1. Shrinkwrap Transmittance Spectrum 261

5.1.2. Preliminary Experiments 261

5.1.3. Alternative Secondary Insert Designs 269

5.1.4. Tertiary Insert Design 270 
Jonathan Tailby

5.1.5. GC Calculation Tests 271

5.1.6. Electronic Gas Pressure Control 289

5.2. Results 292

5.2.1. Complete List of Electrodeposition Samples 292

5.2.2. Complete Spray Coat Mass Data 308

5.2.3. SEM Image Processing Calculations for Electrodeposition on Aluminium 312

5.2.4. SEM Image Processing Calculations for Electrodeposition on $\begin{array}{ll}\text { Palladium } & 316\end{array}$

5.2.5. SEM \& EDS for Spray Coated Samples 317

5.2.6. SEM of Template Electrodeposition on Secondary Insert Tubes 321

5.2.7. Gas Chromatography 324

6. References 363 


\section{Table of Figures}

Figure 1.1.1: Research plan. 35

Figure 1.2.1: Equilibrium proportions for ammonia decomposition at 1 bar...... 41

Figure 1.2.2: Volcano plot for $\log$ rate vs. dissociative $\mathrm{N}_{2}$ adsorption energy. ... 43

Figure 1.2.3: One-dimensional potential energy diagram illustrating from left to right the catalytic decomposition of $\mathrm{NH}_{3}$ over platinum.

Figure 1.2.4: Schematics of traditional reformer and membrane reactor [99]....52

Figure 1.2.5: Schematic representation of the membrane reactor for ammonia decomposition

Figure 1.2.6: Pressure dependence of steam reforming of methane in membrane reactor at $500{ }^{\circ} \mathrm{C}$.

Figure 1.2.7: Comparison of Instantaneous and progressive nucleation models with measured data for cobalt on a copper/glass electrode [122].

Figure 1.2.8: Schematic representation of spillover of a diatomic gas from an adsorbing onto a nonadsorbing surface. 67

Figure 1.2.9: Conversion of cyclohexane by polyfunctional catalysis. 71

Figure 1.2.10: Reaction of cyclohexane with spillover hydrogen. 71

Figure 1.2.11: Spillover reaction tests. HSP-M membrane tubes for the methanol steam reforming reaction at $240{ }^{\circ} \mathrm{C}$. .74

Figure 1.3.1: Sequence of events in the Catalytic Spillover Membrane active system.

Figure 2.2.1: The Electrodeposition cell used in these experiments. 96

Figure 2.2.2: The voltage sources used for electrodeposition. 98

Figure 2.2.3: Tubular electrodeposition set up 104

Figure 2.2.4: Progression of gas reactors. 111

Figure 2.2.5: Sealed Sputnik gas reaction vessel .112

Figure 2.2.6: Open Gas Reaction Vessel 112 
Jonathan Tailby

Figure 2.2.7: Schematic showing the internal structure of the reaction vessel..113 Figure 2.2.8: Schematic showing the gas flow inside the 'Sputnik' gas reaction vessel. 113

Figure 2.2.9: Explorer reactor with rolled stainless steel mesh supporting the sample in the centre of the reactor. 114

Figure 2.2.10: Explorer reactor with stainless steel primary insert holding the sample in the centre of the reactor with reduced volume. .115

Figure 2.2.11: Sample holder for primary insert tube. 116

Figure 2.2.12: Explorer reactor with a secondary insert tube inside the stainless steel primary insert. .116

Figure 2.2.13: Secondary insert tubes. 117

Figure 2.2.14: Nickel mesh secondary insert. 118

Figure 2.2.15: This photograph shows a nickel foam secondary insert compared with an untemplated electrodeposited nickel secondary insert and a pen.......119 Figure 2.2.16: Photograph of the 'Nickel-on-Palladium' tertiary insert. 120 Figure 2.2.17: Image of the steel tube showing the cut-aways under the palladium foil. 121

Figure 2.2.18: Explorer reactor with a tertiary insert tube inside the stainless-steel primary insert. 121

Figure 2.2.19: Photograph of 'Nickel-on-Palladium-no-Permeation' insert...... 122 Figure 2.2.20: Diagram to show the connections between the gas reactor and the mass spectrometer. 126

Figure 2.2.21: SRI GC8610C gas chromatograph. 128

Figure 3.1.1: Current vs. time graphs for electrodeposition of nickel at $-1.6 \mathrm{~V}$ onto aluminium. 148

Figure 3.1.2: Electron micrograph of nickel electrodeposited for $30 \mathrm{~s}$ at $-1.6 \mathrm{~V}$ on aluminium. 
Figure 3.1.3: Current vs. time graphs for electrodeposition of nickel onto aluminium in $0.1 \mathrm{~mol} \cdot \mathrm{L}^{-1} \mathrm{Ni}\left(\mathrm{NO}_{3}\right)_{2}, 30 \mathrm{~s},-0.5--2.5 \mathrm{~V}, \mathrm{pH} 8.3$.

Figure 3.1.4: Back Scatter Electron micrograph of aluminium electrodeposited at $-0.5 \mathrm{~V}$ with $0.1 \mathrm{~mol} \cdot \mathrm{L}^{-1} \mathrm{Ni}\left(\mathrm{NO}_{3}\right)_{2}$, showing little evidence of nickel deposits....151

Figure 3.1.5: Current vs. time graphs for electrodeposition of nickel onto aluminium at varying nickel concentration and $-1.6 \mathrm{~V}$. 152

Figure 3.1.6: XRD showing no nickel detected on the aluminium surface after electrodeposition for $30 \mathrm{~s}$ at $-2.0 \mathrm{~V}$ with the $0.1 \mathrm{~mol} \cdot \mathrm{L}^{-1} \mathrm{Ni}\left(\mathrm{NO}_{3}\right)_{2}$ solution. 153

Figure 3.1.7: SEM of small high surface area deposits on aluminium. 154

Figure 3.1.8: Percentage coverage vs. voltage for nickel electrodeposited on aluminium for $30 \mathrm{~s}$ in $0.1 \mathrm{~mol} \cdot \mathrm{L}^{-1}$ electroplating solution. 157

Figure 3.1.9: Average diameter vs. voltage for nickel electrodeposits on aluminium in $0.1 \mathrm{~mol} \cdot \mathrm{L}^{-1}$ electroplating solution for $30 \mathrm{~s}$. 157

Figure 3.1.10: Electron micrographs for nickel electrodeposited on aluminium showing how quantity and surface complexity of the deposits increased with voltage negativity.

Figure 3.1.11: Current vs. time graph for electrodeposition of nickel from 0.1 mol. $\mathrm{L}^{-1} \mathrm{Ni}\left(\mathrm{NO}_{3}\right)_{2}, 30 \mathrm{~s},-2.0 \mathrm{~V}$ comparing aluminium and palladium substrates.

Figure 3.1.12: $\left(\mathrm{I} / \mathrm{I}_{\max }\right)^{2}$ vs. $\mathrm{t} / \mathrm{t}_{\max }$ of Electrodeposition at $-2.0 \mathrm{~V}$ for the electrodepositions shown in Figure 3.1.11. 160

Figure 3.1.13: Current vs. time graphs comparing electrodepositions of nickel onto aluminium and palladium at multiple voltages. 161

Figure 3.1.14: Electron micrograph of palladium electrodeposited with nickel for $30 \mathrm{~s}$ at $-2.0 \mathrm{~V}$ in the nickel sulfate solution of Table 2.2.1. .162

Figure 3.1.15: SEM micrograph of palladium electrodeposited with nickel. ....163 Figure 3.1.16: Enlarged detail from the same sample of palladium electrodeposited with nickel shown in Figure 3.1.15. .163 
Jonathan Tailby

Figure 3.1.17: Back scatter SEM image of the same location as Figure 3.1.16 ... 164

Figure 3.1.18: X-ray spectrum from EDS analysis as marked in Figure 3.1.17..164

Figure 3.1.19: Back scatter electron micrograph of the palladium electroplated

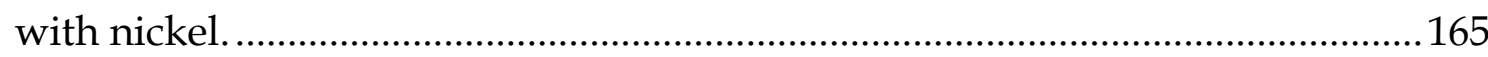

Figure 3.1.20: Nickel elemental map for the same location as Figure 3.1.19.....166

Figure 3.1.21: Graph of percentage area of blistering vs. deposition voltage for electrodeposition of nickel onto palladium..................................................... 167

Figure 3.1.22: XRD trace of palladium electrodeposited with nickel. ................169

Figure 3.1.23: XRD trace of palladium electrodeposited with nickel at $-1.0 \mathrm{~V}$ for 1 hour. 170

Figure 3.1.24: Elemental maps of cross section of electrodeposited palladium. 172

Figure 3.1.25: X-ray spectrum for EDS point analysis showing oxygen and nickel present on the palladium. 172

Figure 3.1.26: Optical micrograph of a spray coated sample which shows where nickel has deposited most heavily at the edges of the droplets. 175

Figure 3.1.27: Optical micrograph of heavy spray coating, showing the overlap of rings and the extra agglomeration that has occurred there. 175

Figure 3.1.28: XRD trace for aluminium sprayed with nickel nanoparticles. ... 176 Figure 3.1.29: High magnification SEM of nickel nanoparticles spray coated onto Steel. 177

Figure 3.1.30: SEM Micrograph of Intersection of multiple drying rings. 177

Figure 3.1.31: Low magnification SEM of circular deposition patterns on a spray coated sample. 178

Figure 3.1.32: Low magnification SEM of circular deposition on spray coat sample with heavy deposition at points of multiple intersection of rings near the centre of the image. 178 
Figure 3.1.33: SEM image of spray coat sample showing both rings and agglomerations.

Figure 3.1.34: SEM image of spray coat sample showing very low density of nickel deposition.

Figure 3.1.35: SEM image of a spray coated palladium sample. 180

Figure 3.1.36: Elemental map of palladium after spray coating 181

Figure 3.1.37: Elemental map of nickel spray coated onto palladium. 181

Figure 3.1.38: Elemental map of oxygen where nickel nanoparticles have been spray coated onto palladium. 182

Figure 3.1.39: Optical micrograph of templates for electrodeposition. Each $10 \mu \mathrm{m}$ circle is a hole that has been burnt through the photosensitive polymer with ultraviolet light. 183

Figure 3.1.40: Current vs. time graphs for templated electrodeposits on steel at $1.2 \mathrm{~V}$. 184

Figure 3.1.41: Estimated volume of nickel vs. time during electrodeposition on templated steel for the same deposition shown in Figure 3.1.40. 184

Figure 3.1.42: Predicted electric current for electrodeposition in a template that overflows then forms a continuous layer. 185

Figure 3.1.43: Series of optical micrographs showing the sequence of steps from template to electrodeposits. 186

Figure 3.1.44: Electron micrograph of the over deposited nickel layer, the side which was formerly attached to the steel substrate. 187

Figure 3.1.45: Current vs. time graphs for templated electrodeposits on steel. 188 Figure 3.1.46: Back scatter electron micrograph of the electrodeposition sample labelled 'Typical Template Deposition' in Figure 3.1.45. 188

Figure 3.1.47: Electron micrograph of a successful templated electrodeposition on steel showing how the deposits were consistent in height and diameter. 189 
Jonathan Tailby

Figure 3.1.48: Higher magnification electron micrograph of a successful deposition showing that they were composed of smaller units aggregated together. 190

Figure 3.1.49: Electron micrograph shows the edge of the same deposition sample as Figure 3.1.47. 190

Figure 3.1.50: Enlargement of the dislodged deposits shown in Figure 3.1.49.191 Figure 3.1.51: Current vs. time graphs for electrodeposition on bare steel at -1.2 V showing the effect of a long delay between preparing the deposition and activating the voltage.

Figure 3.1.52 Predeposition template 193

Figure 3.1.53 Template and deposit after deposition 193

Figure 3.1.54 Nickel deposit after acetone wash has removed the template.....193

Figure 3.1.55: Optical micrograph of the top right point of the main deposit, indicated by the green circle in Figure 3.1.53. Image taken after electrodeposition but before the acetone wash. 193

Figure 3.1.56: Optical micrograph of the top right point of the main deposit, indicated by the green circle in Figure 3.1.54. Image taken after the acetone wash had removed the template. 194

Figure 3.1.57: Optical micrograph of the middle of the sample shown in Figure 3.1.52. Template before deposition. 195

Figure 3.1.58: Optical micrograph of the middle of the sample shown in Figure 3.1.54. After acetone wash. This shows the same region as Figure 3.1.57....... 195 Figure 3.1.59: 400x magnification secondary electron micrograph of templated electrodeposits on a secondary insert. 196

Figure 3.1.60: 800x magnification secondary electron micrograph of templated electrodeposits on a secondary insert. 196

Figure 3.1.61: 800x magnification secondary electron micrograph of templated electrodeposits on a secondary insert. 197 
Figure 3.1.62: Secondary electron image of nickel mesh before ammonia decomposition experiments.

Figure 3.1.63: Back scatter electron image of nickel mesh before ammonia decomposition experiments. 198

Figure 3.1.64: Secondary electron image of nickel mesh after ammonia decomposition experiments. 198

Figure 3.1.65: Back scatter electron image of nickel mesh after ammonia decomposition experiments. 198

Figure 3.1.66: EDS spectrum of nickel mesh before ammonia decomposition experiments

Figure 3.1.67: EDS spectrum of nickel mesh after ammonia decomposition experiments 200

Figure 3.1.68: Optical micrograph of the nickel foam 202

Figure 3.1.69: Optical micrograph of the nickel foam 202

Figure 3.1.70: This XRD trace of the nickel foam confirmed that it contains nickel and no significant quantity of any other crystalline phase. 203

Figure 3.2.1: Mass spectrometry record for a hydrogen permeation experiment with the Sputnik reactor. 204

Figure 3.2.2: Mass spectrometry record for ammonia decomposition experiment with a palladium membrane electrodeposited at $-2.7 \mathrm{~V}$ for $20 \mathrm{~s}$ in the Sputnik reactor. 207

Figure 3.2.3: Mass spectrometry record for argon flushing air out of the reactor at room temperature. 208

Figure 3.2.4: Sample chromatograms for blank steel catalyst temperature series at 300- $500{ }^{\circ} \mathrm{C}$ in the 'Explorer' reactor. 209

Figure 3.2.5: Optical micrographs of the first templated nickel sample to be tested for ammonia decomposition 210 
Jonathan Tailby

Figure 3.2.6: Gas outputs calculated from the GC results plotted against temperature for ammonia decomposition with different catalysts in the Explorer gas reactor.

Figure 3.2.7: Reactor output vs. temperature for hydrogen from samples in the primary insert. .213

Figure 3.2.8: Reactor output vs. temperature for nitrogen from samples in the primary insert. 213

Figure 3.2.9: Reactor output vs. temperature for ammonia from samples in the primary insert. 213

Figure 3.2.10: Ammonia decomposition by catalyst samples in the primary insert. 213

Figure 3.2.11: Plots of the gas output at temperatures from 300 to $450{ }^{\circ} \mathrm{C}$ with the bare steel secondary insert. . 214

Figure 3.2.12: Plot for the decomposition of ammonia against temperature for different catalysts on the secondary inserts from 300 to $450{ }^{\circ} \mathrm{C}$ 216

Figure 3.2.13: Plot for the production of hydrogen against temperature for different catalysts on the secondary inserts from 300 to $450{ }^{\circ} \mathrm{C}$. 217

Figure 3.2.14: Arrhenius plot for Ammonia Decomposition with Secondary Insert Catalysts. 220

Figure 3.2.15: Arrhenius plot of the Hydrogen Formation data for the Secondary Insert Catalysts. 221

Figure 3.2.16: Graph of Specific Reaction Rate vs. Temperature for electrodeposited catalysts on secondary inserts. .225

Figure 3.2.17: Graph of Specific Reaction Rate vs. temperature for Preshaped Catalysts on Secondary Inserts. 225

Figure 3.2.18: Plot of molar output vs. temperature for the for three gases at two different input flows with the templated nickel secondary insert catalyst. ......226 
Figure 3.2.19: Graph of normalised molar output vs. temperature for the for three gases at two different input flows with the templated nickel secondary insert catalyst.

Figure 3.3.1: Rate of ammonia decomposition at $5 \mathrm{~mL} \mathrm{~min}^{-1}$ with four secondary inserts compared with the Nickel-before-Palladium tertiary insert. .230

Figure 3.3.2: hydrogen gas flow in the product gas mixture from ammonia

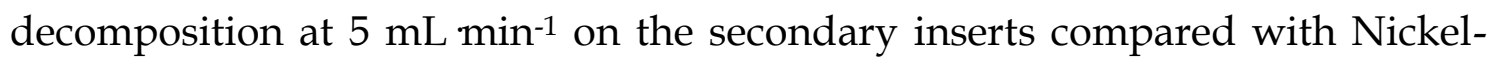
before-Palladium tertiary insert.

Figure 3.3.3: Hydrogen from decomposition of ammonia on four secondary inserts and the nickel foam catalyst with spillover.

Figure 3.3.4: Ammonia decomposition with Nickel-on-Palladium-no-Permeation compared with percentage of ammonia decomposed with secondary inserts. 235 Figure 3.3.5: The ammonia which remained in the retentate after decomposition plotted against temperature. 238

Figure 3.3.6: The rate of ammonia decomposition graphed against temperature for the three tertiary inserts.

Figure 3.3.7: Hydrogen output in the retentate of the tertiary reactors plotted against temperature.

Figure 3.3.8: Hydrogen permeation against temperature for tertiary inserts...241

Figure 3.3.9: Hydrogen flux vs. temperature for tertiary inserts.

Figure 3.3.10: Hydrogen Flux plotted against reaction temperature for tertiary inserts.

Figure 3.3.11: Hydrogen output vs. temperature for tertiary inserts. Retentate outputs are represented by solid lines, permeate outputs by dashed lines. .....243 Figure 3.3.12: Total hydrogen output vs. temperature for tertiary inserts. ........244 Figure 3.3.13: Plot of nitrogen production vs. temperature for tertiary inserts. 245 
Jonathan Tailby

Figure 3.3.14: Hydrogen flux vs. temperature for Nickel-on-Palladium Catalytic Spillover Membrane showing results calculated from GC experiments and by Richardson's Equation 247

Figure 3.3.15: Graph of hydrogen flux vs. inverse temperature showing diffusion limits calculated by Ward and Dao, recalculated here to mark temperatures relevant to this research. 248

Figure 3.3.16: Graph of hydrogen flux vs. temperature comparing the flux values calculated from hydrogen measurements with two models of hydrogen flux; Richardson's and Ward and Dao's. 249

Figure 3.3.17: Average quantity of ammonia decomposed when the ammonia flow was varied from 1 to $6 \mathrm{~mL} \mathrm{~min}{ }^{-1}$ while temperature was maintained at 350 ${ }^{\circ} \mathrm{C}$.

Figure 3.3.18: Hydrogen output in the retentate for the tertiary inserts at $350{ }^{\circ} \mathrm{C}$ when the ammonia flow was varied from 1 to $6 \mathrm{~mL} \mathrm{~min}^{-1}$. 252

Figure 3.3.19: Quantity of hydrogen in the sweep flow of the two permeating tertiary inserts at $350^{\circ} \mathrm{C}$ while ammonia flow varied from 1 to $6 \mathrm{~mL} \mathrm{~min}^{-1}$.... 255 Figure 3.3.20: Hydrogen Flux plotted against ammonia input volumetric flow for

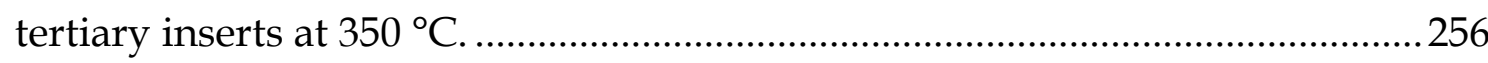

Figure 5.1.1: Percentage Transmittance vs. Wavelength for Shrink Wrap....... 261

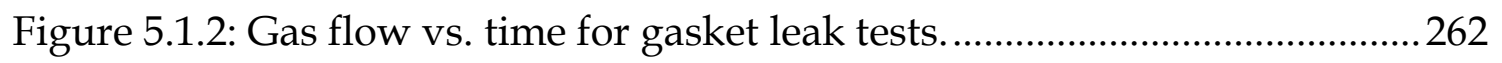

Figure 5.1.3: Percentage gas leak vs. time for the same gasket leak tests shown in Figure 5.1.2. 262

Figure 5.1.4: GC results for Carbon Dioxide flushing nitrogen out of the Explorer reactor. .264

Figure 5.1.5: Temperature calibration for gas reactor with primary insert. ...... 265

Figure 5.1.6: Further temperature calibrations of the primary insert. 265

Figure 5.1.7: Argon Standards Experiments .267 
Figure 5.1.8: Gas chromatogram for sweep argon at $350{ }^{\circ} \mathrm{C}$ inside a tertiary insert with $5 \% \mathrm{H}_{2} / 95 \% \mathrm{~N}_{2}$ in the reaction space.

Figure 5.1.9: Chromatogram for the permeation experiment for the Nickel-onPalladium-No-Permeation. 269

Figure 5.1.10: two designs for space filling sample holders. 269

Figure 5.1.11: design drawing of the tertiary insert. 270

Figure 5.1.12: Temperature Profile inside the primary and tertiary inserts when the external temperature of the reactor is $403^{\circ} \mathrm{C}$ 271

Figure 5.2.1: Electron micrograph of nickel deposited on aluminium at $-1.0 \mathrm{~V}$ for $30 \mathrm{~s}$ in $0.1 \mathrm{~mol} \cdot \mathrm{L}^{-1}$ solution

Figure 5.2.2: Electron micrograph of nickel deposited on aluminium at $-1.5 \mathrm{~V}$ for $30 \mathrm{~s}$ in $0.1 \mathrm{~mol} \cdot \mathrm{L}^{-1}$ solution

Figure 5.2.3: Electron micrograph of nickel deposited on aluminium at $-2.0 \mathrm{~V}$ for $30 \mathrm{~s}$ in $0.1 \mathrm{~mol} \cdot \mathrm{L}^{-1}$ solution 315

Figure 5.2.4: Electron micrograph of nickel deposited on aluminium at $-2.5 \mathrm{~V}$ for $30 \mathrm{~s}$ in $0.1 \mathrm{~mol} \cdot \mathrm{L}^{-1}$ solution 315

Figure 5.2.5: Electron micrograph of nickel deposited on palladium at $-1.0 \mathrm{~V}$ for $30 \mathrm{~s}$. 316

Figure 5.2.6: Electron micrograph of nickel deposited on palladium at $-2.5 \mathrm{~V}$ for $30 \mathrm{~s}$. 316

Figure 5.2.7: SEM image of a spray coated palladium sample. 317

Figure 5.2.8: Elemental map of palladium. Showing decreased palladium in the spray coated areas.

Figure 5.2.9: Elemental map of nickel. Showing increased nickel in the spray coated areas. 318

Figure 5.2.10: Elemental map of oxygen. Showing increased oxygen in the spray coated areas and at surface defects. 
Jonathan Tailby

Figure 5.2.11: High magnification SEM image of a spray coated palladium sample.

Figure 5.2.12: Palladium elemental map showing decreased palladium only at the largest deposits.

Figure 5.2.13: Elemental map of nickel showing increased nickel only at the largest deposits.

Figure 5.2.14: Elemental map of oxygen showing the greatest increase in oxygen was at the largest deposits.

Figure 5.2.15: 6000x magnification secondary electron micrograph of templated electrodeposits on a secondary insert.

Figure 5.2.16: 1600x magnification secondary electron micrograph of templated electrodeposits on a secondary insert. 322

Figure 5.2.17: 800x magnification secondary electron micrograph of templated electrodeposits on a secondary insert. 322

Figure 5.2.18: 1200x magnification secondary electron micrograph of templated electrodeposits on a secondary insert. 322

Figure 5.2.19: 200x magnification secondary electron micrograph of an alternate templated electrodeposit on a secondary insert. 323

Figure 5.2.20: 1200x magnification secondary electron micrograph of an alternate templated electrodeposit on a secondary insert. 323

Figure 5.2.21: 1600x magnification secondary electron micrograph of an alternate templated electrodeposit on a secondary insert. 323

Figure 5.2.22: Decomposition of ammonia with an undeposited stainless steel sample in the primary insert at $400{ }^{\circ} \mathrm{C}$

Figure 5.2.23: Decomposition of ammonia in the primary insert at $400{ }^{\circ} \mathrm{C}$ with a stainless steel sample with templated nickel deposits deposited for 2 minutes at $-0.9 \mathrm{~V}$. .328 
Figure 5.2.24:TCD chromatograms of ammonia decomposition experiments with an undeposited stainless steel secondary insert.

Figure 5.2.25:Typical TCD chromatograms of ammonia decomposition with untemplated nickel deposits on a secondary insert at $300-450{ }^{\circ} \mathrm{C}$. .337

Figure 5.2.26 Representative chromatograms for ammonia decomposition experiments with templated nickel secondary inserts at $300,350,400,450{ }^{\circ} \mathrm{C} .339$ Figure 5.2.27: Typical chromatograms for ammonia decomposition with a nickel mesh catalyst

Figure 5.2.28: Chromatograms for ammonia decomposition with the nickel foam insert showing two injection cycles of 10 minutes each. Each chromatogram represents a different temperature: $\mathrm{a}-350{ }^{\circ} \mathrm{C}, \mathrm{b}-400{ }^{\circ} \mathrm{C}, \mathrm{c}-450{ }^{\circ} \mathrm{C}$. 340

Figure 5.2.29: Arrhenius plot for Ammonia Decomposition with Secondary Insert Catalysts.

Figure 5.2.30: Arrhenius plot of the Hydrogen Formation data for the Secondary Insert Catalysts.

Figure 5.2.31: Arrhenius plot of the Nitrogen Formation data for the Secondary Insert Catalysts. .345

Figure 5.2.32: Chromatograms for the retentate gas mixture after ammonia decomposition with the Nickel-before-Palladium tertiary insert. 347

Figure 5.2.33: Chromatograms for permeate gas mixture after ammonia decomposition with the Nickel-before-Palladium tertiary insert. .348

Figure 5.2.34: Chromatograms for decomposition of ammonia with the foam catalyst in contact with palladium foil (Nickel-on-Palladium-no-Permeation).

Figure 5.2.35: Chromatograms for the retentate of the catalytic spillover membrane when the rate of ammonia flow was retained at $5 \mathrm{~mL} \mathrm{~min}^{-1}$ and the temperature varied from 300 to $450{ }^{\circ} \mathrm{C}$. .350 
Jonathan Tailby

Figure 5.2.36: Chromatogram for permeate from ammonia decomposition reactions with the Nickel-on-Palladium membrane at $400{ }^{\circ} \mathrm{C}$.

Figure 5.2.37: Reproduction of a Plot of calculations by Ward and Dao that predict hydrogen flux at different temperature for three different thicknesses of palladium [112]. 353

Figure 5.2.38: Arrhenius plot of Ammonia Decomposition on Tertiary Insert Nickel Foam Catalysts. 355

Figure 5.2.39: Arrhenius plots for nitrogen formation on the tertiary insert nickel foam catalysts. 356

Figure 5.2.40: Arrhenius plots for retentate hydrogen formation on the tertiary insert nickel foam catalysts. 358

Figure 5.2.41: Arrhenius plots for Permeate hydrogen formation on the tertiary insert nickel foam catalysts. 359

Figure 5.2.42: Arrhenius plots for Total hydrogen formation on the tertiary insert nickel foam catalysts. .360 Figure 5.2.43: Chromatograms for decomposition of ammonia with the foam catalyst in contact with palladium foil (Nickel-on-Palladium-no-Permeation), ammonia flow from 1 to $5 \mathrm{~mL} \cdot \mathrm{min}^{-1}$. 361 


\section{List of Tables}

Table 1.2.1: Sample literature conditions for ammonia decomposition...............44

Table 1.2.2: Transition temperature for permeation limit. .................................55

Table 1.2.3: Permeance at different temperatures for $40 \mu \mathrm{m}$ thick palladium .... 57

Table 1.2.4: Four stages of electrodeposition of nanowires in a template............63

Table 1.2.5: Reaction schemes for dehydrogenation with permeation................. 73

Table 1.2.6: Different steps in multistep models of hydrogen permeation.......... 78

Table 2.1.1: Minimum voltage for relevant electrochemical phenomena ............88

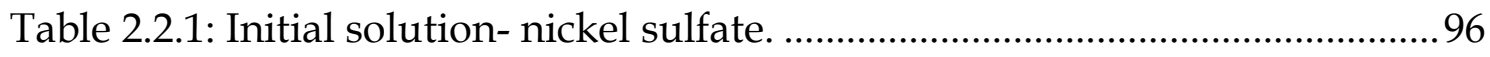

Table 2.2.2: Second electroplating solution, nickel nitrate. ...............................97

Table 2.2.3: Constants for calculating thickness of nickel on palladium............100

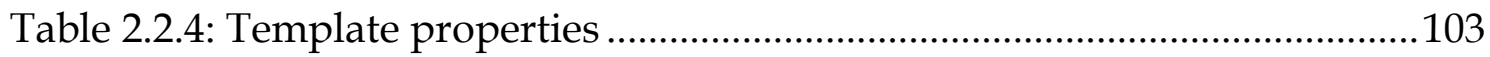

Table 2.2.5: Average mass of catalyst in the secondary insert reaction tests.....125

Table 2.2.6: Average mass of catalyst in the Tertiary insert reaction tests.........125

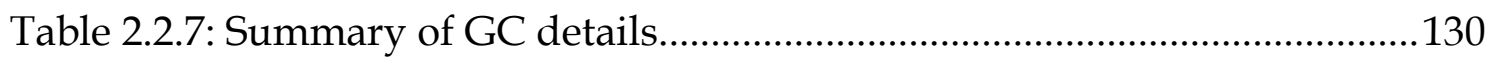

Table 2.2.8: Key variables in the molar flow equations. ....................................132

Table 2.2.9: Assumed relationships in the gas reactor....................................... 132

Table 2.2.10: Key variables in the molar flow equations for permeate. .............137

Table 2.2.11: Maximum hydrogen content of the palladium............................139

Table 2.2.12: Time taken to fill the palladium membranes with hydrogen.......139

Table 3.1.1: Quantitative analysis of 200x SEM of nickel electrodeposited on

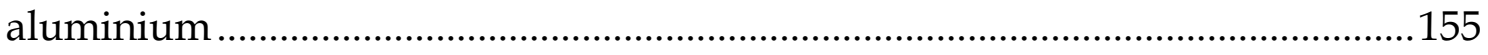

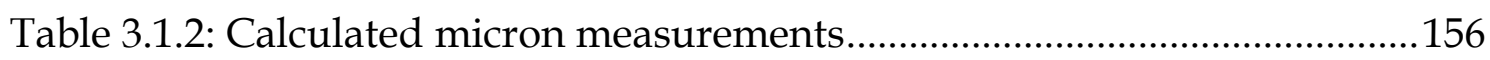

Table 3.1.3: SEM image analysis for concentration and voltage series. .............156

Table 3.1.4: Semiquantitative analysis of point EDS shown in Figure 3.1.18 ....164

Table 3.1.5: Nickel layer thickness calculated from mass data..........................168

Hydrogen From Ammonia By Catalytic Spillover Membrane 23 
Jonathan Tailby

Table 3.1.6: Nickel layer thickness calculated from electrodeposition data................ 168

Table 3.1.7: Quantitative analysis for eds of cross section. ...............................173

Table 3.1.8: Quantification of the elements present in the nickel mesh. ............200

Table 3.2.1: Calculating Data for Arrhenius plots of Ammonia Decomposition

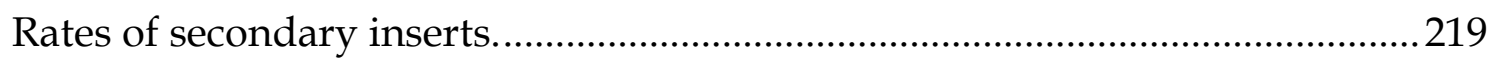

Table 3.2.2: Final calculation of Activation Energy for Secondary Insert Catalysts from Rate of Ammonia decomposition. ........................................................... 220

Table 3.2.3: Calculating Data for Arrhenius plots of Hydrogen Formation Rates

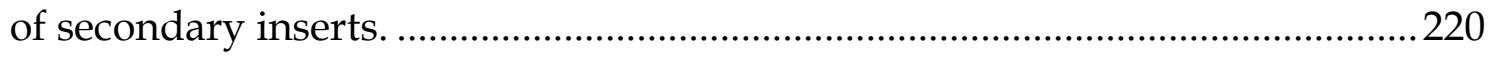

Table 3.2.4: Calculating the Activation Energy for Hydrogen Formation on the

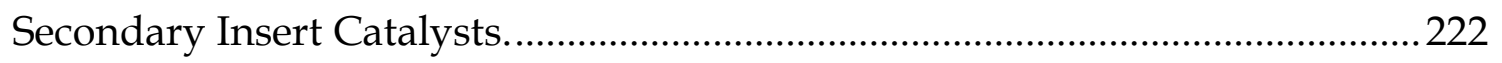

Table 3.2.5: Summary of Activation Energy calculated for each secondary insert from the rate data for the three different species. ............................................. 222

Table 3.2.6: Specific Rate of Reaction for the secondary insert Nickel catalysts

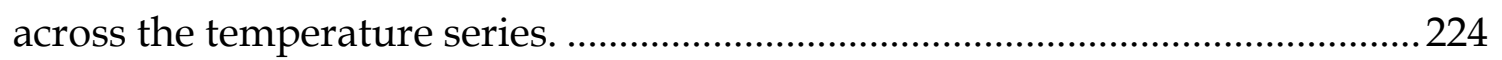

Table 3.3.1: Hydrogen permeate peak areas and calculated values for temperature series on the Nickel-before-Palladium tertiary insert. ................... 229

Table 3.3.2: Calculating ammonia output of the Nickel-before-Palladium tertiary

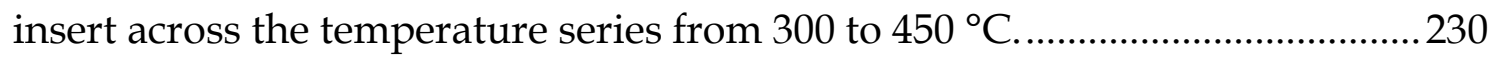

Table 3.3.3: Average peak areas and subsequent calculated values for retentate from ammonia decomposition at 300 to $450{ }^{\circ} \mathrm{C}$ for Nickel-before-Palladium

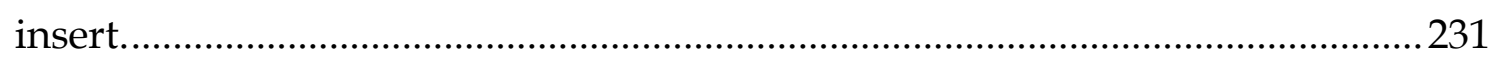

Table 3.3.4 GC Peak measurements and derived calculations for the gas output vs. temperature series of ammonia decomposition experiments on the Nickel-onPalladium-no-Permeation tertiary insert......................................................... 233

Table 3.3.5: Peak area summary with calculated values for ammonia decomposition at $300-450{ }^{\circ} \mathrm{C}$ on the Nickel-on-Palladium tertiary insert....... 237 
Table 3.3.6: Permeate and Flux calculations with full and reduced membrane length.

Table 3.3.7: Summary of Activation Energy calculated for each tertiary insert from the rate data for each different species. Secondary nickel foam is included for comparison. 250

Table 5.1.1: Background data for the calculations. 272

Table 5.1.2: Test calculations for Equation 2.2.5 ...............................................273

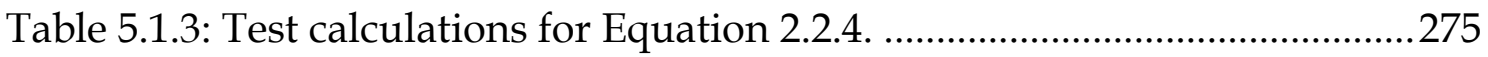

Table 5.1.4: Test calculations for Equation 2.2.6. ................................................2. 277

Table 5.1.5: Additional background for tertiary insert calculations. 279

Table 5.1.6: Test Calculations for Equation 2.2.7, calculating permeation from percentage hydrogen in permeate. 282

Table 5.1.7: Test calculations for Equation 2.2.10, calculating retentate hydrogen flow from percentage hydrogen in retentate.

Table 5.1.8: Test calculations for Equation 2.2.9, calculating retentate nitrogen flow from percentage nitrogen in retentate. 286

Table 5.1.9: Test calculations for Equation 2.2.11, calculating retentate ammonia flow from percentage ammonia in retentate. 288

Table 5.2.1: Complete list of electrodeposition samples. 292

Table 5.2.2: Complete list of spray coat mass data. 309

Table 5.2.3: Quantitative analysis of image data from 1000x magnification SEM of nickel electrodeposited on aluminium to calculate mean deposit size and total coverage. .312

Table 5.2.4: Peak Areas recorded by GC after decomposition experiments with four catalysts at six temperatures in the Explorer Reactor 324

Table 5.2.5: Percentages in reactor output calculated from the peak areas in Table 5.2.4 and the peak areas of standards. .325 
Jonathan Tailby

Table 5.2.6: Molar output calculated from the percentages using equations developed in Section 2.2.12.3. Calculated from the percentages in Table 5.2.5 and

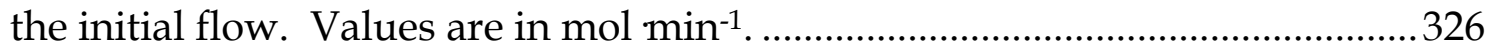

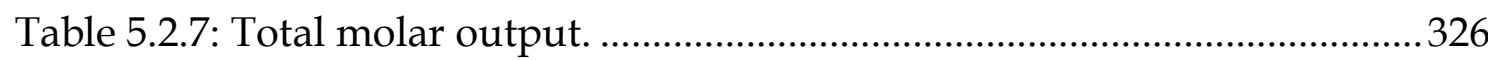

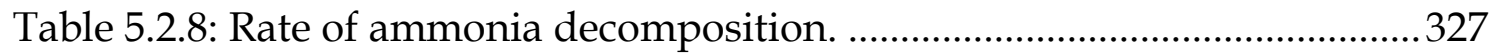

Table 5.2.9: Peak areas recorded by GC after decomposition experiments with three catalysts at 350, 400, 450 and $500{ }^{\circ} \mathrm{C}$ in the Explorer Reactor with the

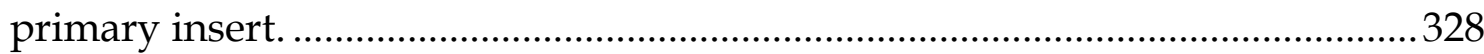

Table 5.2.10: Percentage of gases in output for catalysts in the Explorer reactor.

Table 5.2.11: Molar output of each species for catalysts in the Explorer reactor.

Table 5.2.12: Total gas output of the reaction calculated as the sum of the outputs of all gas species from Table 5.2.11 330

Table 5.2.13: Rate of ammonia decomposition. .330

Table 5.2.14: Measured peak areas for the output gases of the ammonia decomposition with secondary insert catalysts at $300-450{ }^{\circ} \mathrm{C}$. .333

Table 5.2.15: Percentages for the gas output of the decomposition reactions with secondary insert catalysts. 334

Table 5.2.16: Molar ouput of each gas from the decomposition reaction with secondary insert catalysts. .335

Table 5.2.17: Total output of the gas reaction calculated as the sum of the molar output of the three gases from Table 5.2.16. Values in $\mathrm{mol} \mathrm{min}^{-1}$ .336

Table 5.2.18: Calculating Data for Arrhenius plots of Ammonia Rates of secondary inserts. 341

Table 5.2.19: Final calculation of Activation Energy for Secondary Insert Catalysts from Rate of Ammonia decomposition. 
Table 5.2.20: Calculating Data for Arrhenius plots of Hydrogen Formation Rates of secondary inserts.

Table 5.2.21: Calculating the Activation Energy for the Hydrogen Formation on the Secondary Insert Catalysts.

Table 5.2.22: Calculating Data for Arrhenius plots of Nitrogen Formation Rates of secondary inserts

Table 5.2.23: Calculating the Activation Energy for the Nitrogen Formation on the Secondary Insert Catalysts. .346

Table 5.2.24: Flux calculations for the Nickel-on-Palladium Catalytic Spillover Membrane using the Richardson's Equation. 352

Table 5.2.25: Calculating Data for Arrhenius plots of Ammonia decomposition on Tertiary Insert Nickel Foam Catalysts. 354

Table 5.2.26: Calculation of Activation Energy from Arrhenius Plot of Ammonia Decomposition on Tertiary Insert Nickel Foam Catalysts .355

Table 5.2.27: Calculating the data for Arrhenius plots of the rates of nitrogen formation on Tertiary inserts nickel foam catalysts. .355

Table 5.2.28: Calculating the activation energy from the Arrhenius plots for nitrogen formation on tertiary insert nickel foam catalysts. 356

Table 5.2.29: Calculating Data for Arrhenius plots of Retentate Hydrogen formation on Tertiary Insert Nickel Foam Catalysts.

Table 5.2.30: Calculating the activation energy from the Arrhenius plots for retentate hydrogen formation on tertiary insert nickel foam catalysts. 358

Table 5.2.31: Calculating Data for Arrhenius plots of Permeate Hydrogen formation on Tertiary Insert Nickel Foam Catalysts. .358

Table 5.2.32: Calculating the activation energy from the Arrhenius plots for Permeate hydrogen formation on tertiary insert nickel foam catalysts. .359

Table 5.2.33: Calculating Data for Arrhenius plots of Total Hydrogen formation on Tertiary Insert Nickel Foam Catalysts. 360 
Jonathan Tailby

Table 5.2.34: Calculating the activation energy from the Arrhenius plots for Total hydrogen formation on tertiary insert nickel foam catalysts. ............................360

Table 5.2.35: Permeate calculation for Tertiary Insert Flow Series at $350{ }^{\circ} \mathrm{C}$ with

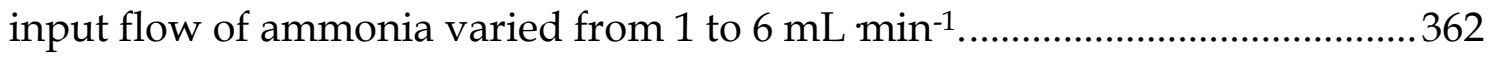




\section{List of Equations}

Equation 1.2.1: Lithium hydride reacted with ammonia ...................................39

Equation 1.2.2: Ammonia decomposition reaction .......................................... 41

Equation 1.2.3: Isotope exchange reaction with ammonia and deuterium. ........47

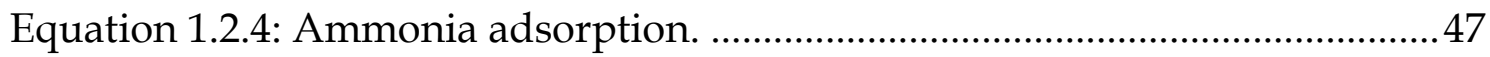

Equation 1.2.5: First dehydrogenation of ammonia.......................................... 47

Equation 1.2.6: Complete dehydrogenation of singly dehydrogenated ammonia.

Equation 1.2.7: Dissociative adsorption of deuterium...................................... 47

Equation 1.2.8: Deuteration of nitrogen dihydride. ......................................... 47

Equation 1.2.9: Dissociation of singly deuterated ammonia.............................. 47

Equation 1.2.10: Associative desorption of singly deuterated hydrogen. ...........47

Equation 1.2.11: Associative desorption of nitrogen..........................................4

Equation 1.2.12: Tamaru model for the rate of Ammonia decomposition. ..........48

Equation 1.2.13: Temken-Pyzhev model for the rate of Ammonia decomposition.

Equation 1.2.14: Rate of ammonia dehydrogenation........................................49

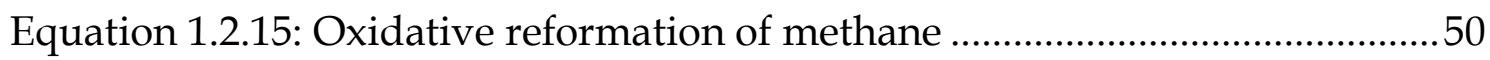

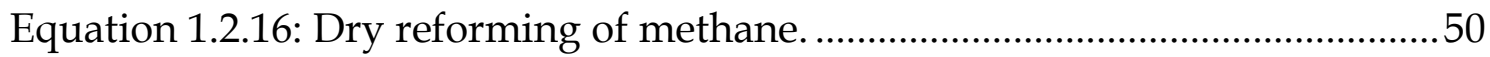

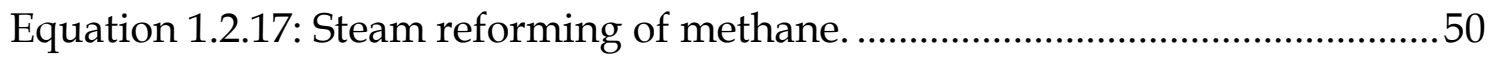

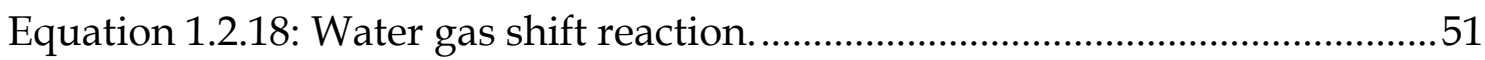

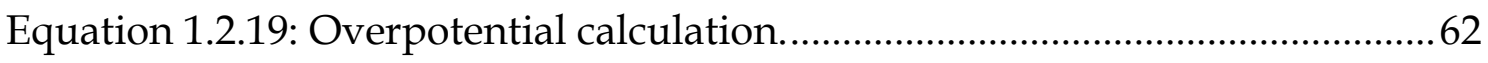

Equation 1.2.20: Trihydrogen ion formation for spillover. ................................6 69

Equation 1.2.21: Richardson's equation for predicting Hydrogen flux. ..............78

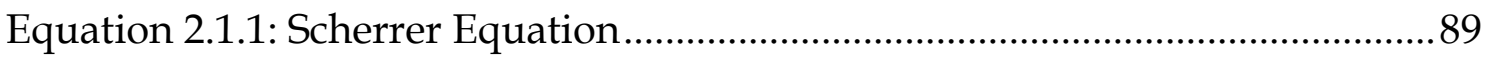


Jonathan Tailby

Equation 2.2.1: Calculating the thickness of nickel on palladium from the

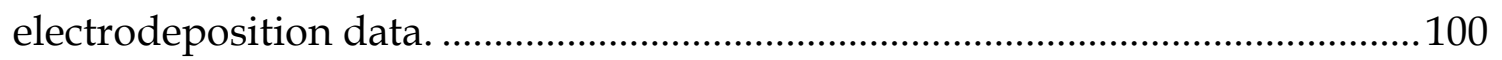

Equation 2.2.2: Chemical equation for ammonia decomposition...................... 131

Equation 2.2.3: Relationship of the final molar flow of nitrogen to the total molar flow 134

Equation 2.2.4: Derivation of an equation for $\mathrm{n}_{\mathrm{NF}}$ from known variables......... 134

Equation 2.2.5: Equation for calculating $\mathrm{n}_{\mathrm{HF}}$ from known variables................. 135

Equation 2.2.6: Deriving an equation to calculate the final flow of ammonia.. 135 Equation 2.2.7: Deriving an Equation to Calculate $\mathrm{n}_{\text {HPerm }}$ in the Permeate........137 Equation 2.2.8: New Expression for Final Total Gas Flow, which now Incorporates a Term for Hydrogen Permeation. 140

Equation 2.2.9: Derivation for the calculation for the total flow of nitrogen in the retentate. 140

Equation 2.2.10: Deriving the Equation to Calculate the Final Molar Flow of Hydrogen. 141

Equation 2.2.11: Calculating the Final Molar Flow of Ammonia in the Retentate 142

Equation 2.2.12: Arrhenius Equation 143

Equation 2.2.13: Alternate form of Arrhenius Equation. 143

Equation 2.2.14: Specific rate of reaction 144 


\section{Definition of Terms}

\begin{tabular}{|l|l|}
\hline Substrate & $\begin{array}{l}\text { In the formation of catalysts, substrate is used to refer to the metal } \\
\text { that the catalyst is deposited on. This definition has been } \\
\text { continued throughout this document, into the sections on gas } \\
\text { reaction. }\end{array}$ \\
\hline Reactant & $\begin{array}{l}\text { The reactant is the starting material of a chemical reaction. In a } \\
\text { catalytic reaction, the term substrate is sometimes used, however } \\
\text { that term is being used to refer to the metal supporting the } \\
\text { catalyst in this work so the term reactant is used for the ammonia } \\
\text { in the gas reactions. }\end{array}$ \\
\hline Reformer & $\begin{array}{l}\text { A reaction vessel where a gas is reformed, particularly one where } \\
\text { a hydrogen containing gas is reacted to release hydrogen. }\end{array}$ \\
\hline
\end{tabular}

\section{List of Abbreviations}

\begin{tabular}{|l|l|}
\hline GC & Gas Chromatograph \\
\hline TCD & Thermal Conductivity Detector \\
\hline EPC & $\begin{array}{l}\text { Electronic Pressure Controller, controls rate of gas flow } \\
\text { through the GC. }\end{array}$ \\
\hline MS & Mass Spectrometer \\
\hline FID & Flame Ionisation Detector \\
\hline SS & Stainless Steel \\
\hline PAA & Porous Anodic Alumina \\
\hline PEM & Polymer Electrolyte Membrane \\
\hline PEM-FC & Polymer Electrolyte Membrane Fuel Cell \\
\hline MSE & $\begin{array}{l}\text { Mercurous Sulfate Reference Electrode a reference } \\
\text { electrode used in some references. }\end{array}$ \\
\hline SCE & $\begin{array}{l}\text { Saturated Calomel Electrode, another reference } \\
\text { electrode used in some references. }\end{array}$ \\
\hline SEM & Scanning Electron Microscope \\
\hline XRD & X-Ray Diffraction \\
\hline
\end{tabular}


Jonathan Tailby

\begin{tabular}{|l|l|}
\hline EDS & Electron Dispersive X-Ray Spectroscopy \\
\hline OM & Optical Microscopy \\
\hline WHSV & $\begin{array}{r}\text { Weight Hourly Space Velocity. } \\
\text { Mass flow of Reactant/Mass of Catalyst }\end{array}$ \\
\hline
\end{tabular}




\section{Introduction}

\subsection{Background:}

One of the challenges in the development of a hydrogen economy is the storage density of hydrogen. An approach to this problem is to store another fuel source that can be converted to hydrogen as needed. Several potential hydrogen carriers such as methane [1, 2], methanol [3, 4], natural gas [5], gasoline [6], diesel fuel[7], aviation jet fuel[7], ethanol[8, 9], and ammonia [10-12] are being explored by different researchers worldwide. The storage and recovery of hydrogen in other fuels is discussed in sections 1.2.1 to 1.2.3 Hydrogen may be used directly as a combusted fuel (with the liberation of both energy and heat) or to supply a fuel cell, with higher efficiency of energy conversion to electricity and with enhanced capacity to use waste heat $[13,14]$. The literature on fuel cells is briefly discussed in section 1.2.5.1.

The gas mixture which results from decomposition of these fuels is not immediately suitable for use in fuel cells. The mixture usually contains byproducts and unreacted gas that can damage the fuel cell components. Hydrogen from organic or petrochemical sources usually contains carbon monoxide and carbon dioxide which damage the precious metal electrodes of Polymer Electrolyte Membrane (PEM) fuel cells [15]. Unreacted ammonia in the hydrogen mixture degrades fuel cell components, even as little as 13 ppm ammonia [11]. One method to purify hydrogen gas is to use a palladium metal filter. Hydrogen gas molecules can dissociate to form atomic hydrogen at the surface of a palladium membrane and these hydrogen atoms diffuse into the metal, the literature of this phenomenon is discussed in section 1.2.7. Hydrogen atoms that diffuse through the palladium will reform as hydrogen gas molecules at the exit face of the membrane. A thin foil of palladium can therefore act as a hydrogen filter with very high selectivity. The problem with large scale use of palladium as a hydrogen filter is the cost and availability of the metal. Research aimed at reducing the amount of palladium required has led to fabrication of thin films supported on porous supports such as stainless steel or alumina. Porous anodic 
alumina has been reportedly used to create palladium foils only 200-300 nm thick which are able to filter hydrogen from hydrogen, nitrogen, carbon dioxide gas mixtures [16].

Not only can palladium membranes be used to purify the hydrogen produced in a gas reactor, they can also be used inside the reactor to increase the amount of hydrogen produced. If the reaction to produce hydrogen is carried out in such a palladium membrane reactor, the continuous removal of hydrogen through the membrane will allow the overall reaction to exceed the equilibrium quantity of hydrogen production [17]. The literature on the use of palladium to make membrane reactors is discussed in section 1.2.4.

The decomposition of a hydrogen carrier is significantly enhanced by the use of a catalyst. Ammonia decomposes with high temperature but the most effective single metal catalyst for the decomposition of ammonia is ruthenium [18-20]. A range of metals can be used, some rare and expensive such as platinum, palladium and iridium [21], some cheap and plentiful such as iron and nickel [19, 22-24].

Direct contact between such a catalyst and a palladium membrane is predicted to increase the rate of hydrogen production and membrane flux by hydrogen 'spillover'[25]. Spillover is diffusion of a species from a surface where that species is formed or easily adsorbed onto another surface the species could not reach directly $[26,27]$, spillover is further discussed in Section 1.2.6. Spillover of hydrogen atoms formed by ammonia decomposition on the catalyst onto the adjacent palladium surface is predicted to increase the rate of both the decomposition reaction and of hydrogen transport through the palladium membrane. Nickel metal is known to be the second most effective single metal catalyst for ammonia decomposition [19] and to be active for hydrogen spillover [28]. Ruthenium is more effective for catalysing ammonia decomposition; however, it is significantly more expensive and available only in smaller quantities. There is less literature evidence of hydrogen spillover with ruthenium than there is with nickel. This research aimed to ensure direct contact 
between the nickel catalyst and the palladium membrane by electrodeposition of the nickel metal catalyst onto the palladium. Electrodeposition literature is discussed in section 1.2.5.2.

The goal of this research is to extend the possibilities for hydrogen storage by combination of catalysis, spillover and filtration with hydrogen transport through a palladium membrane to accelerate the decomposition rate of ammonia gas to high purity hydrogen. Figure 1.1.1 shows the broad outline of this research plan, further details are contained in section 1.3.

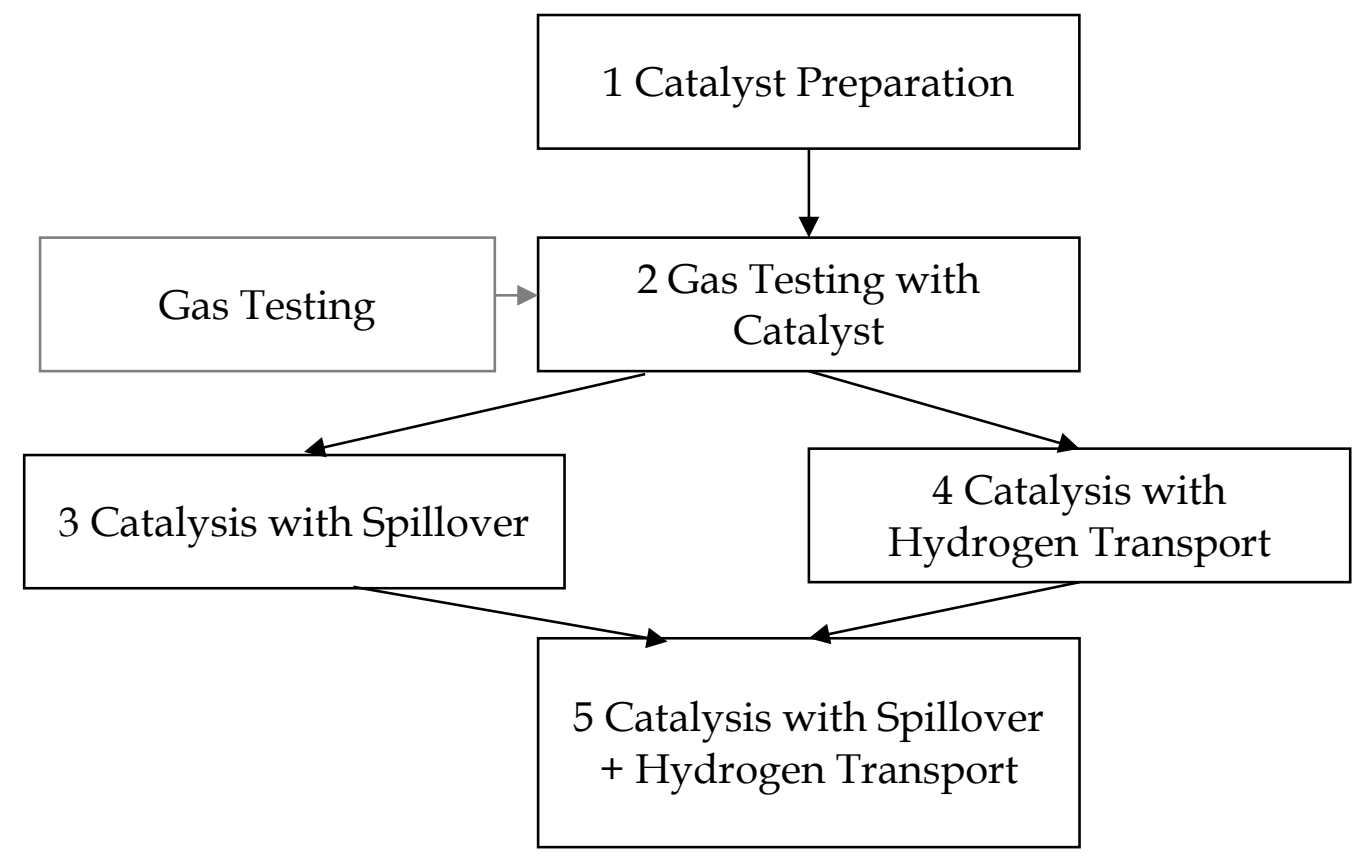

Figure 1.1.1: Research plan.

The first step was to experiment with methods to form a nickel catalyst on a palladium surface, and these methods are described in Sections 2.1.1, 2.1.2, 2.2.2, 2.2.3, 2.2.4 and 2.2.5, with the results given in Section 3.1. The first method explored was electrodepositing nickel from solution, but the nickel formed continuous layers on the palladium surface which were not anticipated to be effective catalysts for ammonia decomposition, therefore a new method was developed. In the second method nickel nanoparticles were spray coated onto the surface. This method produced inconsistent results; therefore, it was abandoned. The third method revisited electrodeposition but with the addition of photolithography to create a template that would shape the electrodeposits 
Jonathan Tailby

into a microscale pattern that was anticipated to be a more effective catalyst than the continuous layers. The products of these formation methods were analysed by multiple analysis methods, including optical microscopy as described in Sections 2.1.3 and 2.2.6; X-ray diffraction, described in Sections 2.1.4 and 2.2.7; and Scanning Electron Microscopy, Sections 2.1.5 and 2.2.8.

The prospective catalysts were tested for effectiveness in ammonia decomposition reactions. The process of gas testing required several redesigns of the gas reactor, as described in Section 2.2.9. The product of the ammonia decomposition was initially analysed with a mass spectrometer, as described in Sections 2.1.6 and 2.2.11, however, this was damaged by the ammonia and was replaced with a gas chromatograph, as described in Sections 2.1.7 and 2.2.12.

The gas reactor was modified, as described in Section 2.2.9.5 to enable ammonia decomposition to be combined with the two processes requiring palladium: spillover and hydrogen permeation. The ammonia decomposition reaction was independently combined with first hydrogen permeation then hydrogen spillover enabling the contribution of each process to the spillover membrane to be resolved. The results for catalysis with hydrogen permeation are reported in Section 3.3.1 and the results for catalysis with spillover are reported in Section 3.3.2. The results for catalysis with hydrogen spillover and permeation are reported in Section 3.3.3 and are compared to all the previous ammonia decomposition results. 


\subsection{Literature Review}

\subsubsection{Gas Reformation}

\subsubsection{Hydrogen as an Energy Carrier}

Hydrogen is seen as a favoured energy carrier because its only combustion product is water, therefore, it is considered environmentally benign. When this combustion is carried out in a fuel cell with recovered heat, energy efficiency can exceed $90 \%$. Because of its low mass, hydrogen has a very high energy density per unit mass $(143 \mathrm{MJ} / \mathrm{kg})$, but because of hydrogen's low storage density it has a low energy density per unit volume [29].

The United States Department of Energy benchmark for on-board hydrogen storage for motor vehicles was 9 wt.\% and storage density of $0.040 \mathrm{~kg} \mathrm{H}_{2} / \mathrm{L}$ hydrogen by $2017[29,30]$. This has since been updated to $6 \mathrm{wt}$ \% for chemical hydrides by 2020 [31]. Compression of hydrogen gas does not achieve this storage density even with high pressure storage vessels of 350-700 bar [7]. Storage as liquid hydrogen is complex and expensive, requiring bulky vessels and using $40 \%$ of the hydrogen energy value for liquefaction [7].

Hydrogen gas has a very low natural abundance, which means it needs to be manufactured for use on an industrial scale. Currently most hydrogen gas is produced by steam reformation of methane [32]. Other fossil fuel processes include thermal cracking of natural gas, partial oxidation of fuels and coal gasification [32]. Water is another source of hydrogen, but none of the conversion methods is yet considered as economic as fossil fuel sources because of the large energy input required. Processes for producing hydrogen gas from water include electrolysis, photolysis, thermolysis, thermochemical hydrolysis [32] and reformation of biogas [33].

Electrolysis of water is becoming more prevalent in areas where electrical energy is cheap and plentiful such as near nuclear power plants or large sustainable energy installations [33]. Electrolysis driven by electricity or heat from nuclear power plants produces no carbon based waste but creates new problems from 
Jonathan Tailby

the management of radioactive waste products [34]. Research into photocatalytic and photoelectric hydrogen production from water is beginning to produce results [35-39].

\subsubsection{Materials for Hydrogen Storage}

Several methods of hydrogen storage in solid materials have been developed that are very safe, but have low mass energy density $[7,40]$. The hydrogen can be adsorbed onto high surface area materials such as nanotubes, graphene or metalorganic frameworks [41]. The hydrogen can be absorbed into hydride materials, either as metal hydrides or as complex inorganic hydrides including amides and borohydrides [41].

Adsorption storage of hydrogen usually involves storage of hydrogen molecules on high surface area, low mass materials [42]. To achieve high specific surface area, the materials are often nanostructured, such as carbon nanotubes, graphene, fullerenes or zeolites [43]. Metal Organic Frameworks (MOFs) are nanostructured materials with metal ion vertices connected by organic linker molecules whose nature and dimensions control the size of nanostructured cages within which the hydrogen or other gas molecules are bound [44].

Metallic hydrides store hydrogen by dissociation of dihydrogen molecules at their surface and absorption of hydrogen atoms into the metal lattice [42]. Palladium is one of the most effective metals for hydrogen absorption and is discussed further in Section 1.2.7. The metals and alloys that are most effective in hydride formation are transition metals, so their high atomic mass reduces the mass density of hydrogen storage [45]. A lighter alternative is $\mathrm{MgH}_{2}$ which can store $7.7 \mathrm{wt} \% \mathrm{H}$ with good reversibility at $0.1 \mathrm{MPa}$ and $300{ }^{\circ} \mathrm{C}$ [43].

Complex hydrides store hydrogen by covalent bonding inside a metal-containing anion. Suitable elements for complex hydrides include boron and light metals such as aluminium, which can achieve higher mass density than is possible with the heavier transition metals that are most effective in metallic hydrides for hydrogen storage [43]. 


\subsubsection{Hydrogen Recovery Processes}

The two main processes for recovery of stored hydrogen are thermolysis and hydrolysis. Thermolysis is extraction of the hydrogen by temperature change, usually heating the material above its storage temperature. Hydrolysis is reaction with water to release hydrogen. Hydrolysis can usually occur closer to ambient temperature than thermolysis, but the products are more difficult to reform into the starting materials for reuse than other hydrogen releasing reactions $[43,46]$. One factor that makes hydrolytic reactions less reversible is that the by-product often forms a slurry which does not readily react with hydrogen gas to reform the starting material to be reused for hydrogen storage.

General considerations for thermolysis include how kinetic and thermodynamic factors affect the properties and working conditions of the material. Thermodynamic properties determine the working temperature and pressure for the material [42]. At a given temperature the material will have an equilibrium hydrogen partial pressure and will absorb or desorb hydrogen to bring the pressure towards equilibrium [42].

Properties of the materials can be tuned by combining different materials[43, 47]. $\mathrm{MgH}_{2}$ has good storage characteristics, but poor desorption pressure. $\mathrm{LiBH}_{4}$ has high hydrogen mass percentage, but is too stable for practical use in thermolytic decomposition. When combined, the decomposition reaction produces $\mathrm{MgB}_{2}$ which destabilises $\mathrm{LiBH}_{4}$, reducing decomposition enthalpy by $25 \mathrm{~kJ} \mathrm{~mol}^{-1} \mathrm{H}_{2}$, which improves the hydrogen output [43]. An alternate explanation for this same phenomenon is that the combination of the $\mathrm{MgH}_{2}$ with the $\mathrm{LiBH}_{4}$ causes decomposition of the $\mathrm{MgH}_{2}$ and it is the two metals form a MgLi alloy before the formation of $\mathrm{MgB}_{2}$. XRD results showed that rehydrogenation reformed the original compounds [48].

An alternative to hydrolysis that may be promising in some areas is to use ammonia as a reactant. Yamamoto has reported experiments with alkali metal hydrides[49]. Lithium hydride reacts according to equation Equation 1.2.1.

Equation 1.2.1: Lithium hydride reacted with ammonia 
$\mathrm{LiH}+\mathrm{NH}_{3} \rightarrow \mathrm{LiNH}_{2}+\mathrm{H}_{2}$

$8.1 \mathrm{wt} \% \mathrm{H}_{2}$ in $\mathrm{LiH}, \Delta \mathrm{H}=-50 \pm 9 \mathrm{kH} \cdot \mathrm{mol}_{\mathrm{H}_{2}}^{-1}$

This reaction is exothermic and can proceed at room temperature. The reverse process can proceed to $>70 \%$ completion at $0.5 \mathrm{MPa}$ hydrogen and $300{ }^{\circ} \mathrm{C}[49]$.

Another approach is to bypass hydrogen storage by in situ hydrogen generation from a fuel such as methane or ammonia $[34,50]$. The advantages of ammonia are the absence of carbon at the point of use, the already developed production and storage infrastructure and the low cost per unit of stored energy [51]. There remains the weakness of a lack of a direct source of ammonia and the need to develop efficient on-board conversion to hydrogen [52]. This research addresses catalytic membrane design in order to improve the rate and purity of ammonia decomposition.

\subsubsection{Ammonia as a Hydrogen Carrier}

Ammonia can be used as a hydrogen carrier because it is more readily stored and transported than pure hydrogen. It liquefies at room temperature under 8 atmospheres [53]. Ammonia can be stored in inorganic solids, which give very high volume density $[50,54]$. When combined with in situ dehydrogenation, this makes ammonia an effective energy carrier. It has a high hydrogen storage capacity (17.7 wt.\%) and energy density (3000 $\left.\mathrm{W} \cdot \mathrm{h} \cdot \mathrm{kg}^{-1}\right)$ [53]. For in situ hydrogen generation ammonia is more economic than methanol [53].

Ammonia is produced synthetically from hydrogen. Industrially, the HaberBosch process reacts hydrogen gas with atmospheric nitrogen at high temperature and pressure. A porous iron catalyst is made by reduction of magnetite [55]. Industrial conditions involve temperatures of 300-500 ${ }^{\circ} \mathrm{C}$ and pressures of 10-20 MPa [55]. Alternative catalysts have been prepared using carbon substrates with barium or potassium promoter such as $\mathrm{Ni} / \mathrm{C}, \mathrm{Ru} / \mathrm{C}$, $\mathrm{Co} / \mathrm{C}$ and $\mathrm{Fe} / \mathrm{C}$ [56]. The main difficulty in production of ammonia from nitrogen gas is the activation of the strong nitrogen-nitrogen triple bond. A recent publication by Pfromm argues that the state of the art Haber-Bosch process has approached its optimum energy efficiency, with the greatest cost locked into the provision of the feedstock hydrogen and energy [57]. 
Alternative synthesis routes are being investigated with the intent of lowering the energy requirements of the process or removing fossil fuels from the supply chain. For example Lan et al. claim to have an electrochemical synthesis method for ammonia directly from air and water under mild conditions [58]. Kuriyama has reported chemical synthesis of ammonia under mild conditions using an iron complex catalyst [59]. Small quantities of ammonia can also be produced cheaply from biological waste streams $[51,60,61]$.

To be effective as a hydrogen carrier, ammonia must be decomposed efficiently. Thermal dissociation is reported not to take place below $400^{\circ} \mathrm{C}$, but to be almost complete at $700-800{ }^{\circ} \mathrm{C}$ and $100 \mathrm{kPa}$ [55]. Equation 1.2.2 shows the equilibrium for ammonia decomposition. Figure 1.2.1 models the equilibrium proportions of ammonia, nitrogen and hydrogen at different temperatures and 1 bar pressure from a starting point of 10 moles of ammonia, as calculated by the computer database HSC Chemistry [62]. This shows that at $300{ }^{\circ} \mathrm{C}$ the decomposition reaction is very near completion and by $450{ }^{\circ} \mathrm{C}$ the equilibrium entirely favours the dissociation products. The barrier to the reaction above $300^{\circ} \mathrm{C}$ must therefore be overwhelmingly kinetic. Hydrogen spillover has been proposed as a kinetic enhancement, which facilitates removal of hydrogen from the active sites on the catalyst by lowering energy barriers [63].

\section{Equation 1.2.2: Ammonia decomposition reaction} $2 \mathrm{NH}_{3} \underset{\leftarrow}{\stackrel{\mathrm{N}}{2}}+3 \mathrm{H}_{2}$

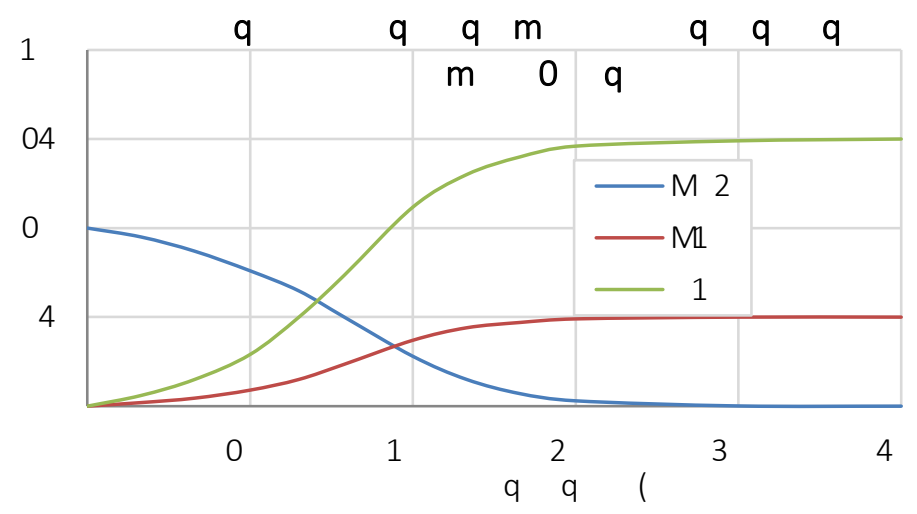

Figure 1.2.1: Equilibrium proportions for ammonia decomposition at 1 bar. From HSC Calculation for Ammonia [62] 


\subsubsection{Catalysts for Ammonia Decomposition}

Ruthenium is one of the most popular catalysts for the ammonia decomposition reaction, although metals such as Fe [64], Co [65], Ni , Ir, Pt [66], Pd, Cu, Rh, Ru and their alloys and compounds have been tested $[19,67]$. Ru shows the highest activity, with an optimal load of 15 wt.\% catalyst $[18,19]$. The catalyst's support affects the activity of the catalyst and active carbon, graphite, carbon nanotubes, alumina, titanium dioxide and magnesium oxide supports have been used with $\mathrm{Ru}$. Carbon nanotubes are effective for $\mathrm{Ru}$ because as well as getting good dispersion, they are electrically conductive to the promoter [68,69]. A promoter is an additional component that enhances the effect of the catalyst, good promoters for ammonia decomposition on $\mathrm{Ru}$ are alkali or alkali earth metals such as $\mathrm{K}, \mathrm{Na}, \mathrm{Li}, \mathrm{Ba}, \mathrm{Cs}, \mathrm{Ca}$, or La because they modify the ruthenium's electronic properties $[12,70]$. Alkali metal promoters have not been shown to improve catalysis of ammonia decomposition with nickel, but transition metals, particularly Ce are effective as promoters for nickel [19]. Ru on C with Cs shows high ammonia decomposition, even below $400{ }^{\circ} \mathrm{C}$ [18]. In contrast Varisli used a mesoporous silicate support, Co catalyst, $\mathrm{KOH}$ promoter and achieved near total conversion at $700{ }^{\circ} \mathrm{C}[71]$.

Li et al. [72-74] prepared a ruthenium catalyst in a bimodal catalytic membrane reactor. The bimodal structure had macroporous a-alumina supporting a hydrogen selective silica membrane. The a-alumina was impregnated first with $\mathrm{Y}$-alumina, second with $\mathrm{Ru}$ catalyst. This structure prevented sintering of the catalyst which would reduce surface area and hence performance.

Simonsen et al. [67] attempted to design a new catalyst from known properties and principles such as Sabatier's principle of optimum adsorption [75]. Simonsen and Boisen used 'Volcano Plots' such as Figure 1.2.2 to propose that an Fe-Ni alloy might have similar properties to $\mathrm{Ru}$, and therefore be highly active as an ammonia decomposition catalyst. These experiments showed an iron-nickel alloy with $20-50 \%$ Ni to be more active than either iron or nickel, but still not as active as $\mathrm{Ru}$ [67]. 

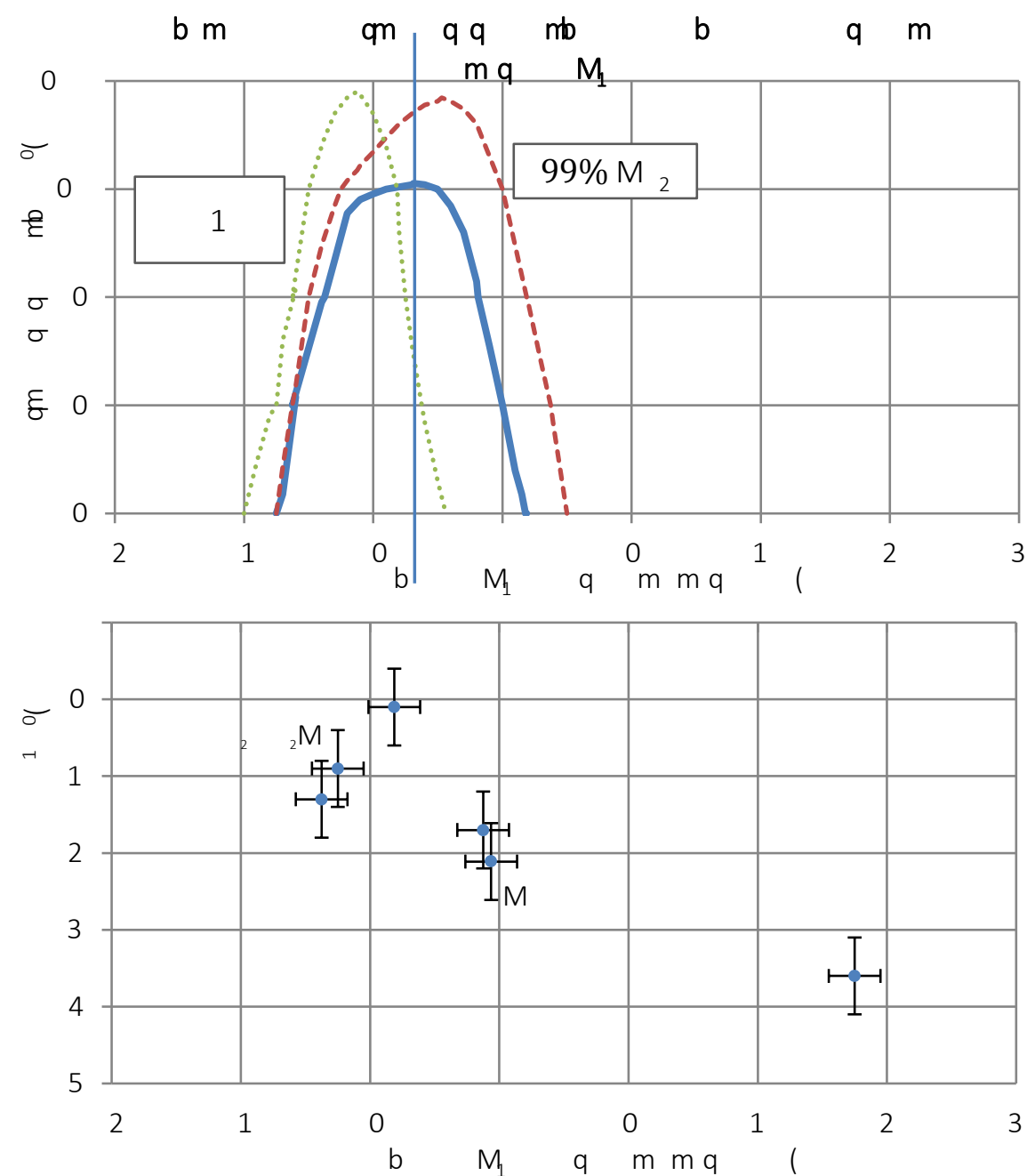

Figure 1.2.2: Volcano plot for log rate vs. dissociative $\mathrm{N}_{2}$ adsorption energy. (Top): Calculated turnover frequencies of ammonia synthesis/decomposition at $773 \mathrm{~K}, 1$ bar, 3:1 $\mathrm{H}_{2} / \mathrm{N}_{2}$ and ammonia 20\% (solid blue line), 0.02\% (dotted green line) and 99\% (dashed brown line).

(Bottom): experimental rates of ammonia decomposition, catalysts as labelled, $773 \mathrm{~K}, 1$ bar, 3:1 $\mathrm{H}_{2} / \mathrm{N}_{2}$ and ammonia $20 \%[67,76]$.

Lakhapatri's [77] investigation of steam reforming of jet fuel examined the causes of the loss of catalytic activity on a catalyst composed of non-alloyed $\mathrm{Ni}$ and $\mathrm{Rh}$ on an alumina support. Sintering of the catalyst by Ostwald ripening reduced the catalytic area and therefore activity. Build-up of $S$ and $C$ by-products was observed to poison the catalyst.

Table 1.2.1 shows catalysts commonly used in ammonia decomposition experiments. The conditions each catalyst was reported to be used in are 
Jonathan Tailby

included on the table, pressure is recorded on the table in the unit reported in by the Author and also standardised to bar to facilitate meaningful comparison between the different values. Iron catalysts similar to the Haber-Bosch process catalysts can be used with high temperature and low pressure so the equilibrium favours nitrogen and hydrogen. Other metal catalysts include: Ru [78], Ir [78], Pd [78], Pt [78], Rh [78], Ni [78], Mo [79], W [79, 80], Co[81], with substrates such as ceria, alumina, zeolites and carbon, particularly carbon nanotubes. These catalysts are commonly used with an alkali or alkaline earth metal promoter [82]. Ruthenium has been repeatedly reported as the most effective single metal for this catalysis $[11,19,20,22,23,53,68,78,79,83-85]$, especially on a carbon substrate with a potassium promoter, although methanation of the carbon substrate can be a problem at high temperature. Ruthenium is an expensive metal, and nickel is a suitable alternative with lower costs [24] and higher production [19], nickel is also reported to be active for hydrogen spillover [28, 63, 86]. The reaction pressures reported in these publications used a variety of units as shown in the column labelled $\mathrm{P}$ (as reported), they are repeated here in a second column where each pressure value has been converted to the same unit labelled P(bar).

\begin{tabular}{|c|c|c|c|c|c|}
\hline Author & Catalyst & $\mathrm{T}\left({ }^{\circ} \mathrm{C}\right)$ & $\begin{array}{l}P \text { (as } \\
\text { reported) }\end{array}$ & P (bar) & Reference \\
\hline Ohtsuka & $\mathrm{Fe}, \mathrm{Ca} / \mathrm{Coal}$ & $750-850$ & ambient & 0.98 & [23] \\
\hline Jedynak & $\mathrm{Fe} / \mathrm{C}$ & 400 & 1 bar & 1 & [22] \\
\hline Hashimoto & $\mathrm{Ru} \mathrm{CeO} 2 / \mathrm{YZ}$ & 300 & $2-4 \mathrm{kPa}$ & 0.02 & [20] \\
\hline Ganley & Metal/Alumina & 580 & $\begin{array}{l}10^{-9} \quad \times 10^{-7} \\
\text { Torr }\end{array}$ & $1.3 \times 10^{-10}$ & [84] \\
\hline Tsai & $\mathrm{Ru}$ & $500-1250$ & $\begin{array}{l}1-2 \quad \times 10^{-6} \\
\text { Torr }\end{array}$ & $1.3 \times 10^{-09}$ & [85] \\
\hline Yin 04 & $\mathrm{Ru} / \mathrm{CNT}$ & $450-550$ & Not reported & & [68] \\
\hline Papapolymerou 97 & $\mathrm{Pt}, \mathrm{Rh}, \mathrm{Pd}, \mathrm{Ir}$ & $500-1900$ & 0.01-1 Torr & 0.00133 & [21] \\
\hline
\end{tabular}




\subsubsection{Mechanism and Kinetics of Ammonia Decomposition}

Catalytic dehydrogenation of ammonia proceeds by breaking the nitrogenhydrogen bonds one by one. This is shown in the energy diagram in Figure 1.2.3.

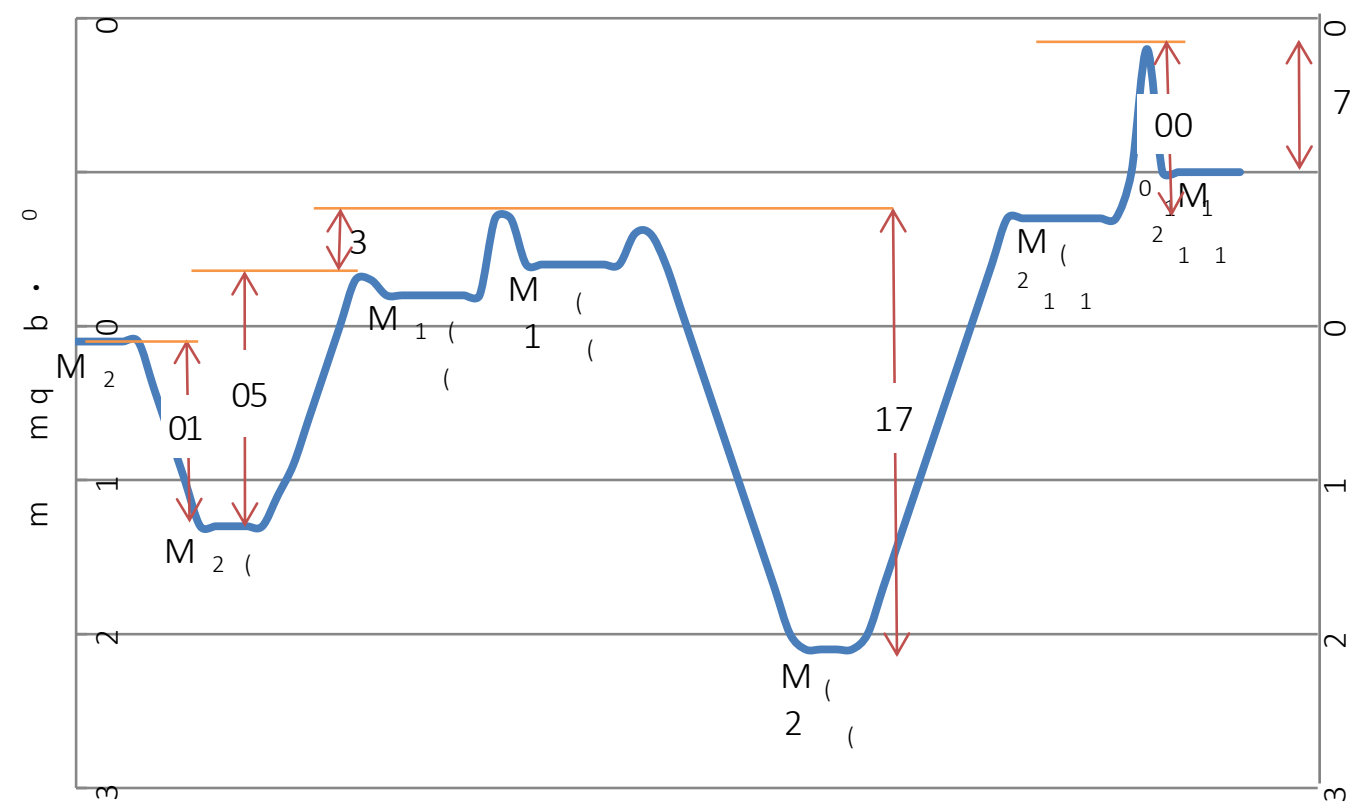

Figure 1.2.3: One-dimensional potential energy diagram illustrating from left to right the catalytic decomposition of $\mathrm{NH}_{3}$ over platinum.

Note that from right to left the diagram shows the catalytic synthesis of $\mathrm{NH}_{3}$ from $\mathrm{N}_{2}$ and $\mathrm{H}_{2}$ [87].

This proposed dissociative mechanism is supported by analysis of isotope exchange reactions of ammonia and deuterium [87]. The subscript " (a)" indicates an adsorbed species. The overall reaction is shown in 
Jonathan Tailby

Equation 1.2.3 and the individual steps that make it up are separated in Equation 1.2.4 to Equation 1.2.11. 
Equation 1.2.3: Isotope exchange reaction with ammonia and deuterium.

$\mathrm{NH}_{3}+\mathrm{D}_{2} \rightarrow \mathrm{NH}_{2} \mathrm{D}, \mathrm{NHD}_{2}, \mathrm{ND}_{3}$

The steps in this reaction are:

Equation 1.2.4: Ammonia adsorption.

$\mathrm{NH}_{3(\mathrm{~g})} \rightarrow \mathrm{NH}_{3(\mathrm{a})}$

Equation 1.2.5: First dehydrogenation of ammonia.

$\mathrm{NH}_{3(\mathrm{a})} \rightarrow \mathrm{NH}_{2(\mathrm{a})}+\mathrm{H}_{(\mathrm{a})}$

Equation 1.2.6: Complete dehydrogenation of singly dehydrogenated ammonia.

$\mathrm{NH}_{2(\mathrm{a})} \rightarrow \mathrm{N}_{(\mathrm{a})}+2 \mathrm{H}_{(\mathrm{a})}$

Equation 1.2.7: Dissociative adsorption of deuterium.

$\mathrm{D}_{2 \mathrm{~g}} \rightarrow 2 \mathrm{D}_{(\mathrm{a})}$

Equation 1.2.8: Deuteration of nitrogen dihydride.

$\mathrm{NH}_{2(\mathrm{a})}+\mathrm{D}_{(\mathrm{a})} \rightarrow \mathrm{NH}_{2} \mathrm{D}_{(\mathrm{a})}$

Equation 1.2.9: Dissociation of singly deuterated ammonia.

$\mathrm{NH}_{2} \mathrm{D}_{(\mathrm{a})} \rightarrow \mathrm{NH}_{2} \mathrm{D}_{(\mathrm{g})}$

Equation 1.2.10: Associative desorption of singly deuterated hydrogen.

$\mathrm{H}_{(\mathrm{a})}+\mathrm{D}_{(\mathrm{a})} \rightarrow \mathrm{HD}_{(\mathrm{g})}$

Equation 1.2.11: Associative desorption of nitrogen.

$2 \mathrm{~N}_{(\mathrm{a})} \rightarrow \mathrm{N}_{2}$ (g)

The diatomic gases are reported to form and desorb in one step. Even at $1000 \mathrm{~K}$ less than $0.1 \%$ of adsorbed ammonia reacts, more than $99.9 \%$ desorbs intact [87].

With ruthenium, the preferred catalyst for the ammonia decomposition reaction, the rate determining step of catalytic decomposition depends on reaction conditions. At low pressure or high temperature, cleavage of the first N-H bond is rate determining, and the subsequent cleavage of the remaining hydrogen bonds follows quickly afterwards [88][85]. At high pressure or low temperature, recombinative desorption of nitrogen is rate determining $[53,69,89]$. For $\mathrm{Ru} / \mathrm{C}$ at low pressure, the rate determining step changes at $650-750 \mathrm{~K}[53,79]$.

The rate of reaction is modelled in two different ways depending on the conditions. At high temperature or low hydrogen partial pressure, rate is 
Jonathan Tailby

dependent on ammonia partial pressure according to the Tamaru model, modelled with Equation 1.2.12[89]:

Equation 1.2.12: Tamaru model for the rate of Ammonia decomposition.

$\gamma=\frac{k \cdot K \cdot P_{\mathrm{NH}_{3}}}{1+K \cdot P_{\mathrm{NH}_{3}}}$

At low temperature or high hydrogen partial pressure, hydrogen inhibits the reaction, which was modelled by Temkin-Pyzhev with Equation 1.2.13 [89]:

Equation 1.2.13: Temken-Pyzhev model for the rate of Ammonia decomposition.

$\gamma=k\left(\frac{P_{N H_{3}}^{2}}{P_{H_{2}}^{3}}\right)^{\alpha}$

Where $\mathrm{\gamma}=$ rate of reaction $\left(\mathrm{mol} \mathrm{s} \mathrm{s}^{-1} \mathrm{~m}^{-3}\right), \mathrm{P}=$ partial pressure, $\mathrm{k}=$ rate parameter $\left(\mathrm{mol} \mathrm{m} \mathrm{m}^{-3} \mathrm{~s}^{-1}\right), \mathrm{K}=$ equilibrium constant for ammonia decomposition, $\mathrm{a}=$ constant Transition between the two models depends on both hydrogen partial pressure and temperature. For $\mathrm{Ni}-\mathrm{Pt} / \mathrm{Al}_{2} \mathrm{O}_{3}$ it is between $520-590{ }^{\circ} \mathrm{C}$ [11] but at a very low partial pressure.

Vilekar et al. [90] have studied the reaction mechanism to determine the rate determining step using data from other studies that used ruthenium and their own data using iron. Comparison of their own Reaction Route Graph with Quasi Steady State models supports nitrogen associative desorption as the rate determining step.

Maier [91] studied ammonia and its dehydrogenated fragments on a $\mathrm{Ru}(0001)$ surface by STM examination and DFT calculations. They found $\mathrm{NH}_{3}$ and $\mathrm{NH}_{2}{ }^{*}$ sit on top of the surface, while $\mathrm{NH}^{*}$ and $\mathrm{N}^{*}$ sit in depressions below the $\mathrm{Ru}$ surface. They also observed formation of $\mathrm{NH}_{3}-\mathrm{nH}$ complexes $(n=1,2,3)$ and $\mathrm{NH}_{3}$ dimers. If the $\mathrm{N}^{*}$ are in depressions, this could reduce their ability to interact, which would explain why the $\mathrm{N}^{*}$ recombination is rate limiting on ruthenium.

A recent kinetic analysis of ammonia decomposition by Takahashi compared nickel with ruthenium and came to the conclusion that they have different rate determining steps [83]. That analysis is relevant to this research because the 
measurements that support the model were taken at ambient pressure and from 300 to $450{ }^{\circ} \mathrm{C}$, the same as the experimental data for this work. It supported the finding that on ruthenium the rate limiting step is recombinative desorption of nitrogen. But for nickel the low desorption of nitrogen gas observed was because the surface coverage of nitrogen was only 0.1 compared to 0.4 on ruthenium. If surface coverage of nitrogen is low, it must be because production of surface nitrogen is slower than removal by recombinative desorption. The reaction that produces surface nitrogen is the dehydrogenation of ammonia, described in that paper as a single step, because after the first hydrogen atom is removed the other two atoms are removed quickly. In that study the rate of ammonia dehydrogenation was expressed according to Equation 1.2.14 [83].

\section{Equation 1.2.14: Rate of ammonia dehydrogenation.}

$r=k \cdot \theta_{\mathrm{NH}_{3}} \cdot \theta_{S}^{3}$

Where $\mathrm{r}$ is the rate of reaction and $\mathrm{k}$ is the kinetic constant. $\theta_{\mathrm{NH}_{3}}$ is the fraction of surface sites with adsorbed ammonia and $\theta_{S}$ is the fraction of surface sites that are vacant. The kinetic constant, $k$, for this reaction was calculated to be nearly an order of magnitude larger for ruthenium than nickel and the activation energies of the rate limiting steps were $123.5 \mathrm{~kJ} \mathrm{~mol}^{-1}$ for ruthenium to 144.0 $\mathrm{kJ} \cdot \mathrm{mol}^{-1}$ and nickel. The presence of hydrogen was determined to inhibit the ammonia decomposition reaction more for a nickel catalyst than for a ruthenium catalyst.

\subsubsection{Other Hydrogen Carrier Reactions}

Catalytic dehydrogenation of ammonia is not the only possibility for in situ hydrogen generation. For dehydrogenation, more than twenty other species have been investigated as hydrogen carriers, including hydrocarbons [92-95], alcohols [3, 96-98] and acetic acid [99, 100]. Other reactions that are being investigated for recovering the hydrogen from these carriers include oxidative dehydrogenation, dry reforming and steam reforming. Water gas shift is often applied to reactions which produce carbon or carbon monoxide to increase hydrogen and carbon dioxide outputs [99]. These reactions each have different 
Jonathan Tailby

advantages in terms of fuel availability, size of reaction vessel, exothermicity and reaction by-products.

The process of oxidative reforming uses oxygen gas to break down fossil fuels. For example, oxidative reforming of methane is described by Equation 1.2.15 [99].

\section{Equation 1.2.15: Oxidative reformation of methane}

$\mathrm{CH}_{4}+1 / 2 \mathrm{O}_{2} \rightarrow \mathrm{CO}+2 \mathrm{H}_{2}$

$\Delta \mathrm{H}=-36 \mathrm{~kJ}^{\mathrm{mol}} \mathrm{mo}^{-1}$

This reaction is exothermic, but the pressure increase (1.5 moles to 3 moles) means that it is not thermodynamically favoured under commercially relevant conditions [99].

Dry reforming of methane uses carbon dioxide as the oxidant. Catalysts for dry reforming include $\mathrm{Co}, \mathrm{Ni}, \mathrm{Ru}, \mathrm{Rh}, \mathrm{Pd}$, and $\mathrm{Pt}$ [99]. Carbon dioxide is reduced to carbon monoxide and carbon. This is shown in the reaction Equation 1.2.16 [99].

Equation 1.2.16: Dry reforming of methane.

$\mathrm{CH}_{4}+\mathrm{CO}_{2} \rightarrow 2 \mathrm{CO}+2 \mathrm{H}_{2}$

$\Delta \mathrm{H}=+247 \mathrm{~kJ} \cdot \mathrm{mol}^{-1}$

In steam reforming, the oxidant is water. The carbon products can include carbon deposits, but excess steam can prevent carbon build up. Reported catalysts include $\mathrm{Rh}, \mathrm{Ru}, \mathrm{Pd}, \mathrm{Pt}, \mathrm{Ni}, \mathrm{Co}, \mathrm{Cu}$ [99]. Steam reforming is effective for a variety of fuels, including methane, methanol, ethanol and acetic acid [99]. The equation for steam reforming of methane is shown in Equation 1.2.17.

Equation 1.2.17: Steam reforming of methane.

$\mathrm{CH}_{4}+\mathrm{H}_{2} \mathrm{O} \underset{\leftarrow}{ } \mathrm{CO}+3 \mathrm{H}_{2}$

$\Delta \mathrm{H}=+206 \mathrm{~kJ} \cdot \mathrm{mol}^{-1}$

The water gas shift reaction uses water to oxidise carbon monoxide, decreasing carbon monoxide output and increasing hydrogen output. This is described by Equation 1.2.18. 


\section{Equation 1.2.18: Water gas shift reaction.}

$\mathrm{CO}+\mathrm{H}_{2} \mathrm{O} \underset{\leftarrow}{\rightleftarrows} \mathrm{CO}_{2}+\mathrm{H}_{2}$

Reported catalysts include metals and oxides of Fe, Cu, Zn, Ce, Cr, Co, Ni [99]. Water gas shift is an exothermic reaction, so at high temperatures it favours the reverse reaction. Water gas shift is commonly combined with another reaction such as steam reforming to increase hydrogen output and decrease the overall endothermicity of steam reforming [7].

The advantage of carbon-based fuels such as methane is their greater natural abundance than hydrogen or ammonia and their established distribution infrastructures.

However, for all reactions that produce hydrogen from carbon-based fuels, $\mathrm{CO}$ or $\mathrm{CO}_{2}$ is one of the by-products. The production of a $\mathrm{CO}_{x}$ gas can potentially damage PEM-FCs by degrading the electrode materials $[11,15]$. Releasing $\mathrm{CO}_{\mathrm{x}}$ gases also cancels the carbon neutral advantage of hydrogen as a fuel. The combination of a first reformation reaction with water gas shift requires both a second reactor and sufficient water supply for the water gas shift, this adds considerable weight to the system. To overcome these disadvantages, carbon fuels can be used for large stationary hydrogen production where system weight is not a factor, and carbon capture can be applied to the output gas. The hydrogen can then be converted into a carbon free secondary fuel such as ammonia for transport applications. These additional steps require greater energy input, however much of this can be applied at the location where hydrogen is formed, enabling ammonia to act sufficiently well if a suitable decomposition catalyst is used.

\subsubsection{Membrane Reactors for Gas Reformation}

\subsubsection{Membrane Reactor Set $U p$}

A membrane reactor is a reaction vessel that uses a membrane inside the reactor to separate one of the products. In this case a palladium based membrane is used to remove hydrogen from the reaction chamber. Palladium membranes are able to achieve up to infinite selectivity for hydrogen by the process described in 
Jonathan Tailby

Section 1.2.775. The traditional natural gas reformer combines multiple reactors, first to reform natural gas into hydrogen and $\mathrm{CO}_{\mathrm{x}}$ gases, followed by an additional reactor to separate the hydrogen from the unwanted by-products. A membrane reactor combines these into a single step with a hydrogen separation membrane inside the reactor where the natural gas is reformed. This is shown schematically in Figure 1.2.4, which compares a traditional reformer with a membrane reactor. Figure 1.2.5 shows a membrane reactor for decomposition of ammonia, with a schematic of the gas species involved.

\section{Traditional Reformer}

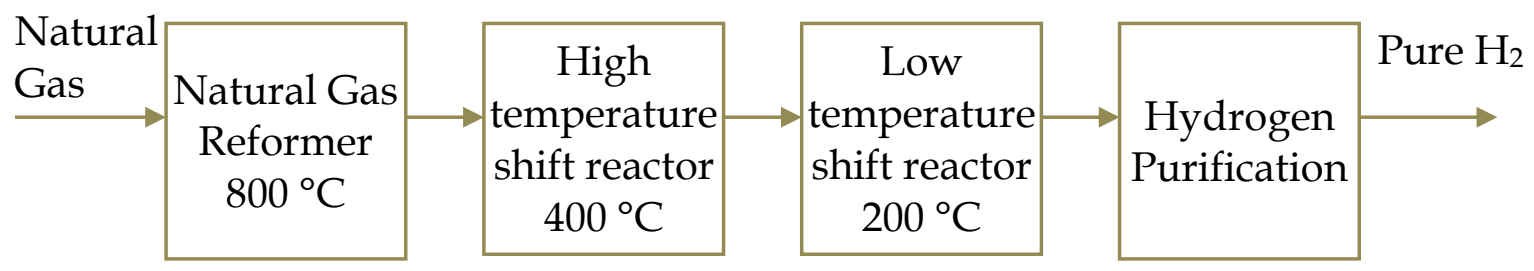

Natural Gas Membrane Reactor $500-600^{\circ} \mathrm{C}$

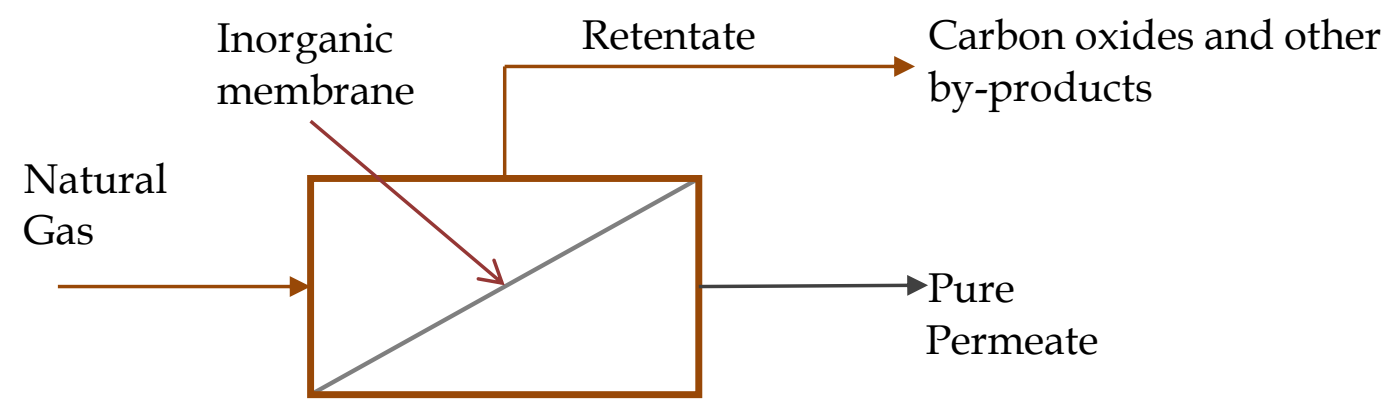

Figure 1.2.4: Schematics of traditional reformer and membrane reactor [99]. 


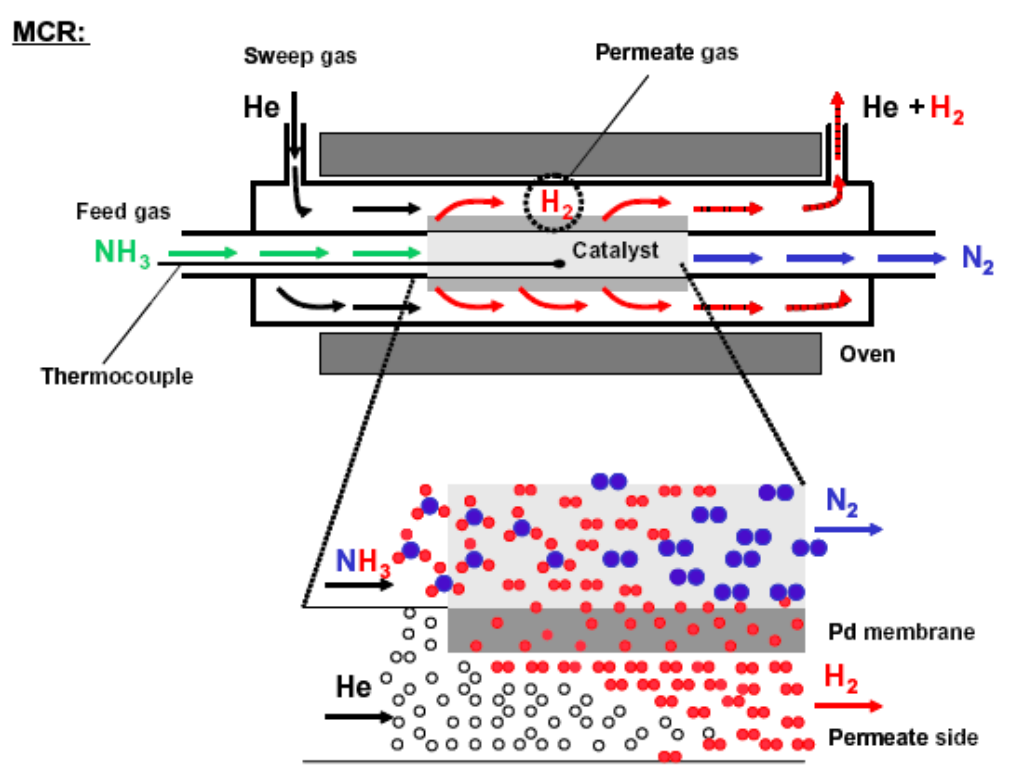

Figure 1.2.5: Schematic representation of the membrane reactor for ammonia decomposition [101].

\subsection{Supported Membranes}

Thin palladium membranes can be constructed on porous supports $[16,102]$. Common supports include porous stainless steel and ceramics such as alumina. Porous alumina has been reported to lose selectivity at $670-770 \mathrm{~K}\left(400-500{ }^{\circ} \mathrm{C}\right)$ because differences in the thermal expansion coefficients cause cracks at the palladium-ceramic interface [103] or because of sintering of fine palladium grains [104]. Porous stainless steel has also been reported to lose effectiveness because of thermal expansion differences and also to lose effectiveness at 700-900 K (427$627^{\circ} \mathrm{C}$ ) because intermetallic diffusion allows the palladium to form new alloys with less permeability [104, 105]. García-García has reported that porous stainless steel supported membranes were still able to operate with high selectivity and permeability after 100 hours of operation at 583-723 K (310-450 $\left.{ }^{\circ} \mathrm{C}\right)[101]$.

\subsubsection{Advantages of a Membrane Reactor}

Continuous removal of hydrogen from the reaction prevents the reaction mixture from reaching equilibrium, so the amount of hydrogen recovered can exceed the quantity predicted by equilibrium at any given temperature $[17,106]$. 
Jonathan Tailby

Additionally, unwanted by-products are less of a problem, such as formation of carbon monoxide from steam reforming of methane [106].

Removal can also improve reaction conditions [107]. The Temkin-Pyzhev model of ammonia decomposition was mentioned in Section 1.2.2.2, with equation Equation 1.2.13 [89]. This model is considered to be dominant at low temperature and high hydrogen partial pressure, and shows that hydrogen inhibits the reaction [107]. If hydrogen gas inhibits the reaction, then its removal will increase the rate of hydrogen production [107].

\subsubsection{Theory of Membrane Reactors}

\subsection{Rate Laws for Membrane Permeation}

The performance of a membrane reactor depends on three main factors: the kinetics of the hydrogen production reaction, efficiency of hydrogen separation and mass transport of hydrogen gas [108]. Under different conditions each of these can be the limiting process. Membrane separation efficiency depends on factors which include thickness, flow direction, flow speed, temperature, pressure and sweep ratio; each of these will be discussed in the subsequent Section 1.2.4.4. Studies by Chen et al. at $773 \mathrm{~K}\left(500^{\circ} \mathrm{C}\right), 2000 \mathrm{kPa}$ and WHSV of 0.3 hour-1 $^{-1}$ [108] have shown that the diffusion of hydrogen gas from catalyst to membrane was calculated to be a limiting process. But gaseous diffusion is one of the processes that could be bypassed by hydrogen spillover.

Optimum conditions for some reactions degrade the palladium membrane because high temperatures damage the membranes, especially very thin membranes [109]. Membrane reactor conditions for these reactions are often a compromise between optimum reaction conditions and conditions that favour membrane stability [99].

The process of hydrogen diffusion through palladium is discussed further in section 1.2.7, which includes a table of the steps in the process. For most palladium membranes, diffusion through the palladium bulk is the rate limiting step [110]. Hydrogen flux is often modelled by using the difference in partial 
pressure between the two sides of the membranes as the driving force $[3,111]$. However, for thinner membranes, bulk diffusion can be faster than surface processes, so dissociative adsorption of hydrogen gas becomes rate limiting [108]. The transition point between bulk limited and surface limited diffusion depends on several factors, most importantly thickness and temperature [112]. The values shown on Table 1.2.2 are taken from the graphs shown in Ward and Dao [112]. One such graph is reproduced as Figure 5.2.37 in Appendix 5.2.7.4. Below these temperatures the permeation is surface limited, above these temperatures it is diffusion limited. The table shows that the transition temperature gets lower as the membrane gets thicker, meaning thinner membranes remain surface limited to a higher temperature. The time taken for hydrogen atoms to diffuse from one surface to the other is greater in the thicker membranes while the time at surface processes remains approximately the same between the different thicknesses. Therefore, the time in diffusion will exceed the time at the surface at a lower temperature for the thicker membranes. When membranes of different thickness are both surface limited, they will have the same flux, however the thicker membrane will change to diffusion limited at a lower temperature and have a lower flux when they are both diffusion limited.

\section{Table 1.2.2: Transition temperature for permeation limit.}

From surface limited to diffusion limited.

\begin{tabular}{|l|l|l|l|}
\hline Membrane Thickness & $1 /$ temperature $\left(\mathrm{K}^{-1}\right)$ & Temperature $(\mathrm{K})$ & Temperature $\left({ }^{\circ} \mathrm{C}\right)$ \\
\hline $\mathbf{1 0 0} \boldsymbol{\mu m}$ & 0.0024 & 417 & 144 \\
\hline $\mathbf{1 0} \boldsymbol{\mu m}$ & 0.0021 & 476 & 203 \\
\hline $\mathbf{1} \mathbf{m}$ & 0.00185 & 541 & 268 \\
\hline
\end{tabular}

\subsubsection{Effect of Relevant Variables on Outcomes from Membrane Reactors}

\subsection{Effect of Direction of Gas Flow on Membrane Reactor}

Comparing the direction of gas flow on the two sides of the membrane reveals two possibilities. Either the two flow in the same direction (co-current) or they flow in opposite directions (counter current). The hydrogen partial pressure on the retentate side will decrease, while on the permeate side it will increase. In a co-current membrane reactor the difference between the hydrogen partial pressures on each side will decrease along the flow, reducing hydrogen 
Jonathan Tailby

permeation. In a counter current reactor, the opposing flows decrease the reduction in hydrogen partial pressure difference along the membrane, thus achieving better hydrogen recovery. This has been shown in several studies, for example Iulianelli et al. [3, 113].

\subsection{Effect of Gas Pressure on Membrane Reactor}

Changes in pressure affect membrane reactor performance in two ways, by the direct effect on the reaction and by the effect on permeation through the membrane. The effect on the extent of the reaction can be positive or negative depending on the reaction [98]. The effect on permeation is always positive, as increased total pressure will increase hydrogen partial pressure which drives permeation [2]. This increased permeation removes more hydrogen, which shifts the equilibrium to favour more hydrogen production [2].

For a reaction where increased pressure has a negative effect on the equilibrium position of the reaction, the overall effect of increased pressure is reported to remain positive in a membrane reactor [98]. Figure 1.2.6 shows the conversion of methane at different pressure. Increased pressure is shown to decrease the equilibrium methane conversion. However, the membrane reactor is shown to exceed this level of conversion and to achieve increased conversion with increased pressure [2]. 


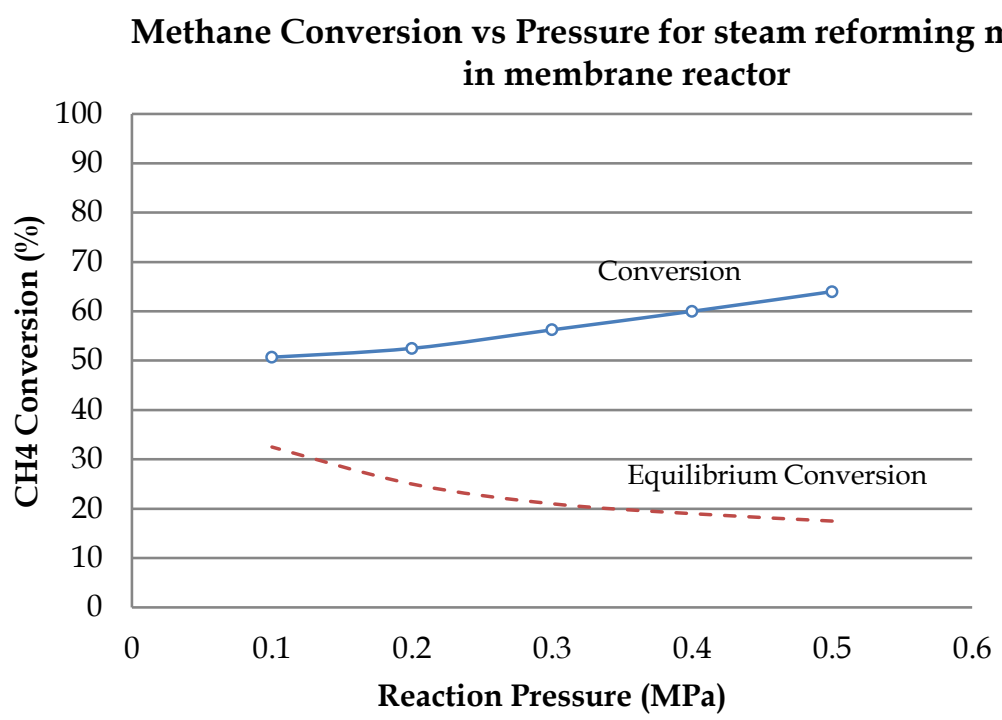

Figure 1.2.6: Pressure dependence of steam reforming of methane in membrane reactor at $500{ }^{\circ} \mathrm{C}$.

Reaction conditions: S/C, 2.0; Space Velocity, 3000 hour-1; sweep flow rate, $0.25 \mathrm{~mol} \cdot$ hour $^{-1}[2]$

\subsection{Effect of Reaction Temperature on Membrane Reactor}

Temperature also affects both permeance and reaction conversion. Most hydrogen producing reactions are endothermic, so an increase in temperature increases hydrogen production $[60,99,114]$. Hydrogen permeance also increases with temperature [112]. Palladium alloys have good permeability at $500{ }^{\circ} \mathrm{C}$ [99], some data for a palladium membrane $40 \mu \mathrm{m}$ thick electroless deposited on porous stainless steel are shown in Table 1.2.3.

\section{Table 1.2.3: Permeance at different temperatures for $40 \mu \mathrm{m}$ thick palladium} [101]

\begin{tabular}{|l|l|l|l|l|l|}
\hline Temperature & 250 & 300 & 350 & 400 & ${ }^{\circ} \mathrm{C}$ \\
\hline Permeance & 4.70 & 6.37 & 8.44 & 10.70 & $\mathrm{~m}^{3} \cdot \mathrm{m}^{-2} \cdot \mathrm{h}^{-1} \cdot \mathrm{atm}^{-1 / 2}$ \\
\hline
\end{tabular}

Methane steam reforming is most efficient at $700-900{ }^{\circ} \mathrm{C}$ where heat transfer is improved and the catalyst performs most effectively [110]. However, this temperature range can degrade an ultra-thin palladium membrane, and in a membrane reactor the temperature of the two processes is often a compromise at $550{ }^{\circ} \mathrm{C}[110]$. 
Jonathan Tailby

\subsection{Effect of Flow rate on Membrane Reactor}

Low flow rate increases contact time between reactant gas and catalyst, so the percentage conversion is increased. This may come at the cost of rate of conversion because less reactant gas is available for conversion to hydrogen.

\subsection{Effect of Sweep ratio on Membrane Reactor}

Sweep ratio is the ratio of sweep gas flow to reactant gas flow. At high hydrogen partial pressure, an increased sweep ratio will increase the hydrogen flux through the membrane. Hydrogen partial pressure decreases on both sides of the membrane with increased sweep flow, but with an increase in partial pressure difference [2]. The partial pressure on the retentate side decreases because the increased hydrogen flux removes hydrogen more quickly [101]. Partial pressure also decreases on the sweep side because the additional sweep gas dilutes the hydrogen gas on the sweep side. Partial pressure decreases on both sides but it decreases more on the sweep side, such that the difference in partial pressure between the retentate side and the sweep side increases, which can increase hydrogen flux through the membrane [115]. However, the increased quantity of hydrogen is spread through a greater quantity of sweep gas, resulting in a more dilute hydrogen mixture [101].

\subsection{Overview of Membrane Reactors}

The ammonia dissociation reaction is favoured by high temperature because it is endothermic [10], and by low pressure because the number of gas molecules doubles (Equation 1.2.2). However, in a membrane reactor, both high temperature and high pressure have been shown to increase permeance and enhance the reaction [99]. Hence, decomposition of ammonia in a membrane reactor should be undertaken at higher pressure than in traditional reactor conditions [2]. Because there is a low rate of adsorption of ammonia onto the catalyst, a low flow rate may increase the proportion of ammonia decomposed, however a higher flow rate may still produce a greater rate of ammonia 
decomposition. A high sweep flow might increase flux through the membrane, but only if factors on the permeate side are rate limiting.

\subsubsection{Electrochemistry}

Two types of electrochemistry were relevant to this research. The aim of the research was to produce hydrogen that was ready for use in fuel cells, therefore, fuel cells are briefly described in Section 1.2.5.1, specifically the proton exchange membranes that are the preferred type of fuel cell for use with hydrogen.

The first method of catalyst preparation that was explored for use in this research was electrodeposition. Electrodeposition is described in Section 1.2.5.2 along with some relevant processes.

\subsubsection{Fuel Cells}

A fuel cell is a device which oxidises a fuel while producing an electric current that can perform work [15]. This involves an electrolyte that allows chemical transfer but requires an external cell to conduct electrons [15]. Proton exchange membranes are the preferred electrolyte for hydrogen fuel cells [13]. PEM fuel cells operate at low temperature compared to most other fuel cells, which improves efficiency and start up time [116]. When heat and power are both recovered, the efficiency can exceed $90 \%$ [15]. The membrane is usually formed from a copolymer of tetrafluoroethylene, perfluororinated vinyl ether and sulfonyl fluoride [15]. Even part per million quantities of ammonia are known to degrade fuel cell performance [11]. One proposed mechanism of this degradation is that the ammonia reacts in the membrane to replace protons with ammonium ions which increases the cell resistance, however this decrease in ion transfer probably only accounts for part of the loss in performance from exposure to ammonia [117].

\subsubsection{Electrodeposition}

Electrodeposition is the process of deposition of material using an electric current. Electrodes that apply a potential to drive a current through a solution containing a metal cation. The cation is reduced at the cathode and deposits of 
Jonathan Tailby

the metal form on the cathode surface [118]. Extended deposition in this way leads to electroplating. Deposits form on the cathode surface by diffusion of the metal atoms which aggregate to form a stable nucleus; this nucleus grows by the addition of further metal atoms [118]. The nucleation and growth characteristics can be modified through changing parameters such as substrate morphology, solution concentration and $\mathrm{pH}$, potential, current density and the mass transfer occurring in the system [119-122]. One characteristic that has been well studied is the size of deposits, and various methods have been used to control this.

\subsection{Nucleation in Electrodeposition}

The process of nucleation during electrodeposition is the initial formation of new deposits and it is controlled by surface energy. The atoms formed by reduction have a high surface energy, so they diffuse and aggregate to decrease surface energy. To nucleate they must reach a critical size where the energy saving of the internal bulk matches the energy of maintaining a surface. This size can be reduced if the surrounding material allows for a low interfacial energy. For example, deposition of a metal onto another metal with similar structure will have a lower interfacial energy and critical size than depositing the same metal onto graphite. For very high energy surfaces such as noble metals, the deposition may occur as layers on the surface rather than individual nuclei [120]. Palladium is a noble metal, so the deposition would be expected to form layers rather than particles that extend out from the surface.

In electrodeposition the nucleation is compared to two model patterns, instantaneous and progressive [122]. For instantaneous nucleation the nuclei form suddenly and simultaneously at the start of the electrodeposition, then no new nuclei are formed [122]. For progressive nucleation, new nuclei are constantly formed as deposition continues [122]. Therefore, for instantaneous nucleation, nucleation and growth occur at separate times which results in sizesimilar deposits. Conversely, for progressive nucleation, nucleation continues to occur while previously formed nuclei grow which results in a wider range of deposit sizes. These two patterns have different characteristic trends in the 
electric current during electrodeposition [122]. Comparing the current curve of an experiment to the model curves can help identify whether the experiment follows an instantaneous or progressive trend. Figure 1.2.7 shows a comparison of the two model curves, and measured data for cobalt on $\mathrm{Cu} /$ glass electrode at $-1 \mathrm{~V}$ (SCE) revealing progressive deposition of the cobalt [122]. The Instantaneous deposition rises more quickly at first but slows this increase, the decrease after the maximum is also slower and finds a steady state at a higher proportion of the maximum.

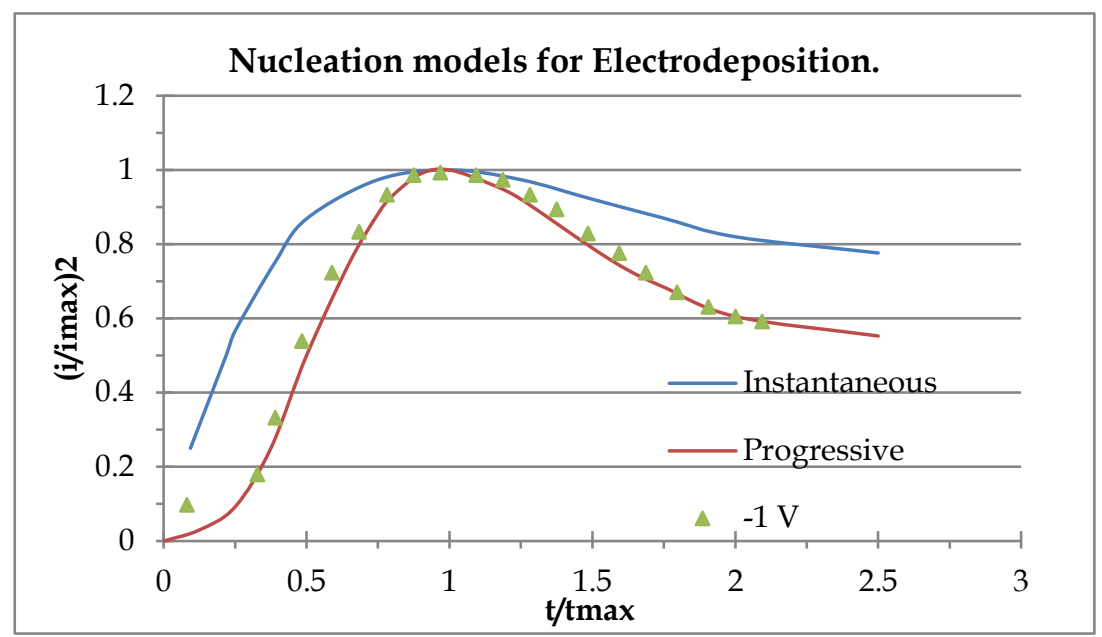

Figure 1.2.7: Comparison of Instantaneous and progressive nucleation models with measured data for cobalt on a copper/glass electrode [122].

\subsection{Substrate Effects in Electrodeposition}

In this work "substrate" is used to refer to the surface that supports the catalyst deposits. In electrodeposition this substrate is the working electrode. Electrode surface conditions affect the progress of deposition. The microscopic detail of the electrode surface is usually rough, with crevices and holes that make it difficult to clean [121]. Further, the crystal properties of the substrate morphology can affect deposition, particularly nucleation [118]. It has been found that more uniform surface morphology results in more evenly distributed initial growth [118]. 
Jonathan Tailby

\subsection{Voltammetry in Electrodeposition}

Reducing the metal ions requires the potential difference at the cathode to exceed the thermodynamic reduction potential of the metal cation. The amount that the potential exceeds the reduction potential is called the overpotential (n) [121]. This overpotential is expressed in Equation 1.2.19.

\section{Equation 1.2.19: Overpotential calculation.}

$\eta=E_{\text {reaction }}-$ Eequilibrium

The overpotential required for a reaction to proceed is commonly regarded as having three components [121]. Ohmic overpotential comes from the resistance of the cell. Activation overpotential is the extra potential needed for the activation energy of the reaction. This can be reduced by application of a catalyst. For example, the activation overpotential for electrolysing water to form hydrogen is lower for a platinum electrode than a carbon electrode because of platinum's catalytic properties for this reaction. The activation overpotential for electrodeposition can be lowered by using a substrate more similar to the deposit, for example electrodeposition of nickel onto steel is lower than the activation for deposition onto carbon with otherwise identical conditions [121]. Concentration overpotential comes from the cation concentration difference between the electrode surface and the bulk solution.

Electrochemistry with palladium has such a low overpotential for hydrogen evolution that it can prevent the electrodeposition of metals. Crousier reports that in codeposition of nickel and molybdenum on palladium, there is no significant deposition by either nucleation or by epitaxial growth [123]. This situation is improved by using basic solutions to decrease the concentration of hydrogen ions, for example the $\mathrm{pH} 8.3$ deposition solution reported to successfully deposit nanonickel on palladium by Zach and Penner [124]. Dunbar et al. [111] report electrodeposition of nickel onto palladium using a commercial sulfamate plating solution and low current density. The work also used a photolithographic template to control the shape of the deposit. Dunbar published that work after the templated electrodeposition reported here had 
been successfully achieved. Dunbar reports a hexagonal honeycomb matrix deposited as a support for an ultrathin palladium membrane, with the goal being the creation of the ultrathin membrane, rather than separated catalyst units on a tubular substrate.

\subsection{Current Density in Electrodeposition}

Current density is an important parameter for controlling the properties of an electrodeposit. It affects rate of deposition and crystallite size which affects mechanical and catalytic properties of the deposit $[125,126]$.

Current is also monitored to gather information about progress of the reaction. If there are no side reactions, current and time can be used to calculate the quantity of material deposited. The current will change through different stages of a reaction, so nucleation and growth stages can be identified, as well as whether nucleation is instantaneous or progressive [127]. In the process of growing nanowires in a template, if the deposition is diffusion limited, there are four stages with different current patterns [127]. These can be used to monitor the progress of a deposition, recognising which stage of the process it has reached by the current behaviour. The four stages are shown in Table 1.2.4. The current increases very rapidly with the initial nucleation, then the current decreases rapidly as the deposition fills the base of the pore and the solution in the pore becomes depleted of metal ions. The current increases slowly while the deposit extends up the length of the pore and the diffusion length for fresh deposition solution decreases. When the deposit first overflows the template, current will increase more quickly while the deposit spreads laterally across the surface of the template, increasing the surface area of the working electrode. The overflow from the deposits will merge together to form a film, at which point the current will become constant while the film thickness increases because the deposition surface area has reached a maximum.

\begin{tabular}{|c|c|c|}
\hline I & Nucleation & Current increases sharply then decreases rapidly. \\
\hline II & Growth & Current increases slowly. \\
\hline
\end{tabular}




\begin{tabular}{|l|l|l|}
\hline III & Wires expand & Current increases. \\
\hline IV & Film formation & Current rises sharply then becomes constant. \\
\hline
\end{tabular}

\subsection{Mass Transport in Electrodeposition}

Mass transport is often the limiting process in electrodeposition [120, 121]. The rate of electron transfer is an exponential function of applied voltage, so it is usually rapid compared to transport of ions from the bulk solution to the electrode surface [121]. When electrodeposition begins, ions at the electrode surface are reduced more quickly than ions are transferred from the bulk to the surface, so the solution around the electrode surface becomes depleted of ions [121]. Transport of ions becomes the limiting process. Depletion is initially around individual deposits, but the depletion zones grow and interact until they form a plane parallel to the surface $[120,127]$. The time of maximum current density correlates with the overlap of depletion zones into a depletion plane[127]. Mass transfer occurs by three modes: migration, convection and diffusion [121]. Migration is transport caused by forces such as electrostatic attraction between cation and cathode [121]. In a solution with background electrolytes this is usually a minor component of mass transport [121]. Convection is caused by density variations in the solution [121] and is particularly affected by cell design. A cell design which increases these density variations, will increase convection. Convection is most important in unstirred cells with a vertical electrode. With vertical wire electrodes there is an initial drop in current, which stabilises in 1-3 minutes [121].

Diffusion is mass transfer which results from concentration differences [121]. As soon as electrodeposition begins, concentration differences appear and diffusion begins. Diffusion is the most influential form of mass transfer in electrochemistry [121].

\subsection{Particle Size Variation in Electrodeposition}

When electrodeposition is used to grow small particles on a surface, there will be variation in the size of these particles. The two main causes are progressive nucleation and interparticle diffusion coupling [120]. In a situation of 
progressive nucleation, more nuclei are formed while others have already grown [122]. Newly formed nuclei will be at the critical size for nucleation, while nuclei that have already had time to grow will be larger. The longer the process is in a progressive nucleation phase, the wider the size variation of the deposits. Separating the nucleation and growth phases in time can reduce the size variation $[120,128]$.

Interparticle diffusion coupling is the result of overlap in the depletion zones around deposits [120]. A three dimensional region depleted of cations forms around each deposit, and diffusion through this zone becomes the limiting step [120]. If two particles have overlapping depletion zones, they will grow more slowly than a particle with no overlap of its diffusion zone [120]. As a result, growth of particles depends on the number and proximity of neighbours. The distribution of particles therefore affects growth rates; randomly distributed particles are more unevenly affected by diffusion coupling than regular arrays [120].

\subsection{Control of Particle Size in Electrodeposition}

Particle synthesis usually aims to produce monodisperse particles, so the factors which cause size variation need to be addressed. Several strategies have been developed to reduce variation in particle size.

An electrochemical method to decrease size variation in deposits is to reduce the overpotential to slow growth [120]. The overpotential is reduced by decreasing the reduction voltage. The voltage necessary for the reaction remains constant if no other factors are changed, hence the over potential, the difference between the reduction voltage and the minimum reduction voltage, is reduced. Interparticle diffusion coupling is less of a problem at lower overpotential. This is because reduction of overpotential reduces growth speed, so diffusion is faster relative to growth speed [120]. This results in less depletion, hence less interparticle diffusion coupling. Decreasing the overpotential also decreases nucleation.

Penner reports deposition of several different metals using a slow growth method with reduced overpotential [120]. An initial nucleation pulse of $500 \mathrm{mV}$ 
vs. MSE overpotential for 5-10 ms produced seed particles of $1 \mathrm{~nm}$. This was followed by a growth pulse with $50-100 \mathrm{mV}$ vs. MSE overpotential for up to 1 minute. The constant current through the growth phase supports the absence of both interparticle diffusion coupling and nucleation [120]. This method produced size-selected mesoscopic particles of metals including $\mathrm{Pt}, \mathrm{Au}, \mathrm{Ag}, \mathrm{Cu}$ $\mathrm{Ni}$ and $\mathrm{Cd}$ as well as $\mathrm{MoO}_{2}[120]$.

Stirring the solution can help improve size similarity if it is stirred on the appropriate scale. A rotating disk electrode does not mix the boundary layer of the electrode surface [124]. Gas evolution at the electrode surface can stir the boundary layer $[120,124]$. Penner showed that -1.2 V vs. MSE nickel deposits on graphite with a nickel nitrate solution were of heterogeneous size, but at -1.6 and $-2.0 \mathrm{~V}$ vs. MSE size uniformity improved to relative standard deviations of $23 \%$ and $14 \%$ [120], this improvement was attributed to hydrogen gas evolution during electrodeposition. If it proves important to deposit size similar particles for this work, then stirring on an appropriate scale could be investigated.

Another method of size control was demonstrated by Dunbar [111]. Photolithography shaped a template that controlled the shape of nickel when it was electro-deposited onto palladium. The template was dissolved in acetone after it had controlled the size and shape of the deposition. This work was reported after a similar process had been developed for this work. Dunbar deposited nickel honeycomb supports on an ultrathin palladium layer. This work deposited an array of individual nickel deposits on a tubular substrate.

\subsubsection{Spillover}

\subsubsection{Definition of Spillover}

Spillover is a process vital to many heterogeneous catalytic reactions [26]. It has been defined thus:

'Spillover involves the transport of an active species sorbed or formed on a first phase onto another phase that does not under the same condition sorb or form the species. The result may be the reaction of this species on the second phase 
with other sorbed gases and/or reaction with and/or activation of the second phase.' [129]

Figure 1.2.8 shows a schematic diagram representing spillover of a molecule adsorbed from the gas phase, and an approximate energy level diagram for the process. Spillover has been observed at distances up to tens of centimetres from the site of production [26, 130] as well as sorption to the second surface [26]. Spillover is an endothermic process, thermodynamically favoured by an increase in entropy[26]. Hydrogen spillover is observed to have a low activation energy [26], 4-5 kJ $\mathrm{mol}^{-1}$ for $\mathrm{Ni} / \mathrm{Al}_{2} \mathrm{O}_{3}$ to $\mathrm{Pd}[28]$.

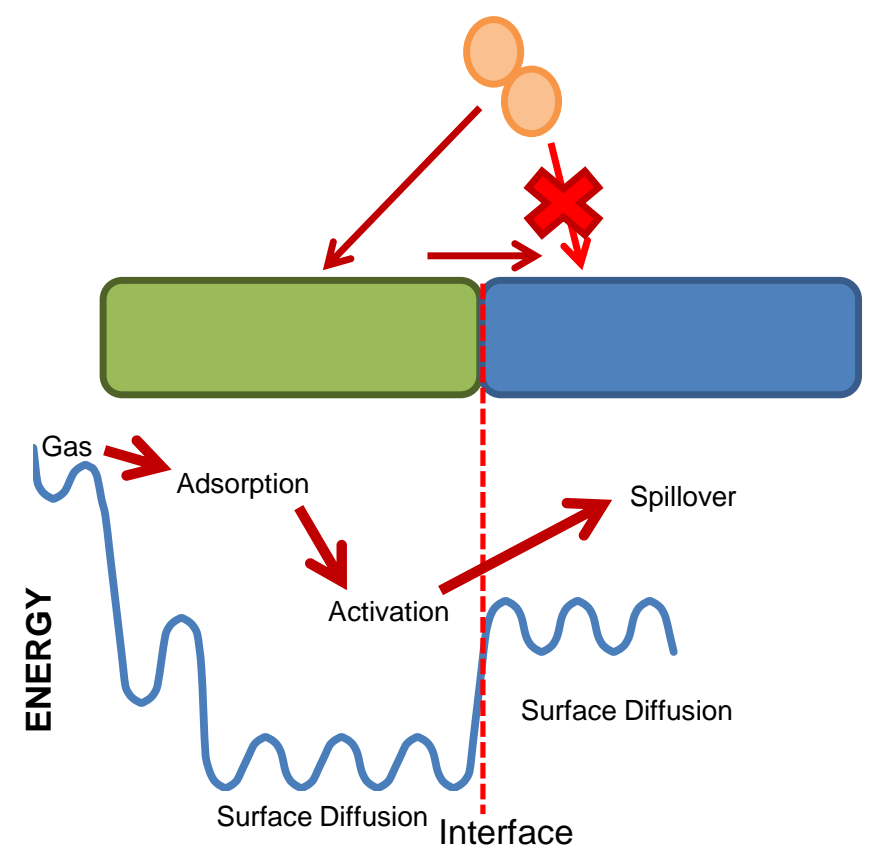

Figure 1.2.8: Schematic representation of spillover of a diatomic gas from an adsorbing onto a nonadsorbing surface.

A reasonable energy level diagram for processes is shown. [26]

\subsubsection{Evidence for Spillover}

Early evidence of the spillover phenomenon was seen in the synthesis and decomposition of $\mathrm{NH}_{3}$ in the 1940s [26]. It was further investigated in 1964 by Khoobiar [131], who reported that $\mathrm{H}_{2}$ reduces $\mathrm{WO}_{3}$ at temperatures greater than $200{ }^{\circ} \mathrm{C}$ causing a colour change. When $\mathrm{Pt} / \mathrm{Al}_{2} \mathrm{O}_{3}$ was present, the same colour change occurred at room temperature because $\mathrm{H}_{2}$ was dissociated on the 
Jonathan Tailby

platinum and $\mathrm{H}$ atoms migrated to the $\mathrm{WO}_{3}$ where they reacted, to cause the colour change [131].

\subsubsection{Properties of Spillover Hydrogen}

Hydrogen is the most commonly reported spillover species. It can be observed by isotope exchange on materials such as silica where surface hydroxyl groups exchange with spillover hydrogen from an activating metal [26]. The nature of spillover hydrogen has been debated with five states proposed: adsorbed $\mathrm{H}_{2}$, dissociated $\mathrm{H}^{*}$ atoms, protonic $\mathrm{H}^{+}$, ion pair $\mathrm{H}^{+}-\mathrm{H}^{-}, \mathrm{H}_{3}$ [129]

\subsection{Evidence for spillover of $\mathrm{H}_{2}$}

Many investigations into hydrogen spillover use gaseous hydrogen as the initial source of the hydrogen. Physisorption of $\mathrm{H}_{2}$ is the first stage of the interaction between hydrogen and the catalyst, but chemisorption requires $\mathrm{H}_{2}$ to dissociate [129].

Most evidence for the nature of spillover hydrogen supports a species other than $\mathrm{H}_{2}$. An infrared spectroscopy study reported by Plisken found two bands, corresponding to the two stable hydrogen isotopes bound to the platinum, $\mathrm{Pt}-\mathrm{H}$ and Pt-D. If the hydrogen was to be bound as dihydrogen, three bands would be expected, corresponding to $\mathrm{Pt}-\mathrm{H}_{2}, \mathrm{Pt}-\mathrm{DH}$ and $\mathrm{Pt}-\mathrm{D}_{2}$ [129].

\subsection{Evidence for spillover of $\mathrm{H}^{*}$}

The presence of atomic hydrogen in spillover hydrogen is often supported by evidence comparing the properties of spillover hydrogen with externally produced $\mathrm{H}^{*}$. Kramer reported results from temperature programmed desorption that showed the same $480{ }^{\circ} \mathrm{C}$ desorption peak for $\mathrm{Pt} / \mathrm{Al}_{2} \mathrm{O}_{3}$ treated with $\mathrm{H}_{2}$ as for metal free $\mathrm{Al}_{2} \mathrm{O}_{3}$ treated with $\mathrm{H}^{*}$. This similarity between surfaces treated with spillover hydrogen and with externally produced $\mathrm{H}^{*}$ does not confirm the spillover hydrogen as $\mathrm{H}^{*}$ because the spillover hydrogen may be affected by interaction with the secondary surface [129]. 


\subsection{Evidence for spillover of $\mathrm{H}^{+}$}

Evidence for hydrogen ions in spillover hydrogen is from reactivity, interaction with magnetic fields and electrochemistry [129]. Levy reported that the activity of catalysts correlated with the proton affinity of the co-catalyst. The increase in reaction rate as proton affinity increases supports the presence of $\mathrm{H}^{+}$in the spillover hydrogen [129]. Magnetic fields were found to affect the H-D exchange of $\mathrm{Pt} /(\mathrm{NaY}-\mathrm{HNaY})$ zeolites. This work suggested that the spillover hydrogen is charged, and is probably $\mathrm{H}^{+}$.

Formation of $\mathrm{H}^{+}$from $\mathrm{H}_{2}$ requires the removal of electrons as well as dissociation. On the surface of the metal these electrons are reported to pass into the conduction band. As the $\mathrm{H}^{+}$spills over onto the new surface it is unlikely to leave the electrons behind as extra electrons in the conduction band of the metal. The alternative is transport of the electron through the interface between the two surfaces. Conductivity changes have been observed for metal oxides, including $\mathrm{TiO}_{2}, \mathrm{SnO}_{2}$ and $\mathrm{ZnO}$, in the presence of spillover hydrogen. Increases in low temperature $\mathrm{n}$ type conductivity is interpreted as transfer of electrons from hydrogen to oxide and adsorption of $\mathrm{H}^{+}$from spillover hydrogen.[129]

\subsection{Evidence for spillover of $\mathrm{H}+-\mathrm{H}-$}

Evidence from some catalytic reactions supports the possibility of a protonhydride pair. An example is the hydroisomerisation of $n$-pentane on a Ptcontaining ZSM-5 zeolite. [129]

\subsection{Evidence for spillover of $\mathrm{H}_{3}$}

From kinetic results of demethoxylation of methoxylated $\mathrm{SiO}_{2}$ with $\mathrm{Pt} / \mathrm{Al}_{2} \mathrm{O}_{3}$ it has been proposed that spillover hydrogen could be $\mathrm{H}_{3}$. This could form by reaction of dissociated hydrogen on the platinum with molecular $\mathrm{H}_{2}$. It has also been proposed that the trihydrogen species could be a $\mathrm{H}_{3}{ }^{+}$ion [129] formed according to Equation 1.2.20

Equation 1.2.20: Trihydrogen ion formation for spillover.

$\mathrm{H}^{+}+\mathrm{H}_{2} \rightarrow \mathrm{H}_{3}^{+}$ 
Jonathan Tailby

\subsection{Roland Model for Hydrogen Spillover}

Most evidence has been gathered to support either atomic or ionic hydrogen in spillover hydrogen. Sometimes there is evidence of each on the same surface. Roland proposed that the two coexist in spillover hydrogen with a ratio that depends on the extent of interaction with the surface [129]. 'Weak' and 'strong' are terms used to refer to the degree of chemisorption, i.e. the degree to which electron transfer can be said to have occurred. $\mathrm{H}^{*}$ is a weakly chemisorbed hydrogen atom which has retained an electron. $\mathrm{H}^{+}$is here strongly chemisorbed, the electron being transferred to the surface [129].

The coexistence of the two states explains apparently contradictory evidence. Evidence for the same system sometimes indicating $\mathrm{H}^{*}$ and sometimes $\mathrm{H}^{+}$can be explained by coexistence of the two H species[129]. Fast exchange between the two states also contributes to results which support both possibilities, or neither conclusively [129].

\subsubsection{Spillover in catalysis}

Spillover is part of the mechanism of catalytic reactions. It has been proposed as an alternative model to polyfunctional catalysis in several organic reactions. For example, the conversion of cyclohexane to methylcyclopentane by polyfunctional catalysis is believed to occur by three steps at different sites on a platinum catalyst. This involves three adsorption/desorption steps with gas phase diffusion of intermediates, as in Figure 1.2.9 [130]. 


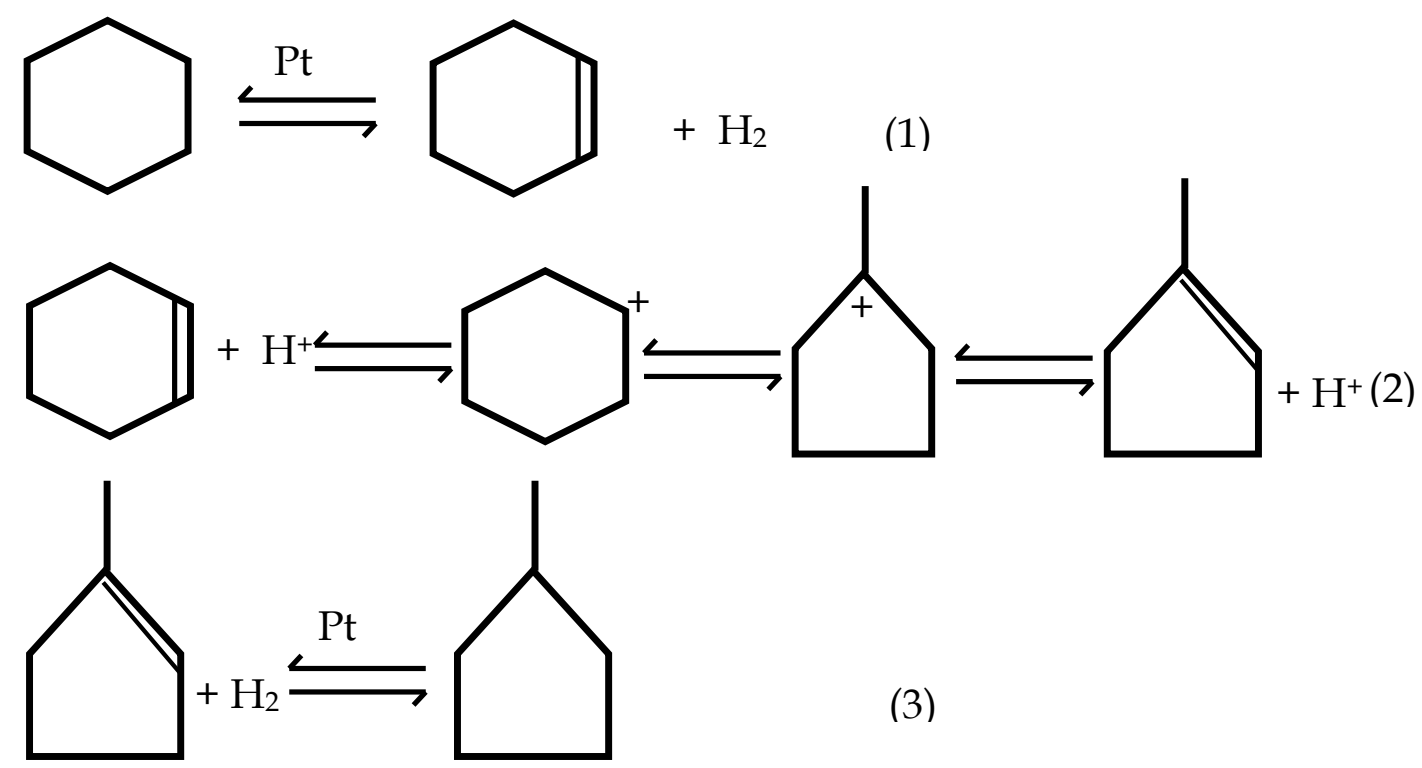

Figure 1.2.9: Conversion of cyclohexane by polyfunctional catalysis.

Spillover of dissociated hydrogen from the platinum catalyst allows the reaction to occur at one site without diffusion of intermediates [130]. Surface diffusion of the olefin intermediate has not been observed. Spillover hydrogen is in an activated form, able to react directly. The spillover reaction is shown in Figure 1.2.10 [130]

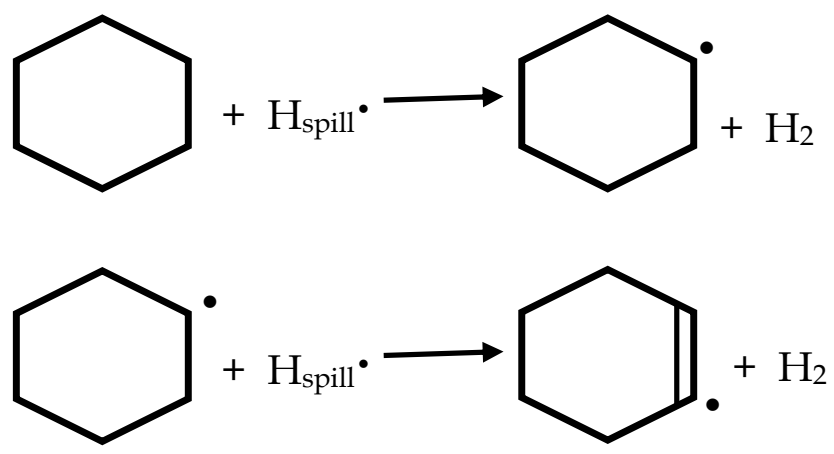

Figure 1.2.10: Reaction of cyclohexane with spillover hydrogen.

In this cyclohexane example, changing the surface medium between the platinum and the acidic reaction sites decreased the rate of reaction. The spillover mechanism explains this because the spillover hydrogen is less able to diffuse across the zeolite surface of the support [130]. The polyfunctional catalyst 
mechanism in Figure 1.2.9 does not explain this because the intervening surface should not affect the gas phase diffusion [130].

Other experiments also support the spillover mechanism more than the polyfunctional catalysis mechanism. Increasing the distance between the two sites does not affect the rate of reaction, which it should do under the polyfunctional catalyst mechanism where gas phase diffusion is the rate limiting step [130]. Reactor set ups with separate reaction sites that would require gas phase diffusion against the gas flow such as those reported by Weigle et al., also continued to produce the final product without significant production of the intermediates required for a polyfunctional mechanism [132].

Baber et al. [133] investigated the effect of single Pd atoms on hydrogen gas. On a $\mathrm{Cu}(111)$ surface, isolated $\mathrm{Pd}$ atoms dissociated hydrogen gas and spillover $\mathrm{H}^{*}$ onto $\mathrm{Cu}$ sites at $420 \mathrm{~K}$. The same did not occur for single Pd atoms on $\mathrm{Au}$ (111), however larger $\mathrm{Pd}$ assemblies were able to spillover $\mathrm{H}^{*}$ to the gold. The results show the effect of an inert substrate on catalysis by $\mathrm{Pd}$ as $\mathrm{H}$ adatoms are thermodynamically stable on $\mathrm{Cu}$ but not on Au.

\subsubsection{Spillover Membrane}

It is reported that enabling hydrogen to spillover from the catalyst to the membrane in a membrane reactor can increase the rate of the reaction [28]. Rei has published that spillover increases the rate of hydrogen production in steam reformation of methanol or $n$-hexane [28]. Table 1.2.5 shows the reaction schemes Rei proposed for the formation and transport of hydrogen. The spillover scheme circumvents two slow steps in the process as hydrogen atoms from the catalyst $\left(\mathrm{CuOZnO}\right.$ or $\left.\mathrm{Ni} / \mathrm{Al}_{2} \mathrm{O}_{3}\right)$ migrate directly from the catalyst surface to the palladium membrane [28]. The activation energy of this spillover is $4-5 \mathrm{~kJ} \mathrm{~mol}^{-1}$ compared with $80-100 \mathrm{~kJ} \mathrm{~mol}^{-1}$ for chemisorption and desorption [28]. 


\section{Table 1.2.5: Reaction schemes for dehydrogenation with permeation} [28]

\begin{tabular}{|c|c|}
\hline A: Conventional scheme: & B: Spillover scheme: \\
\hline $\begin{array}{l}\text { (A-1) Nascent hydrogen atoms } \\
\text { form on the catalyst surface, } \\
\mathrm{RH}+2 S^{*} \rightarrow R S^{*}+H S^{*} \\
\left(S^{*} \text { depicts an active catalyst site on }\right. \\
\text { the catalyst, } \mathrm{R} \text { is the remainder of } \\
\text { the reactant) }\end{array}$ & $\begin{array}{l}\text { (B-1) Nascent hydrogen atoms forms on } \\
\text { the catalyst surface, } \\
\mathrm{RH}+2 \mathrm{~S}^{*} \rightarrow \mathrm{RS}^{*}+\mathrm{HS}^{*}\end{array}$ \\
\hline $\begin{array}{l}\text { (A-2) Desorption of hydrogen from } \\
\text { catalyst surface, } \\
2 \mathrm{HS}^{*} \rightarrow \mathrm{H}_{2}+2 \mathrm{~S}^{*}\end{array}$ & \\
\hline $\begin{array}{l}\text { (A-3) Molecular hydrogen diffuses } \\
\text { through gas phase to membrane } \\
\text { surface for chemisorption }\end{array}$ & \\
\hline $\begin{array}{l}(\mathrm{A}-4) \text { Permeation of chemisorbed } \\
\text { hydrogen. Atoms permeate from } \\
\text { surface to membrane lattice; desorb } \\
\text { from low pressure side of } \\
\text { membrane and diffuse to bulk } \\
\text { phase of vapour, } \\
\mathrm{PdH}^{*}(\mathrm{~S}) \rightarrow \mathrm{PdH}^{*}(\mathrm{M}) \rightarrow \mathrm{PdH}^{*}(\mathrm{~S}-) \rightarrow \\
\mathrm{Pd}^{*}(\mathrm{~S}-)+(1 / 2) \mathrm{H}_{2}\end{array}$ & $\begin{array}{l}\text { (B-2) Nascent hydrogen atoms on the } \\
\text { catalyst surface spillover to membrane } \\
\text { surface for chemisorption directly; } \\
\text { permeate into lattice structure of } \\
\text { membrane and desorb out from the low- } \\
\text { pressure side of membrane, } \\
\mathrm{Hs}^{*}+\mathrm{Pd}(\mathrm{S}) \rightarrow \mathrm{PdH}^{*}(\mathrm{~S}) \rightarrow \mathrm{PdH}^{*}(\mathrm{M}) \rightarrow \\
\mathrm{PdH}^{*}(\mathrm{~S}-) \rightarrow \mathrm{Pd}^{*}(\mathrm{~S}-)+(1 / 2) \mathrm{H}_{2}\end{array}$ \\
\hline
\end{tabular}

This energy shortcut leads to a rate enhancement when the catalyst and membrane are close or have a surface contact. Figure 1.2.11 shows four different reactors tested by Rei et al. [28]. Figure 1.2.11b, c and d each have a $10 \mathrm{~cm}$ stainless steel tube connecting the catalysts to the palladium membrane, in Figure 1.2.11c the steel tube is coated with nickel and in Figure 1.2.11d it is coated with palladium. The nickel and palladium surfaces have low activation energy for hydrogen spillover and produce higher hydrogen flux and greater conversion than the uncoated stainless steel surface or in the absence of a surface contact [28]. This increased membrane flux through spillover is reported to occur even without increasing the hydrogen partial pressure on the reactant side of the membrane [25]. 
Jonathan Tailby

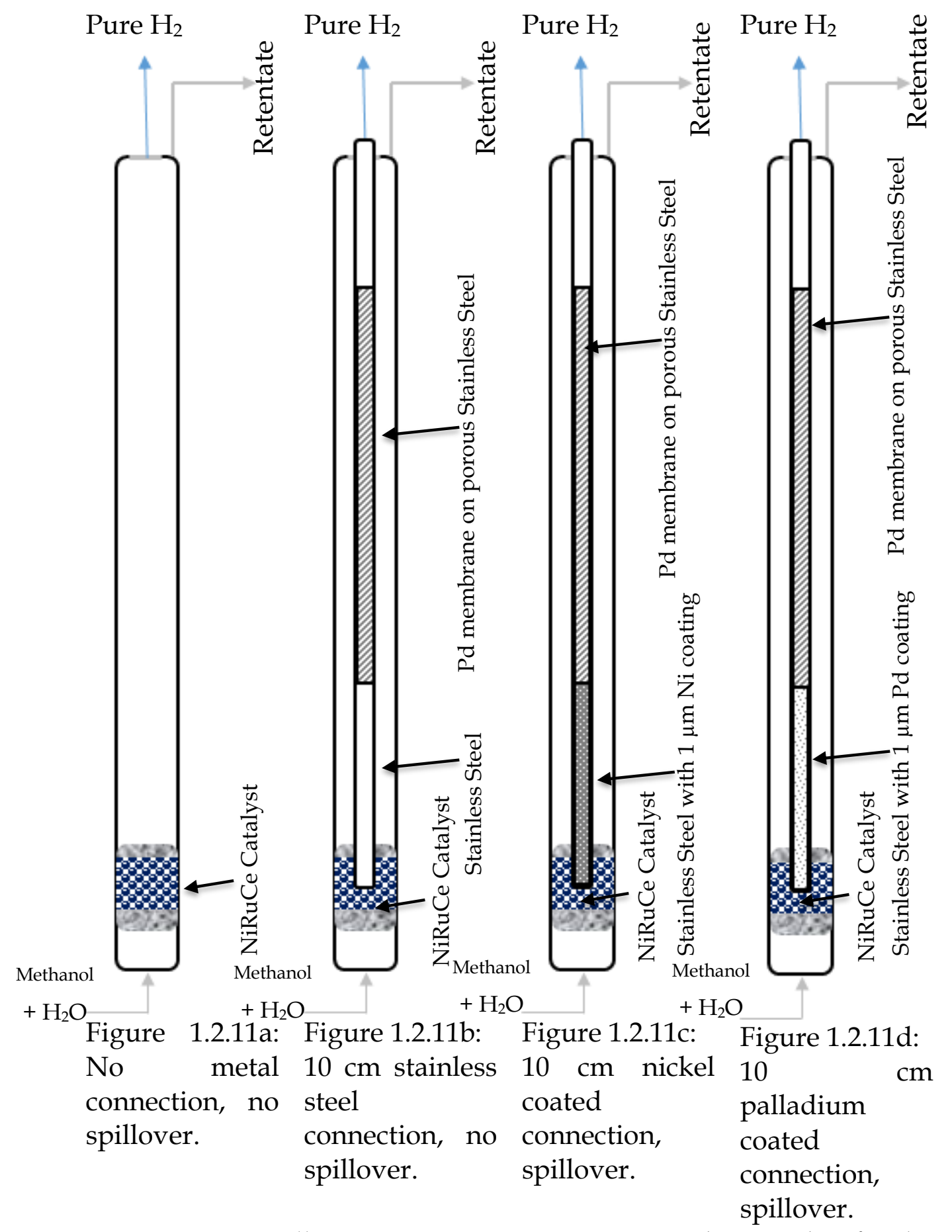

Figure 1.2.11: Spillover reaction tests. HSP-M membrane tubes for the methanol steam reforming reaction at $240^{\circ} \mathrm{C}$.

In a previous study by Rei et al., palladium doping of a $\mathrm{CuO} / \mathrm{ZnO} / \mathrm{Al}_{2} \mathrm{O}_{3}$ catalyst for methanol steam reformation in a membrane reactor increased the turnover frequency of the catalyst [63]. Hydrogen spillover decreased the occupancy time of the hydrogen on the catalytic site and increased the rate of hydrogen production [63]. Studies of ammonia decomposition on several catalysts have 
found desorption of nitrogen to be a slow step, becoming rate limiting when the nitrogen fills reaction sites $[19,53,65,134,135]$. If a suitable desorption surface is put in contact with the catalyst, spillover of nitrogen could enhance the rate of reaction. Palladium has been reported to be a receiver for spillover nitrogen from molybdenum and tungsten [136, 137]. If it can also receive the nitrogen atoms from the nickel catalyst in this research, it will increase the rate that active sites are made available on the catalyst.

\subsubsection{Palladium-Hydrogen}

The enhancement from a palladium membrane reactor comes from the special interaction between hydrogen and palladium. Palladium is able to absorb hydrogen into its metallic structure, allowing it to be used for storage of hydrogen [46, 138-140], or as a highly selective filter [141-143]. Hydrogen is absorbed from a gaseous mixture with a high hydrogen partial pressure at a palladium surface [144], typically into a foil or a sheet. The hydrogen passes out the side of the palladium with a lower hydrogen partial pressure, separating it from all other gases on the absorption side of the foil membrane[105, 145].

\subsubsection{Hydrogen Adsorption by Palladium}

When hydrogen gas adsorbs directly onto a palladium surface, the hydrogen molecules from the gas phase contact the surface. If they hit at an appropriate site with appropriate angle and energy, they can be adsorbed onto that site [146]. The site where adsorption occurs requires a tetrahedral vacancy above the palladium lattice, but clusters of three or more vacancies have a much higher sticking effect [147]. The type and number of available vacancies is related to the atomic topography and crystal faces available [148].

Hydrogen is initially adsorbed as the di-hydrogen molecule, and diffuses on the palladium surface to an octahedral vacancy before it can dissociate into hydrogen atoms. This dissociation is the rate limiting step in the surface processes of hydrogen absorption by palladium. Hydrogen atoms diffuse into the subsurface layer at tetrahedral sites [148]. This involves an extra diffusion step after dissociation and competes for sites with hydrogen molecules adsorbing from the 
Jonathan Tailby

gas phase. The concentration of hydrogen atoms in the subsurface layer is important to the rate of diffusion from surface to subsurface layer [148].

\subsubsection{Palladium Hydrides}

Palladium metal and its hydrides are face centred cubic crystals with tetrahedral and octahedral interstices. The absorbed hydrogen atoms are reported to be resident in the octahedral interstices, but to transfers through the structure via the tetrahedral interstices $[148,149]$. The palladium contributes electron density to the hydrogen from palladium d orbitals. The hydridic character of the trapped hydrogen is shown by an up-field shift in the proton NMR [150]. The palladium lattice expands with the absorption of hydrogen.

At high concentration of dissolved hydrogen, palladium hydrides nucleate within the palladium. Initially there is a low concentration of palladium hydride, so there is no long-range order in the palladium. With greater hydrogen concentration within the palladium, the palladium hydrides nucleate, forming two types of palladium hydride structure.

The two phases of palladium hydride are referred to as alpha $(\alpha)$ and beta $(\beta)$. The a hydride is the dilute form, and is the phase which forms first. Because of its limited capacity, it is better suited to selective permeation of hydrogen. The $\beta$ hydride is more concentrated, and expands much more than the a form. Because of its higher capacity for absorbed hydrogen, it is a better material for hydrogen storage. The dominant phase varies with temperature and hydrogen concentration, with a large region where the two phases coexist. Low concentration favours the a phase and high concentration favours the $\beta$ phase, but the transition point moves, becoming higher with temperature.

The hydride phases have increased volume with respect to the parent palladium metal. Palladium has a lattice parameter of $0.3890 \mathrm{~nm}$, while a $\mathrm{PdH}_{0.07}$ is 0.3894 $\mathrm{nm}$ and $\beta \mathrm{PdH}_{0.67}$ is $0.4025 \mathrm{~nm}$ [148]. The nucleation of hydrides with a higher volume fraction causes plastic deformation of the lattice [151].

The deformation of the lattice and changes in the metal matrix increase the presence of dislocations. This can be measured by positron annihilation 
spectroscopy showing increased dislocation loops in hydrogen loaded samples of palladium [152]. Dislocation density can increase strength and hardness in metals, but decreases ductility.

The two-phase region where the two phases coexist is where most of the rapid changes in volume occur. Formation of a palladium hydride has little effect on volume, and the two-phase region must be traversed as the larger volume $\beta$ phase is formed. The volume changes cause plastic deformation therefore physical changes such as hydrogen embrittlement occur in passing through the two-phase region. The greater amount of this two phase region a sample crosses, the greater the effect of the physical change[153]. The size of the two phase region is related to the difference in volume between the two phases [153].

As temperature increases, the two-phase region gets narrower. At $470 \mathrm{~K}$ it has narrowed significantly and the physical effect of absorption-desorption decreases [153].

Repeated cycles of hydrogen absorption and desorption do not continue to have increasing effect on the physical properties [153]. Most of the build-up of dislocations happens in the first cycle, so there is a major change in physical properties [153]. Subsequent cycles both create and destroy dislocations, so an upper limit is reached after a few cycles with a steady state quantity of dislocation and physical properties [153].

\subsection{Preventing Hydrogen Embrittlement}

The extent of hydrogen embrittlement leading to failure of the palladium membrane can be managed. Above $570 \mathrm{~K}$ the $\beta$ hydride does not form in pure palladium. Hence, if a permeation membrane is not exposed to hydrogen until it is above this critical temperature, there will be less deformation [154, 155]. Alloying with other metals is also a way to stabilise the a hydride and improve permeability. Silver and copper are popular alloying metals; the larger silver atom deforms the palladium lattice, which lowers the temperature where the $\beta$ hydride does not form. The optimum alloy is reported to be 23 atom $\%$ silver 77 
Jonathan Tailby

atom $\%$ palladium $[156,157]$. Alloying the palladium with a less expensive metal also reduces the cost of the membrane.

\subsubsection{Hydrogen Transmission}

The transmission of hydrogen through a palladium membrane can be resolved into several steps. Several similar but non-identical models are reported in the literature and a summary is given in Table 1.2.6. The differences in the models are whether adsorption and dissociation are treated as separate steps or as one combined step, and an equivalent for the reverse step of association and desorption, and the modelling of mass transfer outside the palladium.

\begin{tabular}{|c|c|c|c|c|}
\hline & $\begin{array}{l}\text { e 1.2.6: Different steps } \\
\text { neation. }\end{array}$ & in $\mathrm{mu}$ & ep models & hydrogen \\
\hline & & $\begin{array}{l}\text { Basile08, } \\
\text { Li08 [99, } \\
110]\end{array}$ & $\begin{array}{l}\text { Ward and } \\
\text { Dao99 [112], } \\
\text { Caravella08 } \\
{[142]}\end{array}$ & $\begin{array}{l}\text { McLeod09 } \\
{[158]}\end{array}$ \\
\hline $\mathbf{a}$ & $\begin{array}{l}\text { Bulk to surface mass } \\
\text { transfer in gas }\end{array}$ & & 1 & \\
\hline $\mathbf{b}$ & Adsorption onto Pd surface & 1 & 2 Dissociative & 1 Dissociative \\
\hline c & Hydrogen dissociation & 2 & adsorption & adsorption \\
\hline d & Surface to bulk transition in $\mathrm{Pd}$ & 3 & 3 & 2 \\
\hline e & Diffusion through Pd bulk & 4 & 4 & 3 \\
\hline f & Bulk to surface transition in $\mathrm{Pd}$ & 5 & 5 & 4 \\
\hline g & Hydrogen association & 6 & 6 Associative & 5 Associative \\
\hline $\mathbf{h}$ & Desorption from Pd surface & 7 & desorption & desorption \\
\hline i & $\begin{array}{l}\text { Surface to bulk mass } \\
\text { transfer in gas }\end{array}$ & & 7 & \\
\hline
\end{tabular}

Some researchers, especially those working on membrane reactors, use a single overall model such as Richardson's equation for hydrogen transport through the membrane, covering all the steps in Table 1.2.6. Richardson's equation is written in Equation 1.2.21 [3]:

Equation 1.2.21: Richardson's equation for predicting Hydrogen flux.

$J_{H_{2}}=\frac{P_{e_{0}} \exp \left(-\frac{E_{a}}{R T}\right)\left(\sqrt{p_{H_{2}, r e t}}-\sqrt{p_{H_{2}, p e r}}\right)}{\delta}$ 
Where $J_{H_{2}}$ is the hydrogen flux through the membrane in $\mathrm{mol} \mathrm{m}^{-2} \cdot \mathrm{s}^{-1} ; \mathrm{p}_{H_{2}}$,ret is the partial pressure of hydrogen in the gases retained on the upstream side of the membrane and $\mathrm{p}_{H_{2}, \text { perm }}$ is the partial pressure of hydrogen on the downstream side of the membrane, both pressures in Pa. $\delta$ is the thickness of the membrane. $P_{e_{0}}$ is the preexponential factor in $\mathrm{mol} \cdot \mathrm{m} \cdot \mathrm{s}^{-1} \cdot \mathrm{m}^{-2} \cdot \mathrm{Pa}^{0.5}$ and $E_{a}$ is the apparent activation energy in $\mathrm{kJ} \mathrm{mol}^{-1}$. Equation 1.2.21 assumes difference in partial pressure to be the driving force of diffusion [3], and experimental design that increases this difference improves the efficiency of membrane reactors $[99,159]$. Reactors can increase partial pressure difference with low flow rate[2] and counter flow reactors [3].

Ward and Dao developed a model that individually treats each of the seven steps of transmission [112]. Overall, this model produces results that are similar to Richardson's equation and are diffusion limited with flux inversely proportional to the thickness of the membrane [112]. Caravella produced a development of the Ward and Dao model that changed the driving force of movement through the bulk palladium from partial pressure difference to chemical potential [142] and paid more attention to the mass transport outside the palladium membrane, particularly where a porous support is present [142].

\subsubsection{Rate Limiting Step of Hydrogen transmission through Palladium}

Models and experimental results agree that in the conditions commonly used, diffusion through the bulk palladium is the rate limiting step [158]. Surface processes can be rate limiting in ultra-thin membranes, especially at low temperatures or if the surface is contaminated. Contaminants that affect palladium membranes include mercury, hydrogen sulfide, sulfur dioxide, thiophene, arsenic, unsaturated hydrocarbons, carbon dioxide, carbon monoxide and compounds of sulfur, arsenic, halides and zinc [99]. Some of which are potential impurities in ammonia, including sulfur compounds, $\mathrm{CO}_{x}$ gases, and unsaturated hydrocarbons. Air oxidation of the palladium surface can also reduce these surface processes [99], but this oxide layer can often be removed by reduction. 
Jonathan Tailby

\subsubsection{The Palladium-Hydrogen System and Ammonia Decomposition}

The reactor design proposed for this study had a feed gas of pure $\mathrm{NH}_{3}$ reacting with a catalyst on the surface of the palladium. The hydrogen partial pressure difference commenced at zero and was expected to remain low. Therefore, if a model is used that assumes hydrogen partial pressure is the driver for hydrogen transport, low flux is predicted. However, when each step of the process is modelled individually, as in Table 1.2.6, the prediction supports permeation of hydrogen $\mathrm{NH}_{3}$ decomposition by the catalyst on the membrane.

The catalyst in direct contact with the palladium membrane was predicted to spillover hydrogen produced by ammonia reformation. It was predicted this would be in the form of atomic hydrogen. Spillover then avoids steps b and $\mathrm{c}$ in the transmission process, starting instead with hydrogen atoms spilled over onto the membrane. In the multistage models of Ward and Dao [112] or Caravella [142], the step relying on the feed side hydrogen partial pressure or concentration is step 2, dissociative adsorption. This is one of the steps avoided by hydrogen spillover [63]. These models are however based on net flux from both forward and reverse processes, so the reverse of step 2 was still expected, resulting in a prediction of hydrogen gas in the retained flow gas.

The reverse of steps $b$ and c can be represented by steps $g$ and $h$, associative desorption [142]. This predicts that reverse step $b$ and $c$ has more resistance than step $d$, so more of the hydrogen that gets to the palladium surface will progress into the palladium than will desorb into the retained gas flow [142]. Rei et al [25] have tested other fuels for hydrogen production with spillover membrane reactors, and report increased flux through the membrane without increasing the hydrogen partial pressure on the retentate side.

The catalyst on the membrane partially obscures the surface of the membrane. Studies of surface contamination have shown that under ideal conditions this has little effect on the hydrogen flux, because the flux for the upstream surface processes are orders of magnitude higher than flux through the bulk of the palladium membrane[142]. Further, dissociative adsorption is the step most 
affected by surface contamination, a step which is avoided by hydrogen spillover from the catalyst to the membrane surface.

There is mixed evidence for whether ammonia and nitrogen inhibit hydrogen permeation through the palladium membrane. A study by Lundin et al found no evidence of flux inhibition in palladium membranes at up to $1 \mathrm{MPa}$ of a hydrogen nitrogen gas mixture or a hydrogen ammonia gas mixture [160]. This result is in contrast to another study by Wang et al, who found that hydrogen flux gradually decreased after 100 hours in an equimolar hydrogen nitrogen gas mixture [161]. 
Jonathan Tailby

\subsection{Research Plan}

The overall plan of this research is to design and develop a reactor to accelerate the decomposition of ammonia, based on surface contact between a nickel catalyst and a palladium membrane. This plan is broken down into steps as shown in Figure 1.1.1.

Catalyst preparation began first, and continued while reaction testing gave feedback about the preparation's viability as a catalyst and while the reaction testing set up itself developed and improved.

The plan combines three processes to enhance the reaction: Catalysis, Spillover and Hydrogen Transport through a palladium membrane. These processes were examined in detail in Sections 1.2.2.1, 1.2.4 and 1.2.6. The enhancement to the reaction from each of these three processes is to be quantified separately. This means that when they are combined, the individual contributions of each process can be resolved.

\subsubsection{Catalyst Preparation}

Nickel was selected for use as the catalyst. Although ruthenium is a more active catalyst for this reaction, nickel is a more economic and practical option, being cheaper and having a much higher annual production [19]. The literature contains more information on the activity of nickel in hydrogen spillover than similar activity of ruthenium [28]. There was also considerable experience of the use of nickel in the laboratory where this work was undertaken, both in electrodeposition and with ready-made nanoparticles. All of the catalysis steps relied on the reproducible preparation of the catalyst, so a repeatable method needed to be developed and shown to work.

\subsubsection{Electrodeposition}

The first method proposed for preparing the catalyst was electrodeposition, undertaken using methods detailed in Section 2.2.2. Electric current is passed through a solution with the substrate as the cathode. Metal ions in the solution are reduced to form metal deposits on the substrate, as described in Section 
1.2.5.2. To improve the product, a template was produced by lithography to control the shape of the nickel deposits, as described in Section 2.2.4.

\subsubsection{Spray Coating}

An alternative preparation method was to spray pre-prepared nickel particles onto the metal substrate. This method is detailed in Section 2.2.3.

\subsubsection{Preshaped Nickel}

Two types of preshaped nickel were tested as catalysts. A nickel wire square mesh was wrapped around the secondary insert for one series of experiments. Subsequently, pieces of nickel foam were compressed into the reaction space. This methodology is described in Section 2.2.5.

\subsubsection{Catalyst Characterisation}

For electrodeposited samples the primary characterisation tools were X-ray Diffraction (XRD) and optical microscopy. For spray coated materials a primary characterisation method was by accurate measurement of changes in mass. Selected catalyst samples were examined under Scanning Electron Microscopy (SEM). These techniques are described in Sections 2.1.3 to 2.1.5 with the details of their application in this work in Sections 2.2.6 to 2.2.8.

\subsubsection{Reaction Testing with the Catalyst}

The catalyst samples were tested to compare their activity in the ammonia decomposition reaction. The sequential design, redesign and operation of these reactors is described in detail in Section 2.2.9.

The output from the gas reactor was initially measured with a mass spectrometer. Unfortunately, it was observed that the ammonia damaged the electronics of the mass spectrometer so the measurement device was replaced with a gas chromatograph (GC), as described in Sections 2.2.11 and 2.2.12.

\subsubsection{Catalysis with Spillover}

To measure the influence spillover had on the catalyst, a membrane reactor was constructed with the palladium on top of sealed stainless steel so that it was 
Jonathan Tailby

unable to permeate hydrogen to the inside of the tube. The nickel was located in contact with this palladium to enable spillover. The reaction output was measured by the GC.

\subsubsection{Catalysis with Hydrogen transport}

To measure the influence of hydrogen transport on the catalyst, the processes need to be separated then recombined. This was undertaken by locating the nickel catalyst in the ammonia flow path before the palladium membrane on a tertiary insert, after which the outputs of the reaction side and the permeate side were each analysed by the GC.

\subsubsection{Catalysis with spillover + Hydrogen Transport}

To measure the combined influence of spillover and hydrogen transport a further tertiary insert was designed and fabricated with the nickel catalyst supported on the palladium membrane. The predicted process for catalysed decomposition of ammonia is shown Figure 1.3.1. with hydrogen atoms being removed from ammonia on the nickel catalyst, diffusing onto the palladium surface by spillover then diffusing through the palladium bulk to the exit side of the membrane where the hydrogen recombinatively desorbs into the sweep gas which carries the hydrogen gas to the gas chromatograph.

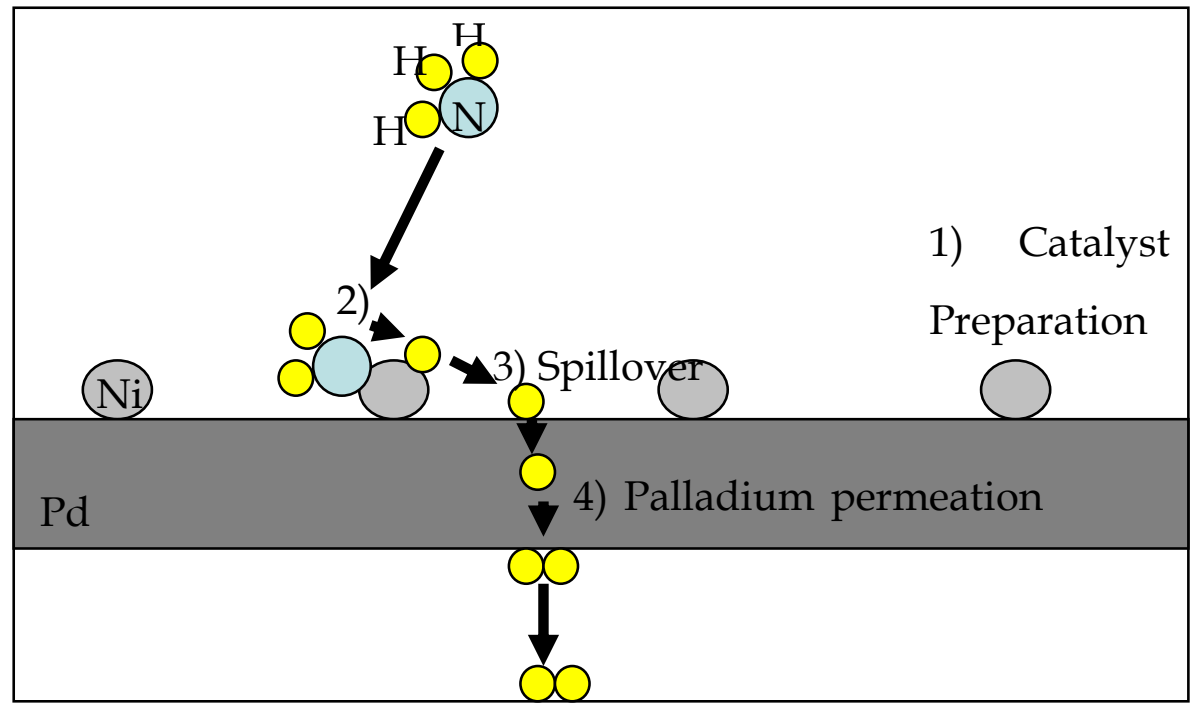

Figure 1.3.1: Sequence of events in the Catalytic Spillover Membrane active system. 


\subsection{Catalysis with Spillover + Hydrogen Transport + Porous} Anodic Alumina

The final stage of the research plan was to investigate substitution of palladium foil with ultrathin palladium membranes supported by porous anodic alumina. Because these membranes are very thin $(<100 \mathrm{~nm} \mathrm{Pd})$, the hydrogen transport should be fast, so it is expected that this membrane would improve the production of hydrogen. A first experiment would be to measure what effect this has on the production and quality of hydrogen from the decomposition of ammonia. A second experiment would be to measure which side of the membrane is better suited to support the catalyst. It is expected that the open side of the membrane will be better than the support side, but experiments would need to confirm this. 
Jonathan Tailby 


\section{Experimental}

This section describes the experimental techniques and methods used in this research. Section 2.1 describes the technical and theoretical background of some of the techniques used in this project. Section 2.2 describes how these methods were applied in this work.

Sections 2.1.1 to 2.1.2 describe the techniques used to prepare the catalyst samples. Sections 2.1.3 to 2.1.5 describe the three main methods used to characterise catalyst samples. Sections 2.1.6 and 2.1.7 describe the two techniques used to analyse the output of the gas reactors and how the data they provided has been interpreted.

Section 2.2.1 summarises the chemicals and materials used in these experiments. Sections 2.2.2 to 2.2.4 are counterparts of sections 2.1.1 to 2.1.2 that give the methodological details used for preparing catalysts. Section 2.2.5 describes relevant details of the preshaped nickel catalysts. Sections 2.2.6 to 2.2.8 are the methodological counter parts to Sections 2.1.3 to 2.1.5 identifying the instruments used in characterising the catalysts and describing how they were applied. Section 2.2.9 describes the sequence of gas reactors and the developments that refined them and led to the final method used to test the potential catalysts for decomposition of ammonia. Section 2.2.10 gives details on the process of catalyst testing with the secondary and tertiary inserts. Sections 2.2.10 and 2.2.12 are the descriptions of the methods used to analyse the products of the gas reaction tests, including the equations that were used to process the raw results from the Mass Spectrometer and the Gas Chromatograph. Some initial tests were made using the Mass Spectrometer, but all of the secondary and tertiary insert experiments used the Gas Chromatograph. Section 2.2.12.4 is a note on a difficulty with the Gas Chromatograph and how it was resolved.

\subsection{Experimental Techniques}

\subsubsection{Electrodeposition}

Electrodeposition was the first method of catalyst preparation explored for this project. This technique involves applying a voltage to a solution nickel ions. The 
Jonathan Tailby

resulting current reduced nickel ions at the cathodic working electrode, which deposits nickel metal onto the working electrode. Variation of the voltage, nickel ion concentration and deposition time changed the quantity and properties of the deposits. More details relating to this process are set out in Section 1.2.5.2.

Table 2.1.1 identifies specific electrochemical phenomena relevant to this electrodeposition. Each phenomenon requires a certain minimum voltage, the minimum voltages are reported in two columns, the first comparing them to a mercurous sulfate reference electrode because this is how they were reported in the source reference [124], the second column compares the minimum voltage to a silver chloride reference electrode because this was the type of reference electrode used in these electrodeposition experiments. Increasing the voltage increases the rate of deposition, but also increases the likelihood of side reactions.

\begin{tabular}{|c|c|c|}
\hline Phenomenon & Voltage vs. MSE (V) & Voltage vs. AgCl electrode (V) \\
\hline Oxidation of $\mathrm{Ni}$ & -0.8 & -0.4 \\
\hline $\begin{array}{l}\text { Ni electrodeposition kinetically } \\
\text { facile \& diffusion controlled }\end{array}$ & -1.0 & -0.6 \\
\hline $\mathrm{H}_{2}$ evolution begins. & -1.2 & -0.8 \\
\hline Voltage used in samples & -2.0 & -1.6 \\
\hline
\end{tabular}

\subsubsection{Lithography}

Photolithography is a microfabrication process that uses light to reproduce a pattern on a surface. A layer of photoactive polymer called a photoresist is deposited onto the clean substrate. A mask aligner with a patterned mask selectively exposes the photoresist to UV light. A developing solution dissolves the photoresist that was exposed to light and causes the remaining photoresist to cease being photoactive. This remaining photoresist now has a pattern matching that of the mask in the mask aligner.

\subsubsection{Optical Microscopy}

Optical microscopy uses lenses to enable sight of features smaller than are visible to the naked eye. Other tools for manipulation of light are included, from simple lamps to complex filters. It is common for modern optical microscope to have an 
electronic camera for connection to a computer for digital recording and manipulation.

\subsection{4. $\quad$ X-Ray Diffraction}

X-ray diffraction (XRD) is one of the key analytical tools of materials chemistry which gives information about the chemical structure and composition of crystalline phases. Collimated monochromatic X-rays irradiate the sample and are diffracted by interaction with the electrons of the atoms in the crystal lattice to give a diffraction pattern that is unique to the crystal structure of the material. A measured diffraction pattern may be compared to the Powder Diffraction File of XRD patterns from the International Centre for Diffraction Data (ICDD) to identify the crystalline materials present in the sample.

Further information that can be obtained by processing the XRD data includes the mean crystallite size. The Scherrer equation calculates crystallite size from the broadening of a relevant peak on the XRD diffractogram.

\section{Equation 2.1.1: Scherrer Equation}

$$
\tau=\frac{K \lambda}{\beta \cos \theta}
$$

In the Scherrer equation, $\tau$ represents the mean size of ordered crystallites. $\mathrm{K}$ is a shape factor chosen to be appropriate to the crystal shape. $\lambda$ is the wavelength of the X-rays used in the experiment. $\beta$ is the width of the peak at half maximum intensity, often referred to as full width half maximum (FWHM), after subtracting the instrumental line broadening, as measured on a standard. $\theta$ is the Bragg angle of the peak in radians. This equation assumes that the only contribution to peak broadening is from the crystallite size. Other factors may exist in the material which cause the calculation to underestimate crystallite size. The Scherrer equation can calculate crystallite sizes below $0.1 \mu \mathrm{m}$, and sometimes between 0.1 and $0.2 \mu \mathrm{m}$ but it is not applicable to crystallites larger than $0.2 \mu \mathrm{m}$. In this research, XRD was one of the methods used to analyse the samples prepared to be catalysts. Further details are included in section 2.2.7. 
Jonathan Tailby

\subsubsection{Scanning Electron Microscopy}

Scanning Electron Microscopy (SEM) is a key analytical technique that was used for analysis of catalyst samples. It was able to detect nickel deposits that were too light or too thin to be detected using XRD and optical microscopy. Further it could also be used to explore the shape and structure of these deposits.

A beam of electrons is directed at the sample and detectors are used to create digital images from either secondary electrons knocked out of orbit or electrons that bounce back from the target. The electron beam is at a shorter wave length than visible light which enables mapping of features smaller than visible by optical microscopy.

The instruments used in this project were also equipped with EDS detectors. This detects X-rays released by electron transitions after the electron beam has altered electron arrangement of atoms in the target, either by excitation or by secondary emission. These transitions and the corresponding X-rays are characteristic of the elements present, and are emitted proportionally to the relative quantities of those elements. This can be used to map the presence or absence of elements over a target area, or to give analysis of a single point. The analysis used in this project was only semi-quantitative because not all of the emitted X-rays arrive at the detector, the $\mathrm{X}$-ray response of different elements are not all equal, and the proportions are calculated from the detected emissions without an independent standard.

\subsubsection{Mass Spectrometry}

Mass spectrometry is an analytical technique that ionises chemical species and separates the ions based on their mass to charge ratio during their passage through a magnetic field. Charged species with high mass to charge are least deflected by the magnetic field while conversely low mass to charge particles are most deflected.

The results give relative quantities of the abundance of the detected species but the data are not fully quantitative. In the present case, the key mass/charge (m/z) signatures are: $\mathrm{H}_{2}(2) ; \mathrm{N}_{2}(14,28) ; \mathrm{NH}_{3}(16,17) ; \mathrm{H}_{2} \mathrm{O}(17,18) ; \mathrm{O}_{2}(16,32) ; \mathrm{Ar}$ 
(40). Although other minor fragments may contribute to the observed mass spectrum, the ratio between fragments within a particular species is fixed and known so that, for example, the source of a signal at m/z 17 can be assigned uniquely to $\mathrm{NH}_{3}$ or $\mathrm{H}_{2} \mathrm{O}$ by considering the relative contribution of signals observed at $\mathrm{m} / \mathrm{z} 16$ and 18 .

\subsubsection{Gas Chromatography}

The main technique used for analysis of the output from the gas reactor during ammonia decomposition experiments was gas chromatography ("GC"). The GC takes a small sample of the gas mixture, separates it into the component gases then measures the quantity of each component.

Individual components of the gas mixture travelled through the column at different rates, therefore they left the column at different times where the TCD registered their presence and indicated their quantity as a signal which was recorded by the computer. The data is displayed as a chromatogram showing peaks which are identified by the time between injection and detection, called retention time and quantified by the peak area compared to an appropriate standard.

The carrier gas for the GC was argon, initially set to 30 psi. The GC is very sensitive to even very small changes in the carrier gas pressure, such changes affect both retention time and thermal conductivity. The carrier gas pushes the sample through the column and the detectors at a fixed rate. In this GC design the carrier gas is also the reference gas for the TCD, therefore, measurements are made relative to argon at 30 psi. The GC had a computer-controlled sampling valve for gas analysis. Continuous gas flow could be fed through gas lines in the valve oven until the sampling valve would inject samples at programmed intervals. A second valve in the valve oven could select the volume of the sample loop, either 2.0 or 0.2 millilitres. The sample is injected into a column for separation. The column itself is packed with material that has a different affinity for different species. The Porapak QS column used in this research had high affinity for ammonia, therefore ammonia moved through the column slowly and had a high retention time. The packing material had a low affinity for hydrogen, 
therefore hydrogen moved quickly through the column and had a low retention time. Three other parameters which enable control of retention time are the rate of carrier gas flow, the length of the column and the column temperature. Clearly a longer column will have longer retention time: the 1 metre Porapak QS which was in the GC when it was first used had a third of the retention time of the 3 metre Porapak QS column which was used for the reaction measurements, when all the other parameters were unchanged. Lower temperature increases retention time. Increased retention time also increases resolution between peaks. Two species with similar retention times may appear as one peak at high temperatures; overlapping peaks at medium temperature and two peaks at low temperature. A low temperature was chosen here to endeavour to resolve the hydrogen and nitrogen peaks even though that meant that ammonia took a long time to completely elute.

Affinity for the column medium also affected the shape of peaks. A species with low affinity for the column medium, and therefore a low retention time, tends to have a narrow peak as the species moves through the column quickly and freely, and all of that species exits the column close together. A species with higher affinity for the column medium, hence a longer retention time, tends to form a wider based peak. With the Porapak Q or QS columns the peaks of ammonia and carbon dioxide tend to rise quite quickly as the first part of that species leaves the column together, but they have long tails while molecules of the species continue to leave the column and the peak slowly decreases to the baseline.

Retention times and relative gas quantities for a GC are established using known standards. The simplest standards are pure gases or commercially available standard mixtures. A $100 \%$ nitrogen standard was used every day to confirm the GC was in running order and was giving consistent results. A standard containing $5 \%$ hydrogen in $95 \%$ nitrogen was checked at the start of each week. 100\% Ammonia standards were also checked.

The GC measurement is independent of the rate of sample flow through the reaction. Measurements are made from a single injection of sample, therefore, if 
sufficient time is allowed between injections for the sample loop to refill then rate of sample flow will have no effect. Flow rate through the sample loop will affect how much time is required to completely fill the sample loop, but will have no other effect on the GC measurement. The sample flow is coming directly from the gas reactor and will be the same as the gas flow through the reactor, therefore any changes in GC measurement will reflect changes in gas composition rather than artefacts of the chromatography procedure.

\subsubsection{Mass Balance}

Full mass balance of these experiments cannot be performed because the gas flow measurements out of the gas reactor was not able to be accurately recorded by the equipment available. Therefore, an accurate comparison of the mass into the system with the mass out cannot be made. Instead, the following equations have been developed to allow for the volume increase resulting from the decomposition of ammonia into hydrogen and nitrogen.

\subsubsection{Standardisation of GC Measurements and Decomposition Mass Balance}

To enable comparison of measurements from different days, each sample measurement was compared to a measurement for a standard of known concentration from the same day as the test measurements. For ammonia and nitrogen this was achieved by dividing the area of the peak in the sample by the peak area of the standard. Hydrogen was standardised to the 5\% hydrogen sample by including a factor of $5 \%$ with the standard in the calculation.

$$
\text { percentage }_{\text {sample }}=\frac{\text { area }_{\text {sample }}}{\text { area }_{\text {standard }}} \times \text { percentage }_{\text {standard }}
$$

The GC response was assumed to be directly proportional to quantity of a species. This was supported by comparison of the two nitrogen samples, the $95 \%$ standard gave a peak area $95 \%$ of the $100 \%$ standard.

Equations were derived to calculate the molar flow of each gas species using the percentage of that gas in the outputs. These equations are explored further in Section 2.2.12.3.1. 
Jonathan Tailby

\subsection{Experimental Method}

\subsubsection{Chemicals and Materials Used}

\subsubsection{Electrodeposition Chemicals}

\begin{tabular}{|l|l|l|l|}
\hline Chemical & Source & Supplier & Purity \\
\hline $\mathrm{NiSO}_{4} \cdot 6 \mathrm{H}_{2} \mathrm{O}$ & $\mathrm{BDH}$ & House Stock & $\geq 98 \%$ \\
\hline $\mathrm{H}_{3} \mathrm{BO}_{3}$ & Cambridge & House Stock & $\geq 99.5$ \\
\hline $\mathrm{NiCl}_{2} \cdot 6 \mathrm{H}_{2} \mathrm{O}$ & Sigma-Aldrich & House Stock & $\geq 98 \%$ \\
\hline $\mathrm{Ni}\left(\mathrm{NO}_{3}\right)_{2} \cdot 6 \mathrm{H}_{2} \mathrm{O}$ & & House Stock & \\
\hline $\mathrm{NH}_{4} \mathrm{Cl}$ & Sigma-Aldrich & House Stock & $\geq 99.5$ \\
\hline $\begin{array}{l}\mathrm{NaCl} \\
\text { Sulfate }\end{array}$ & Sigma-Aldrich & House Stock & $>98 \%$ \\
\hline $\begin{array}{l}\text { Aqueous NH}{ }_{3} \\
\text { Nickel }\end{array}$ & PanReac & House Stock & $28-30 \% \mathrm{NH}_{3}$ \\
\hline $\begin{array}{l}\text { Nickel } \\
\text { Electroplating Solution }\end{array}$ & Made in house & $1.33 \mathrm{molL}^{-1} \mathrm{Ni}^{2+}$ \\
\hline
\end{tabular}

\subsubsection{Substrate and Catalytic Metals}

\begin{tabular}{|l|l|l|l|l|}
\hline Metal & Use & Source & Supplier & Purity \\
\hline Aluminium Foil & $\begin{array}{l}\text { Catalyst } \\
\text { substrate }\end{array}$ & BDH Reagents & House Stock & $99.99 \%$ \\
\hline Palladium Foil & $\begin{array}{l}\text { Catalyst } \\
\text { substrate }\end{array}$ & Alfa Aesar & $\begin{array}{l}\text { Johnson } \\
\text { Matthey }\end{array}$ & $99.9 \%$ Metals \\
\hline Stainless Steel Foil & $\begin{array}{l}\text { Catalyst } \\
\text { Substrate }\end{array}$ & $\begin{array}{l}\text { Catalyst } \\
\text { Substrate }\end{array}$ & House Stock & 316 Stainless \\
\hline Stainless Steel Tube & & & \\
\hline
\end{tabular}




\begin{tabular}{|l|l|l|l|l|}
\hline Metal & Use & Source & Supplier & Purity \\
\hline $\begin{array}{l}\text { Palladium Silver } \\
\text { Foil }\end{array}$ & Membrane & Alfa Aesar & $\begin{array}{l}\text { Johnson } \\
\text { Matthey }\end{array}$ & $\begin{array}{l}99.9 \% \text { Metals } \\
75.25 \text { wt.\% Pd }\end{array}$ \\
\hline Nickel Mesh & Catalyst & Goodfellow & House Stock & \\
\hline Nickel Foam & Catalyst & $\begin{array}{l}\text { Inco Advanced } \\
\text { Technology materials } \\
\text { (Dalian) Co. Lt, Taiwan }\end{array}$ & House Stock & \\
\hline
\end{tabular}

\subsubsection{Gases}

All gases supplied by BOC Scientific.

\begin{tabular}{|l|l|l|}
\hline Gas & Use & Purity \\
\hline Argon & $\begin{array}{l}\text { Sweep gas, inert gas, GC carrier } \\
\text { and reference gas }\end{array}$ & $>99.999 \%$ \\
\hline Nitrogen & GC standard, inert gas & $>99.99 \%$ \\
\hline Hydrogen & GC standard & $>99.98 \%$ \\
\hline $95 \%$ Nitrogen $5 \%$ Hydrogen & GC standard & $95 \% \mathrm{~N}_{2}, 5 \% \mathrm{H}_{2}$ \\
\hline Ammonia & GC standard, Reactant & $>99.9 \%$ \\
\hline
\end{tabular}

\subsubsection{Electrodeposition}

A preliminary experiment was undertaken to gain familiarity with the process. This experiment used the nickel sulfate solution with the composition shown in, Table 2.2.1 which had been developed for a prior experimental programme at IRL. A brass rod was used for the working electrode, $30 \mathrm{~s}$ at $-0.9 \mathrm{~V}$ vs. $\mathrm{AgCl}$ produced a complete nickel coating over the brass where it had been exposed to the solution. This coating strongly adhered and could not be scraped off by a finger nail.

The next experiments tested the conditions necessary to prepare useful catalyst samples. $12 \mathrm{~mm}$ squares of $99.9 \%$ aluminium were placed in accordance with the cell shown in Figure 2.2.1. This exposed a circle of metal $8 \mathrm{~mm}$ in diameter, an 
Jonathan Tailby

area of $0.50 \mathrm{~cm}^{2}$. After deposition, the samples were gently rinsed by placing them in a petri dish of distilled water so any remaining deposition solution would wash off with minimum disturbance to the deposits.
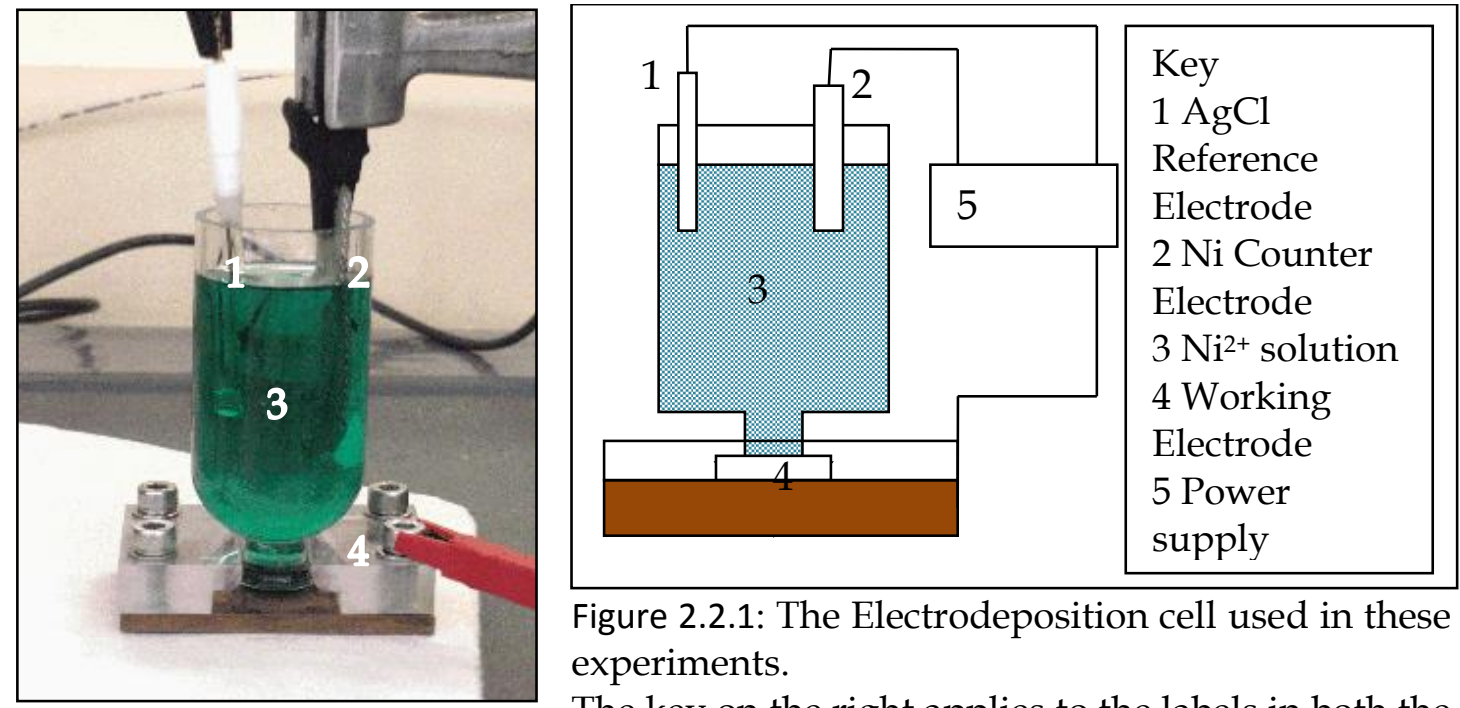

Figure 2.2.1: The Electrodeposition cell used in these experiments.

The key on the right applies to the labels in both the diagram and the photograph.

The electrodeposition solution had the composition shown in Table 2.2.1. The combination of salts produced a buffered solution of $\mathrm{pH} 4.33$ with a nickel ion concentration of 1.33 molL-1 $^{-1}$ Electrodeposition solutions of this composition were already in use by several projects at Industrial Research Laboratory.

\begin{tabular}{|c|c|c|c|}
\hline & $C\left(g^{\prime} L^{-1}\right)$ & Molar mass & $\mathrm{C}\left(\mathrm{mol} \mathrm{L}^{-1}\right)$ \\
\hline $\mathrm{NiSO}_{4} 6 \mathrm{H}_{2} \mathrm{O}$ & 300 & 262.84 & 1.14 \\
\hline $\mathrm{H}_{3} \mathrm{BO}_{3}$ & 45 & 61.83 & 0.73 \\
\hline $\mathrm{NiCl}_{2} 6 \mathrm{H}_{2} \mathrm{O}$ & 45 & 237.69 & 0.19 \\
\hline $\mathrm{Ni}^{2+}$ & & & 1.33 \\
\hline
\end{tabular}

Zach et al. report electrodeposition of nanonickel using a nickel nitrate solution [124] with the composition set out in Table 2.2.2. Application of this new solution did not give measurably improved results in the outcome of this experiment, therefore subsequent experiments used the original sulfate solution of the composition in Table 2.2.1. 
Table 2.2.2: Second electroplating solution, nickel nitrate.

Based on Zach [124].

\begin{tabular}{|llllrl|}
\hline & $\mathbf{C}\left(\mathbf{g} \cdot \mathbf{L}^{-1}\right)$ & Molar mass & $\mathbf{C}\left(\mathbf{m o l} \cdot \mathbf{L}^{-1}\right)$ & Mass for $\mathbf{2 5 0} \mathbf{~ m L}$ \\
\hline $\mathrm{Ni}\left(\mathrm{NO}_{3}\right)_{2} \cdot 6 \mathrm{H}_{2} \mathrm{O}$ & 2.908 & 290.79 & 0.01 & 0.727 & $\mathrm{~g}$ \\
$\mathrm{NH}_{4} \mathrm{Cl}$ & 53.492 & 53.49 & 1.00 & 13.373 & $\mathrm{~g}$ \\
$\mathrm{NaCl}$ & 58.443 & 58.44 & 1.00 & 14.611 & $\mathrm{~g}$ \\
\hline $\mathrm{Ni2}+$ & \multicolumn{5}{c}{0.01} \\
Adjust to $\mathrm{pH} 8.3$ with additional ammonia solution. \\
\hline
\end{tabular}

The three electrical systems used for electrodeposition are shown in Figure 2.2.2. Initial tests were undertaken with a N5751A DC power supply from Agilent Technologies controlled by a computer which ran LabVIEW and recorded data through a datalogger. Later experiments were undertaken using potentiostats, either Autolab PGSTAT128N or Princeton Applied Research PAR 173. The Autolab software recorded the current, whereas the current for the PAR173 depositions was displayed on an external ammeter and recorded by a digital camera. All depositions used an $\mathrm{AgCl}$ reference electrode, a nickel mesh counter electrode and the target substrate as the working electrode.

Voltage

The most common settings for the voltage source during electrodeposition were $-0.9,-1.6$ or $-2.0 \mathrm{~V}$. -0.9 and $-1.6 \mathrm{~V}$ were recommended by Industrial Research staff and $-2.0 \mathrm{~V}$ was the voltage used in the experiments reported by Zach with Nickel nitrate solution [124]. Other settings between 0.5 and $2.5 \mathrm{~V}$ were used as noted. 

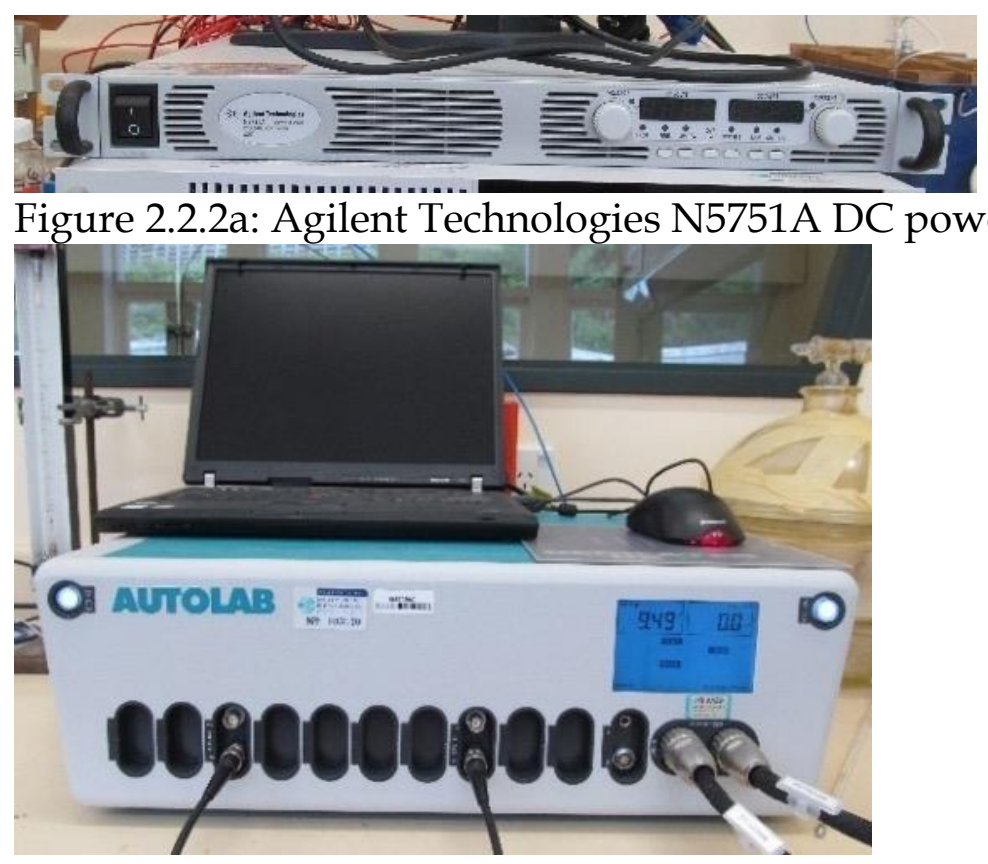

Figure 2.2.2b: Autolab PGSTAT128N Potentiostat

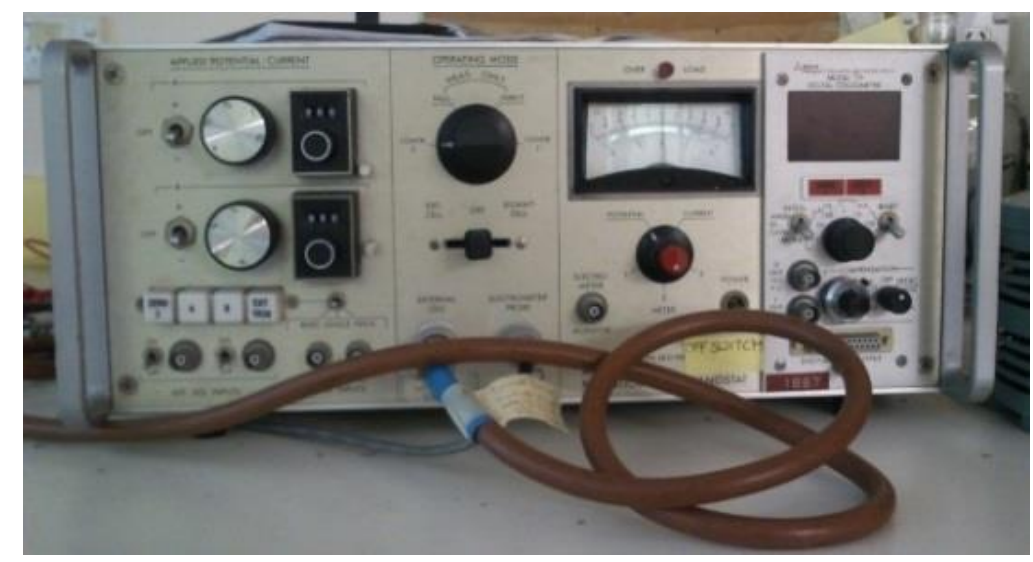

Figure 2.2.2c: Princeton applied research PAR173 Potentiostat Figure 2.2.2: The voltage sources used for electrodeposition.

\subsubsection{Electrodeposition on Aluminium}

In the first exploratory phase, aluminium was substituted for palladium as a nickel deposition substrate. Palladium is an expensive metal so only a limited quantity was available for this research. Therefore, cheaper metals were used as substrates until the electrodeposition process was better understood. Aluminium is a more cost-effective substrate for use during electrodeposition process development. The first metal examined was $0.7 \mathrm{~mm}$ thick $99.99 \%$ 
aluminium sheet supplied by BDH Reagents. The aluminium was cut into 12 $\mathrm{mm}$ squares then degreased by agitation for 10 minutes in a sonicator with ethyl acetate solution.

Experiments to explore the effect of voltage on the outcome were conducted at a range of voltages, from -0.5 to $-2.5 \mathrm{~V}$. These were repeated at least five times each at each $0.5 \mathrm{~V}$ step in this range, recording the current and examining the product under electron microscope. The results are reported in Section 3.1.1. A similar investigation was performed using different dilutions of the nickel nitrate solution in Table 2.2.2 at $-1.6 \mathrm{~V}$. The solution was diluted to $10 \%$ and to $1 \%$ of the concentration shown in Table 2.2.2.

Samples were each electrodeposited at $-1.6 \mathrm{~V}$ vs. a $\mathrm{AgCl}$ reference electrode with the nickel sulfate solution as set out in Table 2.2.1 using the electrodeposition cell shown in Figure 2.2.1.

The products of electrodeposition onto aluminium looked promising for catalysis; therefore, the decision was made to transfer the process to palladium.

\subsubsection{Electrodeposition on Palladium}

The apparent success of electrodeposition of nickel onto aluminium metal sheet encouraged the decision to progress the deposition process onto palladium foil. Islands of nickel deposited on the palladium was seen as optimum for the goal of a catalytic spillover membrane.

During electrodeposition experiments onto palladium, investigation was made into the possibility of electrodeposition for extremely short times or with varying the voltage during the electrodeposition. These showed that the equipment did not provide sufficient time resolution to improve results by these methods.

The details of the deposits are discussed in Section 3.1.1.2. The thickness of the nickel layers on palladium were estimated using the steps in Equation 2.2.1 and the constants in Table 2.2.3. 
Jonathan Tailby

Equation 2.2.1: Calculating the thickness of nickel on palladium from the electrodeposition data.

Charge transferred $=($ Average current $\cdot$ Total time $)$

$$
Q=(I \cdot t)
$$

number of electrons $=($ Charge transferred $/$ Charge on electron $)$

$$
n_{e}=(Q / q)
$$

Quantity of electrons $=($ number of electrons $/$ Avogadro's Number $)$

$$
N_{e}=\left(n_{e} / N_{A}\right)
$$

Quantity of Nickel $=($ Quantity of Electrons $/$ Charge of Nickel ion $)$

$$
N_{N i}=\left(N_{e} / 2\right)
$$

Mass of Nickel $=($ Quantity of $\mathrm{Nickel} \cdot$ relative mass of nickel $)$

$$
m_{N i}=\left(n_{N i} \cdot m r_{N i}\right)
$$

Volume of Nickel $=($ Mass of nickel $/$ Density of nickel $)$

\begin{tabular}{|c|c|c|c|c|c|}
\hline Charge on electron $(\mathbf{q})$ & $-1.602 \times 10^{-19}$ & & $\mathrm{Ni}$ atomic mass $\left(\mathbf{m r}_{\mathbf{N i}}\right)$ & 58.693 & $\mathrm{~g} \cdot \mathrm{mol}^{-1}$ \\
\hline Unit per mole $\left(\mathbf{N}_{\mathrm{A}}\right)$ & $6.022 \times 10^{+23}$ & $\mathrm{~mol}^{-1}$ & Ni density $\left(\boldsymbol{\rho}_{\mathrm{Ni}}\right)$ & 8.91 & $\mathrm{~g} \cdot \mathrm{cm}^{-3}$ \\
\hline Area $(\mathbf{A})$ & 0.50 & $\mathrm{~cm}^{2}$ & & & \\
\hline
\end{tabular}

$$
V_{N i}=\left(m_{N i} / \rho_{N i}\right)
$$

Thickness of Nickel $=($ Volume of nickel $/$ Electrodeposition area $)$

$$
T h_{N i}=\left(V_{N i}{ }_{A}\right)
$$

Table 2.2.3: Constants for calculating thickness of nickel on palladium.

\subsubsection{Spray Coating}

Because electrodeposition produced only continuous layers on palladium an alternative method was undertaken to obtain smaller deposits. This second method was to spray a suspension of nickel nanoparticles onto a warm metal 
substrate. The liquid evaporated leaving the nickel nanoparticles dispersed across the surface of the metal.

The suspension was made by agitation of 0.1 grams of nanonickel powder supplied by QSI Nano in 10 grams of ethanol. This was shaken in a sonicator for 10 minutes to suspend the nickel. A syringe was filled with the suspension then placed on an NE-300 syringe pump from New Era Pump Systems to give controlled delivery of the suspension to a Sonazap Ultrasonic Atomiser from Sonaer. The system was cleaned before and after each use by running a syringe of ethanol without nickel through from the pump to the atomiser and onto a steel foil.

During the spray process the substrate metal was heated to $65^{\circ} \mathrm{C}$ on a hot plate. This is only $13{ }^{\circ} \mathrm{C}$ below the boiling point of ethanol so the microscopic droplets of ethanol evaporated rapidly, leaving the nanonickel behind.

Results were measured by mass difference on a Mettler Vernier microbalance, capable of 6 decimal place resolution. Substrates were weighed before and after spraycoating. Results were also reviewed optically and were found to be inconsistent.

Aluminium had been shown to be a poor model system for testing catalyst formation on palladium, therefore, it was replaced with stainless steel during the spray coating experiments. Stainless steel was chosen because it is readily available in thin sheets.

\subsubsection{Lithography}

When spray coating was proven to be too inconsistent, photolithography was explored as a method to create a template that would allow nickel to be electrodeposited on palladium without forming continuous layers by controlling where nickel could deposit. As with the initial electrodeposition, this templated electrodeposition process was developed using a cheaper metal before applying it to palladium. Aluminium had already been replaced with steel. XRD of the steel showed it to closely resemble the pattern for an iron-nickel alloy, which seemed likely to behave more like palladium in electrodeposition than the 
Jonathan Tailby

aluminium had. Electrodeposition tests with untemplated steel foils showed the formation of complete layers similar to the layers formed on palladium.

\subsubsection{Lithography of Foil Samples}

Before coating, metal substrate samples were washed three times. First in acetone, second in isopropyl alcohol and third in deionised water. When dry, the substrates were spin coated with AZ4533 photoresist. The substrate was placed on a Headway Research PWM32-PS-R790 spin coater which secured the substrate to its stage using vacuum suction while the stage rotated. A drop of AZ4533 was placed in the middle of the substrate. The rapid rotation of the substrate spread the AZ4533 outward to make a thin film across the surface of the substrate. AZ4533 is sensitive to light so the template is prepared in a laboratory that is protected from natural light and fitted with yellow light bulbs. Spin coating onto the square electrodeposition substrates tended to leave the photoresist thickest in the corners, therefore these areas are least likely to have the templated holes fully penetrating the photoresist. The electrodeposition occurred in a circle centred near the middle of the square so the corners with the thicker photoresist were outside the deposition area. This method left a coating approximately $3 \mu \mathrm{m}$ thick in the deposition area. The samples were then heated on an Electronic Micro Systems EMS 1000 hot plate to dry the coating.

The coated samples were placed under a mask with the intended pattern on it then exposed to 365nm UV light in a Quintel Q4000 mask aligner. The exposed photoresist was developed in AZ826 solution. After developing in the AZ826 solution, the areas of photoresist that had been exposed to the UV light through the holes in the mask had dissolved and the remainder of the photoresist had become resistant to photodegradation. This remaining photoresist was the template for electrodeposition.

\subsubsection{Electrodeposition of Templated Samples}

The first templated sample to be electrodeposited was over deposited to allow creation of an example of a current-time graph for templated electrodeposition. The current was predicted to increase when the deposition overflowed the 102 
template as in the publication on growing nanowires in a template by Motoyama [127]. Table 2.2.4 shows the method for calculating the volume of the holes in the template that was used to calculate when the volume of nickel that would be needed to fill the holes in the template.

\begin{tabular}{|l|l|l|}
\hline Table 2.2.4: Template properties \\
\hline Each Hole & Radius $=5 \mu \mathrm{m}=5 \times 10^{-4} \mathrm{~cm}$ & Area $=7.9 \times 10^{-7} \mathrm{~cm}^{2}$ \\
\hline Pattern Unit & Square side $=20 \mu \mathrm{m}=20 \times 10^{-4} \mathrm{~cm}$ & Square Area $=4.0 \times 10^{-6} \mathrm{~cm}^{2}$ \\
\hline Pattern Proportion & Hole $=19.6 \%$ & Template $=80.4 \%$ \\
\hline Deposition Area & Radius $=0.4 \mathrm{~cm}$ & Area $=0.5 \mathrm{~cm}^{2}$ \\
\hline $\begin{array}{l}\text { Exposed deposition } \\
\text { Area }\end{array}$ & $\begin{array}{l}\text { Hole } \% \times \text { total area } \\
=19.6 \% \times 0.5 \mathrm{~cm}^{2}\end{array}$ & Exposed Area $0.10 \mathrm{~cm}^{2}$ \\
\hline Template Volume & $\begin{array}{l}\text { Area } \times \text { Height } \\
=0.10 \mathrm{~cm}^{2} \times 3.3 \times 10^{-4} \mathrm{~cm}\end{array}$ & Volume $=3.26 \times 10^{-5} \mathrm{~cm}^{3}$ \\
\hline
\end{tabular}

The desired product was isolated units of nickel which required deposition to be stopped before reaching the overflow stage. The previous electrodeposition had shown that electrodeposition on palladium was not similar to electrodeposition on aluminium; therefore, aluminium was replaced with stainless steel foil 0.09 mm thick. When satisfactory results were obtained on steel, the method was transferred to palladium. By this time however, the reactor design had progressed to using tubular samples, so the templating process needed further development.

The PAR173 potentiostat used to electrodeposit the templated samples required an extended warm up period for peak performance. It was found that depositing an untemplated stainless steel foil before electrodepositing onto a templated foil improved the current response and the uniformity of the product.

The most uniform sample was deposited after the electrodeposition failed to produce a current. The deposition equipment was examined and no faults were found, but the equipment still produced no current when the voltage was initiated. The equipment was disassembled and reassembled, and subsequently produced a high and steady early current response. The product of this deposition was the most even of all the templated electrodepositions.

The extended exposure to the deposition solution was suspected of having improved the surface conditions of the steel substrate, so a delay between 
Jonathan Tailby

preparation and voltage initiation was introduced as standard procedure. The electrodeposition solution was only a very weak acid, but the two failed electrodepositions may have contributed to reducing a passivating layer or otherwise improving the surface conditions.

\subsubsection{Lithography of Tubular Samples}

By the time the templated electrodeposition process approached readiness on foils, the gas reactor had changed to use tubular inserts. The process therefore needed to be adapted to enable creation of templates on the outside of the secondary insert tubes.

Three secondary insert tubes were electrodeposited without templates to compare their catalytic activity with the templated electrodeposits. The tubes were electrodeposited using the same equipment as the flat squares, modified as shown in Figure 2.2.3. The same nickel sulfate electroplating solution and the same nickel mesh counter electrode were used. The secondary insert tube acted as the working electrode. The untemplated secondary insert tubes were electrodeposited at $-2.0 \mathrm{~V}$ for 3 minutes.

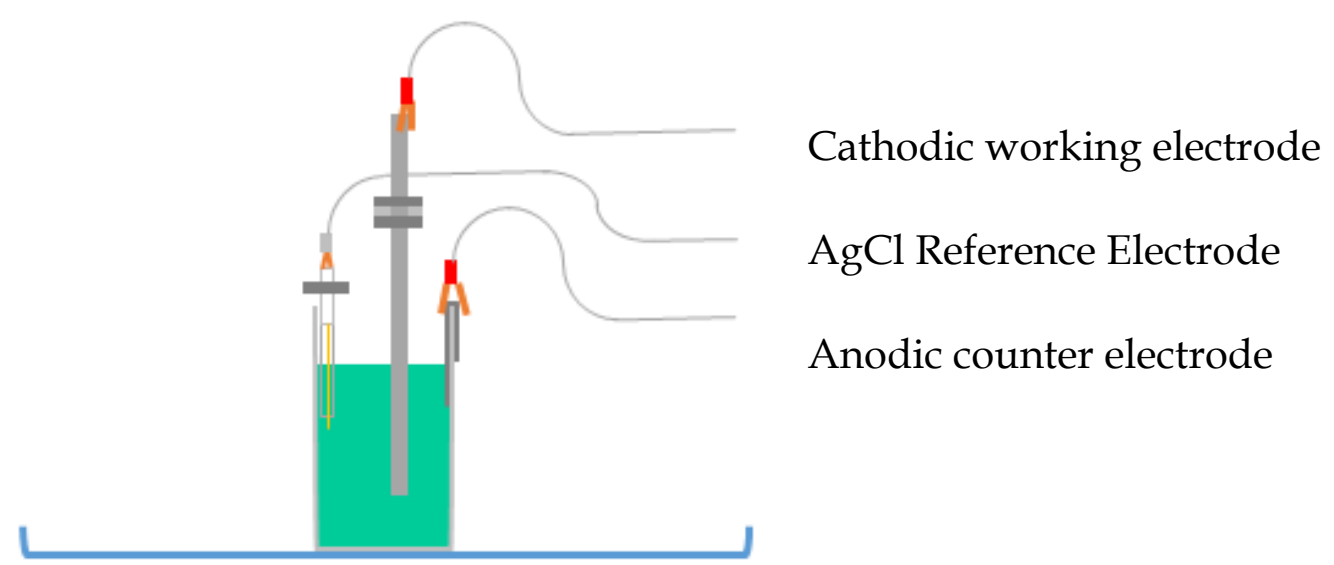

Figure 2.2.3: Tubular electrodeposition set up.

The tubular samples were washed with the same three steps as the metal foil squares described in Section 2.2.4.1 then dried vertically on the hot plate. They were then dip coated in the AZ 4533 photoresist to cover $50 \mathrm{~mm}$ of the $150 \mathrm{~mm}$ stainless steel tube. When the solvent had dried, the photoresist was cured in an 
oven at $80{ }^{\circ} \mathrm{C}$ for 2 hours. The Folex Densotrans HCP diazo film mask was wrapped around the coated tube and secured in place. The masked tube was rotated using a Hitachi DS 14DV electric drill under the mask aligner to expose it to the UV light. The mask was removed then the coating was soaked in the developing solution. The templated tube was electrodeposited using the same set up as the untemplated tubes, as shown in Figure 2.2.1.

The tubular samples were dip coated in the AZ4533 using a computer controlled motorised stage to lower the tubes into the solution then remove them at a constant rate. The tubes were dipped twice, moving at $2 \mathrm{~mm} \mathrm{~s}^{-1}$ with a $3 \mathrm{~s}$ hold at the bottom of each dip and a $4 \mathrm{~s}$ hold at the top between dips. The tubes were quickly inverted and placed on a heated drying platform to evaporate the solvent. They were cured by standing the tubes on a hot plate for 3 minutes to allow heat to be conducted to the coated end so that the end was kept at $100{ }^{\circ} \mathrm{C}$ for at least $50 \mathrm{~s}$. Excess coating solution was removed from the tip of the tube with a cotton bud before it could drip down the side of the tube.

Before use, the masks were heat treated to improve the fit on the steel tube. The mask was rolled and wrapped with a cover paper to protect the pattern on the mask then slipped inside a steel tube which was placed in an oven. The temperature was raised at $20^{\circ} \mathrm{C} \cdot \mathrm{min}^{-1}$ to $150{ }^{\circ} \mathrm{C}$ then held for $30 \mathrm{~min}$. On cooling, the mask retained some of the curve, making it easier to roll tightly around the coated tube without leaving any of the photoresist exposed.

The mask was secured to the tube using shrink wrap. The transmittance spectrum of shrink wrap was first measured to confirm that it would not interfere with the activation of the photoresist, this spectrum is shown in Appendix 5.1.1 (Figure 5.1.1). The wavelength of the exposure is $365 \mathrm{~nm}$ so there is $85.6 \%$ transmittance. The shrink wrap was wound tightly around the mask and heated with a heat gun to make it shrink to achieve an even tighter fit.

Although this templated electrodeposition method was successful on both flat foils and tubular substrates, it was recognised that there would be problems 
Jonathan Tailby

applying it to the insert with the permeation membrane because additional gas tubes attached to the sides of the main tube would interfere with the rotation of the tube under the mask aligner. A new method was proposed, using SUEX film as the templating medium. SUEX is the modern format of the industry standard permanent resist for high aspect ratio photolithography. It is an epoxy resin film with internal photoacid generator produced by DJ Microlaminates. The SUEX film can be templated before being applied to the substrate surface. The film is also available in a variety of thicknesses, all thicker than the layers of photoresist that were being deposited by spin coating and dip coating. The films are also more uniform in thickness so they were predicted to produce more consistent deposits that extend further into the gas space of the reactor.

The SUEX film method was first tested on steel squares using the same square arrays of circles as the coatings but with an increase in the scale of the pattern in some cases from $10 \mu \mathrm{m}$ circles to 15 or $25 \mu \mathrm{m}$ in a film $15 \mu \mathrm{m}$ thick. The outcome was extremely low current measurements and a lack of any detectable nickel on the finished products which suggests that the plating solution could not reach the metal surface to deposit nickel. This was either because the holes in those templates had not fully penetrated the film or because the increased height of the template caused the aspect ratio of the holes to become too narrow which prevented the solution from replenishing its nickel concentration.

This outcome was probably an aberration, that would have been resolved quickly. However, there was no opportunity to retest it at that time.

Because templating the tertiary inserts was not ready at the time, the experiments proceeded with an alternative catalyst. The tertiary inserts were used with nickel foam catalyst materials.

\subsubsection{Preshaped Catalysts}

Two types of preshaped nickel were tested as catalysts, a nickel mesh and a nickel foam. The mesh was first tested by replacing the foil square substrate with a square of mesh, then by pressing the mesh to the foil square. 
With the tubular reactor, the mesh was wrapped around the secondary tube then twisted at the end. This meant that the mesh filled the space between the two tubes. The nickel mesh was house stock believed to originally have been purchased through Goodfellow.

A second preshaped form of nickel was a nickel foam from Inco Advanced Technology materials (Dalian) Co. Lt, Taiwan. The nickel foam was only tested after the change to tubular substrates. The foam was supplied at $3.5 \mathrm{~mm}$ thick but the space between the two tubes was only $1.6 \mathrm{~mm}$, therefore a recess was cut into the external surface of the secondary tube and the foam was compressed into the space between the primary and secondary inserts. The foam was originally $90 \%$ unoccupied by nickel but the compressed foam reduced this unoccupied volume to $79 \%$. When the nickel foam was wrapped around the recess in the tube, the tube was rolled on a bench top to apply a small amount of compression. The tube with the compressed nickel foam was inserted into the outer tube so that the nickel foam completely filled the space between the two tubes for the length of the nickel.

\subsubsection{Optical Microscopy}

Optical microscopy was used throughout this research and was frequently a primary analysis tool, especially during the templated electrodeposition. The 10 $\mu \mathrm{m}$ features of the templates and deposits were beyond the naked eye but visible under optical microscopy.

The microscope used in this research was an Olympus BX60M optical microscopic fitted with five objective lenses ranging from 5-100 x. It was fitted with an Olympus ColorView1 digital camera for viewing and image capture using the AnalySIS software. Low magnification microscopy was used to quickly assess the products of electrodeposition and spray coating. Higher magnification microscopy was less useful because of difficulty with the topography of the samples in the short depth of field at high magnification. 
Jonathan Tailby

\subsubsection{X-Ray Diffraction}

The challenge with these samples was that the deposits were usually thin films or very small aggregations of even smaller crystals dispersed across the substrate. The thin films were best analysed by illuminating the specimens with X-rays at a low incident angle to maximise the path length in the film relative to the substrate. This achieved some satisfactory results. Dispersed aggregations sometimes could not be detected using XRD and required SEM/EDS analytical methods, due to the ability of SEM/EDS to operate with a tighter focus.

In this research, the XRD instrument used was a Bruker D8 with both movable detector and source arms using a cobalt $\mathrm{K}_{\alpha}$ X-ray source. Data was collected with the source arm at $2^{\circ}$ to the sample while the detector arm moved to capture data at different diffraction angles. The collected data was analysed using the EVA software from Bruker and the ICDD powder diffraction file.

The Scherrer equation was applied to the catalysts that could be analysed by XRD using a shape factor of 0.89 and instrumental peak broadening of 0.16 . Catalysts that were analysed this way were the Nickel foam, the nickel mesh and untemplated electrodeposited nickel on a palladium foil and on a stainless steel tube. No rigorous investigation was made into crystallite size because it was not seen as highly relevant to the research as a whole. The crystallite size calculated by the Scherrer equation is not directly related to the size of grains or particles of the material.

\subsubsection{Scanning Electron Microscopy}

Samples for SEM are often given a conductive coating such as carbon or gold to prevent electric charging, that is an accumulation of static electric charges on the specimen surface. The electrodeposition samples were nickel or nickel oxide on metal substrates so they were already conductive and therefore no further coating was necessary.

Four different instruments were used for SEM during this research. The first of the four SEM instruments used was a ZEISS Leo400. This instrument was only used for the very first SEM session of the project. 
The second instrument used was a JEOL JSM-5300LV. The third was JEOL 6500F, this was the primary instrument for examining the flat samples. A fourth SEM became available, this was used for some imaging of flat samples and for all of the electron microscopy on tubular samples, an FEI Quanta 450.

Three of the SEM instruments were equipped with electron dispersive X-ray spectroscopy (EDS) for elemental detection. This was useful for confirming the elemental composition of objects seen in images which assisted in identifying possible causes for the phenomena being observed. All three instruments were used to produce element maps, and the JEOL 6500F and the Quanta 450 were used for standardless semiquantitative analysis of the elements present. The elemental maps show colour where the chosen element is present and absence of colour where the element is absent. Overlaid maps can give useful insights into the nature of the material.

\subsubsection{Cross Section SEM}

When XRD detected nickel on palladium substrates after electrodeposition but no nickel object could be found by top down SEM, it was suspected that nickel was being deposited in a very thin layer. To test this hypothesis, two electrodeposited specimens were stood on their edge. A commercial epoxy was mixed thoroughly then poured around the samples. The epoxy was allowed to harden overnight.

The epoxy encased samples were ground and polished with diamond tools to the level of the metal exposed to nickel electrodeposition. This gave cross section samples that were examined by SEM and EDS using the JEOL JSM-5300LV.

\subsubsection{Quantitative Analysis of SEM Images}

Images from the SEMs were analysed using the Image J software. This was used to calculate total area, average size, and number of nickel deposits on electrodeposited samples. This information about deposition quantity was used to compare samples created under different conditions such as increasing voltage 
Jonathan Tailby

or increasing the concentration of the electroplating solution. The results from these experiments are shown in section 3.1.1.1.

\subsubsection{Gas Reactor Development}

Initial ammonia decomposition experiments were carried out in gas reactors already available in the laboratory. Each reactor was selected based on advice from the team working on membranes for hydrogen permeation at Industrial Research Limited. They were subsequently adapted to improve their design with respect to test specimen presentation and gas flow paths in relation to the results they produced. Figure 2.2.4 illustrates the progression of gas reactors and modifications. Figure 2.2.4a and Figure 2.2.4b are reactors that had been in use at Industrial Research Limited, Figure 2.2.4c to g represent modifications that were made to the available reactor. 


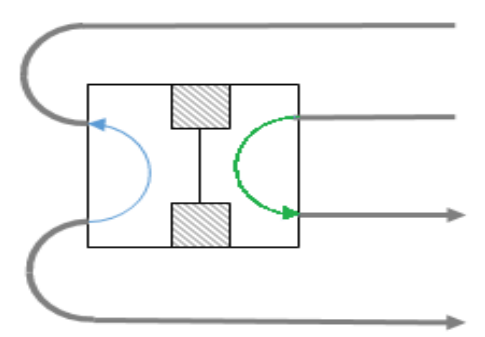

Figure 2.2.4a: Sputnik: the first gas reactor.

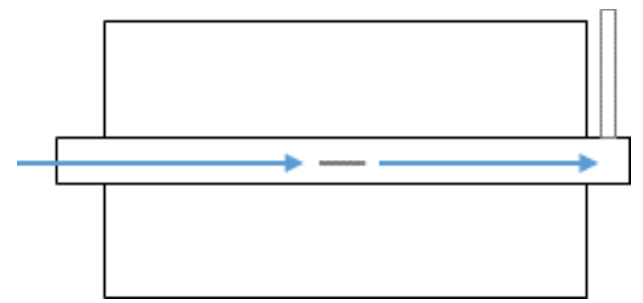

Figure 2.2.4c: Primary insert, reducing the empty space of Figure 2.2.4b.

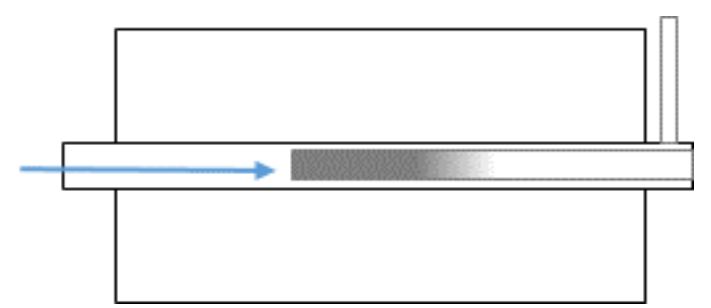

Figure 2.2.4e: Secondary insert reducing the empty space within the primary insert, Figure 2.2.4c.

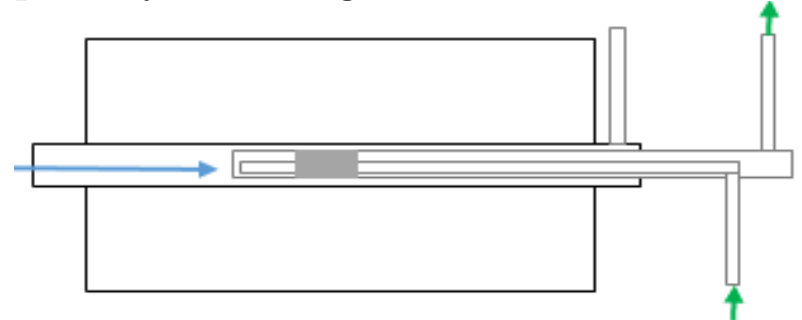

Figure 2.2.4g: Tertiary insert with palladium membrane.

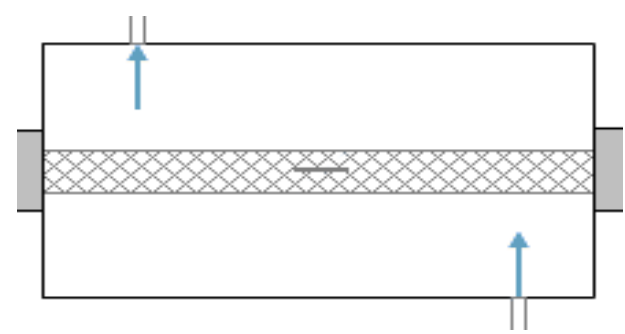

Figure 2.2.4b: Explorer with mesh: the gas reactor with an excess of empty space.

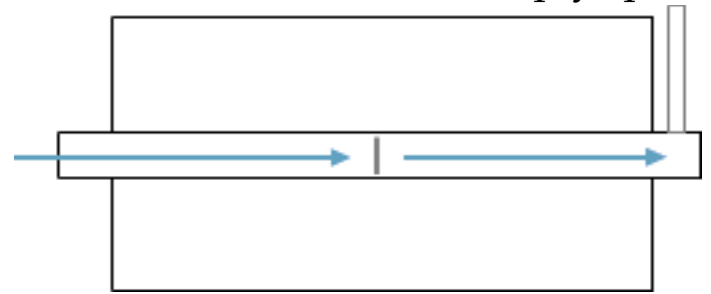

Figure 2.2.4d: Primary insert with the catalyst sample face on to the gas flow to improve contact between catalyst and gas.

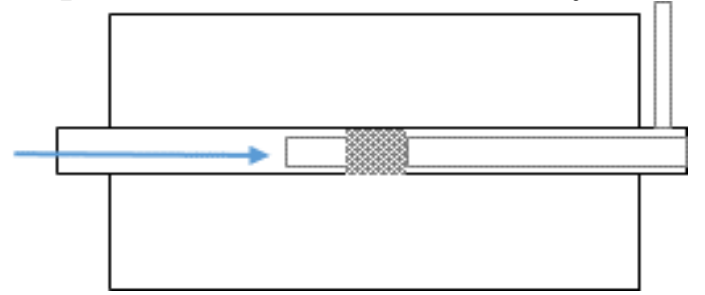

Figure 2.2.4f: Nickel foam to fill the space between the primary and secondary inserts.

Figure 2.2.4: Progression of gas reactors.

From the initial

Sputnik (Figure 2.2.4a) to the Explorer (Figure 2.2.4b) then the progressive inserts that decreased reactor dead space (Figure 2.2.4c-Figure 2.2.4g).

Blue lines represent ammonia and product gas flows, green lines represent sweep gas (argon) and permeate gas flows.

Further details of the first reactor ('Sputnik') are given in Section 2.2.9.1. Preliminary experiments for each stage in the reactor development are described in the relevant subsection of Appendix 5.1.2. 


\subsubsection{Sputnik Reactor}

The initial experiments were performed in a reactor that had previously been used for hydrogen filtration experiments. It consisted of an open tube heater with a two-part sample holder. The two parts closed around the sample and gas flowed in and out of spaces on each side of the sample. Two photographs of this reactor are shown below, the assembled reactor in Figure 2.2.5 and the same reactor disassembled in Figure 2.2.6.

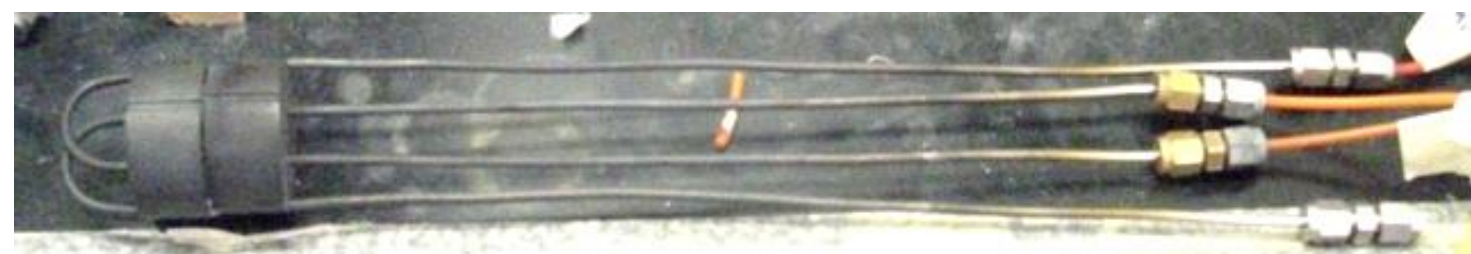

Figure 2.2.5: Sealed Sputnik gas reaction vessel

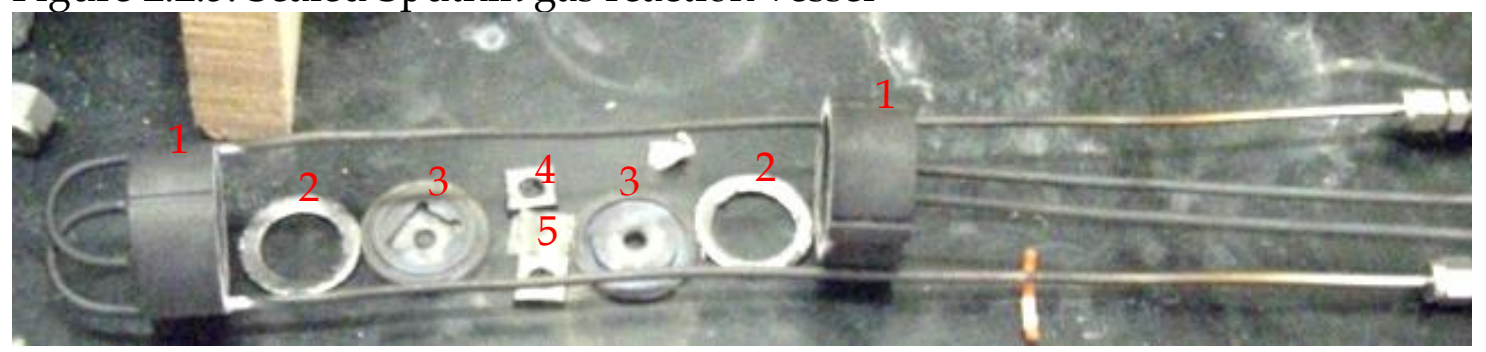

Figure 2.2.6: Open Gas Reaction Vessel

Key: 1) Outer case, 2) Outer gasket, 3) gas cell divider, 4) inner gasket, 5) test sample Figure 2.2.7 shows a cross section of Sputnik reactor when it is closed. The cap screws that held the body of the reactor proved difficult to seal without allowing an air leak between the two halves so it was proposed to use an improved model with a different sealing mechanism. However, this model had a larger heater and there were safety concerns about getting sufficient clearance around the heater inside the fume cupboard so another alternative was designed.

The test sample (yellow) has a cavity on each side with delivery tubes to control the gas content of the cavity. A steel divider (blue) holds the sample in place. Figure 2.2.8 reveals another flaw in the gas reactor. An excess of empty space existed on each side of the membrane for gas to pass through without interacting with either the catalyst or the membrane. 


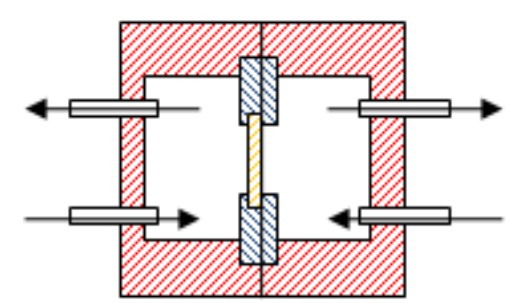

Figure 2.2.7: Schematic showing the internal structure of the reaction vessel.

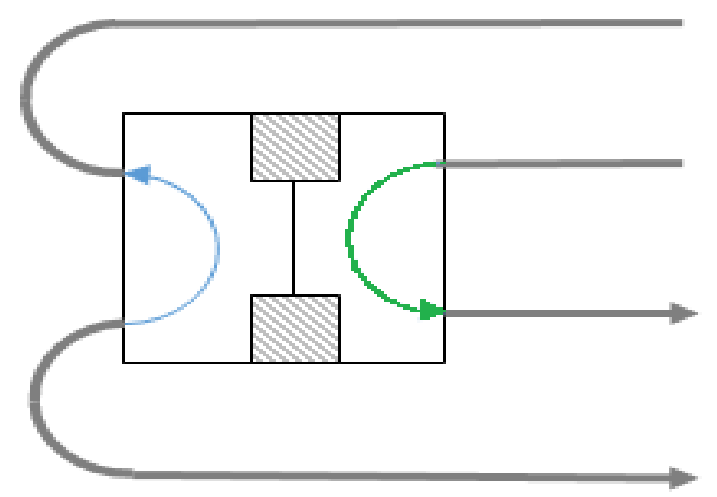

Figure 2.2.8: Schematic showing the gas flow inside the 'Sputnik' gas reaction vessel.

This demonstrates how the gas will flow through the reactor with very little contact with the catalyst sample.

\subsubsection{Explorer Reactor}

A different reactor shape was designed, this time a barrel shaped reactor with a band heater. The initial ammonia decomposition experiments began with the same $12 \times 12 \times 0.09 \mathrm{~mm}$ metal foil squares as the Sputnik experiment, with the sample now suspended inside the reactor using a steel mesh rolled into a tubular cage. This reactor is shown in Figure 2.2.9. The new reactor solved the problem of sealing; however, it increased the problem of excess space for gas to bypass the catalyst. 


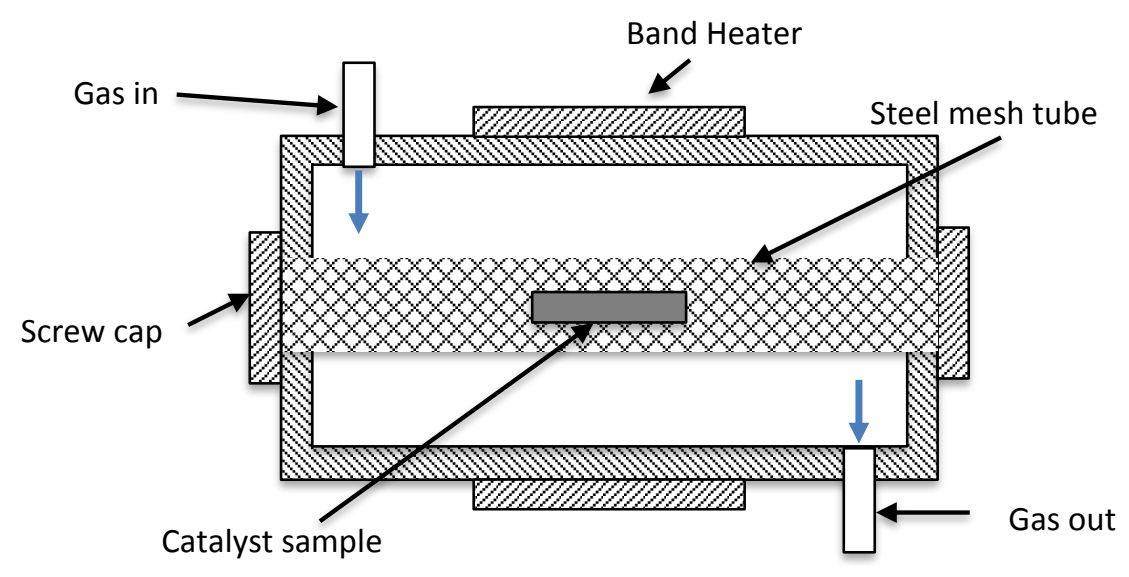

Figure 2.2.9: Explorer reactor with rolled stainless steel mesh supporting the sample in the centre of the reactor.

\subsubsection{Primary Insert}

The decomposition reactions undertaken in the 'Explorer' reactor showed very low levels of conversion and the results were not as consistent as had been expected. To improve both consistency and response time the reactor was redesigned to reduce the empty space in the Explorer reactor where the ammonia was previously able to flow through without contacting the catalyst sample surface. A tube was inserted through the reactor using the end ports that had previously been used for inserting the mesh that held the sample. The gas now flowed through this inside tube, so the effective reactor volume was reduced from 240 to $10 \mathrm{~mL}$. An $\mathrm{N}$ type thermocouple was fitted inside this insert tube so that the reaction temperature could be measured. This tube, that was the first to be inserted into the reactor, was labelled the primary insert tube. Figure 2.2.10 shows a diagram of the Explorer reactor with the primary insert tube. 


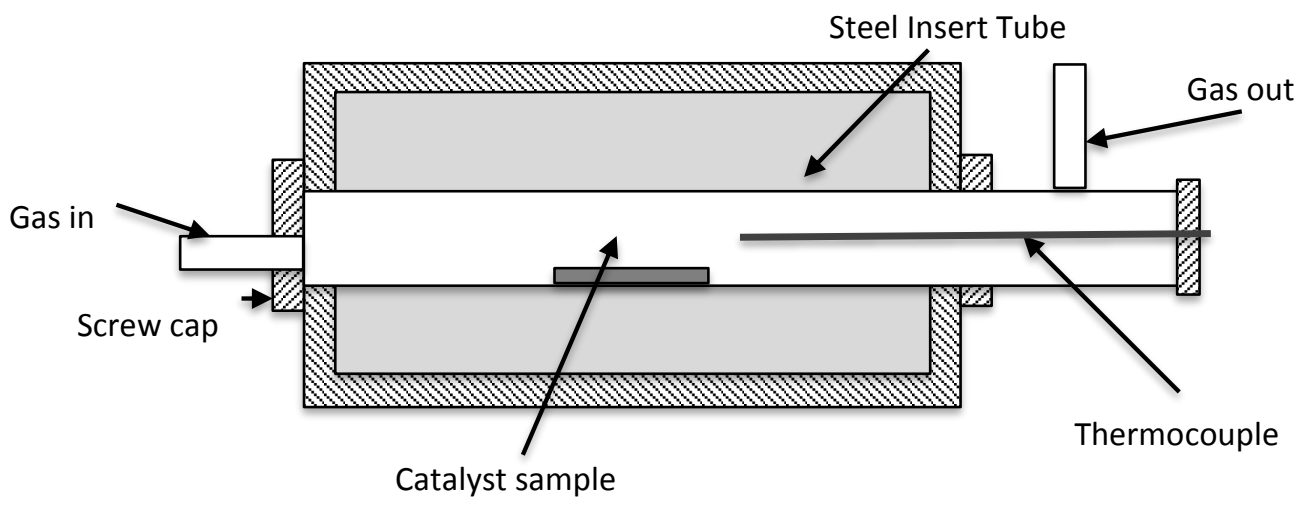

Figure 2.2.10: Explorer reactor with stainless steel primary insert holding the sample in the centre of the reactor with reduced volume.

A preliminary set of ammonia decomposition experiments was undertaken without a catalyst sample to establish a baseline for comparing the catalysts. Ammonia flowed through the reactor insert at a set of internal temperatures: 350 ${ }^{\circ} \mathrm{C}, 400{ }^{\circ} \mathrm{C}, 450{ }^{\circ} \mathrm{C}, 500{ }^{\circ} \mathrm{C}, 550{ }^{\circ} \mathrm{C}, 600^{\circ} \mathrm{C}$ and $650{ }^{\circ} \mathrm{C}$ and the output was injected into the GC in $0.2 \mathrm{~mL}$ samples. Ammonia flow was controlled to $5 \mathrm{~mL} \mathrm{~min}^{-1}$ by a rotameter upstream of the reactor.

\subsection{Primary Insert with Sample Face-on to Gas Flow}

The sample in the primary insert was initially sitting as shown in Figure 2.2.10. This set it side-on to the gas flow, leaving the majority of the cross section open for gas to pass through without interacting with the catalyst sample. To improve sample presentation in the reactor the sample holder shown in Figure 2.2.11 was used to hold the sample face-on to the gas flow. It was anticipated that this would increase the activity of the catalyst as it interacted with a greater proportion of the gas flow. 


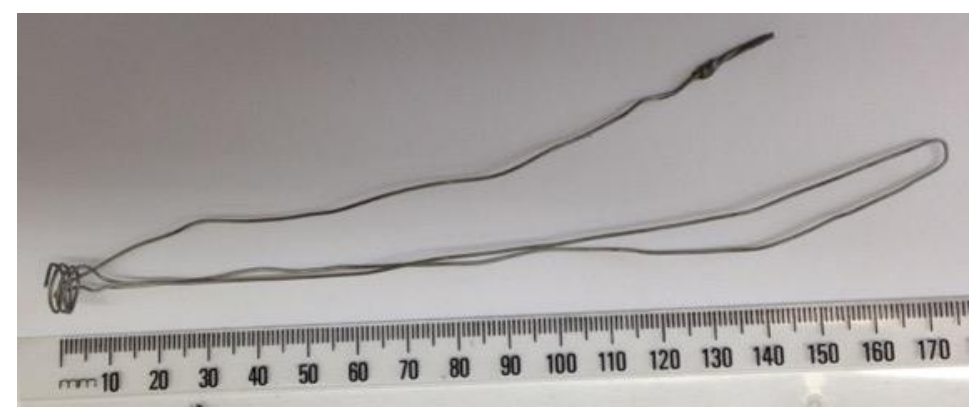

Figure 2.2.11: Sample holder for primary insert tube.

The coil at left held the sample in the hot zone of the furnace while the tail to the right enabled retrieval from the tube.

\subsubsection{Secondary Insert}

The square samples were replaced with stainless steel tubes that were sealed at one end. These tubes, inserted inside the first tube, were labelled the secondary insert tubes. These secondary inserts decreased the reaction zone from the full cross-section of the primary insert to the space between the inner surface of the primary insert and the outer surface of secondary insert. The secondary insert decreased the reactor volume in the hottest zone, from $5.5 \mathrm{~mL}$ to $1.8 \mathrm{~mL}$ and increased the reactive surface exposed to the ammonia. A diagram of the secondary insert tube is shown in Figure 2.2.12.

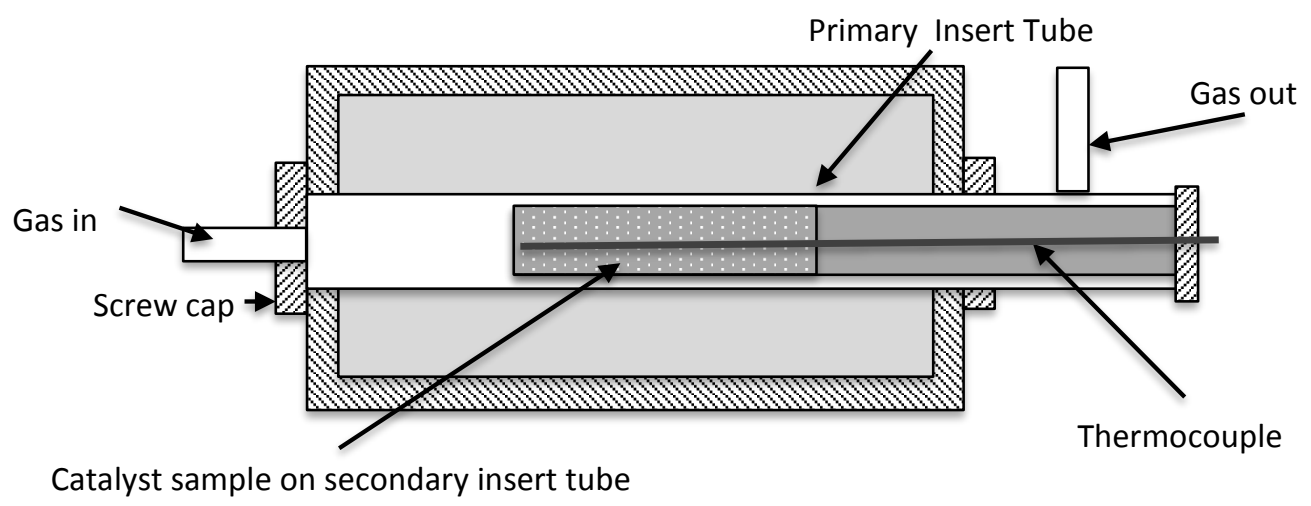

Figure 2.2.12: Explorer reactor with a secondary insert tube inside the stainless steel primary insert.

The catalyst is on the outside of the secondary tube and the ammonia decomposition occurs in the space between the two insert tubes.

The change from foils to tubes was made in consideration of the intention to move to an experimental design where hydrogen could permeate through palladium out of the reaction space. Two alternative designs for the secondary 
insert are discussed in Appendix 5.1.3. The secondary inserts were tubes of stainless steel $150 \mathrm{~mm}$ long and $9.5 \mathrm{~mm}$ outer diameter which were sealed the end intended for the hot zone of the reactor. These secondary inserts are shown in Figure 2.2.13. Figure 2.2.13a is undeposited stainless steel, Figure 2.2.13b has had nickel electrodeposited on the first $50 \mathrm{~mm}$ from the sealed end without a template, and Figure 2.2.13c shows a steel tube with nickel electrodeposited through a template. Figure 2.2.13c is an early example, from when the process was still being developed and the templates were uneven. Later templated electrodepositions were more even. The untemplated electrodeposited insert tubes used had an average of $6.85 \mathrm{mg}$ of nickel deposited which is an average of $0.31 \mu \mathrm{m}$ thick, and the templated electrodeposited insert tubes had an average of $5.41 \mathrm{mg}$ of nickel which is an average of $0.58 \mu \mathrm{m}$ tall.

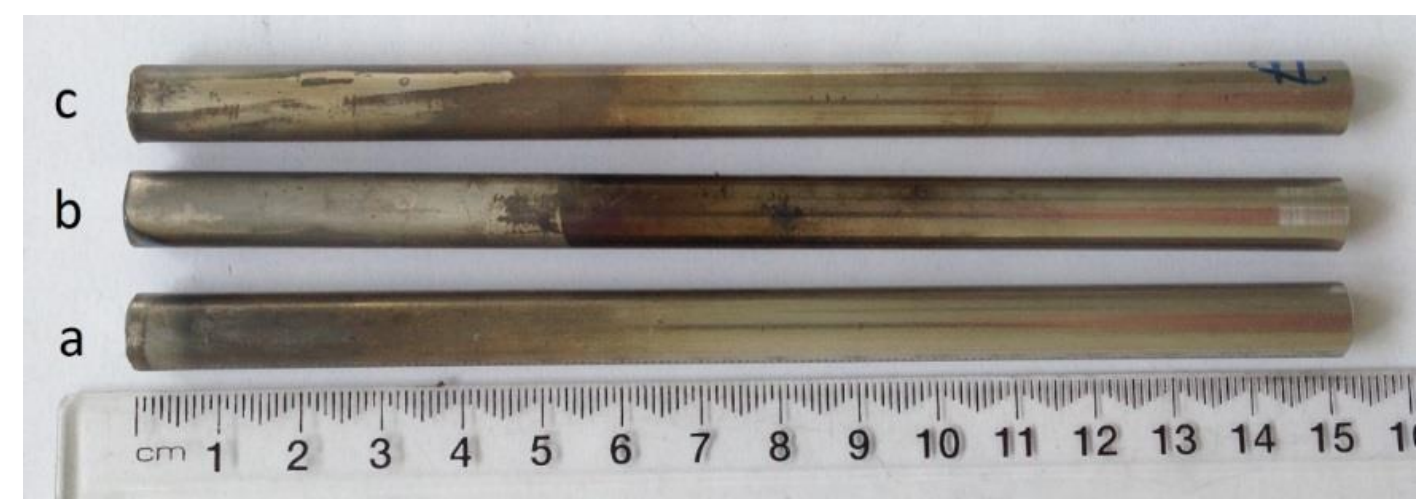

Figure 2.2.13: Secondary insert tubes.

The end at the left is the sealed end intended for the reaction. a) is an undeposited stainless steel tube. b) has untemplated nickel deposited on the first five centimetres from the sealed end. c) has templated nickel deposited on the first five centimetres.

Two nickel catalysts were constructed using prefabricated nickel. A mesh which was wrapped around the reaction end of the secondary insert, as shown in Figure 2.2.14. This mesh was expected to be a more effective catalyst than the electrodeposited nickel because the mesh protruded more into the reaction space than the electrodeposits. This mesh was house stock that was the same as the material in use as a counter electrode in the electrodeposition. It is believed to have originally been sourced from Goodfellow. 


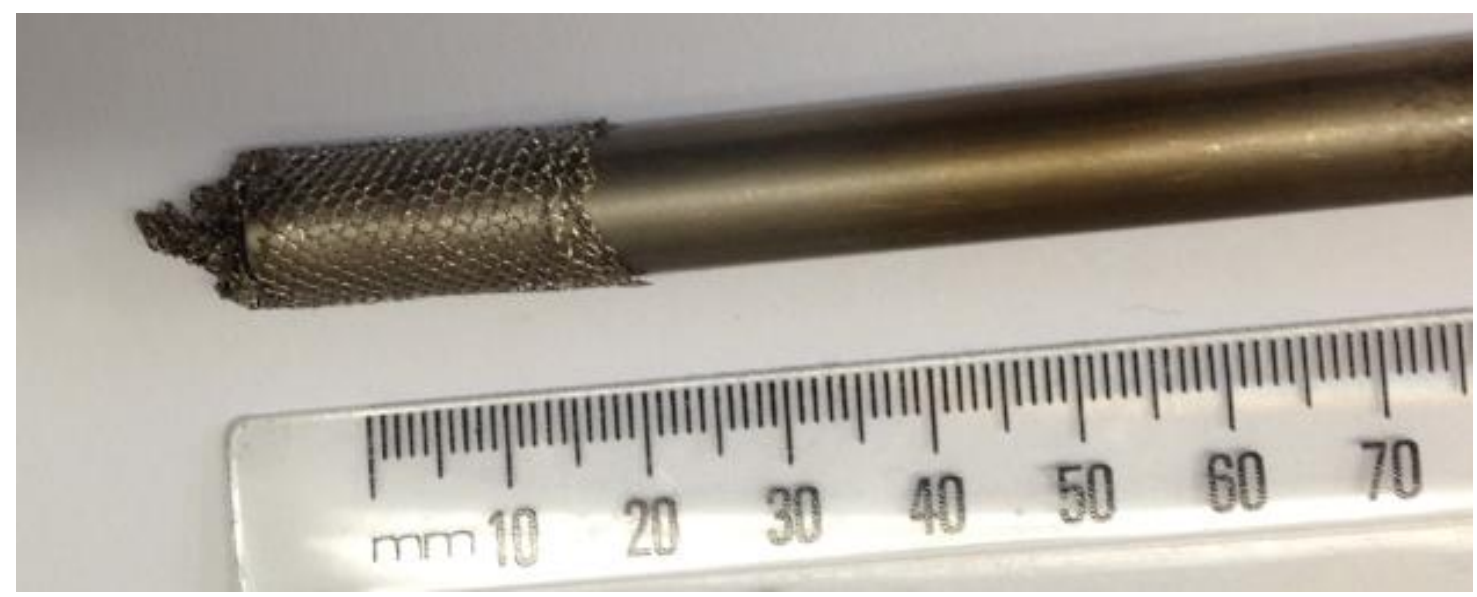

Figure 2.2.14: Nickel mesh secondary insert.

Another secondary insert was cut into so that a section of the tube had a smaller external diameter. Nickel foam was wrapped around that narrow section; however, the nickel foam still required compression to fit inside the primary insert. The foam wrapped inserts were rolled on a bench to compress the foam evenly. The nickel foam secondary insert was pushed into the primary insert with enough force to compress the foam into the intervening space. By this twostep compression the $3.5 \mathrm{~mm}$ thick nickel foam filled the $1.6 \mathrm{~mm}$ space between the primary insert and the recess in the secondary insert. A photograph of the nickel foam insert is shown in Figure 2.2.15. In that figure it is compared with a untemplated electrodeposited secondary insert and a pen on a background of 7 mm ruled writing paper, showing that the nickel foam was shorter than the nickel electrodeposition. The ammonia decomposition results for this foam wrapped insert were expected to show the highest ammonia decomposition because the foam would be most effective at filling the reaction space. The nickel foam was the catalyst most easily applied to the tertiary insert design described in the following section, whereas the process for applying the lithographic template was not successfully adapted to suit the tertiary insert design. This is one example of a difficulty caused by the change from flat to tubular geometry. However, the change had itself been necessitated by practical choices at that time. 


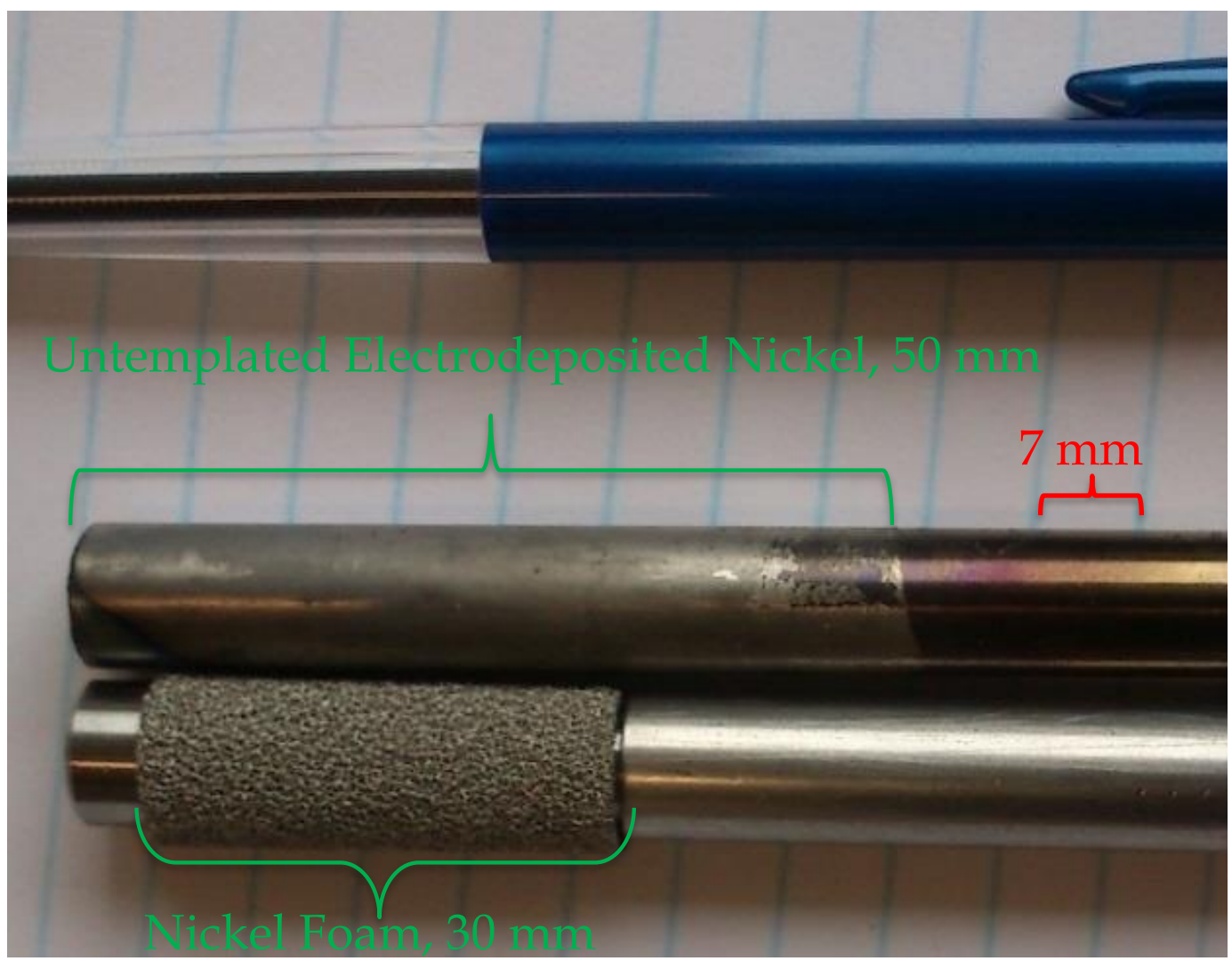

Figure 2.2.15: This photograph shows a nickel foam secondary insert compared with an untemplated electrodeposited nickel secondary insert and a pen.

\subsubsection{Tertiary insert}

After confirming the efficacy of the catalysts, a new insert was designed for permeation experiments. Design drawings for these tertiary inserts are shown in Figure 5.1.11 in Appendix 5.1.4. Photographs of the tertiary inserts for hydrogen permeation experiments are shown in Figure 2.2.16. The 'Nickel-beforePalladium' tertiary insert, shown in Figure 2.2.16a, had the nickel foam catalyst upstream from the palladium membrane. The 'Nickel-on-Palladium' tertiary insert shown in Figure 2.2.16b was the catalytic spillover membrane with the nickel foam wrapped around an insert on top of the palladium membrane. The stainless-steel tube under the palladium was cut through with windows to allow absorbed hydrogen to desorb into the inside of the insert. 


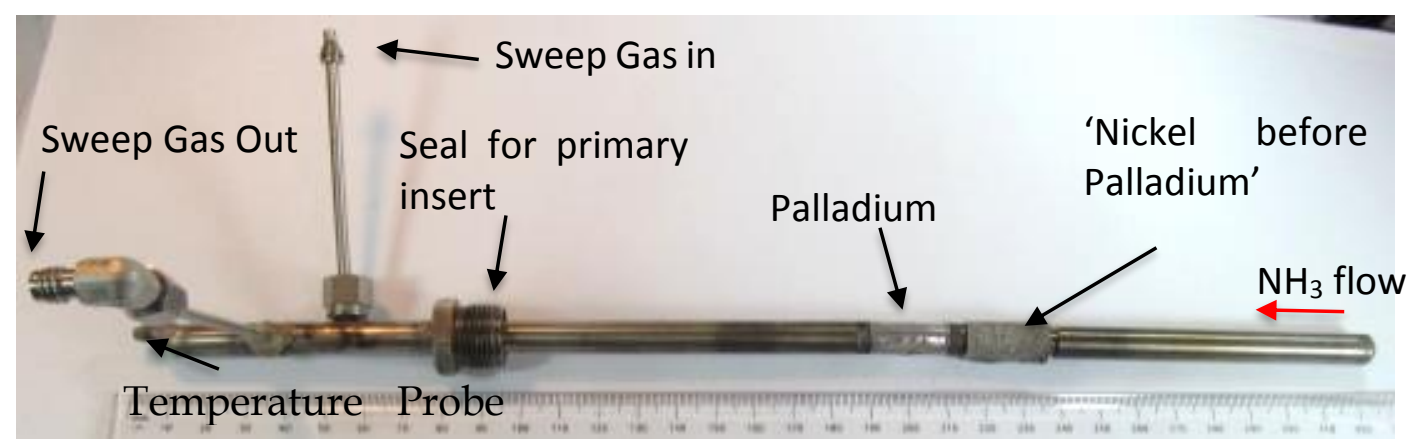

Figure 2.2.16a: Photograph of the 'Nickel-before-Palladium' tertiary insert.

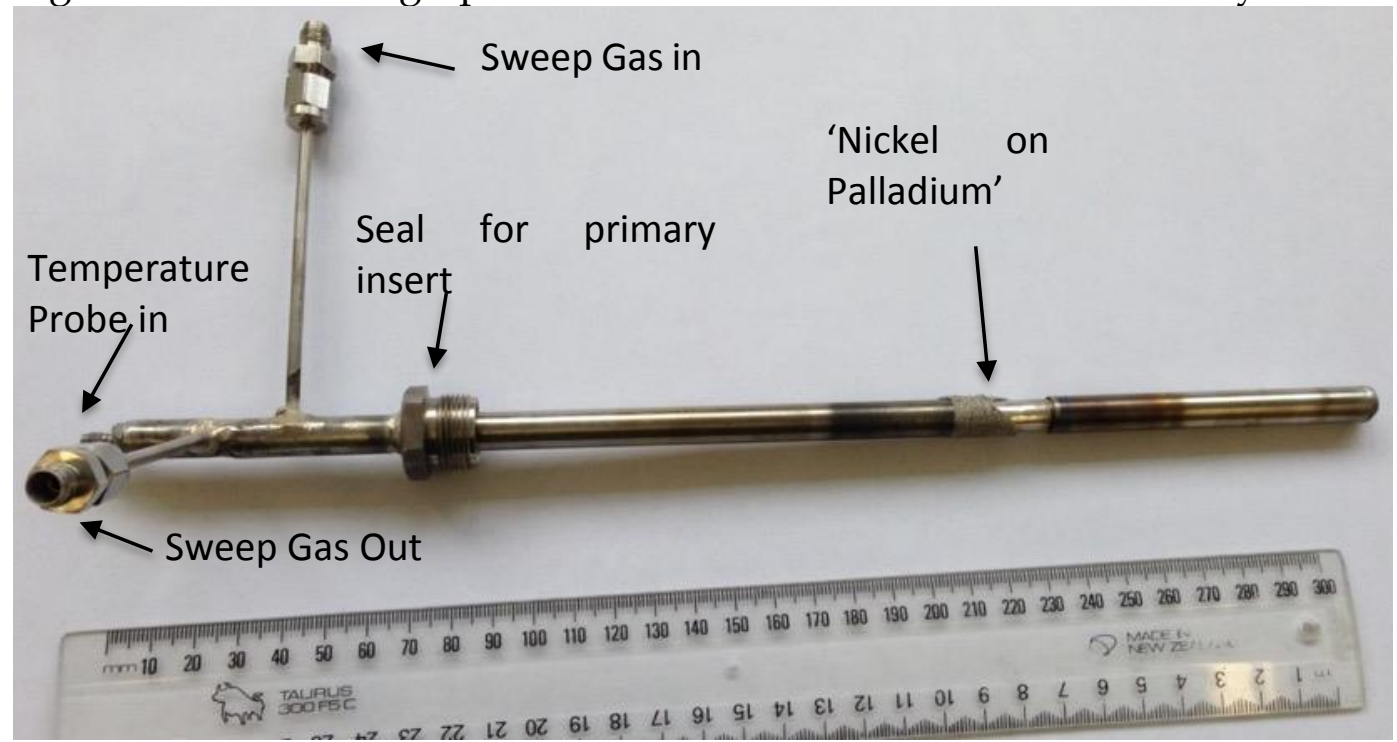

Figure 2.2.16b; Photograph of 'Nickel-on-Palladium' Catalytic Spillover Membrane.

Figure 2.2.16: Photograph of the 'Nickel-on-Palladium' tertiary insert.

Figure 2.2.17 shows two images of the steel structure under the palladium foil, showing how the steel tubes were cut through to make windows $2 \mathrm{~mm}$ wide around the diameter for hydrogen desorption on the inner side. The windows did not extend around the complete circumference. One area was left unmachined where the two ends of the palladium foil were joined to make a tubular sleeve around the steel. This wider steel under the seam improved the seal so that it was gas tight. 


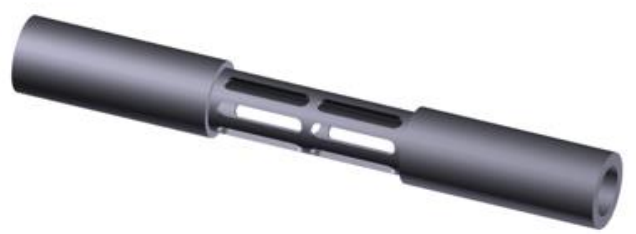

Figure 2.2.17a: narrow struts.

Figure 2.2.17: Image of the steel tube showing the cut-aways under the palladium foil.

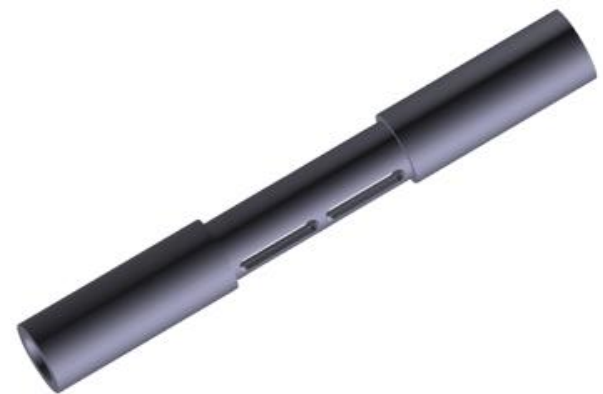

Figure 2.2.17b image of the steel tube showing the extra wide strut under the palladium foil where the seam joined the two edges of the palladium sleeve.

The regions of the steel tube where the catalyst and palladium membrane were placed were selected by measurement of the temperature profiles in the primary insert and, in a prototype, tertiary insert. The temperature profiles are shown in Figure 5.1.12 in Section 5.1.4.

Figure 2.2.18 shows a schematic of the tertiary insert inside the gas reactor. Ammonia entered from the left-hand end marked Gas in and the retentate gas left through the tube labelled Gas out. The nickel foam filled the space between the primary and tertiary tubes.

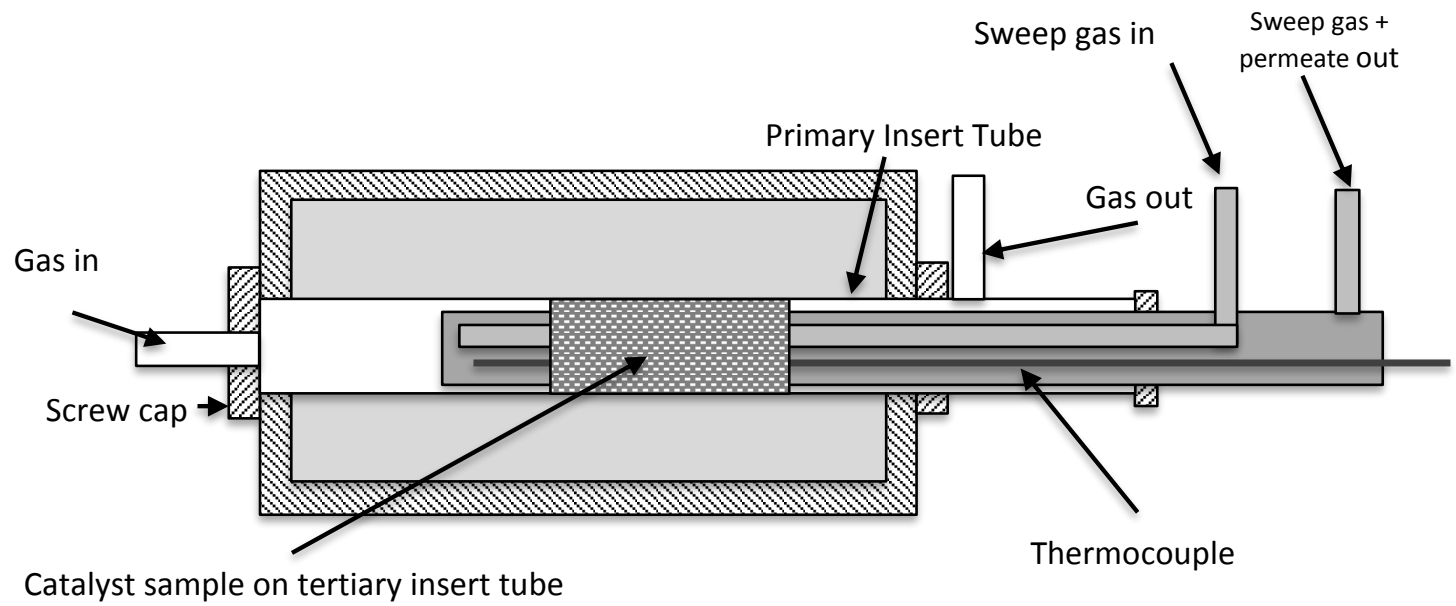

Figure 2.2.18: Explorer reactor with a tertiary insert tube inside the stainless-steel primary insert.

The catalyst is on the outside of the tertiary insert and the ammonia decomposition occurs in the space between the two insert tubes. 
A third tertiary insert design was manufactured which also had both the catalyst and the palladium in zone 1, however this design did not have the windows machined into the steel tube beneath the palladium. This design was intended to enable contact between the nickel and the palladium, but to prevent the hydrogen from permeating through the palladium. Figure 2.2.19 shows a photograph of this 'Nickel-on-Palladium-no-Permeation' insert.

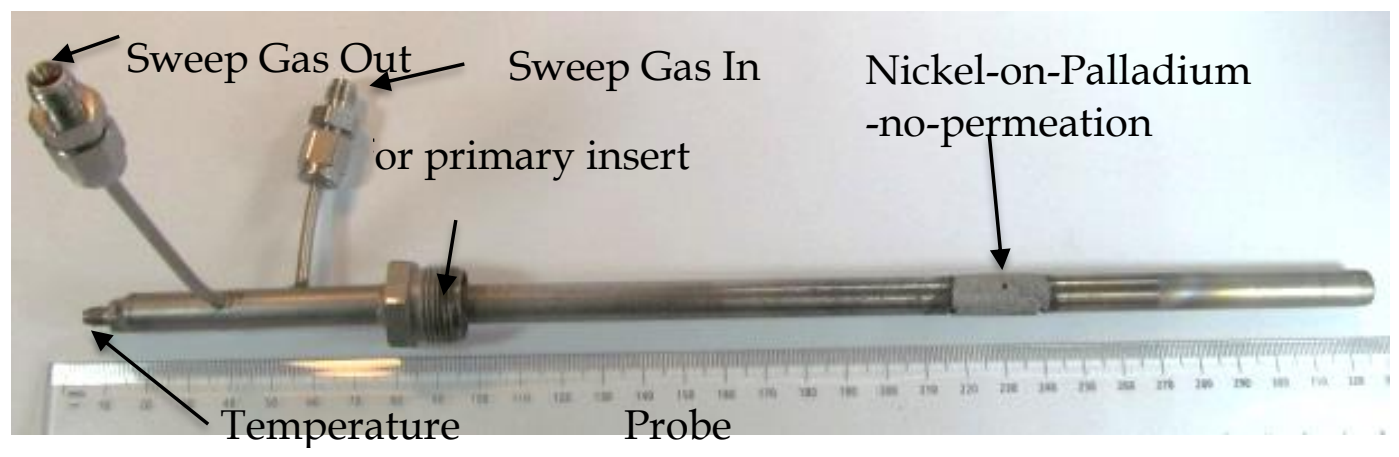

Figure 2.2.19: Photograph of 'Nickel-on-Palladium-no-Permeation' insert. Preliminary experiments for these inserts are described in Appendix 5.1.2.2.3.

The 'Nickel-before-Palladium' tertiary insert experiments aimed to explore the effect of hydrogen permeation on hydrogen production by ammonia decomposition. The literature on the subject suggested that hydrogen permeation would increase both ammonia decomposition and hydrogen production $[73,101,162]$. These experiments aimed to quantify the contribution of hydrogen permeation under the reaction conditions of these experiments for comparison with catalysis with spillover and hydrogen permeation.

Four sets of ammonia decomposition experiments were conducted with the Nickel-before-Palladium tertiary insert. First a set of temperature comparison data was collected at $300,350,400$ and $450{ }^{\circ} \mathrm{C}$, while measuring the composition of the gas that flowed from the reaction side of the palladium membrane. The second data set recorded the composition of the gas that came from the permeation side of the palladium membrane across a range of temperatures. The third set of experiments measured the composition of the gas that flowed out from the reaction side of the palladium membrane when the temperature was held at $350{ }^{\circ} \mathrm{C}$ but the rate of ammonia flow into the reactor was varied through 
$1,2,3,4,5$ and $6 \mathrm{~mL} \cdot \mathrm{min}^{-1}$. The fourth set of experiments repeated the flow variation at $350^{\circ} \mathrm{C}$ but measured the composition of the gas from the permeation side of the palladium membrane.

The same four data sets were collected for the Nickel-on-palladium tertiary insert. The processed data for each of the four sets was compared between the two different tertiary inserts.

When all the data had been collected for both the 'Nickel-before-Palladium' and 'Nickel-on-Palladium' inserts, the same was performed for the 'Nickel-onPalladium with no permeation' insert.

Data for ammonia decomposition was collected for both the temperature variation data set and the flow variation data set, however only the temperature series was recorded for the sweep side, this was sufficient to show that no permeation occurred. The results can be seen in Table 3.3.4.

\subsubsection{Catalyst Testing in the Secondary and Tertiary Inserts}

When Catalyst samples were first inserted into the gas reactor the reactor and catalyst were heated to $550^{\circ} \mathrm{C}$ in ammonia flowing at $5 \mathrm{~mL} \mathrm{~min}^{-}$ 1. After a twenty-minute hold at $550{ }^{\circ} \mathrm{C}$ the reactor was cooled to the target temperature, still in flowing ammonia. The heater was set to cool at $5^{\circ} \mathrm{C} \cdot \mathrm{min}^{-1}$ but the reactor had no forced cooling so the actual cooling ramp was slower. The exact cooling time varied depending on the difference between the preheat temperature and the target temperature, but was always more than 15 minutes. The reactor was held at the target temperature for ten minutes before any measurements were made to allow the reactor to equilibrate at the target temperature and to clear any decomposition products that might remain from reaction at the higher pre-treatment temperature.

This preheating in ammonia reduced any nickel oxide on the nickel surface. Thermodynamic modelling with HSC Chemistry showed that reduction of nickel oxide by ammonia and its decomposition products is favoured above ambient temperature. The high temperature would have overcome any kinetic barriers. The reactor was kept under inert or reducing atmosphere until the 
Jonathan Tailby

catalyst was removed. If the catalyst was returned to the gas reactor after removal then the reductive pre-treatment was repeated.

Gas pressure in the reactor was able to equilibrate with atmospheric pressure, which has a maximum variation of $4 \%$ from 14.7 psi or $101 \mathrm{kPa}$. Ammonia left the cylinder at 10 psi and equilibrated with atmospheric pressure while it flowed to the reactor.

The reactor was controlled to target reaction temperatures from 300 to $450{ }^{\circ} \mathrm{C}$. The band heater and controller used with the tubular reactors maintained temperature to within $3{ }^{\circ} \mathrm{C}$ and the rate of gas flow was too low to cause temperature fluctuations. The heater controller used a thermocouple that inserted through the insulation to contact the reactor close to the heater. A second thermocouple was inserted inside the reactor. The external thermocouple gave feedback to the controller, while the internal thermocouple was used for manual setting of the controller. The fully insulated tubular reactor produced internal temperatures up to $5{ }^{\circ} \mathrm{C}$ higher than the controller setting, therefore the set temperature on the controller was lowered until the temperature reported by the internal thermocouple was exactly the target temperature.

The flow rate of gas into the reactor was controlled by a rotameter manufactured by Aalborg Instruments, model 042-10G. The flow range was 0-7 $\mathrm{mL} \cdot \mathrm{min}^{-1}$ with stated accuracy of $\pm 5 \%$ and repeatability of $\pm 0.25 \%$. The nominal flow calibrated to the same value on a bubble flowmeter.

The sets of catalysts for testing all included some form of blank. In the secondary insert catalysts, this was a secondary insert with no additional nickel. The 316 stainless steel of the primary and secondary inserts would have contained 10-14 $\%$ nickel which could have had some catalytic effect.

At least three samples of each secondary insert catalyst were constructed and tested. Each sample was tested in the reactor at least six times at each setting. If the reading following use of the reactor at a higher temperature had unusually high decomposition compared to other measurements under the same conditions, the high result was considered to have been affected by the remnants 
of the higher temperature and the result was discarded. For example, if the first measurement after the reductive pre-treatment was higher than other measurements with the same temperature and flow, then that first measurement was not included in the set that gave an average value for those settings.

Table 2.2.5 lists the average mass of the nickel catalysts used in the secondary insert reaction tests.

\begin{tabular}{|c|c|}
\hline Catalyst & Average Mass \\
\hline Flat Nickel & $6.85 \times 10-^{3} \mathrm{~g}$ \\
\hline Templated Nickel & $5.41 \times 10^{-3} \mathrm{~g}$ \\
\hline Nickel Foam & $0.43 \mathrm{~g}$ \\
\hline Nickel Mesh & $0.44 \mathrm{~g}$ \\
\hline
\end{tabular}

Samples were tested in a temperature series at $50{ }^{\circ} \mathrm{C}$ intervals from 300 to $450{ }^{\circ} \mathrm{C}$ at $5 \mathrm{~mL} \cdot \mathrm{min}^{-1}$. They were then tested in a flow series from 1 to $6 \mathrm{~mL} \cdot \mathrm{min}^{-1}$ at $350^{\circ} \mathrm{C}$. In the secondary insert temperature series it had appeared that $350{ }^{\circ} \mathrm{C}$ produced the greatest difference between the different catalysts under the first method of processing the data. Under the more sophisticated data processing that is presented here this is no longer the case.

There was insufficient palladium for additional models of each tertiary insert. Each sample was tested ten times over multiple days. Unusually high measurements after the reductive pre-treatment were discarded, as with the secondary insert catalysts. Also, if the first measurements of hydrogen permeation were unusually low compared to subsequent measurements, those abnormally low measurements were assumed to be before hydrogen had sufficiently penetrated the membrane and were discarded. Table 2.2.6 lists the mass of nickel foam catalyst on each of the Tertiary inserts used in the gas reactions.

\begin{tabular}{|c|c|}
\hline Catalyst & Average Mass \\
\hline Nickel before Palladium & $0.38 \mathrm{~g}$ \\
\hline Nickel on Palladium & $0.43 \mathrm{~g}$ \\
\hline
\end{tabular}




\begin{tabular}{|l|l|}
\hline $\begin{array}{l}\text { Nickel on Palladium no } \\
\text { Permeation }\end{array}$ & $0.44 \mathrm{~g}$ \\
\hline
\end{tabular}

\subsubsection{Mass Spectrometry for Reaction Analysis}

In this work, initial ammonia gas decomposition experiments were analysed by mass spectrometer. The instrument used was a Dycor Dymaxion DM100M Quadrupole Mass Spectrometer, manufactured by Ametek, attached to a computer running the Dycor 2000 software. In the present case, the key mass/charge (m/z) signatures are: $\mathrm{H}_{2}(2) ; \mathrm{N}_{2}(14,28) ; \mathrm{NH}_{3}(16,17) ; \mathrm{H}_{2} \mathrm{O}(17,18)$; $\mathrm{O}_{2}$ (16, 32); $\mathrm{Ar}(40)$.

Figure 2.2.20 shows a diagram of how the gas reactor was connected to the mass spectrometer. The coloured lines represent $1 / 8^{\prime \prime}$ polymer tubes. The Sputnik gas reactor had two lines where gas entered the reactor and two lines where gas exited the reactor, one pair each for ammonia and for an argon sweep gas. The two exit lines entered opposite ports of a switching valve. The two remaining ports of the switching valve were connected to gas lines that vented into the fume cupboard. One of these waste lines had a side port that sampled for the capillary tube that led to the mass spectrometer. Manually turning the 4-port switching valve changed which gas output went directly to the waste line and which gas was sampled for the mass spectrometer. This enabled either the retentate or the permeate to be analysed.

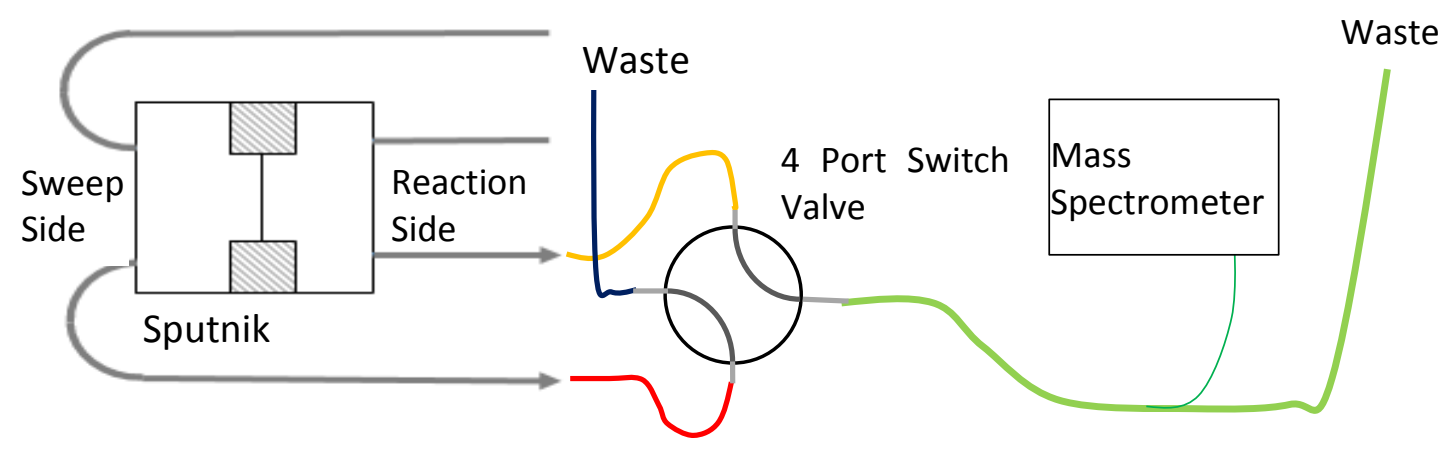

Figure 2.2.20: Diagram to show the connections between the gas reactor and the mass spectrometer.

The two gas outputs from the gas reactor went into a switching valve so that the mass spectrometer could sample from either the reaction output or from the sweep side with any permeate hydrogen. 
Decomposition experiments followed, with the ammonia side of the palladium membrane being analysed to investigate the potential for detecting a difference between catalysed and uncatalysed ammonia decomposition experiments. Before positive decomposition results were confirmed it was observed that the mass spectrometer registered positive for ammonia even when it could not be present. Regrettably the mass spectrometer was found to have damaged electronics requiring expensive repairs. It was determined that ammonia was the most likely cause, being the only corrosive gas that was known to have flowed through the mass spectrometer before it malfunctioned. As a precaution, ammonia gas analysis in the mass spectrometer was discontinued. Subsequent gas experiments were analysed with a gas chromatograph which demonstrated much greater tolerance to ammonia corrosion while providing good discrimination between the key gas species.

\subsubsection{Gas Chromatography for Reaction Analysis}

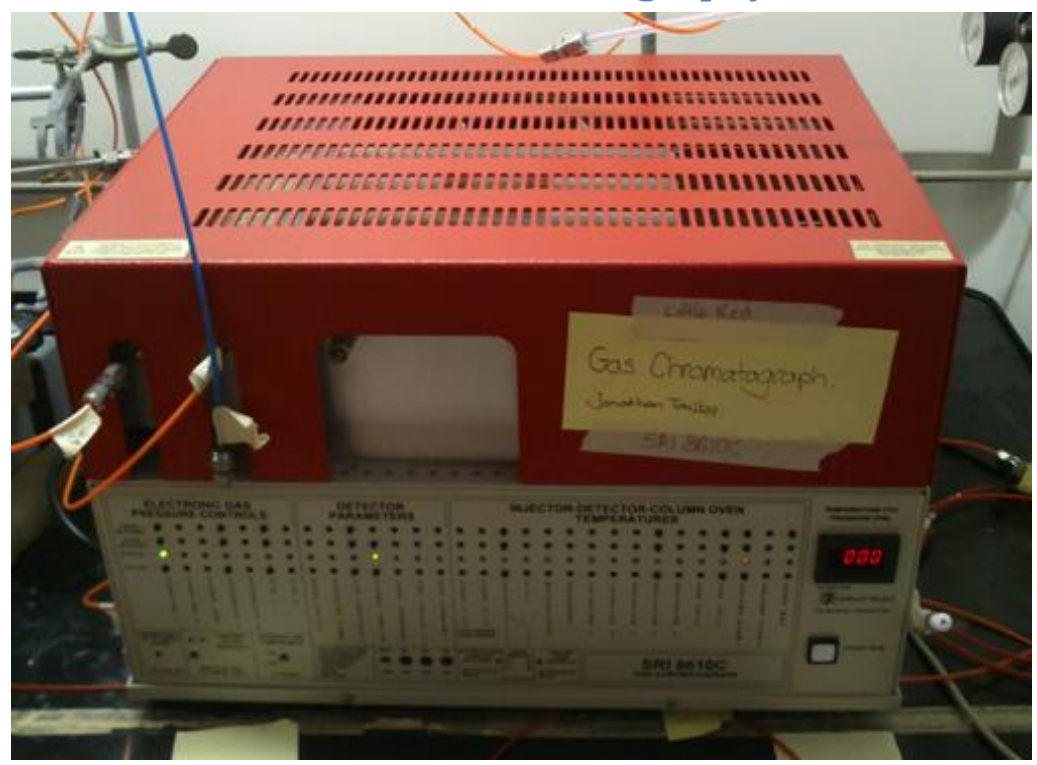

a: SRI $8610 \mathrm{C}$ GC with top cover closed. 


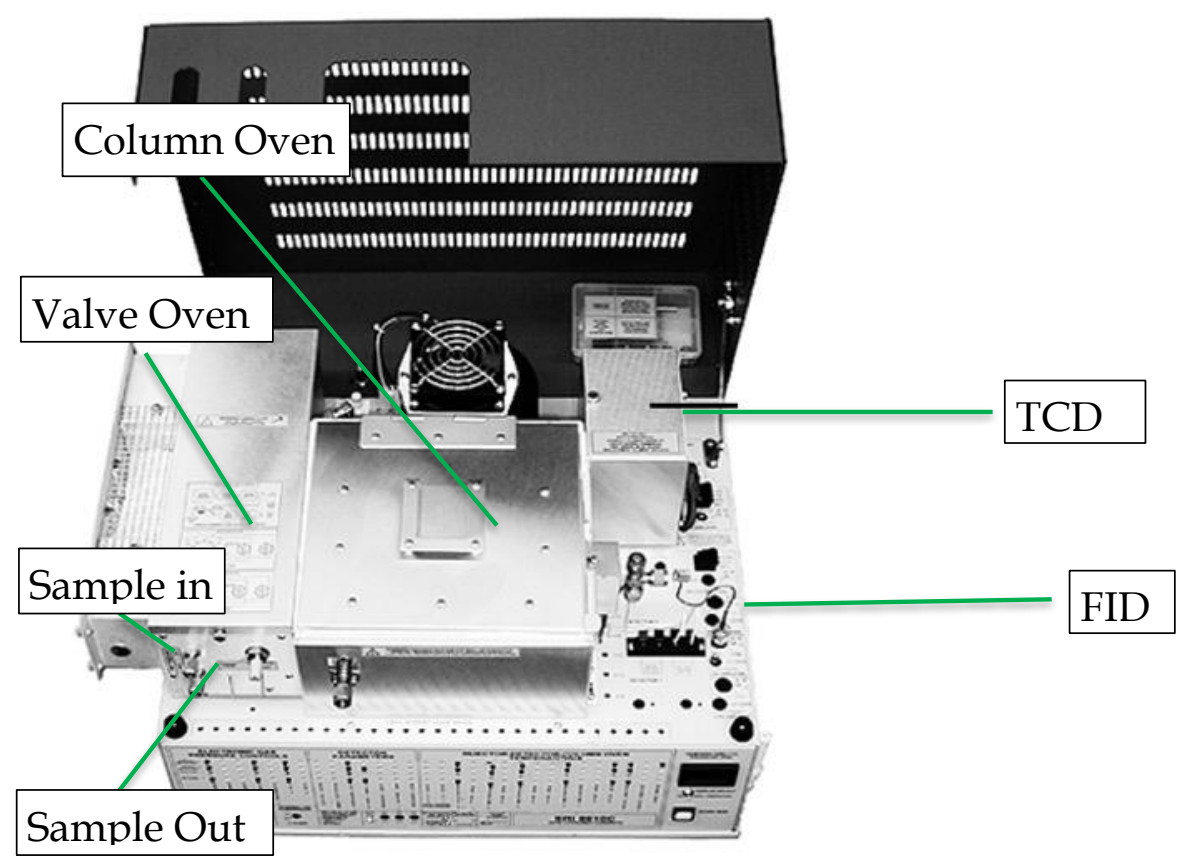

b: SRI GC8610C with top lid open and key elements labelled.

Figure 2.2.21: SRI GC8610C gas chromatograph.

All of the external connections on the left of the images are 1/8" Swagelok.

The specific GC used in these experiments was an SRI Instruments SRI 8610C00 using its thermal conductivity detector (TCD). Figure 2.2.21 shows two images of the instrument. The instrument had a computer-controlled sampling valve so sample gas could flow through the sample loop until a programmed injection turned the valve, which allowed the carrier gas to push a fixed volume of sample into the column. The GC connected to the experiment in the same way as the mass spectrometer, as shown in Figure 2.2.20, replacing the mass spectrometer with the GC.

Experiments were conducted to find an optimum temperature for using the GC with the gases relevant to this research. This showed that $30^{\circ} \mathrm{C}$ was the lowest temperature setting at which the column oven would maintain a constant temperature so it was retained throughout the experimental programme, except in the tertiary insert experiments. For the tertiary insert ammonia decomposition experiments, a special programme was introduced that used a column oven temperature of $30^{\circ} \mathrm{C}$ until the nitrogen had completely left the column at three minutes after injection. After three minutes the programme raised the column 
oven temperature at $20^{\circ} \mathrm{C} \cdot \mathrm{min}^{-1}$ to $100^{\circ} \mathrm{C}$. Prior to these experiments the carrier gas pressure had been reduced from the 30 psi of the secondary insert experiments to $25 \mathrm{psi}$; this extended the time ammonia required to completely leave the column at $30{ }^{\circ} \mathrm{C}$ to more than thirty minutes. The increased temperature accelerated the movement of ammonia through the column so that ammonia began leaving the column before the column temperature reached 100 ${ }^{\circ} \mathrm{C}$ and had completely left the column approximately 9 minutes after it had first begun to leave the column. At that time (15.5 minutes) the temperature began to decrease at $20^{\circ} \mathrm{C} \cdot \mathrm{min}^{-1}$ back to $30^{\circ} \mathrm{C}$. With extra time allowed to let the column temperature equilibrate at $30^{\circ} \mathrm{C}$, this temperature programme reduced the time to measure the composition of the gas mixture from ammonia decomposition from over thirty minutes to twenty minutes.

This GC was fitted with a flame ionisation detector (FID) and a thermal conductivity detector (TCD). The FID is well suited for detecting carbon containing species but not for any of the gases of interest in these experiments so the hydrogen fuel for the FID was disconnected and the FID was not considered further. The TCD is able to detect any species except its own reference gas. The TCD is a Wheatstone bridge of four resistors which produces a voltage difference resulting from the different rate of heat loss between the resistors in the reference gas and the resistors in the sample gas. By choosing a reference gas independent of those gases of relevance, the TCD can detect any gas.

Because of concern that ammonia had the potential to damage the GC electronics, which it was already suspected of having done to the mass spectrometer, the GC was flushed with nitrogen or argon after each day if ammonia was used. First the inert gas was run through the sample loop at high flow for 10 minutes, then a series of injections flushed the electronics and showed that ammonia was no longer present inside the GC.

Until after the secondary insert measurements were completed, the GC was checked daily with the nitrogen standard and weekly with the $5 \%$ hydrogen/95\% nitrogen standard to confirm the consistency of its measurements 
Jonathan Tailby

For the tertiary insert experiments, hydrogen/nitrogen and ammonia standards were measured daily.

In the secondary and tertiary insert experiments the area of the ammonia peak was measured to 3 minutes after the maximum. The peak continued for more than three times this length, however it was more efficient to use only the first three minutes of data due to interference in the electrical supply of the GC which caused loss of data. The majority of the peak area was in this first three minutes, and using only the first three minutes enabled the successful collection of more decomposition results than could have been collected by requiring the full peak. Comparison between the peaks to three minutes, the peaks to six minutes and the peaks to nine minutes gave outcomes within $0.4 \%$ difference of the standardised percentages, however the peaks to 3 minutes enabled collection of more results because interference at 5 minutes past the maximum would ruin the 6 and 9 minute area calculations but not the 3 minute calculation. For this reason the peak areas were calculated to 3 minutes past the maximum.

\subsubsection{Summary of GC details}

Table 2.2.7 contains a summary of the GC operation details for this research.

With the tertiary inserts the column temperature ran at $30^{\circ} \mathrm{C}$ for two minutes, until all of the hydrogen and nitrogen had left the column. The column temperature then increased by $20{ }^{\circ} \mathrm{C} \cdot \mathrm{min}^{-1}$ to $100{ }^{\circ} \mathrm{C}$ to accelerate the rate that ammonia travelled through the column. At 15 minutes the column cooled back to $30^{\circ} \mathrm{C}$.

\begin{tabular}{|l|l|}
\hline Table 2.2.7: Summary of GC details \\
\hline Instrument & SRI8610C \\
\hline Carrier gas & $\begin{array}{l}\text { Ar initially } 30 \text { psi (EPC local set point 35 psi, } \\
\text { Computer set point 29 psi, Input 29 psi), later 26 psi } \\
\text { (EPC disconnected, Input 26 psi) }\end{array}$ \\
\hline Column type & Porapak QS $3 \mathrm{~m}$ \\
\hline Column temperature & temperature $30^{\circ} \mathrm{C}$ (tertiary inserts with 30-100 $\left.{ }^{\circ} \mathrm{C}\right)$ \\
\hline TCD temperature & $100^{\circ} \mathrm{C}(106)$ \\
\hline Valve oven temperature & $30^{\circ} \mathrm{C}(36)$ \\
\hline Injection volume & $0.2 \mathrm{~mL}$ \\
\hline
\end{tabular}




\subsubsection{Preliminary Experiments}

Preliminary experiments were designed to test the functionality and response of the instrument then to identify the optimal settings for the various parameters. Details and results of these are included in Appendix 5.1.2.

\subsubsection{Molar Flow Calculations}

This section explores the derivations of equations to calculate the molar output of each gas species from the percentage output of that gas and the gas input.

\subsection{Terms and Assumptions}

The starting point for the derivation of the equations was to set out the quantities relevant to the calculation, which ones were known and unknown and the relationships that exist between them. This section sets out these terms and assumptions.

Initially, ammonia gas at 1 atmosphere of pressure entered the reactor at room temperature, with the volumetric flow controlled by a rotameter set to 1, 2, 3, 4 or $5 \mathrm{~mL} \cdot \mathrm{min}^{-1}$. When the gas entered the reactor there could be changes in temperature and point pressure that could affect the volumetric flow. Throughout these changes molar flow would remain constant.

However, molar flow will change when the ammonia decomposes. The total molar flow of all gases will increase at decomposition because there are twice as many product molecules as the number of decomposed ammonia molecules, as shown in Equation 2.2.2. For the palladium membrane experiments, the permeation of hydrogen through the membrane would decrease the hydrogen on the retentate side, reducing the total molar flow there, but that permeation would increase the total flow on the permeate side.

Equation 2.2.2: Chemical equation for ammonia decomposition. $2 \mathrm{NH}_{3} \rightleftarrows \mathrm{N}_{2}+3 \mathrm{H}_{2}=\mathrm{NH}_{3} \rightleftarrows \frac{1}{2} \mathrm{~N}_{2}+\frac{3}{2} \mathrm{H}_{2}$

\section{Key Variables}

Table 2.2.8 lists the important variables used to calculate molar flows in the ammonia decomposition. 
Jonathan Tailby

The variable nHPerm, the molar rate of hydrogen permeation, is listed as both unknown and derived. In the calculations of permeate flow it is the target unknown variable, however, in the retentate flow calculations it is assumed to be the respective value from the permeate calculations, hence a derived variable.

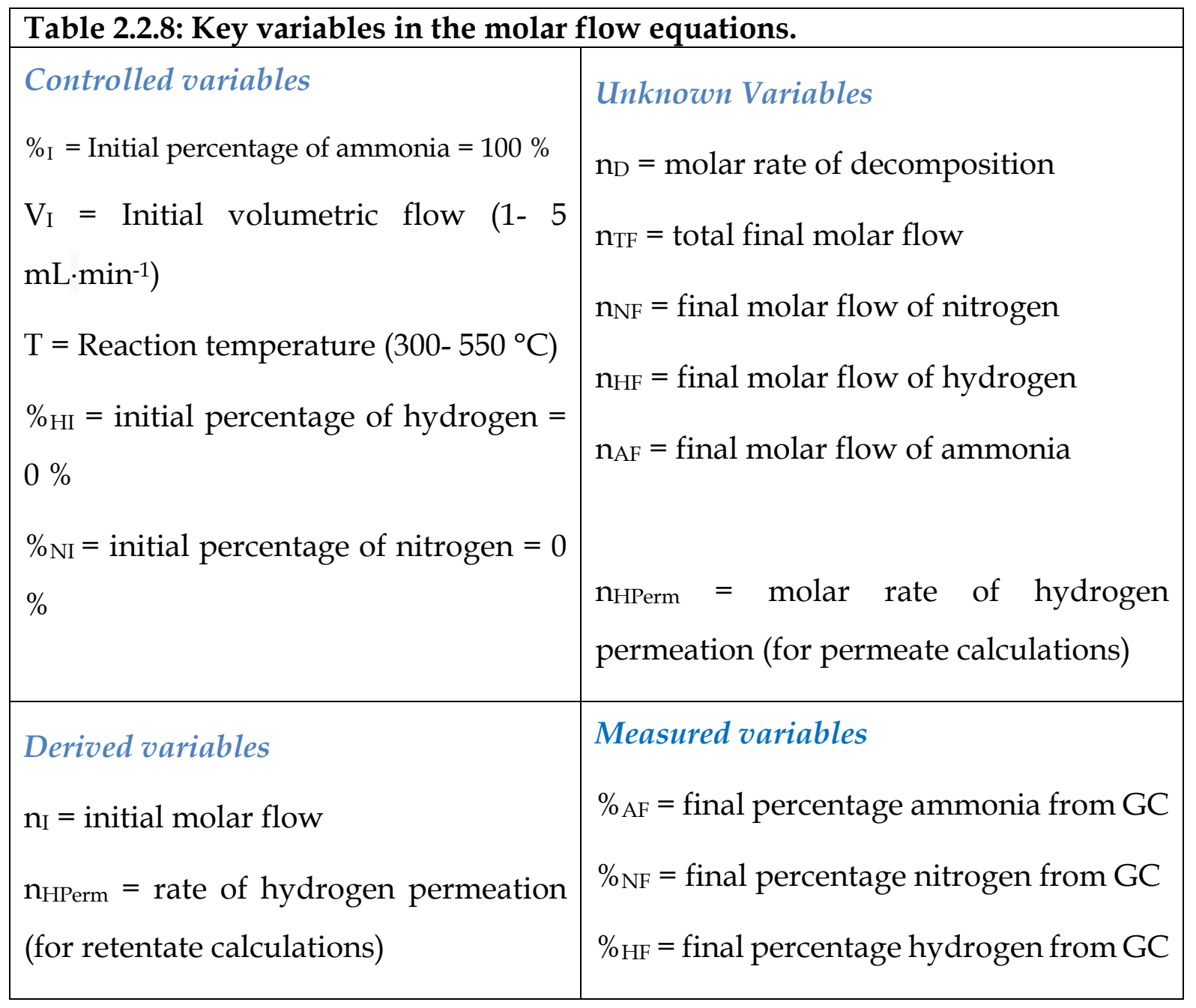

\section{Assumed Relationships}

Table 2.2.9 lists some assumed relationships between quantities that are used to develop equations for the calculating the molar flow of each species from the gas chromatograph measurement and the initial flow.

\section{Table 2.2.9: Assumed relationships in the gas reactor.}

Final flow of nitrogen is equal to the final percentage of $n_{N F}=\%_{N F} \times n_{T F}$ nitrogen multiplied by the total final flow. 


\begin{tabular}{|l|c|}
\hline $\begin{array}{l}\text { Total final flow }=\text { the sum of the flows of the } \\
\text { constituent species. }\end{array}$ & $n_{T F}=n_{A F}+n_{N F}+n_{H F}$ \\
\hline $\begin{array}{l}\text { Final ammonia flow is equal to the initial } \\
\text { ammonia flow minus the rate of } \\
\text { decomposition }\end{array}$ & $n_{A F}=n_{I}-n_{D}$ \\
\hline $\begin{array}{l}\text { Final nitrogen flow is equal to half the rate of } \\
\text { decomposition. }\end{array}$ & $n_{N F}=\frac{1}{2} n_{D}$ \\
\hline $\begin{array}{l}\text { Final hydrogen flow is equal to three times } \\
\text { half the rate of decomposition. }\end{array}$ & $n_{H F}=\frac{3}{2} n_{D}$ \\
\hline $\begin{array}{l}\text { Total final flow is equal to initial flow less rate of } \\
\text { decomposition, add half the rate of decomposition for } \\
\text { nitrogen formation, add three halves rate of } \\
\text { decomposition for hydrogen formation. }\end{array}$ & $n_{T F}=n_{I}-n_{D}+\frac{1}{2} n_{D}+\frac{3}{2} n_{D}$ \\
\hline $\begin{array}{l}\text { Simplify the previous equation to total final flow is } \\
\text { equal to initial flow plus the rate of decomposition. }\end{array}$ & $n_{T F}=n_{I}+n_{D}$ \\
\hline
\end{tabular}


Jonathan Tailby

\subsection{Calculating Molar Flow without Palladium Membrane}

In the experiments without a palladium membrane the only event that is expected to change the molar flow is the decomposition of ammonia. This will produce a molar quantity of nitrogen and hydrogen greater than the molar quantity of ammonia that decomposes (see Equation 1.2.2).

The proportion of each gas was measured at the gas chromatograph which was compared with a known standard to give a percentage of gas. The final molar flow of the gas is equal to the final percentage of that gas in the total molar flow, expressed in Equation 2.2.3.

Equation 2.2.3: Relationship of the final molar flow of nitrogen to the total molar flow

$n_{N F}=\%_{N F} \times n_{T F}$

$\mathrm{mol} \cdot \mathrm{min}^{-1}=$ unitless proportion $\mathrm{x} \mathrm{mol} \cdot \mathrm{min}^{-1}$

Although $\mathrm{n}_{\mathrm{TF}}$ is not initially known, the assumptions in Table 2.2.9 enable a new equation to be derived, as shown in Equation 2.2.4.

Equation 2.2.4: Derivation of an equation for $n_{N F}$ from known variables.

Because $n_{T F}=n_{I}+n_{D}$ and $n_{N F}=\%_{N F} \times n_{T F}$

$n_{N F}=\%_{N F} \times\left(n_{I}+n_{D}\right)$

Because $n_{D}=\%_{D} \times n_{I}$

$n_{N F}=\%_{N F} \times\left(n_{I}+\%_{D} \times n_{I}\right)$

Because $\%_{D}=2 \times \%_{N F}$

$n_{N F}=\%_{N F} \times\left(n_{I}+2 \times \%_{N F} \times n_{I}\right)$

$\mathrm{mol} \cdot \mathrm{min}^{-1}=$ unitless proportion $\mathrm{x}\left(\mathrm{mol} \cdot \mathrm{min}^{-1}+\right.$ unitless ratio $\mathrm{x}$ unitless proportion $\left.\mathrm{x} \mathrm{mol} \cdot \mathrm{min}^{-1}\right)$

$=$ unitless proportion $\mathrm{x}\left(\mathrm{mol} \cdot \mathrm{min}^{-1}+\mathrm{mol} \cdot \mathrm{min}^{-1}\right)$

$=$ unitless proportion $\mathrm{x}\left(\mathrm{mol} \cdot \mathrm{min}^{-1}\right)$

$=\operatorname{mol} \cdot \mathrm{min}^{-1}$

The units of measurement in the calculation follow through to match the unit of measurement of the calculated quantity. 
This equation enables the final molar flow of nitrogen to be calculated from the final percentage of nitrogen and the initial gas flow. The derivation for the final molar flow of hydrogen follows a similar pattern to Equation 2.2.4, differing only in the last step, because $\%_{D}=2 / 3 \% n_{H F}$. This produces Equation 2.2.5.

Equation 2.2.5: Equation for calculating $\mathbf{n}_{\mathrm{HF}}$ from known variables.

$n_{H F}=\%_{H F}\left(n_{I}+\frac{2}{3} \times \%_{H F} \times n_{I}\right)$

When Equation 2.2.4 was tested with simulated data for the whole numbers $0 \leq \% \mathrm{D}$ $\leq 45$ and initial flow of $2.01 \times 10^{-04} \mathrm{~mol} \cdot \mathrm{min}^{-1}$, which was the flow used for the experiments which tested a range of temperatures, thirty-two values returned exactly the same as the simulated data. This data is shown in Appendix 5.1.5.2. For the remaining thirteen values the difference between the simulated values and the calculated values were less than $1.4 \times 10^{-20} \mathrm{~mol} \cdot \mathrm{min}^{-1}$, compared to the smallest value being more than $1 \times 10^{-6} \mathrm{~mol} \cdot \mathrm{min}^{-1}$. Equation 2.2.5 was tested on the same data and produced fifteen values with non-zero differences less than $5.5 \times 10^{-20} \mathrm{~mol} \cdot \mathrm{min}^{-1}$. Which compares to the smallest calculated value of $3.05 \times 10^{-6} \mathrm{~mol} \cdot \mathrm{min}^{-1}$. The magnitude of the difference between calculations and the apparent errors, was deemed acceptable, so the equations were accepted. The test calculations are shown in Appendix 5.1.5.1.

The derivation of an equation for ammonia started with the same initial relationship, but required some different steps to produce a useful equation. The derivation used to produce an equation for calculating the final molar flow of ammonia is shown in Equation 2.2.6.

Equation 2.2.6: Deriving an equation to calculate the final flow of ammonia. $n_{A F}=\%_{A F} n_{T F}$

$$
\begin{aligned}
& \text { Because } n_{T F}=n_{I}+n_{D} \\
& n_{A F}= \%_{A F}\left(n_{I}+n_{D}\right) \\
& \text { Because } n_{D}=\%_{D}+n_{I} \\
& n_{A F}=\%_{A F}\left(n_{I}+\%_{D} \times n_{I}\right)
\end{aligned}
$$$$
\text { Because } \%_{D}=\%_{A I}-\%_{A F}
$$ 
Jonathan Tailby

$n_{A F}=\%_{A F}\left(n_{I}+\left(\%_{A I}-\%_{A F}\right) \times n_{I}\right)$

Substitute units of measurements into the final equation to test whether they match. $\mathrm{mol} \cdot \mathrm{min}^{-1}=$ unitless proportion $\mathrm{x}\left(\mathrm{mol} \cdot \mathrm{min}^{-1}+(\right.$ unitless proportion - unitless proportion) $\mathrm{x} \mathrm{mol} \cdot \mathrm{min}^{-1}$ )

$$
\begin{aligned}
& =\text { unitless proportion } \times\left(\mathrm{mol} \cdot \mathrm{min}^{-1}+(\text { unitless proportion }) \times \mathrm{mol} \cdot \mathrm{min}^{-1}\right) \\
& =\text { unitless proportion } \mathrm{x}\left(\mathrm{mol} \cdot \mathrm{min}^{-1}+\mathrm{mol} \cdot \mathrm{min}^{-1}\right) \\
& =\text { unitless proportion } \mathrm{x} \mathrm{mol} \cdot \mathrm{min}^{-1} \\
& =\mathrm{mol} \cdot \mathrm{min}^{-1}
\end{aligned}
$$

The units of measurement follow through the calculation to match the unit of measurement for the calculated quantity.

Equation 2.2.6 was tested with the same simulated data as Equation 2.2.4 and Equation 2.2.5 as shown in Appendix 5.1.5.3. Of the nine test calculations that did not produce exactly the starting number, the greatest difference was $5.42 \times 10^{-20}$ $\mathrm{mol} \cdot \mathrm{min}^{-1}$, compared to the smallest calculated value of $1.60 \times 10^{-4} \mathrm{~mol} \cdot \mathrm{min}^{-1}$. Equations were produced for calculating the molar flow of each of the gas species detected by the gas chromatograph. In the absence of measurements of the flow leaving the reactor, the results of these calculations were used to compare the different decomposition experiments.

\subsection{Calculations for Tertiary Insert Results}

The three equations produced in Section 2.2.12.3.2 calculated the molar flow when the only event to affect the molar flow was the decomposition of ammonia. In the experiments with a palladium membrane, there was the potential for changing the molar flow of hydrogen by permeation through the membrane. The previous equations did not account for permeation and new equations had to be developed.

\subsection{Permeate Side Calculation}

The permeate side of the palladium membranes had the key variables listed in Table 2.2.10. Initially the flow was the pure argon sweep gas, but as the hydrogen permeated, the total molar flow would increase and hydrogen would appear in the 
gas chromatograph measurements. The argon sweep gas did not appear in the gas chromatograph measurements because it was both the carrier gas and the reference gas for the gas chromatograph.

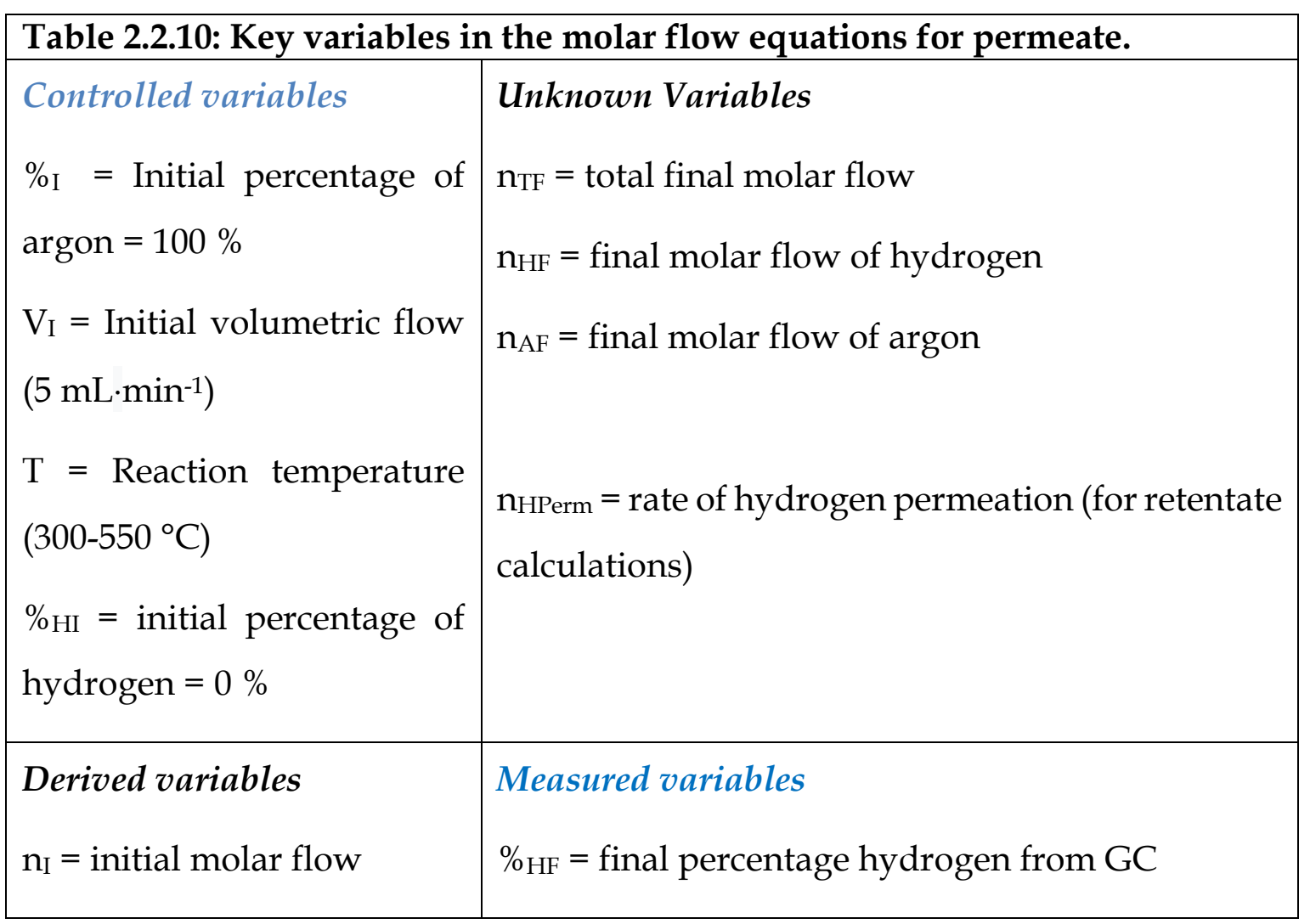

An equation for calculating nHPerm on the permeate side from the known quantities can be derived using the method shown in Equation 2.2.7

Equation 2.2.7: Deriving an Equation to Calculate $\mathbf{n}_{H P e r m}$ in the Permeate $n_{\text {HPerm }}=\%_{\text {HPerm }} \times n_{T}$

Because total gas flow equals the sum of all gas flows, $n_{T}=n_{A r}+n_{H P e r m}$ $n_{\text {HPerm }}=\%_{H \text { Perm }} \times\left(n_{\text {Ar }}+n_{\text {HPerm }}\right)$

expanding the bracket on the right-hand side

$n_{\text {HPerm }}=\%_{\text {HPerm }} \times n_{\text {Ar }}+\%_{\text {HPerm }} \times n_{\text {HPerm }}$ subtract $\%_{\text {HPerm }} \times n_{\text {HPerm }}$ from both sides.

$n_{\text {HPerm }}-\%_{\text {HPerm }} \times n_{\text {HPerm }}=\%_{\text {HPerm }} \times n_{\text {Ar }}$

factorise the left-hand side 
Jonathan Tailby

$n_{\text {HPerm }}\left(1-\%_{\text {HPerm }}\right)=\%_{H \text { Herm }} \times n_{\text {Ar }}$

divide both sides by the bracket on the left-hand side

$n_{\text {HPerm }}=\frac{\%_{H P e r m} \times n_{A r}}{1-\%_{H P e r m}}$

This equation was tested with 100 values of $\%$ HPerm up to $33 \%$, using the model shown in Appendix 5.1.5.4. This returned exactly the starting value in seventy five cases. In the remaining twenty five cases, the difference between the calculated $\mathrm{n}_{\text {HPerm }}$ and the starting $\mathrm{n}_{\text {HPerm }}$ was less than $1.5 \times 10^{-17}$. This equation was accepted as sufficiently accurate.

\subsection{Retentate Side Calculation}

Initially the $\mathrm{n}_{\mathrm{HF}}$ values for the retentate side would be significantly larger than the $\mathrm{n}$ HPerm values for the permeate side. When hydrogen begins to diffuse through the palladium to the permeate side the nHPerm values for the permeate side will begin to increase. Potentially the system will reach an equilibrium where the palladium membrane is desorbing palladium at the same rate as it is absorbing it and the nHPerm be equal to the $\mathrm{n}_{H F}$.

Before any samples were assayed in the gas chromatograph the reactor temperature was allowed to come to a steady state with flowing ammonia for at least twenty minutes. Each experiment is recorded as an average of multiple measurements taken on the same day, each measurement requiring more than twenty minutes, therefore, each data point represents more than an hour, sometimes up to six hours of continuous ammonia flow. The maximum hydrogen capacity of the membranes is calculated in Table 2.2.11 $3.5 \times 10^{-4}$ moles of hydrogen for the Nickel-beforePalladium tertiary insert and $2.9 \times 10-4$ moles of hydrogen for the Nickel-onPalladium insert. 
Table 2.2.11: Maximum hydrogen content of the palladium.

\begin{tabular}{|l|l|l|l|l|}
\cline { 2 - 5 } \multicolumn{1}{c|}{} & $\begin{array}{l}\text { OD } \\
\mathbf{m m}\end{array}$ & $\begin{array}{l}\text { Circumference } \\
\mathbf{m m}\end{array}$ & Length $\mathbf{m m}$ & $\begin{array}{l}\text { Area } \\
\left.\mathbf{( m m}^{\mathbf{2}}\right)\end{array}$ \\
\hline $\begin{array}{l}\text { Ni- } \\
\text { before-Pd }\end{array}$ & 9.21 & 28.9 & 20.2 & 585.0 \\
\hline Ni-on-Pd & 7.79 & 24.5 & 20.2 & 494.8 \\
\hline
\end{tabular}

\begin{tabular}{|c|c|c|c|c|c|}
\hline & $\begin{array}{l}\text { Area } \\
\left(\mathrm{mm}^{3}\right)\end{array}$ & Thickness & $\begin{array}{l}\text { Volume of } \\
\operatorname{Pd}\left(\mathrm{mm}^{3}\right)\end{array}$ & $\begin{array}{l}\begin{array}{l}\multicolumn{2}{l}{\text { Volume }} \\
\text { of } \\
\left(\mathrm{cm}^{3}\right)\end{array} \\
\end{array}$ & $\begin{array}{l}\text { Density } \\
\mathrm{g} \mathrm{cm}^{-3}\end{array}$ \\
\hline $\begin{array}{l}\mathrm{Ni}- \\
\text { before-Pd }\end{array}$ & 585.0 & 0.1 & 58.5 & 0.0585 & 12.023 \\
\hline Ni-on-Pd & 494.8 & 0.1 & 49.4 & 0.0495 & 12.023 \\
\hline
\end{tabular}

\begin{tabular}{|l|l|l|l|l|}
\cline { 2 - 5 } \multicolumn{1}{c|}{} & $\begin{array}{l}\text { Mass } \\
\text { of } \mathbf{P d}\end{array}$ & $\begin{array}{l}\text { Molar Mass of } \\
\mathbf{P d}\end{array}$ & Moles of $\mathbf{P d}$ & Moles $\mathbf{H}$ in $\mathbf{P d}_{\mathbf{9 5}} \mathbf{H}_{\mathbf{5}}$ \\
\hline $\begin{array}{l}\text { Ni- } \\
\text { before-Pd }\end{array}$ & 0.703 & 106.42 & 0.00661 & 0.000331 \\
\hline Ni-on-Pd & 0.595 & 106.42 & 0.00559 & 0.000279 \\
\hline
\end{tabular}

Table 2.2.12 compares the hydrogen capacity of the membranes with the hydrogen permeation to estimate the time it would take to completely fill the membrane. This calculation assumes the hydrogen content of the membrane increased at the average rate it left the downstream side shown for the Nickel-before-Palladium insert in Table 3.3.1 and for the Nickel-on-Palladium membrane in Table 3.3.5. From the calculated times shown in Table 2.2.12 it is reasonable to expect that in these experiments the membranes approached equilibrium conditions during the measurements at 400 and $450{ }^{\circ} \mathrm{C}$ and thus the nHPerm values for the retentate and the permeate were equal. The adsorption and desorption of the membrane is less likely to have reached an equilibrium for the 300 and $350{ }^{\circ} \mathrm{C}$ measurements.

Table 2.2.12: Time taken to fill the palladium membranes with hydrogen.

\begin{tabular}{|c|c|c|c|c|c|}
\hline Temperature & 300 & 350 & 400 & 450 & ${ }^{\circ} \mathrm{C}$ \\
\hline \multicolumn{6}{|c|}{ Nickel-before-Palladium } \\
\hline Permeation & $1.27 \mathrm{E}-08$ & 7.23E-07 & 4.60E-06 & 1.70E-05 & mol $\mathrm{min}^{-1}$ \\
\hline $\begin{array}{ll}\text { Time } & \text { to } \\
\text { Capacity } & \end{array}$ & 26000 & 457 & 72.0 & 19.5 & minutes \\
\hline \multicolumn{6}{|c|}{ Nickel-on-Palladium } \\
\hline Permeation & $2.49 \mathrm{E}-07$ & $2.59 \mathrm{E}-06$ & 1.46E-05 & 5.76E-05 & mol min $^{-1}$ \\
\hline $\begin{array}{l}\text { Time to } \\
\text { Capacity }\end{array}$ & 1120 & 108 & 19.1 & 4.85 & minutes \\
\hline
\end{tabular}


Jonathan Tailby

The retentate flows were calculated by including a negative term for the permeation of hydrogen, $\mathrm{n}_{\mathrm{HPerm}}$ measured in $\mathrm{mol} \cdot \mathrm{min}^{-1}$ into the total gas flow, as shown in Equation 2.2.7. When a value for this term was needed, the respective value for the $\mathrm{n}_{\text {HPerm }}$ in the retentate was used.

Equation 2.2.8: New Expression for Final Total Gas Flow, which now Incorporates a Term for Hydrogen Permeation.

$n_{T F}=n_{I}+n_{D}-n_{H P e r m}$

The derivations for the equations to calculate final molar gas flow had to be changed to allow for this variation. Equation 2.2.9 shows the derivation for the equation to calculate the total flow of nitrogen in the retentate. Initially, known relationships between the terms are combined, then algebraic rearrangements separate the known terms from the unknown term.

Equation 2.2.9: Derivation for the calculation for the total flow of nitrogen in the retentate.

$n_{N F}=\%_{N F}\left(n_{T F}\right)$

Because now $n_{T F}=n_{I}+n_{D}-n_{\text {HPerm }}$

$n_{N F}=\%_{N F}\left(n_{I}+n_{D}-n_{H P e r m}\right)$

Because $n_{D}=2 \times n_{N F}$

$n_{N F}=\%_{N F}\left(n_{I}+2 \times n_{N F}-n_{H P e r m}\right)$

$n_{N F}=\%_{N F} \times n_{I}+\%_{N F} \times 2 \times n_{N F}-\%_{N F} \times n_{H P e r m}$

$n_{N F}-\%_{N F} \times 2 \times n_{N F}=\%_{N F} \times n_{I}-\%_{N F} \times n_{H P e r m}$

$n_{N F}\left(1-\%_{N F} \times 2\right)=\%_{N F} \times n_{I}-\%_{N F} \times n_{H P e r m}$

$n_{N F}=\frac{\%_{N F} \times n_{I}-\%_{N F} \times n_{H P e r m}}{\left(1-\%_{N F} \times 2\right)}$

$n_{N F}=\frac{\%_{N F} \times\left(n_{I}-n_{H P e r m}\right)}{\left(1-\%_{N F} \times 2\right)}$

$n_{N F}=\frac{\%_{N F}\left(n_{I}-n_{H P e r m}\right)}{1-2 \times \%_{N F}}$ 
The equation that resulted from Equation 2.2.9 was put through the test calculations for $0 \leq \%_{\mathrm{D}} \leq 40$. The results from these calculations are shown in Appendix 5.1.5.6. Equation 2.2.10 shows the derivation for an equation to calculate the total flow of hydrogen in the retentate. This required an additional hydrogen permeation term where the production of hydrogen is substituted for the decomposition of ammonia.

\section{Equation 2.2.10: Deriving the Equation to Calculate the Final Molar Flow of} Hydrogen.

$n_{H F}=\%_{H F}\left(n_{T F}\right)$

Because now $n_{T F}=n_{I}+n_{D}-n_{H P e r m}$

$n_{H F}=\%_{H F}\left(n_{I}+n_{D}-n_{H P e r m}\right)$

Because $n_{D}=\frac{2}{3} \times\left(n_{H F}+n_{H P e r m}\right)$

$n_{H F}=\%_{H F}\left(n_{I}+\frac{2}{3} \times\left(n_{H F}+n_{H P e r m}\right)-n_{H P e r m}\right)$

$n_{H F}=\%_{H F} n_{I}+\%_{H F} \times \frac{2}{3} \times\left(n_{H F}+n_{H P e r m}\right)-n_{H P e r m} \times \%_{H F}$

$n_{H F}=\%_{H F} n_{I}+\%_{N F} \times \frac{2}{3} \times n_{H F}+\%_{H F} \times \frac{2}{3} \times n_{H P e r m}-n_{H P e r m} \times \%_{H F}$

$n_{H F}=\%_{H F} n_{I}+\%_{H F} \times \frac{2}{3} \times n_{H F}+\%_{H F} \times \frac{2}{3} \times n_{H P e r m}-n_{H P e r m} \times \%_{H F}$

$n_{H F}-\%_{H F} \times \frac{2}{3} \times n_{H F}=\%_{H F} n_{I}-\frac{1}{3} \times n_{H P e r m} \times \%_{H F}$

$n_{H F}\left(1-\%_{H F} \times \frac{2}{3}\right)=\%_{H F} n_{I}-\frac{1}{3} \times n_{H P e r m} \times \%_{H F}$

$n_{H F}=\frac{\%_{H F} n_{I}-\frac{1}{3} \times n_{H P e r m} \times \%_{H F}}{\left(1-\%_{H F} \times \frac{2}{3}\right)}$

Rearrange right hand side.

$n_{H F}=\frac{\%_{H F}\left(n_{I}-\frac{1}{3} n_{H P e r m}\right)}{1-\frac{2}{3} \times \%_{H F}}$ 
Jonathan Tailby

One test of the equation is whether the calculated answer is measured in the same units as the desired quantity. This is tested here by substitution of the units of measurement into the formula and simplifying it down.

$\mathrm{mol} \cdot \mathrm{min}^{-1}=\left(\right.$ unitless proportion $\mathrm{x}\left(\mathrm{mol} \cdot \mathrm{min}^{-1}-\mathrm{mol} \cdot \mathrm{min}^{-1}\right) /($ unitless - unitless ratio $\mathrm{x}$ unitless proportion $)$

$$
\begin{aligned}
& =\left(\text { unitless proportion } \times\left(\mathrm{mol} \cdot \mathrm{min}^{-1}\right) / \text { unitless }\right) \\
& =\mathrm{mol} \cdot \mathrm{min}^{-1}
\end{aligned}
$$

The resultant unit of measurement matches the unit of measurement for the desired quantity. This is an important mark of the validity of the equation.

When Equation 2.2.10 was put through the test calculations for $0 \leq \%_{\mathrm{D}} \leq 40,16$ results differed from the original value, and the greatest difference was $4.07 \times 10^{-20}$, compared to a minimum $\mathrm{n}_{\mathrm{HF}}$ value of $2.59 \times 10^{-6} \mathrm{~mol} \cdot \mathrm{min}^{-1}$.

Equation 2.2.11 shows the derivation to produce the equation that was used for calculating the final molar flow of ammonia.

\section{Equation 2.2.11: Calculating the Final Molar Flow of Ammonia in the Retentate}

$n_{A F}=\%_{A F} n_{T F}$

Because $n_{T F}=n_{I}+n_{D}-n_{H P e r m}$

$n_{A F}=\%_{A F}\left(n_{I}+n_{D}-n_{H P e r m}\right)$

Because $n_{D}=n_{I}-n_{A F}$

$n_{A F}=\%_{A F}\left(n_{I}+n_{I}-n_{A F}-n_{H P e r m}\right)$

$n_{A F}=\%_{A F}\left(2 n_{I}-n_{A F}-n_{H P e r m}\right)$

$n_{A F}=\%_{A F}\left(2 n_{I}-n_{H P e r m}\right)-\%_{A F} n_{A F}$

$n_{A F}+\%_{A F} n_{A F}=\%_{A F}\left(2 n_{I}-n_{H P e r m}\right)$

$n_{A F}\left(1+\%_{A F}\right)=\%_{A F}\left(2 n_{I}-n_{H P e r m}\right)$

$n_{A F}=\frac{\%_{A F}\left(2 n_{I}-n_{H P e r m}\right)}{\left(1+\%_{A F}\right)}$

Equation 2.2.11 was also tested by substitution of the units of measurement into the formula.

$\mathrm{mol} \cdot \mathrm{min}^{-1}=\left(\right.$ unitless proportion $\mathrm{x}\left(\right.$ unitless ratio $\left.\left.\mathrm{x} \mathrm{mol} \cdot \mathrm{min}^{-1}-\mathrm{mol} \cdot \mathrm{min}^{-1}\right)\right) /($ unitless + unitless proportion $)$ 


$$
\begin{aligned}
& =\left(\text { unitless proportion } \mathrm{x} \mathrm{mol} \cdot \mathrm{min}^{-1}-\mathrm{mol} \cdot \mathrm{min}^{-1}\right) / \text { unitless } \\
& =\left(\mathrm{mol} \cdot \mathrm{min}^{-1}-\mathrm{mol} \cdot \mathrm{min}^{-1}\right) / \text { unitless } \\
& =\mathrm{mol} \cdot \mathrm{min}^{-1} / \text { unitless } \\
& =\mathrm{mol} \cdot \mathrm{min}^{-1}
\end{aligned}
$$

The resultant unit of measurement matches the unit of measurement for the desired quantity. This equation also has this important mark of the validity of an equation.

Equation 2.2.11 was put through the test calculations for $0 \leq \%_{\mathrm{D}} \leq 40,17$ results differed from the original value, and the greatest difference was $2.71 \times 10^{-20}$, compared to a minimum $\mathrm{n}_{\mathrm{AF}}$ value of $1.21 \times 10^{-4} \mathrm{~mol} \cdot \mathrm{min}^{-1}$.

The error in the test calculations for the equations to calculate the final molar flow of each species in the retentate were comparable to the error in the calculations used without hydrogen permeation, so these retentate equations were also accepted for this work. Data processed using these equations was used to compare the outcomes of the different decomposition and permeation experiments, as shown in Sections 3.2 and 3.3.

\subsubsection{Calculating Arrhenius Activation Energy}

The activation energy was calculated from the rates of reaction using the Arrhenius Equation, shown in Equation 2.2.12.

\section{Equation 2.2.12: Arrhenius Equation}

$k=A \cdot e^{-\frac{E_{a}}{R \cdot T}}$

Where $\mathrm{k}$ is the rate coefficient; $\mathrm{A}$ is a preexponential factor which is taken to be a constant; $E_{a}$ is the activation energy; $\mathrm{R}$ is the universal gas constant and $\mathrm{T}$ is the temperature in degrees Kelvin.

An alternate but equivalent form of the equation is shown in Equation 2.2.13.

\section{Equation 2.2.13: Alternate form of Arrhenius Equation}

$\ln k=\ln A-\frac{E_{a}}{R} \cdot \frac{1}{T}$ 
The rate results from Section 3.2.2.4 were used to create Arrhenius plots with $y=$ $\ln k$ and $x=\frac{1}{T}$. The straight lines produced by these graphs conformed to the general formula $y=m \cdot x+c$, where $c=\ln A$ and $m=-\frac{E_{a}}{R}$. Therefore $E_{a}=-R \cdot m$. Values for activation energy were calculated for all rate values including ammonia decomposition, nitrogen formation and hydrogen formation for all secondary and tertiary inserts. The steps for these calculations are shown in Appendices 5.2.7.3.1 and 5.2.7.4.1, with the outcomes summarised in Sections 3.2.2.4.6 and 3.3.3.1.1.

\subsubsection{Specific Rate of Reaction}

The rate of reaction per mass of catalyst, specific rate of reaction, was calculated by dividing the rate of reaction by the mass of catalyst as per Equation 2.2.14.

\section{Equation 2.2.14: Specific rate of reaction}

$r_{\text {specific }}=\frac{r}{m}$

Where $\mathrm{r}$ is the rate of reaction in $\mathrm{mol} \mathrm{min}^{-1}$ and $\mathrm{m}$ is the mass of catalyst in $\mathrm{g}$, $\mathrm{r}_{\text {specific }}$ is the specific rate of reaction in $\mathrm{mol} \mathrm{min}^{-1} \mathrm{~g}^{-1}$. Conversion to specific rate enables more valid comparison with reaction rates reported by other researchers. Calculated specific rates of reaction for the secondary insert catalysts are included in Section 3.2.2.4.6.

\subsubsection{Electronic Gas Pressure Control}

The rate of carrier gas flow through the GC column was controlled by the carrier gas pressure at the entrance to the column. The GC had an electronic gas pressure control (EPC) which was intended to keep the carrier gas pressure constant, thereby ensuring constant carrier gas flow through the column and the detectors. Significant problems with the EPC arose after the secondary insert experiments had been completed. These were resolved before the tertiary insert experiments commenced by cutting into the gas line from the EPC to the TCD reference gas and connecting the gas input to the TCD line. This completely bypassed the EPC so the EPC could have no effect on the gas flow rate and the carrier gas pressure depended entirely on the regulator which controlled the input pressure of argon. To improve the consistency of the input pressure, the Norgren R07-200-RNKG single stage 
regulator was replaced with the Victor 8PT 2700 two stage regulator. This kept a more stable pressure, but the operating procedure was altered so that subsequent measurements were always standardised with measurements from the same day before being compared with measurements from other days.. Further details of the problems and solutions are included in Appendix 5.1.6. 
Jonathan Tailby 


\section{Results and Discussion}

This section brings together the outcomes from the methods and processes described in section 2. Each subsection contains representative results for a different set of experiments.

Section 3.1 focuses on the methods that were used to create potential catalyst samples and the analytical methods used to characterise them. Representative results are shown and interpretation of these results is discussed. This section is divided into subsections based on the different methods tested to form catalysts.

Section 3.2 contains representative results for experiments to test the efficacy of the catalyst samples in ammonia decomposition. The same section contains graphs which compare the apparent effect of the different catalysts and explanation for why nickel foam was chosen as the catalyst for the subsequent tertiary insert experiments.

Section 3.3 contains results for experiments to test the enhancement of the catalyst by hydrogen transport out of the reactor and by hydrogen spillover away from the catalyst. This section discusses these results with comparison to the previous two sections and proposes explanations for the observed outcomes.

\subsection{Catalyst Preparation}

\subsubsection{Electrodeposition Results}

Initially, electrodeposition was explored as a method for forming a nickel catalyst on palladium. Test electrodeposition experiments on aluminium revealed nanostructured microscale nickel deposits with the potential to be a high surface area catalyst for ammonia decomposition. When this process was transferred to palladium, the deposition topography results were very different. The higher electric currents during electrodeposition reflected faster nickel formation which quickly spread across the surface to create a continuous layer. Continuous layers were not expected to be as effective for catalysing ammonia decomposition as discrete islands of nickel, so electrodeposition was halted and an alternative method for catalyst preparation explored. 


\subsubsection{Electrodeposition on Aluminium}

Aluminium metal was used for initial electrodeposition. Aluminium was a cost-effective substitute for palladium during process development. The current vs. time graphs shown in Figure 3.1.1 shows that the electrodepositions had a rapid early increase in current, the two that were allowed to continue past $30 \mathrm{~s}$ peaked then decreased in current before a slow increase.

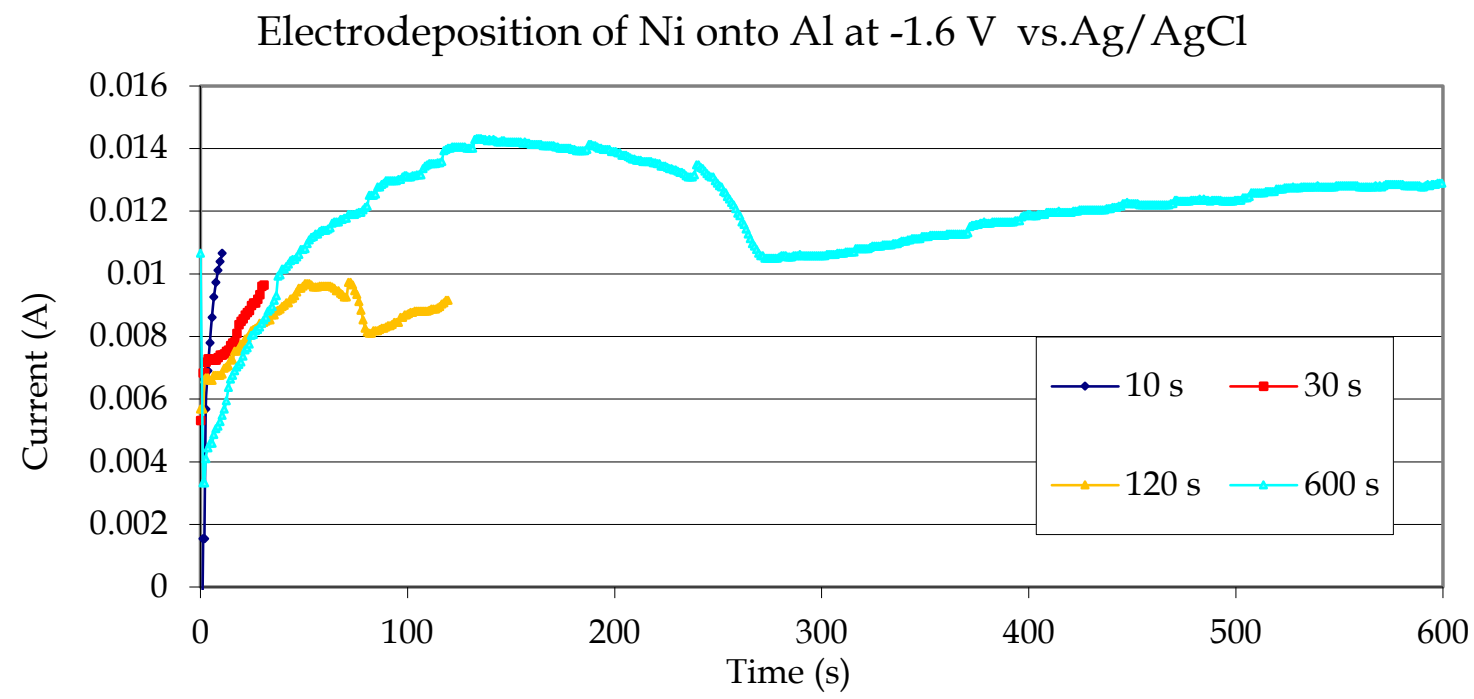

Figure 3.1.1: Current vs. time graphs for electrodeposition of nickel at $-1.6 \mathrm{~V}$ onto aluminium.

Comparison of this measured data to the ideal curves for the nucleation models shown in Section 1.2.5.2.1 suggest that these electrodepositions followed a progressive model of nucleation, newly deposited nuclei formed while pre-existing nuclei continued to grow. Progressive nucleation means that over time the size distribution of nickel deposits would have increased. To ensure electrodeposits of a similar size, the electrodeposition would have to have been stopped quickly, before there had been time for the first electrodeposits to grow and new small nuclei to form. An Alternate electrodeposition regime could have been a two-stage process with a short high voltage nucleation pulse followed by a longer low voltage growth phase with enough overpotential to grow deposits but not enough to form new deposits could have been used to grow large deposits that are still similar in size as described in Section 1.2.5.2.7. 
Figure 3.1.2 shows an SEM image from the $30 \mathrm{~s}$ deposition shown in Figure 3.1.1. This backscatter electron image shows the nickel deposits as brighter than the aluminium background. The right side of the micrograph shows grey shapes marked by a yellow arrow that are likely to be salts left over from electrodeposition. EDS showed that these shapes contained more nickel than the aluminium substrate, but the counterion in the salt would have brought down the average atomic mass in the salts so that they do not show as brightly in the back-scatter micrograph as the nickel metal. The sample shown in Figure 3.1.2 had been rinsed, but subsequent samples were soaked in distilled water to remove the nickel salts. The left side of the micrograph shows that the nickel deposits tend to form in defects on the foil surface such as pits and scratches, for example the line marked by a red arrow. This would be expected if those defects have thinned the oxide coating on the aluminium, thereby decreasing the electrical resistance in those areas.

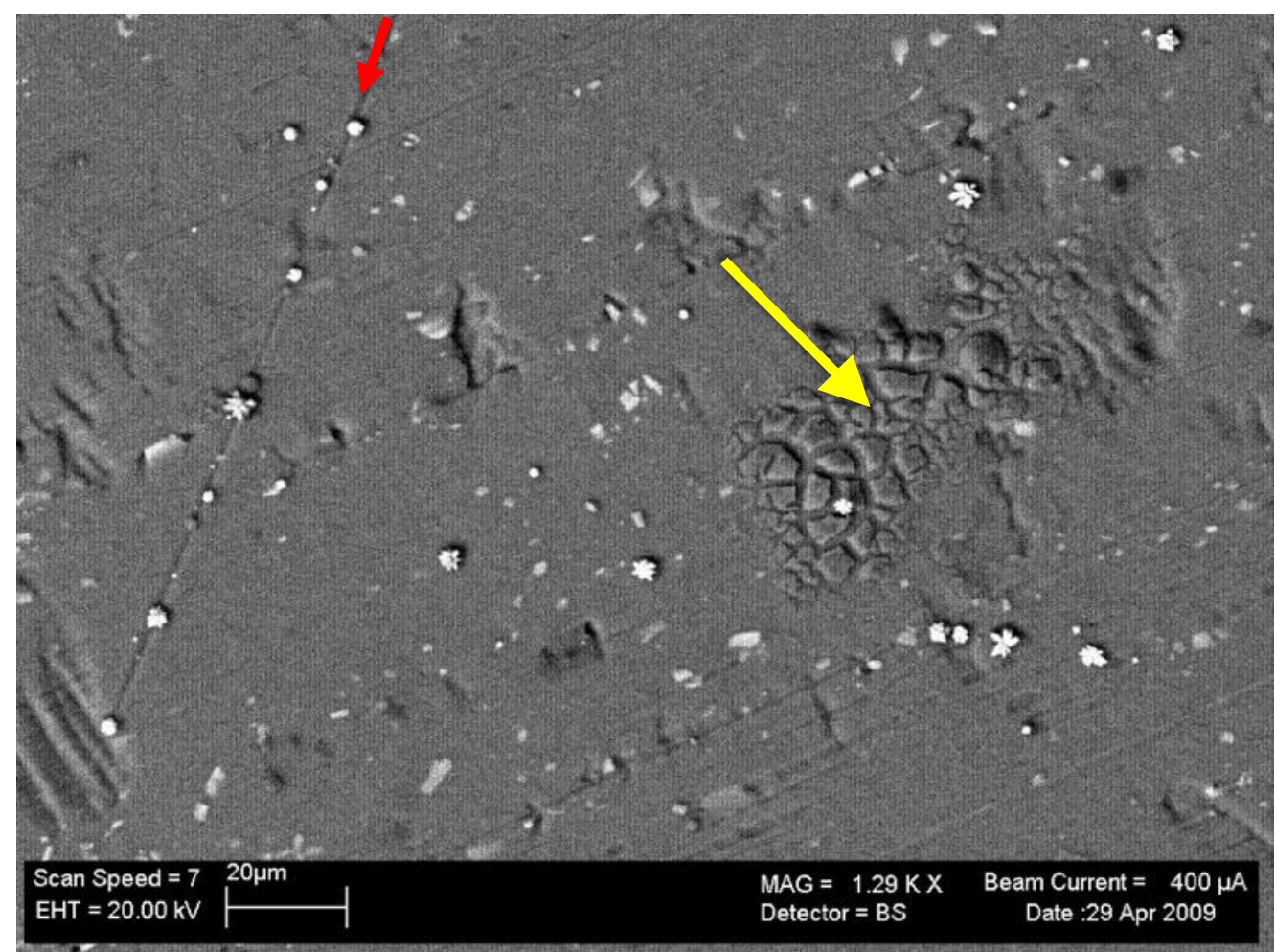

Figure 3.1.2: Electron micrograph of nickel electrodeposited for $30 \mathrm{~s}$ at $-1.6 \mathrm{~V}$ on aluminium.

Figure 3.1.3 shows current vs. time graphs for electrodeposition of nickel onto aluminium at -0.5 to $-2.5 \mathrm{~V}$. The $-0.5 \mathrm{~V}$ electrodeposition shown does not rise above $0 \mathrm{~mA}$. The electron micrograph for a $-0.5 \mathrm{~V}$ electrodeposition sample shown in 
Jonathan Tailby

Figure 3.1.4 shows very little evidence of nickel deposits compared to the more negative voltage depositions. From the absence of current and the few deposits compared to more negative voltages it was concluded that $-0.5 \mathrm{~V}$ was below an activation threshold for nickel deposition on aluminium. The other voltages show increased current with more negative voltage. The higher current is most likely related to increased nickel deposition. It could be related to an unwanted side reaction, such as electrolytic decomposition of water to hydrogen and oxygen [163] also see Table 2.1.1, however no gas production was observed .

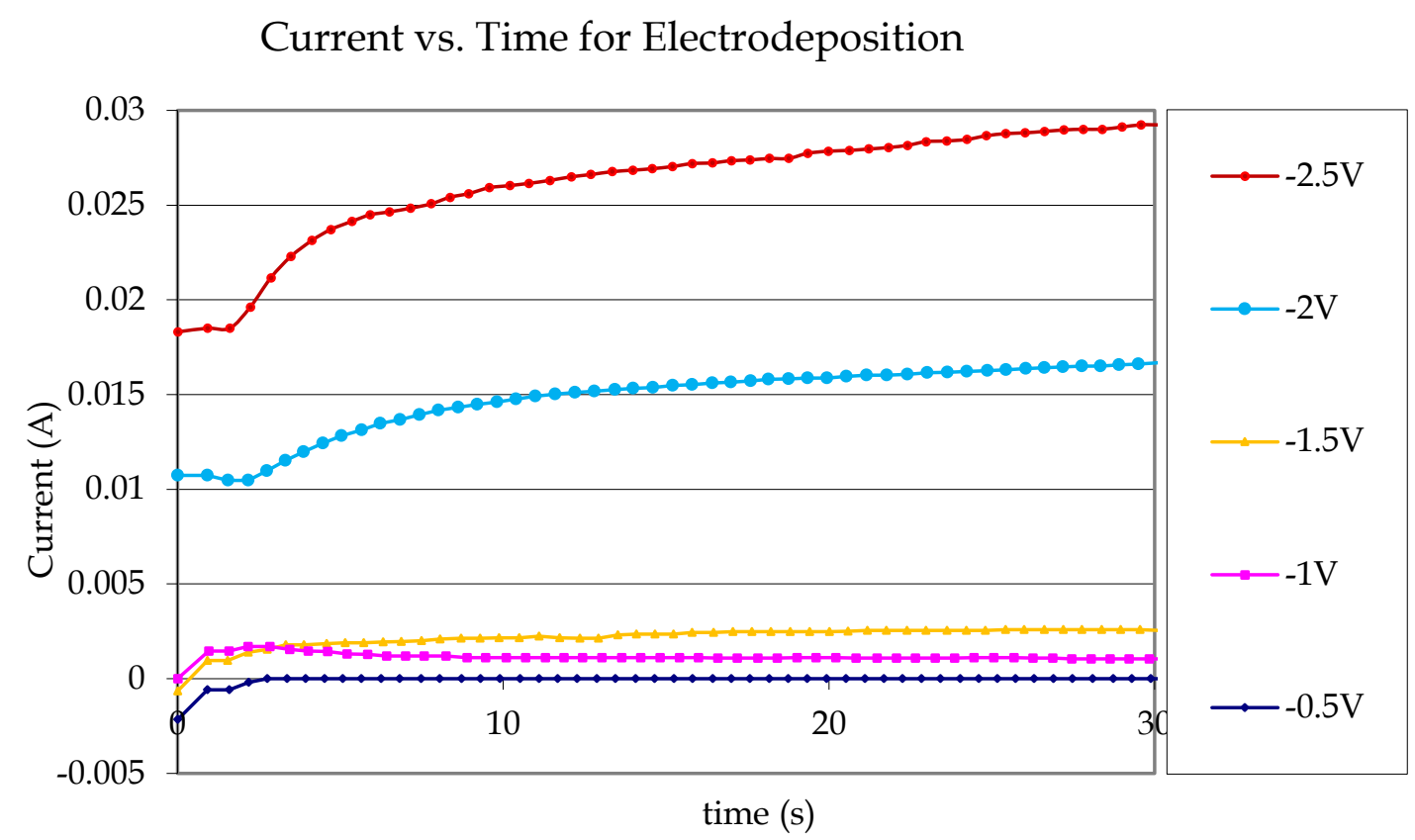

Figure 3.1.3: Current vs. time graphs for electrodeposition of nickel onto aluminium in $0.1 \mathrm{~mol} \cdot \mathrm{L}^{-1} \mathrm{Ni}\left(\mathrm{NO}_{3}\right)_{2}, 30 \mathrm{~s},-0.5--2.5 \mathrm{~V}, \mathrm{pH} 8.3$. 


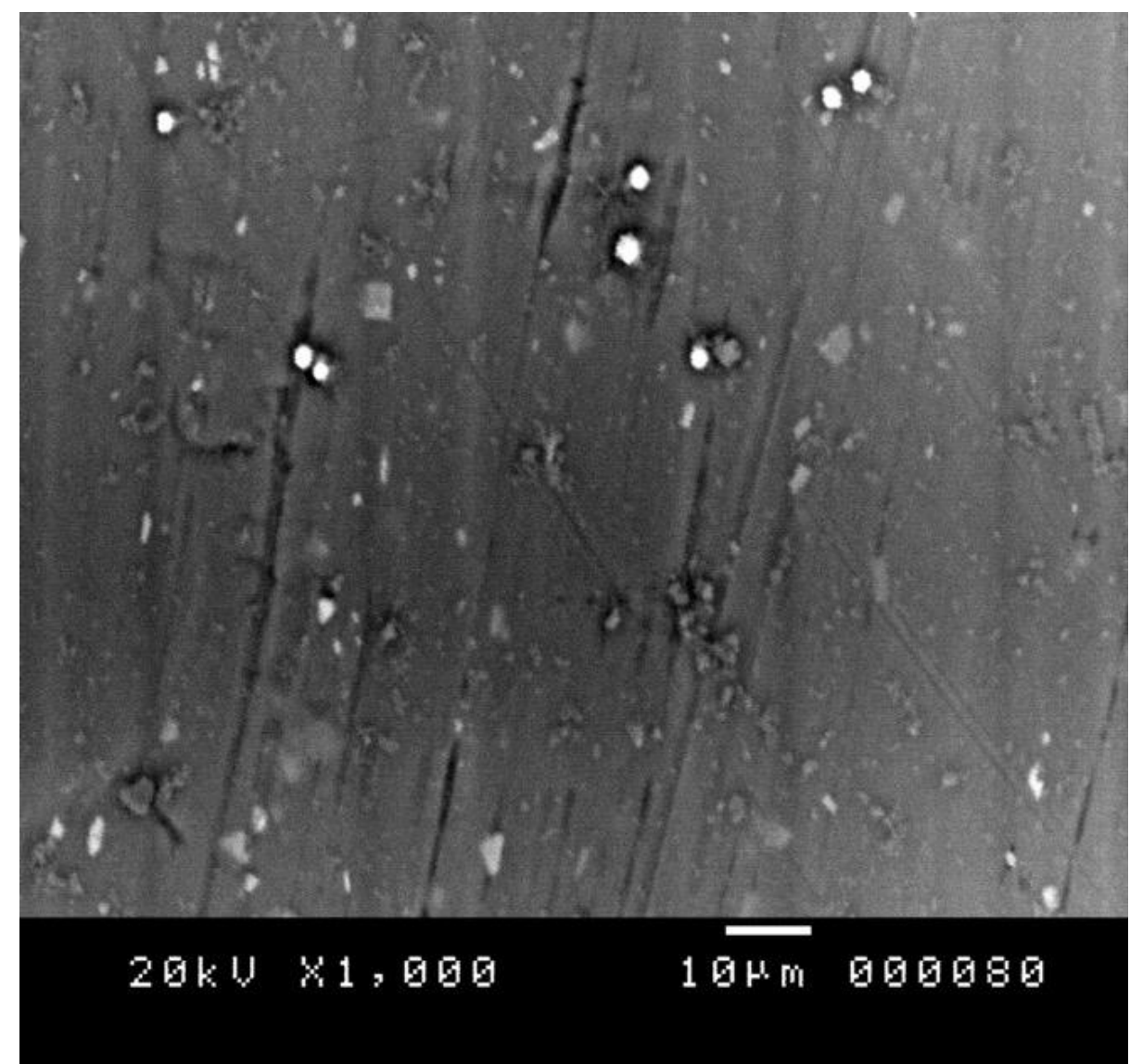

Figure 3.1.4: Back Scatter Electron micrograph of aluminium electrodeposited at $-0.5 \mathrm{~V}$ with $0.1 \mathrm{~mol} \cdot \mathrm{L}^{-1} \mathrm{Ni}\left(\mathrm{NO}_{3}\right)_{2}$, showing little evidence of nickel deposits.

The white circles in the top half of the image are nickel deposits, the grey background, including the irregular shapes, is the aluminium substrate

Figure 3.1.5 shows the current vs. time graphs for electrodeposition of nickel onto aluminium at $-1.6 \mathrm{~V}$ with three different dilutions of the nickel nitrate electroplating solution with the formulation in Table 2.2.2. The solution with $0.01 \mathrm{~mol} \cdot \mathrm{L}^{-1}$ nickel ions produced the lowest current and is therefore expected to have the least amount of deposited nickel. The electrodeposition that used the solution with $1.0 \mathrm{~mol} \cdot \mathrm{L}^{-1}$ nickel ions had the highest early current response, which decreased after an initial peak. The electrodeposition with the $0.1 \mathrm{~mol} \cdot \mathrm{L}^{-1}$ nickel ions had an early current response between the other two concentrations, but caught up to the $1.0 \mathrm{~mol} \cdot \mathrm{L}^{-1}$ sample at approximately $30 \mathrm{~s}$. After $30 \mathrm{~s}$ the 1.0 and $0.1 \mathrm{~mol} \cdot \mathrm{L}^{-1} \mathrm{samples}$ had similar current responses. From this electric current data, the sample deposited with the $1.0 \mathrm{~mol} \cdot \mathrm{L}^{-1}$ solution was predicted to have many deposits of varying sizes. The sample deposited with the $0.1 \mathrm{~mol} \cdot \mathrm{L}^{-1}$ solution was expected to have fewer but larger deposits. The sample deposited with the $0.01 \mathrm{~mol} \cdot \mathrm{L}^{-1}$ solution was predicted to have very little deposition. 
The initial high current of the high concentration experiment can be explained by the availability of nickel ions at the deposition surface, however the later decrease to the same level can be explained if that local concentration at the deposition surface was not replenished any faster than the $0.1 \mathrm{~mol} \cdot \mathrm{L}^{-1}$ electrodeposition resulting in localised depletion and decreased deposition[164]. The electrodeposition equipment used in these experiments did not allow for the solution to be stirred at the scale necessary to address this problem.

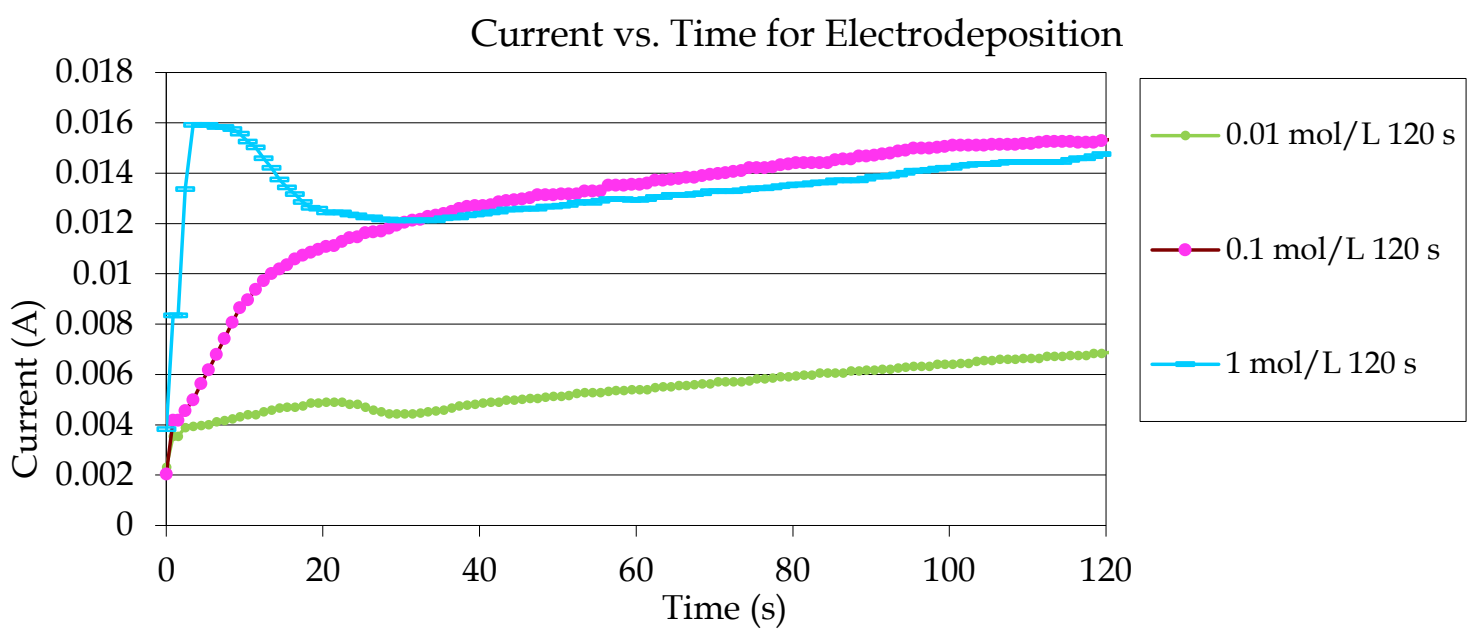

Figure 3.1.5: Current vs. time graphs for electrodeposition of nickel onto aluminium at varying nickel concentration and $-1.6 \mathrm{~V}$.

Figure 3.1.6 shows an XRD trace for one of the aluminium samples electrodeposited with nickel. All of the peaks correspond to the pattern for aluminium and none of them match the expected peaks for nickel. The XRD is not detecting any nickel however, nickel is visible in the SEM, and confirmed by EDS with the SEM. The nickel deposits must be too small and diffuse for XRD to detect them. Some later depositions did produce deposits that were detected by XRD, as shown in Figure 3.1.23, which suggests that the nickel was crystalline and potentially detectable by XRD, however SEM images such as Figure 3.1.4 and Figure 3.1.7 show that the nickel is in very small and highly dispersed units which are not easily detected by XRD. 


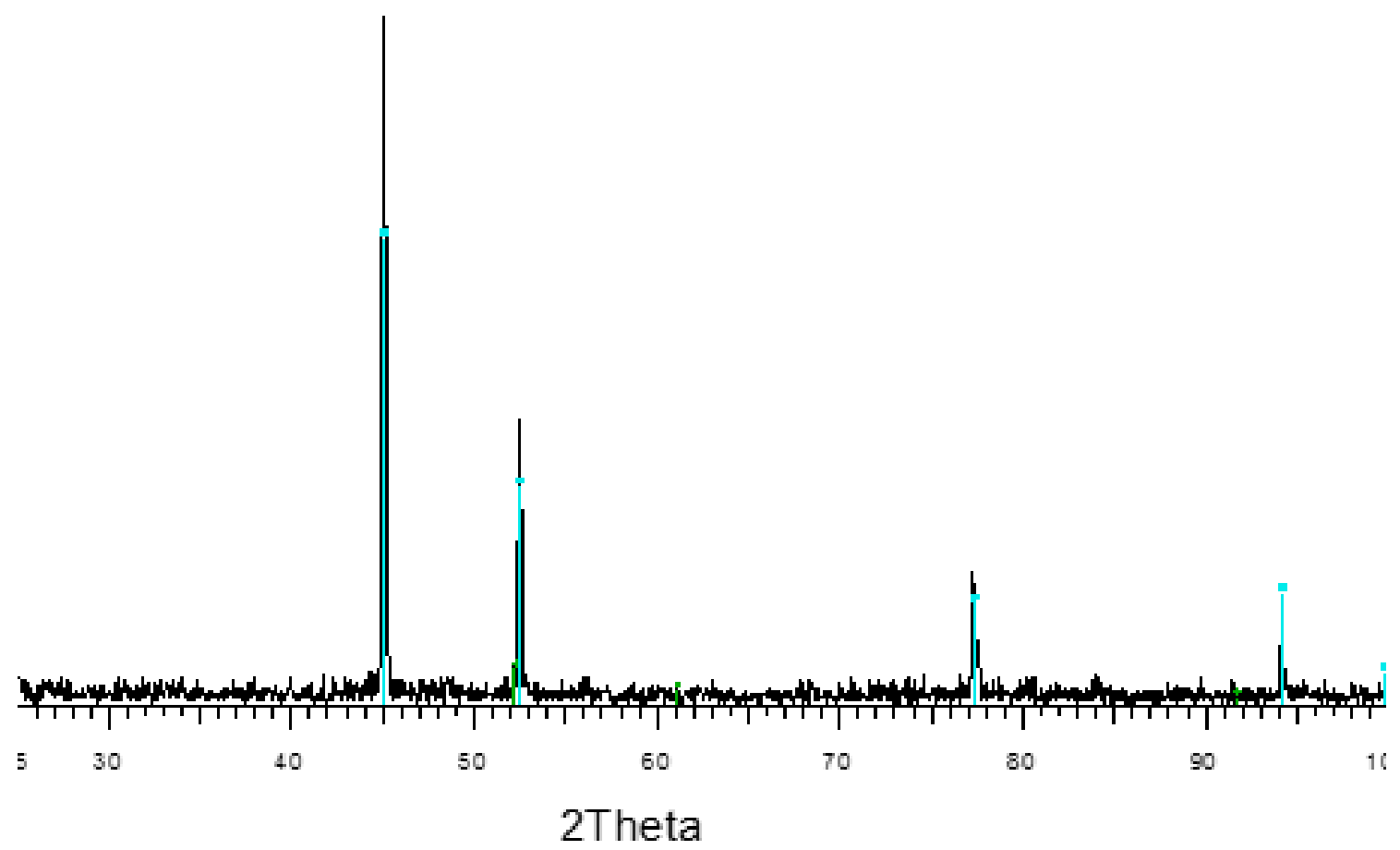

Figure 3.1.6: XRD showing no nickel detected on the aluminium surface after electrodeposition for $30 \mathrm{~s}$ at $-2.0 \mathrm{~V}$ with the $0.1 \mathrm{~mol} \cdot \mathrm{L}^{-1} \mathrm{Ni}\left(\mathrm{NO}_{3}\right)_{2}$ solution.

The peaks on the XRD trace match the pattern for aluminium shown in blue, but there are no additional peaks to match the pattern for nickel shown in green.
Aluminium after electrodeposition
- Aluminium, Al
- Nickel, Ni

Figure 3.1.7 shows an electron micrograph of the same sample. Many high surface area nickel deposits are visible in the micrograph. The micrograph shows that electrodeposition on aluminium created structures that may be of value as a catalyst. 


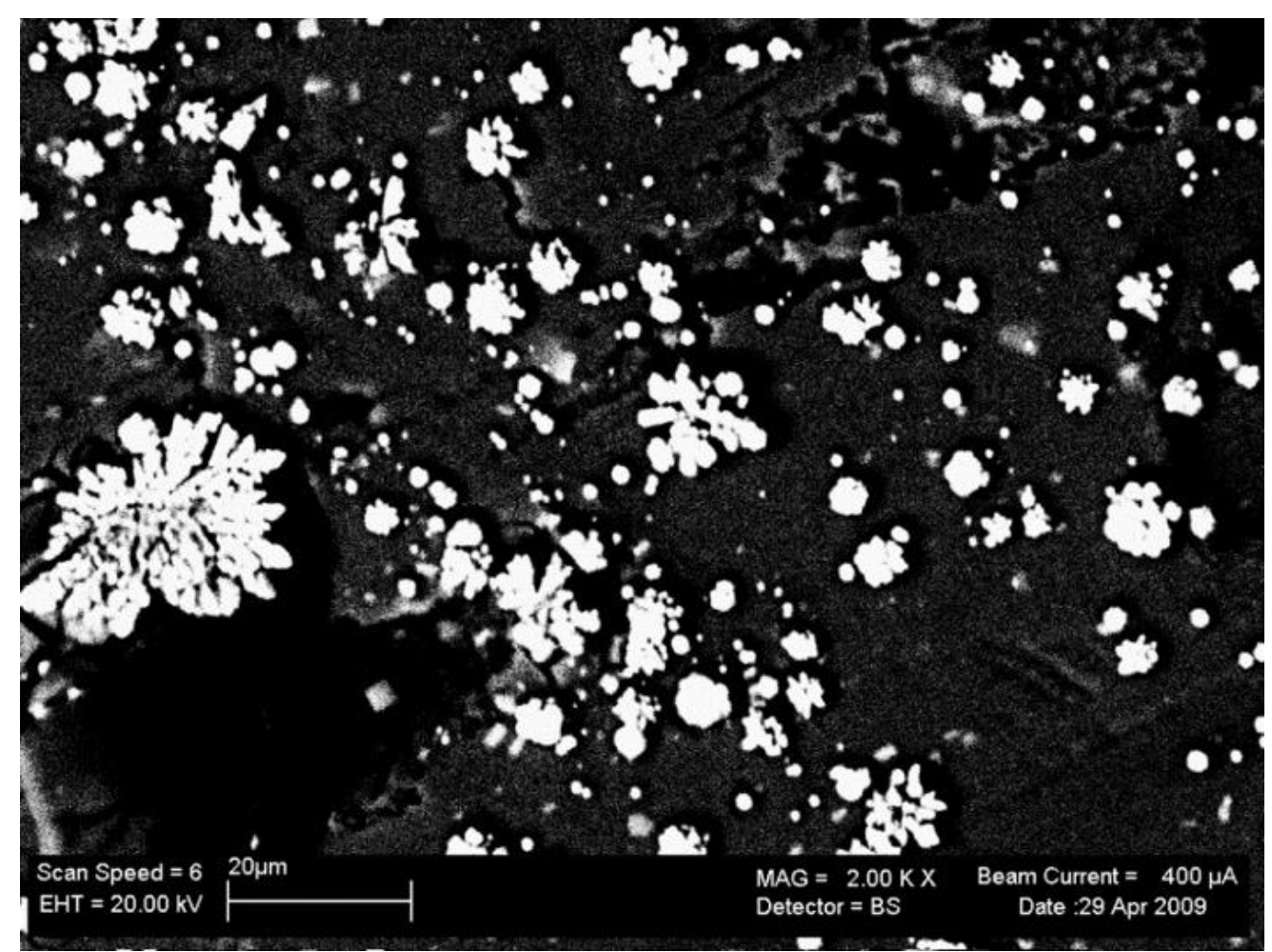

Figure 3.1.7: SEM of small high surface area deposits on aluminium.

This is the same sample that provided the XRD trace shown in Figure 3.1.6, electrodeposited for $30 \mathrm{~s}$ at $-2.0 \mathrm{~V}$ with the $0.1 \mathrm{~mol} \cdot \mathrm{L}^{-1} \mathrm{Ni}\left(\mathrm{NO}_{3}\right)_{2}$ solution.

Image analysis of micrographs was used to gather the data shown in Table 3.1.1 and graphed in Figure 3.1.8 and Figure 3.1.9.

Table 3.1.1 shows the data and calculations for quantifying the nickel deposition seen in the SEM images collected at 200x magnification. The table shows a concentration series, each electrodeposited at $-1.6 \mathrm{~V}$, with the electroplating solution concentration varying from 0.01 to $1.0 \mathrm{molL}^{-1}$ and $\mathrm{pH}$ standardised to 6 . The table also shows a voltage series with each sample electrodeposited in a $0.1 \mathrm{~mol} \cdot \mathrm{L}^{-1}$ electroplating solution but the voltage increasingly negative from -0.5 to $-2.5 \mathrm{~V}$ in $0.5 \mathrm{~V}$ steps. Table 3.1.1 shows the measurements obtained by analysis of SEM images with the ImageJ software [165]. 


\begin{tabular}{|c|c|c|c|c|c|}
\hline \multirow{2}{*}{\multicolumn{6}{|c|}{$\begin{array}{l}\text { Table 3.1.1: Quantitative analysis of 200x SEM of nickel electr } \\
\text { Calculating mean deposit size and total coverage. The curr } \\
\text { electrodeposition of these samples are shown in Figure } 3.1 .3 \text { a } \\
\text { Pixel measurements of deposits } \\
\end{array}$}} \\
\hline & & & & & \\
\hline $\begin{array}{l}\text { Nickel } \\
\text { Concentration }\end{array}$ & $\begin{array}{l}\text { Number of } \\
\text { Deposits }\end{array}$ & $\begin{array}{l}\text { Average Area } \\
\text { of deposits } \\
\text { (pixels) }\end{array}$ & $\begin{array}{l}\text { Area standard } \\
\text { deviation }\end{array}$ & $\begin{array}{l}\text { Average } \\
\text { Diameter of } \\
\text { deposits } \\
\text { (pixels) }\end{array}$ & $\begin{array}{l}\text { Diameter } \\
\text { standard } \\
\text { deviation }\end{array}$ \\
\hline $0.01 \mathrm{~mol} \cdot \mathrm{L}^{-1}$ & 199 & 40.5 & 24.5 & 6.95 & 1.82 \\
\hline $0.01 \mathrm{~mol} \cdot \mathrm{L}^{-1}$ & 402 & 50.6 & 29.4 & 7.76 & 2.05 \\
\hline $0.1 \mathrm{~mol} \mathrm{~L}^{-1}$ & 319 & 95.0 & 88.1 & 10.1 & 4.45 \\
\hline $0.1 \mathrm{~mol}^{-\mathrm{L}^{-1}}$ & 269 & 85.9 & 69.7 & 9.73 & 3.85 \\
\hline $0.1 \mathrm{~mol} \cdot \mathrm{L}^{-1}$ & 512 & 82.7 & 70.1 & 9.56 & 3.73 \\
\hline $1.0 \mathrm{~mol} \cdot \mathrm{L}^{-1}$ & 67 & 77.1 & 45.9 & 9.49 & 2.89 \\
\hline \multicolumn{6}{|l|}{ Voltage } \\
\hline$-0.5 \mathrm{~V}$ & 181 & 43.9 & 27.6 & 7.19 & 2.05 \\
\hline$-1.0 \mathrm{~V}$ & 221 & 43.5 & 36.0 & 7.13 & 2.13 \\
\hline$-1.5 \mathrm{~V}$ & 155 & 105 & 79.2 & 10.7 & 4.33 \\
\hline$-2.0 \mathrm{~V}$ & 615 & 106 & 101. & 10.6 & 4.73 \\
\hline$-2.5 \mathrm{~V}$ & 1390 & 89.6 & 195 & 9.51 & 4.86 \\
\hline$-2.5 \mathrm{~V}$ & 1210 & 93.5 & 114 & 9.86 & 4.68 \\
\hline 0 & 50 & 42.9 & 31.8 & 7.07 & 2.15 \\
\hline 0 & 66 & 41.4 & 26.1 & 6.99 & 1.95 \\
\hline 0 & 29 & 36.9 & 18.8 & 6.68 & 1.55 \\
\hline
\end{tabular}

Table 3.1.2 shows the values from Table 3.1.1 converted to microns using a ratio of 298 pixels to 100 microns which was derived from the scale bar in the images. Table 3.1.3 show the averages of the calculated micron values for each sample that was examined in this session, to the right of these are the electrodeposition conditions relevant to that sample. 


\begin{tabular}{|c|c|c|c|c|c|c|}
\hline \multicolumn{7}{|c|}{$\begin{array}{l}\text { Table 3.1.2: Calculated micron measurements } \\
\text { The measurements in pixels from the previous table were converted into } \\
\text { measurements in microns using a linear conversion derived from the } \\
\text { relationship } 100 \text { microns }=298 \text { pixels. }\end{array}$} \\
\hline \multicolumn{7}{|c|}{ Conversion value: 298 pixels $=100$ microns; total area $=2194088$} \\
\hline \begin{tabular}{|l|} 
Nickel \\
Solution \\
Concentration
\end{tabular} & $\begin{array}{l}\text { Area } \\
\text { average } \\
\left(\mu \mathrm{m}^{2}\right)\end{array}$ & $\begin{array}{l}\text { Area } \\
\text { standard } \\
\text { deviation }\end{array}$ & $\begin{array}{l}\text { Diameter } \\
\text { average } \\
(\mu \mathrm{m})\end{array}$ & $\begin{array}{l}\text { Diameter } \\
\text { standard } \\
\text { deviation }\end{array}$ & $\begin{array}{l}\text { Total } \\
\text { deposit } \\
\text { Area }\end{array}$ & $\begin{array}{l}\text { Percentage } \\
\text { Area }\end{array}$ \\
\hline $0.01 \mathrm{~mol} \cdot \mathrm{L}^{-1}$ & 4.56 & 2.76 & 1.75 & 0.46 & 907 & $0.37 \%$ \\
\hline $0.01 \mathrm{~mol} \cdot \mathrm{L}^{-1}$ & 5.70 & 3.32 & 1.95 & 0.52 & 2290 & $0.93 \%$ \\
\hline $0.1 \mathrm{~mol} \cdot \mathrm{L}^{-1}$ & 10.7 & 9.92 & 2.53 & 1.12 & 341 & $1.38 \%$ \\
\hline $0.1 \mathrm{~mol} \cdot \mathrm{L}^{-1}$ & 9.67 & 7.85 & 2.44 & 0.97 & 2600 & $1.05 \%$ \\
\hline $0.1 \mathrm{~mol} \cdot \mathrm{L}^{-1}$ & 9.31 & 7.89 & 2.40 & 0.94 & 4770 & $1.93 \%$ \\
\hline $1.0 \mathrm{~mol} \cdot \mathrm{L}^{-1}$ & 8.69 & 5.16 & 2.38 & 0.73 & 582 & $0.24 \%$ \\
\hline \multicolumn{7}{|l|}{ Voltage } \\
\hline$-0.5 \mathrm{~V}$ & 4.94 & 3.11 & 1.81 & 0.515 & 894. & $0.36 \%$ \\
\hline$-1.0 \mathrm{~V}$ & 4.90 & 4.05 & 1.79 & 0.535 & 1080 & $0.44 \%$ \\
\hline$-1.5 \mathrm{~V}$ & 11.8 & 8.91 & 2.69 & 1.08 & 1830 & $0.74 \%$ \\
\hline$-2.0 \mathrm{~V}$ & 11.9 & 11.4 & 2.66 & 1.19 & 7330. & $2.97 \%$ \\
\hline$-2.5 \mathrm{~V}$ & 10.1 & 21.9 & 2.39 & 1.22 & 14000 & $5.68 \%$ \\
\hline$-2.5 \mathrm{~V}$ & 10.5 & 12.8 & 2.48 & 1.18 & 12800 & $5.17 \%$ \\
\hline 0 & 4.83 & 3.58 & 1.78 & 0.54 & 241 & $0.10 \%$ \\
\hline 0 & 4.66 & 2.93 & 1.76 & 0.490 & 307 & $0.12 \%$ \\
\hline 0 & 4.16 & 2.12 & 1.68 & 0.389 & 121 & $0.05 \%$ \\
\hline
\end{tabular}

Table 5.2.3 in Appendix 5.2.3 shows equivalent data to Table 3.1.1 to Table 3.1.3 but for SEM micrographs taken at 1000x magnification. Because the micrographs had the same number of pixels but at a higher magnification, the ratio had changed to 148 pixels to 10 microns.

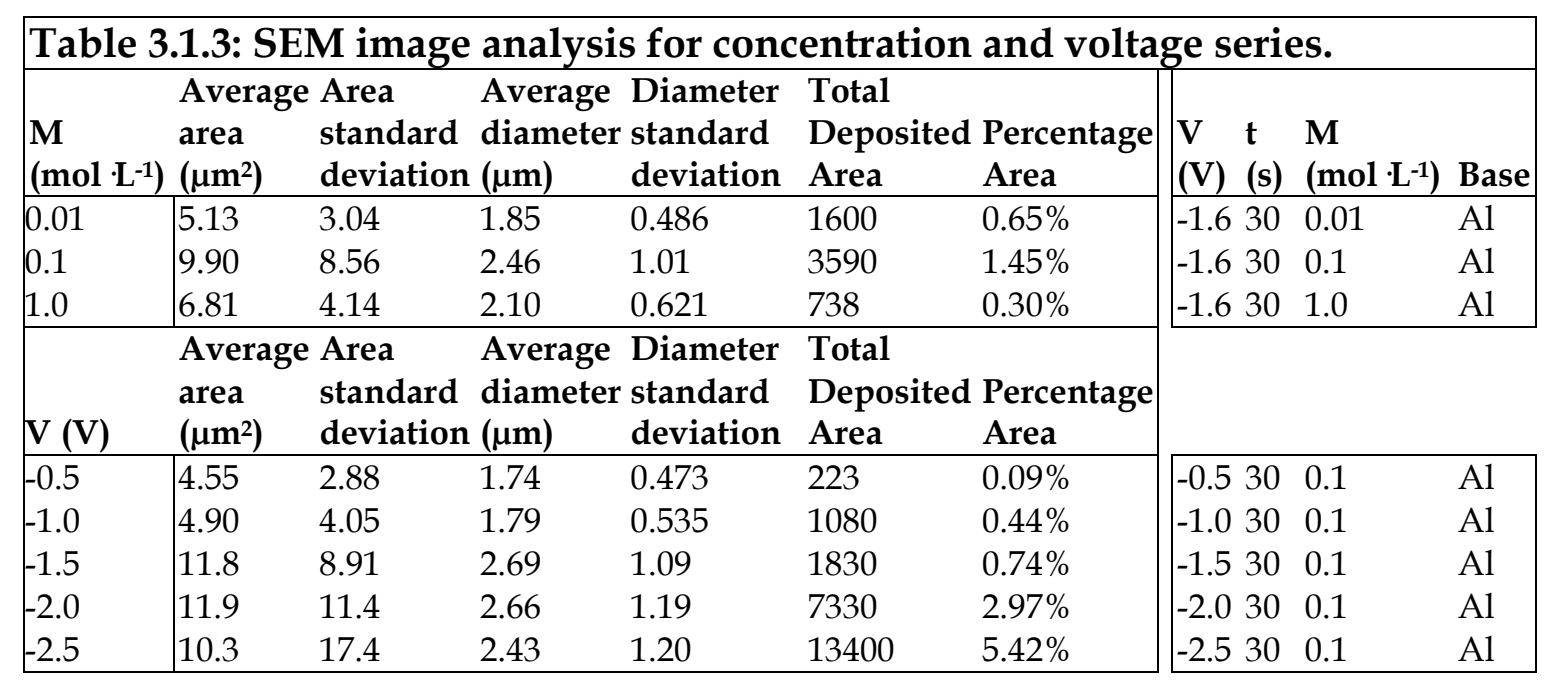

Figure 3.1.8 graphs the total area of deposits using the data from images taken at 200x magnification. This shows that the total area covered with deposits increased 
when the voltage became more negative. This matches the more rapid early current increase because it corresponds to more nucleation at more negative voltage.

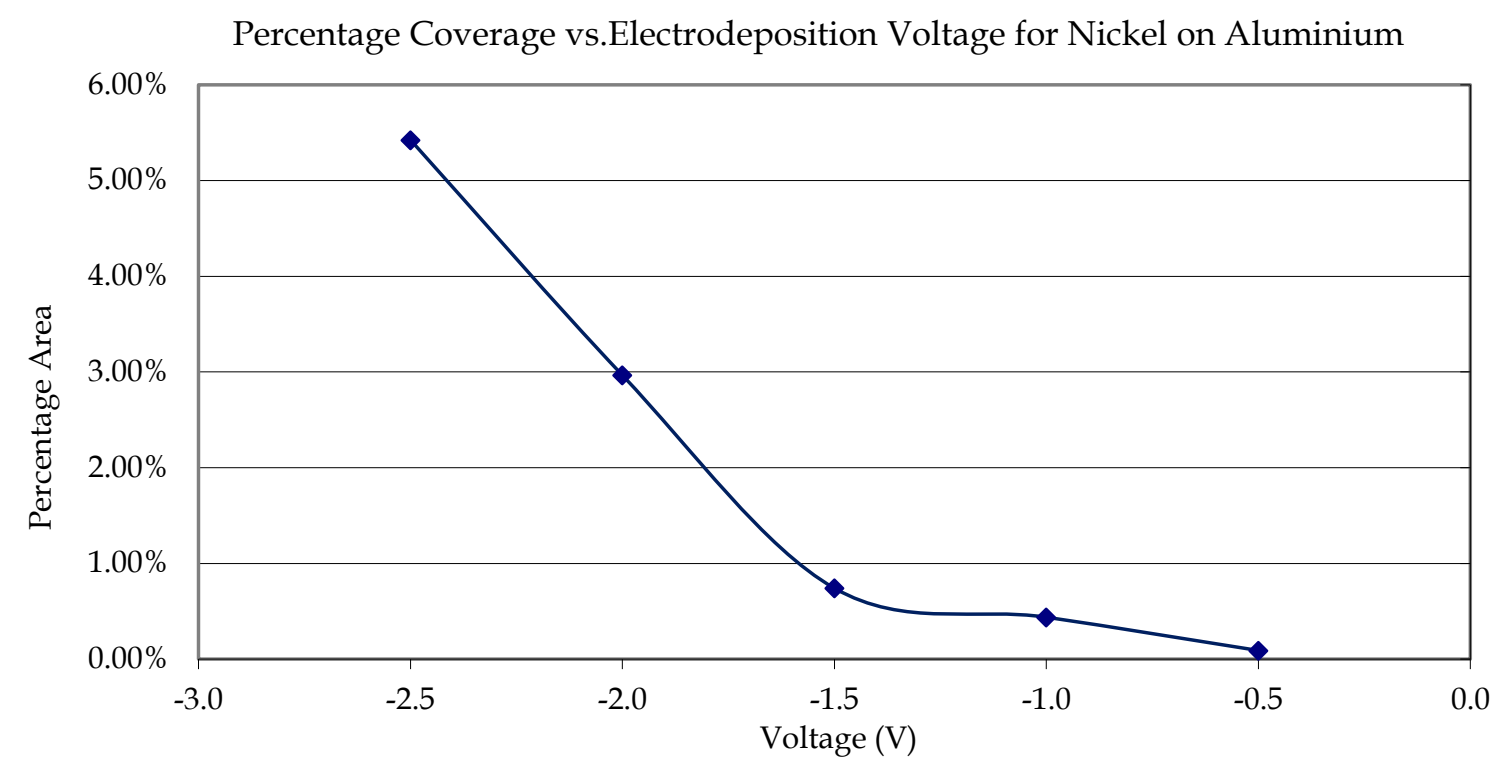

Figure 3.1.8: Percentage coverage vs. voltage for nickel electrodeposited on aluminium for $30 \mathrm{~s}$ in $0.1 \mathrm{~mol} \cdot \mathrm{L}^{-1}$ electroplating solution.

Calculated from electron micrographs taken at 200x magnification.

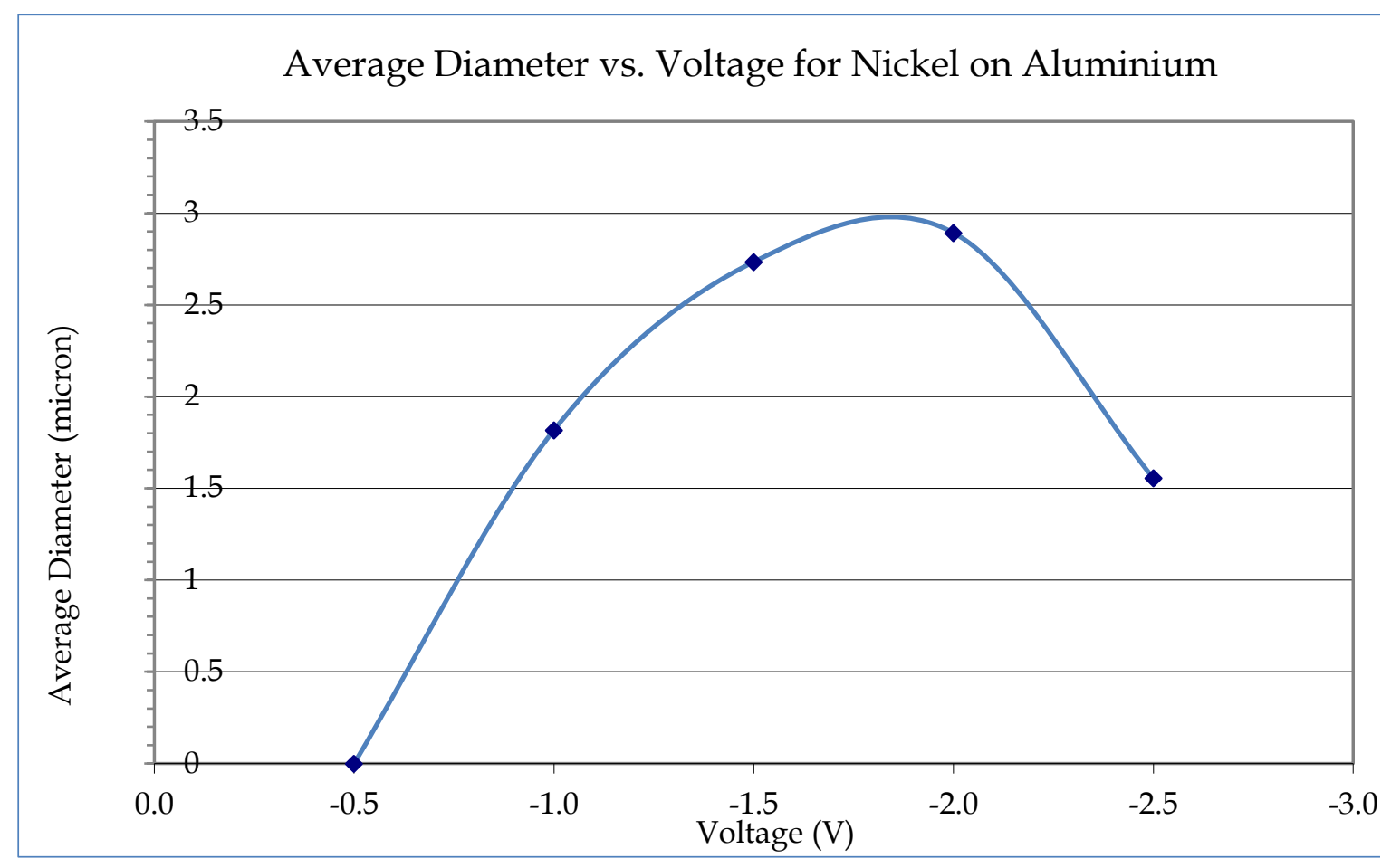

Figure 3.1.9: Average diameter vs. voltage for nickel electrodeposits on aluminium in $0.1 \mathrm{~mol} \cdot \mathrm{L}^{-1}$ electroplating solution for $30 \mathrm{~s}$.

Calculated from electron micrographs taken at 1000x magnification. 
Jonathan Tailby

Figure 3.1.9 shows the average size of the nickel deposits graphed against the deposition voltage using data from images taken at 1000x magnification. This shows that the average deposit size reaches a maximum between -1.5 and $-2.0 \mathrm{~V}$. Together with Figure 3.1.8, this data suggest that $-2.5 \mathrm{~V}$ may be the best voltage for catalyst formation of those tested because more nickel is deposited and it is in smaller deposits, therefore it is expected to have the most nickel surface area. The surface area of deposits is further increased by the greater surface roughness of deposits from more negative voltages as shown in Figure 3.1.10.

Sample SEM images for electrodeposition at -1.0 to $-2.5 \mathrm{~V}$ are shown in Figure 3.1.10, a sample image for -0.5 was shown in Figure 3.1.4. The coral-like shapes of some of these deposits is related to the increased nucleus formation at higher voltage having led to aggregations of smaller deposits rather than individual overgrown deposits with the surface area smoothed over by the longer growth.

The nickel deposits shown in Figure 3.1.10 appear to have a high surface area and were expected to have good potential for use as ammonia decomposition catalysts. This was considered to be a success, so the decision was made progress to electrodeposition on palladium. 


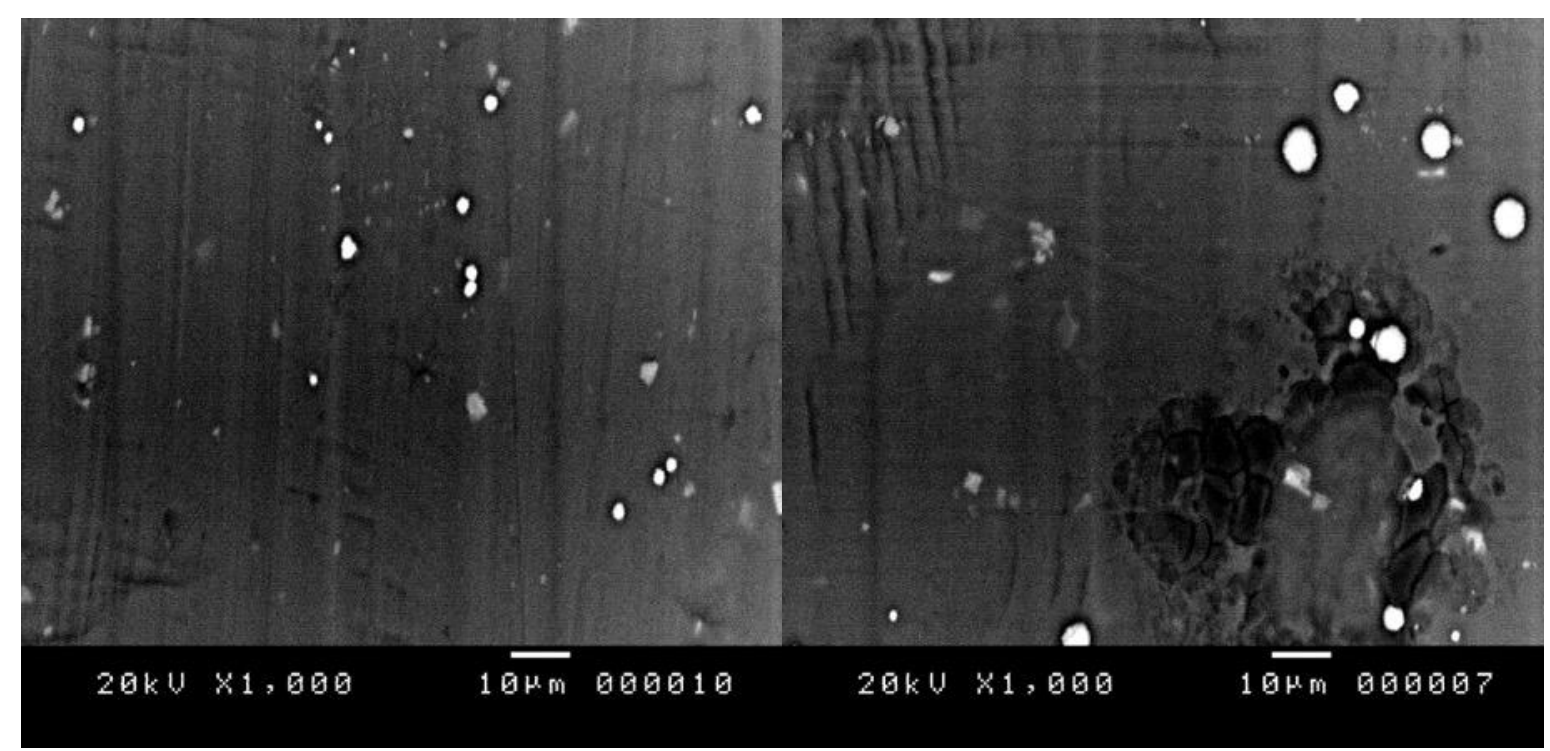

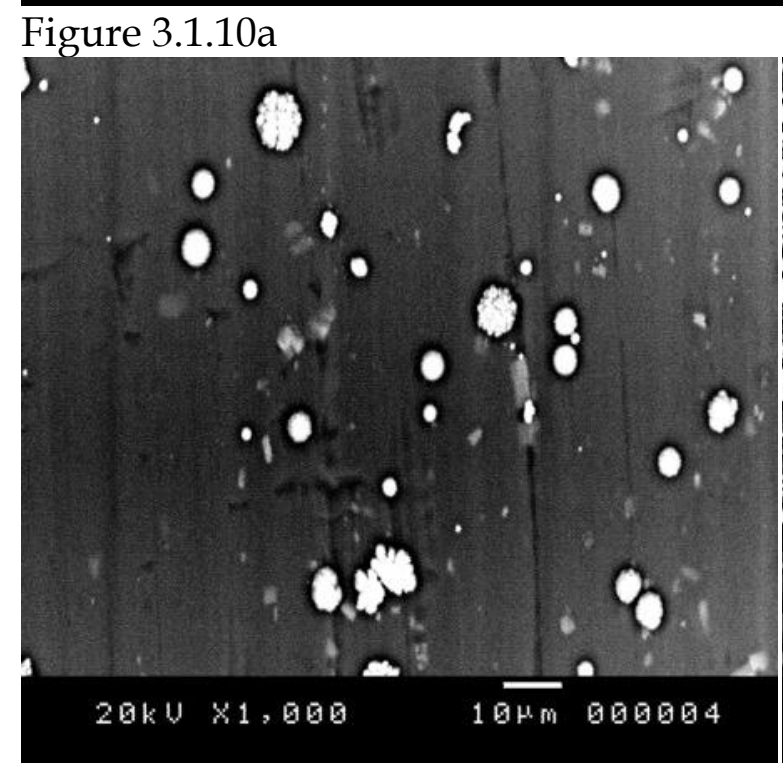

Figure 3.1.10c
Figure 3.1.10b

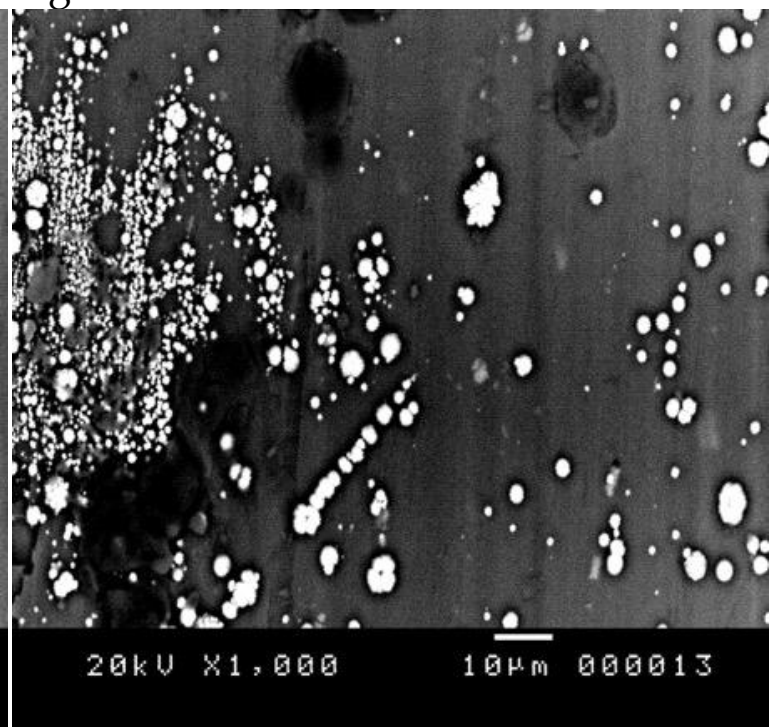

Figure 3.1.10d

Figure 3.1.10: Electron micrographs for nickel electrodeposited on aluminium showing how quantity and surface complexity of the deposits increased with voltage negativity.

Electrodeposited for $30 \mathrm{~s}$ in $0.1 \mathrm{~mol} \cdot \mathrm{L}^{-1} \mathrm{Ni}^{2+}$ at $-1.0,-1.5,-2.0,-2.5 \mathrm{~V}$ respectively.

\subsubsection{Electrodeposition on Palladium}

The response of palladium to electrodeposition was quite different to that of aluminium. First, the current produced was higher, as can be seen in Figure 3.1.11. The current vs. time graph shown in Figure 3.1.11 shows palladium following a pattern more typical of instantaneous nucleation compared to the continuous nucleation of aluminium. This is better illustrated by the graph in Figure 3.1.12 
Jonathan Tailby

which plots $\left(I / I_{\max }\right)^{2}$ against $t / t_{\max }$ where the square of the current divided by the maximum current has been plotted against the time divided by the time at which maximum current occurred as in Figure 1.2.7. The palladium plot rises quickly then settles at a steady height, characteristic of instantaneous nucleation. The aluminium plot does not rise as quickly to its maximum, then descends before settling at a steady value, which is characteristic of continuous nucleation.

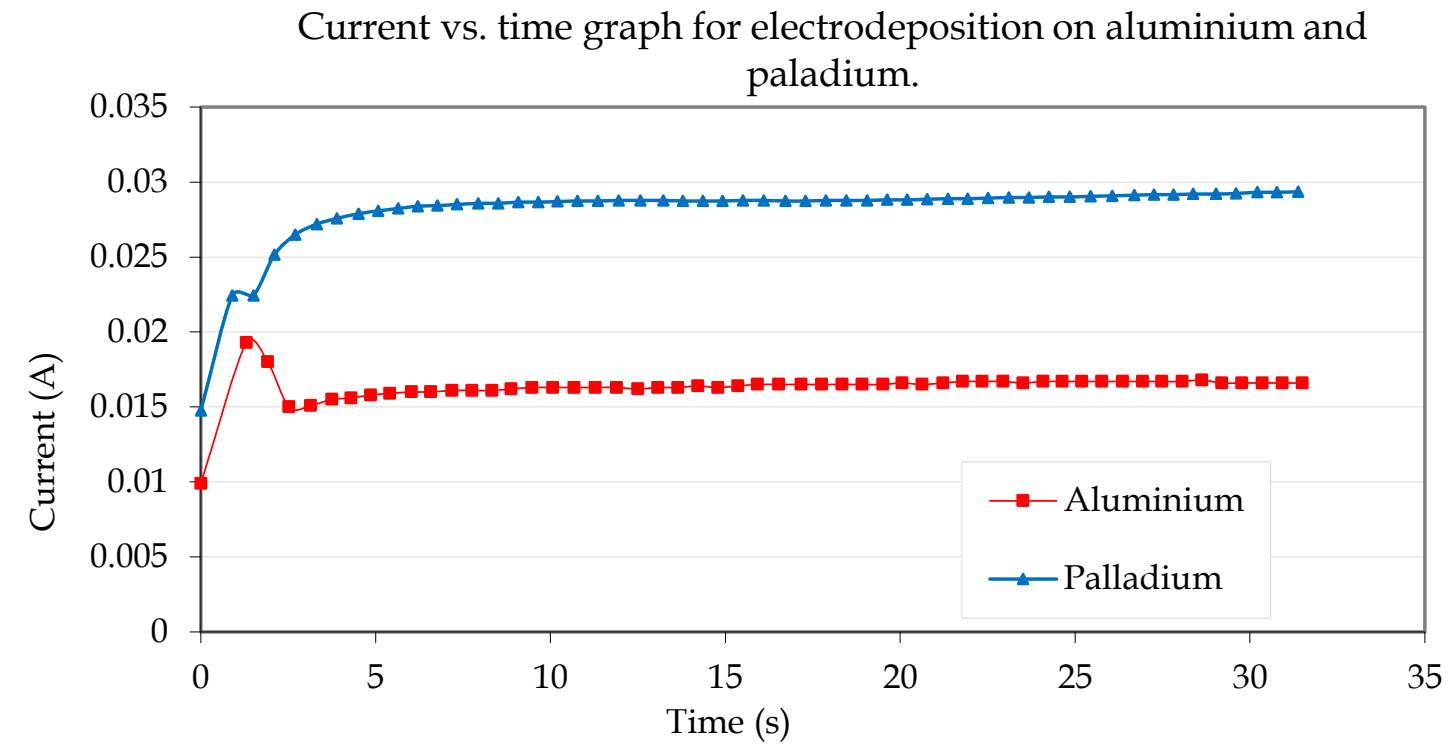

Figure 3.1.11: Current vs. time graph for electrodeposition of nickel from 0.1 $\mathrm{mol} \cdot \mathrm{L}^{-1} \mathrm{Ni}\left(\mathrm{NO}_{3}\right)_{2}, 30 \mathrm{~s},-2.0 \mathrm{~V}$ comparing aluminium and palladium substrates.

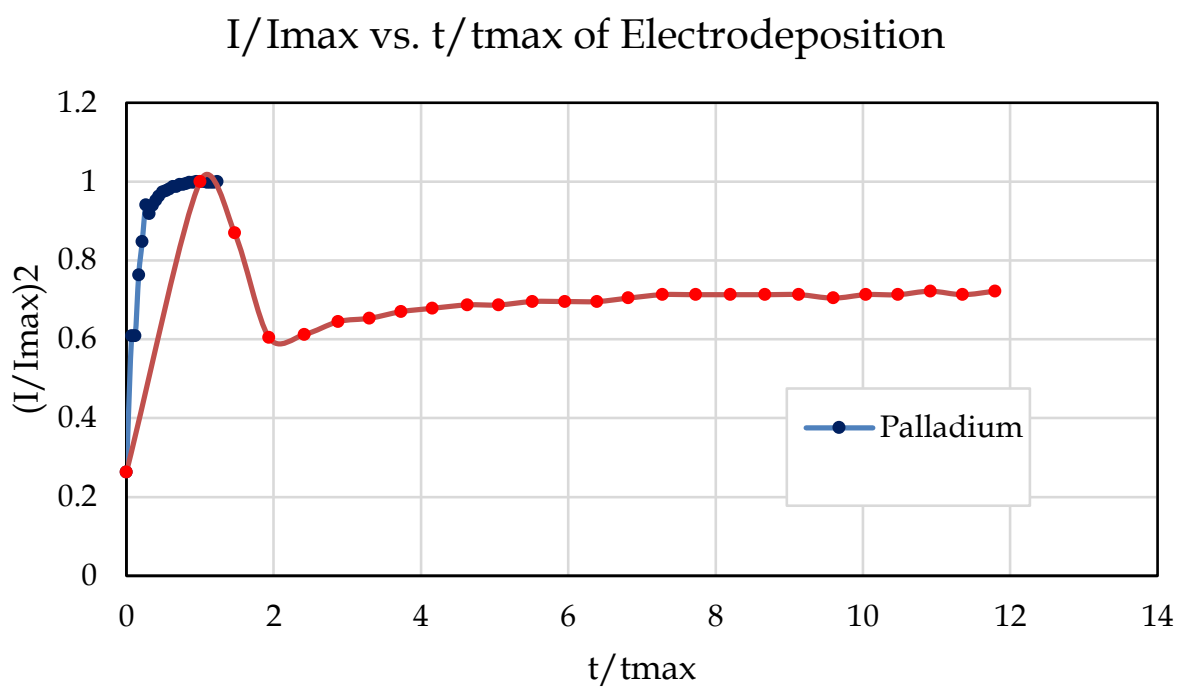

Figure 3.1.12: $\left(\mathrm{I} / \mathrm{I}_{\max }\right)^{2}$ vs. $\mathrm{t} / \mathrm{t}_{\max }$ of Electrodeposition at $-2.0 \mathrm{~V}$ for the electrodepositions shown in Figure 3.1.11. 
Figure 3.1.13 displays further current vs. time graphs, showing that the difference between the current responses of the two metals increased at more negative voltage. Because of this it was expected that there would be more nickel on the palladium samples than on their aluminium equivalents.

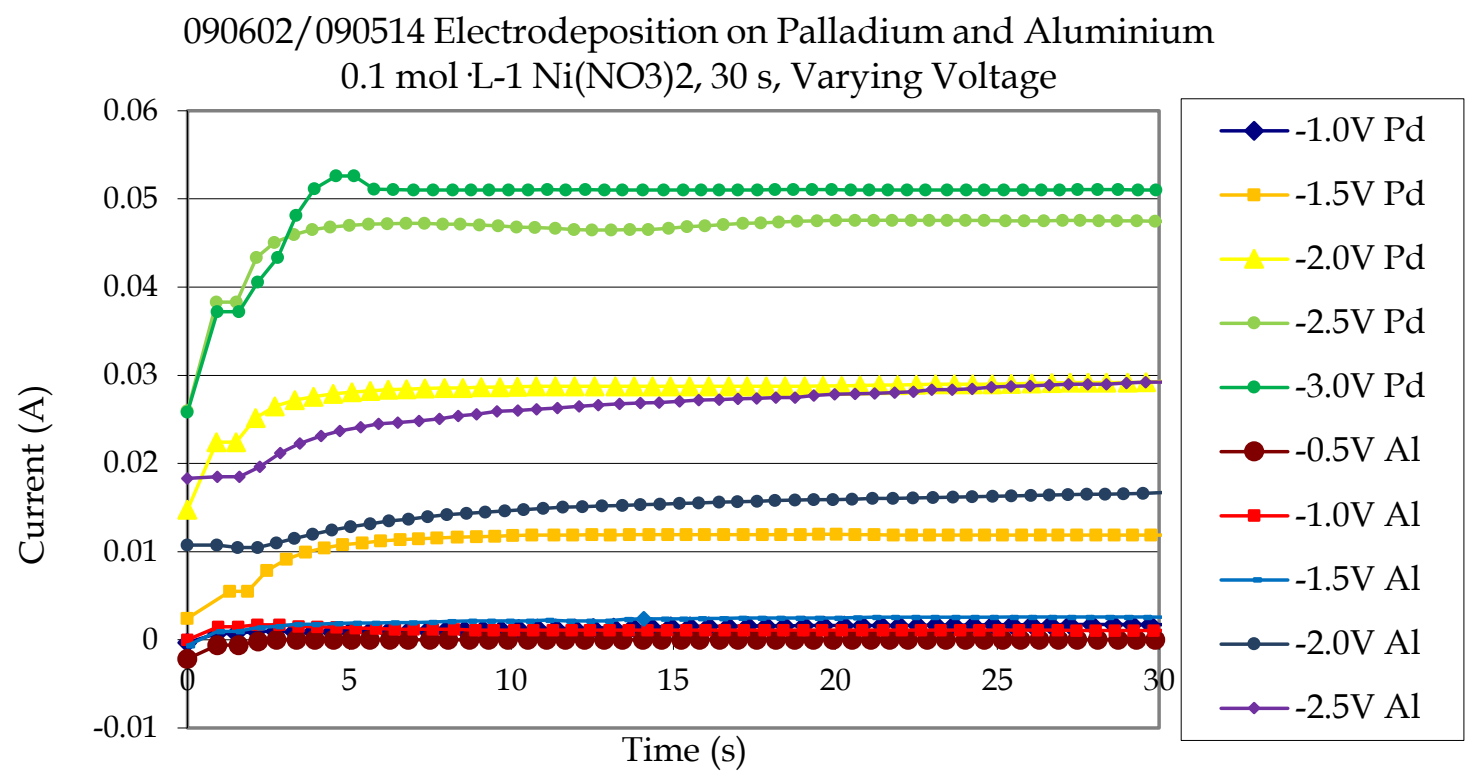

Figure 3.1.13: Current vs. time graphs comparing electrodepositions of nickel onto aluminium and palladium at multiple voltages.

SEM on the deposited palladium found no obvious nickel deposits, and certainly not of the kind that were expected, such as those which were clearly evident on aluminium samples seen in Figure 3.1.10., Figure 3.1.14 shows an electron micrograph of nickel electrodeposited palladium. The black spots did not occur outside the electrodeposition area and increased in size as the deposition voltage was increased; they were therefore interpreted as relevant to the electrodeposition. However, they do not resemble the deposits from electrodeposition of nickel on aluminium, such as those shown in Figure 3.1.10, and the EDS reveals the same weak nickel signal from the area surrounding the black spots as from the black spots themselves. This suggested that the black spots were related to electrodeposition but were not themselves the nickel deposits, which required further investigation. Additional electron micrographs are included in Appendix 5.2.4. 


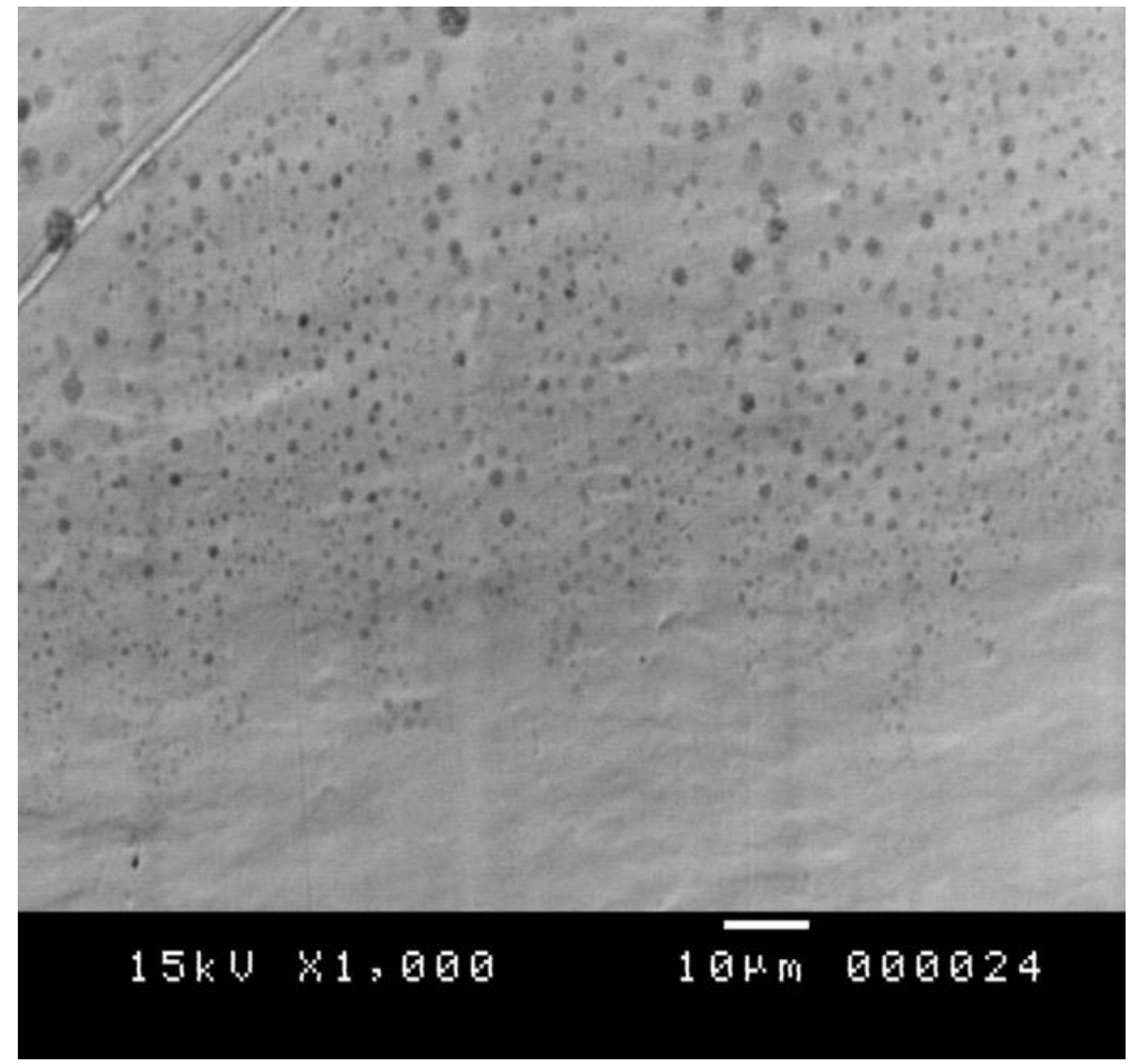

Figure 3.1.14: Electron micrograph of palladium electrodeposited with nickel for $30 \mathrm{~s}$ at -2.0 V in the nickel sulfate solution of Table 2.2.1.

These black spots are not present outside the electrodeposition area therefore they are likely to be relevant to the electrodeposition, but they do not resemble the deposits created by electrodeposition on aluminium.

The sample with the most of these black spots was examined using the JEOL 6500F SEM to get higher resolution images. Two clearer images are shown in Figure 3.1.15 and Figure 3.1.16 along with a back scatter image in Figure 3.1.17 and quantitative EDS analysis of a single point in Figure 3.1.18 and Table 3.1.4. The EDS showed only palladium in the bright circle that fills most of the left side of the Figure 3.1.16; nickel and palladium were present throughout the rest of the image but no other elements were present.

Figure 3.1.17 shows the same location as Figure 3.1.16, but Figure 3.1.17 is a backscatter electron micrograph instead of a secondary emission electron micrograph. The backscatter electron microscopy is more sensitive to atomic mass; therefore, the dark regions were interpreted as palladium coated with a layer of nickel and the light regions were interpreted as uncovered palladium or having a thinner nickel layer. The EDS for the point marked above the centre of Figure 3.1.17 
was quantitatively analysed, and the results are shown in Figure 3.1.18 and Table

\subsection{4.}

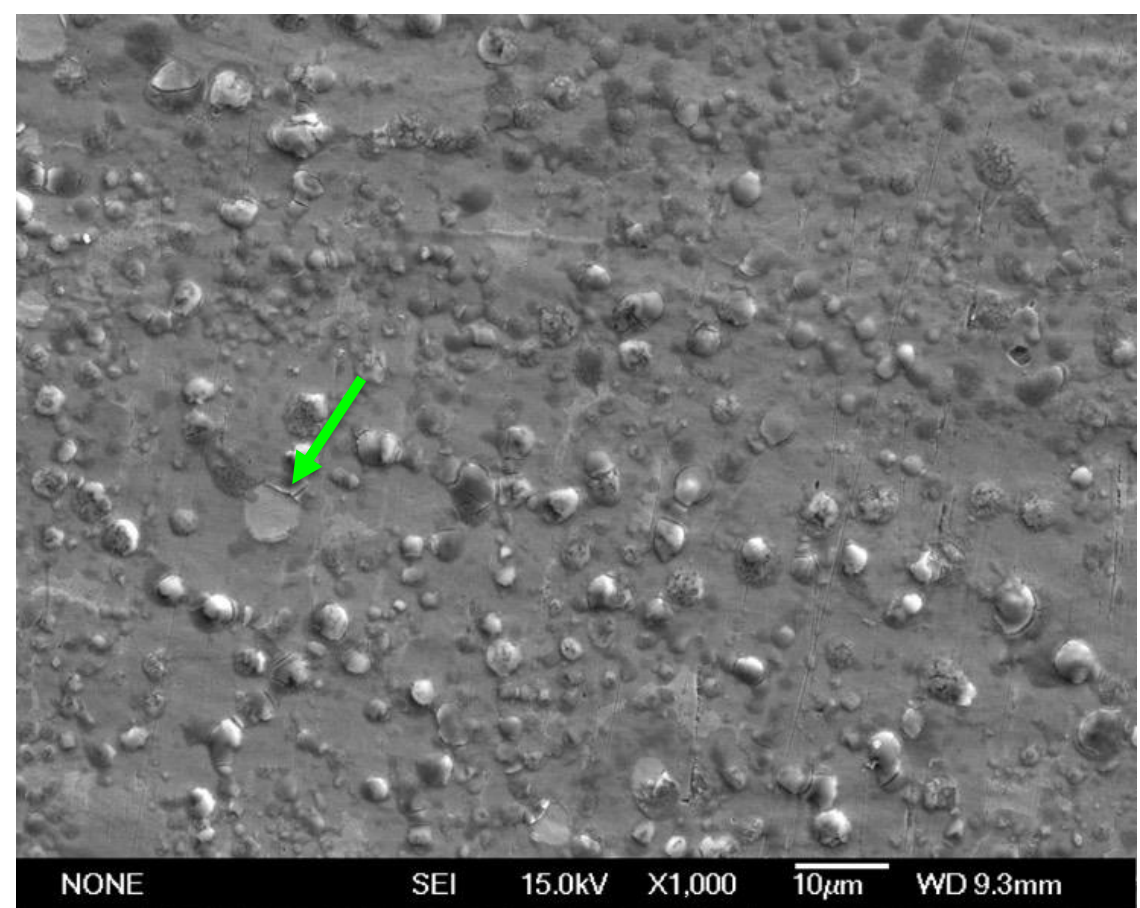

Figure 3.1.15: SEM micrograph of palladium electrodeposited with nickel.

These features do not resemble the deposits produced by electrodeposition on aluminium. The area below the green arrow is enlarged in Figure 3.1.16.

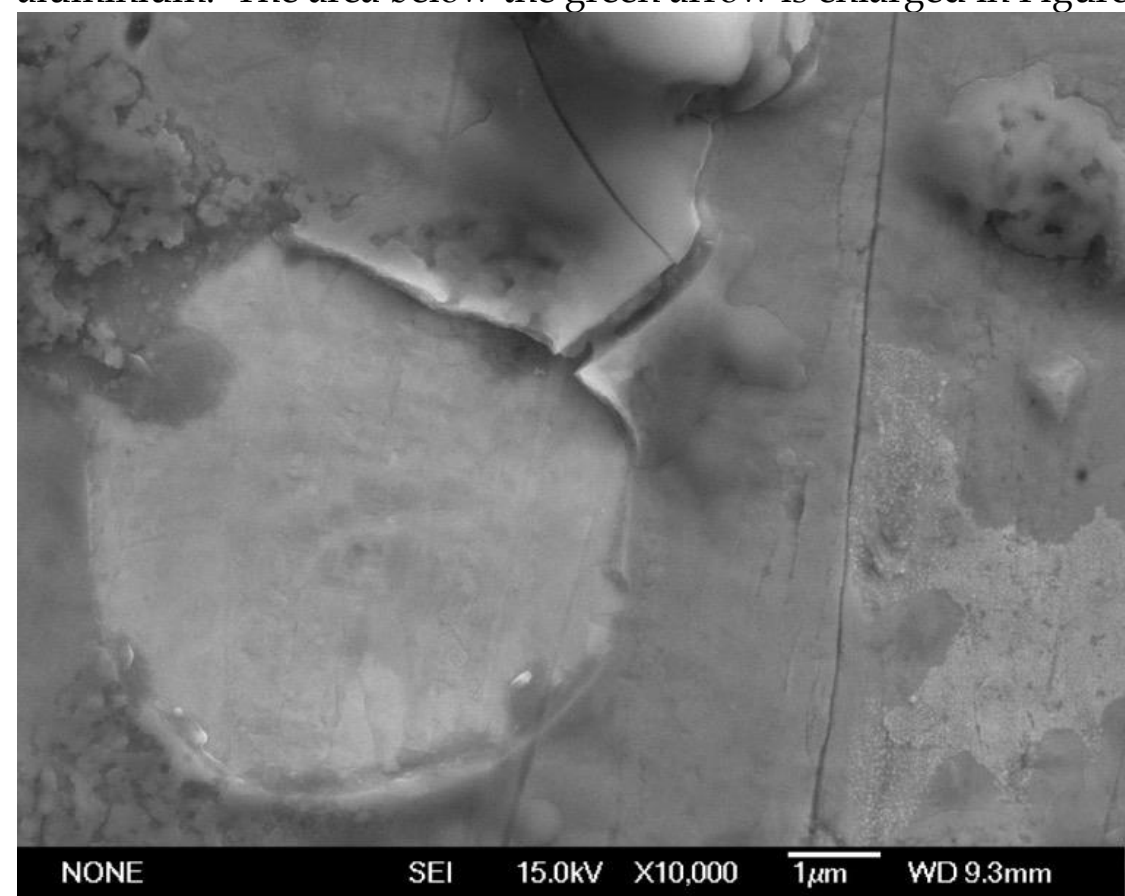

Figure 3.1.16: Enlarged detail from the same sample of palladium electrodeposited with nickel shown in Figure 3.1.15.

The EDS for this image detected only palladium in the large circular feature on the left, but nickel and palladium in the area above the circle. This circle is interpreted as a hole in the continuous nickel layer and the smaller features as bubbles in the continuous layer 


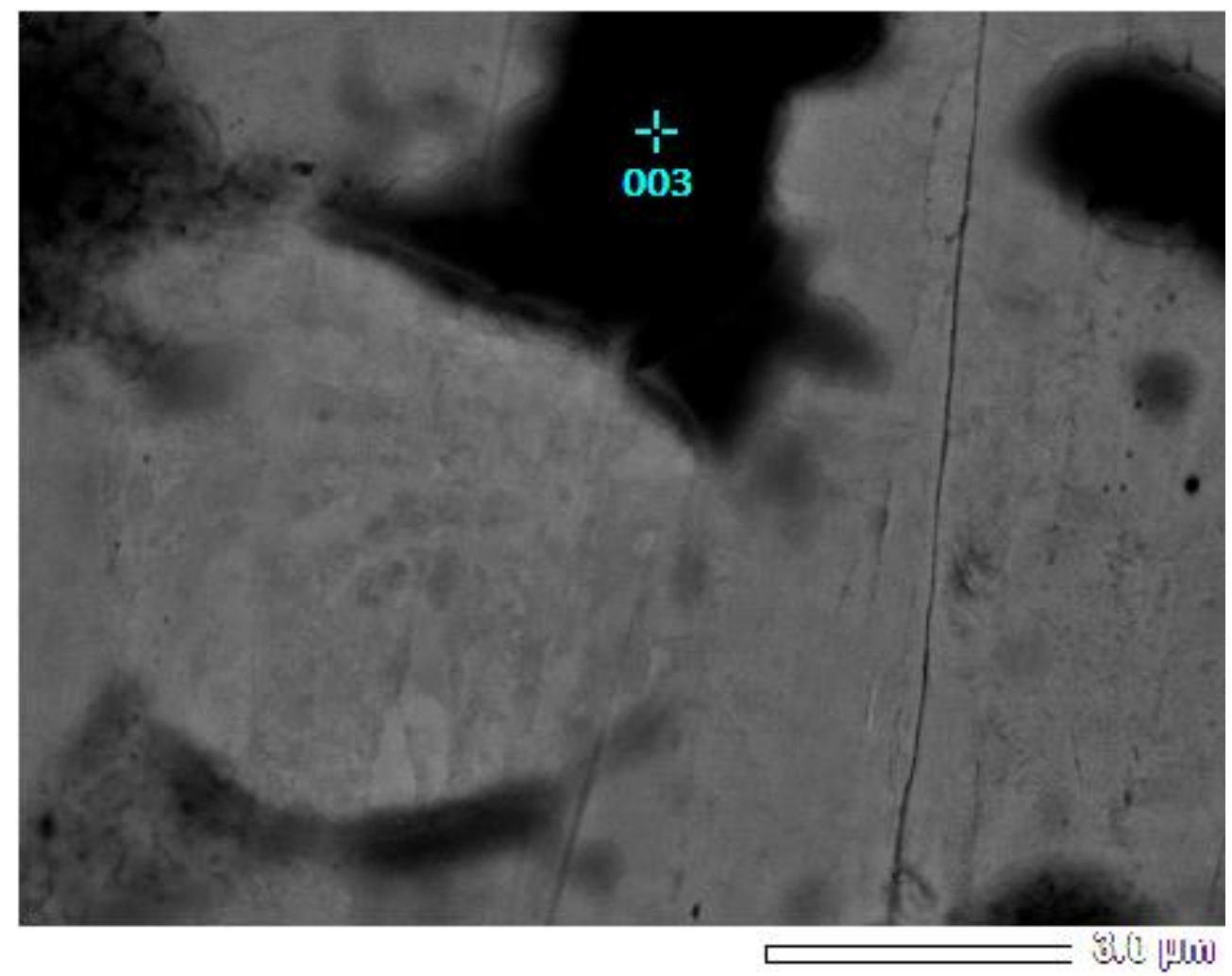

Figure 3.1.17: Back scatter SEM image of the same location as Figure 3.1.16 Semiquantitative analysis for the point marked 003 is shown in Figure 3.1.18 and Table 3.1.4. The peaks are compared with each other for relative proportions but have no external standard for full quantitative analysis.

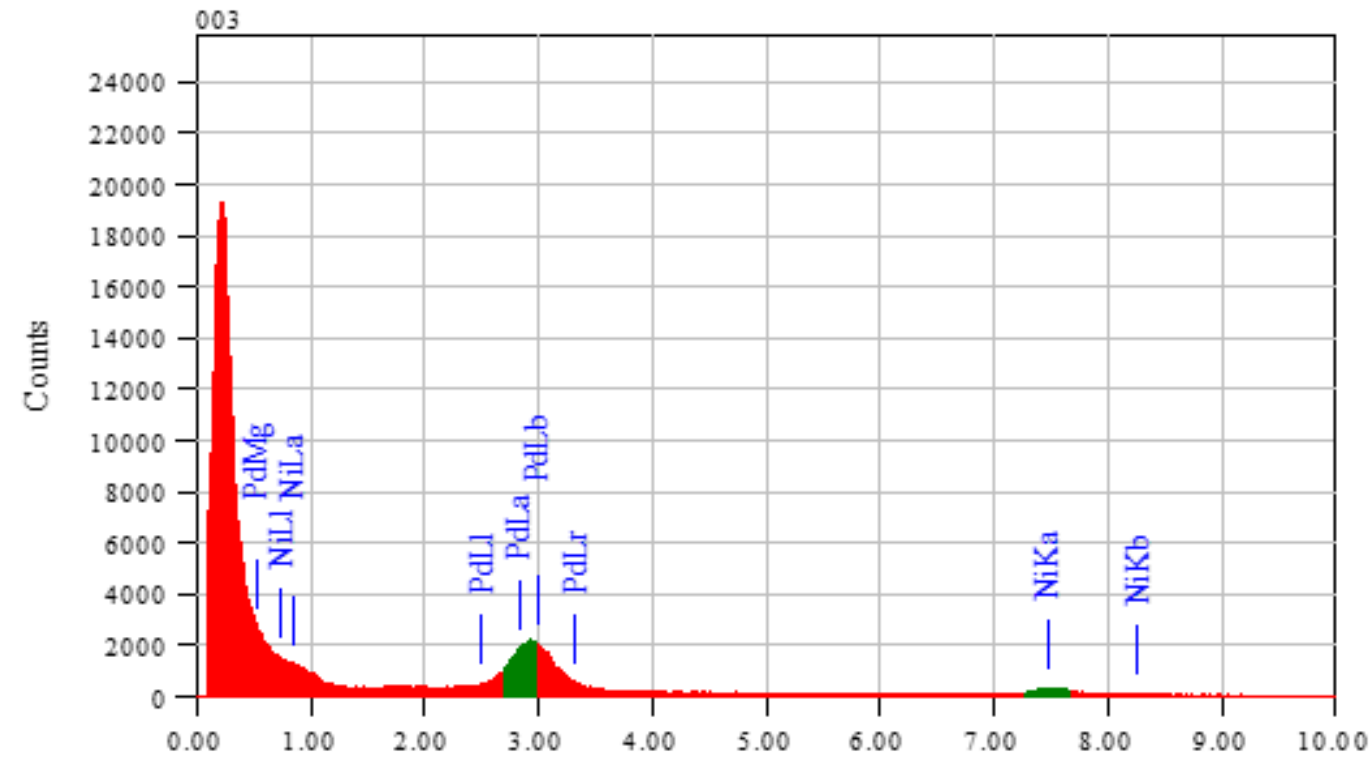

$\mathrm{keV}$

Figure 3.1.18: X-ray spectrum from EDS analysis as marked in Figure 3.1.17.

Table 3.1.4: Semiquantitative analysis of point EDS shown in Figure 3.1.18

\begin{tabular}{|l|l|l|l|l|l|}
\hline Element & keV & Mass\% & Counts & Atom\% & K \\
\hline $\begin{array}{l}\text { Ni K } \\
\text { (Ref.) }\end{array}$ & 7.471 & 53.93 & 693.71 & 67.98 & 1.0000 \\
\hline Pd L & 2.838 & 46.07 & 690.70 & 32.02 & 0.8579 \\
\hline Total & & 100.00 & & 100.00 & \\
\hline
\end{tabular}


Figure 3.1.19 and Figure 3.1.20 show SEM and EDS images for a palladium foil electrodeposited with nickel at $-2.0 \mathrm{~V}$ for $30 \mathrm{~s}$.

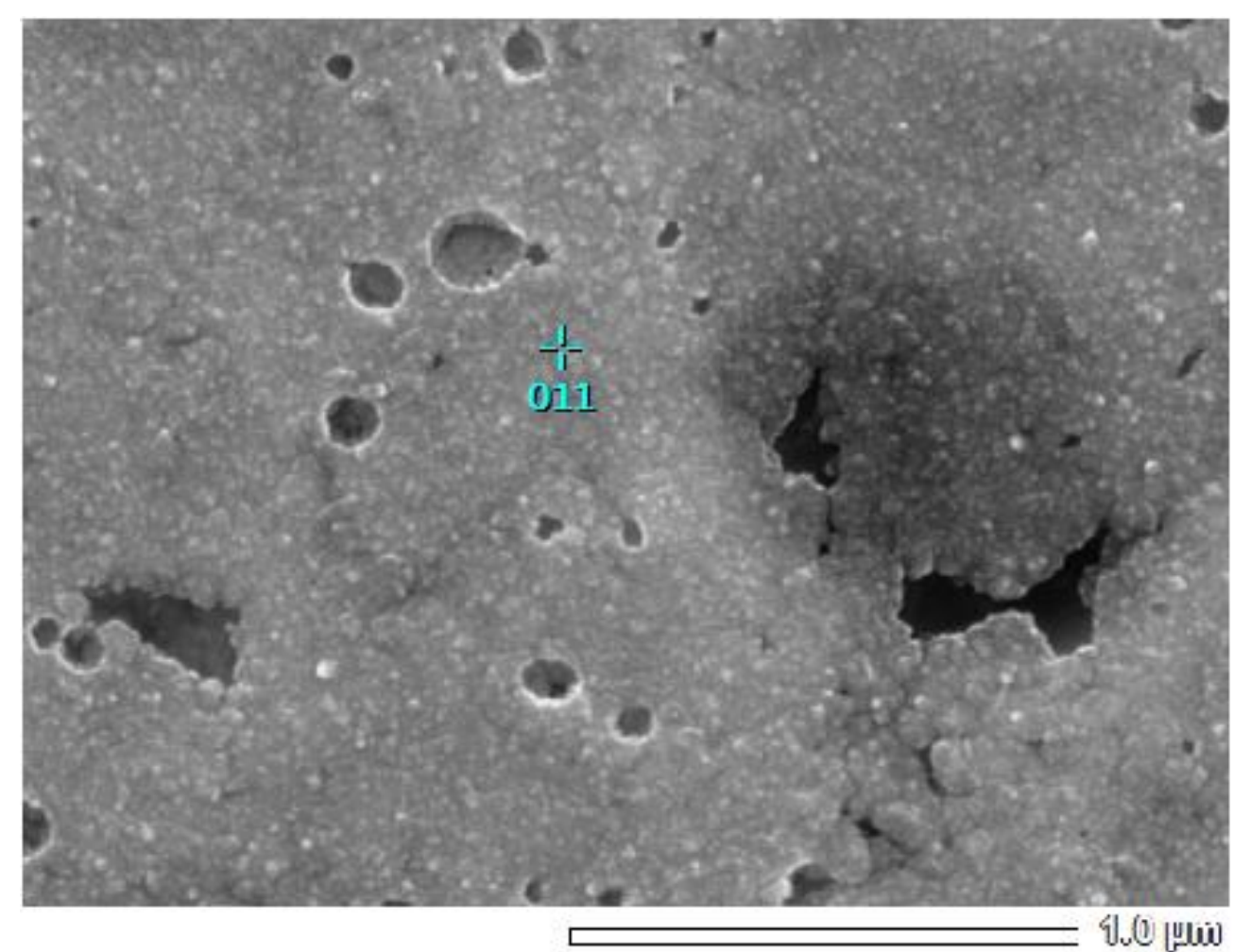

Figure 3.1.19: Back scatter electron micrograph of the palladium electroplated with nickel. 


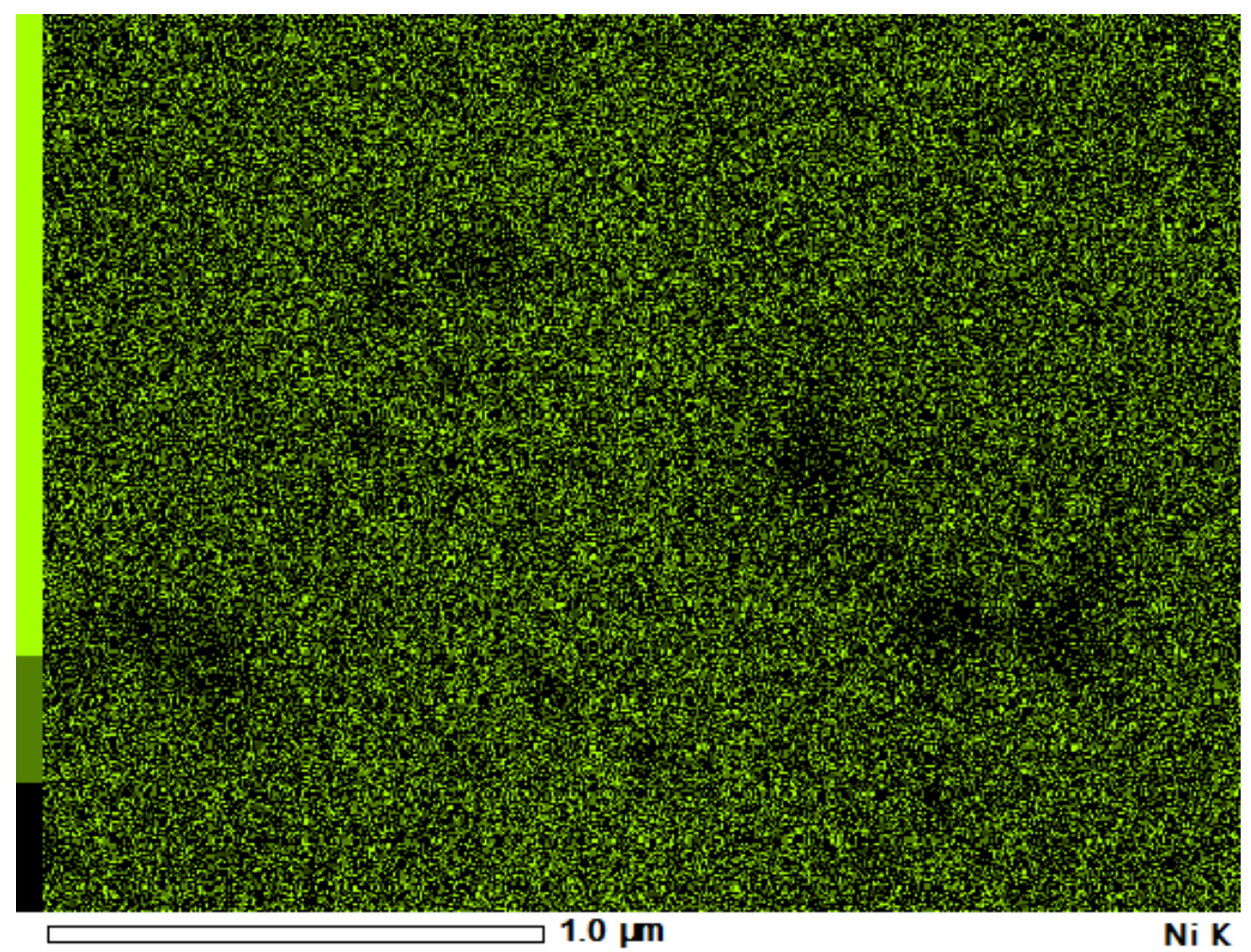

Figure 3.1.20: Nickel elemental map for the same location as Figure 3.1.19. The green marks points that show positive for nickel.

From these images, it was proposed that the nickel deposited on the palladium as a continuous layer that was prone to blistering. Possible mechanisms for formation of these blisters could have been a drying effect; or gas formation, either during formation or release of hydrogen absorbed into the palladium during the electrodeposition. If it was hydrogen production, then a lower voltage could reduce the blistering. This appears to be the case as is seen in Figure 3.1.21 which shows the proportion of the area covered with these features to have increased as the deposition voltage became more negative. If the problem was hydrogen trapped in the palladium underneath the nickel then the blistering might be prevented if the nickel was deposited as discrete particles, which would leave uncovered surface for the gas to desorb from without disturbing the deposits. 


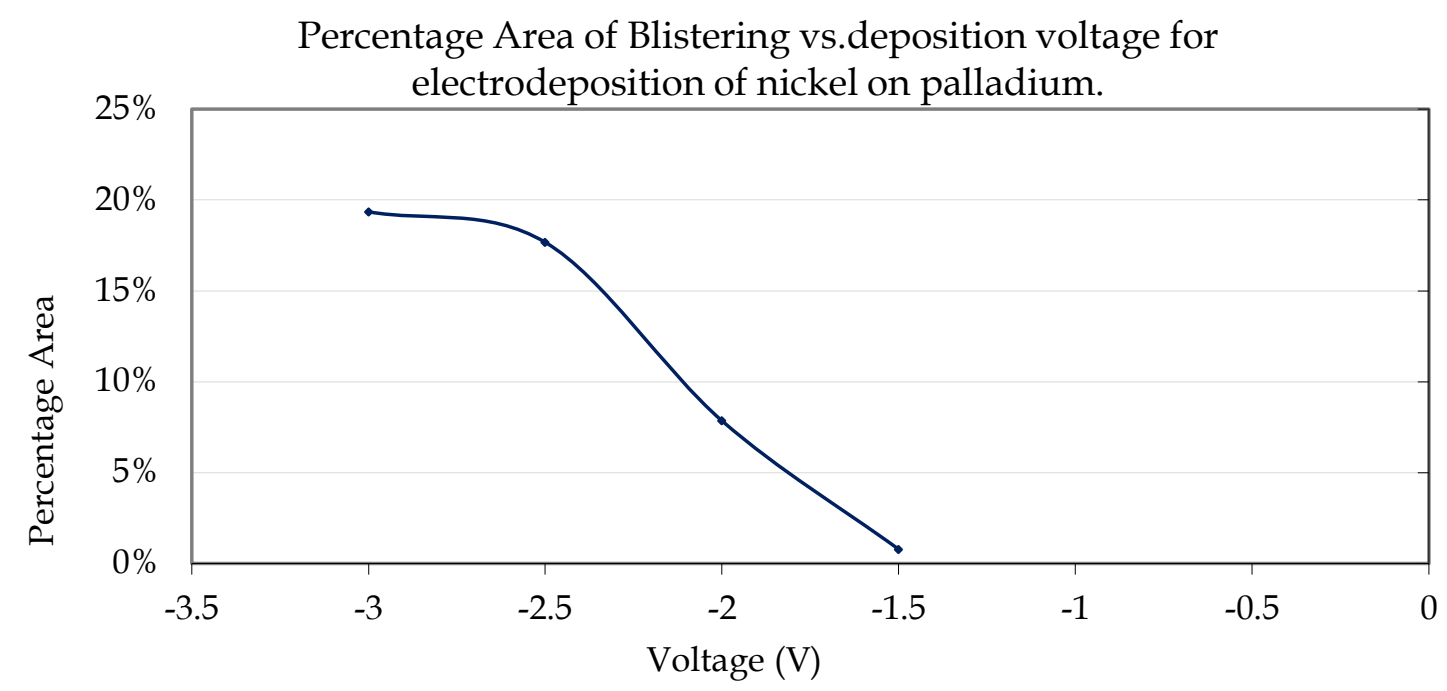

Figure 3.1.21: Graph of percentage area of blistering vs. deposition voltage for electrodeposition of nickel onto palladium.

This shows that there was more blistering with increasingly negative voltage.

In another attempt to confirm the presence of nickel, palladium samples were weighed before and after electrodeposition. This was challenging because the expected mass gain was four orders of magnitude smaller than the substrate mass. Table 3.1.5 displays the mass data and estimates the thickness of the nickel layer from the mass difference. Table 3.1.6 calculates the thickness from the electrodeposition data following the steps of Equation 2.2.1. Mass measurements using a 5 decimal place balance were abandoned as a useful source of information because the measurements were less than their error. 
Jonathan Tailby

Table 3.1.5: Nickel layer thickness calculated from mass data.

Samples deposited in the nickel sulfate solution from Table 2.2.1, with time and voltage given.

\begin{tabular}{|c|c|c|c|c|c|}
\hline \multicolumn{6}{|c|}{ Thickness of Electrodeposited Nickel on Palladium calculated from Mass Data } \\
\hline $\begin{array}{l}\text { mass before } \\
\text { (g) }\end{array}$ & $\begin{array}{l}\text { mass after } \\
(\mathrm{g})\end{array}$ & $\begin{array}{l}\text { Difference } \\
(\mathrm{g})\end{array}$ & $\begin{array}{ll}\begin{array}{l}\mathrm{Ni} \\
\left(\mathrm{cm}^{3}\right)\end{array} & \text { volume } \\
\end{array}$ & $\begin{array}{l}\text { Layer } \\
\text { thickness }(\mathrm{cm})\end{array}$ & $(\mu \mathrm{m})$ \\
\hline 0.18133 & 0.18151 & 0.00018 & 0.0000202 & 0.0000402 & 4.0 \\
\hline 0.17438 & 0.17528 & 0.0009 & 0.000101 & 0.000201 & 20 \\
\hline 0.18882 & 0.1702 & -0.01862 & & & \\
\hline 0.18456 & 0.18552 & 0.00096 & 0.000108 & 0.000214 & 21 \\
\hline 0.18115 & 0.18204 & 0.00089 & 0.0000999 & 0.000199 & 20 \\
\hline 0.18123 & 0.18132 & 0.00009 & 0.0000101 & 0.0000201 & 2.0 \\
\hline
\end{tabular}

Table 3.1.6: Nickel layer thickness calculated from electrodeposition data.

\begin{tabular}{|c|c|c|c|c|c|c|c|c|c|c|}
\hline \multicolumn{11}{|c|}{ Maximum Nickel Thickness calculated from Electrical Data } \\
\hline Voltage & \begin{tabular}{|l|} 
average \\
current
\end{tabular} & total time & \begin{tabular}{|l|} 
charge \\
transferred
\end{tabular} & Electrons & mole $\mathrm{e}^{-}$ & mole Ni & $\mathrm{m} \mathrm{Ni}$ & V Ni & Thickness & Thickness \\
\hline$-2.5 \mathrm{~V}$ & $\begin{array}{l}\mathrm{A} \\
0.0286\end{array}$ & $\begin{array}{l}\text { S } \\
20.06\end{array}$ & $\begin{array}{l}\text { C } \\
-0.574\end{array}$ & $3.58 \mathrm{E}+18$ & 5.95E-06 & 2.97E-06 & $\begin{array}{l}\mathrm{g} \\
1.75 \mathrm{E}-04\end{array}$ & $\begin{array}{l}\mathrm{cm}^{3} \\
1.96 \mathrm{E}-05\end{array}$ & $\begin{array}{l}\text { cm } \\
3.90 \mathrm{E}-05\end{array}$ & $\begin{array}{l}\mu \mathrm{m} \\
0.39\end{array}$ \\
\hline$-2.5 \mathrm{~V}$ & 0.0351 & 20.25 & -0.710 & $4.43 \mathrm{E}+18$ & 7.36E-06 & 3.68E-06 & 2.16E-04 & 2.43E-05 & 4.83E-05 & 0.48 \\
\hline$-2.5 \mathrm{~V}$ & 0.0335 & 30.52 & -1.024 & $6.39 \mathrm{E}+18$ & 1.06E-05 & 5.31E-06 & 3.11E-04 & 3.50E-05 & $6.96 \mathrm{E}-05$ & 0.70 \\
\hline$-2.5 \mathrm{~V}$ & 0.0359 & 35.18 & -1.262 & $7.88 \mathrm{E}+18$ & 1.31E-05 & $6.54 \mathrm{E}-06$ & 3.84E-04 & 4.31E-05 & 8.57E-05 & 0.86 \\
\hline & $-6.02 E-05$ & 35.51 & 0.002 & $-1.33 E+16$ & $-2.21 \mathrm{E}-08$ & $-1.11 \mathrm{E}-08$ & $-6.50 \mathrm{E}-07$ & -7.30E-08 & $-1.45 \mathrm{E}-07$ & 0.00 \\
\hline$-2.5 \mathrm{~V}$ & 0.0172 & 15.03 & -0.258 & $1.61 \mathrm{E}+18$ & 2.68E-06 & 1.34E-06 & 7.86E-05 & 8.82E-06 & 1.75E-05 & 0.18 \\
\hline$-2.5 \mathrm{~V}$ & 0.0146 & 20.38 & -0.297 & $1.85 \mathrm{E}+18$ & 3.08E-06 & $1.54 \mathrm{E}-06$ & 9.03E-05 & 1.01E-05 & 2.03E-05 & 0.20 \\
\hline$-3.0 \mathrm{~V}$ & 0.00188 & 20.24 & -0.038 & 2.37E+17 & 3.94E-07 & 1.97E-07 & 1.16E-05 & 1.30E-06 & $2.60 \mathrm{E}-06$ & 0.03 \\
\hline$-2.5 \mathrm{~V}$ & $6.29 \mathrm{E}-05$ & 20.42 & -0.001 & $8.02 \mathrm{E}+15$ & 1.33E-08 & 6.66E-09 & 3.91E-07 & 4.39E-08 & $8.78 \mathrm{E}-08$ & 0.00 \\
\hline
\end{tabular}


Initially the suspected nickel layer on palladium was not detectable by XRD as seen in Figure 3.1.22. The simplest explanation would be that there is no nickel, but the SEM evidence is counter to this. Accepting that nickel is present, it could be that it was insufficiently crystalline to register in XRD. Nickel metal is usually a face centred cubic crystal and the electrodeposition conditions were not extraordinary so it seems an unlikely explanation that it was not crystalline. More likely, crystalline nickel is present but with insufficient quantity of material for the XRD to detect. An electrodeposition sample deposited for one hour at $-1.0 \mathrm{~V}$ tested positive for nickel on low angle XRD. That XRD trace is included as Figure 3.1.23 and has the same reference patterns as Figure 3.1.22, marked in the same colours. The long electrodeposition that created the sample with the XRD trace in Figure 3.1.23 would have increased the size of the nickel crystallites and the thickness of the nickel layer, increasing the interaction between nickel and the x-rays.

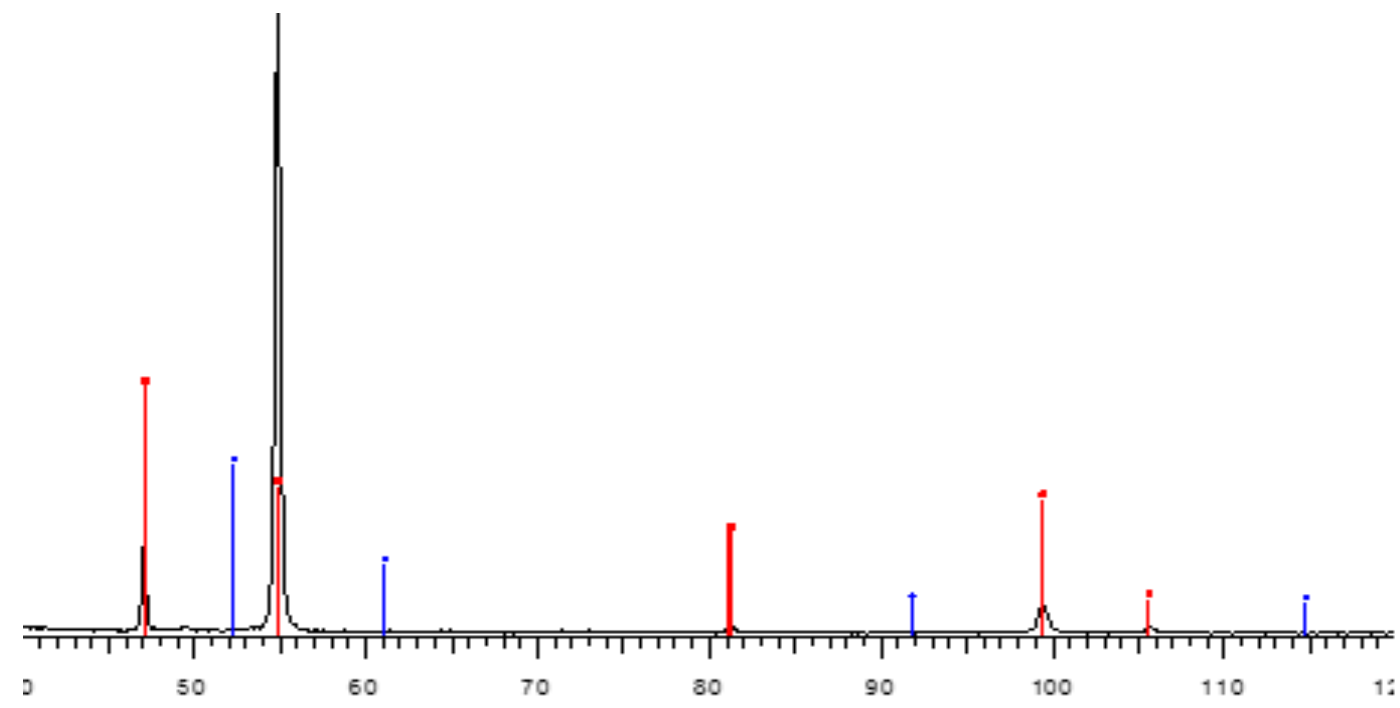

2Theta

Palladium after electrodeposition $\square$ Palladium, Pd $\bullet$ Nickel, Ni

Figure 3.1.22: XRD trace of palladium electrodeposited with nickel.

The trace has peaks matching the palladium pattern marked in red but none which match the nickel peaks marked in blue. 


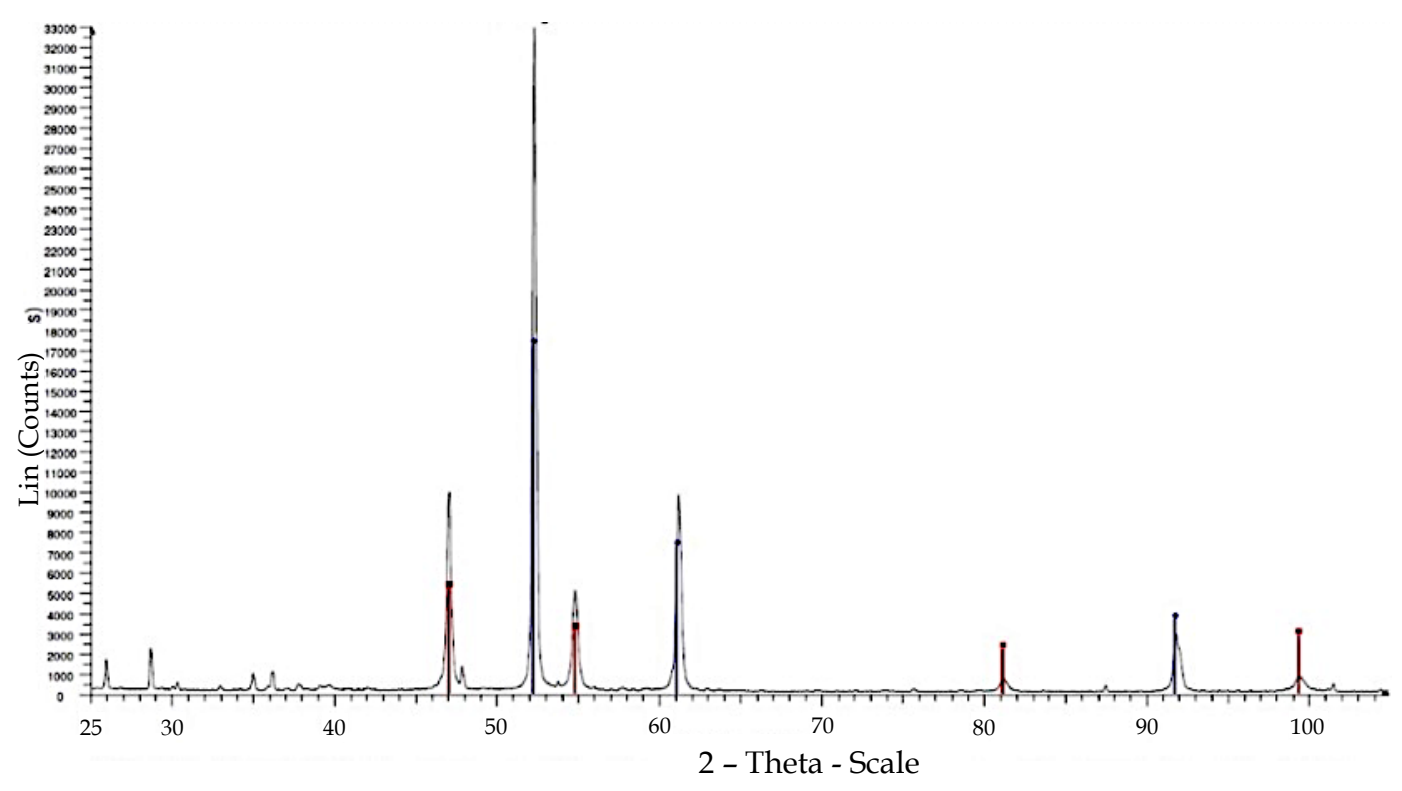

Palladium after electrodeposition $\quad \square$ Palladium, Pd $\bullet$ Nickel, Ni

Figure 3.1.23: XRD trace of palladium electrodeposited with nickel at $-1.0 \mathrm{~V}$ for 1 hour.

The trace has peaks which match the palladium pattern marked in red and the nickel pattern marked in blue. This was the first electrodeposition sample to show up Nickel in the XRD trace.

Figure 3.1.23 shows that nickel is present and the Scherrer equation calculates crystallite size of the nickel to be $290 \AA$. Any nickel present is not clearly visible in the SEM images that were taken up to this point. To make the nickel visible in the SEM, two electrodeposited samples were prepared for crosssection SEM as described in Section 2.2.8.1.

The result of one sample is shown in Figure 3.1.24. Figure 3.1.24a shows a backscatter SEM micrograph of the region showing that the top right of the image has much heavier elements than the bottom left. This was interpreted to mean that the image showed the boundary between the heavy palladium metal and the lighter elements that composed the epoxy. The map of palladium beside the micrograph shows red where palladium is present, which indicated a very large concentration of palladium in the top right corner that matched the heavy element shown in the back-scatter micrograph. Figure 3.1.24b shows the map of nickel in green, this map shows the highest concentration of nickel was at the edge of the palladium. The additional nickel throughout the map was attributed to nickel redistributed during the grinding process. The map in blue shows oxygen, which was most concentrated in the bottom left of the 
image, the additional oxygen throughout the image was interpreted as a combination of oxidation and epoxy spread by the same grinding process that dispersed the nickel. Figure 3.1.24c shows the palladium, nickel and oxygen elemental maps overlaid together to show more clearly that the largest concentration of nickel is at the boundary between the epoxy and the palladium.

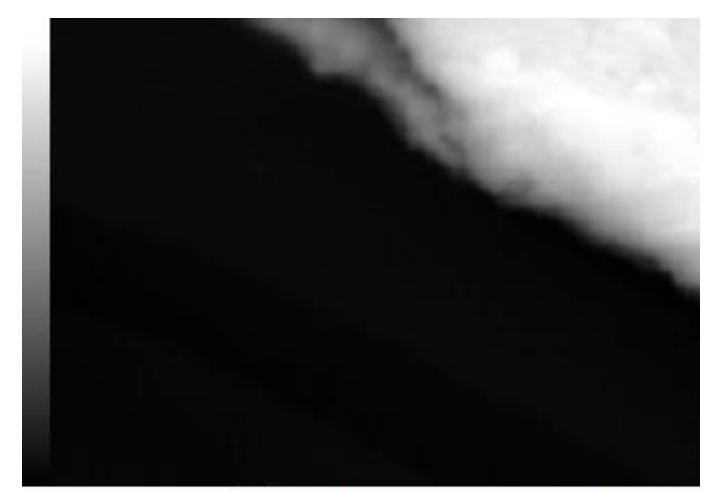

$0.5 \mu \mathrm{m}$

IMG1

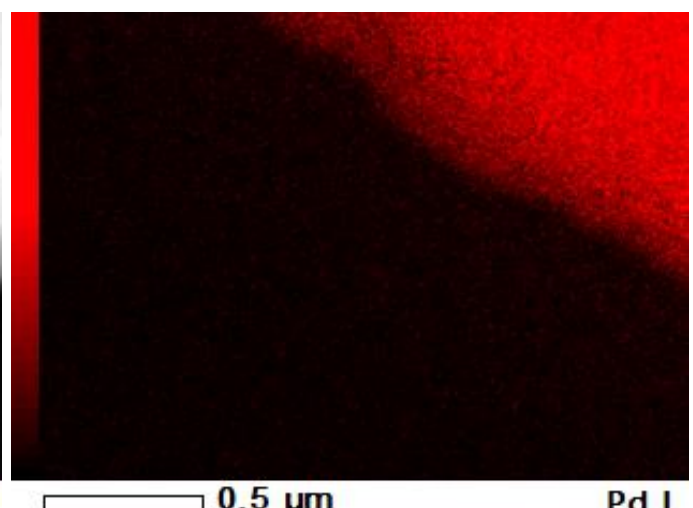

Figure 3.1.24a: Back scatter electron micrograph of the border between the palladium and the epoxy and an elemental map which shows that the palladium was located in the top right of the image

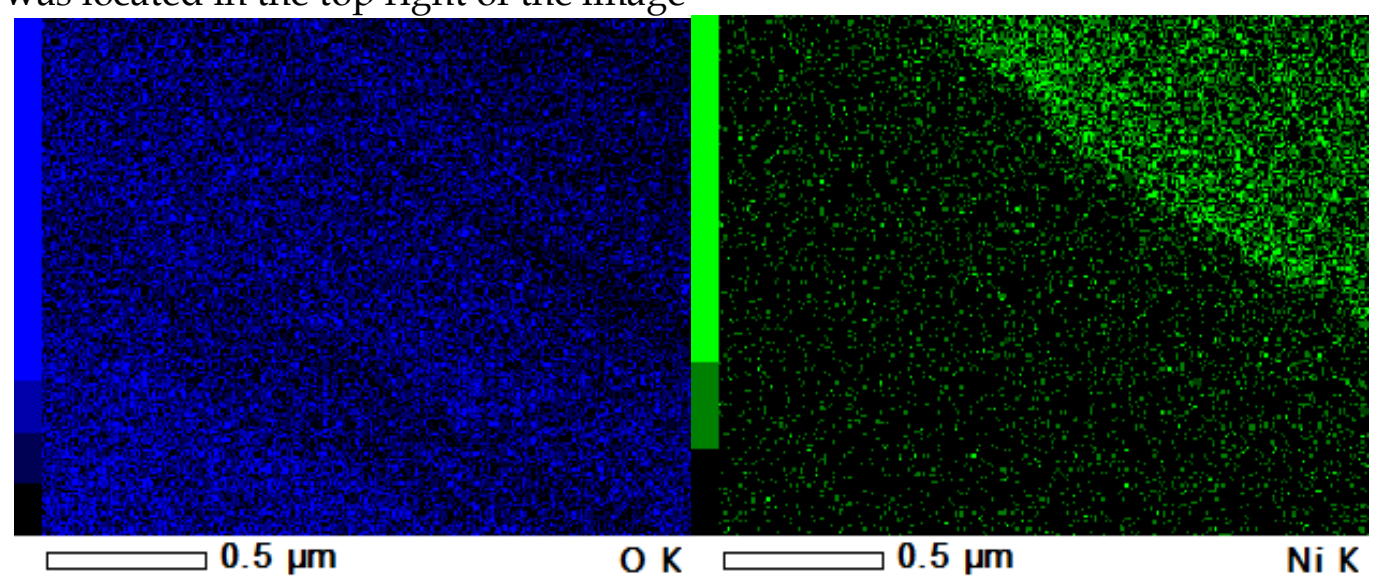

Figure 3.1.24b: An elemental map which shows the oxygen to have been most concentrated in the bottom left of the image and an elemental map of nickel which shows the nickel to have been most concentrated at the border between the palladium and the epoxy. 


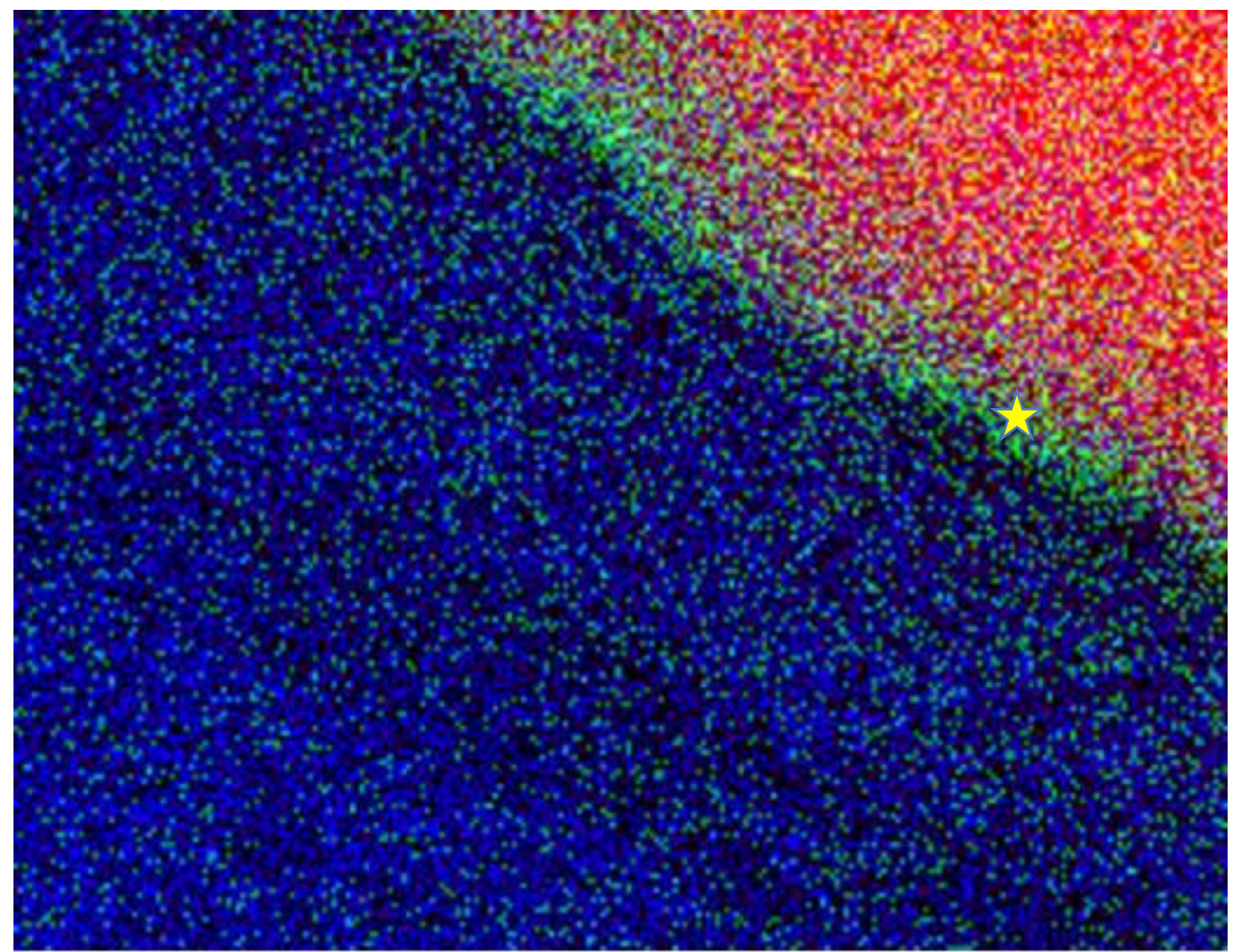

\section{$0.5 \mu \mathrm{m}$}

Figure 3.1.24c: Overlay of the EDS maps which shows that the greatest concentration of nickel was at the boundary between the palladium and the epoxy. Figure 3.1.24: Elemental maps of cross section of electrodeposited palladium.

The point marked with a yellow star on Figure 3.1.24c was assessed semiquantitatively by EDS. The results are shown in Figure 3.1.25 and Table 3.1.7. The results showed that this particular point has a relatively high proportion of nickel compared to other points on the substrate.

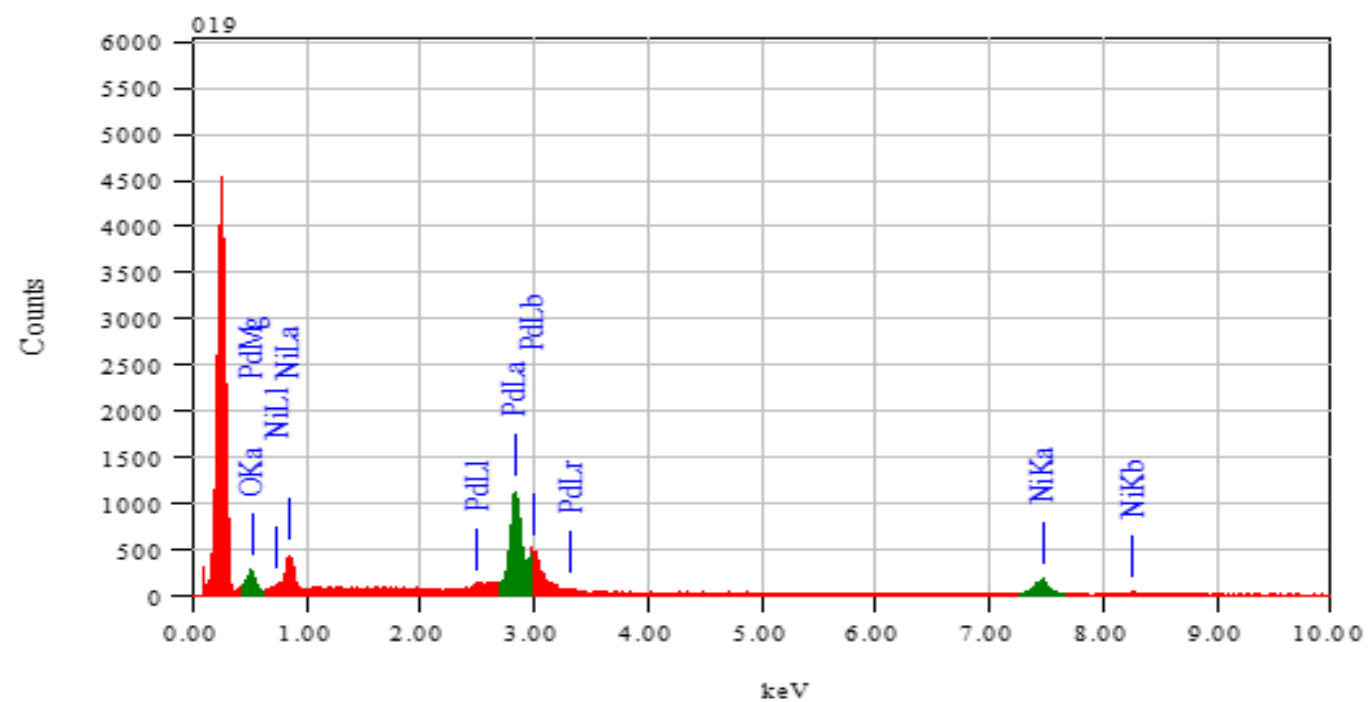

Figure 3.1.25: X-ray spectrum for EDS point analysis showing oxygen and nickel present on the palladium. 
Table 3.1.7: Quantitative analysis for eds of cross section.

\begin{tabular}{|l|l|l|l|l|l|l|}
\hline Element & keV & Mass\% & Counts & Sigma & Atom\% & K \\
\hline & & & & & & \\
\hline O K & 0.525 & 2.63 & 1048.35 & 0.07 & 13.09 & 0.2718 \\
\hline Ni K & 7.471 & 22.81 & 2123.17 & 0.56 & 31.00 & 1.1656 \\
\hline $\begin{array}{l}\text { Pd L } \\
\text { (Ref.) }\end{array}$ & 2.838 & 74.57 & 8090.89 & 0.81 & 55.90 & 1.0000 \\
\hline Total & & 100.00 & & & 100.00 & \\
\hline
\end{tabular}

These electrodeposition experiments did not succeed in forming a product suitable for use as an ammonia decomposition catalyst with a palladium membrane, therefore, an alternate method of catalyst formation was explored. The next method of catalyst formation that was explored was spray coating nickel nanoparticles onto the metal substrate. 
Jonathan Tailby

\subsection{2. $\quad$ Spray Coating}

The second method tested to form a suitable catalyst was spray coating of nickel nanoparticles onto the metal substrate.

Samples were examined by optical microscopy; the dominant features this revealed were the ring formations that can be seen in Figure 3.1.26. These arise as ethanol migrates to the edge of the evaporating droplet carrying the suspended nanoparticles with it. Areas of heavy spraying have many overlapping rings from subsequent drops, with the heaviest nickel deposits where the rings intersect, illustrated by the optical micrograph shown in Figure 3.1.27. Another feature that is visible in Figure 3.1.26 and Figure 3.1.27 is that a ring with a much larger diameter also had a much thicker ring than a ring with a small diameter. This correlation would be because a larger drop would spread out to cover a larger area and would also contain more suspended nickel, significantly more because the quantity of nickel is proportional to the volume of the drop and therefore to the cube of the radius while the circumference of the ring left by the drop would be directly proportional to the radius. The same processes would be active in both small and large drops to migrate the nickel to the edges [166] so that a larger drop would produce a thicker ring. 


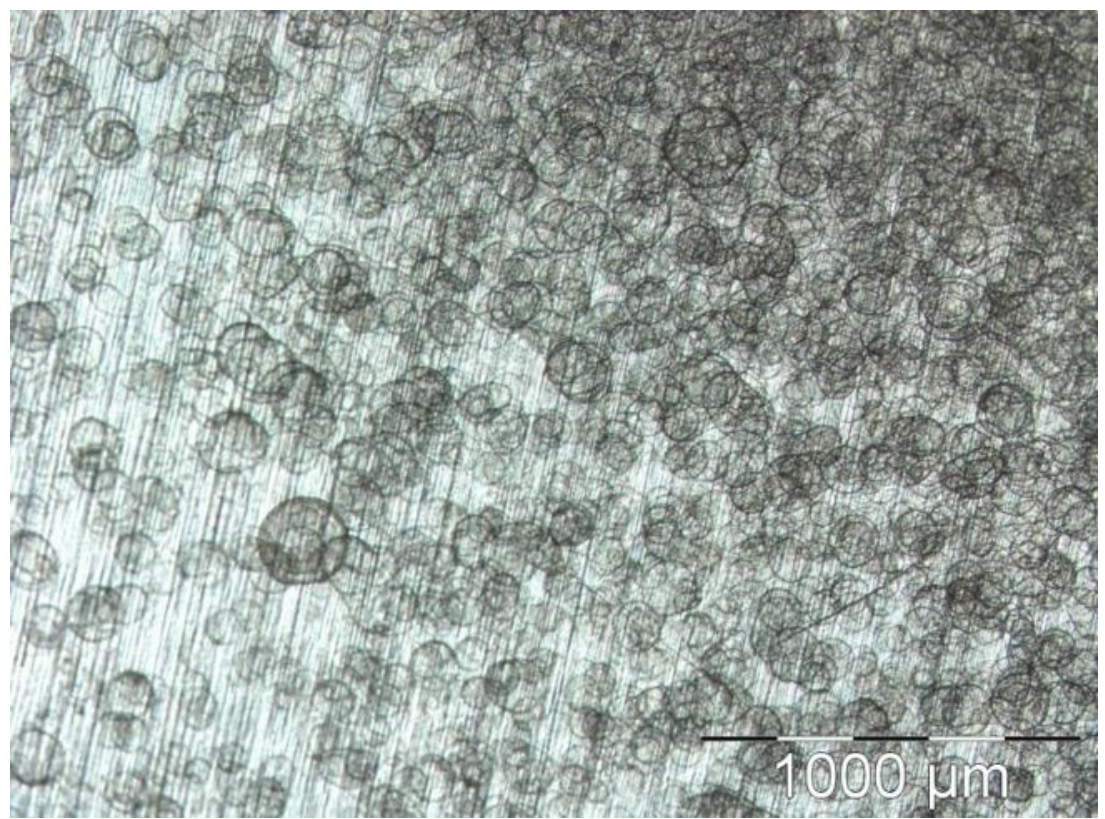

Figure 3.1.26: Optical micrograph of a spray coated sample which shows where nickel has deposited most heavily at the edges of the droplets.

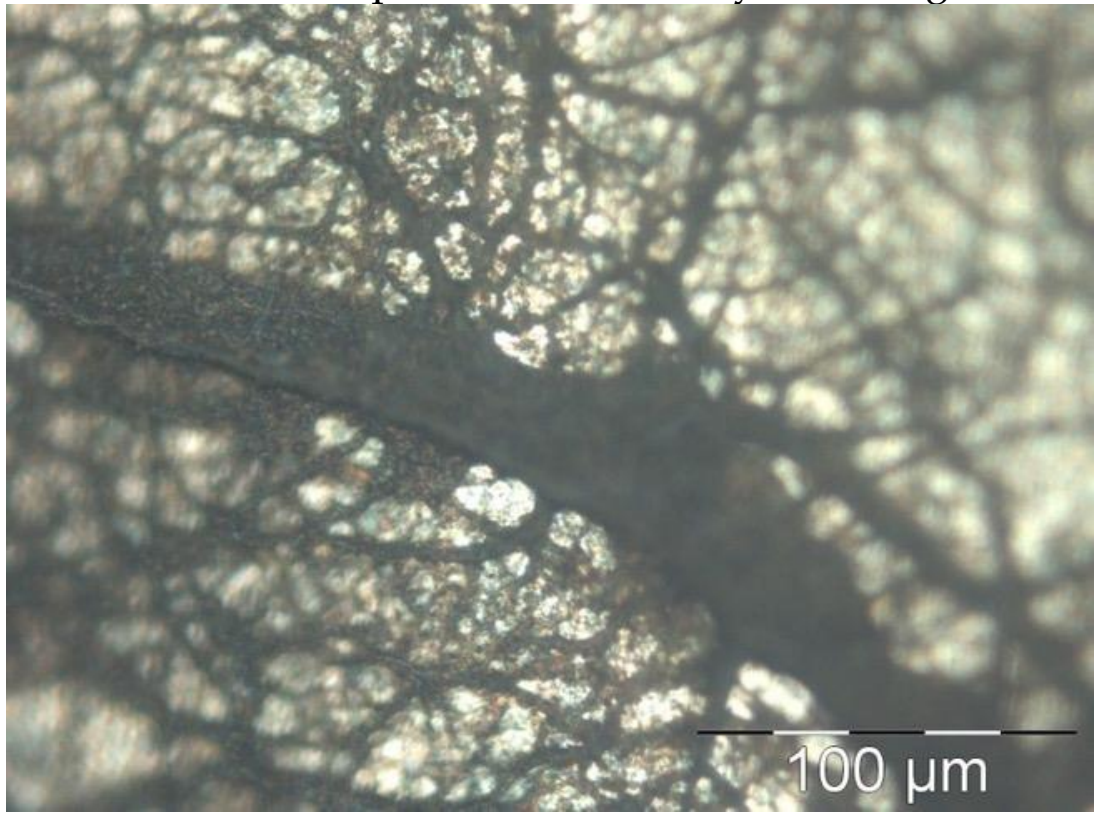

Figure 3.1.27: Optical micrograph of heavy spray coating, showing the overlap of rings and the extra agglomeration that has occurred there.

Early spray coating samples were examined by XRD, and a sample XRD trace is shown in Figure 3.1.28. The nickel nanoparticles must have been too small and too well dispersed to be detected by this technique. 
Jonathan Tailby

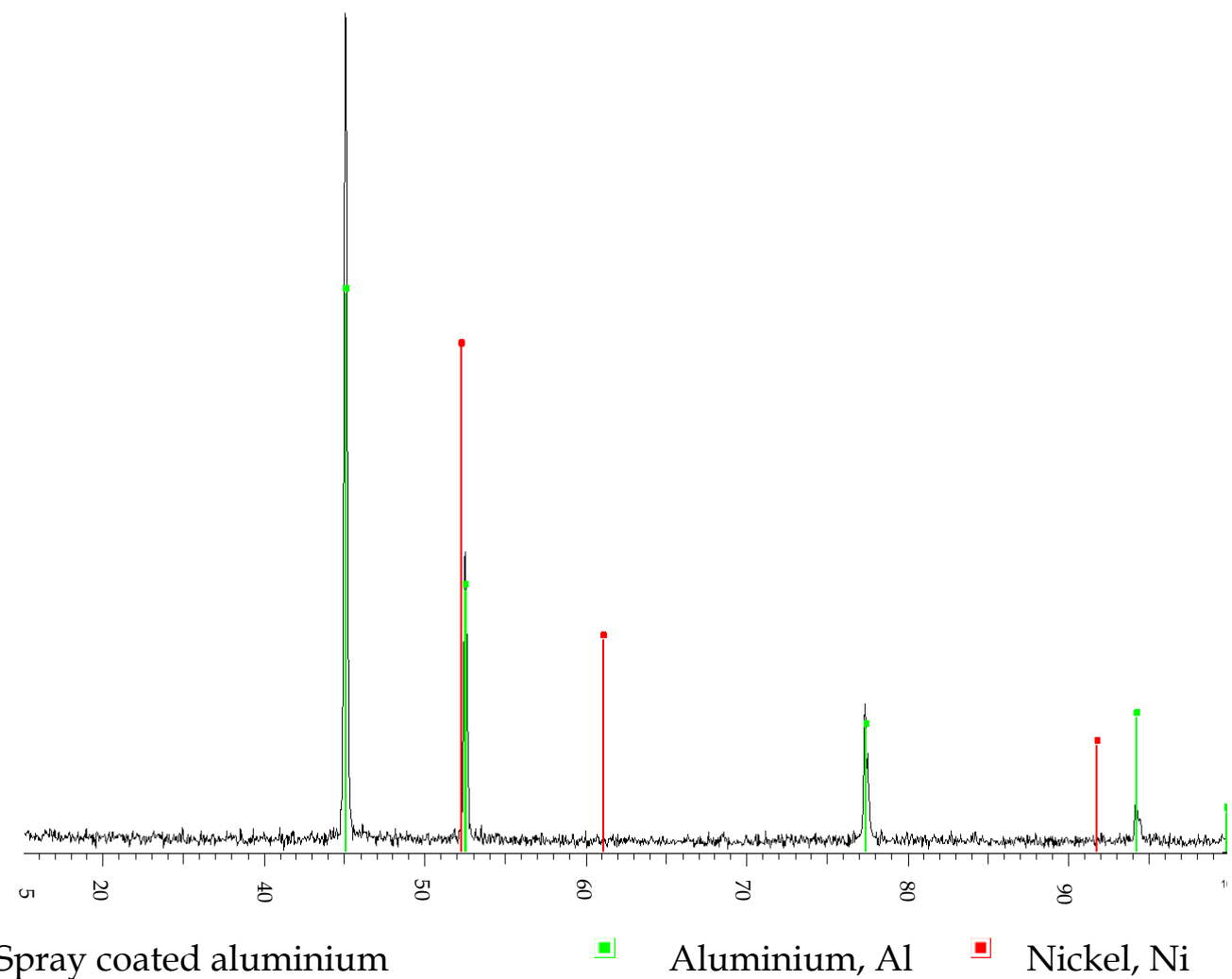

Figure 3.1.28: XRD trace for aluminium sprayed with nickel nanoparticles.

The peaks on the trace match the pattern for aluminium marked in green, but the only peak which matches the pattern for nickel marked in red is the peak at $52.5^{\circ} 2 \theta$ which also matches the aluminium pattern.

SEM imaging with EDS analysis confirmed these dark rings as predominantly nickel and oxygen, as expected for these nanoparticles. A selection of images is shown from Figure 3.1.29 to Figure 3.1.38. 


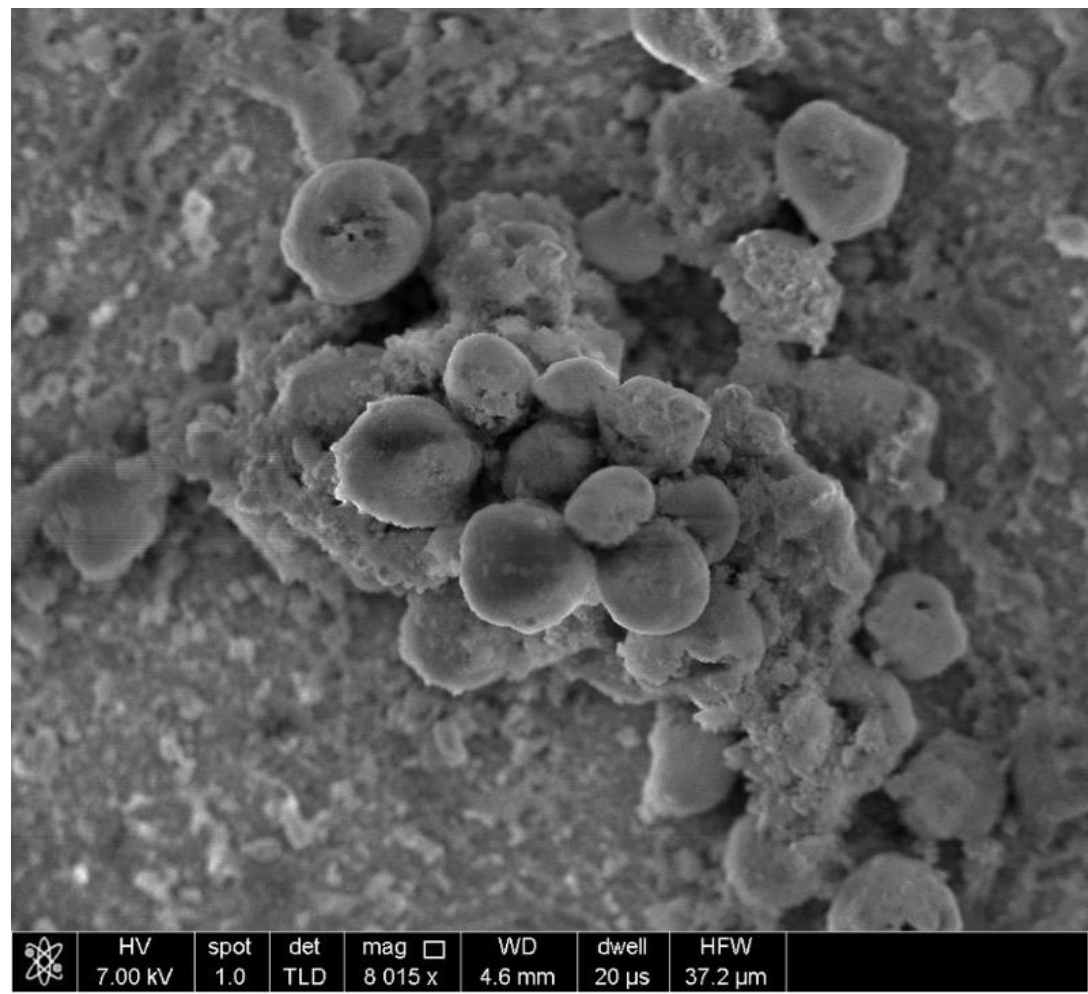

Figure 3.1.29: High magnification SEM of nickel nanoparticles spray coated onto Steel.

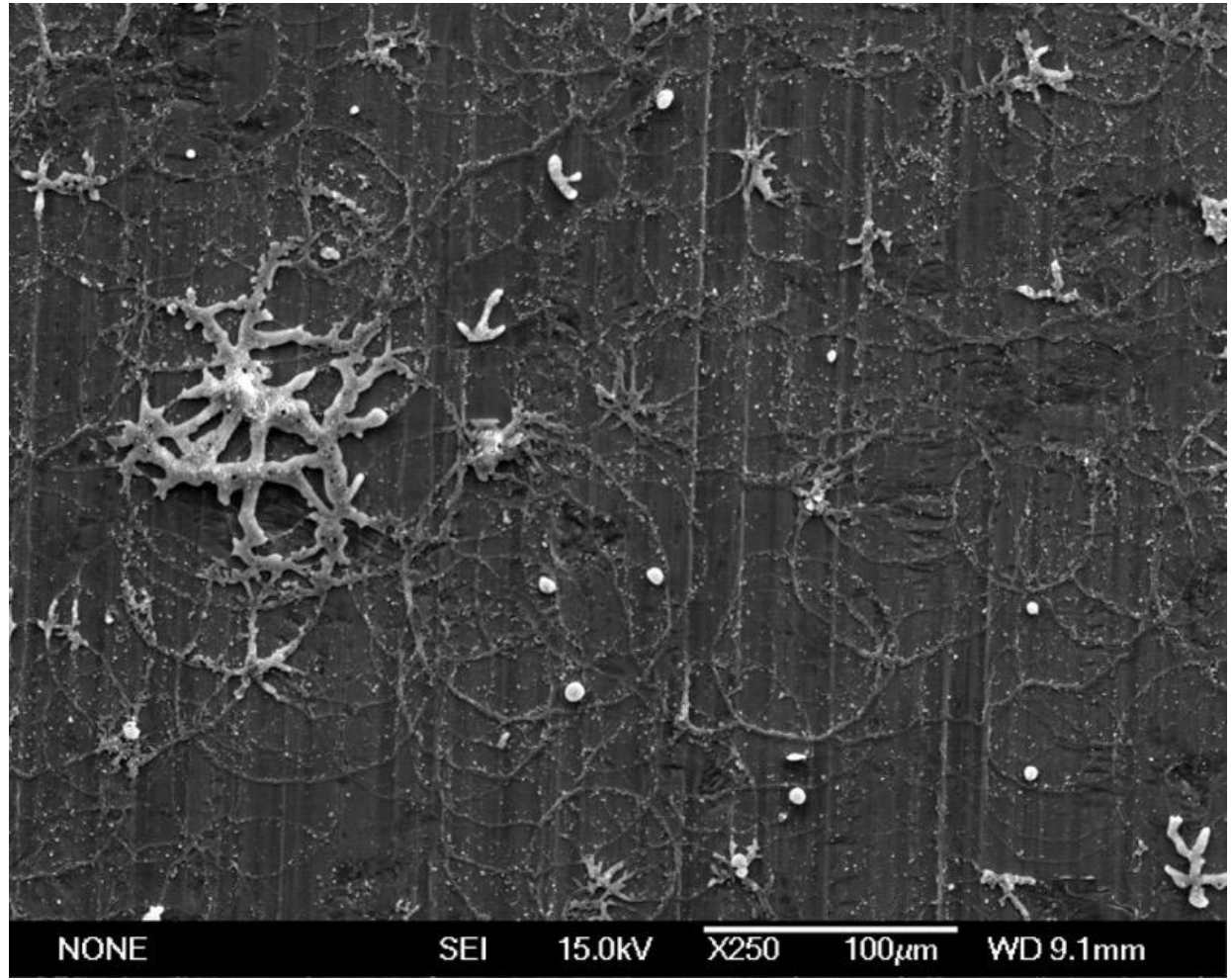

Figure 3.1.30: SEM Micrograph of Intersection of multiple drying rings. 


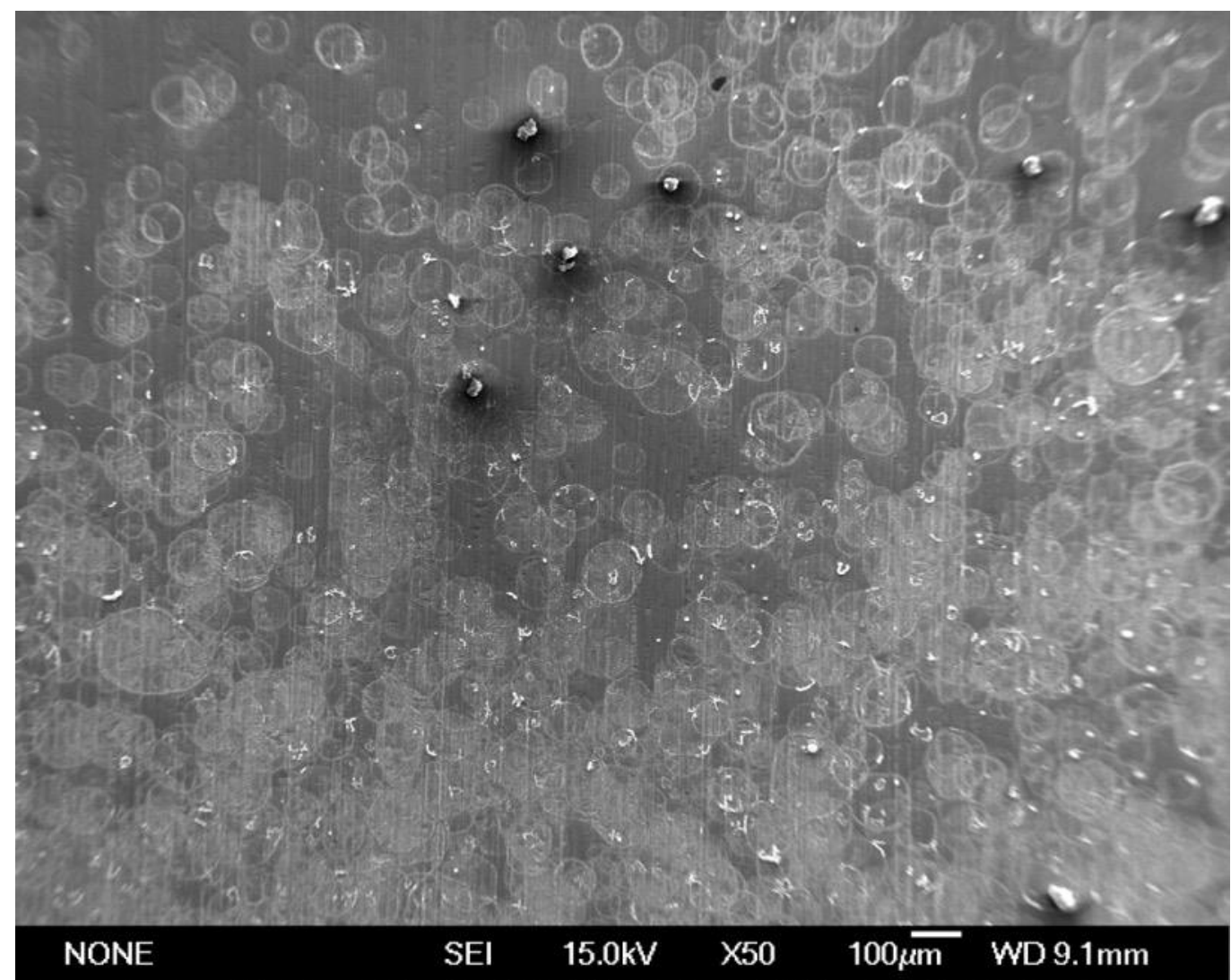

Figure 3.1.31: Low magnification SEM of circular deposition patterns on a spray coated sample.

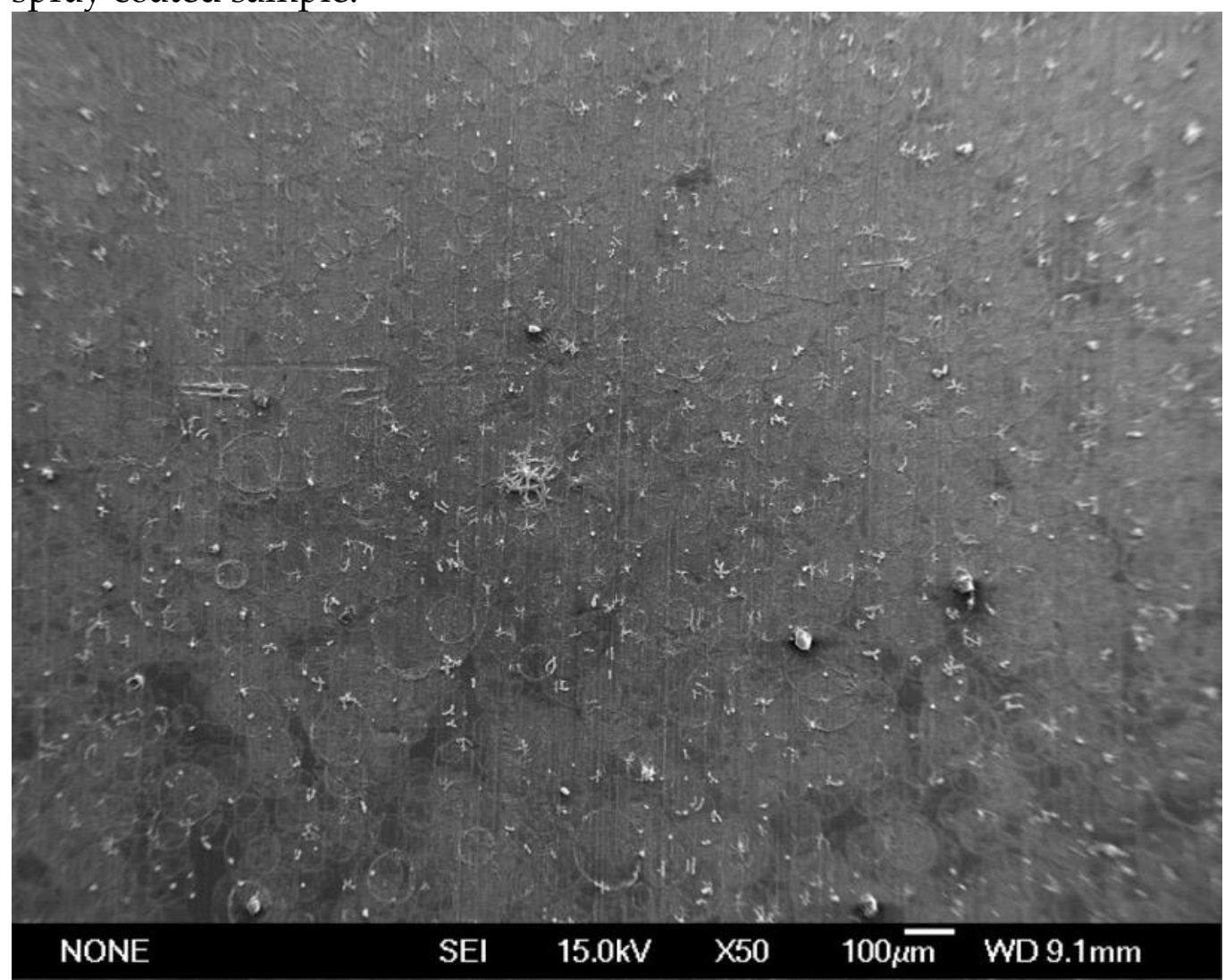

Figure 3.1.32: Low magnification SEM of circular deposition on spray coat sample with heavy deposition at points of multiple intersection of rings near the centre of the image. 


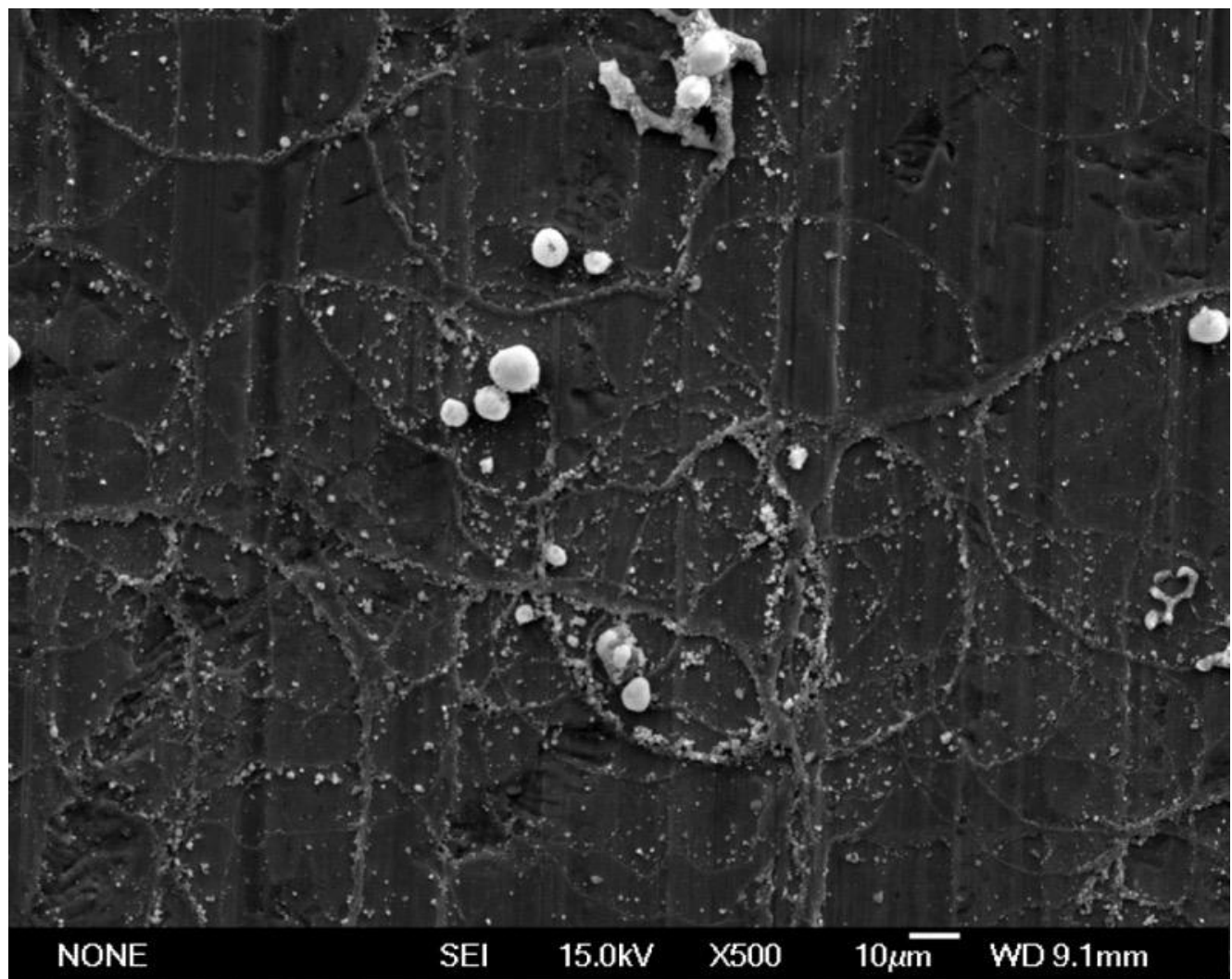

Figure 3.1.33: SEM image of spray coat sample showing both rings and agglomerations.

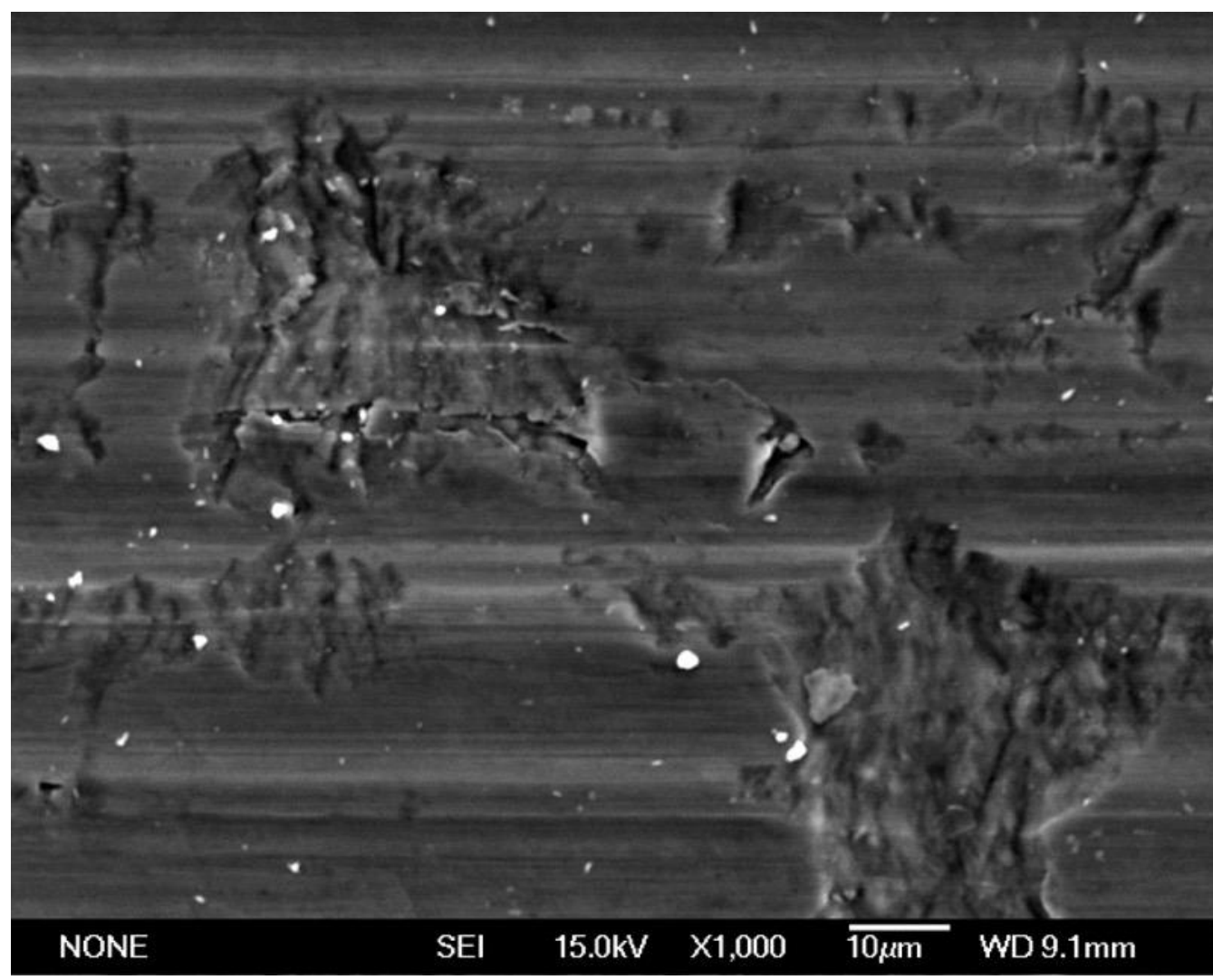

Figure 3.1.34: SEM image of spray coat sample showing very low density of nickel deposition. 
Jonathan Tailby

Figure 3.1.35 to Figure 3.1.38 show SEM with EDS for spray coated palladium. Similar EDS for two more samples are shown in Appendix 5.2.5. The dark regions on the elemental maps for palladium match the deposits visible in the micrographs and the lightest regions on the nickel and oxygen element maps. This was interpreted as confirming the deposits as being composed of small particles of nickel and oxygen.

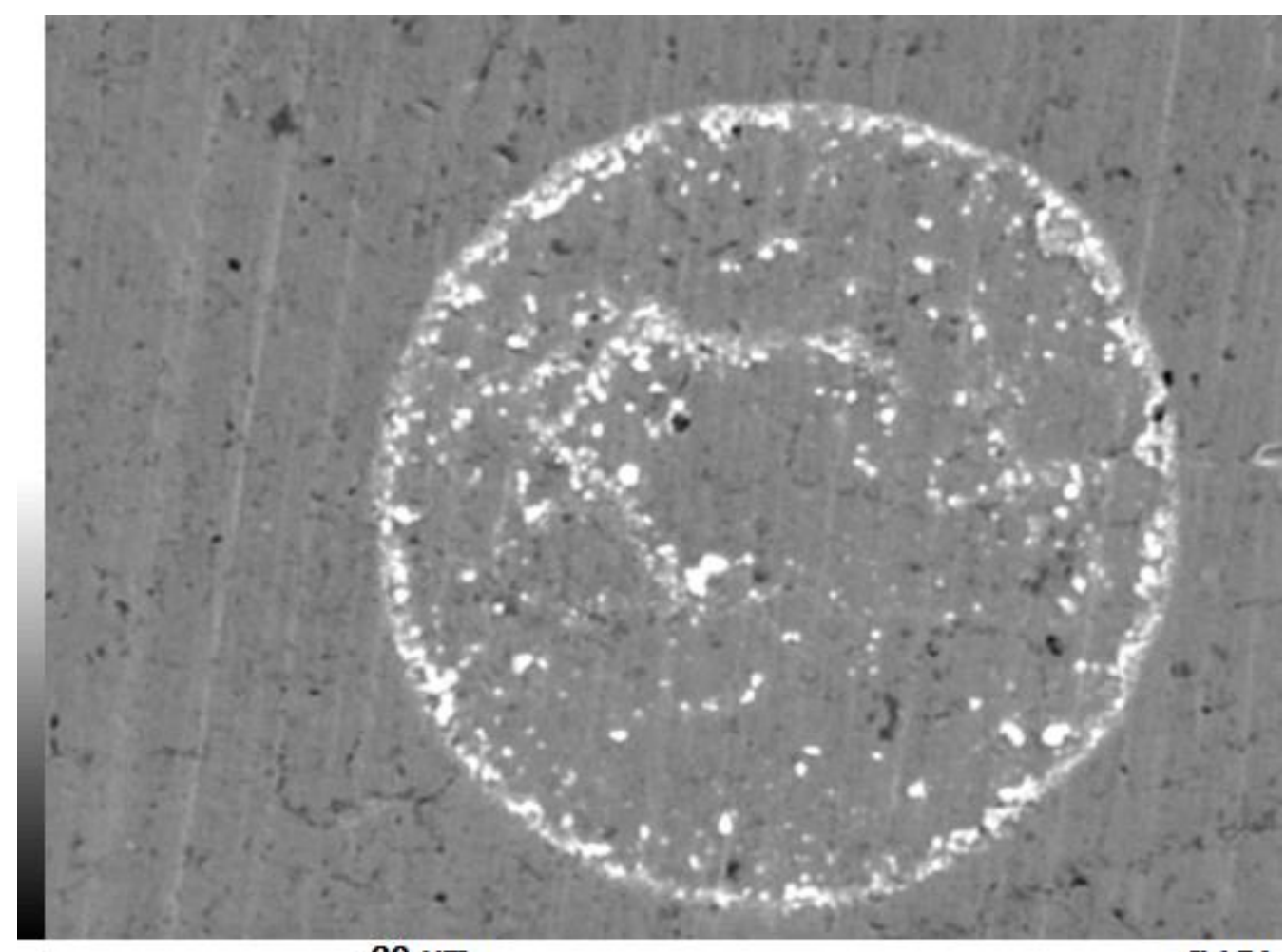

$$
30 \mu \mathrm{m}
$$

IMG1

Figure 3.1.35: SEM image of a spray coated palladium sample.

The white spots are the nickel particles and the grey background is the palladium substrate. 


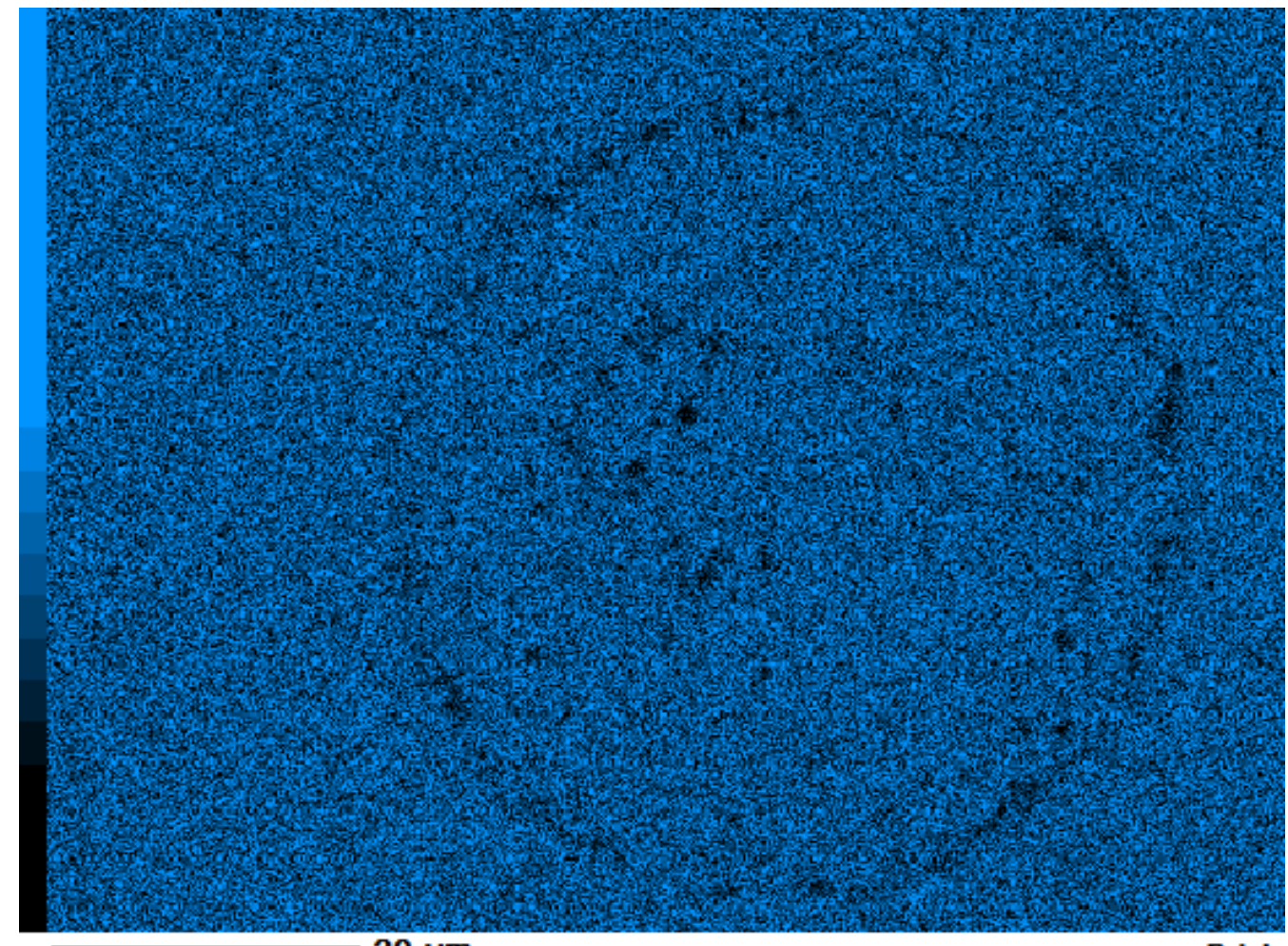

Figure 3.1.36: Elemental map of palladium after spray coating Palladium is marked in blue. The diminished palladium in the spray coated areas shows where nickel masks the palladium signal.

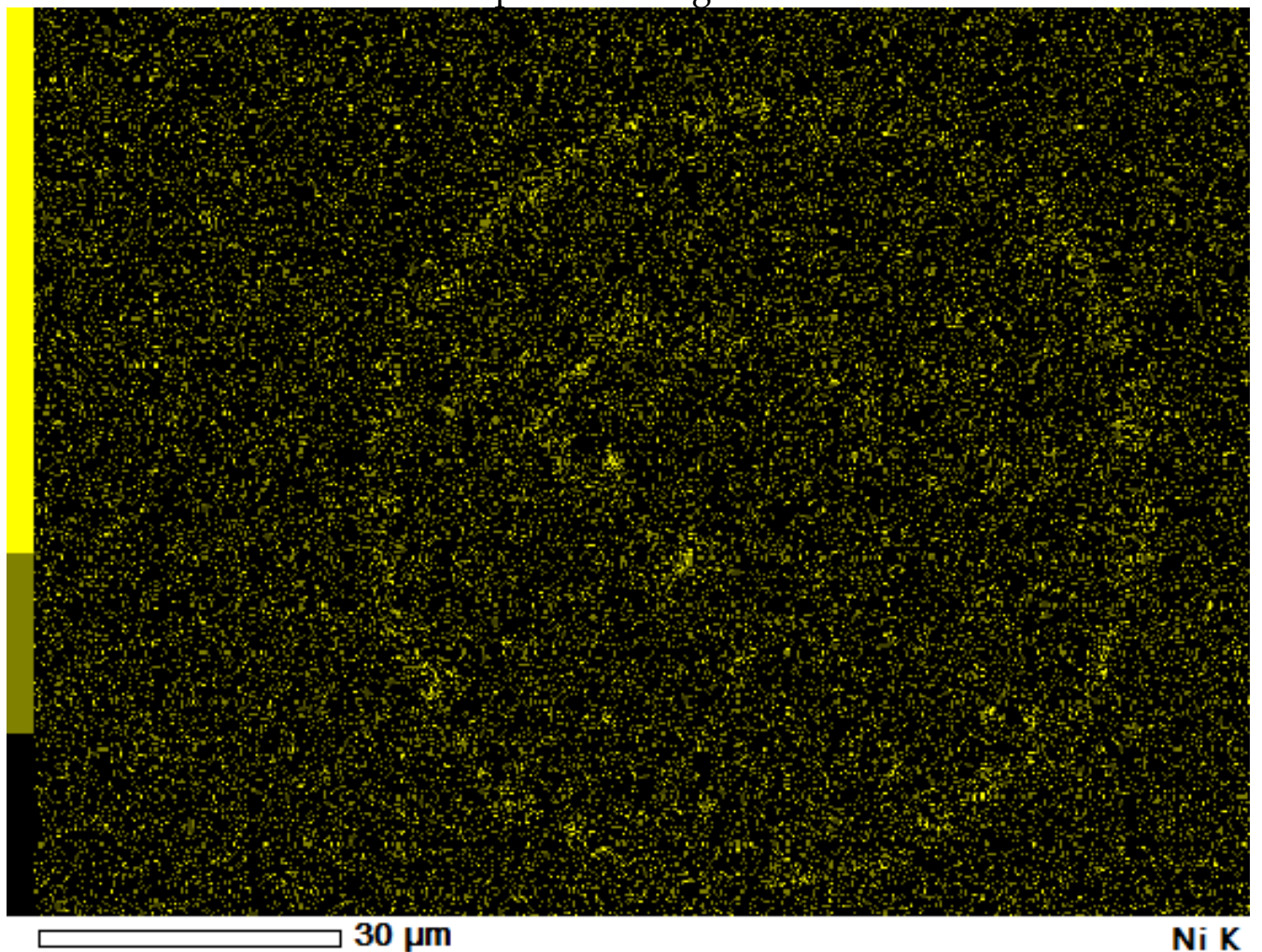

Figure 3.1.37: Elemental map of nickel spray coated onto palladium.

The nickel is marked in yellow. The yellow ring shows increased nickel in the spray coated areas. 


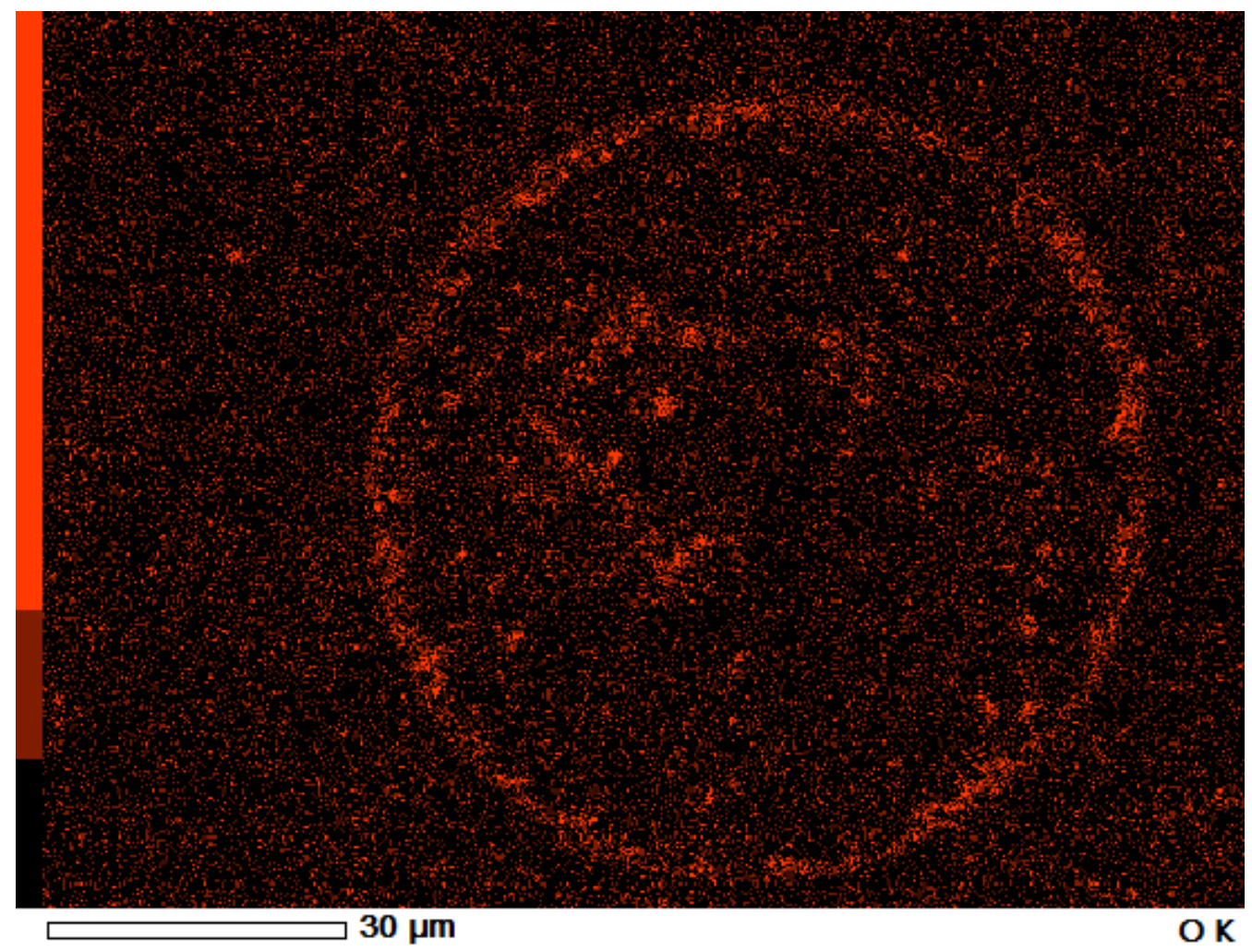

Figure 3.1.38: Elemental map of oxygen where nickel nanoparticles have been spray coated onto palladium.

The oxygen, shown in red, has increased in the spray coated areas.

The inconsistency of this method was most likely because the nickel would accumulate and block the spray nozzle, sometimes reducing the spray output but other times dripping larger drops onto the substrate. This inconsistency made the method too unreliable so an alternate methodology was explored.

The high oxide content of the nanoparticles could have been a problem, however it could have been remedied by in situ reduction.

\subsubsection{Electrodeposition with Lithographic Templates}

Electrodeposition was reconsidered as a possible method for catalyst formation by the use of templates to force the electrodeposition into a pattern. This method is described in Section 2.2.4 starting on page 101.

\subsubsection{Templated Electrodeposition On Foils}

Figure 3.1.39 shows an optical micrograph of a template on flat steel made by photolithography. Each circle is a hole through the AZ24533 polymer to the steel below. The dark ring around each circle is because the holes taper in, to be slightly smaller diameter at the bottom than the top. The diameter of the 
holes was larger when high magnification microscopy was focused on the template surface than when focused on the metal substrate.

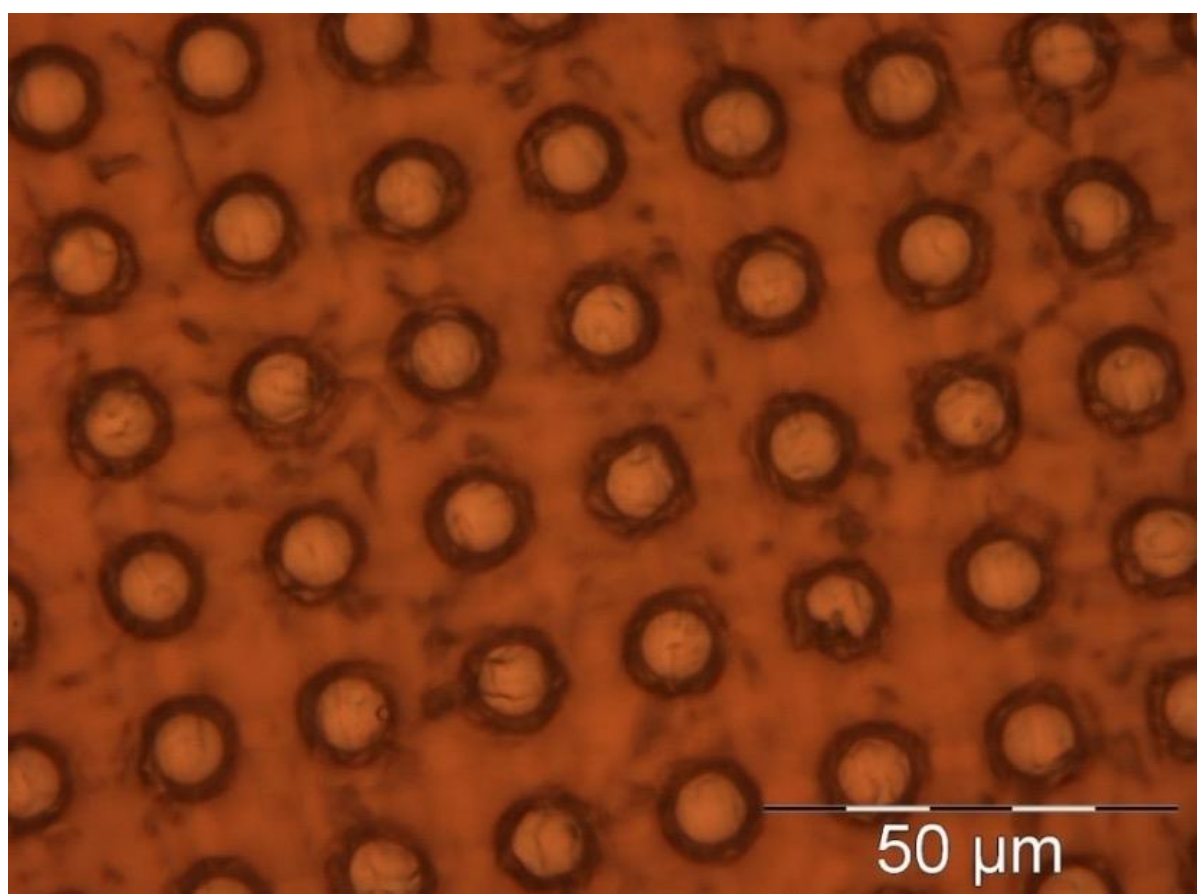

Figure 3.1.39: Optical micrograph of templates for electrodeposition. Each $10 \mu \mathrm{m}$ circle is a hole that has been burnt through the photosensitive polymer with ultraviolet light.

The first templated electrodeposition explored the electrical characteristics of the process by intentionally overdepositing the template. Figure 3.1.40 shows the current vs. time graph for this overdeposition. The two plots on the graph both represent the same electrodeposition, but the $1 \mathrm{~s}$ plot represents current measurements taken at $1 \mathrm{~s}$ intervals and the $10 \mathrm{~s}$ plot represents measurements taken at $10 \mathrm{~s}$ intervals.

The rapid current increase until $17 \mathrm{~s}$ (the red dashed 'Nucleation' line) shows that the electrodeposition primarily forms new nuclei (as discussed in Section 1.2.5.2.1), then the decrease in current from $17 \mathrm{~s}$ shows that it enters a stage where those nickel nuclei firstly grow and then agglomerate (also refer to Section 1.2.5.2.1). Figure 3.1.41 shows the estimated volume of nickel that has been produced over time, based on the current measurements shown in Figure 3.1.40. A horizontal line at $3.26 \times 10^{-5} \mathrm{~cm}^{3}$ on Figure 3.1.41 represents the volume of the holes in the template as calculated in Table 2.2.4. The red dashed line at $450 \mathrm{~s}$ represents the point where the volume of nickel was equal to the 
Jonathan Tailby

volume of the holes in the template; further electrodeposition caused the nickel deposits to overflow the template. A vertical line at $450 \mathrm{~s}$ is also shown on Figure 3.1.40, the current had been expected to show a more dramatic change from this point, as described in the publication on growing nanowires in a template by Motoyama [127].

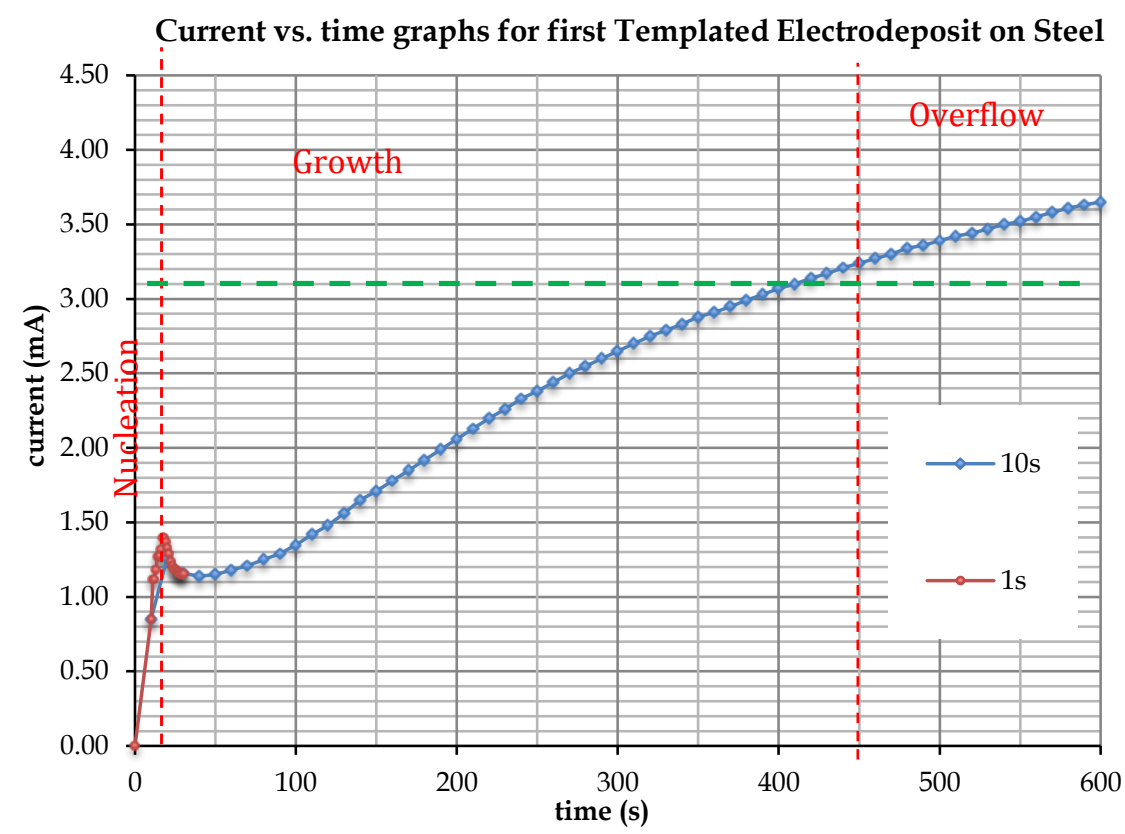

Figure 3.1.40: Current vs. time graphs for templated electrodeposits on steel at -1.2 V.

The blue line shows a deposition which overflowed the template, an electron micrograph of which is shown Figure 3.1.44.

Estimated Volume of Nickel vs. Time during Electrodeposition

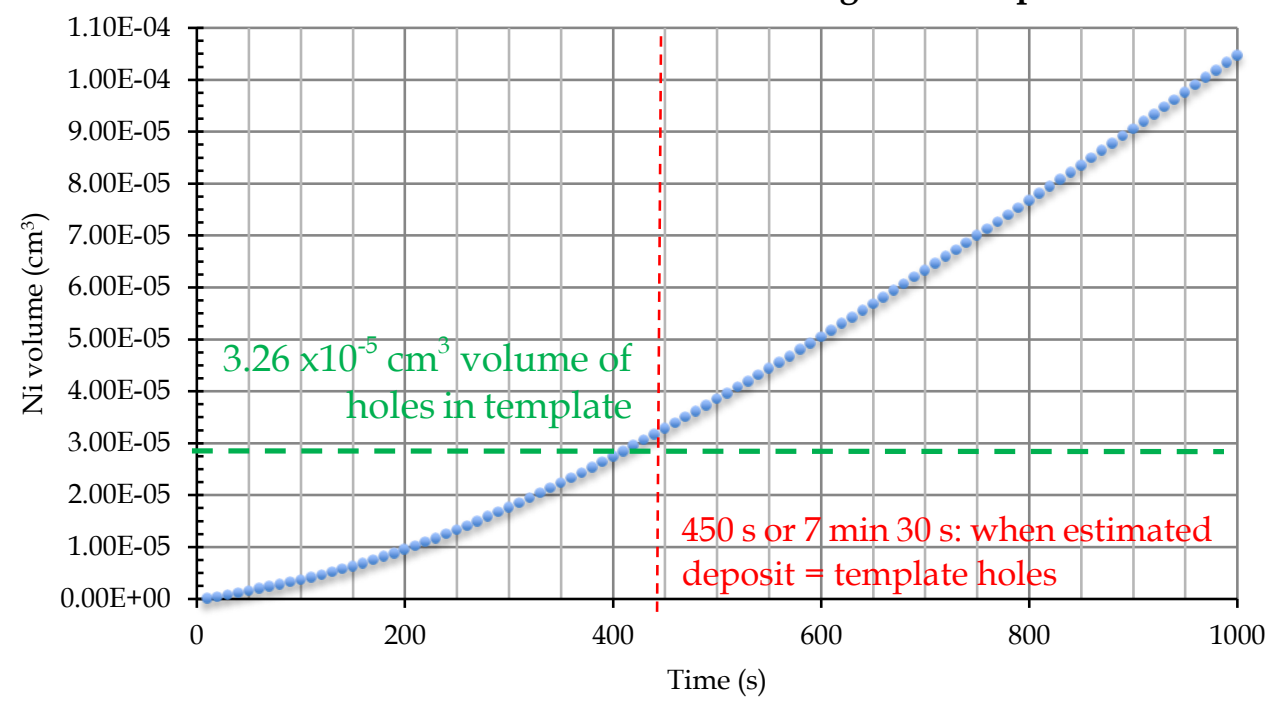

Figure 3.1.41: Estimated volume of nickel vs. time during electrodeposition on templated steel for the same deposition shown in Figure 3.1.40. 
Figure 3.1.42 shows the current vs. time graph from Figure 3.1.40 repeated with the addition of a green line showing the predicted current after $450 \mathrm{~s}$. This prediction is based on a publication by Motoyama [127] about growing nanowires in a template.

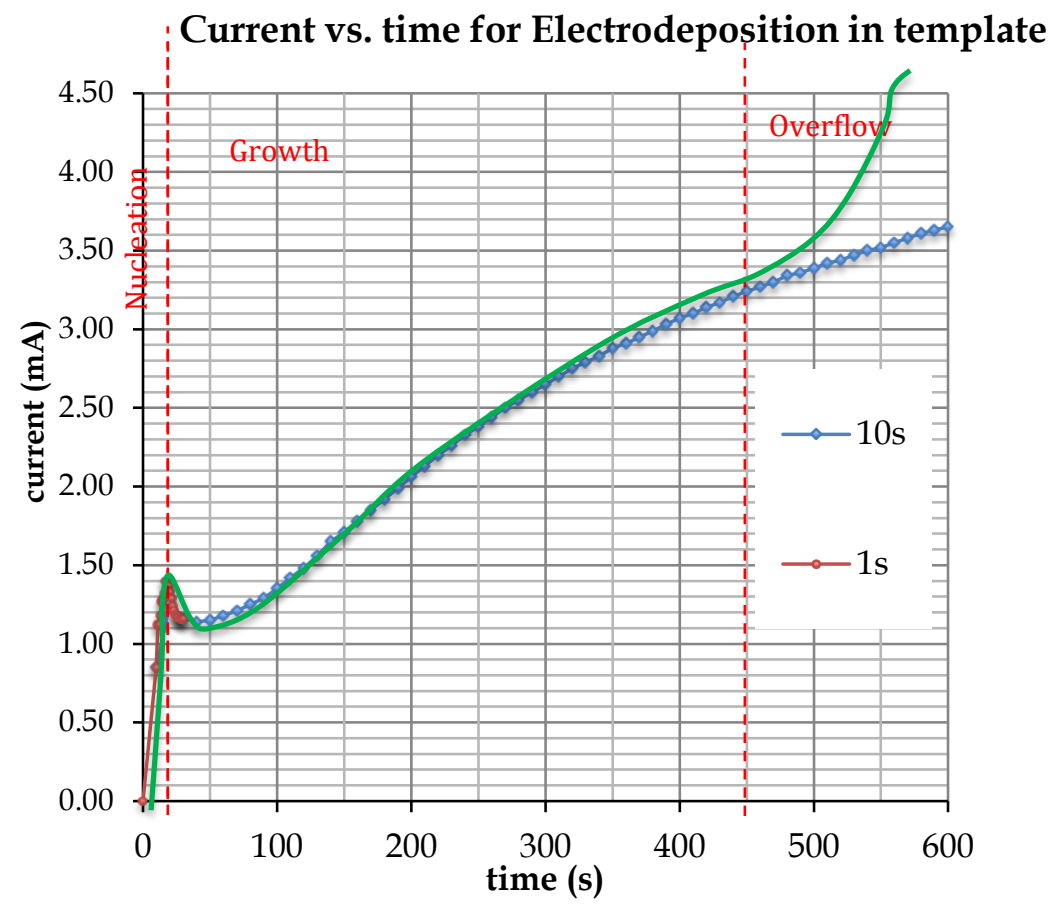

Figure 3.1.42: Predicted electric current for electrodeposition in a template that overflows then forms a continuous layer.

The prediction based on Motoyama [127] is marked in green.

Figure 3.1.43 shows a series of optical micrographs that follow the sequence in the production of a templated electrodeposit. Figure 3.1.43a shows the template before electrodeposition, the dark region around each hole is the tapering sides of the hole as the hole gets narrower towards the base. The holes are $10 \mu \mathrm{m}$ in diameter and aligned in a square array with $20 \mu \mathrm{m}$ from centre to centre. Figure $3.1 .43 \mathrm{~b}$ shows the template after electrodeposition. The holes now contain nickel deposits. The image shows the edge of the deposited region so the left half has deposits beginning to overflow the template and the right half has empty holes. The final product is shown in Figure 3.1.43c. The template has been dissolved by submerging the whole sample in acetone, leaving the free-standing nickel deposits. Figure $3.1 .43 \mathrm{c}$ is 
Jonathan Tailby

focused at the level of the shorter deposits so both the lower substrate and the taller deposits are out of focus.

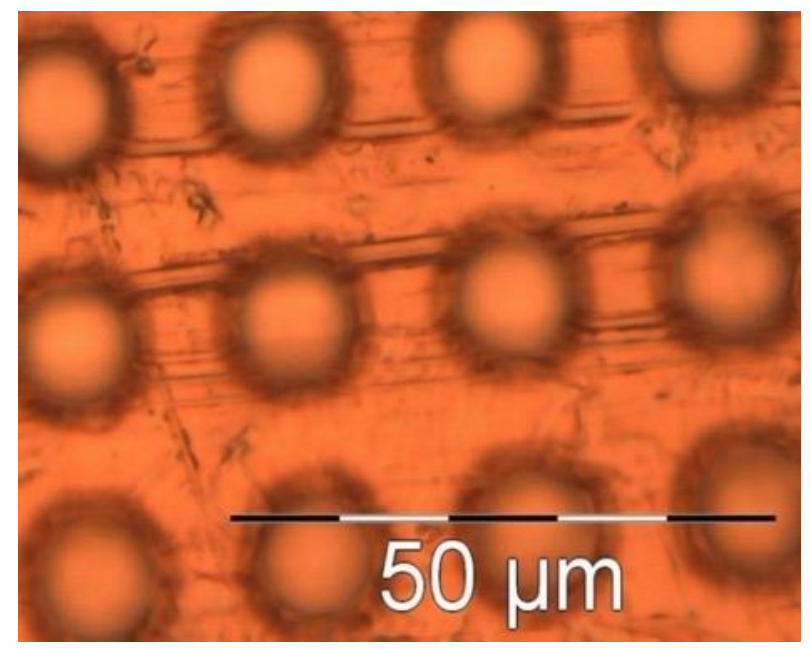

Figure 3.1.43a: The photoresist template before electrodeposition.

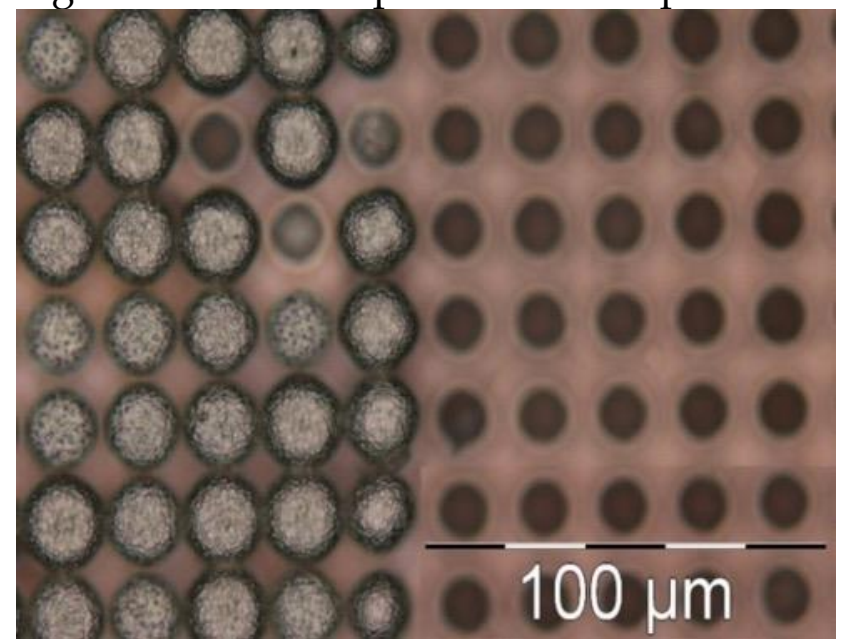

Figure 3.1.43b: The photoresist template at the edge of the deposition area after electrodeposition. The photo resist on the left side of the image contains electrodeposited nickel.

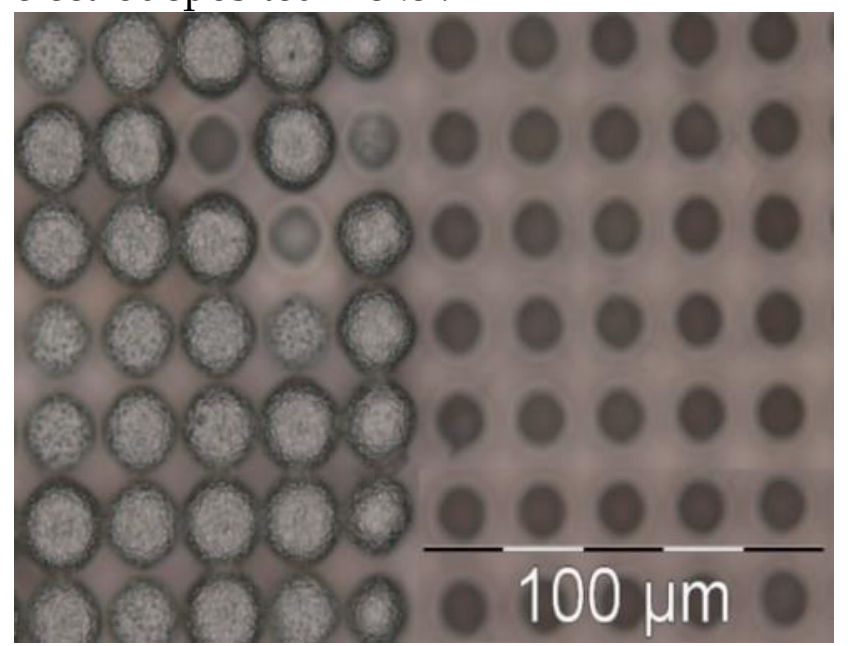

Figure 3.1.43c: Nickel Deposits after the template has been dissolved.

Figure 3.1.43: Series of optical micrographs showing the sequence of steps from template to electrodeposits. 
The electron micrograph in Figure 3.1.44 shows that the overflowed deposits have joined up to make a layer. That layer peeled off when the photoresist template dissolved. Complete nickel layers on steel and palladium had already shown a tendency to delaminate when they were thick enough to be clearly visible to the eye, indicating low adherence between the substrate and electrodeposit. This peeling is probably increased by acetone under the layer lifting it off or the partially dissolved template delaminating and lifting the electrodeposit The acetone must have penetrated beneath the nickel layer to dissolve the template.

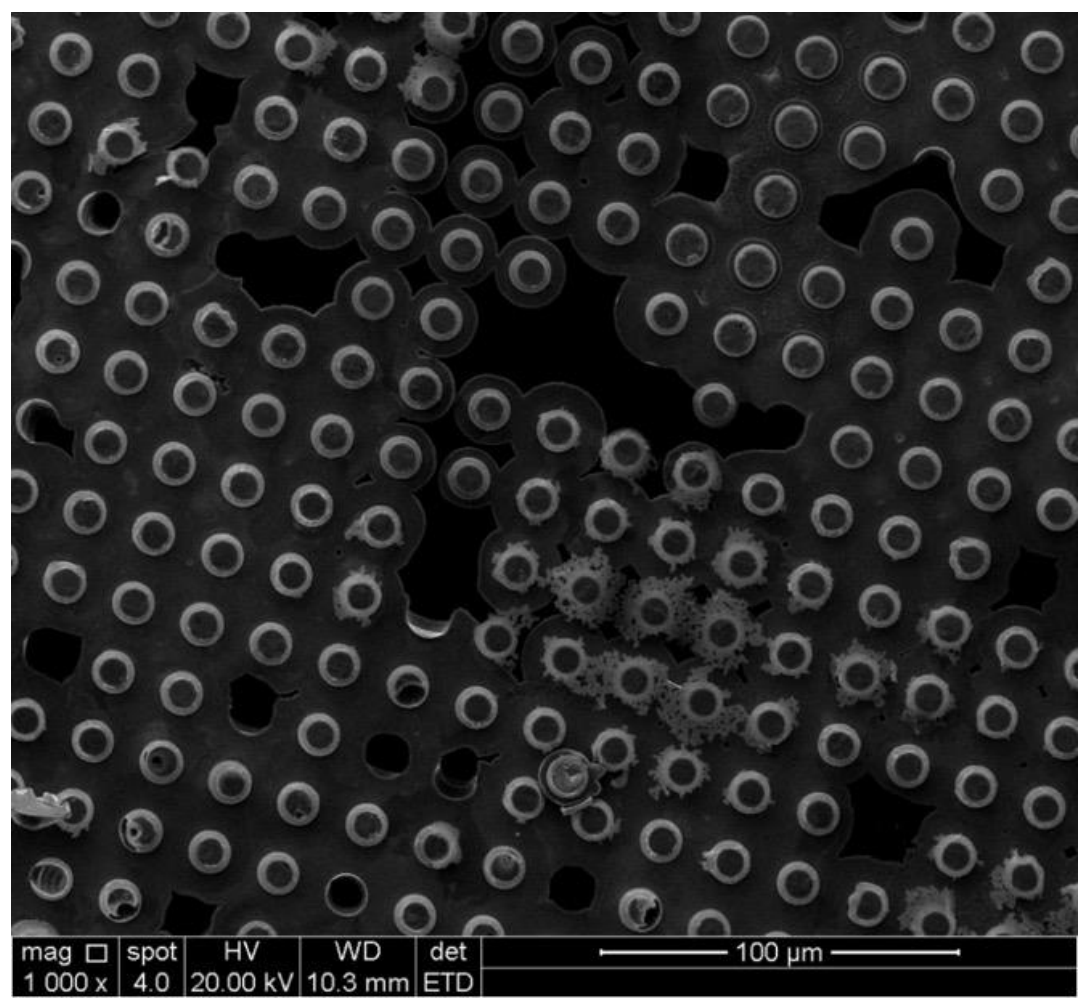

Figure 3.1.44: Electron micrograph of the over deposited nickel layer, the side which was formerly attached to the steel substrate.

(the deposit detached when the template was dissolved)

The desired product is isolated units of nickel, this required deposition to be stopped before reaching the overflow stage. The first experiment that overdeposited the template became a precedent that set the upper time limit for subsequent electrodepositions. A more typical current graph is shown in Figure 3.1.45. Deposition current started similarly to Figure 3.1.40, but it was stopped before it could form a continuous layer. The graph of current has the 
Jonathan Tailby

same sharp early peak that declined then increased more slowly. The more rapid increase in current after $300 \mathrm{~s}$ for the sample drawn in red was interpreted as the deposition beginning to overflow the template so the deposition was terminated.

Figure 3.1.46 shows an electron micrograph of the sample labelled 'typical template deposition' in Figure 3.1.45 which reveals uneven deposition, some of which has overflowed the template to the point of allowing deposits to join to their nearest neighbours. Of the small deposits, most appear to have failed to fill the template, but some show evidence of being the remains of larger deposits which broke off after deposition was ended.

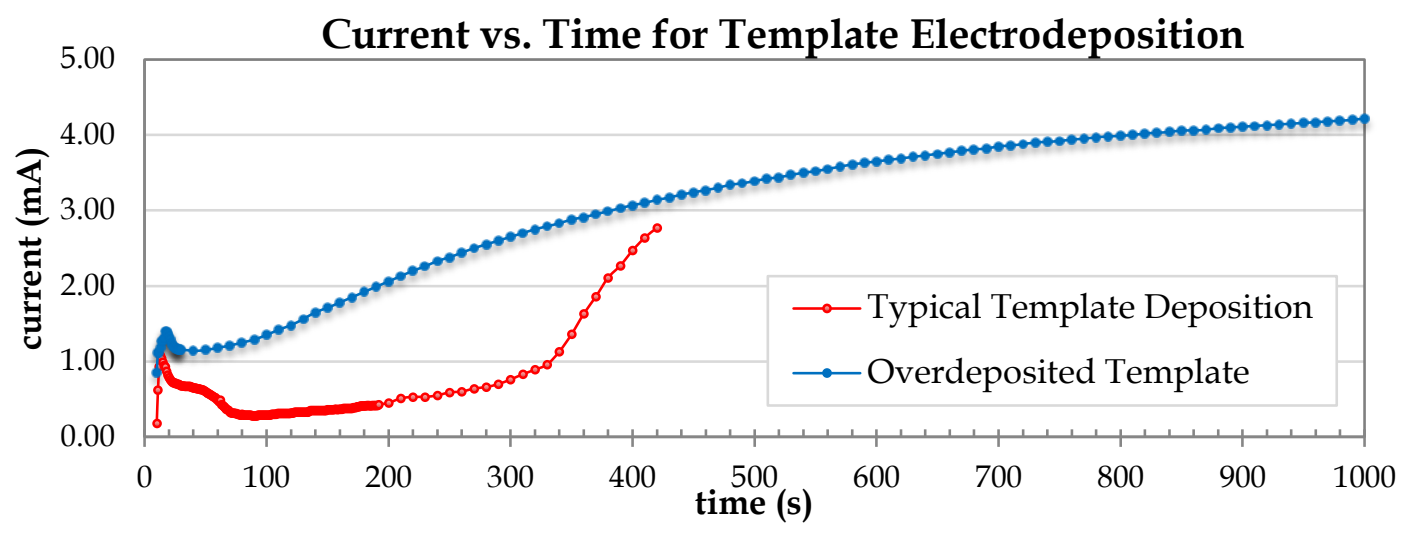

Figure 3.1.45: Current vs. time graphs for templated electrodeposits on steel. The blue shows a deposition which overflowed the template, the same one as Figure 3.1.46. The red shows a later deposition which was stopped before reaching the overflow point, shown in Figure 3.1.46.

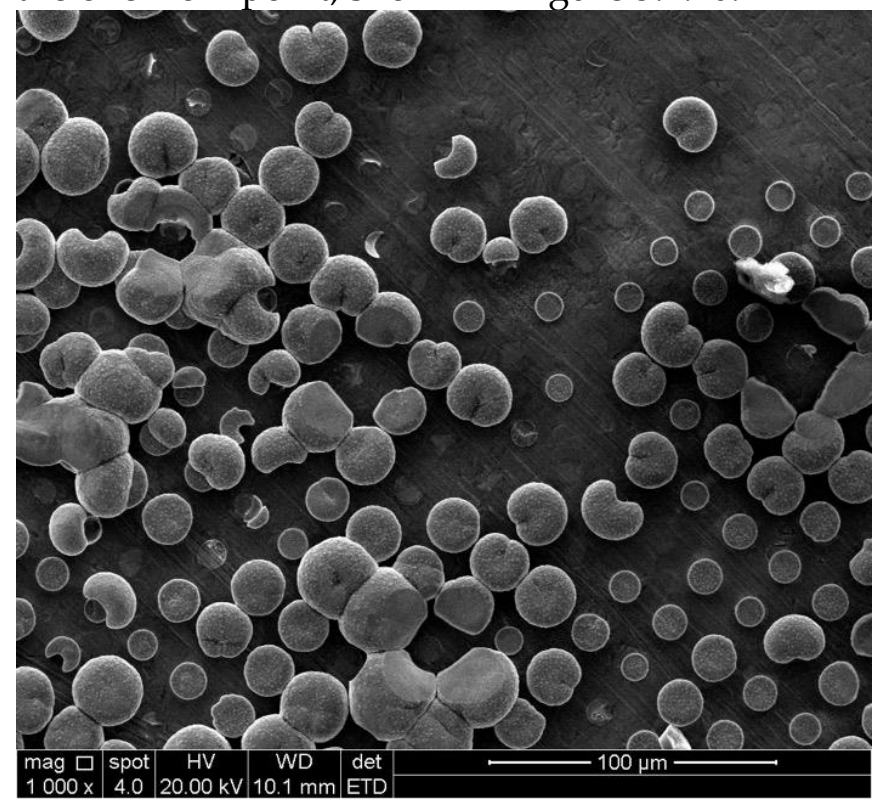

Figure 3.1.46: Back scatter electron micrograph of the electrodeposition sample labelled 'Typical Template Deposition' in Figure 3.1.45. 
Figure 3.1.47 and Figure 3.1.48 show a successful deposit with even and regular nickel depositions. The process that formed this successful deposition began with two failed attempts. The deposition experiment was set up, but no current was produced when the voltage was initiated. The experimental set up was examined and no errors were found, but the voltage again produced no current. The experiment was then disassembled and the substrate removed. When reassembled, the successful deposition proceeded with a high initial current. This high initial current indicates extremely rapid nucleation, which would lead to the even deposition. The high current was thought to have been caused by the extended exposure to the solution before electrodeposition having improved the surface conditions of the metal substrate, perhaps by acidic reduction although the electrodeposition solution was only mildly acidic. A delay between preparation and voltage initiation was introduced as standard procedure.

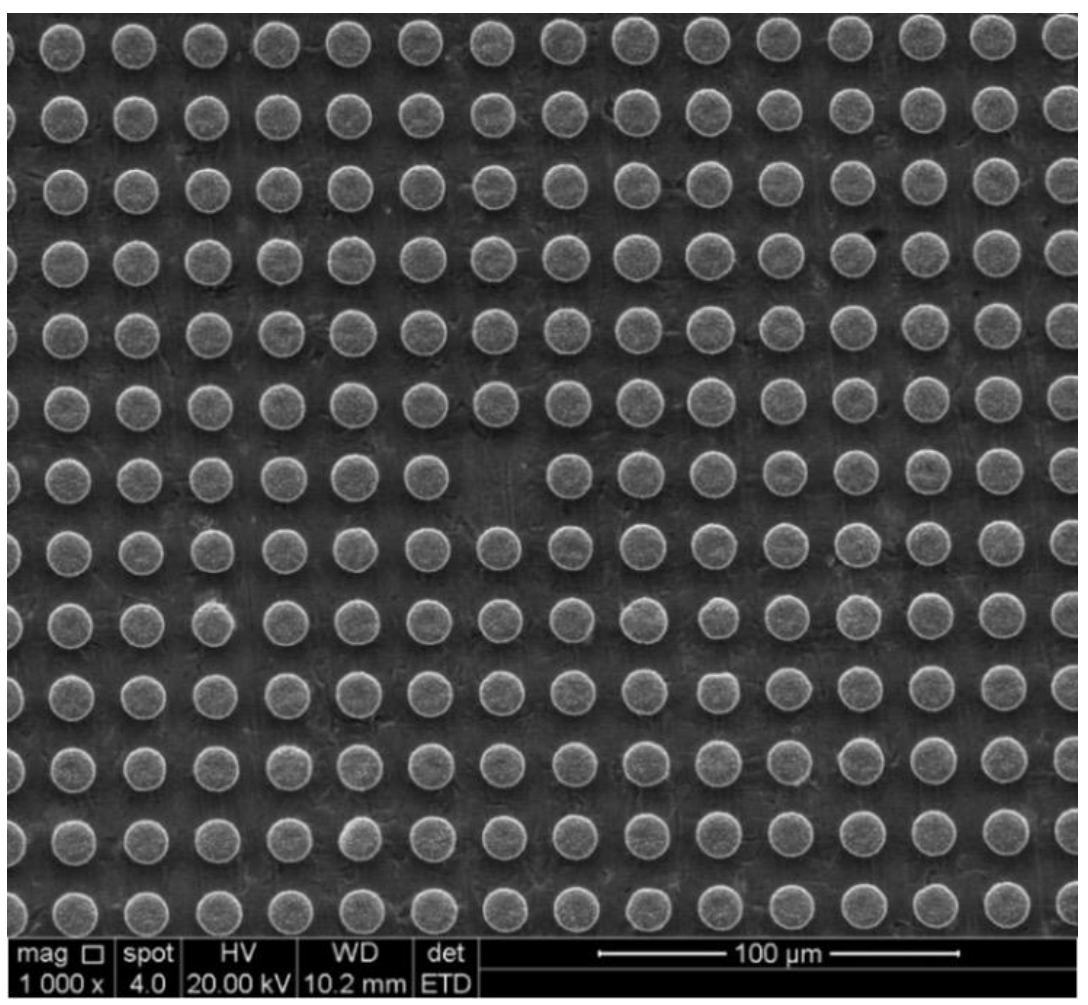

Figure 3.1.47: Electron micrograph of a successful templated electrodeposition on steel showing how the deposits were consistent in height and diameter.

Faults of even a single deposit such as the absence shown in this image were rare, except at the edges of the deposition area, as shown in Figure 3.1.49.

At the edge of the deposition circle for the sample shown in Figure 3.1.47 some of the nickel deposits were knocked over, as shown in Figure 3.1.49 and Figure 
Jonathan Tailby

3.1.50. These deposits were probably dislodged by the $\mathrm{O}$ ring at the bottom of the deposition glass as it was removed. The toppled deposits give a side view confirming the tapering shape and allowing the height to be estimated at $3 \mu \mathrm{m}$.

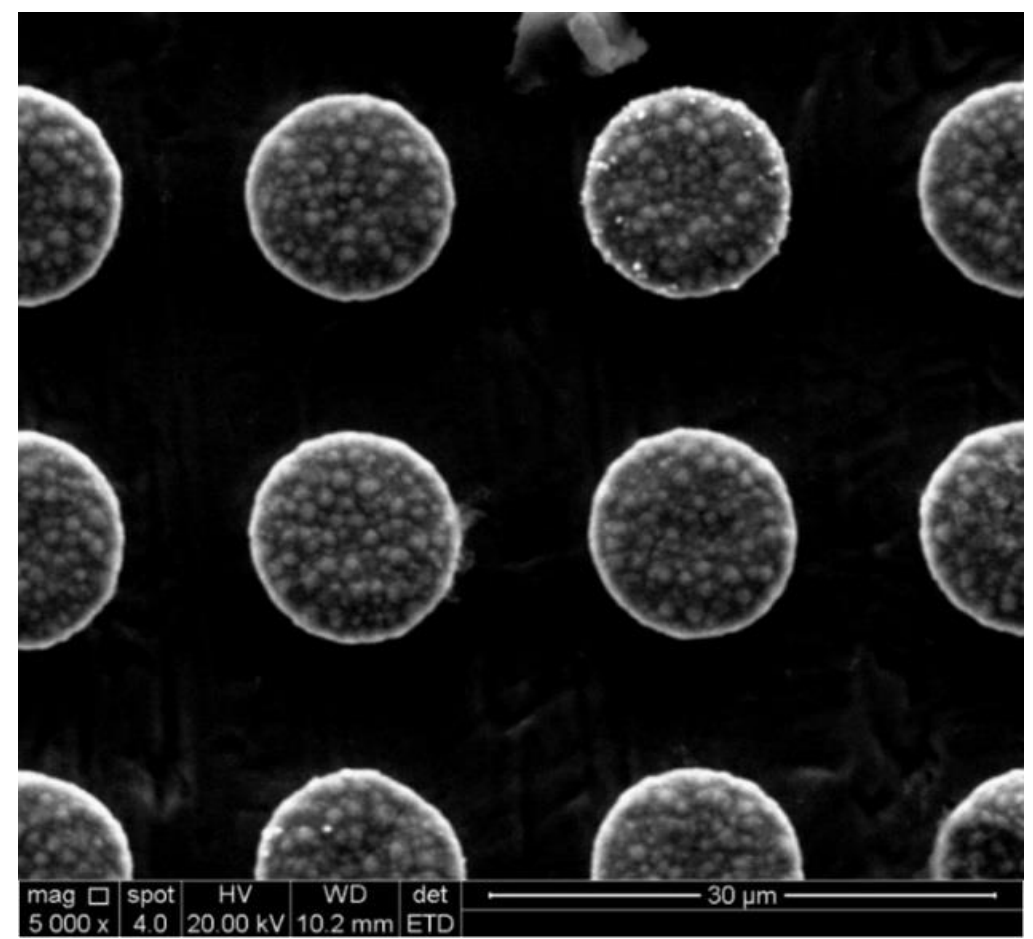

Figure 3.1.48: Higher magnification electron micrograph of a successful deposition showing that they were composed of smaller units aggregated together.

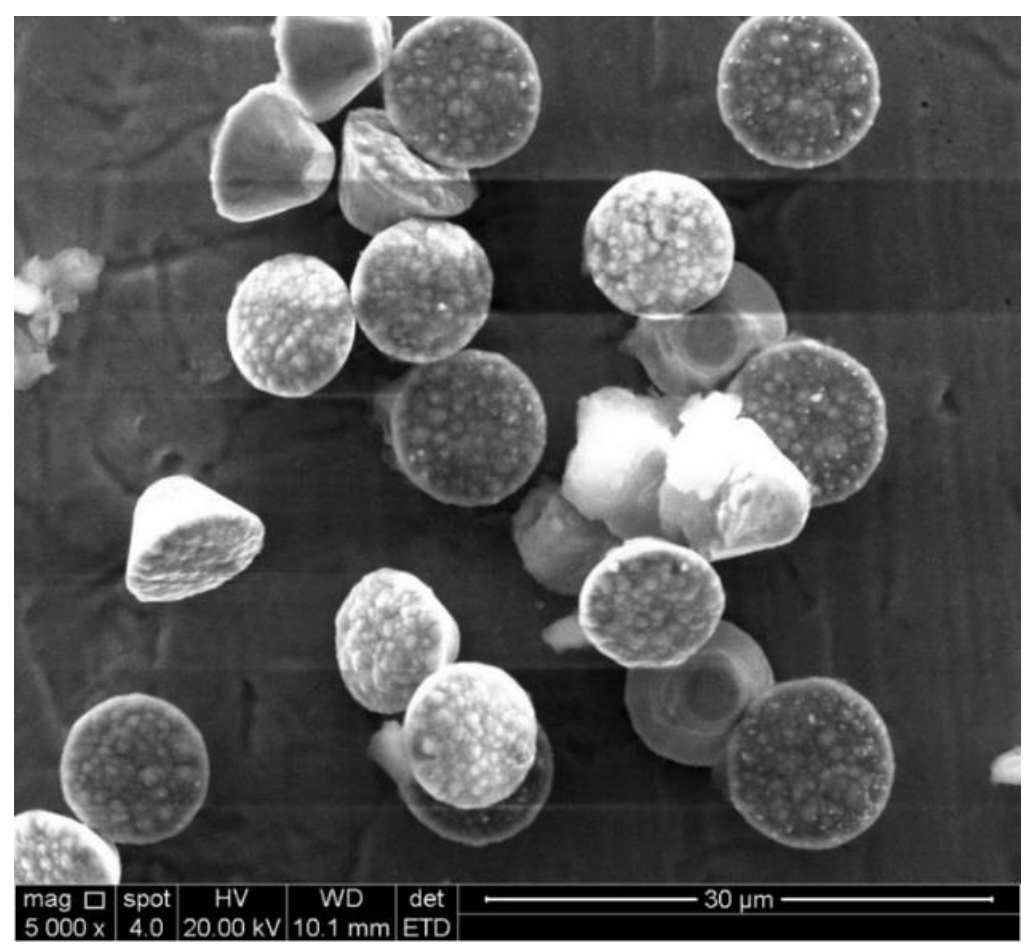

Figure 3.1.49: Electron micrograph shows the edge of the same deposition sample as Figure 3.1.47.

An enlargement from the top left of this image is shown in Figure 3.1.50. 


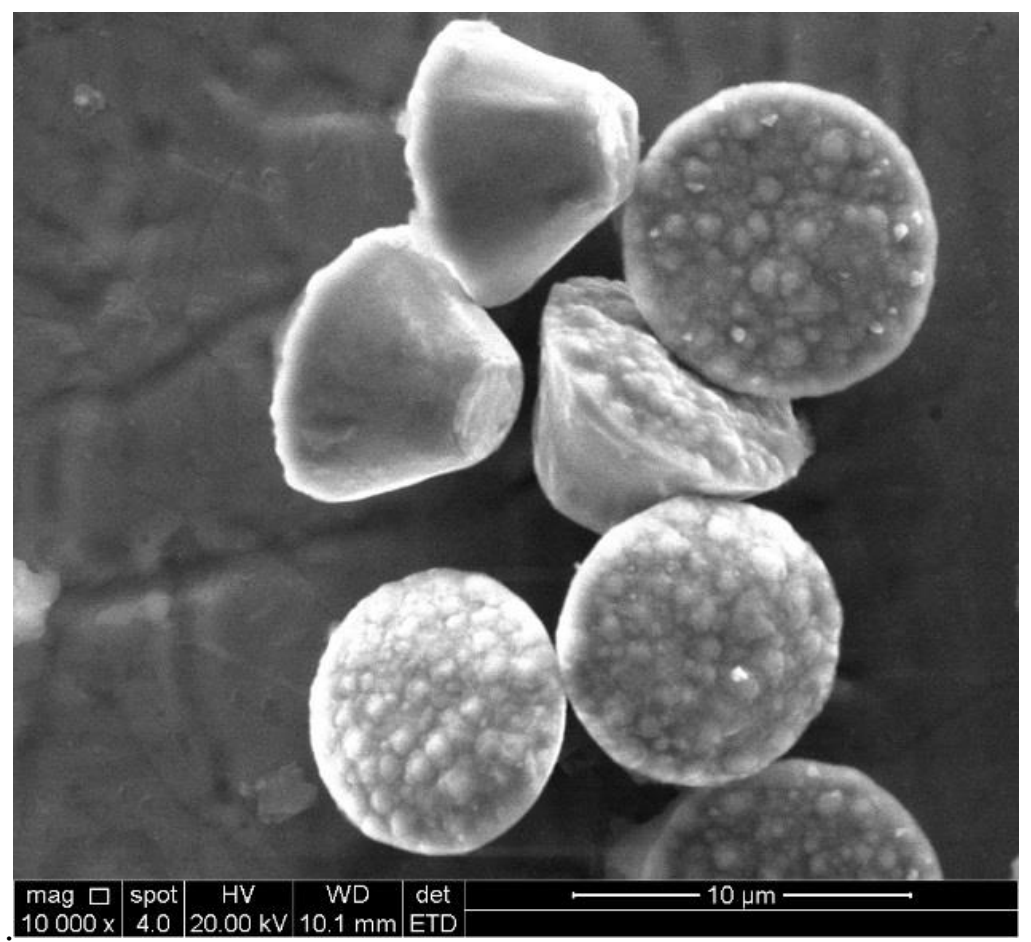

Figure 3.1.50: Enlargement of the dislodged deposits shown in Figure 3.1.49

The effect of a delay on activating voltage was first tested using bare steel, shown in Figure 3.1.51. The depositions are labelled with their number in order and the number of minutes between set up and voltage initiation, hold. These results showed that initial electric current increased and became stable more quickly with each subsequent deposition without regard to the presence or absence of a delay. The fourth and fifth depositions are almost identical which shows that the order of deposition had more effect than the length of delay. It had already been established as standard procedure to discard a preparatory deposition before commencing a deposition experiment, so this result confirms the value of that practice. 


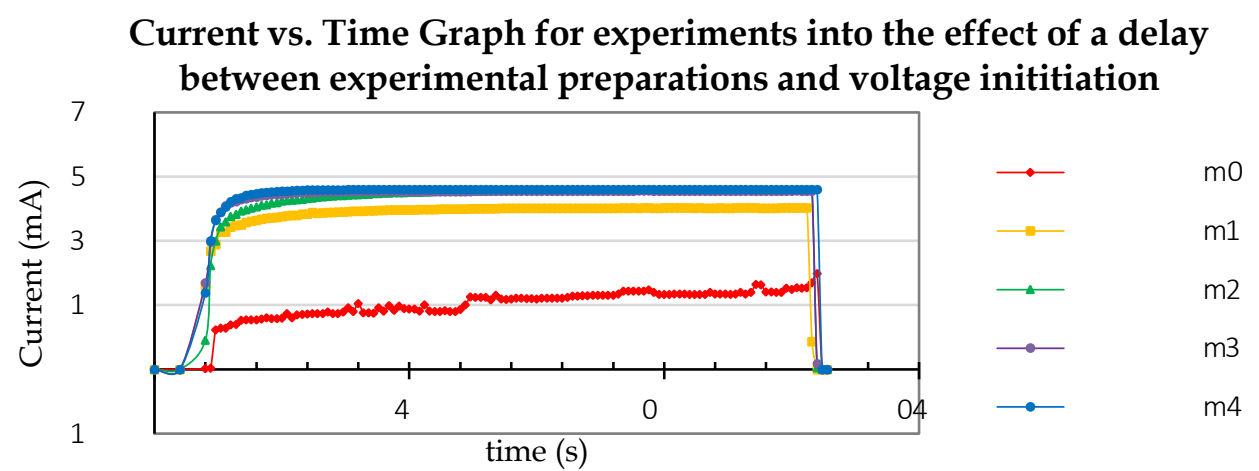

Figure 3.1.51: Current vs. time graphs for electrodeposition on bare steel at -1.2 V showing the effect of a long delay between preparing the deposition and activating the voltage.

Deposition number gives the order in which the samples were deposited, hold is the number of minutes between set up and initiation of the voltage. The order of deposition had more effect on the deposition current than the hold length.

The templated electrodeposits on palladium were not as even as the templated electrodeposits on steel. This was caused by the templates on the palladium substrate being less even. The microfabrication laboratory that prepared the templates acknowledged this unevenness and examined the process to ascertain and resolve the cause of these uneven templates. During that time the uneven templates were experimented on to predict how the characteristics of the template would affect the deposition.

Figure 3.1.52 to Figure 3.1.54 show three photographs of the same $12 \mathrm{~mm} \times 12$ $\mathrm{mm}$ palladium foil substrate: first of the template before deposition; second of the template with the deposit inside and third of the substrate and deposit after acetone had washed off the template. The template shows the typical brown corners of the spin coated templates which are thicker towards the corners. An irregular pattern is visible in the membrane, which, when overlaid with the circular deposition area, matches the white shapes visible in the photographs taken after deposition microscopy and confirms these whitish areas to be composed of the deposits.

Figure 3.1.55 and Figure 3.1.56 show a pair of optical micrographs that focus on the area inside the green circle on Figure 3.1.53 and Figure 3.1.54. This point was not a feature of interest during the predeposition microscopy, therefore no micrograph of the area before deposition is available. It is clear that the 
central region with nickel deposits in Figure 3.1.55 and Figure 3.1.56 is the tip of the deposit in Figure 3.1.53 and Figure 3.1.54.

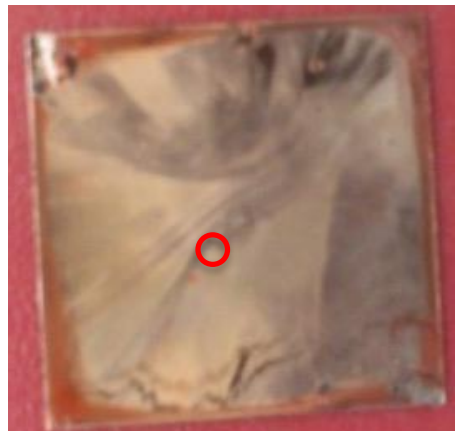

Figure 3.1.52 Predeposition template

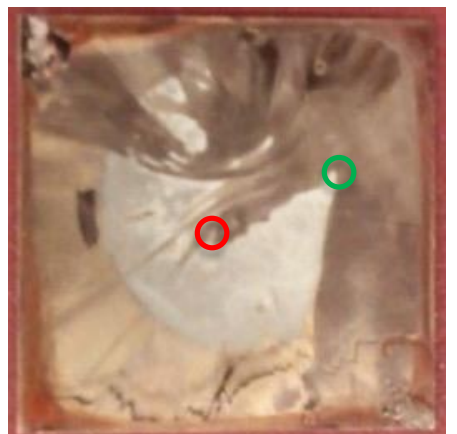

Figure 3.1.53 Template and deposit after deposition

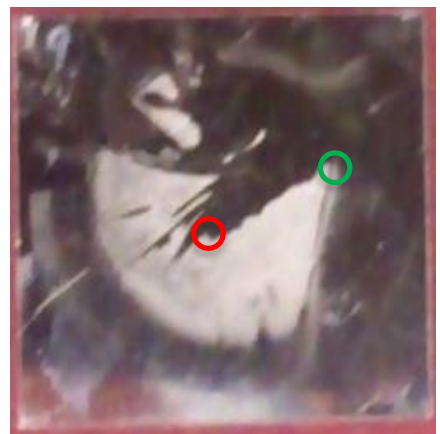

Figure 3.1.54 Nickel deposit after acetone wash has removed the template.

Figure 3.1.52 to Figure 3.1.54: comparisons for a single template from the template before deposition through to the nickel deposit on $12 \mathrm{~mm} \times 12 \mathrm{~mm}$ palladium foil square.

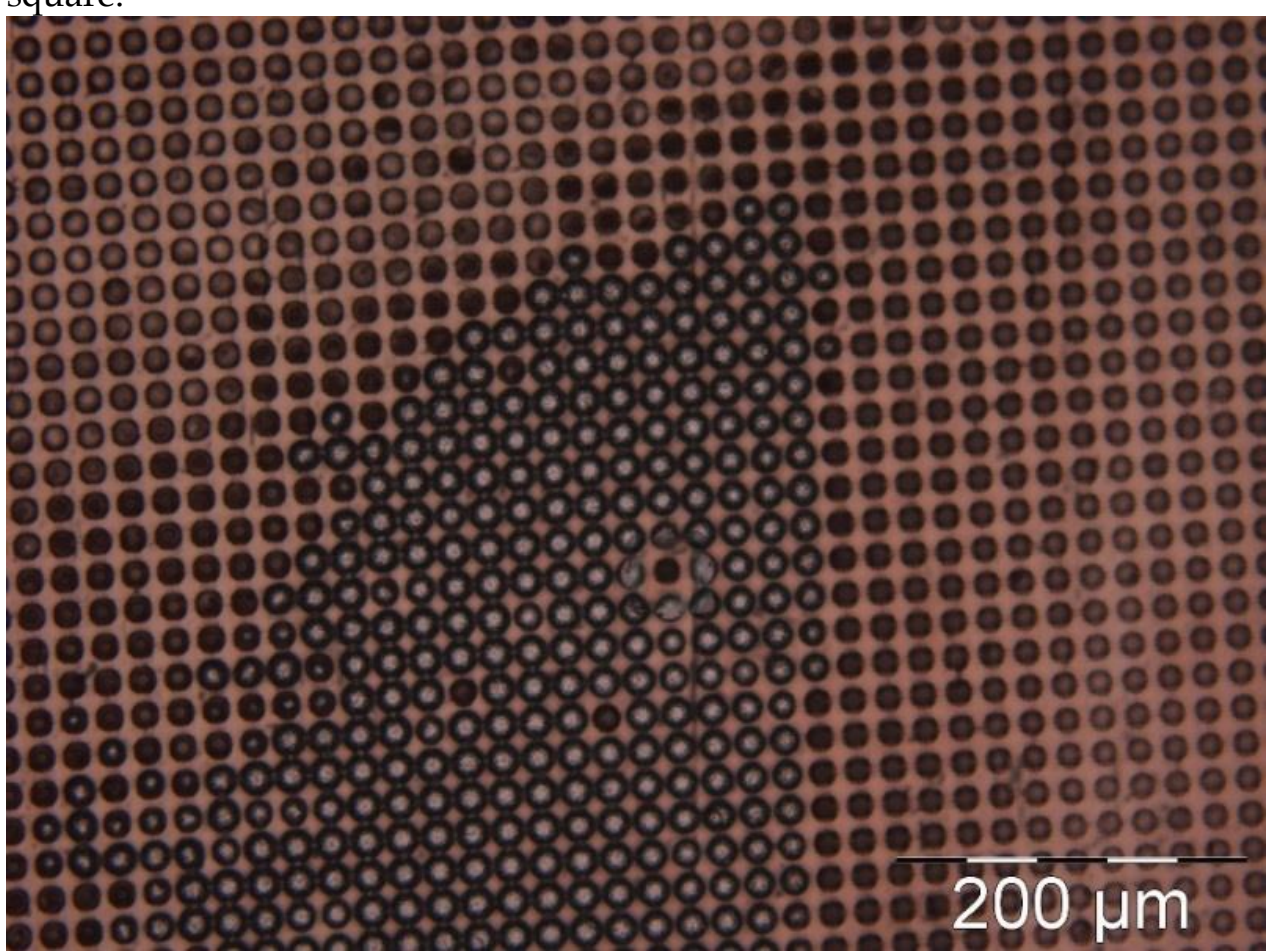

Figure 3.1.55: Optical micrograph of the top right point of the main deposit, indicated by the green circle in Figure 3.1.53. Image taken after electrodeposition but before the acetone wash. 


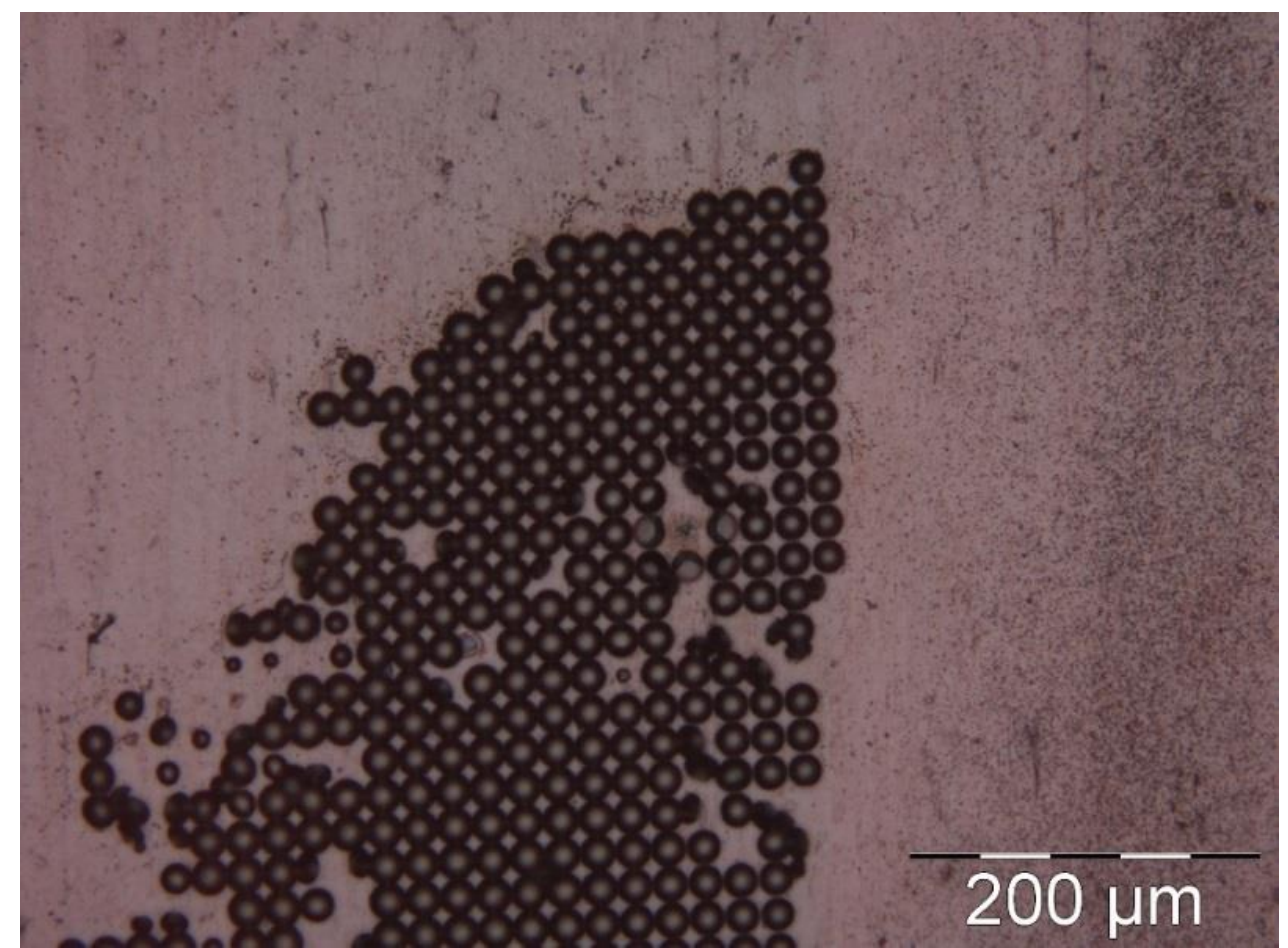

Figure 3.1.56: Optical micrograph of the top right point of the main deposit, indicated by the green circle in Figure 3.1.54. Image taken after the acetone wash had removed the template.

Figure 3.1.57 and Figure 3.1.58 show optical micrographs of the middle of the sample shown in Figure 3.1.52 to Figure 3.1.54, as indicated by the red circle. The images are taken of approximately the same location, such that some common features can be identified. The large triangle in the top left corner of Figure 3.1.57 where the pattern is almost invisible at this magnification is visible in Figure 3.1.58 as the best deposited region in the field of view. With the information from Figure 3.1.52 to Figure 3.1.58 it is evident that the outcome of electrodeposition is influenced by the quality of the template and some predictions about the final deposits could be made with appropriate observation of the template and sufficient understanding of the processes involved. 


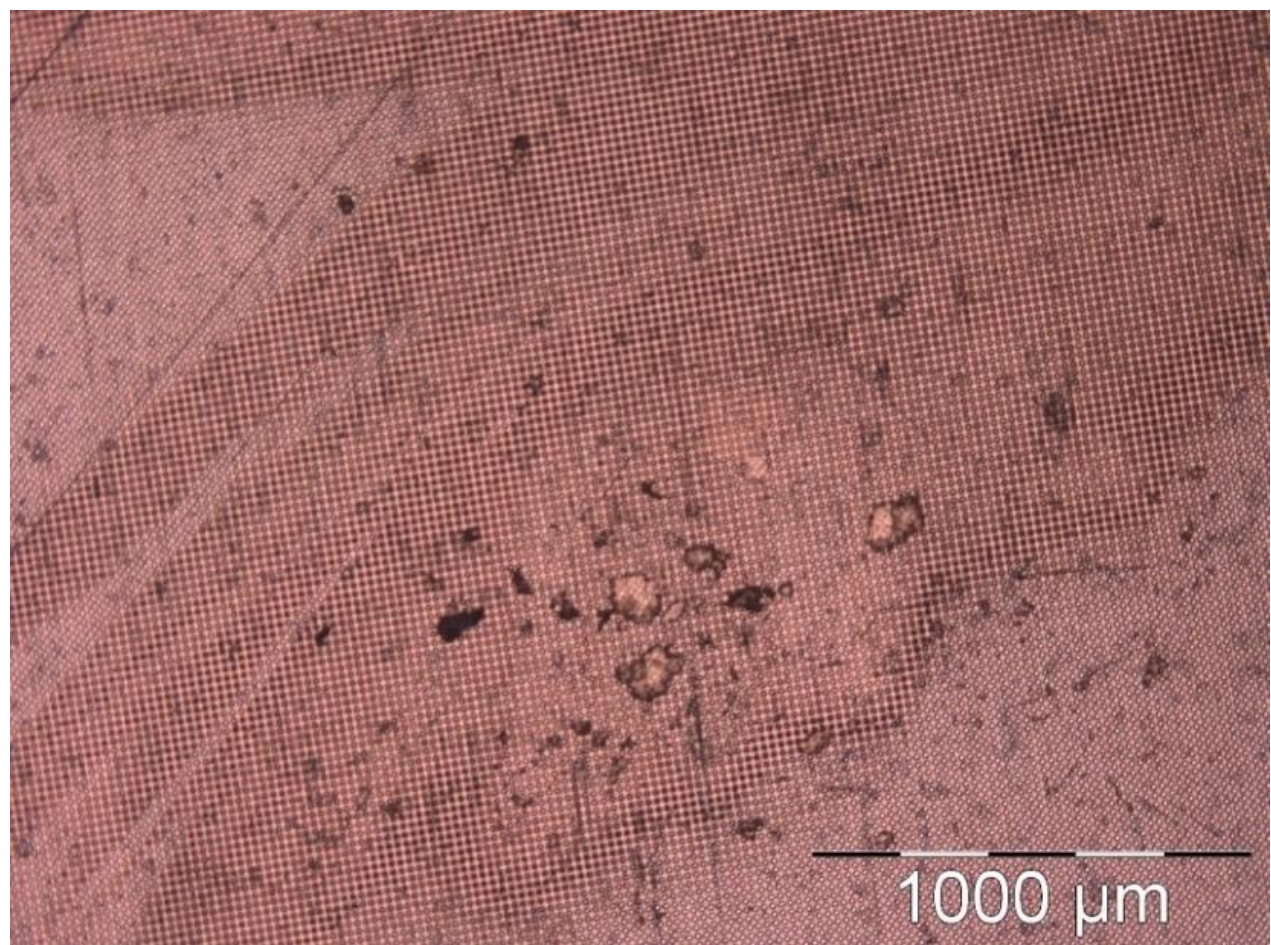

Figure 3.1.57: Optical micrograph of the middle of the sample shown in Figure 3.1.52. Template before deposition.

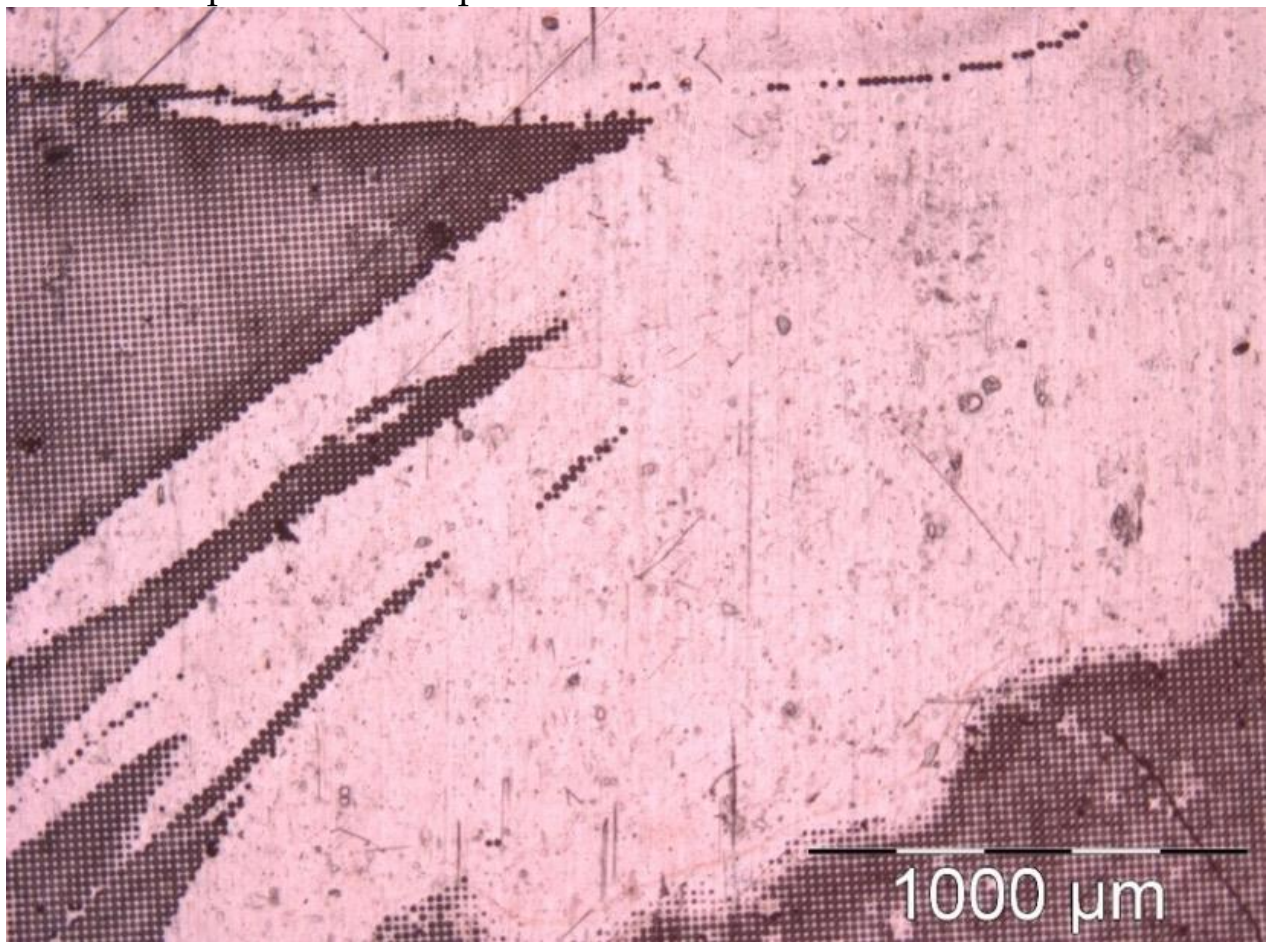

Figure 3.1.58: Optical micrograph of the middle of the sample shown in Figure 3.1.54. After acetone wash. This shows the same region as Figure 3.1.57.

\subsubsection{Templated Electrodeposition on Tubular Substrates}

By the time the templated electrodeposition had been developed the gas reaction process had progressed to using tubular catalyst supports. The 
Jonathan Tailby

templated electrodeposition process was transferred to work on the secondary insert tubes. The first templated secondary inserts had very uneven templates which produced uneven deposits. Figure 3.1.59 to Figure 3.1.61 show SEM images of a templated secondary insert that demonstrate the variability of the pattern. This secondary insert was tested as an ammonia decomposition catalyst as discussed in Sections 2.2.10 and 3.2.2.4.3. The process for templating these tubes was improved, as discussed in Section 2.2.4.3, but it could not be applied to the tertiary inserts because the additional tubes for sweep gas meant the tertiary inserts could not rotate under the UV exposure lamp. Further illustrative SEM images of the templated secondary insert are shown in Appendix 5.2.6. Overall the pattern of the deposition on the alternate secondary insert was not as uniform as the insert shown in Figure 3.1.59 to Figure 3.1.61.

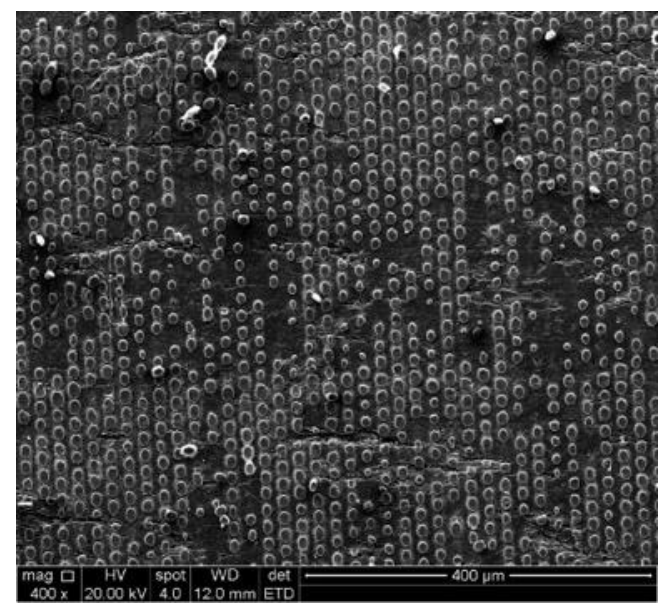

Figure 3.1.59: 400x magnification secondary electron micrograph of templated electrodeposits on a secondary insert.

This image shows successfully templated electrodeposition over most of the field of view.

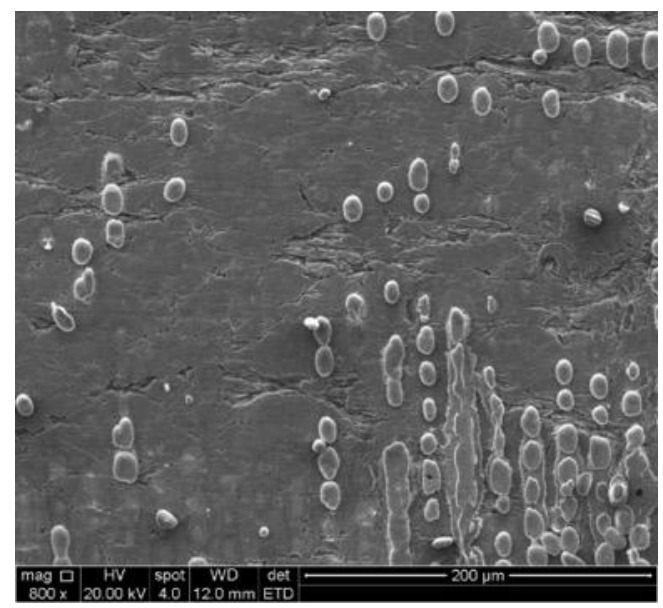

Figure 3.1.60: 800x magnification secondary electron micrograph of templated electrodeposits on a secondary insert.

This image shows significant quantities of absent deposits and elongation of the electrodeposits that are present, until some are beginning to agglomerate. 
Figure 3.1.59 to Figure 3.1.61: SEM images of a secondary insert for the gas reactor with templated electrodeposits showing a mix of successfully and unsuccessfully templated regions.

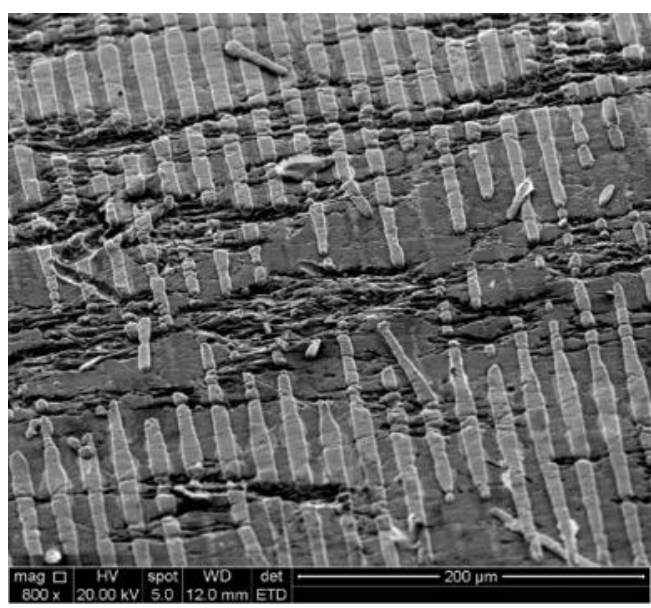

Figure 3.1.61: 800x magnification secondary electron micrograph of templated electrodeposits on a secondary insert.

This image shows linear deposits with some absences.

\subsubsection{SUEX membranes for Templated Electrodeposition}

The method described in Section 2.2.4.3 was unsuitable to be adapted to the tertiary inserts. For this reason, experiments were undertaken on SUEX films that can be templated before being applied to the substrate. Preliminary experiments with SUEX membranes on flat steel substrates produced less than $0.001 \mathrm{~mA}$ and there were no visible traces of nickel on the substrate when viewed under the microscope. The two most likely causes for this result were that the thicker membrane had not been fully penetrated by the lithography which prevented contact between substrate and solution, or that the greater aspect ratio of the holes in the thicker SUEX membrane caused the nickel concentration at the deposition surface to replenish too slowly.

The templated electrodeposition was shown to work on both steel and palladium foils and on steel tubes, however it was seen that the current method for templating tubular substrates could not be applied to the tertiary inserts. Two more methods of catalyst preparation were tested that could be adapted more easily to the tertiary inserts. These last methods involved using preshaped nickel to create catalysts that would protrude more into the reaction space which was predicted to improve their effectiveness as catalysts. 
Jonathan Tailby

\subsubsection{Preshaped Nickel}

\subsubsection{Nickel Mesh}

The nickel mesh was examined by XRD and by SEM with EDS. Figure 3.1.62 to Figure 3.1.65 show electron micrographs of the nickel mesh before and after ammonia decomposition experiments. Figure 3.1.62 shows a secondary electron image of the nickel mesh before the ammonia decomposition experiments. Figure 3.1.63 shows the same location in a back-scatter electron image. Figure 3.1.64 and Figure 3.1.65 show the nickel mesh after the ammonia decomposition experiments in secondary electron and back scatter electron images.

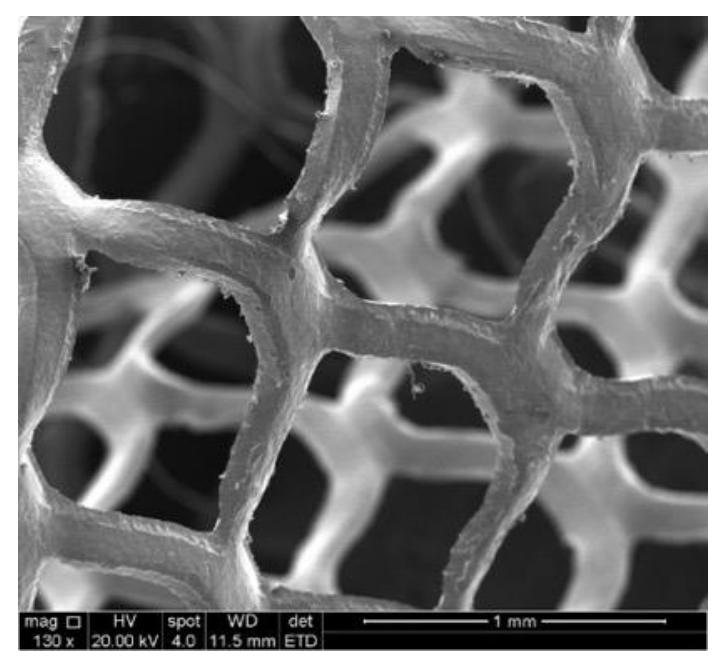

Figure 3.1.62: Secondary electron image of nickel mesh before ammonia decomposition experiments.

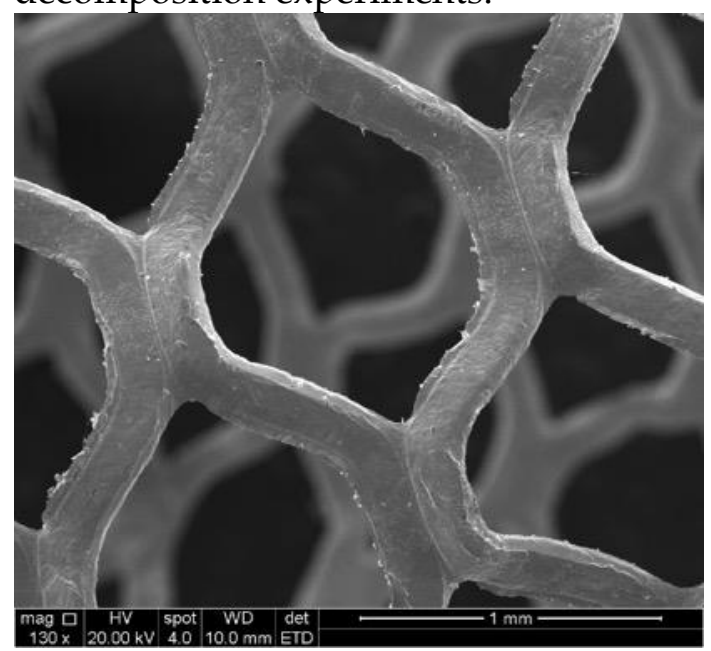

Figure 3.1.64: Secondary electron image of nickel mesh after ammonia decomposition experiments.

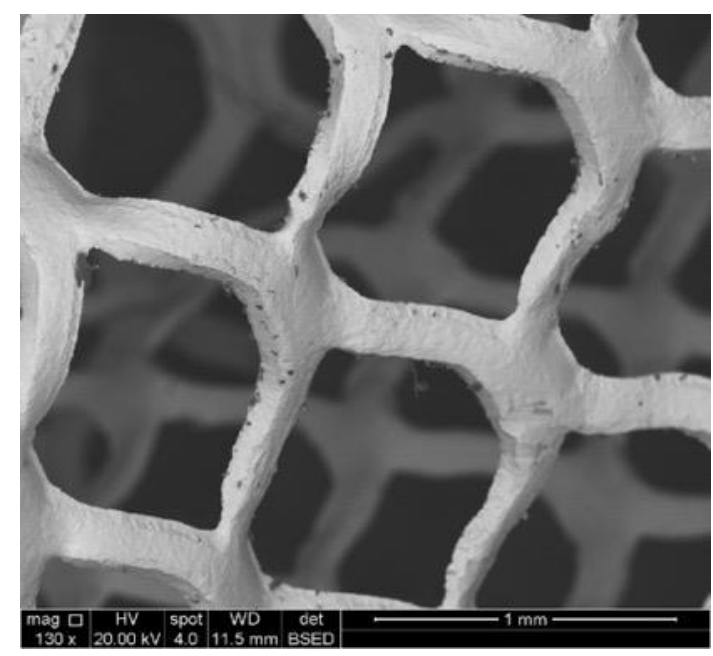

Figure 3.1.63: Back scatter electron image of nickel mesh before ammonia decomposition experiments.

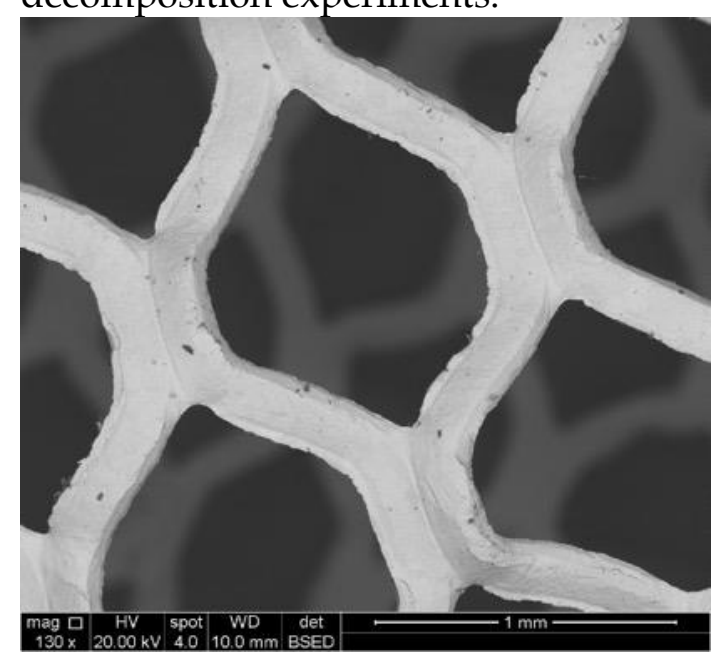

Figure 3.1.65: Back scatter electron image of nickel mesh after ammonia decomposition experiments. 
The EDS spectra that correspond to the locations shown in Figure 3.1.62 and Figure 3.1.65 are shown in Figure 3.1.66 and Figure 3.1.67. The quantities of various elements present in the nickel mesh before and after ammonia decomposition experiments was calculated from the EDS data as shown in Table 3.1.8. The decrease in the non-nickel elements is difficult to see in the EDS spectra, but the decrease in carbon and oxygen after ammonia decomposition is clearer in the calculated quantities. The calculated atom percentage of oxygen was below the average error both before and after, decreasing from approximately half the error to nearer a quarter. The atom percentage of nickel increased from an average of $61.4 \%$ to an average of 83.5 $\%$ with an overall average error of only $2.2 \%$. The high error in the small measurements was expected in these standardless semiquantitative analyses.

Decreases in carbon and oxygen on the nickel mesh during ammonia decomposition would have resulted from side reactions with the hydrogen produced by the decomposition. Carbon reacts with hydrogen to form methane and leaves the reactor; the oxygen will form water.

This decrease in oxygen content supports the expectation that a high temperature treatment with ammonia will reduce nickel oxide, removing a surface oxide to leave the nickel metal exposed, which is reported in the literature to be a more active catalyst than the oxide [19]. 


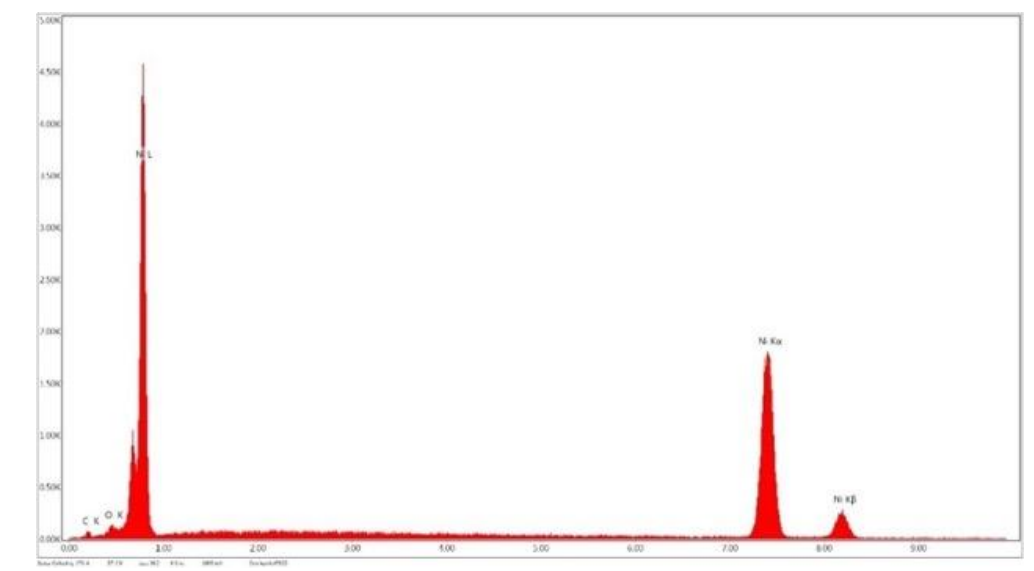

Figure 3.1.66: EDS spectrum of nickel mesh before ammonia decomposition experiments.

Nickel peak heights are $\mathrm{L} 4.6 \mathrm{~K}, \mathrm{~K}_{\mathrm{\alpha}} 1.8 \mathrm{~K}$ and $\mathrm{K}_{\beta} 0.29 \mathrm{~K}$. Other visible peaks are Carbon $0.06 \mathrm{~K}$ and Oxygen $0.14 \mathrm{~K}$.

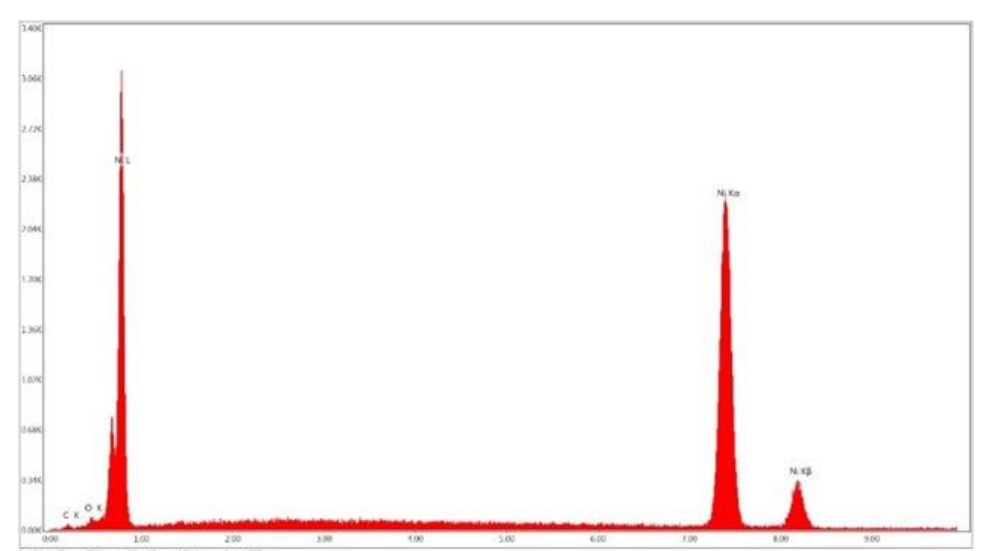

Figure 3.1.67: EDS spectrum of nickel mesh after ammonia decomposition experiments.

Identified Nickel peaks are $\mathrm{L} 3.1 \mathrm{~K}, \mathrm{~K}_{\alpha} 2.3 \mathrm{~K}$ and $\mathrm{K}_{\beta} 0.35 \mathrm{~K}$. Other visible peaks are Carbon $\mathrm{K} 0.05 \mathrm{~K}$ and Oxygen $\mathrm{K} 0.1 \mathrm{~K}$.

Table 3.1.8: Quantification of the elements present in the nickel mesh. These are for the same two samples before and after testing for catalytic activity in ammonia decomposition.

\begin{tabular}{|l|l|l|l|}
\hline Element & $\begin{array}{l}\text { Weight } \\
\%\end{array}$ & $\begin{array}{l}\text { Atomic } \\
\%\end{array}$ & $\begin{array}{l}\text { Error } \\
\%\end{array}$ \\
\hline \multicolumn{4}{|l|}{ Precatalysis } \\
\hline C K & 7.00 & 25.16 & 16.61 \\
\hline O K & 3.31 & 8.93 & 12.47 \\
\hline Ni K & 89.68 & 65.91 & 2.23 \\
\hline \multicolumn{4}{|l}{ Precatalysis } \\
\hline C K & 9.94 & 32.28 & 14.52 \\
\hline O K & 4.44 & 10.82 & 12.22 \\
\hline Ni K & 85.62 & 56.9 & 2.26 \\
\hline
\end{tabular}

\begin{tabular}{|l|l|l|l|}
\hline Element & $\begin{array}{l}\text { Weight } \\
\%\end{array}$ & $\begin{array}{l}\text { Atomic } \\
\%\end{array}$ & $\begin{array}{l}\text { Error } \\
\%\end{array}$ \\
\hline Post catalysis \\
\hline C K & 2.63 & 11.38 & 22.04 \\
\hline O K & 1.00 & 3.25 & 16.96 \\
\hline Ni K & 96.37 & 85.37 & 2.14 \\
\hline Post catalysis \\
\hline C & 3.51 & 14.67 & 18.64 \\
\hline O & 1.20 & 3.77 & 16.52 \\
\hline Ni & 95.29 & 81.56 & 2.14 \\
\hline
\end{tabular}


When the Scherrer equation was applied to the XRD diffractograms, the crystallite size of the unused mesh was calculated to be $999 \AA$. When the Scherrer equation was applied to a diffractogram of the mesh that had been used in the catalyst tests, the results indicated that the crystallites were larger than the $0.2 \mu \mathrm{m}$ limit of the equation. This can be explained by high temperature aggregation of the crystallites removing small crystallites and creating more large crystallites.

A photograph of the nickel mesh insert is shown in Figure 2.2.14. The nickel mesh covered approximately $23 \mathrm{~mm}$ length of the steel insert with another 6 $\mathrm{mm}$ of twisted mesh at the front end. The nickel foam was $28 \mathrm{~mm}$ long. It can be seen in Figure 2.2.13 that the area covered by the nickel electrodeposits was greater than the area covered by the nickel mesh.

\subsubsection{Nickel Foam}

A second prefabricated form of nickel was a nickel foam from Inco Advanced Technology materials (Dalian) Co. Lt, Taiwan. The nickel foam was assessed by both XRD and optical microscopy. Figure 3.1.68 and Figure 3.1.69 show two optical micrographs of the nickel foam, which show the three-dimensional network of interlinked nickel that leaves more of the enclosed volume empty than occupied, $90 \%$ of the volume was unoccupied in the supplied foam and $79 \%$ was unoccupied in the foam as used. Figure 3.1.70 shows an XRD trace for the nickel foam compared to a pattern for nickel. The only significant peaks in the trace match the two in the pattern which confirmed the foam as nickel metal with no significant quantity of other crystalline material. 


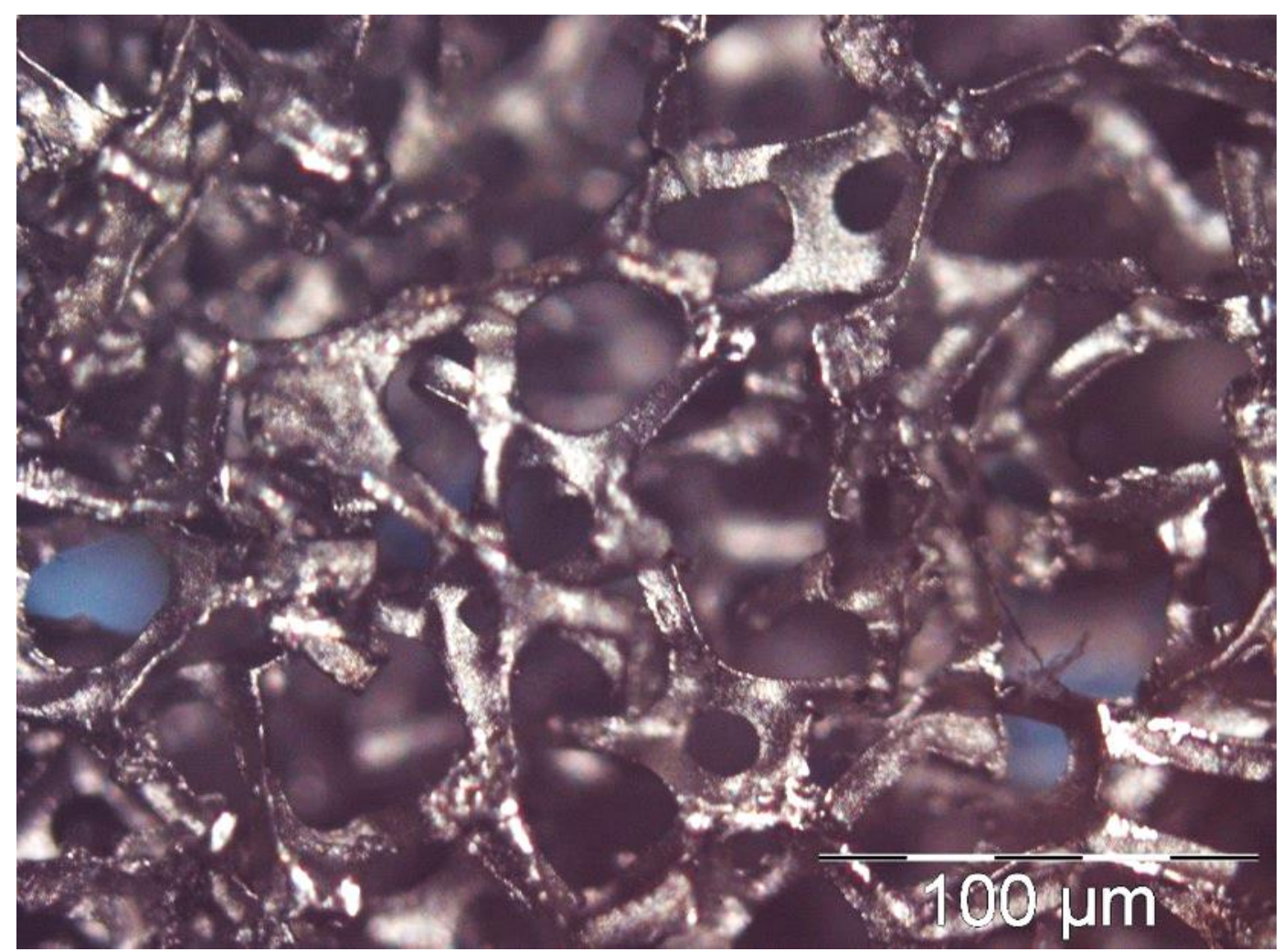

Figure 3.1.68: Optical micrograph of the nickel foam.

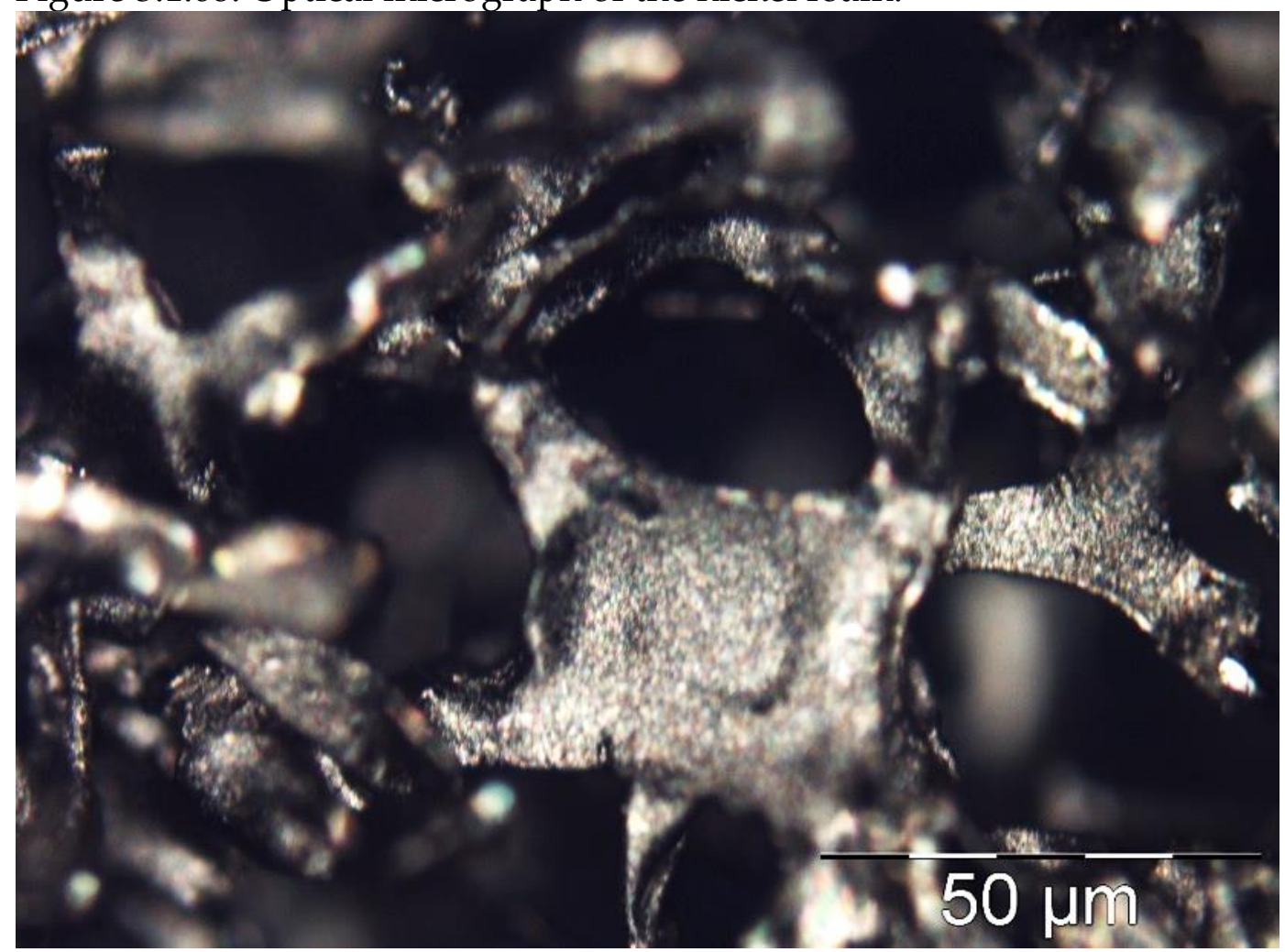

Figure 3.1.69: Optical micrograph of the nickel foam. 


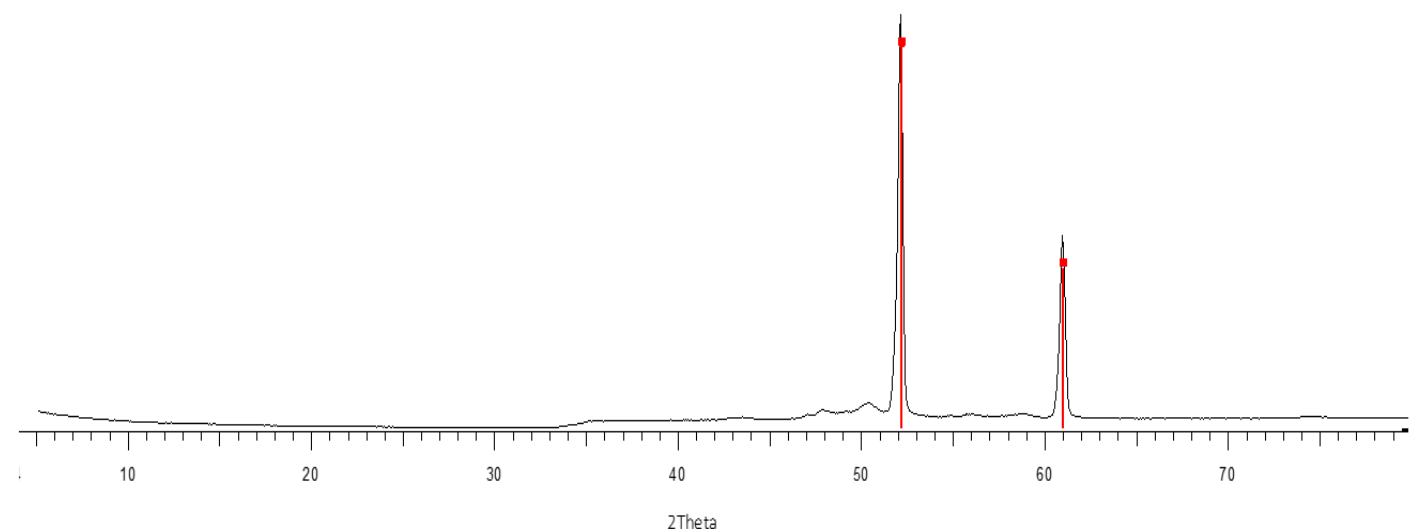

Nickel Foam

- Nickel, $\mathrm{Ni}$

Figure 3.1.70: This XRD trace of the nickel foam confirmed that it contains nickel and no significant quantity of any other crystalline phase.

Although the foam could be said to be occupying the entire volume of its length inside the reactor, it can be seen from Figure 3.1.68 and Figure 3.1.69 that the majority of that space is empty of nickel, however the complex network of nickel would have made it unlikely that an ammonia molecule would pass through the foam without making contact with the nickel.

Figure 3.1.70 shows the diffractogram for the nickel foam confirming that there are no other crystalline phases. The crystallite size calculated from this diffractogram with the Scherrer equation was $1230 \AA$. 
Jonathan Tailby

\subsection{Reaction Testing with Catalysts}

\subsubsection{Mass Spectrometry Results}

The first gas reactions were monitored by a mass spectrometer, as detailed in Sections 2.1.6 and 2.2.11. Before beginning ammonia decomposition testing, palladium foils were tested for hydrogen permeation in the Sputnik reactor detailed in Section 2.2.9.1. A mass spectrometry record from one of these experiments is shown in

Figure 3.2.1.

Mass Spectrometry Record for hydrogen permeation experiment
tate to

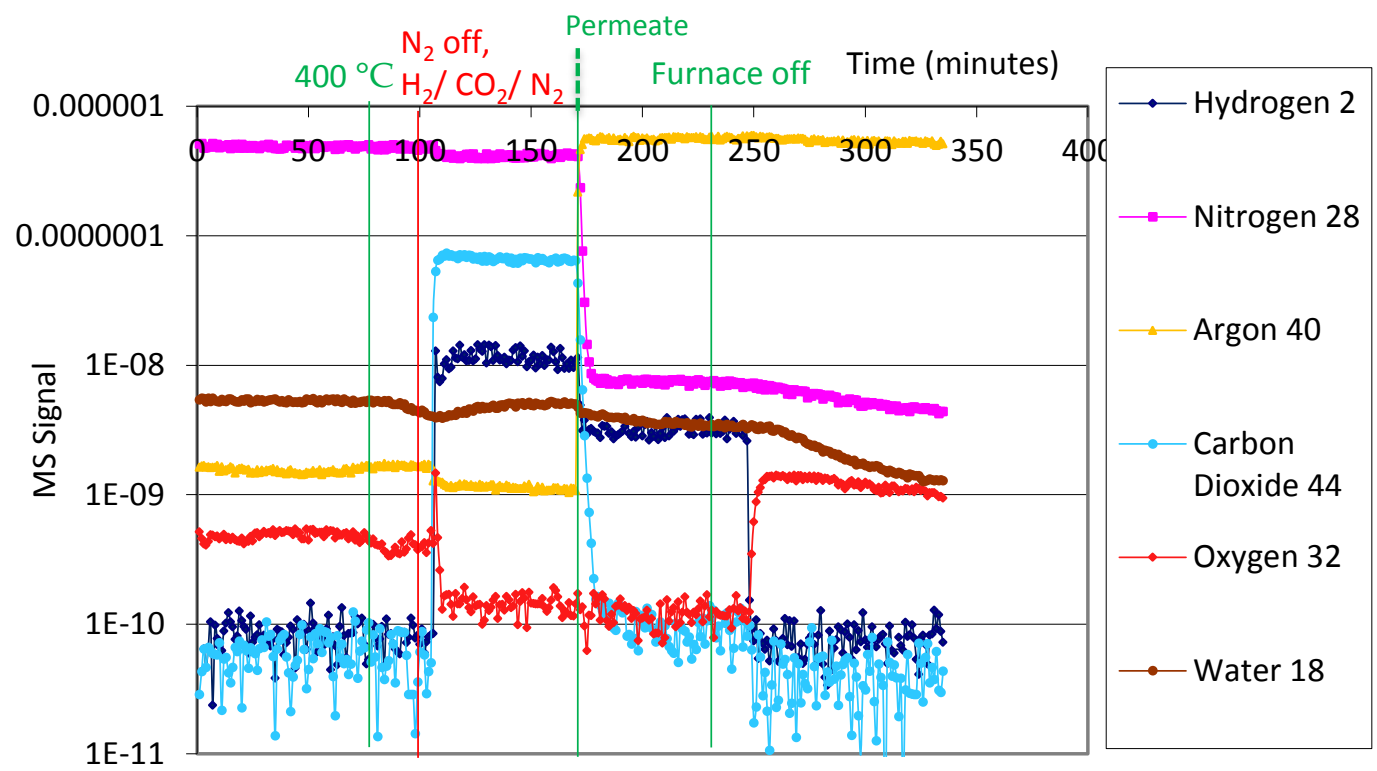

Figure 3.2.1: Mass spectrometry record for a hydrogen permeation experiment with the Sputnik reactor.

The palladium foil membrane had no nickel catalyst. The mass spectrometer initially sampled from the retentate gas, then switched to permeate at 170 minutes. Initially nitrogen gas was fed to the retentate side of the reactor, at 100 minutes this was changed to a mixture of hydrogen, nitrogen and carbon dioxide. An argon sweep gas was fed to the permeate side of the reactor throughout the experiment.

Initially nitrogen gas was fed into the reactor on the same side as the mass spectrometer was analysing. Under the nitrogen atmosphere, the reactor was heated to $400{ }^{\circ} \mathrm{C}$ over 80 minutes then held at $400{ }^{\circ} \mathrm{C}$. 
At 100 minutes the nitrogen gas was exchanged for a 10\% hydrogen /10\% carbon dioxide $/ 80 \%$ nitrogen mixture. At 170 minutes the valve that controlled which output gas was analysed by the mass spectrometer was switched to analyse the permeated hydrogen in an argon sweep gas. At 230 minutes the furnace was turned off, which caused the reactor temperature to decrease.

The presence of gases other than nitrogen before 100 minutes indicates that there were problems with sealing the two halves of the reactor. The oxygen must have been entering from outside the reactor at the point where the two halves meet. The argon could be crossing between the two halves because it was flowing on the permeate side of the reactor, and it was present in greater quantity than the oxygen, which would not be possible if it was only coming from the air.

The mass spectrometry record shows that hydrogen and carbon dioxide both significantly increased after the change in feed gas at 100 minutes, from levels where they could be dismissed as noise to levels that were clearly significant. At 170 minutes, when the mass spectrometer changed to sampling the permeate instead of the retentate, both nitrogen and carbon dioxide decreased significantly more than hydrogen indicating that the hydrogen was permeating through the palladium membrane, carbon dioxide returned to noise level and the level of nitrogen could suggest a leak. Argon increased significantly.

After hydrogen permeation had been confirmed ammonia decomposition experiments began, with the ammonia side of the membrane being analysed to investigate the potential for detecting a difference between catalysed and uncatalyzed ammonia decomposition.

Figure 3.2.2 shows the mass spectrometer record for ammonia decomposition with a palladium foil that had been electrodeposited in $0.01 \mathrm{~mol} \mathrm{~L}^{-1}$ electroplating solution at $-2.5 \mathrm{~V}$ for $20 \mathrm{~s}$ for the catalyst sample. Temperature 
Jonathan Tailby

increased from room temperature at approximately $4.75^{\circ} \mathrm{C} \mathrm{min}^{-1}$ to $400{ }^{\circ} \mathrm{C}$ in 80 minutes.

At 70 minutes oxygen decreased and carbon dioxide increased. If the starting room temperature was approximately $20^{\circ} \mathrm{C}$, at 70 minutes the reactor temperature would have been approximately $350{ }^{\circ} \mathrm{C}$, which is sufficient for most carbon-based materials to burn. This change in gas composition was interpreted as combustion of the gaskets that sealed around the metals parts inside the reaction vessel. Combustion would consume oxygen and produce carbon dioxide, which would cause the observed the observed changes. Because there is no other change in gas composition, particularly no influx of air that would have been visible by increased nitrogen and argon signals, the gaskets must have been damaged but not destroyed. This would be expected because the compressed fibres in the gaskets were not flammable, therefore only the binder would be expected to burn.

At 130 minutes the sampling valve was switched so the mass spectrometer analysed the permeate instead of the retentate, at this time every gas except argon decreased. The argon increased because it was the sweep gas on the permeate side. Ammonia was expected to decrease because it was the input gas on the retentate side, hydrogen and nitrogen were expected to decrease because these were the products of any ammonia decomposition that might have been occurring. That carbon dioxide decreased, suggests that the gaskets on the permeate side of the reactor were less damaged by combustion than the retentate side, perhaps having been protected by the argon flow. 


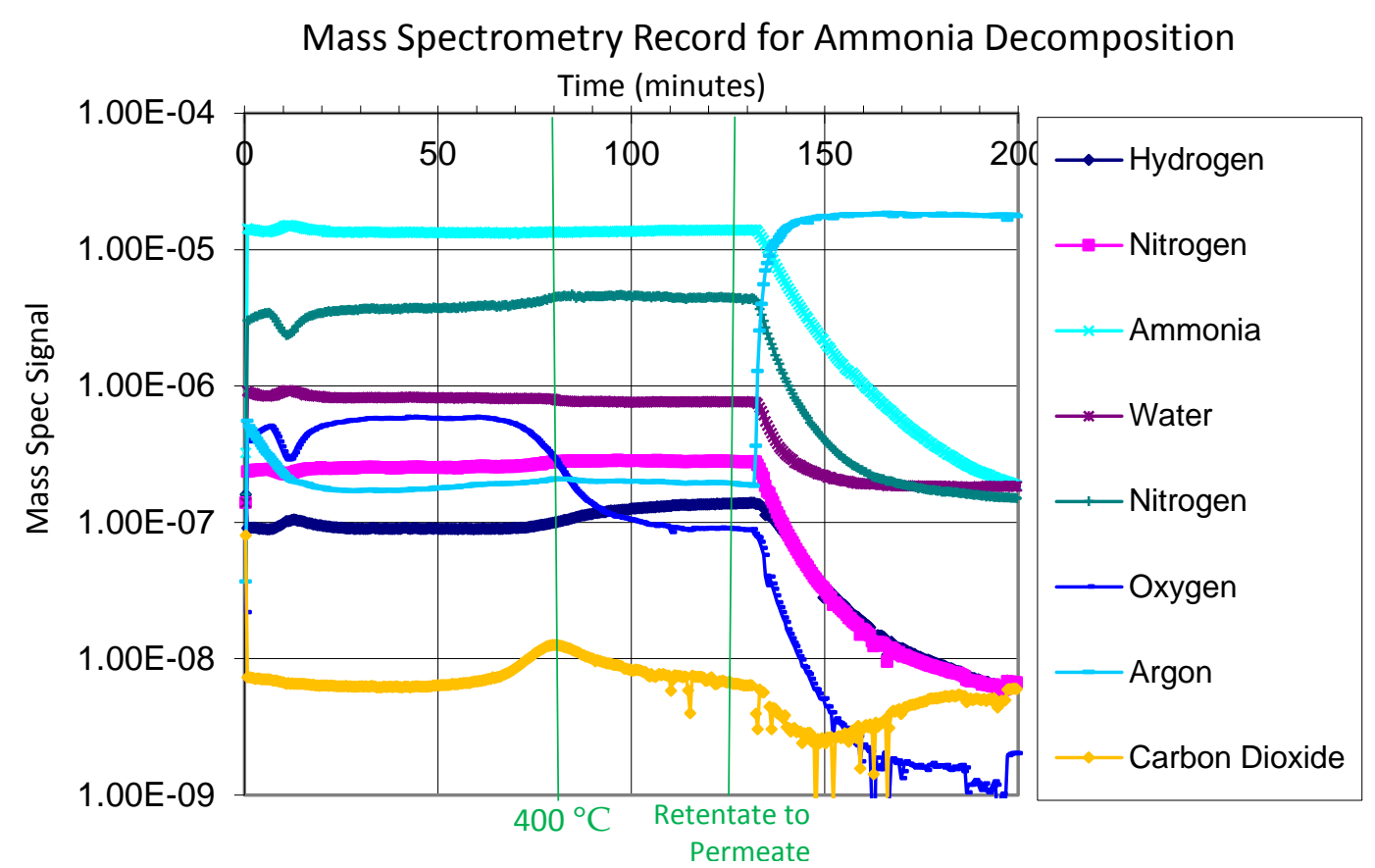

Figure 3.2.2: Mass spectrometry record for ammonia decomposition experiment with a palladium membrane electrodeposited at $-2.7 \mathrm{~V}$ for $20 \mathrm{~s}$ in the Sputnik reactor.

Temperature increased at $4.75^{\circ} \mathrm{C} \cdot \mathrm{min}^{-1}$ to $400{ }^{\circ} \mathrm{C}$, then held at $400{ }^{\circ} \mathrm{C}$ for the remainder of the experiment. Initially the mass spectrometer sampled from the retentate. At 130 minutes the valve was changed to make the mass spectrometer sample from the permeate.

Before positive results were confirmed for catalysis it was observed that the mass spectrometer registered positive for ammonia even when it could not be present.

Figure 3.2.3 shows a mass spectrometer record of argon flushing air from the gas reaction vessel recorded at that time. The trace for ammonia is large enough and consistent enough to be considered meaningful except that there is no reason for ammonia to be present. This was interpreted as damage to the electronics in the Mass Spectrometer as described in Section 2.2.11. 
Mass Spectrometry Record of Empty Cell with Rogue Ammonia

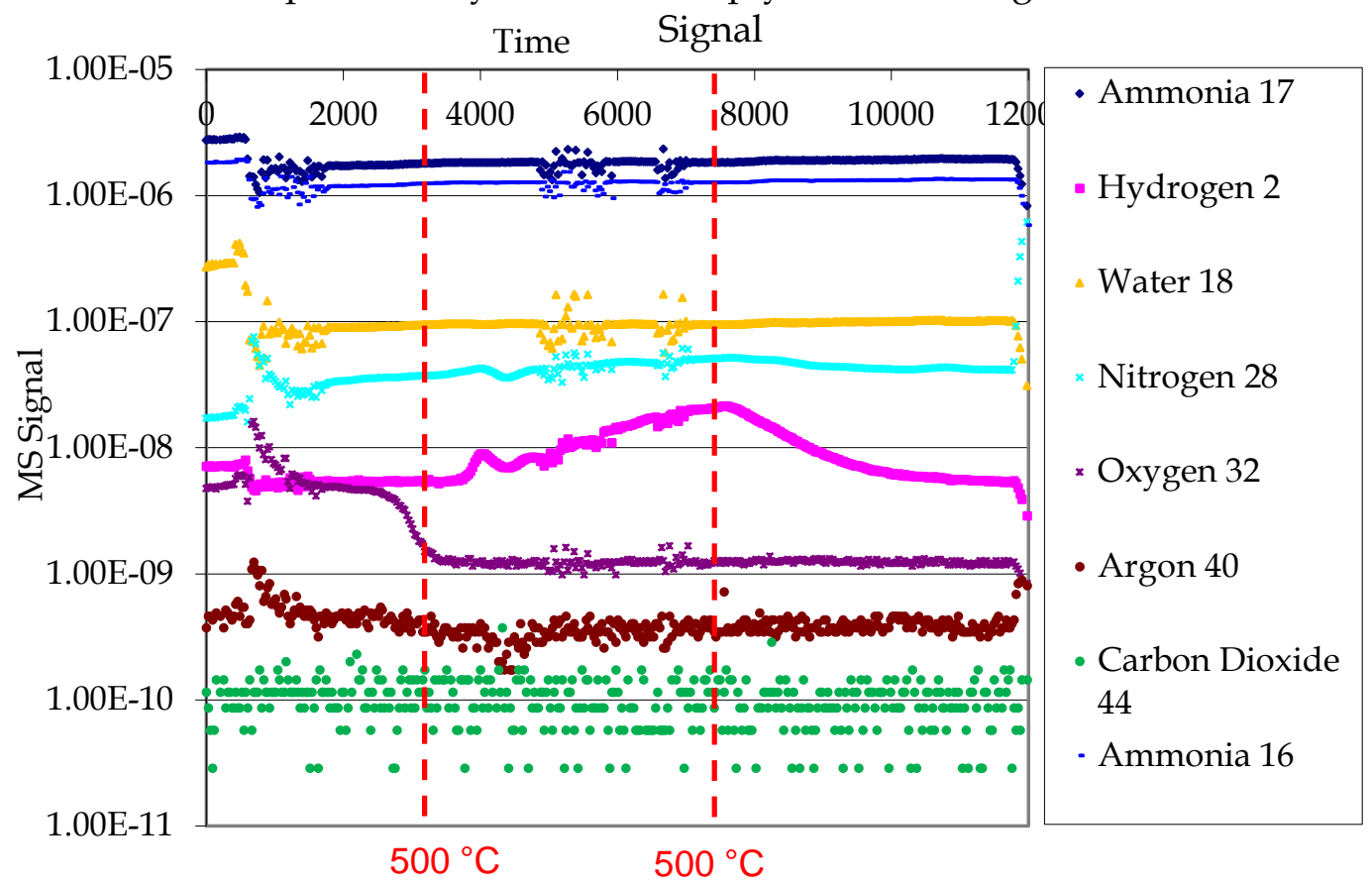

Figure 3.2.3: Mass spectrometry record for argon flushing air out of the reactor at room temperature.

The presence of ammonia in this record was attributed to damage to the electronics caused by the ammonia.

\subsubsection{Reactor Development}

\subsubsection{Sputnik}

A satisfactory seal could not be consistently achieved with this reactor. The Sputnik reactor was therefore replaced with the Explorer reactor, as described in Section 2.2.9.

\subsubsection{Explorer: Whole Reactor}

Ammonia decomposition experiments were conducted at five temperatures rising in fifty degrees increments from $300{ }^{\circ} \mathrm{C}$ to $500{ }^{\circ} \mathrm{C}$ with a square of blank stainless steel instead of a catalyst. Results were recorded with a gas chromatograph as detailed in Sections 2.1.7 and 2.2.12. A typical chromatogram for ammonia decomposition at each of these temperatures is shown in Figure 3.2.4. Each chromatogram shows a five minute gas chromatography record for an ammonia decomposition experiment. The 
ammonia peak at 3.1 minutes decreased with temperature. Hydrogen at 0.6 minutes and nitrogen at 0.7 minutes increased with temperature. This confirms that ammonia decomposition increased with temperature.
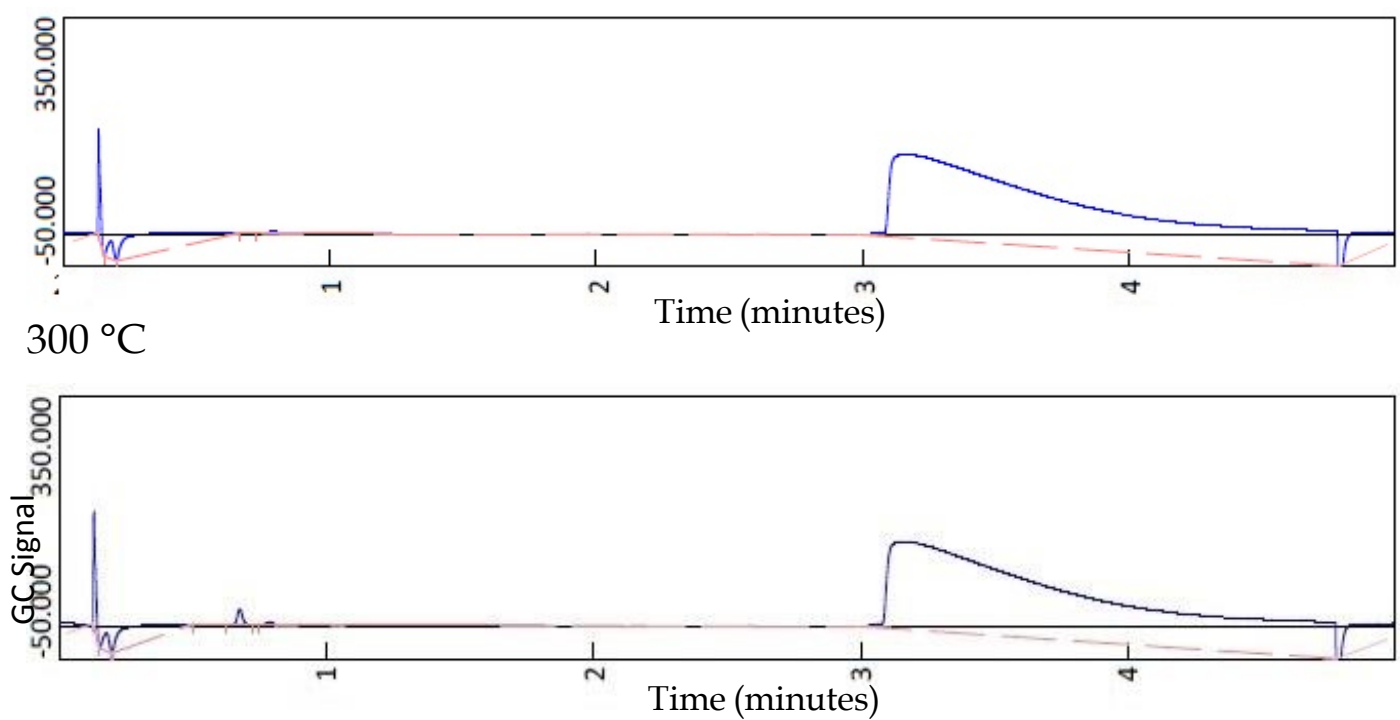

$350{ }^{\circ} \mathrm{C}$
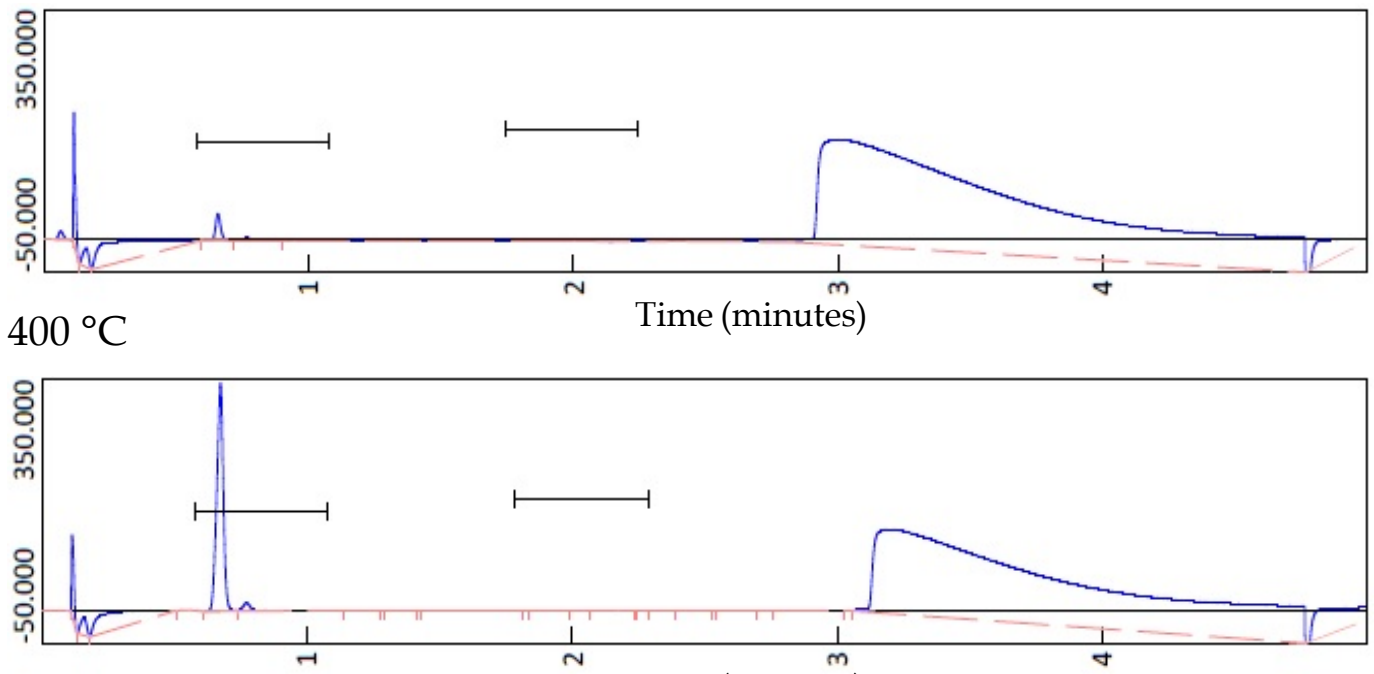

$450{ }^{\circ} \mathrm{C}$

Time (minutes)

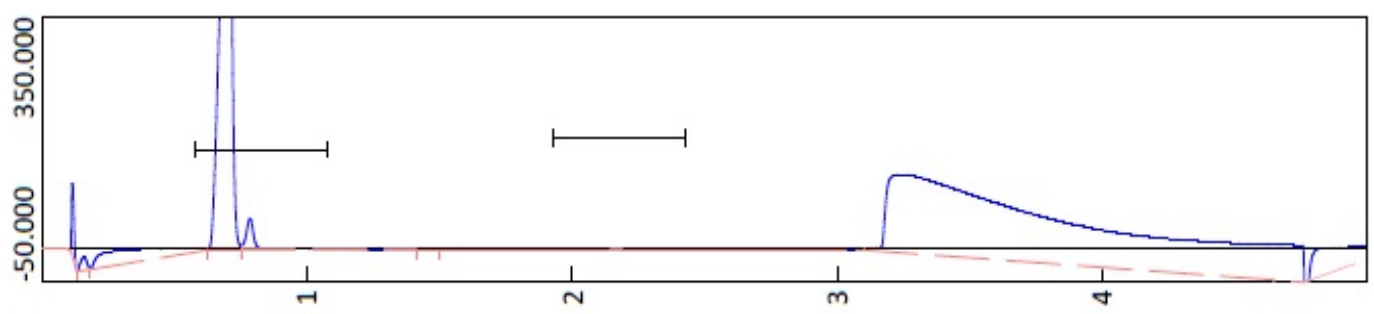

$500{ }^{\circ} \mathrm{C}$

Time (minutes)

Figure 3.2.4: Sample chromatograms for blank steel catalyst temperature series at $300-500{ }^{\circ} \mathrm{C}$ in the 'Explorer' reactor.

The ammonia peak at 3.1 minutes after injection decreased with temperature. Hydrogen at 0.6 minutes and nitrogen at 0.7 minutes increased with temperature. 
Jonathan Tailby

The measurements for all temperatures were below equilibrium hydrogen and nitrogen production for their respective temperatures, so there must have been a kinetic limit to the ammonia decomposition. A kinetic limitation supports the expectation that a catalyst would enhance the rate of ammonia decomposition.

After these experiments, the steel sample was taken out and examined. It had been browned and blackened by the process but this discolouration was a thin layer that was readily scraped off with steel but not with a plastic tool.

Next in this comparison set, a nickel deposited steel sample was tested. For this first catalyst test, a sample with unevenly deposited template sample was selected, the selected sample had been deposited with a template at $-0.9 \mathrm{~V}$ for 600 s. Optical micrographs for this sample are shown in Figure 3.2.5.
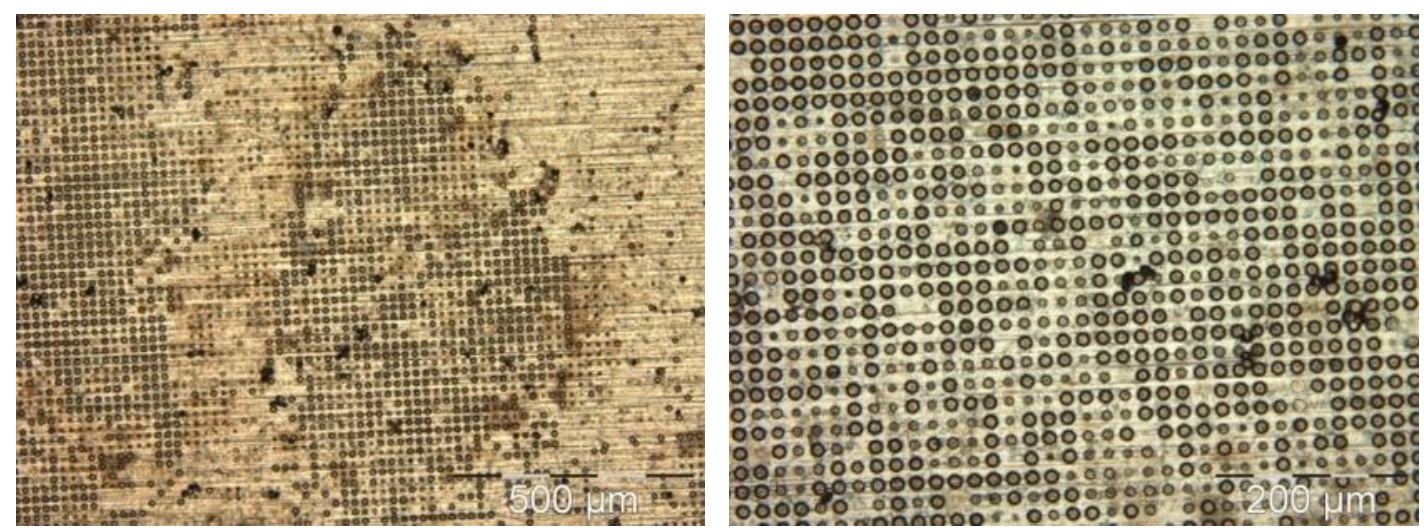

Figure 3.2.5: Optical micrographs of the first templated nickel sample to be tested for ammonia decomposition.

The templated steel had been electrodeposited at $-0.9 \mathrm{~V}$ for $600 \mathrm{~s}$. The image on the left was taken at x100 magnification and the image on the right at x200.

Table 5.2.5 in Appendix 5.2.7.1 summarise the gas chromatography data and calculate the output quantities of each of the gases. These outputs have been plotted against temperature in

Figure 3.2.6.

The reactor output results for the ammonia decomposition experiments comparing the catalytic effectiveness of the different samples at a range of temperatures are plotted against temperature in 
Figure 3.2.6 to give a summary for comparison. It is clear that temperature increased the ammonia decomposition because hydrogen and nitrogen signals increased and the ammonia signal decreased. Compared with blank steel, nickel deposited steel has increased the ammonia decomposition.

The comparison is less clear for blank palladium and deposited palladium, which could be because of the better quality of nickel deposition on the nickel/stainless steel sample compared to that of the nickel/palladium sample. There is no significant improvement in nickel/palladium over nickel/stainless steel, which may also be because of the better quality of the depositions on steel than on palladium.

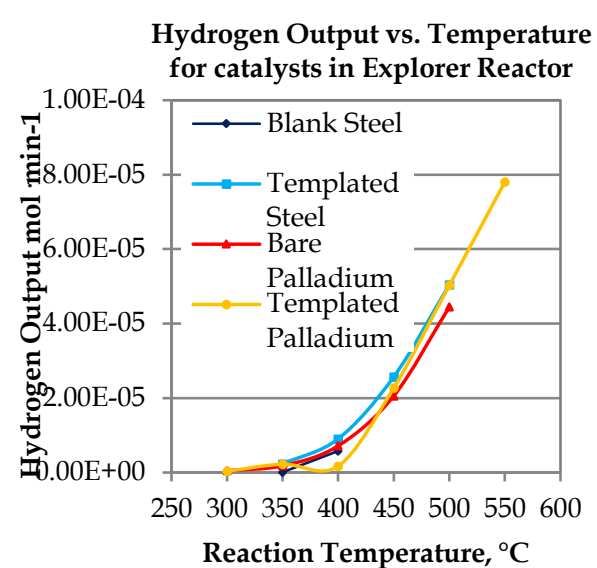

Figure 3.2.6a

Ammonia Remnant vs. Temperature for catalysts in Explorer Reaction

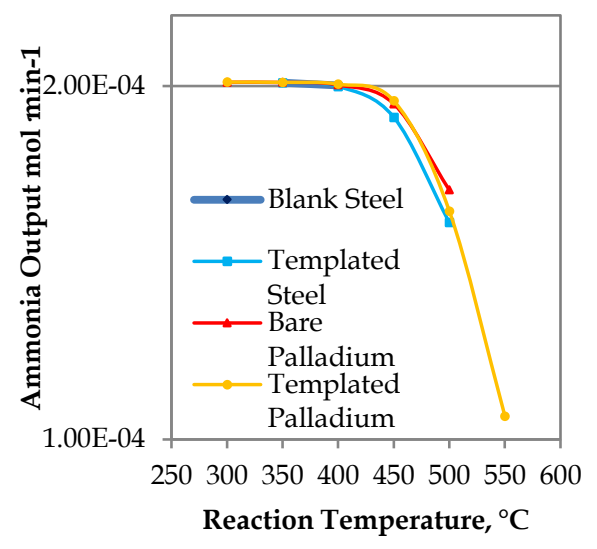

Figure 3.2.6c

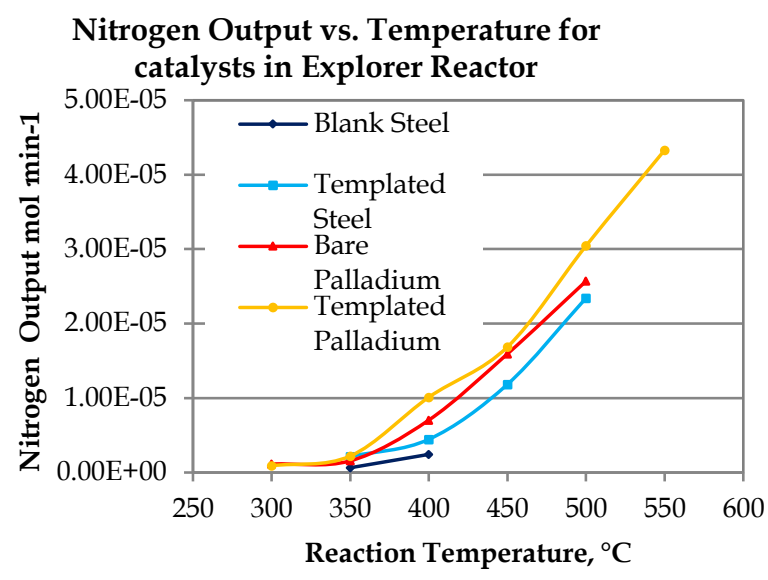

Figure 3.2.6b

Figure 3.2.6: Gas outputs calculated from the GC results plotted against temperature for ammonia decomposition with different catalysts in the Explorer gas reactor.

Figure 3.2.6a shows the hydrogen product and

Figure 3.2.6b the nitrogen.

Figure 3.2.6c shows the remaining ammonia. Hydrogen and nitrogen both increased with temperature and ammonia decreased, indicating increased decomposition at higher temperature. 
Jonathan Tailby

\subsubsection{Explorer: Primary Insert}

In an attempt to increase interaction between samples and gas flow, the samples were supported face on to the gas flow. Chromatograms for some initial ammonia decomposition experiments with samples supported face on to the gas flow are shown in Figure 5.2.22 and Figure 5.2.23.

Figure 3.2.7 to Figure 3.2.9 show graphs of gas output vs. temperature for the gas flows from Table 5.2.9 to Table 5.2.13 in Appendix 5.2.7.2. Figure 3.2.7 shows the output of hydrogen, Figure 3.2.8 shows the output of nitrogen, and Figure 3.2.9 shows the output of ammonia. Figure 3.2.10 shows ammonia decomposition plotted against temperature. Ammonia decomposition has been calculated by the difference between ammonia input and ammonia output. The two catalyst samples were approximately equally effective and each less effective than the empty sample holder. The lack of difference between deposited and undeposited steel was attributed to a continuing excess of empty space in the reactor relative to the quantity of nickel. 


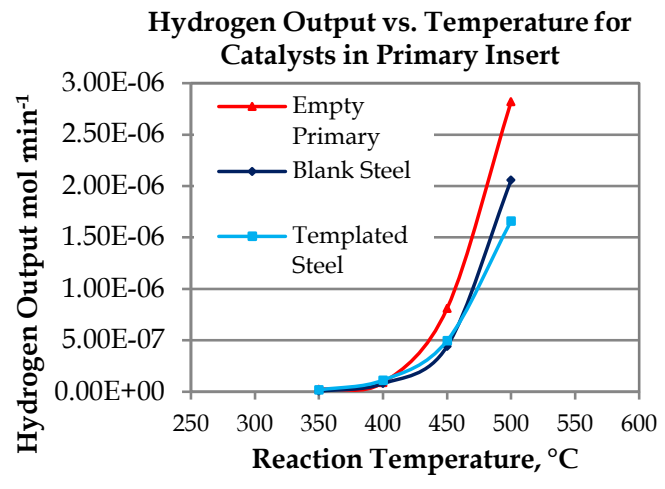

Figure 3.2.7: Reactor output vs. temperature for hydrogen from samples in the primary insert.

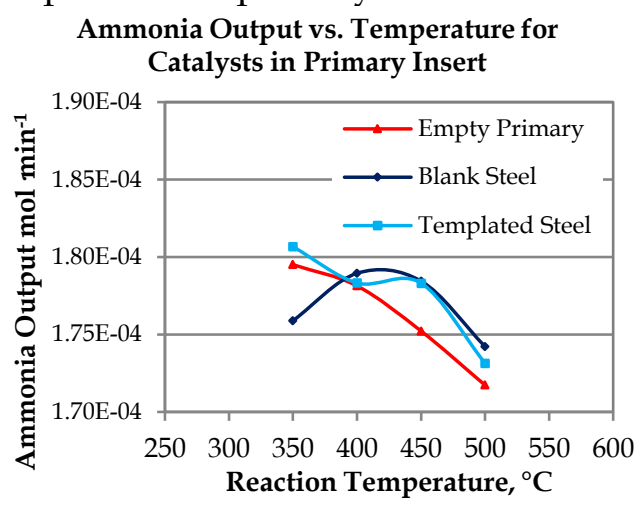

Figure 3.2.9: Reactor output vs. temperature for ammonia from samples in the primary insert.

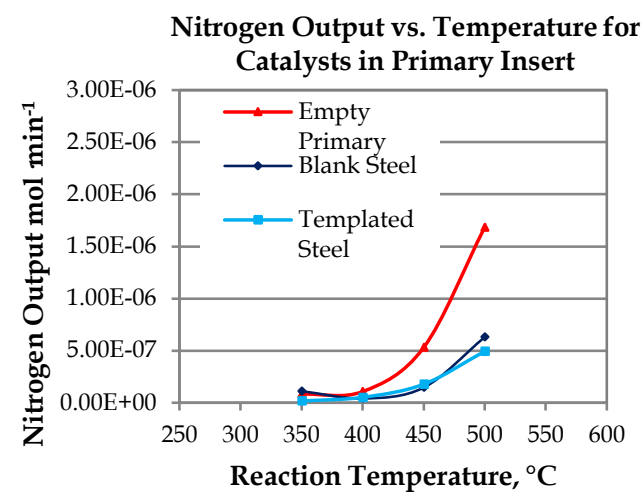

Figure 3.2.8: Reactor output vs. temperature for nitrogen from samples in the primary insert.

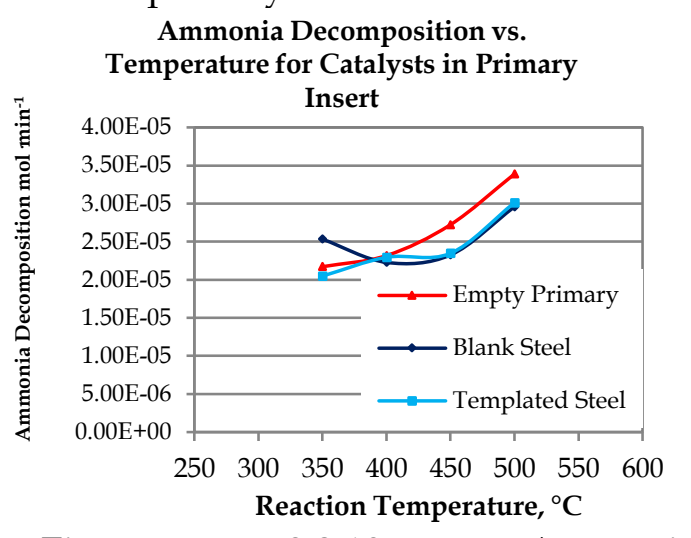

Figure 3.2.10: Ammonia decomposition by catalyst samples in the primary insert.

\subsubsection{Explorer with Secondary Insert}

The secondary insert was a tube inserted inside the primary insert tube to further reduce the reactor volume. The reaction occurred in the space between the outer diameter of the secondary insert and the inner diameter of the primary insert, therefore, the catalysts were placed on the outside of secondary inserts. The first secondary insert was the stainless steel tube with no additional catalyst, subsequently five different catalysts were tested following details set out in Section 2.2.10. The results of the ammonia decomposition experiments are collected in this section. 
Jonathan Tailby

\subsection{Undeposited Stainless Steel Secondary Insert}

A bare stainless steel secondary insert was tested in ammonia decomposition experiments to give a baseline for comparison of the catalyst samples and to confirm whether or not they were indeed acting as a catalyst. Ammonia decomposition experiments were conducted at 300, 350, 400, 450, 500, 550 and $600^{\circ} \mathrm{C}$.

Chromatograms for the ammonia decomposition with an undeposited stainless steel secondary insert are shown in Appendix 5.2.7.3. Table 5.2.14 summarises the peak data from the chromatograms and Table 5.2.15 calculates the gas output from the chromatograph measurements for the secondary inserts. Figure 3.2.11 shows plots of the average gas output of hydrogen, nitrogen and ammonia with the bare steel secondary insert across a range of temperatures from 300 to $450{ }^{\circ} \mathrm{C}$.

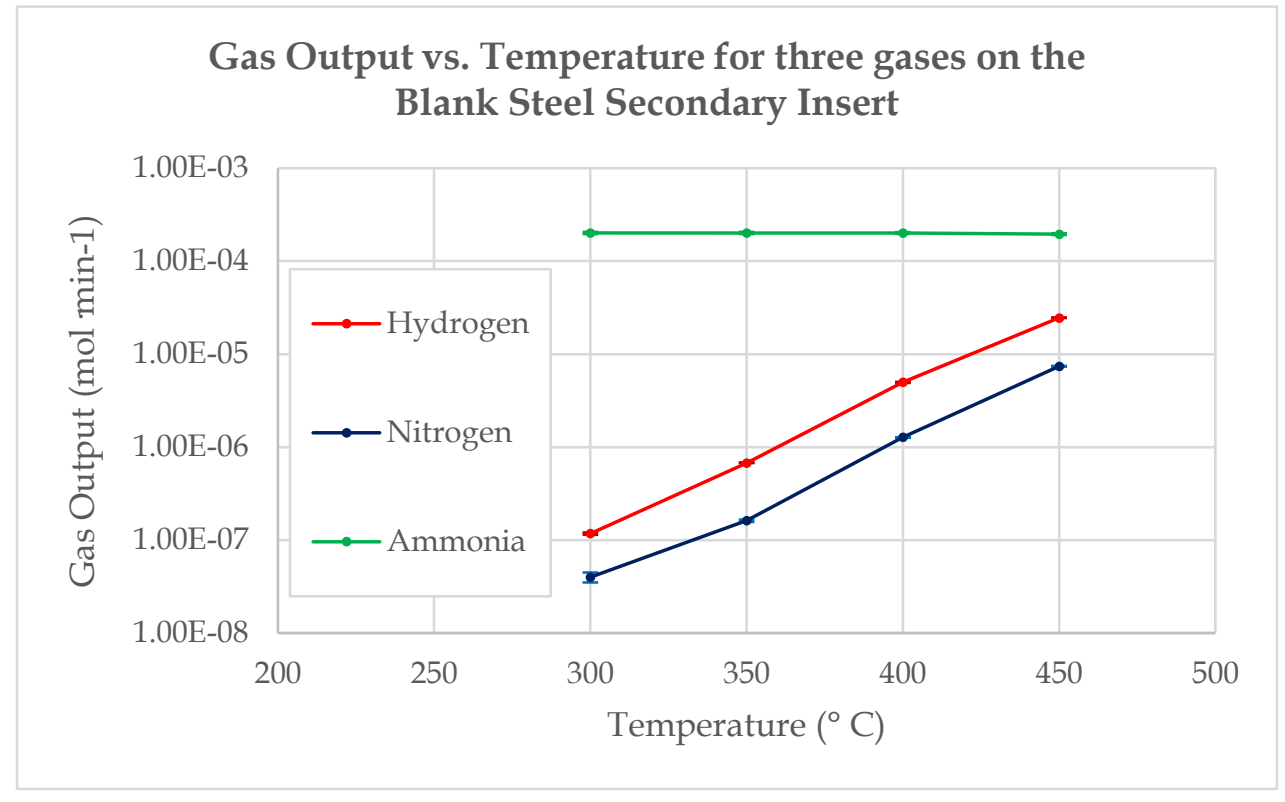

Figure 3.2.11: Plots of the gas output at temperatures from 300 to $450{ }^{\circ} \mathrm{C}$ with the bare steel secondary insert.

\subsection{Untemplated Nickel Deposits on Secondary Insert}

Secondary inserts deposited with untemplated nickel were tested in ammonia decomposition experiments at 300, 350, 400 and $450{ }^{\circ} \mathrm{C}$. Chromatograms for the full temperature range are shown in Figure 5.2.25: in Appendix 5.2.7.3. 


\subsection{Templated Nickel Deposits on Secondary Insert}

TCD chromatograms for ammonia decomposition experiments on the templated nickel deposited secondary insert are included in Figure 5.2.26 Appendix 5.2.7.3. The template nickel electrodeposits were more effective catalysts for the ammonia decomposition reaction than the untemplated electrodeposits. The preformed nickel catalysts were subsequently tested to investigate if their greater protrusion into the reaction space would improve catalysis. The method of template formation on the secondary insert could not be replicated on the tertiary inserts, therefore this catalyst was not taken forward to the palladium experiments.

\subsection{Nickel Mesh on Secondary Insert}

Chromatograms for the gas reaction testing of the nickel mesh secondary inserts are shown in Figure 5.2.27 in Appendix 5.2.7.3. Calculated values for the gas outputs of these reactions are included in the graphs of Section 3.2.2.4.6

\subsection{Nickel Foam on Secondary Insert}

The nickel foam insert was expected to be the most effective catalyst because it filled the reaction space most effectively therefore ammonia molecules were less likely to pass through the reaction space without contacting the catalyst. Chromatograms for the reaction testing of the nickel foam catalyst are included in Appendix 5.2.7.3. in Figure 5.2.28. The peak areas of the chromatogram shown in Figure 5.2.28 and the calculated gas outputs included in the graphs in Section 3.2.2.4.6 show that this expectation has not been met. One possible explanation for this would be if the electrodepositions favour exposing one particular crystal face and the nickel foam exposes another, with the electrodeposition face being the more active in ammonia decomposition catalysis. 
Jonathan Tailby

\subsection{Secondary Insert Summary}

When all of the catalysts that had been tested were compared, it was clear that the templated electrodeposits were the best catalyst for ammonia decomposition on the secondary inserts. Figure 3.2.12 shows the decomposition of ammonia plotted against reaction temperature for four of the catalysts tested on the secondary insert.

\section{Ammonia decomposition vs. temperature for Catalysts on Secondary Inserts}

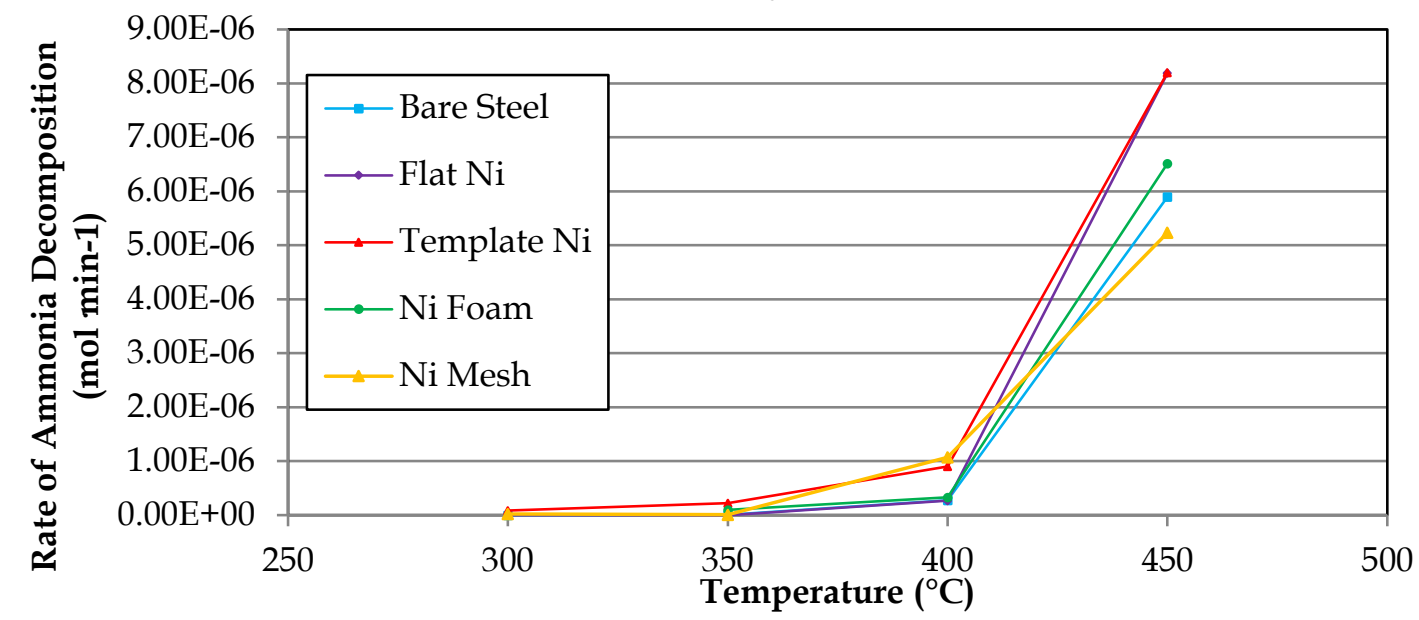

Figure 3.2.12: Plot for the decomposition of ammonia against temperature for different catalysts on the secondary inserts from 300 to $450{ }^{\circ} \mathrm{C}$.

Figure 3.2.13 shows the rate hydrogen production plotted against temperature for four of the catalysts tested on the secondary inserts. Each of the catalysts achieved better decomposition than bare steel under some conditions, however they are still not effective enough to help the reaction approach equilibrium. Similar to the percentage of ammonia decomposed, the templated nickel electrodeposits produced the highest result for nearly all temperatures. 
Hydrogen production vs. Temperature for Secondary Insert Catalysts

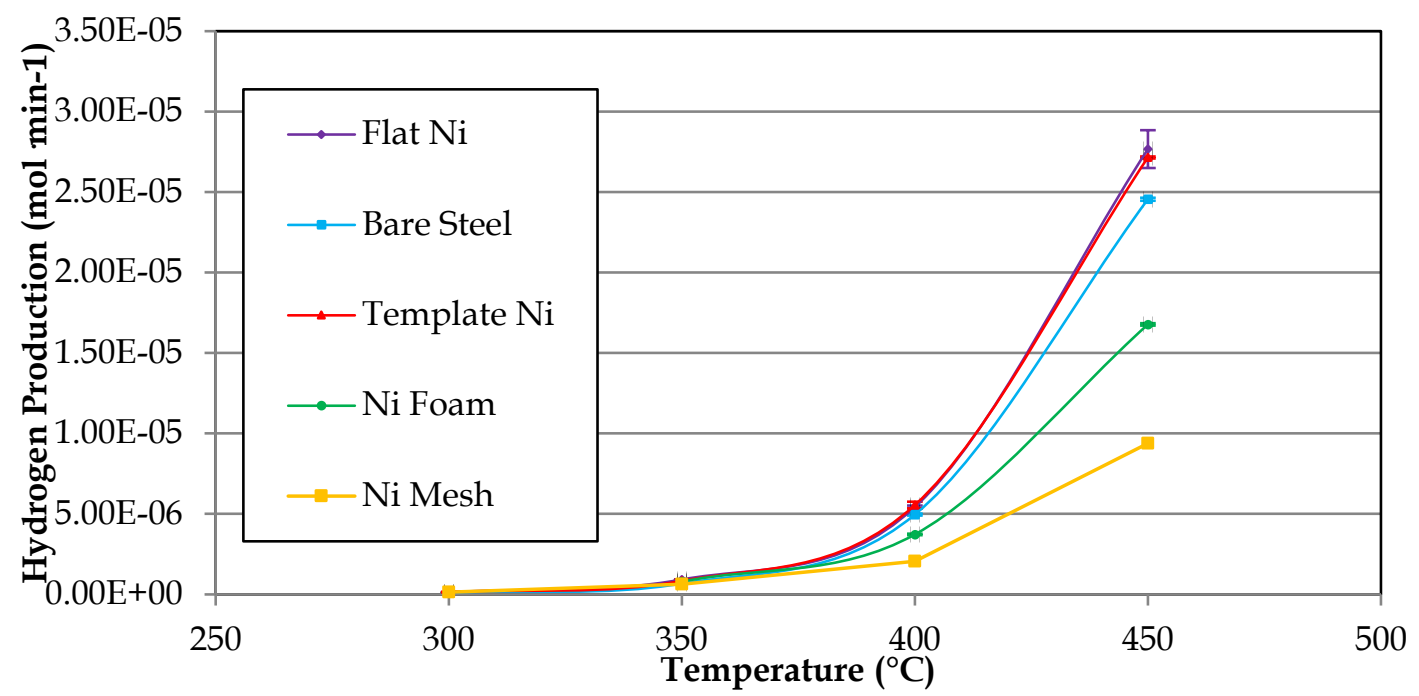

Figure 3.2.13: Plot for the production of hydrogen against temperature for different catalysts on the secondary inserts from 300 to $450{ }^{\circ} \mathrm{C}$.

The nickel foam was one of the least effective catalysts, however it was the best choice for application in the next stage of the research because it could be most easily wrapped onto the tertiary insert. For this practical reason, nickel foam is used in the tertiary insert experiments which explored the effects of combining the nickel catalyst with palladium in different arrangements. The templated nickel electrodeposits would have been preferred but the method for making them could not be applied to the tertiary insert design at this time, and the untemplated nickel was expected to be unsuitable because of the continuous layer.

\section{Arrhenius Activation Energy}

Activation energy for these catalysts was calculated using the Arrhenius method as described in Section 2.2.12.4. Log of the Reaction rate for each catalyst was plotted against the inverse of the temperature in degrees Kelvin. The slope from the plotted line was multiplied by the negative of the gas constant to calculate activation energy. The calculation process for each catalyst is included in Appendix 5.2.7.3.1. The calculation of two exemplar gas species is shown here. Table 3.2.1 calculates the data needed to construct the Arrhenius plot for the rate of ammonia decomposition with the secondary insert catalysts. The Arrhenius plot of that ammonia data is shown in Figure 
Jonathan Tailby

3.2.14. Table 3.2.2 calculates the activation energy from the information gained from the Arrhenius plot. Table 3.2.3, Figure 3.2.15 and 
Table 3.2.4 repeat this calculation process for the rate of hydrogen formation by ammonia decomposition on the secondary insert catalysts.

Table 3.2.1: Calculating Data for Arrhenius plots of Ammonia Decomposition Rates of secondary inserts.

\begin{tabular}{|c|c|c|c|c|c|}
\hline Temperature $\left({ }^{\circ} \mathrm{C}\right)$ & 300 & 350 & 400 & 450 & \\
\hline Temperature (K) & 573 & 623 & 673 & 723 & \\
\hline $\mathrm{T}^{-1}$ & $1.75 \mathrm{E}-03$ & $1.61 \mathrm{E}-03$ & 1.49E-03 & 1.38E-03 & $\mathrm{K}^{-1}$ \\
\hline Flat Nickel & $5.41 \mathrm{E}-11$ & $3.73 \mathrm{E}-10$ & $2.72 \mathrm{E}-07$ & 8.19E-06 & $\mathrm{mol} \cdot \mathrm{min}^{-1}$ \\
\hline $\ln k$ & $-2.36 \mathrm{E}+01$ & $-2.17 \mathrm{E}+01$ & $-1.51 \mathrm{E}+01$ & $-1.17 \mathrm{E}+01$ & \\
\hline Bare Steel & $1.36 \mathrm{E}-09$ & 2.80E-09 & 2.77E-07 & $5.90 \mathrm{E}-06$ & $\mathrm{~mol} \cdot \mathrm{min}^{-1}$ \\
\hline $\ln k$ & $-2.04 \mathrm{E}+01$ & $-1.97 \mathrm{E}+01$ & $-1.51 \mathrm{E}+01$ & $-1.20 \mathrm{E}+01$ & \\
\hline Template Nickel & 8.54E-08 & 2.22E-07 & 9.02E-07 & $8.20 \mathrm{E}-06$ & $\mathrm{~mol} \cdot \mathrm{min}^{-1}$ \\
\hline $\ln k$ & $-1.63 E+01$ & $-1.53 E+01$ & $-1.39 E+01$ & $-1.17 \mathrm{E}+01$ & \\
\hline Nickel Foam & & $9.76 \mathrm{E}-08$ & 3.29E-07 & $6.52 \mathrm{E}-06$ & $\mathrm{~mol} \cdot \mathrm{min}^{-1}$ \\
\hline $\ln k$ & & $-1.61 E+01$ & $-1.49 \mathrm{E}+01$ & $-1.19 \mathrm{E}+01$ & \\
\hline
\end{tabular}




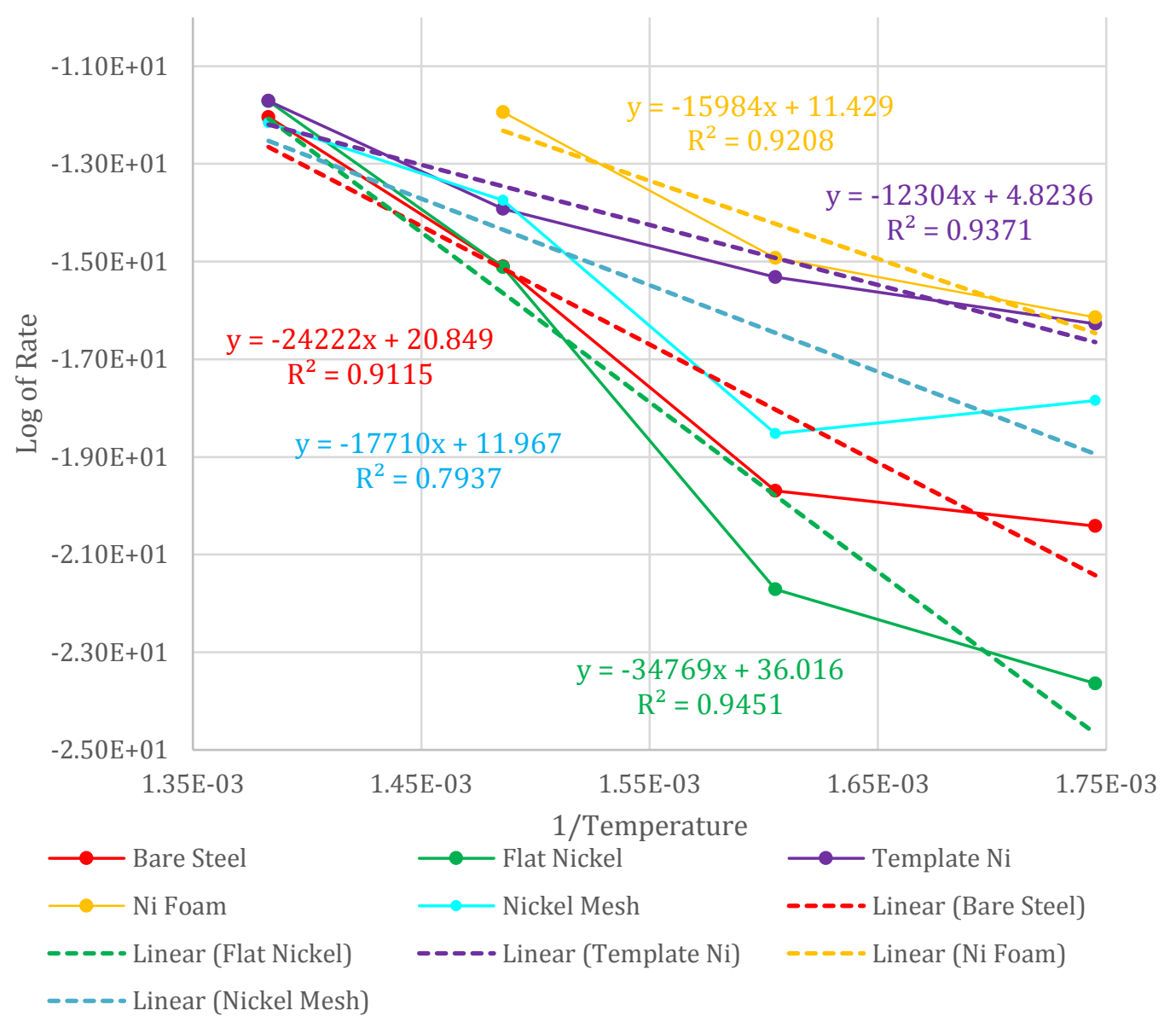

Figure 3.2.14: Arrhenius plot for Ammonia Decomposition with Secondary Insert Catalysts.

Table 3.2.2: Final calculation of Activation Energy for Secondary Insert Catalysts from Rate of Ammonia decomposition.

\begin{tabular}{|l|l|l|l|l|}
\hline Catalyst & Intercept & Slope & $-\mathbf{R}$ m & $\mathbf{E}_{\mathbf{a}}$ \\
\hline Flat Ni & 36.0 & -34769 & 289069 & 289 \\
\hline Bare Steel & 20.8 & -24222 & 201382 & 201 \\
\hline Template Ni & 4.8 & -12304 & 102295 & 102 \\
\hline Nickel Mesh & 12.0 & -17710 & 147241 & 147 \\
\hline Ni Foam & 11.4 & -15984 & 132891 & 133 \\
\hline & & & $\mathrm{J} \mathrm{mol}^{-1}$ & $\mathrm{~kJ} \mathrm{~mol}^{-1}$ \\
\hline
\end{tabular}

Table 3.2.3: Calculating Data for Arrhenius plots of Hydrogen Formation Rates of secondary inserts.

\begin{tabular}{|l|l|l|l|l|l|}
\hline Temperature $\left({ }^{\circ} \mathrm{C}\right)$ & 300 & 350 & 400 & 450 & \\
\hline Temperature $(\mathrm{K})$ & 573 & 623 & 673 & 723 & \\
\hline $\mathrm{T}^{-1}$ & 0.001745 & 0.001605 & 0.001486 & 0.001383 & $\mathrm{~K}^{-1}$ \\
\hline
\end{tabular}




\begin{tabular}{|c|l|l|l|l|l|}
\hline Flat Nickel & $1.37 \mathrm{E}-07$ & $9.26 \mathrm{E}-07$ & $5.43 \mathrm{E}-06$ & $2.77 \mathrm{E}-05$ & $\mathrm{~mol} \mathrm{~min}^{-1}$ \\
\hline $\ln k$ & $-1.58 \mathrm{E}+01$ & $-1.39 \mathrm{E}+01$ & $-1.21 \mathrm{E}+01$ & $-1.05 \mathrm{E}+01$ & \\
\hline Bare Steel & $1.17 \mathrm{E}-07$ & $6.77 \mathrm{E}-07$ & $4.96 \mathrm{E}-06$ & $2.46 \mathrm{E}-05$ & $\mathrm{~mol} \mathrm{~min}^{-1}$ \\
\hline $\ln k$ & $-1.60 \mathrm{E}+01$ & $-1.42 \mathrm{E}+01$ & $-1.22 \mathrm{E}+01$ & $-1.06 \mathrm{E}+01$ & \\
\hline Template Nickel & $1.44 \mathrm{E}-07$ & $8.42 \mathrm{E}-07$ & $5.56 \mathrm{E}-06$ & $2.71 \mathrm{E}-05$ & $\mathrm{~mol} \mathrm{~min}^{-1}$ \\
\hline $\ln k$ & $-1.58 \mathrm{E}+01$ & $-1.40 \mathrm{E}+01$ & $-1.21 \mathrm{E}+01$ & $-1.05 \mathrm{E}+01$ & \\
\hline Nickel Foam & & $7.93 \mathrm{E}-07$ & $3.73 \mathrm{E}-06$ & $1.68 \mathrm{E}-05$ & $\mathrm{~mol} \mathrm{~min}^{-1}$ \\
\hline $\ln k$ & & $-1.40 \mathrm{E}+01$ & $-1.25 \mathrm{E}+01$ & $-1.10 \mathrm{E}+01$ & \\
\hline Nickel Mesh & $1.41 \mathrm{E}-07$ & $6.25 \mathrm{E}-07$ & $2.07 \mathrm{E}-06$ & $9.39 \mathrm{E}-06$ & $\mathrm{~mol} \mathrm{~min}^{-1}$ \\
\hline $\ln k$ & $-1.58 \mathrm{E}+01$ & $-1.43 \mathrm{E}+01$ & $-1.31 \mathrm{E}+01$ & $-1.16 \mathrm{E}+01$ & \\
\hline
\end{tabular}

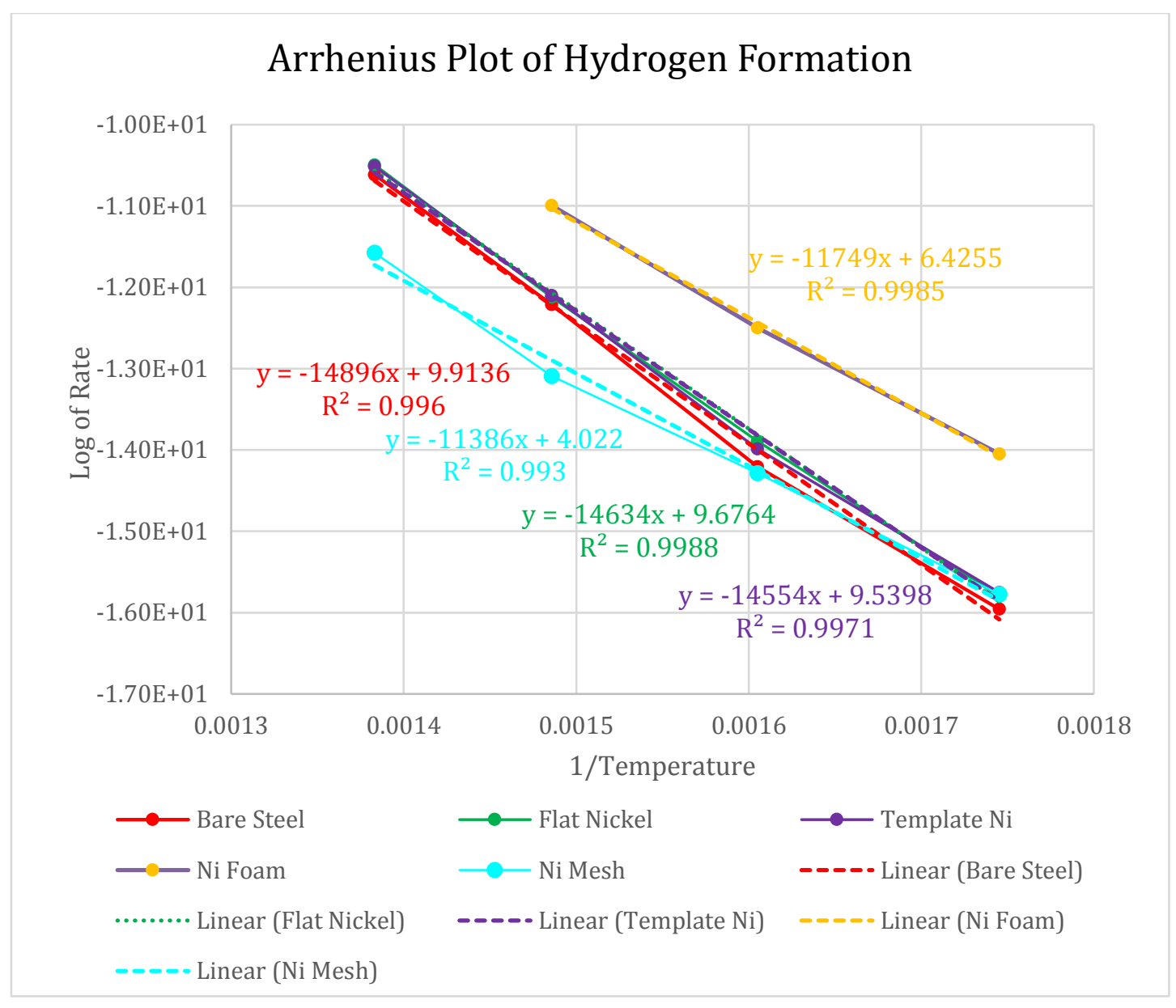

Figure 3.2.15: Arrhenius plot of the Hydrogen Formation data for the Secondary Insert Catalysts. 
Table 3.2.4: Calculating the Activation Energy for Hydrogen Formation on the Secondary Insert Catalysts.

\begin{tabular}{|c|c|c|c|c|}
\hline Catalyst & Intercept & Slope & $-\mathrm{R} \mathrm{m}$ & $\mathrm{E}_{\mathrm{a}}$ \\
\hline Flat Ni & 9.6764 & -14634 & 121667 & 121.7 \\
\hline Bare Steel & 9.9195 & -14896 & 123845 & 123.8 \\
\hline Template Ni & 9.5398 & -14554 & 121002 & 121.0 \\
\hline Ni Foam & 6.4255 & -11749 & 97681 & 97.7 \\
\hline \multirow[t]{2}{*}{ Ni Mesh } & 4.022 & -11386 & 94663 & 94.7 \\
\hline & & & $\mathrm{J} \cdot \mathrm{mol}^{-1}$ & $\mathrm{~kJ} \mathrm{~mol}^{-1}$ \\
\hline
\end{tabular}

The fitted trend lines on Figure 3.2.14 and Figure 3.2.15 are displayed with equations, including an $\mathrm{R}^{2}$ value to indicate how well the line fits the data. For the ammonia plots the lowest value is 0.7937 and the highest 0.9451 , while the hydrogen plots range from 0.993 to 0.9988 . The high $\mathrm{R}^{2}$ values indicate that the Arrhenius model fits exceptionally well for the hydrogen gas formation, but less accurately for the ammonia decomposition. High $\mathrm{R}^{2}$ values also show that the activation energy calculated from the rate of hydrogen production can be confidently compared with values published elsewhere.

The less accurate fit for the ammonia data to the Arrhenius model could mean that the reaction involves multiple steps.

Table 3.2.5 shows the Arrhenius activation energy results for each secondary insert catalyst calculated from the reaction results for each gas species.

Table 3.2.5: Summary of Activation Energy calculated for each secondary insert from the rate data for the three different species.

\begin{tabular}{|l|l|l|l|}
\hline & & & $\mathrm{kJ} \cdot \mathrm{mol}^{-1}$ \\
\hline Species: & Ammonia & Nitrogen & Hydrogen \\
\hline Flat Ni & 289.1 & 108.2 & 121.7 \\
\hline Bare Steel & 201.4 & 121.3 & 123.8 \\
\hline Template Ni & 102.3 & 109.2 & 121.0 \\
\hline Ni Foam & 132.9 & 104.2 & 97.7 \\
\hline Ni Mesh & 147.2 & 94.7 & 94.7 \\
\hline
\end{tabular}

Only one value for a nickel catalyst is higher than the activation energy for the plain steel, this is also the only result that is outside the range of results seen 
in the literature. The nickel foam was consistently one of the catalysts with a low activation energy.

Previous work on the activation energy of ammonia decomposition has found that the activation energy depends on both the composition and presentation of the catalyst. Takahashi reported calculating the activation energy for the subprocesses in ammonia decomposition and found that the maximum activation energy occurred at different steps for nickel $\left(144 \mathrm{~kJ}^{\mathrm{mol}}{ }^{-1}\right.$ at dehydrogenation) and ruthenium (127 $\mathrm{kJ} \mathrm{mol}^{-1}$ at nitrogen desorption) [83]. Takahashi claimed that this shows that the rate determining step is occurring at different steps for different catalysts. Ganley has published attempts to estimate activation energy from other properties that are known, and presented a calculated activation energy for $\mathrm{N}-\mathrm{H}$ bond breaking of $130 \mathrm{~kJ} \mathrm{~mol}^{-}$ ${ }^{1}$ [84], approximately the same as the ammonia decomposition on nickel foam value in Table 3.2.5. Activation energies that have been claimed for other metals include $155 \mathrm{~kJ} \mathrm{~mol}^{-1}$ for iron, $117 \mathrm{~kJ} \mathrm{~mol}^{-1}$ for platinum and $110 \mathrm{~kJ} \mathrm{~mol}$ 1 for palladium.

Choudhary reports different activation energies for different forms of the same metals, with $180 \mathrm{~kJ} \mathrm{~mol}^{-1}$ for nickel films and $209 \mathrm{~kJ} \mathrm{~mol}^{-1}$ for nickel wires, both higher than all the results calculated in this work except the value for flat nickel calculated from ammonia decomposition [78]. Chellappa published a figure for nickel wires similar to Choudhary, $211 \mathrm{~kJ} \mathrm{~mol}^{-1}$ [11]. Chellappa's work was aimed at comparing the catalytic activity of a nickel catalyst with a nickel-platinum catalyst. Activation energy was one parameter discussed, with platinum wires stated to be $88 \mathrm{~kJ} \mathrm{~mol}^{-1}$ and the nickel-platinum catalyst to be $196 \mathrm{~kJ} \mathrm{~mol}^{-1}$. This shows the combination with platinum had lowered the activation energy of the nickel. That study finely crushed the precursors of the two metals together, whereas the nickel on palladium tertiary inserts in this work have two metals separate but in contact.

The range of values calculated in this present work is comparable to the range of values in the literature. 
Jonathan Tailby

\section{Specific Rate of Reaction}

The rate of reaction was standardised to the quantity of nickel catalyst. Table 3.2.6 shows the resulting standardised rates in $\mathrm{mol} \cdot g_{\text {catalyst }}^{-1} \mathrm{~min}^{-1}$ for ammonia decomposition and for the production of nitrogen and hydrogen. These results are plotted in Figure 3.2.16 and

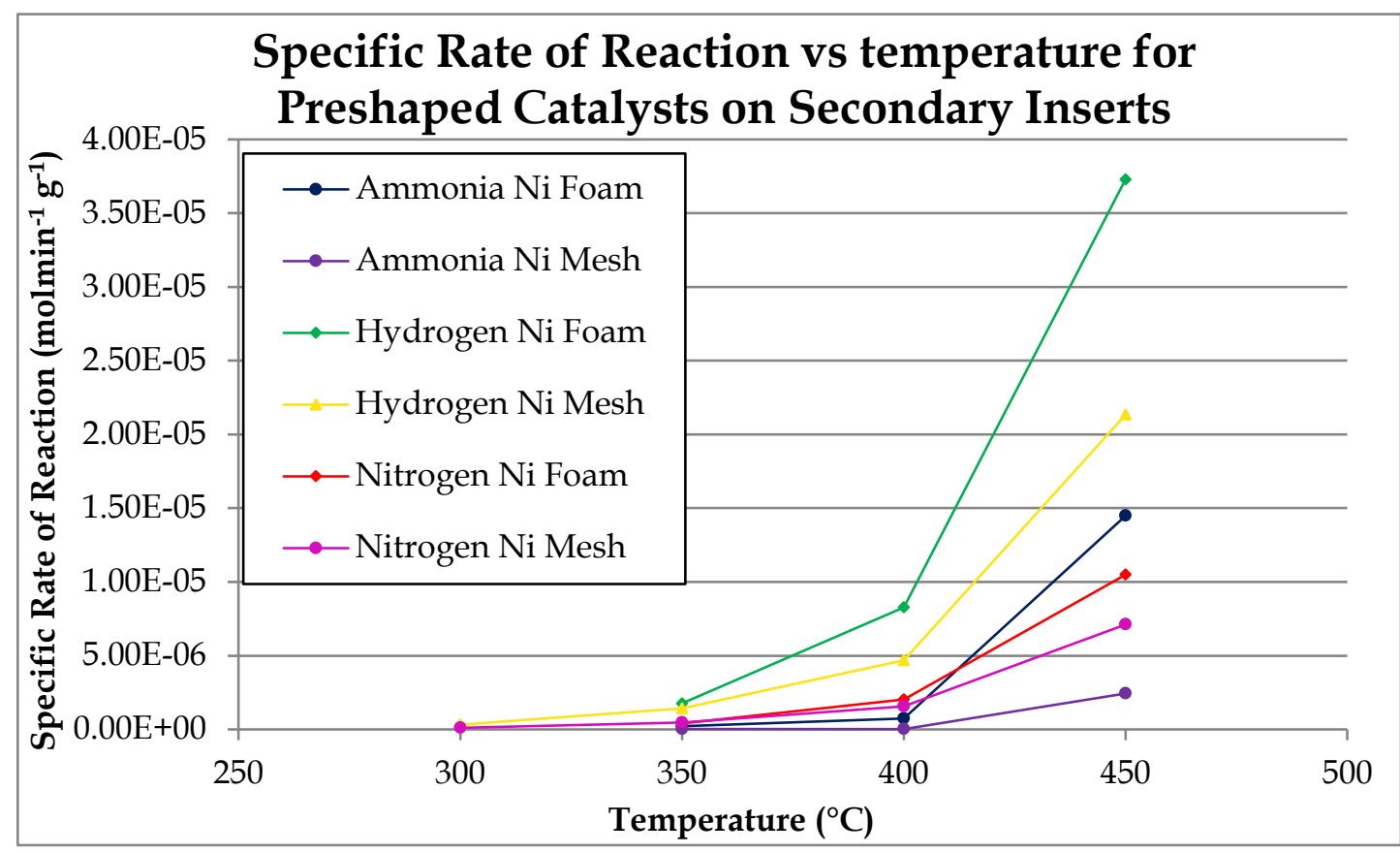

Figure 3.2.17. The electrodeposited nickel catalysts and the preshaped nickel catalysts have been separated into two graphs because the differences in magnitude are better plotted at different scales.

Table 3.2.6: Specific Rate of Reaction for the secondary insert Nickel catalysts across the temperature series.

\begin{tabular}{|l|l|l|l|l|}
\hline Ammonia Decomposition & $\mathbf{3 0 0}{ }^{\circ} \mathbf{C}$ & $\mathbf{3 5 0}^{\circ} \mathbf{C}$ & $\mathbf{4 0 0}{ }^{\circ} \mathbf{C}$ & $\mathbf{4 5 0}{ }^{\circ} \mathbf{C}$ \\
\hline Flat Nickel & $7.90 \mathrm{E}-09$ & $5.45 \mathrm{E}-08$ & $3.97 \mathrm{E}-05$ & $1.20 \mathrm{E}-03$ \\
\hline Template Nickel & $1.58 \mathrm{E}-05$ & $4.10 \mathrm{E}-05$ & $1.67 \mathrm{E}-04$ & $1.52 \mathrm{E}-03$ \\
\hline Nickel Mesh & $4.04 \mathrm{E}-08$ & $2.05 \mathrm{E}-08$ & $2.44 \mathrm{E}-06$ & $1.19 \mathrm{E}-05$ \\
\hline Nickel Foam & & $2.17 \mathrm{E}-07$ & $7.3 \mathrm{E}-07$ & $1.45 \mathrm{E}-05$ \\
\hline Hydrogen Formation & $\mathbf{3 0 0}{ }^{\circ} \mathbf{C}$ & $350^{\circ} \mathbf{C}$ & $\mathbf{4 0 0}{ }^{\circ} \mathbf{C}$ & $\mathbf{4 5 0}{ }^{\circ} \mathbf{C}$ \\
\hline Flat Nickel & $2.01 \mathrm{E}-05$ & $1.35 \mathrm{E}-04$ & $7.93 \mathrm{E}-04$ & $4.04 \mathrm{E}-03$ \\
\hline Template Nickel & $2.66 \mathrm{E}-05$ & $1.56 \mathrm{E}-04$ & $1.03 \mathrm{E}-03$ & $5.02 \mathrm{E}-03$ \\
\hline Nickel Mesh & $3.20 \mathrm{E}-07$ & $1.42 \mathrm{E}-06$ & $4.69 \mathrm{E}-06$ & $2.13 \mathrm{E}-05$ \\
\hline Nickel Foam & & $1.76 \mathrm{E}-06$ & $8.28 \mathrm{E}-06$ & $3.73 \mathrm{E}-05$ \\
\hline Nitrogen Formation & $\mathbf{3 0 0}{ }^{\circ} \mathbf{C}$ & $350^{\circ} \mathbf{C}$ & $400^{\circ} \mathbf{C}$ & $\mathbf{4 5 0}{ }^{\circ} \mathbf{C}$ \\
\hline Flat Nickel & $1.03 \mathrm{E}-05$ & $3.58 \mathrm{E}-05$ & $2.07 \mathrm{E}-04$ & $1.13 \mathrm{E}-03$ \\
\hline Template Nickel & $1.32 \mathrm{E}-05$ & $4.19 \mathrm{E}-05$ & $2.90 \mathrm{E}-04$ & $1.43 \mathrm{E}-03$ \\
\hline Nickel Mesh & $1.07 \mathrm{E}-07$ & $4.73 \mathrm{E}-07$ & $1.56 \mathrm{E}-06$ & $7.11 \mathrm{E}-06$ \\
\hline
\end{tabular}




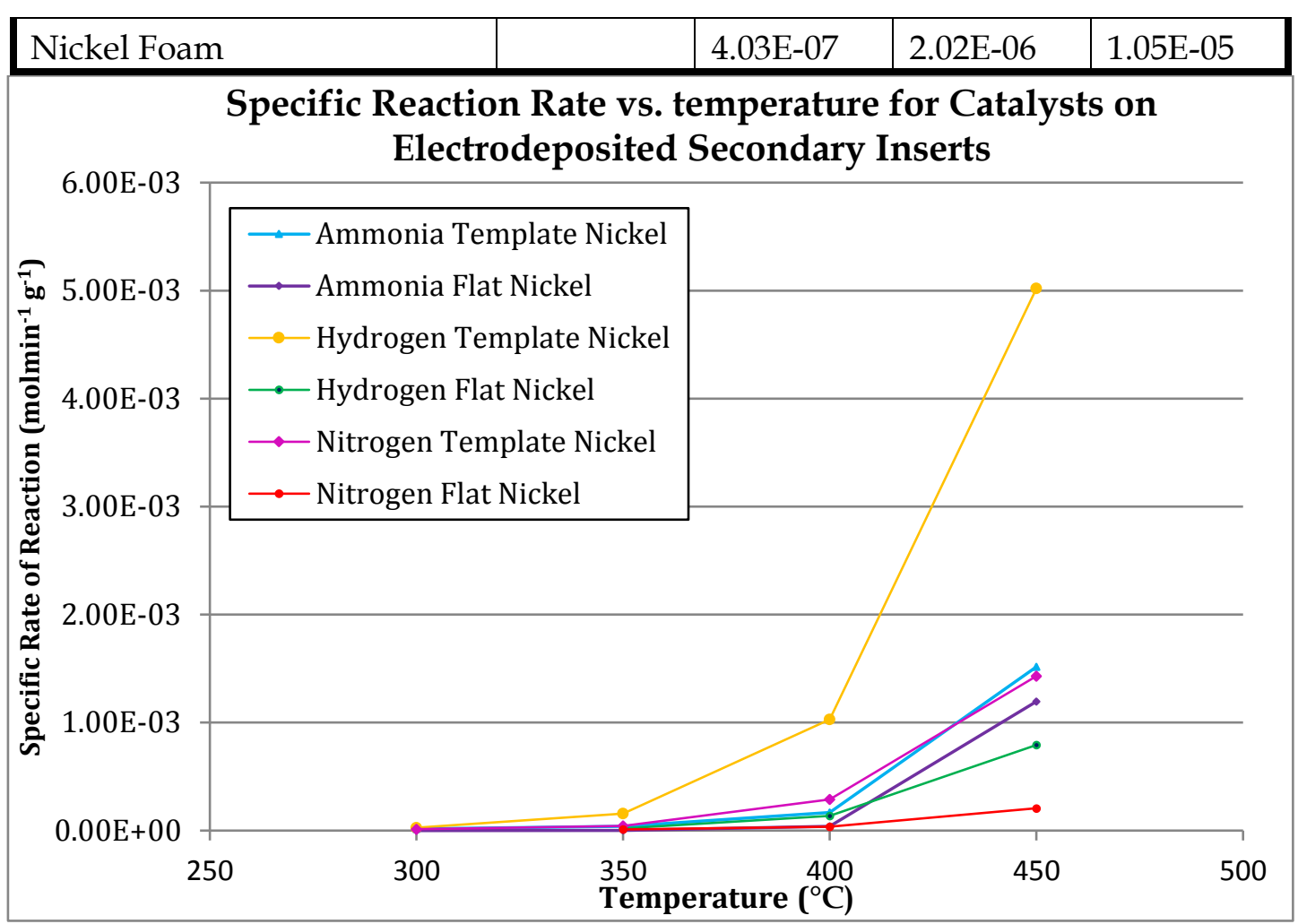

Figure 3.2.16: Graph of Specific Reaction Rate vs. Temperature for electrodeposited catalysts on secondary inserts.

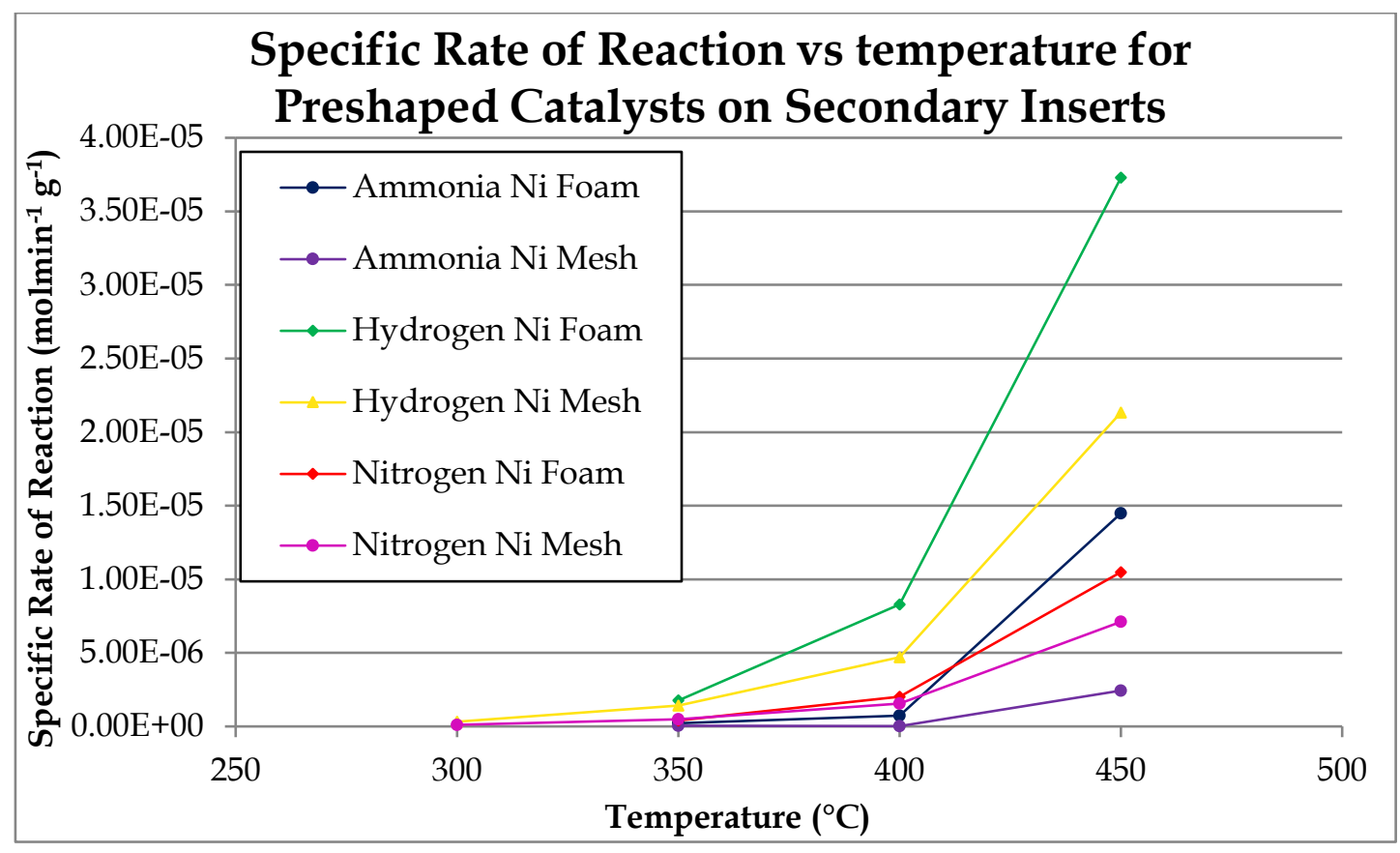

Figure 3.2.17: Graph of Specific Reaction Rate vs. temperature for Preshaped Catalysts on Secondary Inserts.

Podila reports producing 6.2 to $6.6 \times 10^{-3} \mathrm{~mol} \cdot g_{\text {catalyst }}^{-1} \cdot \mathrm{min}^{-1}$ on Co and CoMoN at $600{ }^{\circ} \mathrm{C}$ [65]. The electrodeposited catalysts were close to these values at $450{ }^{\circ} \mathrm{C}$, and particularly the template may have surpassed Podila's values at $600{ }^{\circ} \mathrm{C}$. Further specific rate data that has been reported is $3.35 \times 10^{-}$ 
Jonathan Tailby

${ }^{2} \mathrm{~mol} \cdot g_{\text {catalyst }}^{-1} \cdot \mathrm{min}^{-1}$, for ruthenium on carbon nanotubes at $450-550{ }^{\circ} \mathrm{C}$ [53].

Ruthenium is widely reported to be a better catalyst than nickel, and carbon to be a particularly effective support for ruthenium [19, 53, 69, 70].It is therefore to be expected that ruthenium in its optimum configuration is a more effective catalyst than the nickel in this study which has not yet been optimised, especially when the ruthenium is reacted at $100{ }^{\circ} \mathrm{C}$ higher temperature.

\subsection{Secondary Insert Flow Comparison}

Figure 3.2.18 shows a plot of molar output vs. temperature for three gases and two input flows with the templated nickel secondary input catalyst. The output of each gas is greater for the higher input results. The ammonia is greater because there was more to begin with, and the product gases are greater because the reaction is not at its maximum conversion, therefore a greater availability of gas results in greater conversion.

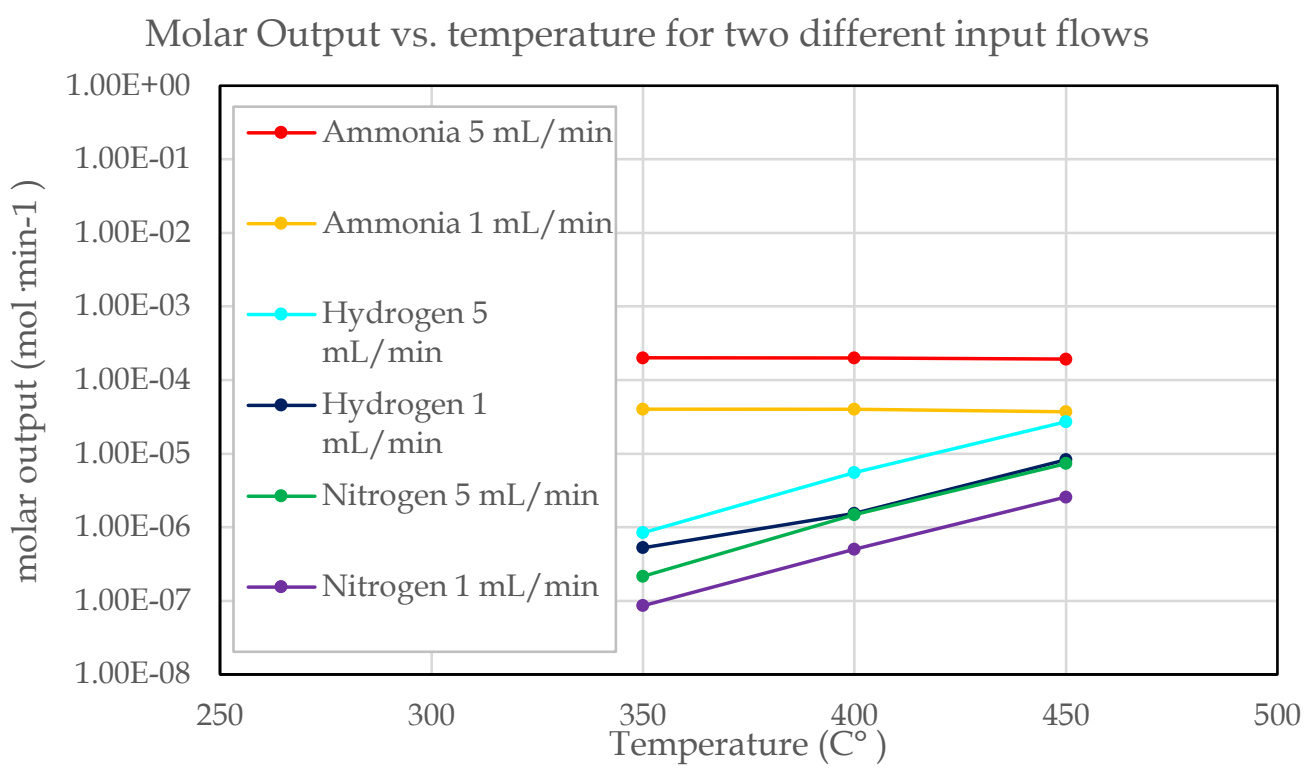

Figure 3.2.18: Plot of molar output vs. temperature for the for three gases at two different input flows with the templated nickel secondary insert catalyst.

Figure 3.2.19 shows a graph of the normalised molar output vs. temperature for the templated nickel secondary catalyst at $1 \mathrm{~mL} \cdot \mathrm{min}^{-1}$ and $5 \mathrm{~mL} \cdot \mathrm{min}^{-1}$. The $1 \mathrm{~mL} \mathrm{~min}^{-1}$ data has been multiplied to allow for the greater availability of ammonia in the $5 \mathrm{~mL} \mathrm{~min}^{-1}$ experiment. This has brought the two ammonia 
outputs too close to be distinguished. The product gases have greater values at low flow under this normalisation. This is consistent with the greater proportion of decomposition at the cost of the lower quantity of decomposition that had been predicted, by analogy to the published work on membrane reactors mentioned in Section 1.2.4.4.4. It was therefore expected that this effect of greater proportion for lesser quantity would become more pronounced in the palladium enhanced catalysts of the tertiary inserts.

\section{Normalised Molar Output vs. temperature for two different input flows}

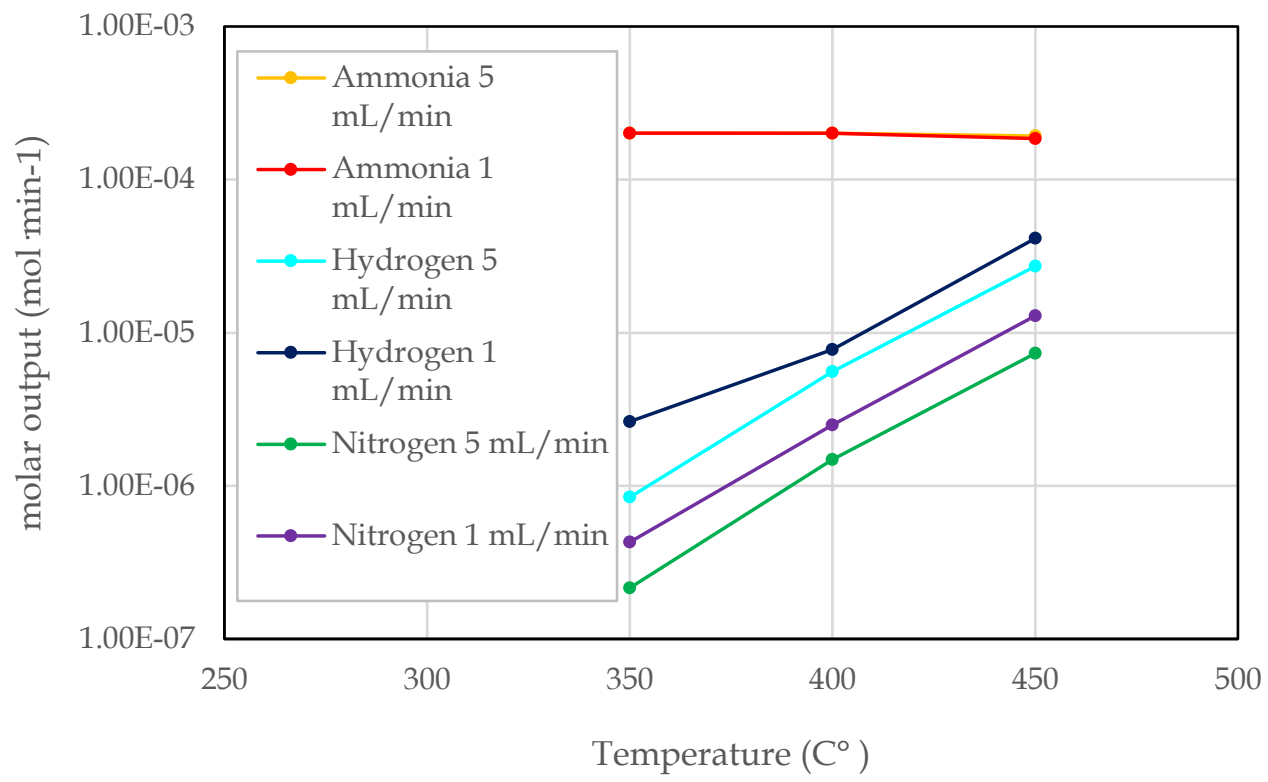

Figure 3.2.19: Graph of normalised molar output vs. temperature for the for three gases at two different input flows with the templated nickel secondary insert catalyst. 
Jonathan Tailby

\subsection{Reaction Testing with Enhanced Catalysts}

The experiments testing the combinations of catalysis with hydrogen permeation and spillover used inserts in the primary insert that resembled the secondary inserts but with extra connections, as discussed in Section 2.2.9.5. Because these tubes represented the secondary insert but with another tube inside, they were labelled tertiary inserts.

\subsubsection{Catalyst with Permeation but no Spillover}

Chromatograms for the retentate from ammonia decomposition experiments with the nickel-before-palladium catalyst at $300,350,400$ and $450{ }^{\circ} \mathrm{C}$ are shown in Figure 5.2.32 in Appendix 5.2.7.4. Table 3.3.1 to Table 3.3.3 summarise the chromatogram data and calculate output quantities for the three gases. These outputs have been used to plot the graphs Figure 3.3.1 and Figure 3.3.2 to compare the effect of the Nickel-before-Palladium catalyst with the secondary insert catalysts.

The palladium membrane after the nickel foam catalyst was able to absorb hydrogen from outside the tertiary insert, where the ammonia decomposition reaction occurred, then permeate it through and desorb it inside the tertiary insert. Inside the tertiary insert the argon sweep gas carried the hydrogen out of the reactor to the GC. Chromatograms for this permeate gas are shown in Figure 5.2.33 in Appendix 5.2.7.4.

The area of the hydrogen peaks increased with temperature which showed increased permeation. Increased temperature can increase the quantity of permeate hydrogen by two mechanisms. The greater proportion of ammonia decomposition at increased temperature increases the partial pressure of hydrogen on the reaction side, thereby increasing the hydrogen partial pressure difference between the two sides of the membrane, which is an important driving force for hydrogen permeation [99]. The second mechanism is acceleration of the diffusion through the membrane by the increased temperature the effect of temperature on a membrane reactor was discussed in Section 1.2.4.4.3. Both partial pressure and temperature are factors in 
Richardson's Equation, Equation 1.2.21, for calculating hydrogen flux through a palladium membrane discussed in Section 1.2.7.3.

Table 3.3.1 shows the average peak areas for the hydrogen in the permeate output from decomposition experiments with permeation at a range of temperatures from 300 to $450^{\circ} \mathrm{C}$. Subsequent lines on the table follow through to calculate a rate of hydrogen output from the permeate side. Sweep gas flow on the permeate side of the membrane was $100 \%$ argon at $5 \mathrm{~mL} \cdot \mathrm{min}^{-1}=2.01 \mathrm{x}$ $10^{-4} \mathrm{~mol} \mathrm{~min}^{-1}$. Ammonia input to the reaction side of the membrane was $100 \%$ ammonia also at $5 \mathrm{~mL} \cdot \mathrm{min}^{-1}=2.01 \times 10^{-4} \mathrm{~mol} \mathrm{~min}^{-1}$.

The percentage of hydrogen in the permeate was calculated using the hydrogen standard, the $5 \%$ hydrogen standard with a peak area of 547.

The hydrogen output in the permeate was calculated using Equation 2.2.7 from Section 2.2.12.3.3. and the molar flow of sweep gas.

\begin{tabular}{|l|l|l|l|l|l|}
\hline \multicolumn{6}{|c|}{$\begin{array}{l}\text { Table 3.3.1: Hydrogen permeate peak areas and calculated values for } \\
\text { temperature series on the Nickel-before-Palladium tertiary insert. }\end{array}$} \\
\hline Temperature & $\mathbf{3 0 0}$ & $\mathbf{3 5 0}$ & $\mathbf{4 0 0}$ & $\mathbf{4 5 0}$ & ${ }^{\circ} \mathrm{C}$ \\
\hline GC Peak & 0.69 & 39.17 & 244.70 & 853.95 & area \\
\hline Percentage & $0.0063 \%$ & $0.36 \%$ & $2.24 \%$ & $7.81 \%$ & $\%$ \\
\hline $\begin{array}{l}\text { Hydrogen } \\
\text { Output }\end{array}$ & $1.27 \times 10^{-08}$ & $7.23 \times 10^{-07}$ & $4.60 \times 10^{-06}$ & $1.70 \times 10^{-05}$ & $\mathrm{~mol} \mathrm{~min}^{-1}$ \\
\hline
\end{tabular}

Table 3.3.2 contains the outcome of the calculations for ammonia output from the Nickel-before-Palladium tertiary insert in the reactor at 300 to $450{ }^{\circ} \mathrm{C}$. The percentages are calculated from a standard of $100 \%$ ammonia measured on the same day as experiment, these standards are listed in the row marked 100\% standard. Ammonia output is calculated using Equation 2.2.11 from Section 2.2.12.3.3. The permeation term for that equation is supplied from Table 3.3.1., initial flow was $2.01 \times 10^{-4} \mathrm{~mol} \mathrm{~min}^{-1}$ and the percentage of ammonia is taken from the preceding line in this table. 


\begin{tabular}{|c|c|c|c|c|c|}
\hline \multicolumn{6}{|c|}{$\begin{array}{l}\text { Table 3.3.2: Calculating ammonia output of the Nickel-before-Palladium } \\
\text { tertiary insert across the temperature series from } 300 \text { to } 450^{\circ} \mathrm{C} \text {. }\end{array}$} \\
\hline Temperature & 300 & 350 & 400 & 450 & ${ }^{\circ} \mathrm{C}$ \\
\hline GC Peak & 4403.4 & 4335.7 & 4210.73 & 3676.2 & area \\
\hline $100 \%$ Standard & 4419.1 & 4365.6 & 4344.4 & 4338.2 & area \\
\hline Percentage & $99.6 \%$ & $99.3 \%$ & $96.9 \%$ & $84.7 \%$ & $\%$ \\
\hline Ammonia Output & $2.01 \times 10^{-4}$ & $2.00 \times 10^{-4}$ & $1.96 \times 10^{-4}$ & $1.77 \times 10^{-4}$ & mol min $^{-1}$ \\
\hline $\begin{array}{l}\text { Ammonia } \\
\text { Decomposition }\end{array}$ & $3.64 \times 10^{-7}$ & $1.05 \times 10^{-6}$ & $5.41 \times 10^{-6}$ & $2.44 \times 10^{-5}$ & $\mathrm{~mol} \mathrm{~min}^{-1}$ \\
\hline
\end{tabular}

Figure 3.3.1 shows graphs of the rate of ammonia decomposition for the Nickel-before-Palladium tertiary insert and for the secondary inserts vs. temperature.

Figure 3.3.1 shows evidence at all temperatures of enhancement in the ammonia decomposition with the Nickel-before-Palladium tertiary insert decomposing more ammonia than the each of the secondary inserts. This fits with literature evidence that hydrogen permeation can enhance the rate of ammonia decomposition by both thermodynamic [101, 162] and kinetic [107, 167] mechanisms. Above $300{ }^{\circ} \mathrm{C}$ the Nickel-before-Palladium tertiary membrane exceeded all the secondary inserts in accelerating ammonia decomposition.

Ammonia Decomposition vs. Temperature for Nickel-Before-

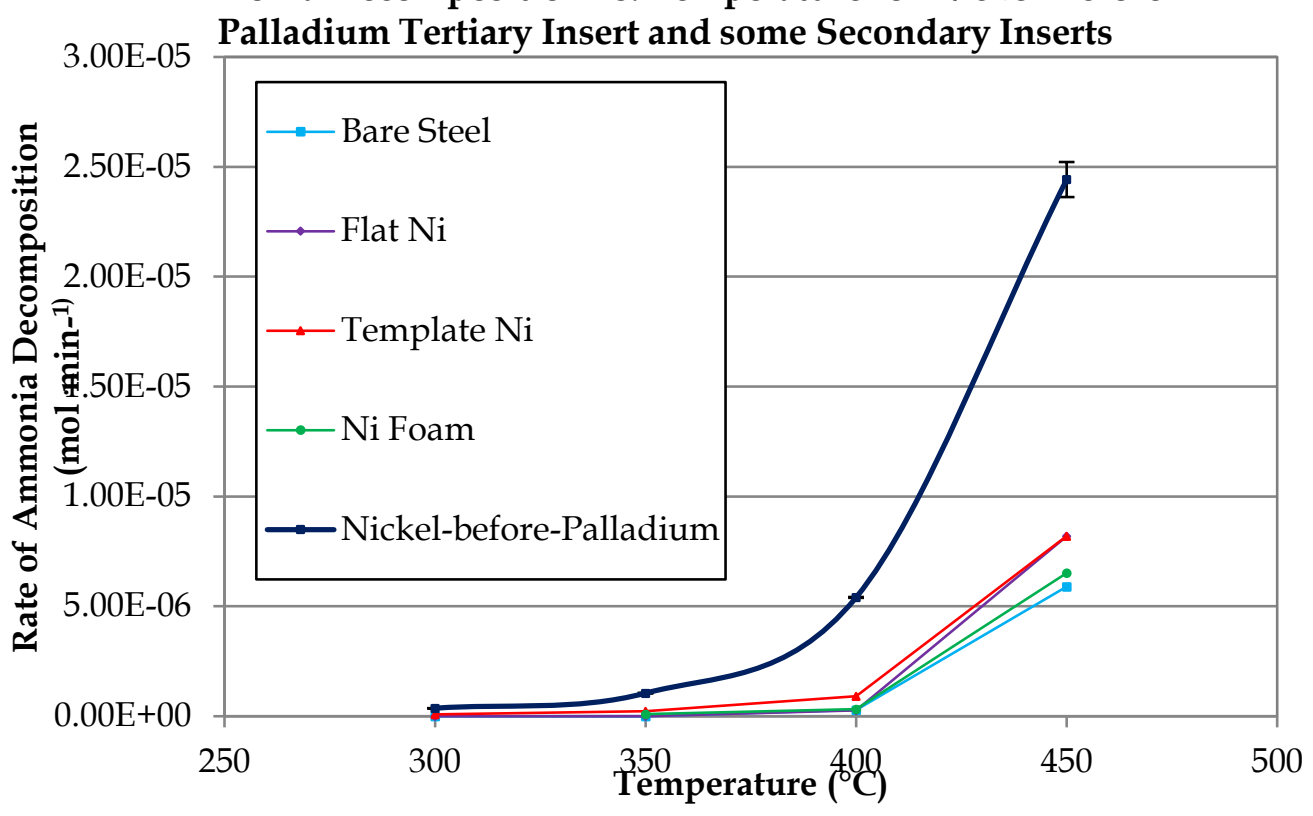

Figure 3.3.1: Rate of ammonia decomposition at $5 \mathrm{~mL} \cdot \mathrm{min}^{-1}$ with four secondary inserts compared with the Nickel-before-Palladium tertiary insert. 
Table 3.3.3 contains the outcome for the calculation of retentate hydrogen output for the Nickel-before-Palladium tertiary insert at $300-450{ }^{\circ} \mathrm{C}$. The GC peak area is the average of peak measurements for these experiments. The standard is the average of all measurements of the 5\% standard from the week these measurements were recorded. The percentage is calculated from the sample peak area and the standard peak area. Hydrogen permeate is taken from Table 3.3.1. The hydrogen retentate is calculated using Equation 2.2.10 from section 2.2.12.3.3. Total hydrogen is calculated as the sum of the permeate and the retentate.

Table 3.3.3: Average peak areas and subsequent calculated values for retentate from ammonia decomposition at 300 to $450{ }^{\circ} \mathrm{C}$ for Nickel-before-Palladium insert.

\begin{tabular}{|c|c|c|c|c|c|}
\hline Temperature & 300 & 350 & 400 & 450 & ${ }^{\circ} \mathrm{C}$ \\
\hline GC Peak & 18.1 & 35.4 & 347.6 & 1205.0 & area \\
\hline $5 \%$ Standard & 536.3 & 536.3 & 536.3 & 536.3 & area \\
\hline Percentage & $0.17 \%$ & $0.33 \%$ & $3.24 \%$ & $11.23 \%$ & $\%$ \\
\hline $\begin{array}{l}\text { Hydrogen } \\
\text { Permeate }\end{array}$ & $1.27 \mathrm{E}-08$ & 7.23E-07 & 4.60E-06 & 1.70E-05 & $\mathrm{mol} \cdot \mathrm{min}^{-1}$ \\
\hline $\begin{array}{l}\text { Hydrogen } \\
\text { Retentate }\end{array}$ & 3.40E-07 & 6.65E-07 & $6.74 \mathrm{E}-06$ & 2.55E-05 & $\mathrm{mol} \cdot \mathrm{min}^{-1}$ \\
\hline $\begin{array}{l}\text { Total Hydrogen } \\
\text { Output }\end{array}$ & 3.53E-07 & 1.39E-06 & $1.13 \mathrm{E}-05$ & 4.25E-05 & $\mathrm{mol} \cdot \mathrm{min}^{-1}$ \\
\hline
\end{tabular}

Figure 3.3.2 shows graphs of the quantity of hydrogen in the gas flow out of the reactor for the Nickel-before-Palladium tertiary insert and for the secondary inserts plotted against temperature. The total hydrogen output of the Nickel-before-Palladium Tertiary insert has exceeded the hydrogen output of every secondary insert at every temperature. 
Rate of Hydrogen Production vs. Temperature for Secondary Inserts and Ni-before-Pd Tertiary Insert

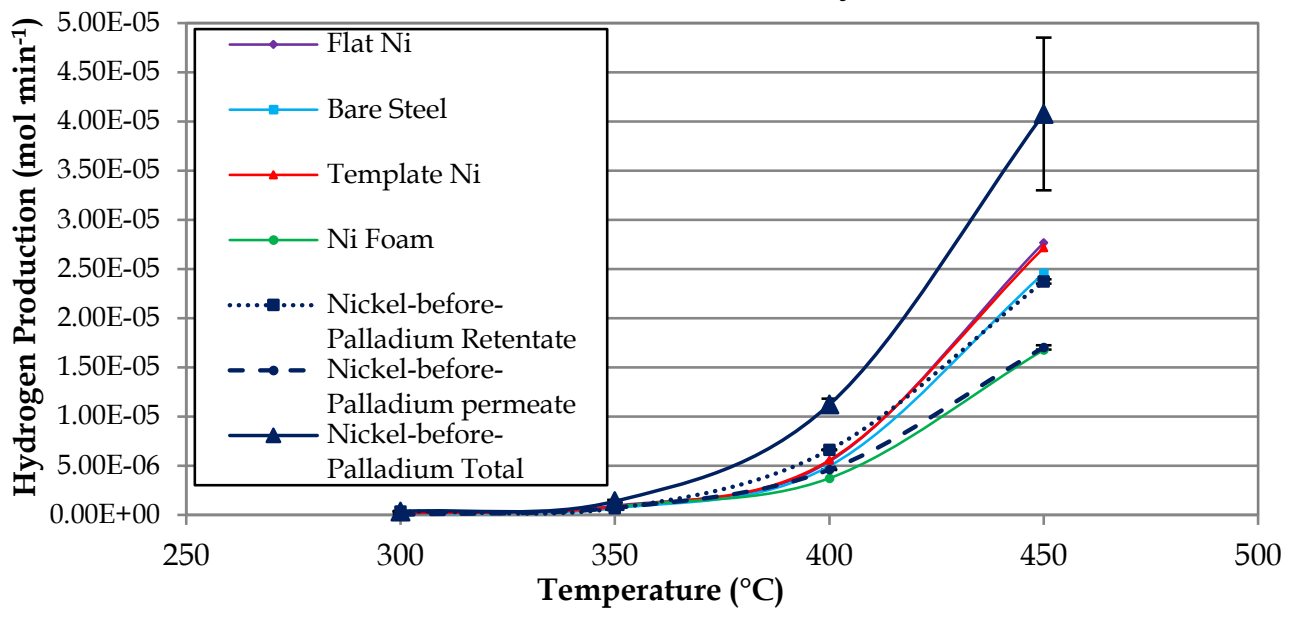

Figure 3.3.2: hydrogen gas flow in the product gas mixture from ammonia

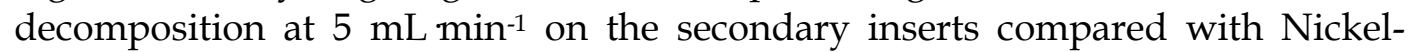
before-Palladium tertiary insert.

The experiments reported in this section have selectively permeated hydrogen from the reaction side of the membrane to the sweep side. Figure 3.3.1 shows that this permeation has increased the ammonia decomposition and Figure 3.3.2 shows that it has increased the quantity of hydrogen produced, however more hydrogen has been retained than has permeated.

\subsubsection{Catalyst with Spillover but no Permeation}

For the tertiary insert experiments the GC column was programmed to hold for three minutes at $30{ }^{\circ} \mathrm{C}$ then increase at $20^{\circ} \mathrm{C} \mathrm{min}-1$ until it reached $100{ }^{\circ} \mathrm{C}$. It held at $100{ }^{\circ} \mathrm{C}$ until 15.5 minutes, when all the ammonia had left the column, then decreased at $20^{\circ} \mathrm{C} \cdot \mathrm{min}^{-1}$ to $30^{\circ} \mathrm{C}$. The baseline signal was affected by additional noise while the temperature was changing, this effect settled down when the temperature became steady again. There was small variation between 3 and 6.5 minutes while the column heated, some of which can be seen in Figure 5.2.34d (in Appendix 5.2.7) between 3 and 4 minutes; the variation when the column cooled again after 15.5 minutes was much more significant.

Figure 5.2.34 in Appendix 5.2.7.4 shows a typical TCD chromatogram series from the temperature comparison series for the Nickel-on-Palladium-noPermeation tertiary insert. Each chromatogram shows 16 minutes of a 21 
minute injection cycle. Table 3.3.4 summarises the peak area measurements for this temperature series of ammonia decomposition experiments with the Nickel-on-Palladium-no-Permeation and shows derived values calculated from those measurements.

\begin{tabular}{|c|c|c|c|c|c|}
\hline Temperature & 300 & 350 & 400 & 450 & ${ }^{\circ} \mathrm{C}$ \\
\hline Permeate Peak & 0 & 0 & 0 & 0 & area \\
\hline Ammonia Peak & 4293.4 & 4371.2 & 4147.4 & 3534.6 & area \\
\hline Ammonia Standard & 4450.8 & 4450.8 & 4450.8 & 4450.8 & area \\
\hline Ammonia Percent & $96.46 \%$ & $97.91 \%$ & $93.18 \%$ & $78.45 \%$ & $\%$ \\
\hline Ammonia Flow & $2.01 \times 10^{-4}$ & $2.01 \times 10^{-4}$ & $2.00 \times 10^{-4}$ & $1.92 \times 10^{-4}$ & mol min-1 \\
\hline $\begin{array}{l}\text { Ammonia } \\
\text { Decomposition }\end{array}$ & $2.51 \times 10^{-7}$ & $8.82 \times 10^{-8}$ & $9.35 \times 10^{-7}$ & $9.34 \times 10^{-6}$ & mol min-1 \\
\hline Retentate Peak & 41.2 & 104.9 & 528.4 & 1948.1 & area \\
\hline Hydrogen Standard & 547.1 & 548.8 & 547.1 & 549.5 & area \\
\hline Hydrogen Percent & $0.38 \%$ & $0.96 \%$ & $4.83 \%$ & $17.72 \%$ & $\%$ \\
\hline Hydrogen Output & $7.60 \times 10^{-7}$ & $1.93 \times 10^{-6}$ & $1.00 \times 10^{-5}$ & $3.99 \times 10^{-5}$ & $\mathrm{~mol} \mathrm{~min}^{-1}$ \\
\hline Nitrogen Peak & 1.6 & 4.0 & 17.6 & 82.4 & area \\
\hline Nitrogen Standard & 971.3 & 967.6 & 965.4 & 971.3 & area \\
\hline Nitrogen Percent & $0.16 \%$ & $0.39 \%$ & $1.73 \%$ & $8.06 \%$ & $\%$ \\
\hline Nitrogen Output & $3.21 \times 10^{-7}$ & $7.95 \times 10^{-7}$ & $3.59 \times 10^{-6}$ & $1.88 \times 10^{-5}$ & mol min-1 \\
\hline
\end{tabular}

Figure 3.3.3 shows graphs of the quantity of hydrogen produced by the secondary insert catalysts and the Nickel-on-Palladium-no-Permeation tertiary catalyst plotted against temperature. These results show that the Nickel-on-Palladium-no-Permeation insert produced more hydrogen at each temperature than any of the secondary inserts, even compared with the template nickel deposited secondary insert.

The improvement of the nickel foam from a low performing catalyst without palladium to the best in this series when in contact with palladium is explained by hydrogen spillover, a mechanism that has been suggested elsewhere in the literature [63, 168, 169]. An alternative explanation such as electronic modification of the nickel by palladium is unlikely given the macroscopic scale of the contact between the two metals. The hydrogen atoms that formed on the nickel foam during ammonia decomposition would have been able to spillover to the palladium. Hydrogen will have desorbed more quickly from 
Jonathan Tailby

the palladium surface because of the lower activation energy of associative desorption on palladium $\left(41.8 \mathrm{~kJ} \mathrm{~mol}^{-1}\right.$ [112]) than on nickel $\left(109.0 \mathrm{~kJ} \mathrm{~mol}^{-1}\right.$ [83]). There is also evidence in the literature that spillover hydrogen may be more loosely bound to the surface than hydrogen formed in situ [170].

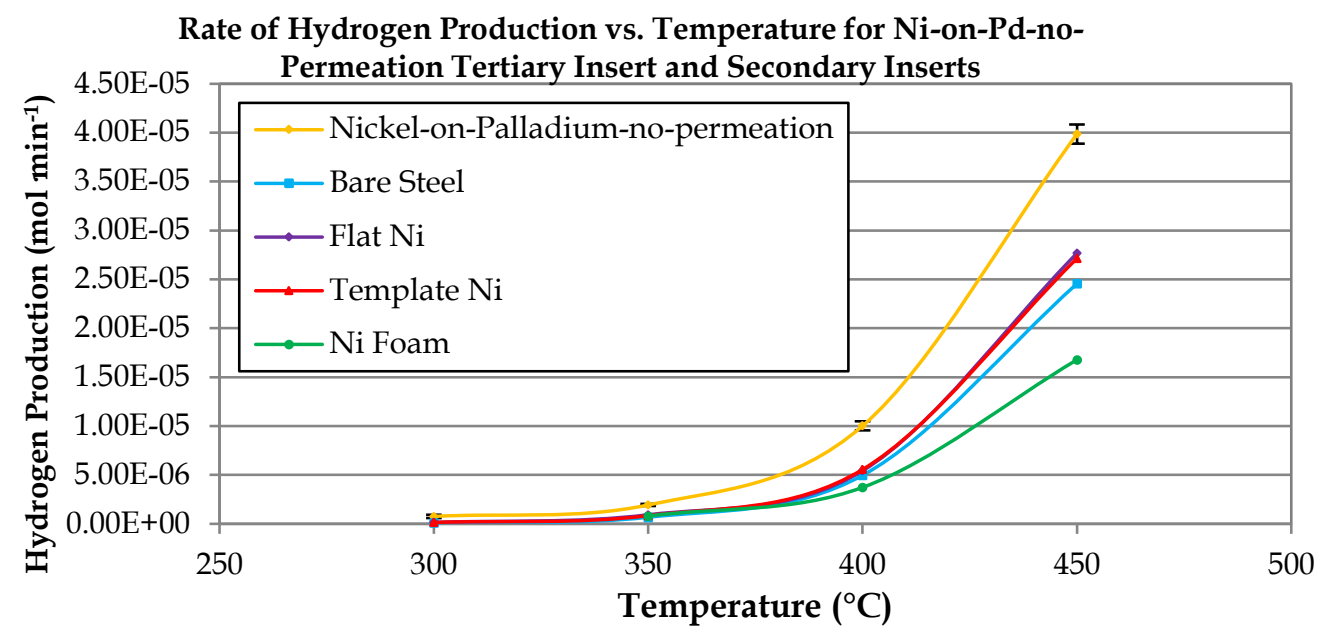

Figure 3.3.3: Hydrogen from decomposition of ammonia on four secondary inserts and the nickel foam catalyst with spillover.

Nickel-on-Palladium-no-Permeation produced more hydrogen at all four temperatures tested.

Figure 3.3.4 shows graphs of the ammonia decomposition against temperature for the secondary insert catalysts and the Nickel-on-Palladium-withoutPermeation tertiary insert. The Nickel-on-Palladium was superior to the nickel foam secondary catalyst in terms of ammonia decomposition at all temperatures. It was equal or superior to the templated electrodeposits at every temperature. This was interpreted as the result of hydrogen spillover from nickel catalyst to the palladium accelerating the rate at which the hydrogen vacated the active sites on the nickel, increasing the effectiveness of the catalyst $[168,171]$. 


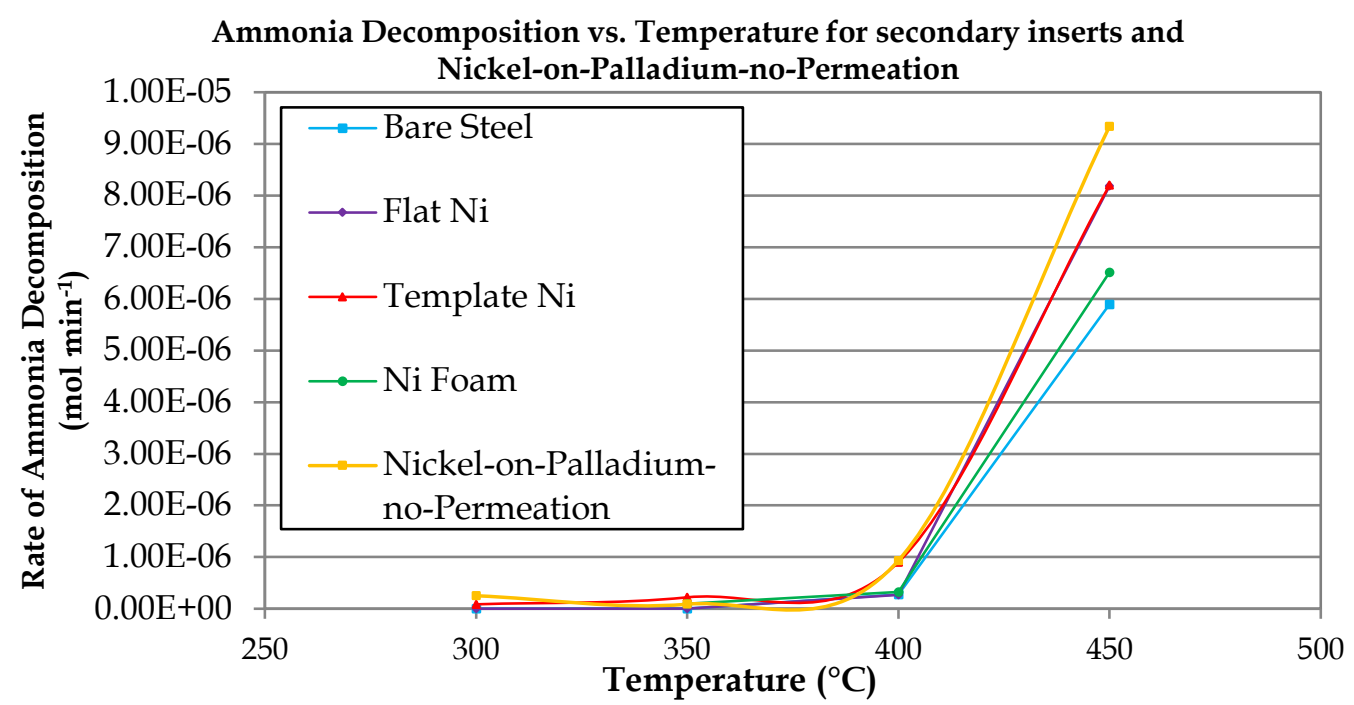

Figure 3.3.4: Ammonia decomposition with Nickel-on-Palladium-no-Permeation compared with percentage of ammonia decomposed with secondary inserts.

Research in other groups has shown that acceleration of catalysts by platinum or palladium is sometimes associated with alteration of the electronic properties; for example, the special catalytic properties of nickel-palladium particles in hydrogenation reactions published by Leppert, L., R. Kempe, and S. Kümmel [172]. In that case the palladium and nickel atoms were intermingled in the model structures so that the two metals were in much closer association than the nickel foam pressed to the palladium foil. In the experiments reported here, therefore, the same electronic interaction is not expected and spillover is a better explanation of the improved reactivity of the catalyst. The palladium-nickel catalysts in the reference [172] were being utilised for hydrogenation reactions, effectively the opposite of ammonia decomposition in which hydrogen is removed from the nitrogen atoms. It is already recorded in the literature that the optimum catalyst for a reaction is not always the optimum for the reverse. For example, the ruthenium catalysts that are the current state of the art for ammonia decomposition are not as highly effective for ammonia synthesis because of changes in the binding energies of hydrogen and nitrogen at different hydrogen to ammonia ratios [67]. 
Jonathan Tailby

A second set of experiments was conducted with the Nickel-on-Palladium-noPermeation insert, which was a flow experiment where the temperature was maintained at $350{ }^{\circ} \mathrm{C}$ while the flow rate of the ammonia into the gas reactor varied through 1,3 , and $5 \mathrm{~mL} \cdot \mathrm{min}^{-1}$.

Figure 5.2.43 shows typical TCD chromatograms for the flow experiments with the Nickel-on-Palladium-no-Permeation insert. Decreasing the rate of ammonia flow was found to decrease the proportion of ammonia in the remnant gas, and to increase the proportion of product gases, however the rate of ammonia decomposition decreased because more reactant was available.

\subsubsection{Catalyst with Spillover and Hydrogen Permeation}

The goal of this research was to produce and test a catalytic spillover membrane for ammonia decomposition. The 'Nickel-on-Palladium' inserts reported on in this section were that product and these experiments reveal that they were effective in achieving their purpose.

\subsubsection{Temperature Series}

The first comparison sets that were measured with the tertiary insert with the nickel foam in contact with a palladium permeation membrane were temperature sets for both retentate and permeate. Retentate chromatograms for the full $300-450{ }^{\circ} \mathrm{C}$ temperature range are shown in Figure 5.2.35 in Appendix 5.2.7.4. The product peaks increased when the temperature was increased but the ammonia peak decreased when the temperature was increased.

Chromatograms for the permeate from the full range of experiments in the temperature series are shown in

Figure 5.2.36 in Appendix 5.2.7.4. Each chromatogram records eight threeminute injection cycles. The quantity of hydrogen that permeated increased with temperature. This was expected because hydrogen production increases with temperature and literature models of permeation show the rate increased with increased temperature. 
Table 3.3.5 contains a summary of the peak area measurements from the ammonia decomposition experiments at $300-450{ }^{\circ} \mathrm{C}$ and calculates output rates for each gas on the Nickel-on-Palladium catalytic spillover membrane tertiary insert. This confirms that the products increased and the ammonia decreased with temperature.

Table 3.3.5: Peak area summary with calculated values for ammonia decomposition at $300-450{ }^{\circ} \mathrm{C}$ on the Nickel-on-Palladium tertiary insert.

\begin{tabular}{|c|c|c|c|c|c|}
\hline Temperature & 300 & 350 & 400 & 450 & ${ }^{\circ} \mathrm{C}$ \\
\hline \multirow{4}{*}{$\begin{array}{l}\text { Permeate Peak } \\
\text { Hydrogen } \\
\text { Standard } \\
\text { Permeate Percent } \\
\text { Rate of Permeation }\end{array}$} & 12.4 & 132.2 & 732.1 & 2681.5 & area \\
\hline & 501.2 & 516.7 & 522.4 & 538.0 & area \\
\hline & $0.1 \%$ & $1.3 \%$ & $7.0 \%$ & $24.9 \%$ & $\%$ \\
\hline & 2.49E-07 & $2.59 \mathrm{E}-06$ & $1.46 \mathrm{E}-05$ & $5.76 \mathrm{E}-05$ & mol 1 \\
\hline \multirow{5}{*}{$\begin{array}{l}\text { Ammonia Peak } \\
\text { Ammonia } \\
\text { Standard } \\
\text { Ammonia Percent } \\
\text { Ammonia Flow } \\
\text { Ammonia } \\
\text { Decomposition }\end{array}$} & 4430.7 & 4327.6 & 4050.5 & 3038.1 & area \\
\hline & 4441.6 & 4419.2 & 4399.7 & 43 & $\operatorname{arc}$ \\
\hline & 99.76 & $97.93 \%$ & $91.41 \%$ & 69.05 & $\%$ \\
\hline & $2.01 \mathrm{E}-04$ & $1.98 \mathrm{E}-04$ & $1.90 \mathrm{E}-04$ & $1.59 \mathrm{E}-04$ & $\mathrm{~mol} \mathrm{r}$ \\
\hline & $3.02 \mathrm{E}-07$ & $2.64 \mathrm{E}-06$ & $1.06 \mathrm{E}-05$ & 4.18E-05 & mol min-1 \\
\hline Retentate Peak & 12.4 & 132.2 & 32.1 & 2681.5 & area \\
\hline \multirow{2}{*}{$\begin{array}{l}\text { Hydrogen Percent } \\
\text { Hydrogen Output }\end{array}$} & $0.1 \%$ & $1.3 \%$ & $7.0 \%$ & $24.9 \%$ & $\%$ \\
\hline & $2.49 \mathrm{E}-\mathrm{C}$ & $2.59 \mathrm{E}-0$ & $1.46 \mathrm{E}-05$ & $5.76 \mathrm{E}-05$ & $\mathrm{~mol} \mathrm{t}$ \\
\hline \multirow{4}{*}{$\begin{array}{l}\text { Nitrogen Peak } \\
\text { Nitrogen Standard } \\
\text { Nitrogen Percent } \\
\text { Nitrogen Output }\end{array}$} & 1.3 & 7.1 & 39.9 & 152.1 & area \\
\hline & 965.2 & 967.4 & 965.3 & 958.1 & area \\
\hline & $0.13 \%$ & $0.69 \%$ & $3.93 \%$ & $15.08 \%$ & $\%$ \\
\hline & $2.64 \mathrm{E}-07$ & $1.41 \mathrm{E}-06$ & 8.37E-06 & 3.97E-05 & mol min $^{-1}$ \\
\hline
\end{tabular}

\subsection{Temperature Series Summary}

Figure 3.3.5 shows the amount of ammonia in the retentate graphed against temperature for the three different tertiary inserts. The gas that entered the reactor was one hundred percent ammonia at $2.01 \times 10^{-4} \mathrm{~mol} \mathrm{~min}^{-1}$ in all cases, all of the decrease is assumed to have been caused by decomposition of ammonia. 


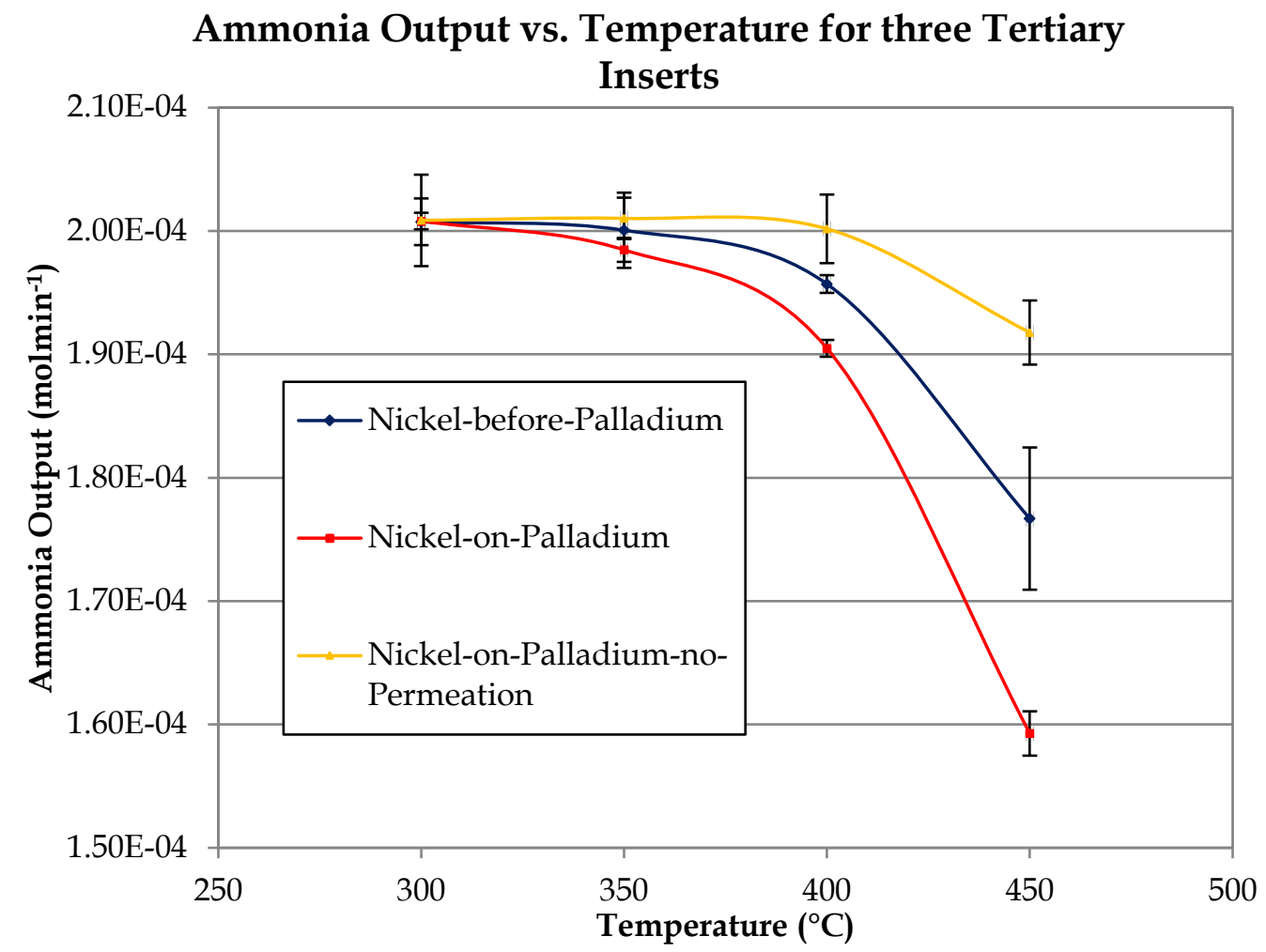

Figure 3.3.5: The ammonia which remained in the retentate after decomposition plotted against temperature.

The difference between the output ammonia and the input is graphed as decomposition of ammonia in Figure 3.3.6. The difference between the three tertiary inserts is magnified from the previous graph because of the change in scale. At $300{ }^{\circ} \mathrm{C}$ all three tertiary inserts had a negligible amount of decomposition, from 350 to $450{ }^{\circ} \mathrm{C}$ the catalytic spillover membrane decomposed the most ammonia while the Nickel-on-Palladium-noPermeation decomposed least and the Nickel-before-Palladium was intermediate of the other two. These results show that both spillover and permeation through a palladium membrane can enhance catalytic decomposition of ammonia across a range of temperatures.

Figure 3.3.6 shows that individually, permeation had greater effect on decomposition than spillover, but the combined effect was greater than either separate phenomenon. 
Ammonia Decomposition vs. Temperature for three Tertiary

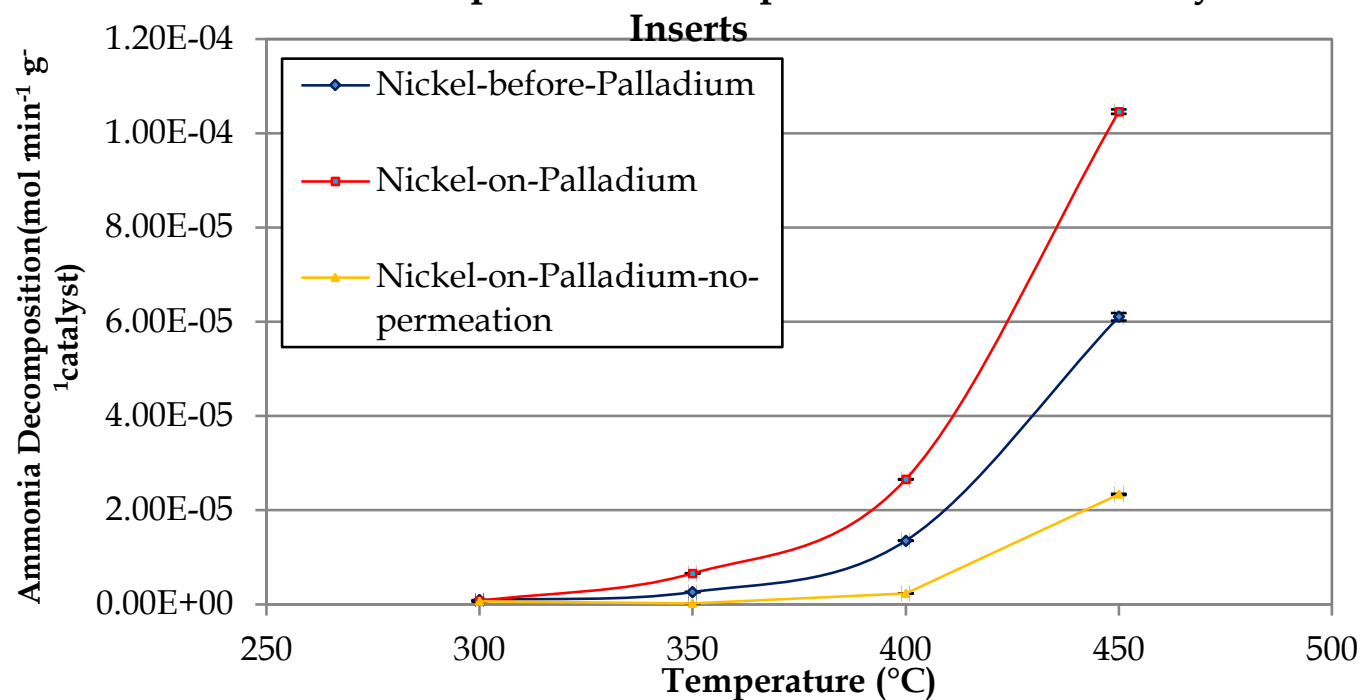

Figure 3.3.6: The rate of ammonia decomposition graphed against temperature for the three tertiary inserts.

Figure 3.3.7 shows the retentate hydrogen output of the tertiary reactors plotted against temperature, all tertiary catalysts have produced more hydrogen as the temperature increased with all three having negligible production at 300 ${ }^{\circ} \mathrm{C}$. Nickel-on-Palladium was the most effective across the range of temperatures measured above $300{ }^{\circ} \mathrm{C}$. The Nickel-on-Palladium-noPermeation had the second highest retentate hydrogen production above 300 ${ }^{\circ} \mathrm{C}$. This suggests that spillover is a more influential phenomenon than permeation for retentate hydrogen production.

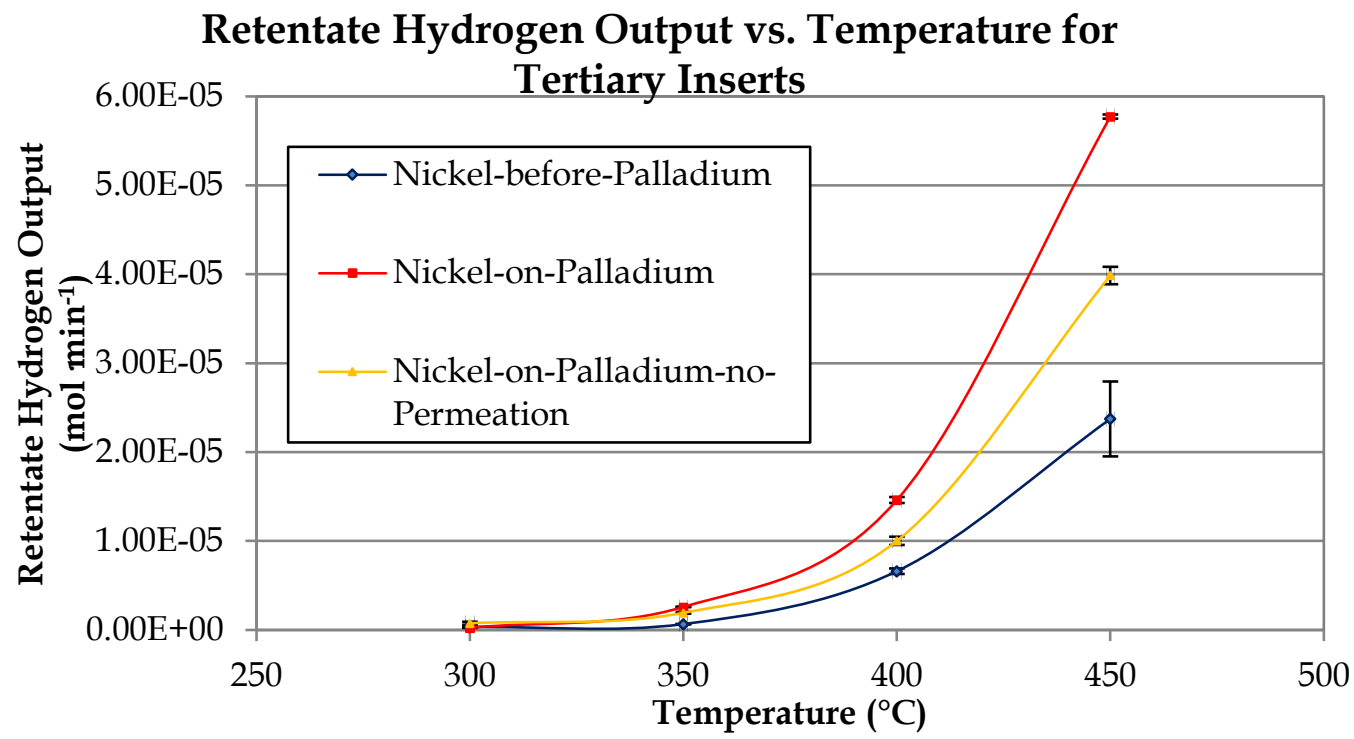

Figure 3.3.7: Hydrogen output in the retentate of the tertiary reactors plotted against temperature. 
Figure 3.3.8 is a plot of hydrogen permeation against temperature for the tertiary inserts. The nickel foam without permeation was designed to prevent permeation and is included here to confirm that no hydrogen permeated. At $350{ }^{\circ} \mathrm{C}$ the Nickel-on-Palladium has greater hydrogen permeation than the Nickel-before-Palladium, however at 400 and $450{ }^{\circ} \mathrm{C}$ the hydrogen permeation of the Nickel-before-Palladium exceeds that of the Nickel-on-Palladium. This gives evidence that the combination of spillover and permeation can be better than permeation alone in certain conditions, however at the higher temperatures spillover seems to have increased retentate hydrogen more than permeate.

Figure 3.3.9 shows a graph of hydrogen flux through the palladium membrane vs. temperature for the tertiary inserts. The hydrogen permeation values shown in Figure 3.3.8 were divided by the area of the palladium membrane, minutes converted to seconds, and hydrogen molecules converted to atoms to calculate flux in moles of hydrogen atoms per second per square centimetre. The Nickel-before-Palladium membrane had a greater palladium area because the membrane of the Nickel-on-Palladium membrane was deeper set in the insert tube, giving it a smaller diameter. Both inserts appear to have exceeded the models of Ward and Dao above $300{ }^{\circ} \mathrm{C}$ [112]. Those models predict hydrogen diffusion in the bulk palladium of the membrane to be rate limiting for membranes of this thickness above $137^{\circ} \mathrm{C}$. That model even assumes a partial pressure difference of 0.5 bar, greater than could occur inside the reactor for these experiments, which would predict a lower flux for these experiments. The zero flux of the Nickel-on-Palladium-no-Permeation insert has been omitted from Figure 3.3.9. 


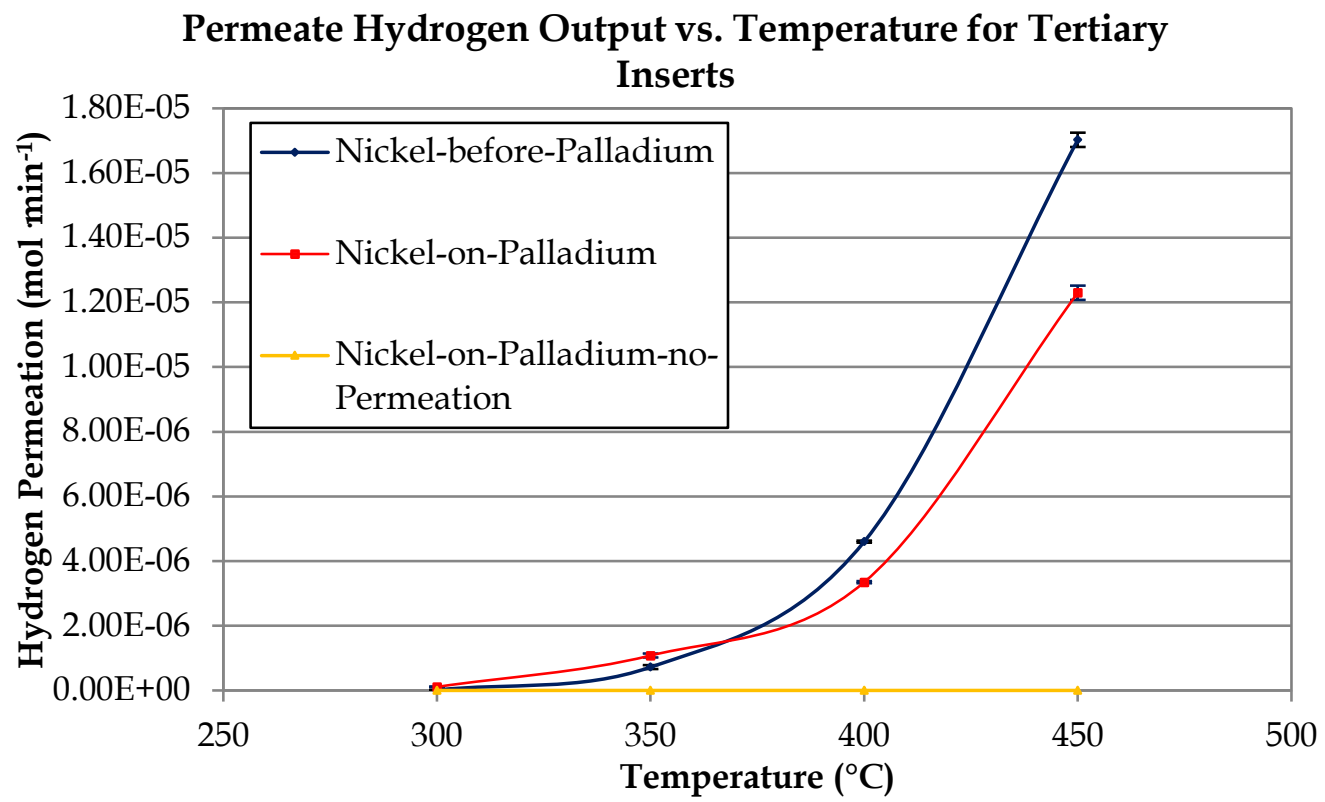

Figure 3.3.8: Hydrogen permeation against temperature for tertiary inserts.

Hydrogen Flux vs. Temperature for Tertiary Inserts

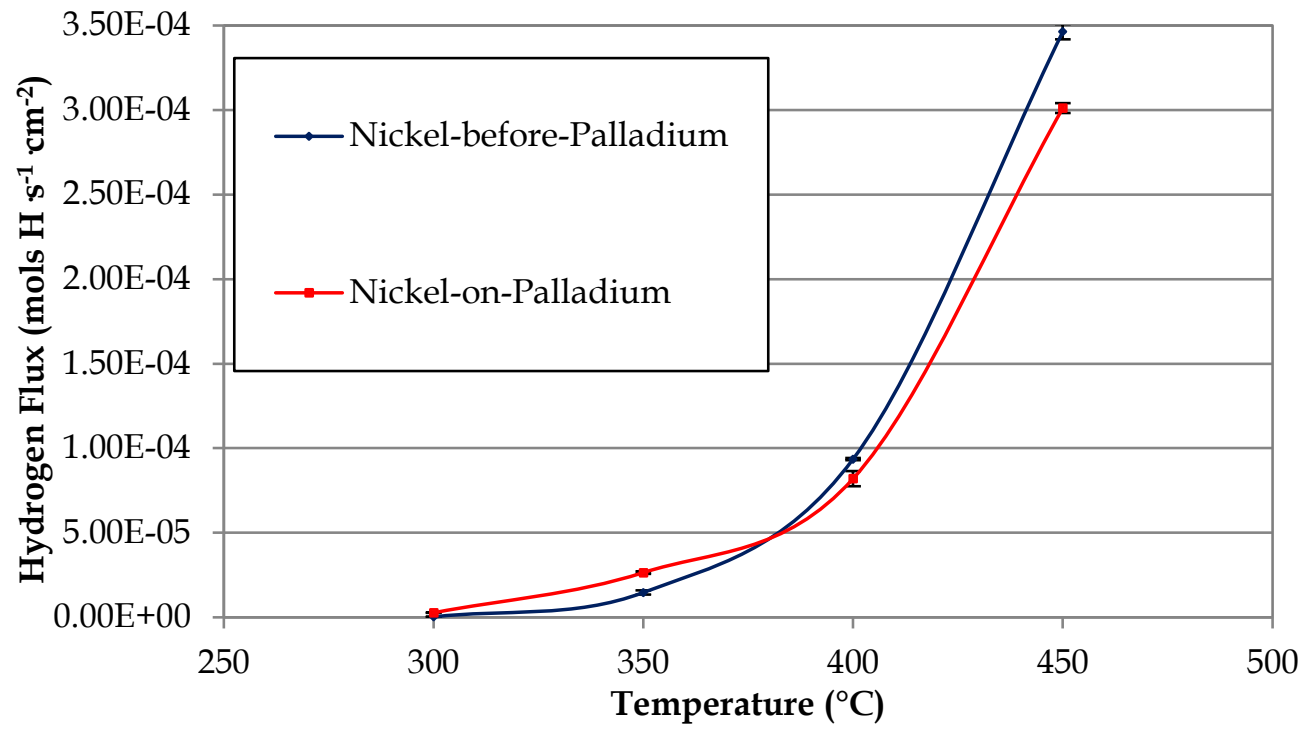

Figure 3.3.9: Hydrogen flux vs. temperature for tertiary inserts.

The permeation shown in Figure 3.3.8 has been turned into flux relative to the palladium area.

The nickel foam on the catalytic spillover membrane had greater linear compression than the nickel foam before the palladium membrane. The palladium upstream from the nickel catalyst would have been less active in permeation than the palladium downstream from the nickel, it is possible that this upstream palladium was completely inactive. Table 3.3.6 shows the palladium surface area for the two permeating tertiary inserts. On this table 
Jonathan Tailby

$3 b^{*}$ represents Nickel-on-Palladium with a reduced area that assumes that the upstream palladium is inactive and calculates only for the area of palladium downstream from nickel. This reduced area results in a new, higher, value for flux as shown in Table 3.3.6. $3 b^{*}$. This new flux value shows that spillover clearly increased the rate of hydrogen flux at temperatures greater than 300 ${ }^{\circ} \mathrm{C}$, as shown in Figure 3.3.10.

\begin{tabular}{|c|c|c|c|c|c|c|c|c|}
\hline & $\begin{array}{l}\text { OD } \\
\mathrm{mm}\end{array}$ & $\begin{array}{l}\text { circumference } \\
\text { mm }\end{array}$ & $\begin{array}{l}\text { length } \\
\mathrm{mm}\end{array}$ & $\begin{array}{l}\text { area } \\
\text { mm }^{2}\end{array}$ & $\begin{array}{l}\text { area } \\
\mathrm{cm}^{2}\end{array}$ & & $\begin{array}{l}\mathrm{mm}^{2} \\
\text { Nickel }\end{array}$ & $\mathrm{cm}^{2}$ \\
\hline $\begin{array}{l}\text { Ni- } \\
\text { before- } \\
\text { Pd }\end{array}$ & 9.21 & 28.9 & 20.2 & 585 & 5.85 & $3 b^{*}$ & & \\
\hline $\begin{array}{l}\text { Ni-on- } \\
\text { Pd }\end{array}$ & 7.79 & 24.4 & 20.2 & 494 & 4.94 & $\begin{array}{l}\text { active } \\
\text { area }\end{array}$ & 233 & 2.33 \\
\hline
\end{tabular}

\begin{tabular}{|c|c|c|c|c|c|c|}
\hline & Temperature: & 300 & 350 & 400 & 450 & ${ }^{\circ} \mathrm{C}$ \\
\hline \multirow[t]{2}{*}{ Ni-before-Pd } & Permeate & $1.26 \mathrm{E}-08$ & 7.23E-07 & $4.6 \mathrm{E}-06$ & $1.7 \mathrm{E}-05$ & $\mathrm{~mol} \cdot \mathrm{min}^{-1}$ \\
\hline & Permeation & 2.15E-09 & $1.22 \mathrm{E}-07$ & $7.8 \mathrm{E}-07$ & $2.89 \mathrm{E}-06$ & $\mathrm{~mol} \mathrm{~min}^{-1} \mathrm{~cm}^{-2}$ \\
\hline Ni-before-Pd & Flux & $2.58 \mathrm{E}-07$ & 1.47E-05 & $9.36 \mathrm{E}-05$ & $3.46 \mathrm{E}-04$ & $\mathrm{~mol}_{\mathrm{H}} \mathrm{s}^{-1} \cdot \mathrm{cm}^{-2}$ \\
\hline \multirow[t]{2}{*}{ Ni-on-Pd } & Permeate & $1.10 \mathrm{E}-07$ & $1.08 \mathrm{E}-06$ & $3.35 \mathrm{E}-06$ & $1.23 \mathrm{E}-05$ & $\mathrm{~mol} \cdot \mathrm{min}^{-1}$ \\
\hline & Permeation & 2.25E-08 & $2.21 \mathrm{E}-07$ & $6.83 \mathrm{E}-07$ & $2.51 \mathrm{E}-06$ & $\mathrm{~mol} \min ^{-1} \mathrm{~cm}^{-2}$ \\
\hline & $\begin{array}{l}\text { Flux, full } \\
\text { membrane }\end{array}$ & 2.70E-06 & $2.65 \mathrm{E}-05$ & 8.2E-05 & 3.01E-04 & $\mathrm{mol}_{\mathrm{H}} \mathrm{s}^{-1} \cdot \mathrm{cm}^{-2}$ \\
\hline Ni-on-Pd & \begin{tabular}{|l} 
Permeation, \\
reduced \\
membrane
\end{tabular} & 4.72E-08 & 4.62E-07 & 1.43E-06 & $5.26 \mathrm{E}-06$ & $\mathrm{~mol} \mathrm{~min}^{-1} \mathrm{~cm}^{-2}$ \\
\hline Ni-on-Pd & $\begin{array}{l}\text { Flux, reduced } \\
\text { membrane }\end{array}$ & 5.66E-06 & $5.54 \mathrm{E}-05$ & $1.72 \mathrm{E}-04$ & 6.31E-04 & $\mathrm{mol}_{\mathrm{H}} \mathrm{s}^{-1} \cdot \mathrm{cm}^{-2}$ \\
\hline $\begin{array}{l}\text { Ni-on-Pd no } \\
\text { permeation }\end{array}$ & Permeation & $x$ & $\mathrm{x}$ & $\mathrm{x}$ & $\mathrm{x}$ & \\
\hline
\end{tabular}




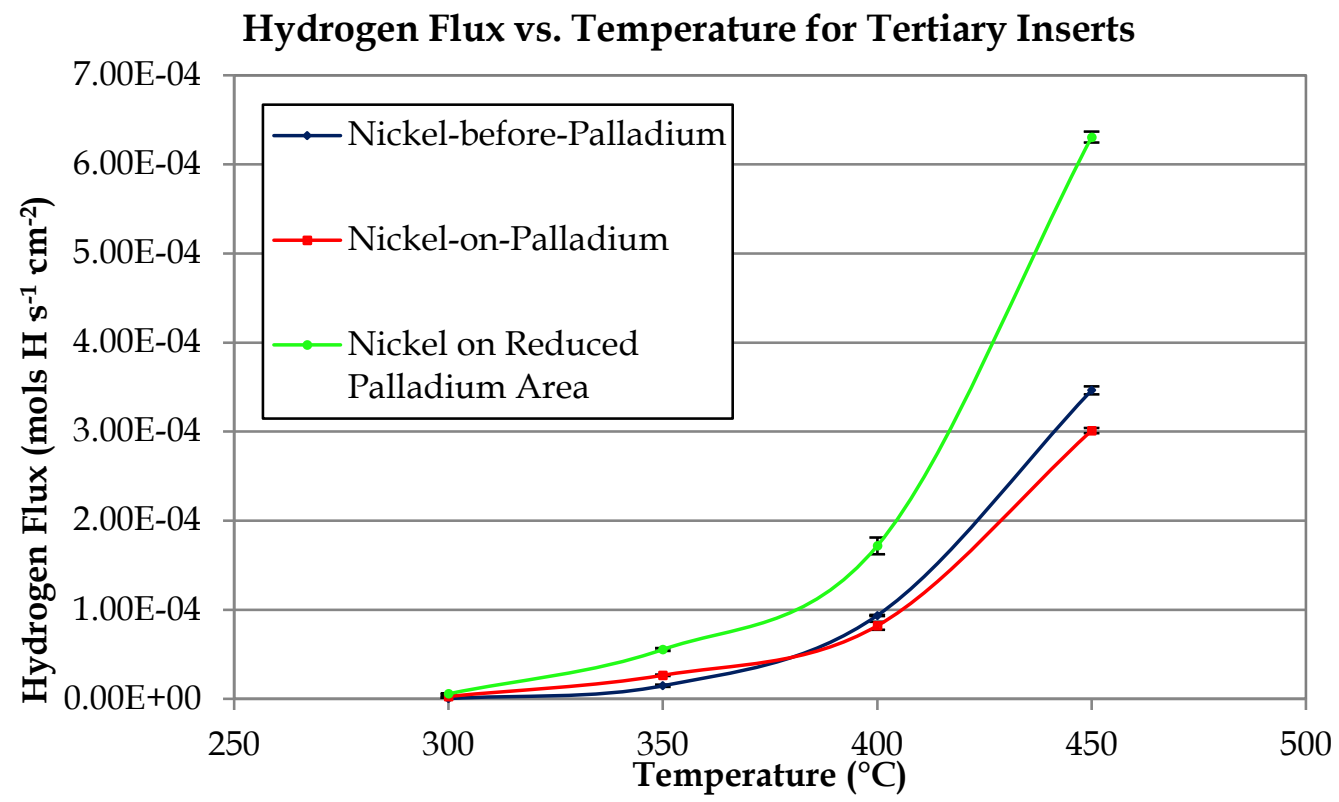

Figure 3.3.10: Hydrogen Flux plotted against reaction temperature for tertiary inserts.

The red line represents the flux for the Nickel-on-Palladium catalytic spillover membrane calculated for the full palladium area and the green line calculates the flux for only the area after nickel catalyst.

Figure 3.3.11 shows the graphs of retentate and permeate hydrogen on the same axes. This shows that the hydrogen produced was more likely to be retained than to permeate for both the Nickel-on-Palladium and the Nickel-beforePalladium, however the difference between the retentate and permeate was greater for the Nickel-on-Palladium than for the Nickel-before-Palladium.

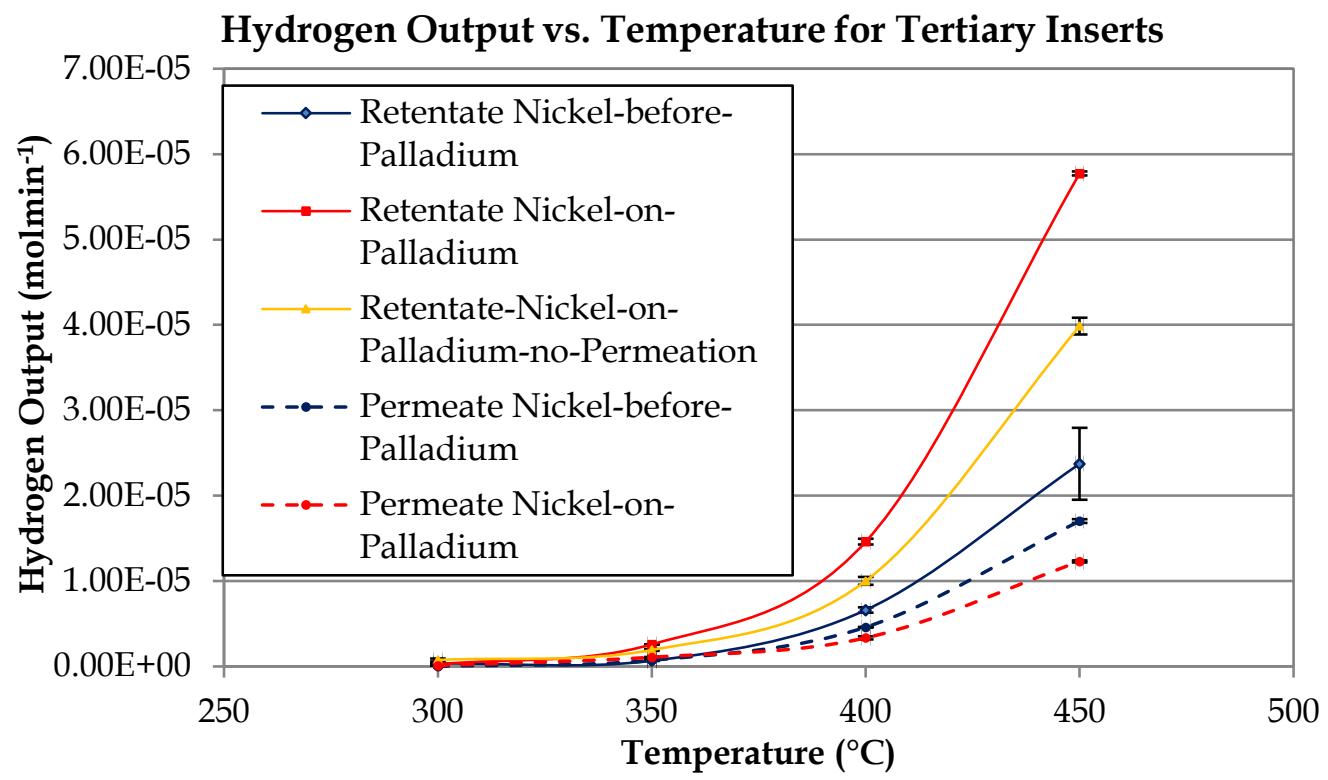

Figure 3.3.11: Hydrogen output vs. temperature for tertiary inserts. Retentate outputs are represented by solid lines, permeate outputs by dashed lines. 
Jonathan Tailby

Figure 3.3.12 graphs the sum of the retentate and permeate of each tertiary insert against temperature for a total hydrogen output. At $300{ }^{\circ} \mathrm{C}$ all three inserts produced negligible hydrogen. From $350{ }^{\circ} \mathrm{C}$ the Nickel-on-Palladium insert produced more hydrogen than the other inserts, the majority of which has already been shown to be retained on the reaction side of the palladium membrane. The Nickel-on-Palladium-no-Permeation and the Nickel-beforePalladium produced very similar quantities of total hydrogen from 350 to 450 ${ }^{\circ} \mathrm{C}$. The catalytic spillover membrane has produced more hydrogen as predicted, however, it had also been expected to permeate more hydrogen which it did not. The most likely explanation for the permeation of the catalytic spillover membrane failing to exceed that of the catalyst with adjacent membrane is that the rate limiting step for the hydrogen permeation was the diffusion of hydrogen within the bulk palladium. Diffusion is frequently cited as the slowest step in permeation through membranes under most operating conditions [158], for example, Ward and Dao have published that diffusion is limiting for $100 \mu \mathrm{m}$ membranes at temperature above $127^{\circ} \mathrm{C}$ [112]. The palladium membranes used in these experiments were $100 \mu \mathrm{m}$ thick.

Total hydrogen output vs. Temperature for three Tertiary Inserts

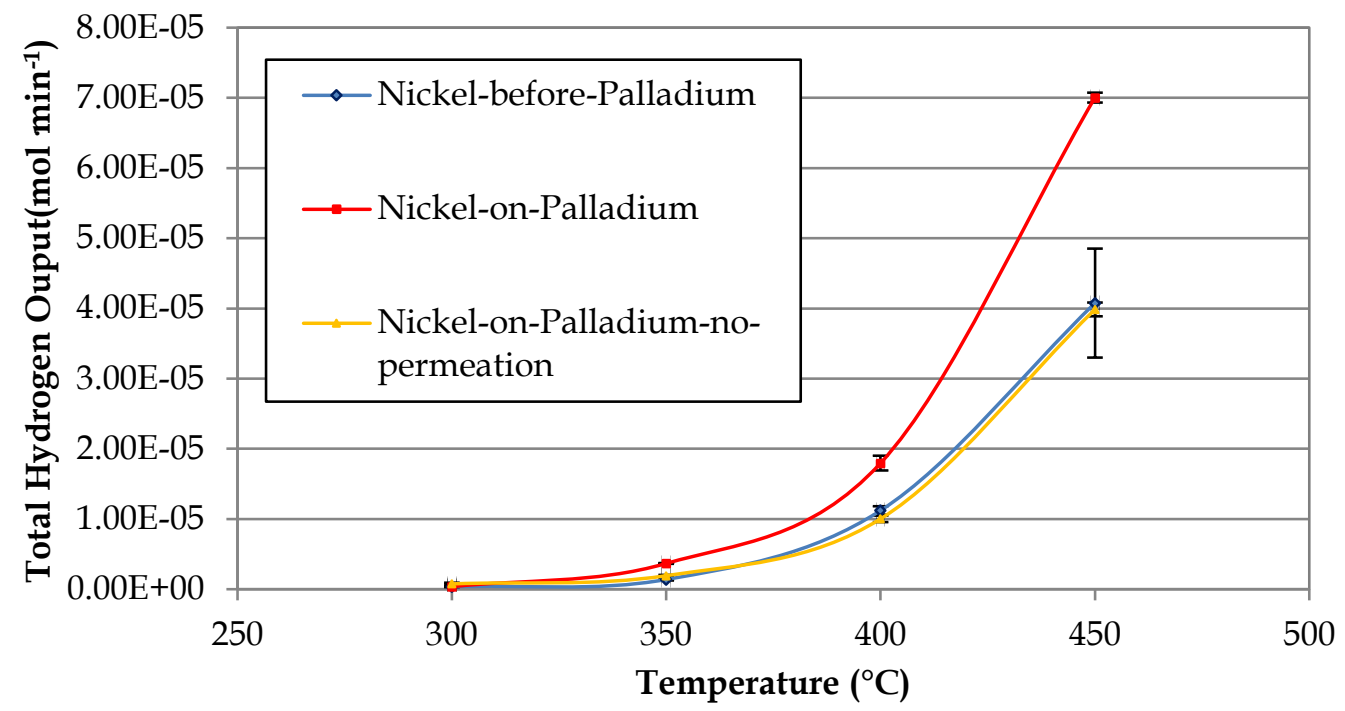

Figure 3.3.12: Total hydrogen output vs. temperature for tertiary inserts.

Figure 3.3.13 shows the production of nitrogen plotted against the reaction temperature for the three tertiary inserts. At $300{ }^{\circ} \mathrm{C}$ the three different inserts 
have produced negligible quantities of nitrogen. Above $300^{\circ} \mathrm{C}$ the Nickel-onPalladium insert produced more nitrogen than the other inserts. At $350{ }^{\circ} \mathrm{C}$ and greater temperature the Nickel-before-Palladium and the Nickel-onPalladium-no-Permeation produced very similar quantities of nitrogen. This is the same pattern as for hydrogen production, which was expected because the two product gases come from the same source, however the Nickel-onPalladium-no-Permeation insert decomposed the least ammonia. The difference in the no-Permeation insert's apparent performance for decomposition and product output could be related to spillover of both hydrogen and nitrogen to the palladium giving it a larger desorption surface.

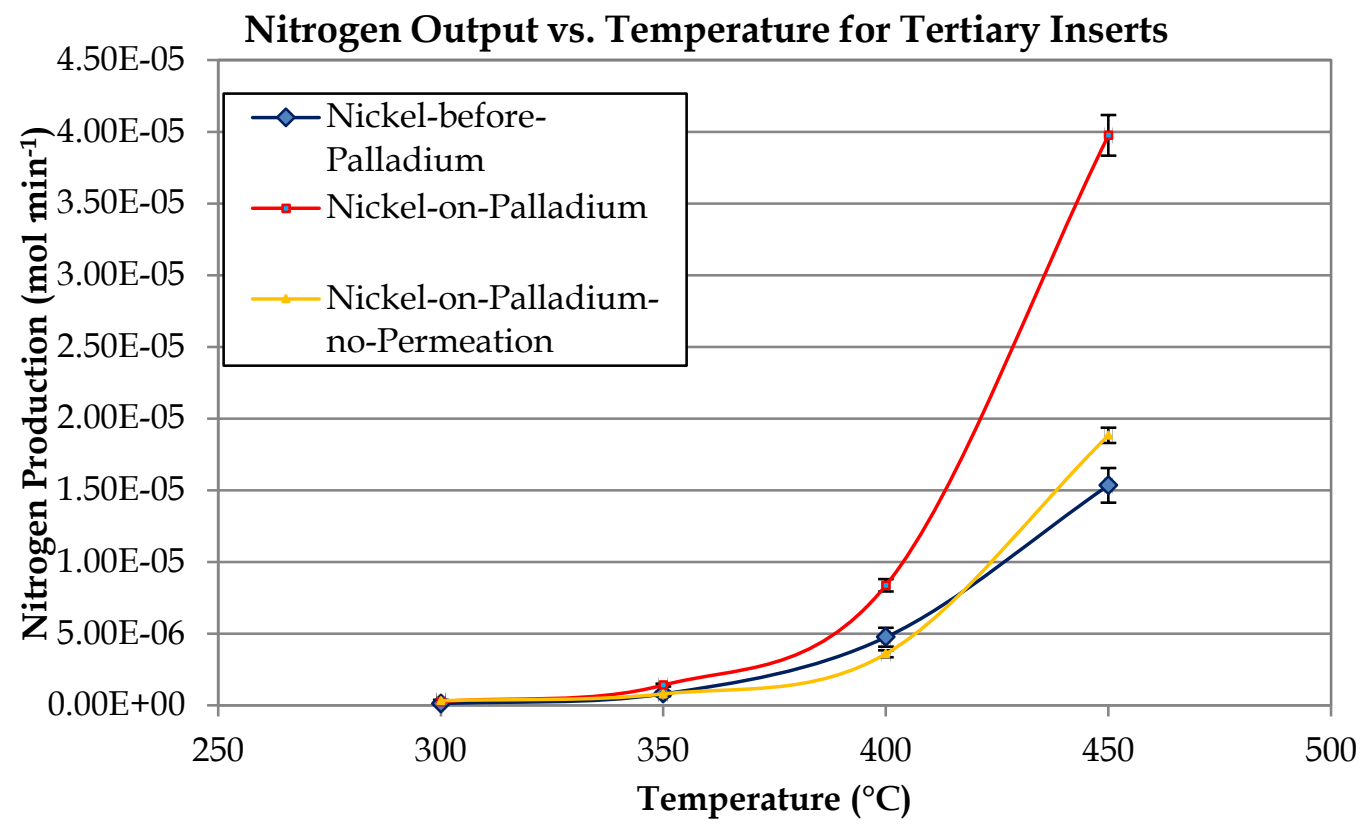

Figure 3.3.13: Plot of nitrogen production vs. temperature for tertiary inserts.

Slow diffusion through the membrane could explain why the Nickel-onPalladium insert is no better than the Nickel-before-Palladium insert for hydrogen flux, however, it does not explain why it is worse at 400 and $450{ }^{\circ} \mathrm{C}$. The decrease in permeation could be because of a reduction in the effective palladium surface. The foam itself, covers some of the palladium, however, the nickel foam is $79 \%$ empty space therefore this would have covered a small proportion of the surface, and diffusion limited permeation would be less affected by a small decrease in the surface processes. 
Other possibilities are that the spillover hydrogen is too weakly bound to the palladium surface, which could make the activation energy for desorption is lower than for movement from surface to subsurface. It has been reported in the literature that spillover hydrogen is more weakly bound to the surface than hydrogen on the surface where it has initially sorbed or been formed [170] this could enable a higher rate of hydrogen desorption than had been expected.

After the experiments were completed it was seen that the nickel foam on the palladium membrane was compressed towards the exit end of the membrane more than the Nickel-before-Palladium. Two effects of this were that it exposed more than a quarter of the membrane before the ammonia could interact with the catalyst. This exposed portion of the membrane would have been unable to adsorb hydrogen from the gas phase because the gas passing it did not yet contain hydrogen. It could also be that the higher temperature experiments provided newly formed hydrogen atoms with greater available energy to quickly desorb into the retentate. These speculative explanations would require further investigation to determine if any of them is valid.

The palladium before the nickel foam in the Nickel-on-Palladium insert would have been less active in hydrogen absorption. If it is assumed to be completely inactive, a new flux for the reduced area after the nickel can be calculated as shown in Table 3.3.6. To confirm whether permeation was compromised by the compression of the nickel foam, the flux values calculated from the permeation measurements were compared to the flux predicted by the Richardson Equation included earlier as Equation 1.2.21. In one reference article for this equation [99] the constants are given as minima and maxima. The calculations were completed with all combinations of these minimum and maximum values as shown in Table 5.2.24 in Appendix 5.2.7.4 on page 352. The minimum and maximum results of the Richardson Equation at each temperature are plotted on Figure 3.3.14. The flux for the Nickel-on-Palladium Catalytic Spillover Membrane permeation results, calculated for the full area of the palladium are less than the minimum predicted by Richardson's Equation at each temperature except $450^{\circ} \mathrm{C}$. However, the flux calculated for 
the same permeation with only the palladium area under the nickel foam is greater than the maximum predicted by Richardson's Equation at each temperature except at $300{ }^{\circ} \mathrm{C}$. This suggests that the reality is an intermediate case where the palladium before the nickel is less active in permeation than the palladium under the nickel but it is not completely inactive. If the flux is more than the flux calculated for the full area, then it will be more than the flux for the Nickel-before-Palladium membrane.

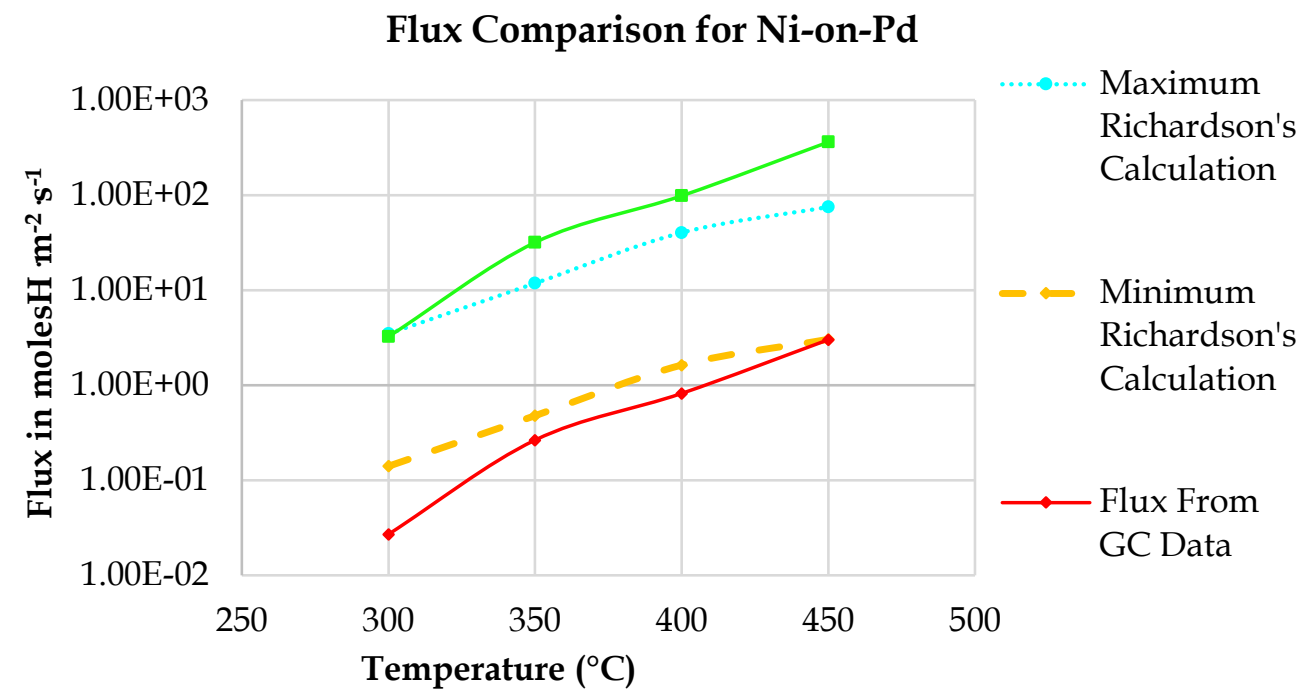

Figure 3.3.14: Hydrogen flux vs. temperature for Nickel-on-Palladium Catalytic Spillover Membrane showing results calculated from GC experiments and by Richardson's Equation.

Another literature source that the measured flux can be compared to is the work of Ward and Dao [112]. Their numerical modelling for hydrogen permeation calculated diffusion limits for flux at three different thicknesses of palladium and desorption limit of flux which varied more by temperature. A graph from this paper is reproduced as Figure 5.2.37 in appendix 5.2.7.4 and a version of the graph redrawn to highlight the temperatures and membrane thickness relevant to this work is shown in Figure 3.3.15. The calculations that developed the data for Figure 3.3.15 are included in appendix 5.2.7.4. 


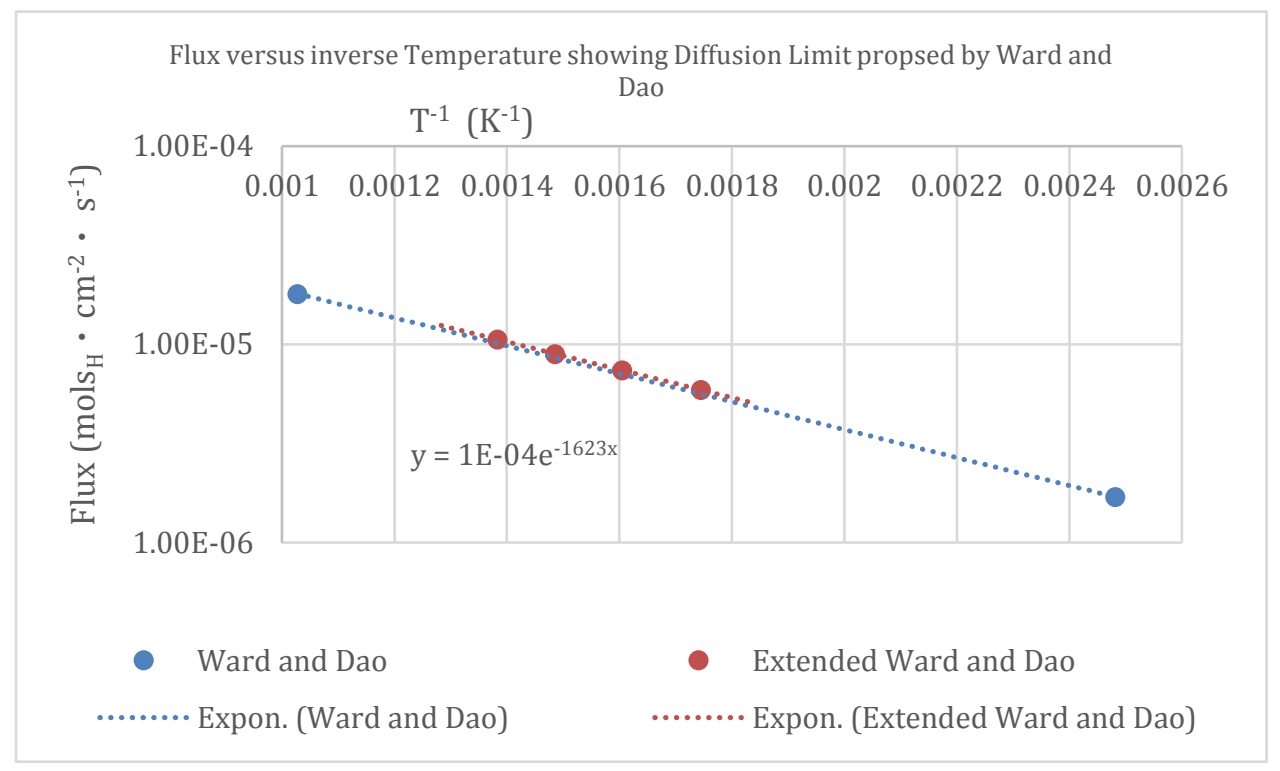

Figure 3.3.15: Graph of hydrogen flux vs. inverse temperature showing diffusion limits calculated by Ward and Dao, recalculated here to mark temperatures relevant to this research.

Figure 3.3.16 graphs flux vs. temperature similar to Figure 3.3.14 but including both the Richardson equation flux and the diffusion limit calculated from the Ward and Dao model. Richardson's equation exceeded Ward and Dao's limit even with the parameters that gave the lowest flux values in Richardson's equation. All of the measured values exceeded the Ward and Dao limit above $300{ }^{\circ} \mathrm{C}$, with only the nickel-before-palladium at $300{ }^{\circ} \mathrm{C}$ below the diffusion limit calculated by Ward and Dao. In the work cited here Ward and Dao referred to eight published experimental studies, which all supported the diffusion limits calculated for their respective thicknesses of palladium.

The models of Richardson and Ward and Dao are theoretical, and however successful previously, may not perfectly represent the actual system. It is possible that the reactors used here, particularly the catalytic spillover membrane have facilitated some small steps in the process that have enabled the overall process to exceed anticipated flux. This is a result worthy of further investigation. 


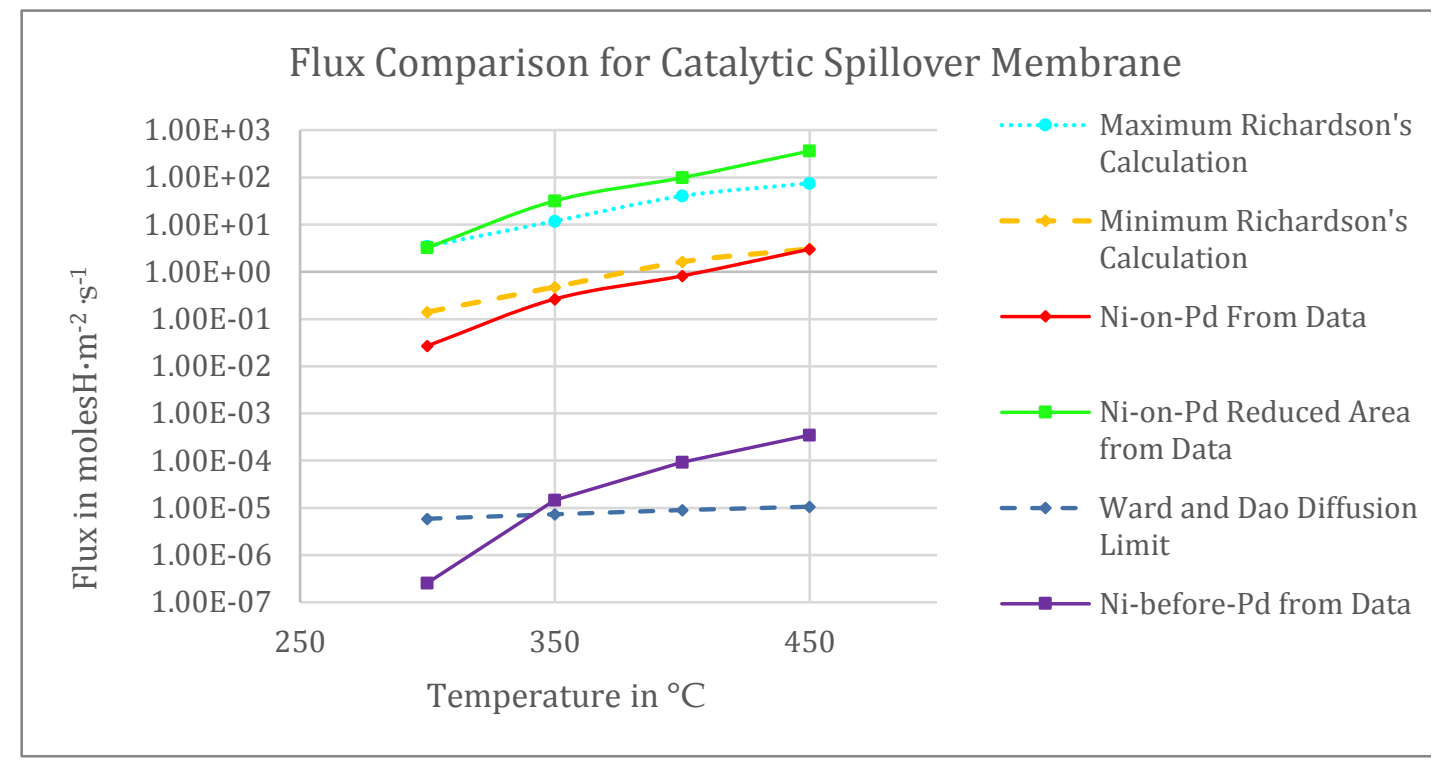

Figure 3.3.16: Graph of hydrogen flux vs. temperature comparing the flux values calculated from hydrogen measurements with two models of hydrogen flux; Richardson's and Ward and Dao's.

Across all temperatures in this series: 300, 350, 400 and $450{ }^{\circ} \mathrm{C}$ the Nickel-onPalladium insert produced the most retentate hydrogen and the most nitrogen. The model that produced the most permeate hydrogen varied by temperature.

At $350{ }^{\circ} \mathrm{C}$ Nickel-on-Palladium decomposed the most ammonia and permeated the most hydrogen. At 400 and $450{ }^{\circ} \mathrm{C}$ a lower proportion of the hydrogen permeated. Nickel-on-Palladium decomposed the most ammonia but did not permeate the most hydrogen. However, the flux for the full palladium area of the Nickel-on-Palladium and the Nickel-before-Palladium inserts were very similar. If the palladium of the Nickel-on-Palladium insert that was before the start of the nickel catalyst is considered inactive for flux, then the flux for the remaining palladium is significantly higher than for the Nickel-before-Palladium insert.

\section{Arrhenius Activation Energy}

The activation energy for the reactions with the tertiary inserts have been calculated by the Arrhenius method as described in Section 2.2.12.4. The calculation steps are included in Appendix 5.2.7.4.1. Table 3.3.7 shows the 
Jonathan Tailby

results for the three inserts and the nickel foam secondary insert for comparison.

Table 3.3.7: Summary of Activation Energy calculated for each tertiary insert from the rate data for each different species. Secondary nickel foam is included for comparison.

\begin{tabular}{|llllll|}
\hline & $\mathrm{n}_{\mathrm{A}}$ & $\mathrm{n}_{\mathrm{N}}$ & $n_{H_{\text {ret }}}$ & $n_{H_{\text {perm }}}$ & $n_{H_{\text {tot }}}$ \\
Nickel Before & 97.3 & 113.8 & 102.5 & 164.0 & 112.2 \\
Nickel On & 112.0 & 115.6 & 125.1 & 105.9 & 92.4 \\
Nickel on no Perm & 87.0 & 93.4 & 92.4 & $\mathrm{x}$ & 92.4 \\
Nickel foam secondary & 132.9 & 104.2 & & & 97.7 \\
\hline
\end{tabular}

The activation energy values for all three tertiary inserts calculated from the rate of ammonia decomposition are lower than the equivalent value for the nickel foam secondary insert.

Activation energy values were calculated from the different rates of hydrogen formation with the tertiary insert catalysts. These may not be directly comparable because the hydrogen gas is forming through different mechanisms. The retentate hydrogen of the two permeating catalysts may be comparable with each other, but not with the catalyst which does not permeate because the retentate hydrogen of the two permeating catalysts has been reduced by permeation.

The activation energy calculated from the rate of hydrogen permeation must be a value for the combined processes of decomposition and permeation.

Total hydrogen $\left(n_{H_{t o t}}\right)$ is the best hydrogen value to compare with the secondary insert because both retentate and permeate only represent part of the rate of hydrogen formation.

The activation energy for the two tertiary insert catalysts with contact between the nickel and the palladium are identical, both lower than the respective value for the tertiary insert with no contact between nickel and palladium. This suggests that the contact has affected the reaction mechanism, and it is proposed in this work that spillover is the cause of this change.

Activation energy for hydrogen permeation is higher than for hydrogen retention with the nickel before palladium tertiary insert. However, with the 
nickel on palladium tertiary insert the activation energy for hydrogen permeation is lower than for hydrogen retention. It is proposed that this could be related to the changes in mechanism provided by spillover, however no further investigation into the outcome was made at the time.

\subsubsection{Flow Series}

Table 5.2.35 in Appendix 5.2.7.4.2 calculates the rate of permeation for the Flow Series with the Tertiary Inserts.

Figure 3.3.17 shows the quantity of ammonia decomposed with the three different tertiary inserts at $350^{\circ} \mathrm{C}$ with the rate of ammonia input flow varying from 1 to $6 \mathrm{~mL} \mathrm{~min}^{-1}$. The temperature was set at $350{ }^{\circ} \mathrm{C}$ because in the secondary inserts this temperature had produced the greatest difference between catalysts, and for the tertiary insert temperature series it was the temperature where the hydrogen permeation of the Nickel-on-Palladium insert exceeded that of the Nickel-before-Palladium insert.

Ammonia Decomposition vs. Input Flow for Tertiary Inserts

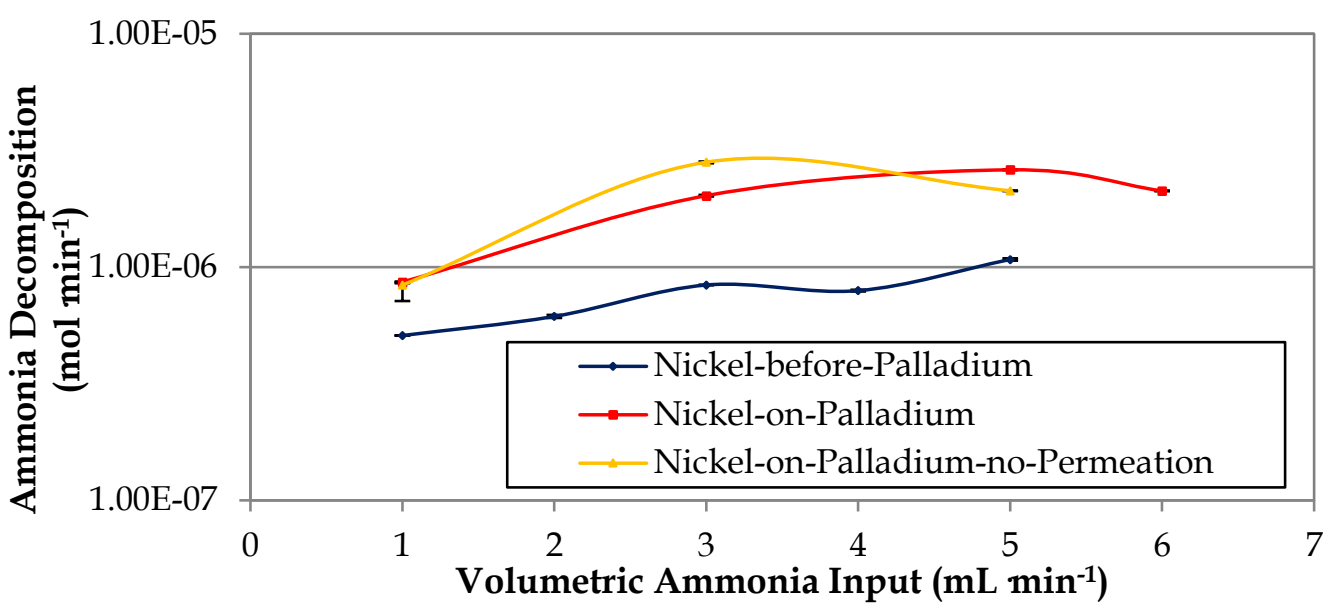

Figure 3.3.17: Average quantity of ammonia decomposed when the ammonia flow was varied from 1 to $6 \mathrm{~mL} \mathrm{~min}-1$ while temperature was maintained at $350{ }^{\circ} \mathrm{C}$.

In this flow series, the Nickel-on-Palladium decomposed more ammonia than the other inserts at $5 \mathrm{~mL} \mathrm{~min}^{-1}$. The Nickel-on-Palladium-no-permeation was the highest at $3 \mathrm{~mL} \mathrm{~min}-1$ and equal to the nickel foam on palladium at 1 $\mathrm{mL} \mathrm{min}^{-1}$. The Nickel-before-Palladium decomposed the least ammonia at 
Jonathan Tailby

each flow where it was measured, this is consistent with the $350{ }^{\circ} \mathrm{C}$ measurement in the temperature series shown in Figure 3.3.7.

Figure 3.3.18 shows a graph of the output of hydrogen in the retentate mixture and in the permeate mixture each against the input ammonia gas flow at 350 ${ }^{\circ} \mathrm{C}$. The curves appear to be increasing as input flow increases. This is logical given that increasing the flow will increase the quantity of ammonia available to decompose and produce the hydrogen, therefore if the proportion of ammonia decomposed does not decrease too quickly when flow increased, greater ammonia input will result in greater hydrogen output, as was seen in in the secondary insert results of Section 3.2.2.4.7 and predicted from Section 1.2.4.4.4.

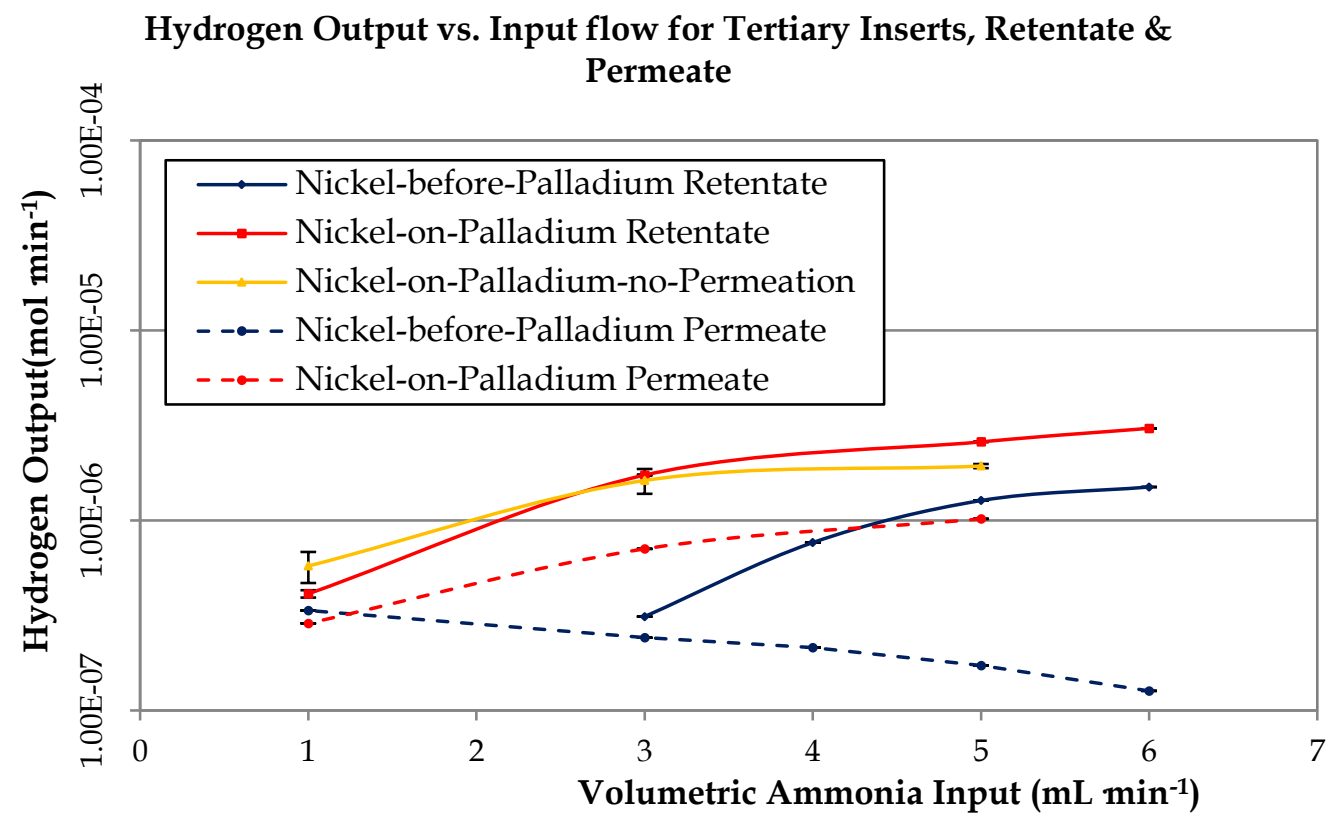

Figure 3.3.18: Hydrogen output in the retentate for the tertiary inserts at $350{ }^{\circ} \mathrm{C}$ when the ammonia flow was varied from 1 to $6 \mathrm{~mL} \mathrm{~min}^{-1}$.

Nickel-on-Palladium-no-Permeation produced the most retentate hydrogen at $1 \mathrm{~mL} \mathrm{~min}^{-1}$. Nickel-on-Palladium produced the most retentate hydrogen at 5 and $6 \mathrm{~mL} \cdot \mathrm{min}^{-1}$. At $3 \mathrm{~mL} \mathrm{~min}^{-1}$ the retentate hydrogen for Nickel-onPalladium and Nickel-on-Palladium-no-Permeation are within the calculated error of each other. Nickel-before-palladium has the lowest retentate hydrogen. At $1 \mathrm{~mL} \cdot \mathrm{min}^{-1}$ Nickel-before-Palladium has higher permeate 
hydrogen than Nickel-on-Palladium however at every higher flow that was measured the Nickel-on-Palladium insert had higher permeate hydrogen. In the temperature series, Nickel-on-Palladium had higher permeate at $350{ }^{\circ} \mathrm{C}$, therefore it had been expected to have higher permeate for this flow series at $350^{\circ} \mathrm{C}$.

The retentate hydrogen for the Nickel-on-Palladium insert is particularly low. From the temperature series data it would be expected to be the highest set of measurements on the graph but it is instead the lowest.

The permeate output for the two inserts remains two orders of magnitude below the sweep gas flow of $2.0 \times 10^{-4} \mathrm{~mol} \mathrm{~min}^{-1}$; however, the $5 \mathrm{~mL} \mathrm{~min}^{-1}$ measurements have each changed from the $350{ }^{\circ} \mathrm{C}$ measurement in the temperature variation data set. The Nickel-on-Palladium measurement at 5 $\mathrm{mL} \mathrm{min}^{-1}$ has decreased from $1.08 \times 10^{-6}$ to $1.02 \times 10^{-6}$, but the Nickel-beforePalladium insert has decreased from $7.23 \times 10^{-7}$ to $1.73 \times 10^{-7}$. For the Nickelon-Palladium catalytic spillover membrane, the hydrogen permeation increased when the flow increased, which is the same trend as for the retentate. The hydrogen outputs for the Nickel-on-Palladium insert and the Nickelbefore-Palladium insert preferentially leave the reactor by retention rather than permeation across all of the input flows tested.

This reactor allowed for the measurement of the retentate and the permeate hydrogen, however it does not allow for measurement of the quantity of hydrogen inside the palladium membrane. If diffusion through the membrane is rate limiting for the permeation, which could be expected from the thickness of the membrane and work such as Ward and Dao[112], then the quantity of hydrogen inside the membrane at any time would be predicted to be an appreciable quantity. A maximum amount can be calculated from the mass of palladium because at this temperature the palladium hydride is limited to the alpha conformation with a maximum of 5 atom $\%$ hydrogen, as detailed in Section 1.2.7.2.1. 
Jonathan Tailby

\subsection{Flow Series Summary}

These flow series results show that the improvement to ammonia combination of permeation and spillover can exceed each separate phenomenon for increasing the rate of ammonia decomposition.

The relative quantities of permeate hydrogen from Nickel-on-Palladium and Nickel-before-Palladium, suggest that the spillover may have affected the rate limiting step in the process at $350{ }^{\circ} \mathrm{C}$, likely by accelerating a step on the surface of the palladium where hydrogen enters the membrane. Reference back to the Temperature Series, particularly Figure 3.3.11 suggests that this effect was decreased at higher temperature.

It could be that the greater hydrogen production from the ammonia decomposition on the Nickel-on-Palladium insert was directed into the membrane by spillover, but hydrogen diffusion through the bulk palladium was the rate limiting step. Therefore more hydrogen was contained in the palladium membrane of Nickel-on-Palladium insert, slowly diffusing through to the permeate. This spillover would also increase turn over frequency on the catalyst as reactive sites become free more quickly, enabling greater ammonia decomposition.

Figure 3.3.19 shows a graph of the total hydrogen output of the tertiary inserts graphed against the volumetric flow of the ammonia input. The Nickel-onPalladium produced the most total hydrogen across the range of flows measured. The Nickel-on-Palladium-no-Permeation produced the second greatest quantity of hydrogen, none of which could permeate through to the sweep gas. The Nickel-before-Palladium insert produced the least total hydrogen across the whole range of flows. The two Nickel on Palladium inserts decomposed much closer quantities of ammonia than the quantities of hydrogen they produced. The Nickel-before-Palladium insert decomposed the least ammonia and produced the least total hydrogen. 


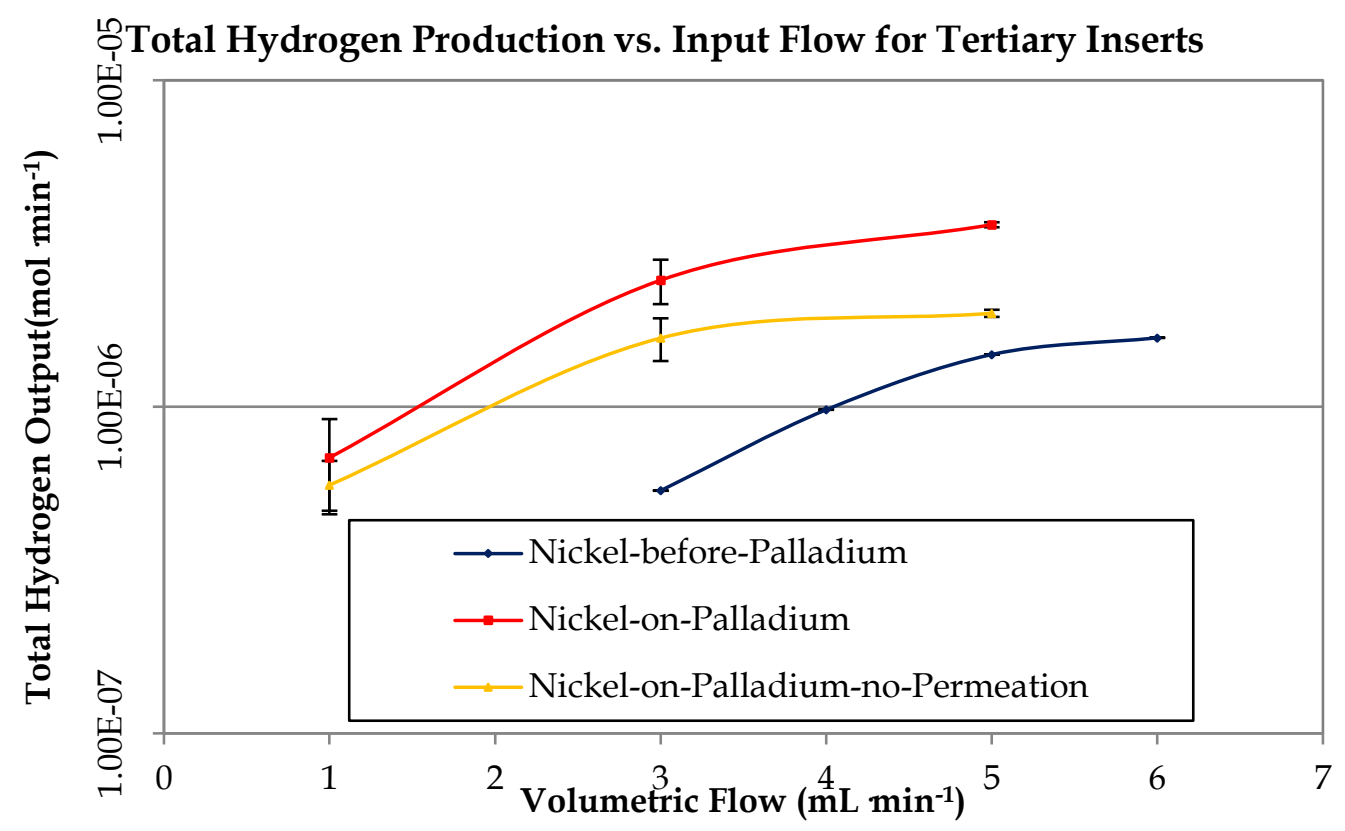

Figure 3.3.19: Quantity of hydrogen in the sweep flow of the two permeating tertiary inserts at $350{ }^{\circ} \mathrm{C}$ while ammonia flow varied from 1 to $6 \mathrm{~mL} \mathrm{~min}{ }^{-1}$.

The hydrogen permeation in $\mathrm{mol} \mathrm{min}^{-1}$ was converted into flux in $\mathrm{mol}_{\mathrm{H}} \cdot \mathrm{s}^{-1} \mathrm{~m}^{-2}$. Two conversions were calculated for Nickel-on-Palladium, one for the total palladium area and one for the reduced palladium area that included only the palladium after the first nickel catalyst as, the same as on page 243 .

Figure 3.3.20 shows that at $350{ }^{\circ} \mathrm{C}$ the Nickel-on-Palladium insert had greater flux than the Nickel-before-Palladium across all of the input flows tested, more significantly so if the flux is calculated only for the palladium are after the nickel catalyst begins. 


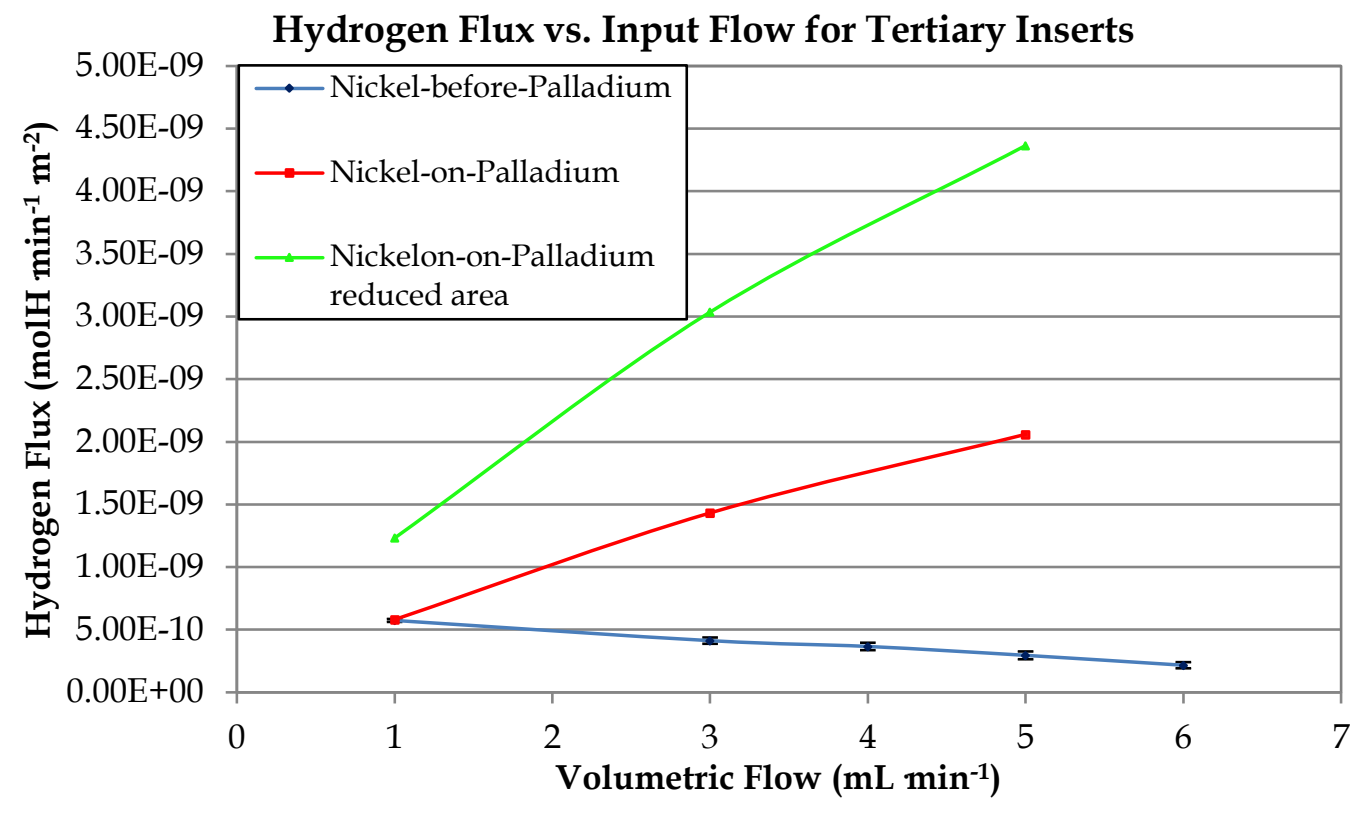

Figure 3.3.20: Hydrogen Flux plotted against ammonia input volumetric flow for tertiary inserts at $350{ }^{\circ} \mathrm{C}$.

The red line represents the flux for the catalytic spillover membrane calculated for the full palladium area and the green line calculates the flux for only the area after nickel catalyst. 


\section{Final Summary}

This research aimed to create a catalytic spillover membrane and evaluate its effectiveness in producing hydrogen by catalytic decomposition of ammonia. Improvements to this reaction and recovery of the hydrogen produced will extend the possibility of ammonia as a hydrogen carrier.

Nickel catalysts on the surface of steel tube substrates were produced by four different methods. Reactors were designed and built to test these potential catalysts for ammonia decomposition over a temperature range up to $500{ }^{\circ} \mathrm{C}$. A nickel foam catalyst was subsequently retested for ammonia decomposition in three scenarios: in contact with palladium foil, the Nickel-on-Palladium-noPermeation insert; in a reactor adjacent to a palladium membrane, the Nickelbefore-Palladium insert; and in contact with a palladium membrane, the Nickel-on-Palladium membrane. The Nickel-on-Palladium catalytic spillover membrane appears to have been the best combination for ammonia decomposition. This is supported by the temperature variation results at greater than $300{ }^{\circ} \mathrm{C}$ and the flow variation results at $350{ }^{\circ} \mathrm{C}$. These results suggest there is value in a nickel-on-palladium catalytic spillover membrane, but further investigation is needed to optimise the conditions to exploit the effect.

Nickel foam in contact with a palladium surface accelerated the decomposition reaction by allowing spillover to transport hydrogen away from the active site of the catalyst more quickly than the catalyst alone could.

A hydrogen permeation membrane increased ammonia decomposition and selectively filtered hydrogen. There are reports in the literature of inhibition of the decomposition reaction by hydrogen [89], therefore selective removal of hydrogen from the reactor accelerates the reaction and drives the ammonia decomposition equilibrium towards completion.

The Nickel-on-Palladium catalytic spillover membrane increased ammonia decomposition and hydrogen flux, which opens up further avenues of research to better take advantage of the phenomenon. 
Jonathan Tailby

Before this project, it was known that nickel was one possible metal catalyst for ammonia decomposition [84]. While the project was active, it was reported that nickel is the second best single metal catalyst for ammonia decomposition [19]. This project has shown that hydrogen spillover from nickel to palladium increases the rate of ammonia decomposition further than the catalyst alone.

Palladium membranes had been shown to increase the decomposition of ammonia in a membrane reactor [101]. This project has shown that spillover from nickel catalyst to palladium membrane further increases the rate of ammonia decomposition over and above the rate with catalyst and membrane without a spillover connection. It was also shown that the spillover connection does not require complex atomic level integration of catalyst and secondary surface, the macroscopic interaction of the nickel foam on palladium foil was sufficient. At $350{ }^{\circ} \mathrm{C}$ and the Catalytic Spillover Membrane permeated more hydrogen than the nickel foam before the palladium membrane.

It was observed that positioning the palladium before the nickel on Catalytic Spillover Membrane generated less active hydrogen permeation than the palladium after the start of the nickel, which is consistent with the permeation being dependent on the difference in hydrogen partial pressure as the literature suggests. These results show that spillover has increased flux through the palladium membrane.

This project developed several catalysts that could potentially be applied on a palladium membrane. One catalyst was used to show that the combination of spillover and a palladium membrane enhances catalytic decomposition and hydrogen permeation more than either spillover or membrane permeation alone. 


\subsection{Future Work}

In this research, the hydrogen diffusion through the membrane step of hydrogen permeation was found to cause the rate limit in the overall process that combined hydrogen production by catalytic decomposition of ammonia, hydrogen spillover and hydrogen separation by permeation through a dense palladium membrane.

An extension of the research would be to develop a variation with ultrathin palladium membranes so that the membrane diffusion step becomes less of a barrier. Recent work undertaken at this laboratory has demonstrated that a nanoscaled palladium membrane can be deposited across the collinear pores of an anodised alumina substrate allowing the palladium to be suspended as a continuous 50-100 nm film supported by the alumina pore array [16]. Prototype membranes demonstrated $100 \%$ selectivity for hydrogen transport from mixed gas sources. The extreme thinness of this supported membrane enables large reduction of the diffusion time for hydrogen gas transport through the palladium. While the dihydrogen dissociation/reformation processes at the entry and exit surfaces of the membrane remain as potential rate limiting processes, the reduction of the membrane thickness by some four orders of magnitude confers major benefits in terms of membrane costs and hydrogen transport rates. Although technically challenging to implement, the integration of the novel reactor design and process knowledge from the current thesis work with an adapted ultrathin palladium membrane design could provide a big step forward in enabling a practical technology outcome from this materials science study. With such a change, another step in the process would then become rate limiting, which would be expected to be either recombinative desorption of nitrogen or dehydrogenation of ammonia. Addition of up to $30 \mathrm{wt} . \%$ cerium has been shown to facilitate nitrogen desorption on ruthenium, and cerium oxide has been shown to enhance the decomposition of ammonia on nickel [173]. If recombinative desorption of nitrogen became the limiting step, 
Jonathan Tailby

experiments with these could explored. Also palladium itself has the potential to receive spillover nitrogen $[136,137]$, which could alleviate this potential bottleneck. If dehydrogenation of ammonia becomes rate limiting then alternate catalysts could be explored, either ruthenium or an iron-nickel alloy [67].

As well as thinning the membrane, alternative membranes could be tested. Several other non-palladium metals, including nickel, are known to allow hydrogen atoms to permeate, but none are as effective as palladium at dissociating hydrogen molecules. If hydrogen atoms are being produced by ammonia decomposition then nickel or nickel-vanadium membranes could be tested. However, a palladium coating on the desorption surface could still be necessary to facilitate associative desorption of hydrogen [174].

Expanding the system analysis is another important direction for future work. This should include life on-stream measurements and catalyst poisoning resistance, specifically with reference to common impurities in industrial ammonia. The analysis could also include efficiency calculations for the decomposition.

Further work on the templating method could enable realisation of the initial plan of electrodepositing nickel onto palladium. Using SUEX photolithographic film to template electrodeposition of nickel onto palladium would be a good extension to the project that would be expected to increase ammonia decomposition and thus hydrogen output. 


\section{Appendices}

\subsection{Method}

\subsubsection{Shrinkwrap Transmittance Spectrum}

Figure 5.1.1 shows the transmittance spectrum of the shrink wrap used for securing the lithography mask around the stainless-steel tube. The wavelength used in the photolithography was $365 \mathrm{~nm}$, which has a transmittance of $85.6 \%$.

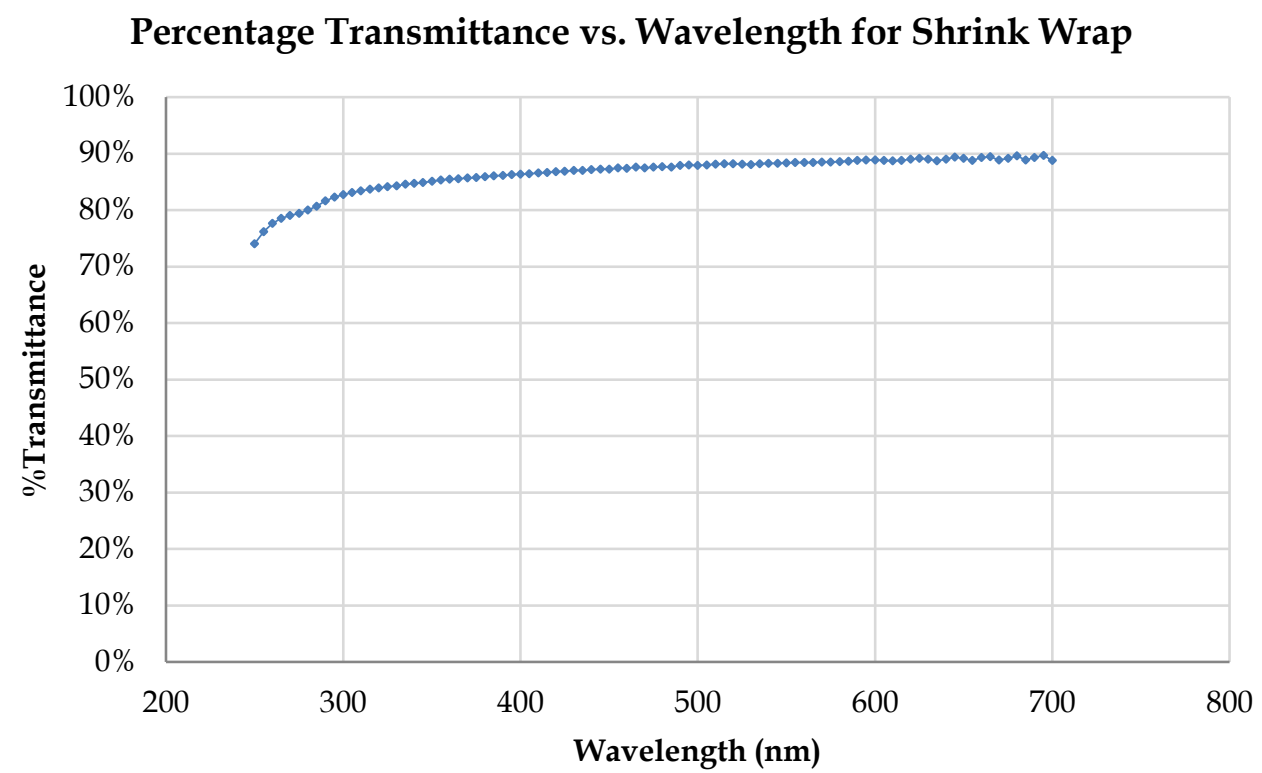

Figure 5.1.1: Percentage Transmittance vs. Wavelength for Shrink Wrap.

\subsubsection{Preliminary Experiments}

\subsubsection{Sputnik}

The first experiments with the sputnik reactor used polished copper rings as gaskets between the halves of the gas reaction vessel, for the next experiment these were replaced with compressed fibre (CF) gaskets. Figure 5.1.2 and Figure 5.1.3 shows records for two leak tests of the $\mathrm{CF}$ gaskets at high temperature. Flow into the reactor was set at $85 \mathrm{sccm}$, then the flow out was measured. Temperature was increased at 20 ${ }^{\circ} \mathrm{C} \mathrm{min}^{-1}$ to 400 . At $400{ }^{\circ} \mathrm{C}$ it was held for 50 minutes before heating further to $600{ }^{\circ} \mathrm{C}$. An apparent failure at $400{ }^{\circ} \mathrm{C}$ recovered then leaked more slowly until $600{ }^{\circ} \mathrm{C}$. The 
Jonathan Tailby

repeat test gave improved results, leaking less than $20 \%$ even at $600{ }^{\circ} \mathrm{C}$. The $\mathrm{CF}$ gaskets were used for the experiments shown in Figure 3.2.1 and Figure 3.2.2.

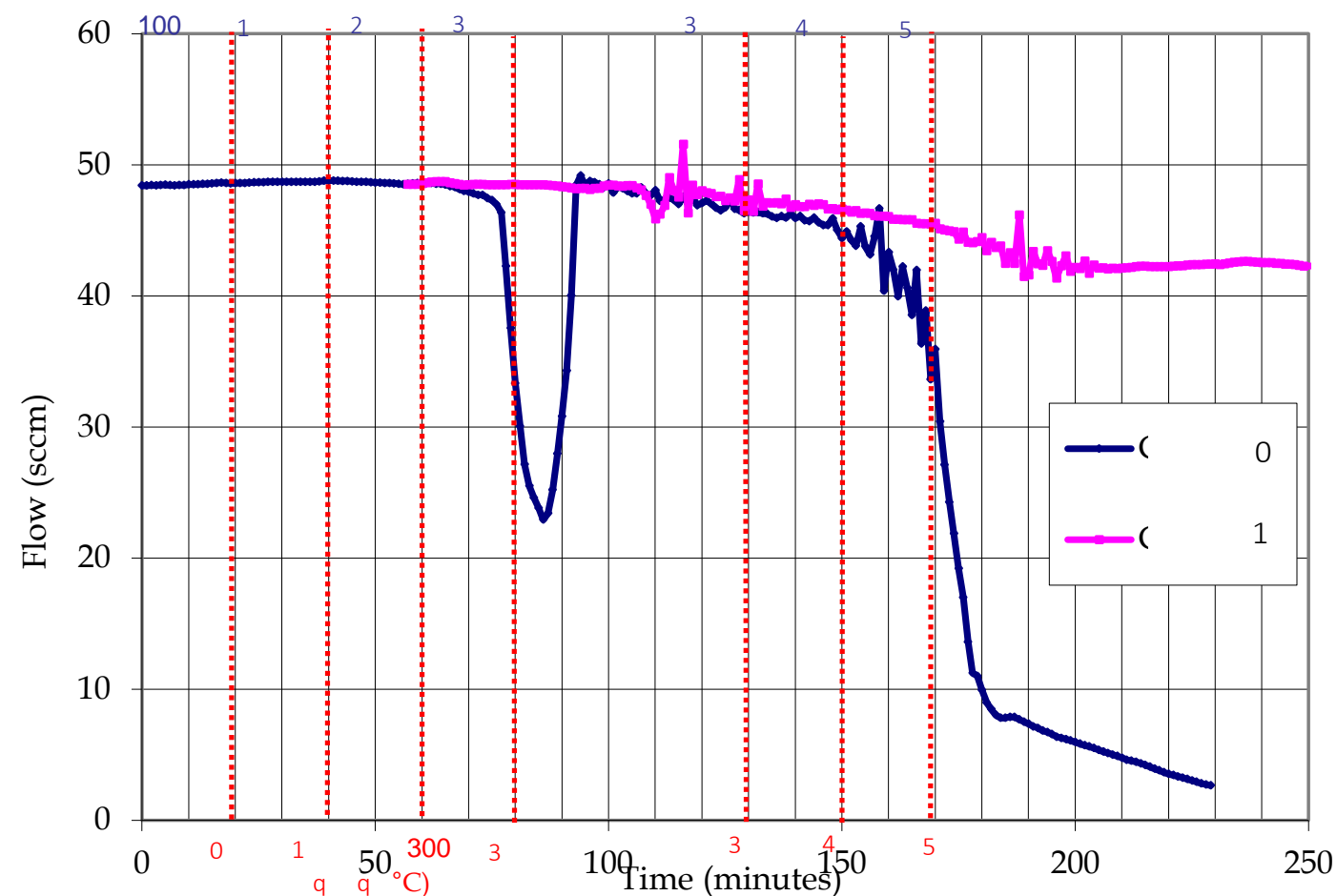

Figure 5.1.2: Gas flow vs. time for gasket leak tests.

Temperature was increased at $5{ }^{\circ} \mathrm{C} \cdot \mathrm{min}^{-1}$ to $400{ }^{\circ} \mathrm{C}$ then held for 50 minutes before heating again to $600{ }^{\circ} \mathrm{C}$.

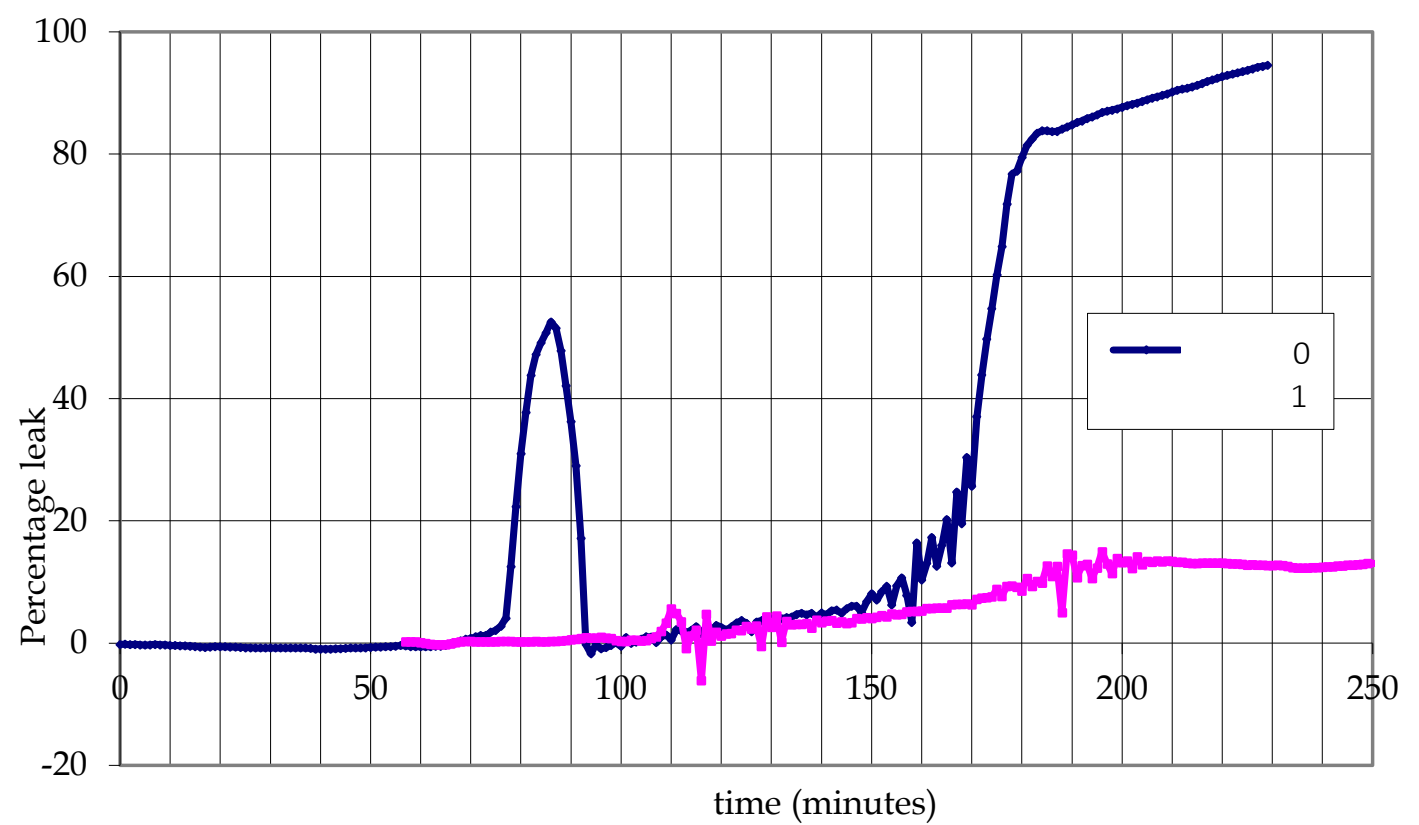

Figure 5.1.3: Percentage gas leak vs. time for the same gasket leak tests shown in Figure 5.1.2.

This is the same data as fig 3.2.1.4a recalculate to show the gas loss as percentages. 


\subsubsection{Explorer}

\subsection{Whole Reactor}

A preliminary experiment combined the new reactor and the GC to measure the time to completely flush one gas from the reactor with another gas. GC measurements were made while carbon dioxide 5 psi, $10 \mathrm{~mL} \mathrm{~min}^{-1}$ flowed from the source tap, through the reactor to the GC then to vent in the fume cupboard. These injections were continued until only carbon dioxide registered on the GC. At this point the carbon

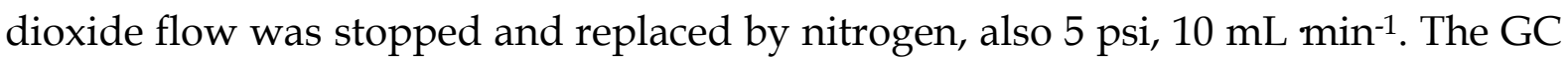
commenced recording and the times were measured until the first appearance of nitrogen and until the output was only nitrogen. This process was repeated several times, alternating between carbon dioxide and nitrogen flows. Figure 5.1.4 shows a set of GC results from when carbon dioxide flushed out nitrogen. The visible peaks, from left to right, are the injection of sample into the column at 0.1 minutes, nitrogen at 0.8 minutes and carbon dioxide at 1.8 to 2.2 minutes. Carbon dioxide appeared in the first injection ( 3 minutes), but it was not until the eighth injection cycle (24 minutes) that the gas output of the reactor was completely free of nitrogen.

Nitrogen reappeared in the chromatogram injection 10, initiated 6 minutes after the gas flow was switched back from carbon dioxide to nitrogen.

After injection 3 the sample loop did not reopen immediately therefore the sample loop for injection 4 was only partially filled and both sample peaks in chromatogram 4 are smaller than both 3 and 5 . 

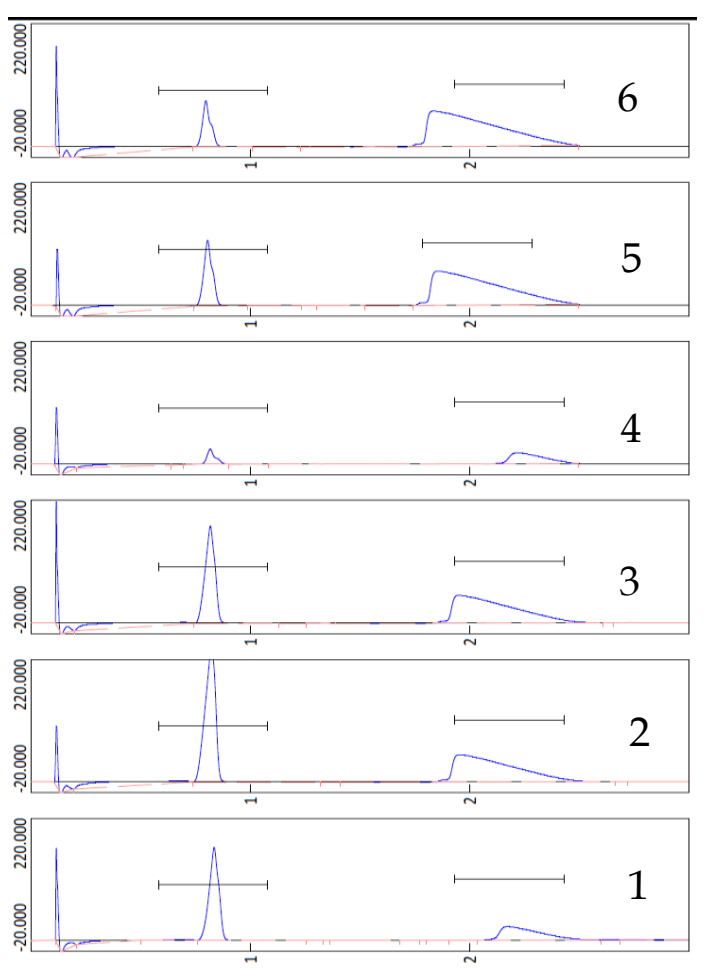
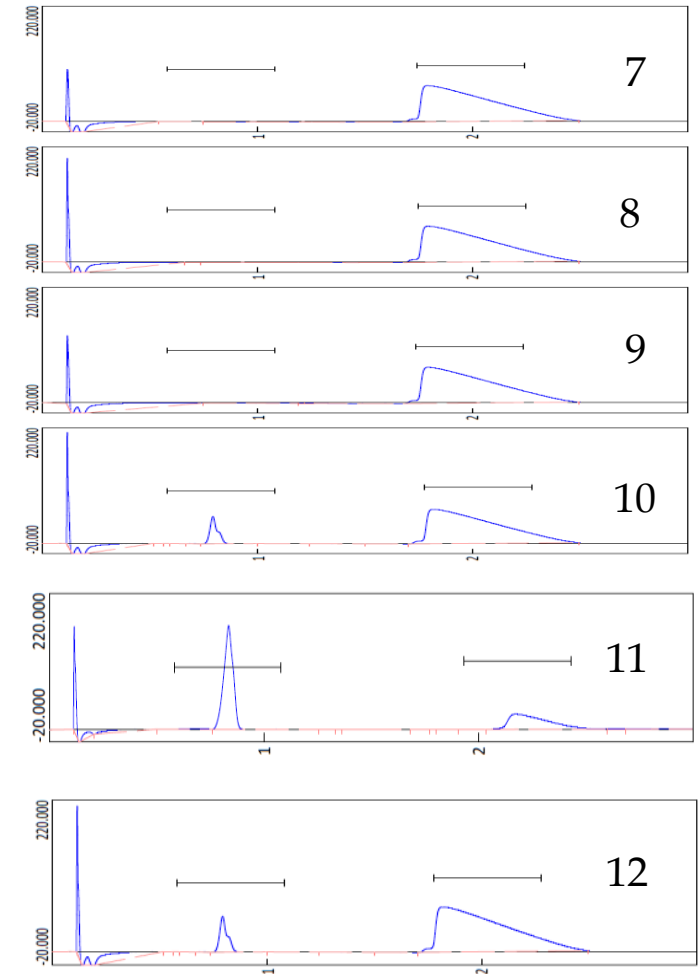

Figure 5.1.4: GC results for Carbon Dioxide flushing nitrogen out of the Explorer reactor.

Injections every three minutes as numbered, from bottom left up to top left, then from top right to bottom right. The peaks visible in each injection are from left to right: injection at 0.1 , nitrogen at 0.8 and carbon dioxide at 1.8 to 2.2.

\subsection{Primary Insert}

A reference calibration was undertaken between the external temperature probe that was connected to the heater controller and the internal probe. The first comparison found a difference of $70{ }^{\circ} \mathrm{C}$ at external temperature $350{ }^{\circ} \mathrm{C}$. Because of the size of this difference between the external temperature and the internal temperature, extra insulation was added to the outside of the reactor. Thick aluminium foil was wrapped around the original Kaowool insulation to reduce heat loss from the air flow of the fume cupboard and additional Kaowool was added outside the aluminium. A second calibration was undertaken that showed the difference between the external and internal temperature measurements reduced to $30^{\circ} \mathrm{C}$ at external temperature $350{ }^{\circ} \mathrm{C}$ and also showed that the temperatures were more stable, varying by less than $3{ }^{\circ} \mathrm{C}$ at $350{ }^{\circ} \mathrm{C}$ instead of up to $7^{\circ} \mathrm{C}$ at $350^{\circ} \mathrm{C}$ for the first temperature series. These calibrations for nitrogen $10 \mathrm{psi}, 20 \mathrm{~mL} \mathrm{~min}^{-1}$ are plotted in Figure 5.1.5. Further calibrations at 


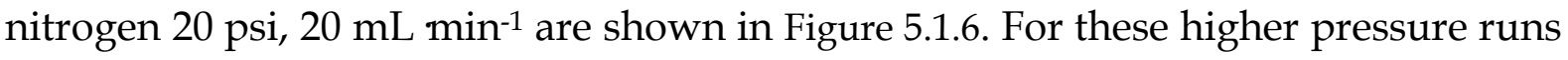
the internal temperature was observed to be greater than the external temperature, but only $20^{\circ} \mathrm{C}$ at external temperature $350^{\circ} \mathrm{C}$.

Internal Temperature vs. External Temperature for Primary Insert

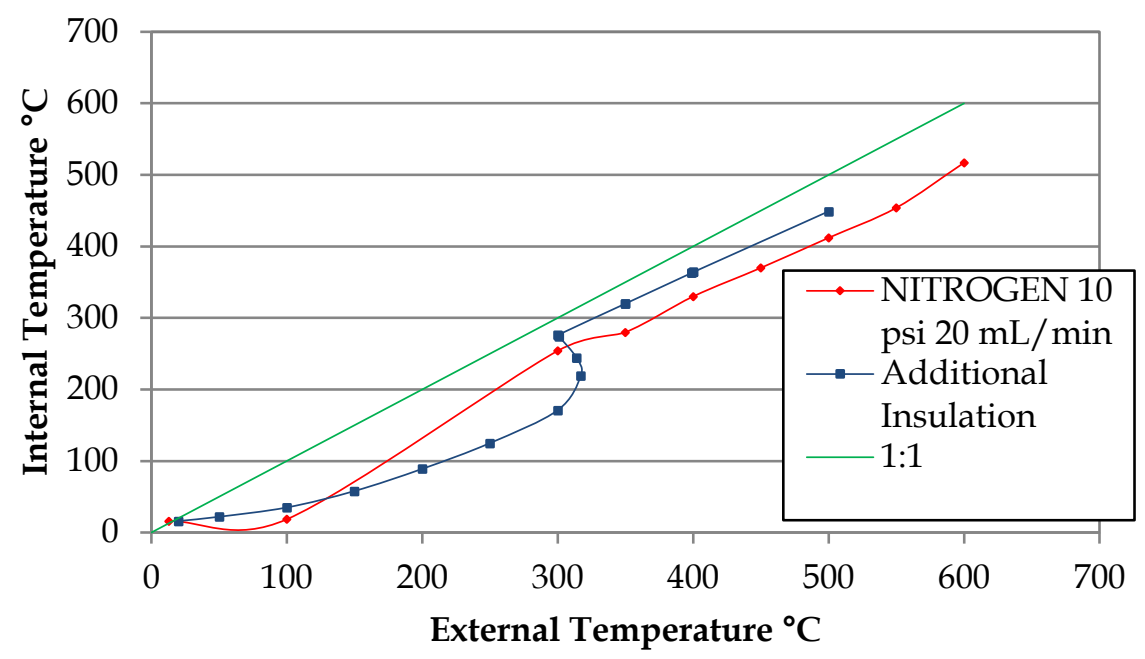

Figure 5.1.5: Temperature calibration for gas reactor with primary insert.

The green line indicates where they would be equal. The internal temperatures in the first set of measurement was too far below the external temperatures, so additional insulation was added and a second set of measurements was made with decreased temperature difference above $300^{\circ} \mathrm{C}$.

Internal Temperature vs. External Temperature for Primary Insert

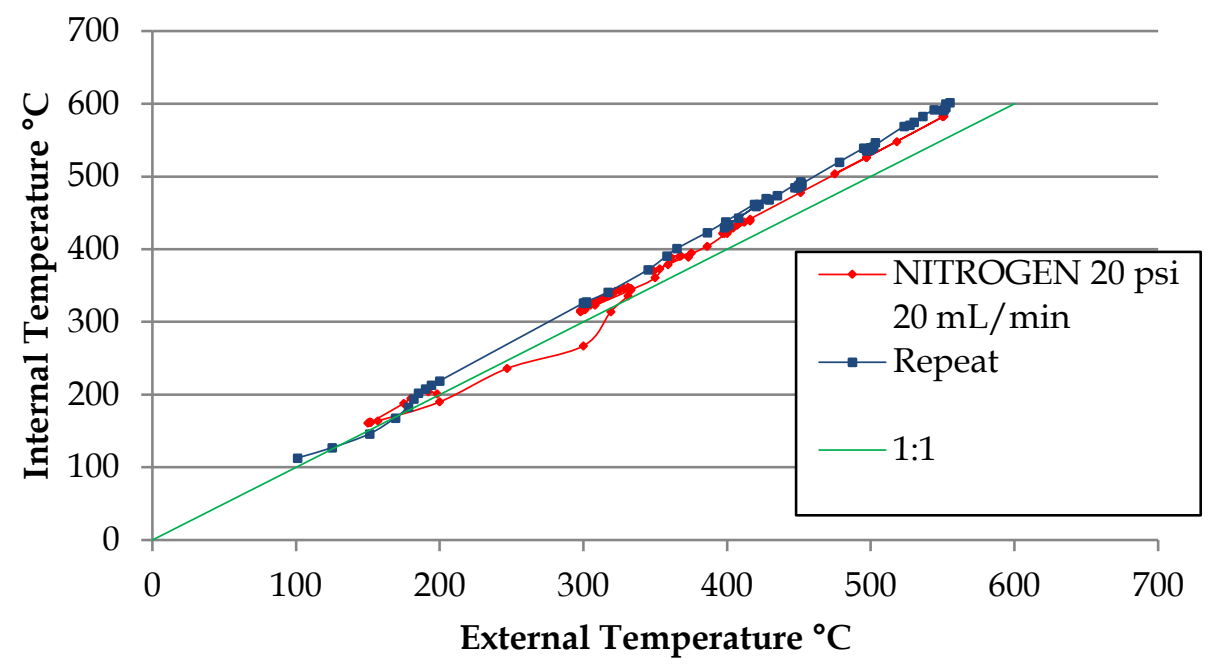

Figure 5.1.6: Further temperature calibrations of the primary insert.

The gas pressure was increased to 20 psi. The internal temperature became much closer to the external temperature and from external temperature of $300{ }^{\circ} \mathrm{C}$ the internal temperature was greater than the external temperature. 


\subsection{Tertiary Insert}

The new tertiary inserts were tested for gas leaks in the palladium membrane. Argon from the sweep side inside the insert was flowed to the gas chromatograph while nitrogen flowed between the primary and tertiary inserts. The gas chromatograph record was compared to one for argon flowing through the primary insert outside the tertiary insert, as shown in Figure 5.1.7a and b. The two chromatograms each show the same pattern, five three minute cycles of a 0.5 minute wait to fill the sample loop, then a tall, sharp injection peak.

At 1.8 minutes there is a small peak with an even smaller shoulder afterwards. This retention time of 1.32 minutes (1.8-0.5) matches the retention time of nitrogen, the small shoulder immediately afterwards is in the expected region for the oxygen in air. The average area for each peak and its shoulder was 1.2, because the two measurements were taken on the same instrument within minutes of each other, the peak areas are directly comparable. The small peak representing an air leak in the rotameter or earlier did not change between the inside or the outside of the main tube, hence there was no leak between the nitrogen and argon gas flows in this experiment. At 3 minutes there is a negative peak caused by the sample loop reopening. The cycle repeats, adding 3 minutes to the time of each event: reinjecting at 3.5 minutes, air peak at 4.8 minutes and negative peak at 6 minutes and again until 15 minutes although the negative peak at 15 minutes is off the record.

Figure 5.1.7c shows an enlargement of the air peak from the fifth cycle of the chromatogram shown in Figure 5.1.7b which was recorded with argon flowing through the tertiary insert to the GC. This peak was already present in the argon supplied to the tertiary insert, and has neither increased nor decreased. This enlargement clearly shows the shoulder on the right-hand side of the peak. This shoulder is from oxygen, which has a retention time similar to the retention time of nitrogen. In the manufacturer's information on the retention time of Porapak packed columns, for a $1 \mathrm{~m} \times 2.3 \mathrm{~mm}$ ID stainless steel column, $80-100$ mesh at $30{ }^{\circ} \mathrm{C}$, with 
$25 \mathrm{~mL} \mathrm{~min}^{-1}$ He carrier gas both nitrogen and oxygen are listed as having 0.24 minutes retention time for $Q$ type packing material [175]. The peak shape shown in Figure 5.1.7c is characteristic of air whenever it was seen on the GC throughout this research.

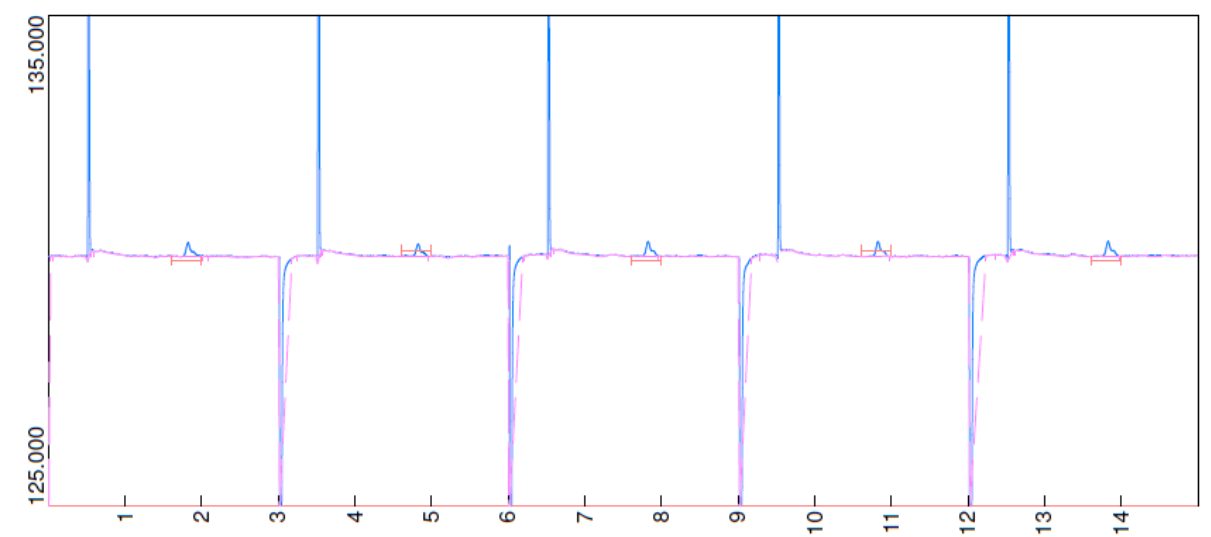

Figure 5.1.7a: GC record for Ar through the primary insert.

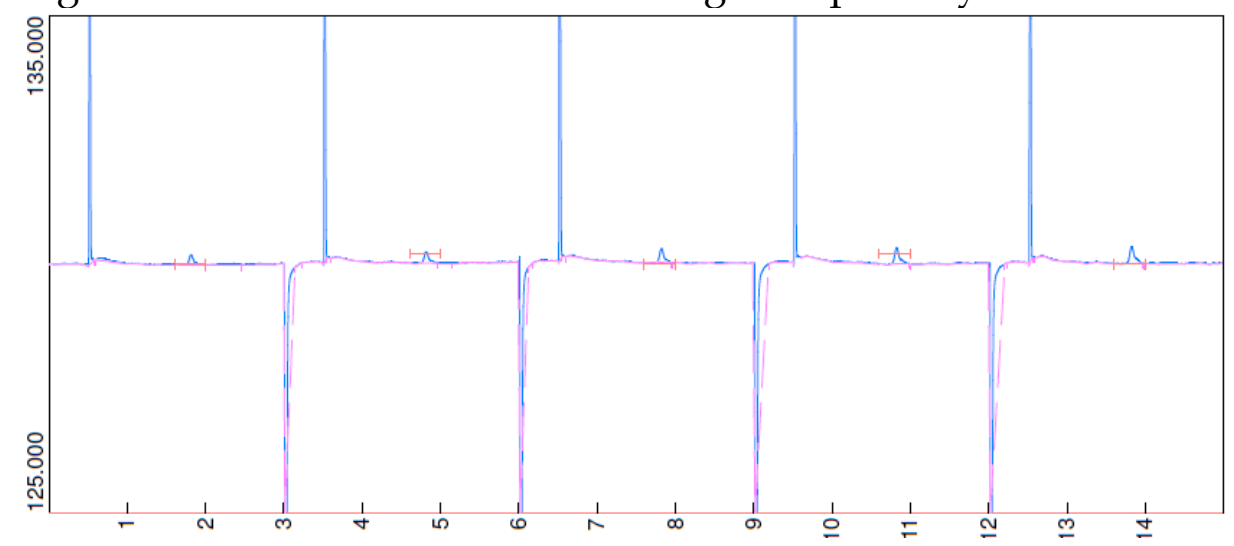

Figure 5.1.7b: GC record for Ar through tertiary insert showing only the air leak in the delivery system.

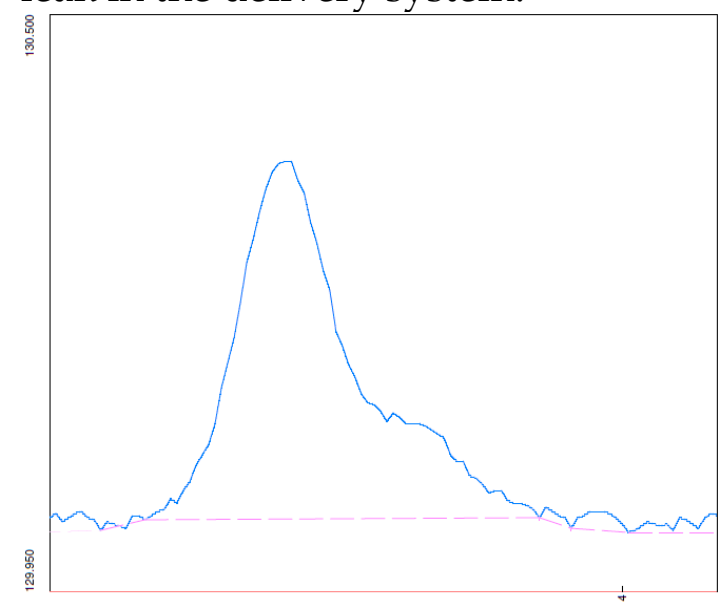

Figure 5.1.7c: Enlargement of the fifth sample peak in the chromatogram for Ar through the tertiary insert, at 13.8 minutes.

Figure 5.1.7: Argon Standards Experiments

Another preliminary experiment was to observe hydrogen transport across the membrane on the tertiary insert. The gas chromatograph record is shown in Figure 
5.1.8. The peaks at $0.5,3.5,6.5$ and 9.5 minutes are from injection of sample into the column. The negative peaks at 3, 6, 9 and 12 minutes are the sample loop opening to be refilled. The presence of hydrogen peaks 1.08 minutes after each injection confirmed hydrogen permeation through the tertiary insert.The 5\% hydrogen/95\% nitrogen mixture flowed outside the tertiary insert at $350{ }^{\circ} \mathrm{C}$. Argon flowed through the inside and then to the gas chromatograph.; this has the same small air peaks as the two gas chromatograph records in Figure 5.1.7 from the same leak in the argon delivered to the reactor, but the chromatogram also has larger hydrogen peaks at retention times of 1.08 minutes. Hydrogen content of the gas increased inside the reactor but nitrogen did not, indicating gas permeation rather than a gas leak. Therefore, the tertiary insert was performing its function and the ammonia decomposition with hydrogen permeation experiments were commenced.

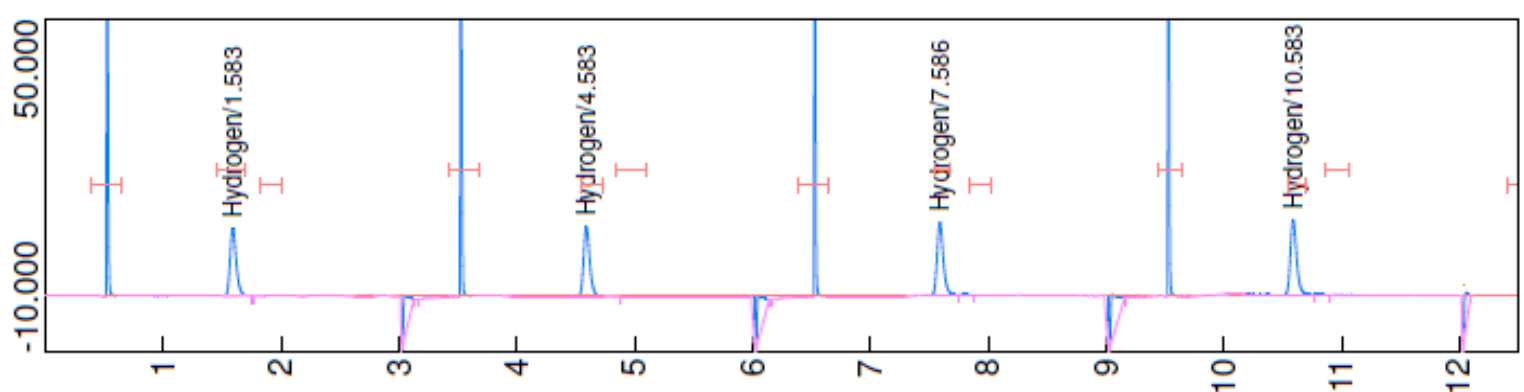

Figure 5.1.8: Gas chromatogram for sweep argon at $350{ }^{\circ} \mathrm{C}$ inside a tertiary insert with $5 \% \mathrm{H}_{2} / 95 \% \mathrm{~N}_{2}$ in the reaction space.

\section{Nickel-on-Palladium-No-Permeation Insert, Permeation Test}

This tertiary insert was designed without holes through the steel underneath the palladium to prevent permeation of hydrogen through to the sweep gas inside. One set of permeation experiments was conducted with this insert, to show that hydrogen could not permeate through the occluded palladium. Figure 5.1.9 shows a chromatogram for this permeation experiment. Each injection cycle has an injection peak at 0.5 minutes, a negative peak for the opening of the sample loop at 3.0 minutes and a small air peak at 1.8 minutes the same as chromatogram for the sweep gas when fed directly from the rotameter to the GC.

The small peak at 1.8 minutes shows that the air leak in the argon supply to the reactor had returned. If a hydrogen peak had been present, it would have been expected to be significantly larger than the nitrogen and oxygen peaks that overlap at 1.8 minutes. 


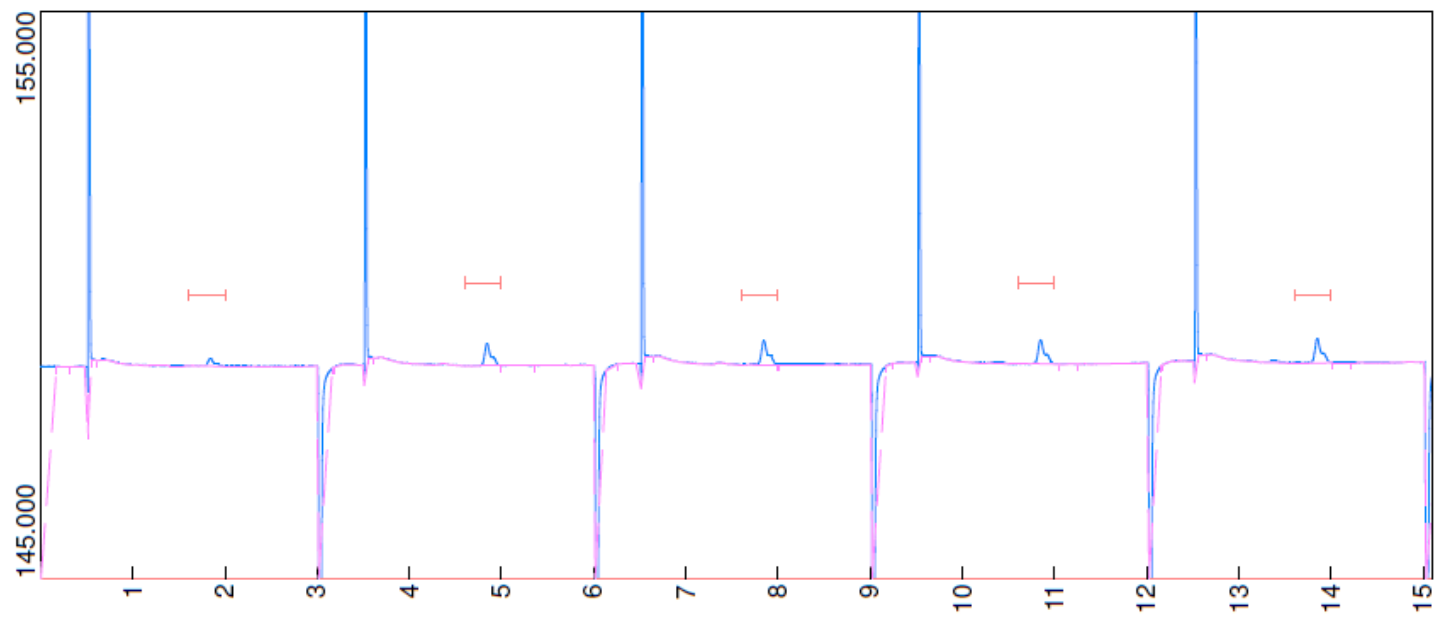

Figure 5.1.9: Chromatogram for the permeation experiment for the Nickel-onPalladium-No-Permeation.

\subsubsection{Alternative Secondary Insert Designs}

Two designs for new space filling sample holders to hold the catalyst in the best position while reducing the gas volume are shown in Figure 5.1.10. Design one was a divided rod with struts holding two solid half cylinders $2 \mathrm{~mm}$ apart. The spaces between the struts were $13 \mathrm{~mm}$ long arranged in such a way to enable four spaces to sit four samples of the same type in line, filling the bottom half of the gap and leaving a $1 \mathrm{~mm} \times 12 \mathrm{~mm}$ space for gas flow. This was designed to be made by cutting a $12 \mathrm{~mm}$ diameter stainless steel rod longitudinally and welding the struts in place. Design two changed this concept by varying the height of the samples, alternating high and low to perturb gas flow and obscure any clear paths for gas to flow. The tubular design that was chosen was initially seen as simpler because of the relative ease of construction and because a pathway forward to developing permeation modules could be seen. This choice did however create new challenges in the catalyst development.

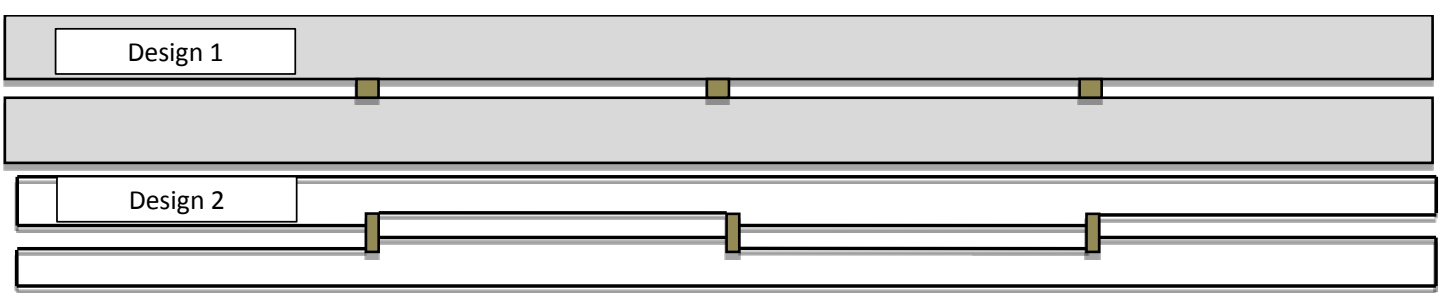

Figure 5.1.10: two designs for space filling sample holders. 


\subsubsection{Tertiary Insert Design}

A design drawing for the tertiary insert is shown in Figure 5.1.11. The main tube, drawn in blue on Figure 5.1.11, replaced the secondary insert but sealed to the primary insert. This new insert extended backwards from that seal with two 1/8" Swagelok tubes to allow a sweep gas to flush out any permeate. Because of the tube that ran inside the main tube to deliver sweep gas to the reaction end of the main tube, marked in red on Figure 5.1.11, the whole design was named the 'tertiary insert'. The ammonia reaction was intended to take place between the outer tube of this insert and the primary insert outside it, analogous to the secondary insert.

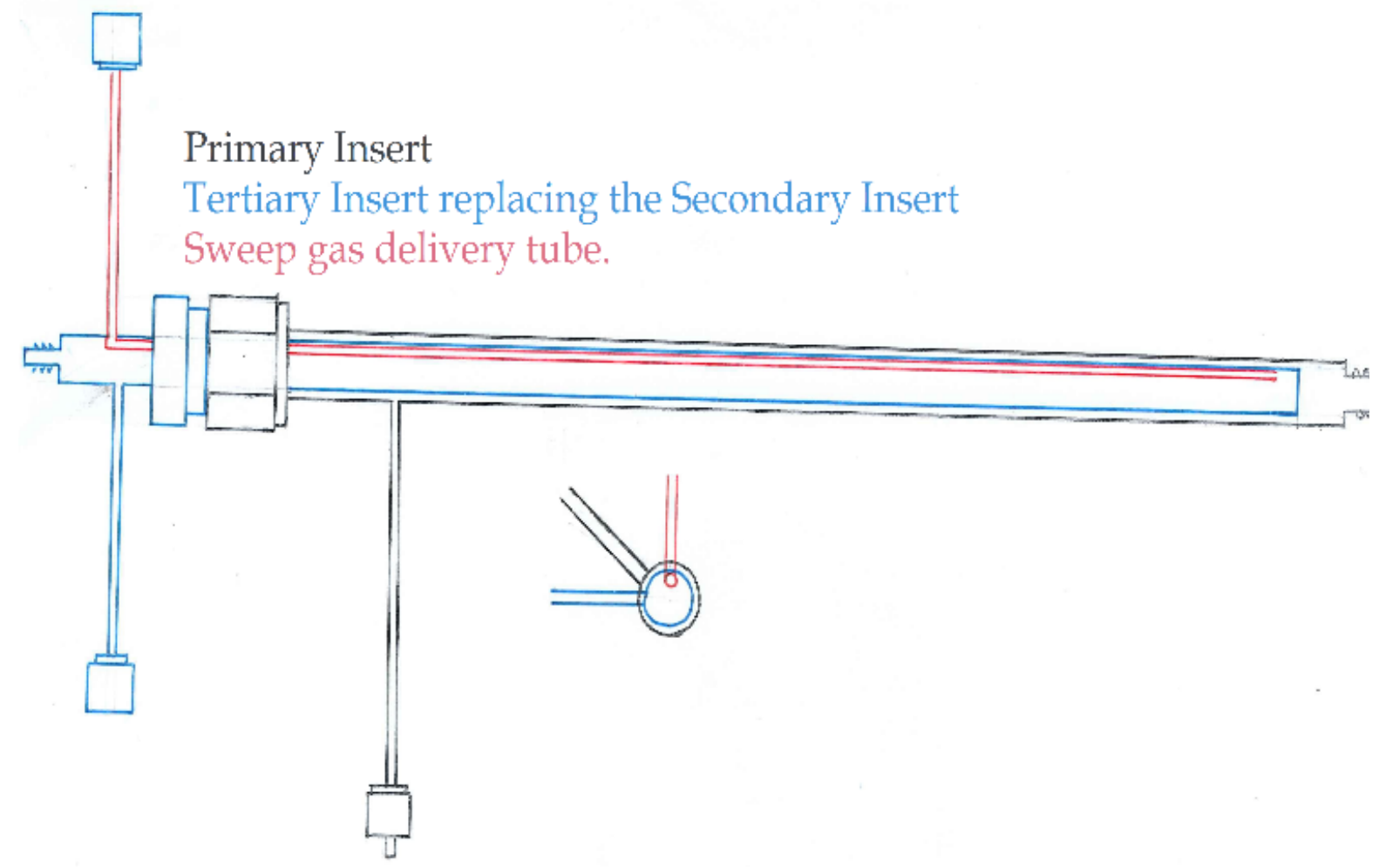

Figure 5.1.11: design drawing of the tertiary insert.

Two further changes were made to the design in Figure 5.1.11: the right angle in the sweep gas tube was replaced with a curving tube because right angle was too difficult to manufacture without creating a gas leak, and the 1/16" Swagelok connection at the outside end where the temperature probe sealed to the tertiary insert was moved off centre so the sweep gas tube did not prevent the probe from reaching the reaction point of the insert. 
The regions of the tube for replacement with palladium and addition of catalyst were chosen by measuring temperature profiles in the primary insert and, in a prototype tertiary insert. Figure 5.1.12 shows the temperature profile for the empty primary insert and a prototype tertiary insert when the external temperature of the reactor is $403{ }^{\circ} \mathrm{C}$ with the replacement regions marked. Two different permeation designs were fabricated, one with a nickel foam catalyst in zone 1 and palladium in zone 2 and a second with the palladium in zone 1 and the nickel foam catalyst also in zone 1, directly on top of the palladium. Nickel foam was chosen as the catalyst because the lithographic process for templating could not be adapted to suit the tertiary inserts. The tertiary inserts used are shown photographically in Figure 2.2.16.

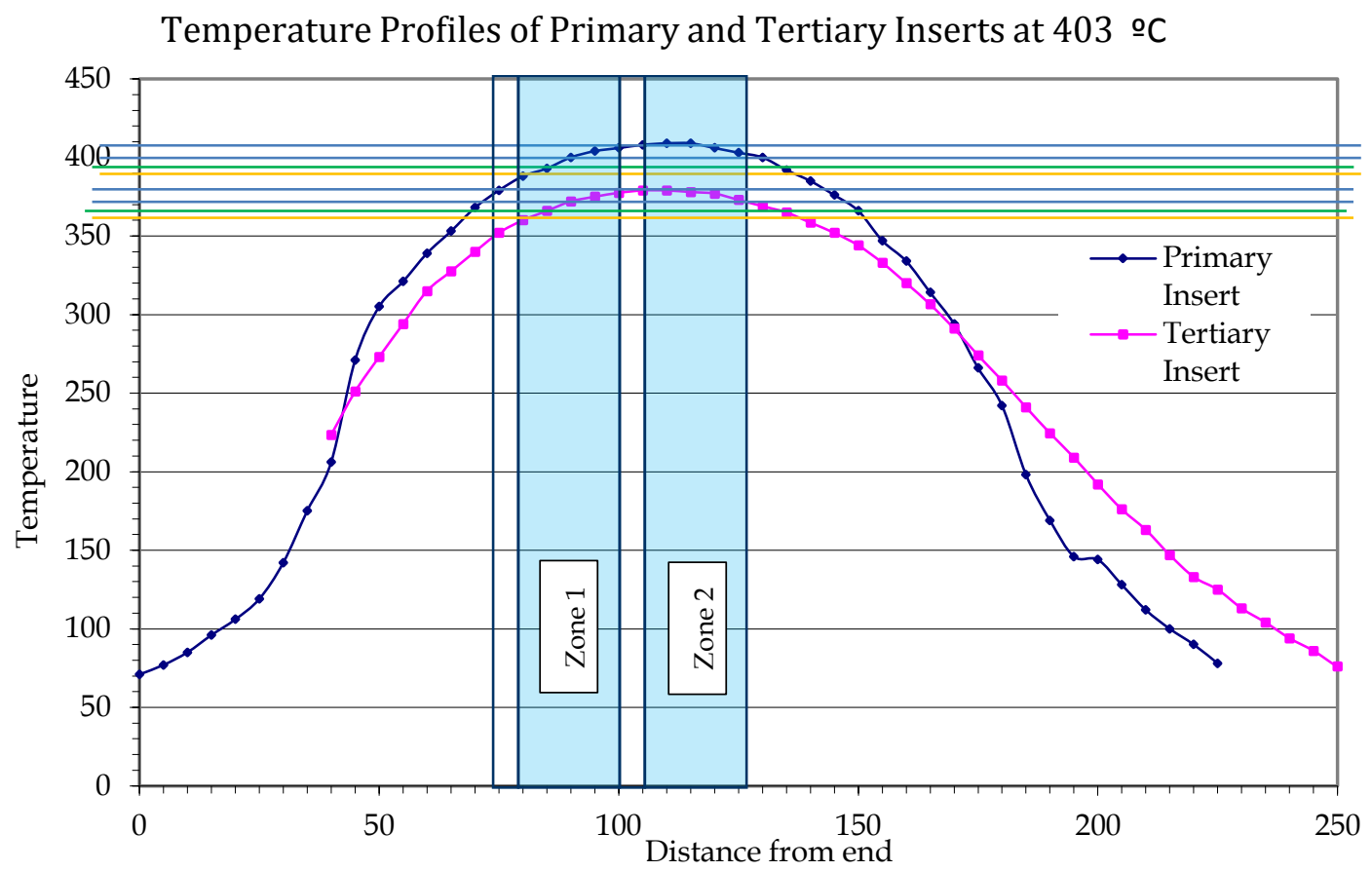

Figure 5.1.12: Temperature Profile inside the primary and tertiary inserts when the external temperature of the reactor is $403^{\circ} \mathrm{C}$

\subsubsection{GC Calculation Tests}

Section 2.2.12.3.2 developed equations for calculating the molar flow of each gas species from the gas chromatograph measurements for the secondary inserts.

Table 5.1.1 shows the data used to establish the model for these calculations and the subsequent sections test the equations in by comparing them to back calculations of the model. If the equations prove reliable, they will be helpful for interpreting 
Jonathan Tailby

measurements, when the background data equivalent to Table 5.1 .1 will not be available.

Table 5.1.1: Background data for the calculations.

\begin{tabular}{|c|c|c|c|c|c|c|c|c|c|}
\hline & & & & & & & & & \\
\hline & flow & ition & D & n Molar Flov & $\begin{array}{l}\text { NPercentage } \\
\text { Nitrogen }\end{array}$ & $\begin{array}{l}\text { Molar Flon } \\
\text { Nitrogen }\end{array}$ & $\begin{array}{l}\text { centage } \\
\text { drogen }\end{array}$ & \begin{tabular}{|l} 
Molar \\
Flow \\
Hydrogen
\end{tabular} & A \\
\hline $\mathrm{AI}$ & $\mathrm{n}_{\mathrm{AI}}$ & $\%_{\mathrm{D}}$ & $n_{D}$ & $n_{\mathrm{AF}}$ & $\%_{\mathrm{NF}}$ & $n_{\mathrm{NF}}$ & $\%_{\mathrm{HF}}$ & $n_{\mathrm{HF}}$ & $\mathrm{n}_{\mathrm{TF}}$ \\
\hline $0 \%$ & 2.01E-04 & $0 \%$ & $0.00 \mathrm{E}+00$ & 2.01E-04 & $0.0 \%$ & $0.00 \mathrm{E}+00$ & $0.0 \%$ & $0.00 \mathrm{E}+00$ & $2.01 \mathrm{E}-04$ \\
\hline $0 \%$ & -04 & $1 \%$ & 2.0 & $1.99 \mathrm{E}-04$ & $0.5 \%$ & $1.02 \mathrm{E}-06$ & $1.5 \%$ & $3.02 \mathrm{E}-06$ & 2.00 \\
\hline $0 \%$ & 2.01E-04 & $2 \%$ & $4.02 \mathrm{E}-06$ & 1.97E-04 & $1.0 \%$ & $2.05 \mathrm{E}-06$ & $3.0 \%$ & $6.03 \mathrm{E}-06$ & E-04 \\
\hline $0 \%$ & $2.01 \mathrm{E}-04$ & $3 \%$ & 6.03E-06 & 1.95E-04 & $1.5 \%$ & $3.11 \mathrm{E}-06$ & $4.5 \%$ & $9.05 \mathrm{E}-06$ & $\mathrm{E}-04$ \\
\hline $0 \%$ & 2.01E-04 & $4 \%$ & 8.04E-06 & $1.93 \mathrm{E}-04$ & $2.0 \%$ & $4.18 \mathrm{E}-06$ & $6.0 \%$ & $1.21 \mathrm{E}-05$ & 2.0 \\
\hline $0 \%$ & 2.01E-04 & $5 \%$ & 1.01E-05 & $1.91 \mathrm{E}-04$ & $2.5 \%$ & $5.28 \mathrm{E}-06$ & $7.5 \%$ & $1.51 \mathrm{E}-05$ & \\
\hline $0 \%$ & 2.01E-04 & $6 \%$ & $1.21 \mathrm{E}-05$ & $89 \mathrm{E}-04$ & $3.0 \%$ & $6.40 \mathrm{E}-06$ & $9.0 \%$ & $1.81 \mathrm{E}-05$ & $8-04$ \\
\hline $0 \%$ & 2.01E-04 & $7 \%$ & 1.41E-05 & $1.87 \mathrm{E}-04$ & $3.5 \%$ & 7.53E-06 & $10.5 \%$ & 2.11E-05 & 2.15E-04 \\
\hline $0 \%$ & 2.01E-04 & $8 \%$ & 1.61E-05 & $1.85 \mathrm{E}-04$ & $4.0 \%$ & 8.69E-06 & $12.0 \%$ & 2.41E-05 & 04 \\
\hline $0 \%$ & 2.01E-04 & $9 \%$ & $1.81 \mathrm{I}$ & -04 & $4.5 \%$ & $9.86 \mathrm{E}-06$ & $13.5 \%$ & 2.71E-05 & -04 \\
\hline $00 \%$ & 2.01E-04 & $10 \%$ & 2.01E-05 & $1.81 \mathrm{E}-04$ & $5.0 \%$ & 1.11E-05 & $15.0 \%$ & $3.02 \mathrm{E}-05$ & 2.21E-04 \\
\hline $0 \%$ & 2.01E-04 & $11 \%$ & 2.21E-05 & $1.79 \mathrm{E}-04$ & $5.5 \%$ & $1.23 \mathrm{E}-05$ & $16.5 \%$ & 3.32E-05 & -04 \\
\hline $0 \%$ & 2.01E-04 & $12 \%$ & $2.41 \mathrm{E}-05$ & -04 & $6.0 \%$ & $1.35 \mathrm{E}-05$ & $18.0 \%$ & $3.62 \mathrm{E}-05$ & \\
\hline $100 \%$ & 2.01E-04 & $13 \%$ & 2.61E-05 & 1.75E-04 & $6.5 \%$ & $1.48 \mathrm{E}-05$ & $19.5 \%$ & 3.92E-05 & 2.27E-04 \\
\hline $00 \%$ & 2.01E-04 & $14 \%$ & 2.82E-05 & $1.73 \mathrm{E}-04$ & $7.0 \%$ & $1.60 \mathrm{E}-05$ & $21.0 \%$ & $4.22 \mathrm{E}-05$ & 2.2 \\
\hline $100 \%$ & 2.01E-04 & $15 \%$ & 3.02E-05 & $1.71 \mathrm{E}-04$ & $7.5 \%$ & $1.73 \mathrm{E}-05$ & $22.5 \%$ & 4.52E-05 & 04 \\
\hline $0 \%$ & 2.0 & $16 \%$ & 3.2 & & $8.0 \%$ & -05 & 24.1 & & 04 \\
\hline $100 \%$ & 2.01E-04 & $17 \%$ & 3.42E-05 & $1.67 \mathrm{E}-04$ & $8.5 \%$ & 2.00E-05 & $25.5 \%$ & $5.13 \mathrm{E}-05$ & $2.35 \mathrm{E}-04$ \\
\hline $100 \%$ & 2.01E-04 & $18 \%$ & 3.62E-05 & $1.65 \mathrm{E}-04$ & $9.0 \%$ & $2.14 \mathrm{E}-05$ & $27.0 \%$ & 5.43E-05 & -04 \\
\hline $100 \%$ & 2.01E-04 & $19 \%$ & 5 & -04 & 9 & 2.27E-05 & 28. & 5.73E-05 & \\
\hline $100 \%$ & $2.01 \mathrm{E}-04$ & $20 \%$ & 4.02E-05 & $1.61 \mathrm{E}-04$ & $10.0 \%$ & $2.41 \mathrm{E}-05$ & $30.0 \%$ & $6.03 \mathrm{E}-05$ & $2.41 \mathrm{E}-04$ \\
\hline $100 \%$ & 2.01E-04 & $21 \%$ & 4.22E-05 & $1.59 \mathrm{E}-04$ & $10.5 \%$ & 2.56E-05 & $31.5 \%$ & 6.33E-05 & 2.43E-04 \\
\hline $100 \%$ & 2.01E-04 & $22 \%$ & $4.42 \mathrm{E}-05$ & -04 & $11.0 \%$ & 2.70E-05 & $33.0 \%$ & $6.64 \mathrm{E}-05$ & \\
\hline $00 \%$ & 2.01E-04 & $23 \%$ & 4.63E-05 & $1.55 \mathrm{E}-04$ & $11.5 \%$ & 2.84E-05 & $34.5 \%$ & 6.94E-05 & 2.47E-04 \\
\hline $100 \%$ & 2.01E-04 & $24 \%$ & 4.83E-05 & $1.53 \mathrm{E}-04$ & $12.0 \%$ & 2.99E-05 & $36.0 \%$ & 7.24E-05 & 2.49E-04 \\
\hline $100 \%$ & 2.01E-04 & $25 \%$ & 5.03E-05 & $1.51 \mathrm{E}-04$ & $12.5 \%$ & $3.14 \mathrm{E}-05$ & $37.5 \%$ & 7.54E-05 & 04 \\
\hline $100 \%$ & 2.01E-04 & $26 \%$ & $5.23 \mathrm{E}-05$ & $1.49 \mathrm{E}-04$ & $13.0 \%$ & 3.29E-05 & $39.0 \%$ & $7.84 \mathrm{E}-05$ & $2.53 \mathrm{E}-04$ \\
\hline $100 \%$ & 2.01E-04 & $27 \%$ & $5.43 \mathrm{E}-05$ & 1.47E-04 & $13.5 \%$ & 3.45E-05 & $40.5 \%$ & $8.14 \mathrm{E}-05$ & $2.55 \mathrm{E}-04$ \\
\hline $100 \%$ & 2.01E-04 & $28 \%$ & 5.63E-05 & $1.45 \mathrm{E}-04$ & $14.0 \%$ & $3.60 \mathrm{E}-05$ & $42.0 \%$ & $8.45 \mathrm{E}-05$ & 2.57E-04 \\
\hline $100 \%$ & 2.01E-04 & $29 \%$ & 5.83E-05 & $1.43 \mathrm{E}-04$ & $14.5 \%$ & $3.76 \mathrm{E}-05$ & $43.5 \%$ & $8.75 \mathrm{E}-05$ & $2.59 \mathrm{E}-04$ \\
\hline $100 \%$ & 2.01E-04 & $30 \%$ & $6.03 \mathrm{E}-05$ & $1.41 \mathrm{E}-04$ & $15.0 \%$ & 3.92E-05 & $45.0 \%$ & $9.05 \mathrm{E}-05$ & 2.61E-04 \\
\hline $100 \%$ & 2.01E-04 & $31 \%$ & $6.23 \mathrm{E}-05$ & $1.39 \mathrm{E}-04$ & $15.5 \%$ & $4.08 \mathrm{E}-05$ & $46.5 \%$ & 9.35E-05 & 2.63E-04 \\
\hline $100 \%$ & 2.01E-04 & $32 \%$ & $6.44 \mathrm{E}-05$ & $1.37 \mathrm{E}-04$ & $16.0 \%$ & $4.25 \mathrm{E}-05$ & $48.0 \%$ & $9.65 \mathrm{E}-05$ & $2.65 \mathrm{E}-04$ \\
\hline $100 \%$ & 2.01E-04 & $33 \%$ & 6.64E-05 & 1.35E-04 & $16.5 \%$ & $4.41 \mathrm{E}-05$ & $49.5 \%$ & $9.95 \mathrm{E}-05$ & 2.67E-04 \\
\hline $100 \%$ & 2.01E-04 & $34 \%$ & $6.84 \mathrm{E}-05$ & $1.33 \mathrm{E}-04$ & $17.0 \%$ & $4.58 \mathrm{E}-05$ & $51.0 \%$ & $1.03 \mathrm{E}-04$ & 2.69E-04 \\
\hline $100 \%$ & 2.01E-04 & $35 \%$ & 7.04E-05 & $1.31 \mathrm{E}-04$ & $17.5 \%$ & $4.75 \mathrm{E}-05$ & $52.5 \%$ & $1.06 \mathrm{E}-04$ & $2.71 \mathrm{E}-04$ \\
\hline $0 \%$ & 2.01E-04 & $36 \%$ & 7.24E-05 & $1.29 \mathrm{E}-04$ & $18.0 \%$ & $4.92 \mathrm{E}-05$ & $54.0 \%$ & 1.09E-04 & $.74 \mathrm{E}-04$ \\
\hline
\end{tabular}




\begin{tabular}{|c|c|c|c|c|c|c|c|c|c|}
\hline $\begin{array}{l}\text { Percentage } \\
\text { Ammonia }\end{array}$ & $\begin{array}{l}\text { Initial } \\
\text { Molar flow }\end{array}$ & $\begin{array}{l}\text { Percentage } \\
\text { Decomposition }\end{array}$ & $\begin{array}{l}\text { Molar Rate o } \\
\text { n Decompositio }\end{array}$ & $\begin{array}{l}\text { of Final } \\
\text { on|Molar Flow } \\
\text { Ammonia }\end{array}$ & $\begin{array}{l}\text { Final } \\
\text { wercentag } \\
\text { Nitrogen }\end{array}$ & $\begin{array}{l}\text { Final } \\
\text { e Molar Flon } \\
\text { Nitrogen }\end{array}$ & $\begin{array}{l}\text { Final } \\
\text { w Percentage } \\
\text { Hydrogen }\end{array}$ & \begin{tabular}{|l} 
Final \\
Molar \\
Flow \\
Hydrogen
\end{tabular} & \begin{tabular}{|l} 
Final Total \\
Molar Flow
\end{tabular} \\
\hline$\%_{\mathrm{AI}}$ & $\mathrm{n}_{\mathrm{AI}}$ & $\%_{D}$ & $n_{D}$ & $\mathrm{n}_{\mathrm{AF}}$ & $\%_{N F}$ & $n_{N F}$ & $\%_{\mathrm{HF}}$ & $\mathrm{n}_{\mathrm{HF}}$ & $\mathrm{n}_{\mathrm{TF}}$ \\
\hline $100 \%$ & 2.01E-04 & $37 \%$ & 7.44E-05 & 1.27E-04 & $18.5 \%$ & 5.10E-05 & $55.5 \%$ & $1.12 \mathrm{E}-04$ & $2.76 \mathrm{E}-04$ \\
\hline $100 \%$ & 2.01E-04 & $38 \%$ & 7.64E-05 & $1.25 \mathrm{E}-04$ & $19.0 \%$ & 5.27E-05 & $57.0 \%$ & $1.15 \mathrm{E}-04$ & $2.78 \mathrm{E}-04$ \\
\hline $100 \%$ & 2.01E-04 & $39 \%$ & $7.84 \mathrm{E}-05$ & 1.23E-04 & $19.5 \%$ & $5.45 \mathrm{E}-05$ & $58.5 \%$ & $1.18 \mathrm{E}-04$ & $2.80 \mathrm{E}-04$ \\
\hline $100 \%$ & $2.01 \mathrm{E}-04$ & $40 \%$ & 8.04E-05 & $1.21 \mathrm{E}-04$ & $20.0 \%$ & 5.63E-05 & $60.0 \%$ & $1.21 \mathrm{E}-04$ & 2.82E-04 \\
\hline $100 \%$ & 2.01E-04 & $41 \%$ & $8.25 \mathrm{E}-05$ & 1.19E-04 & $20.5 \%$ & 5.81E-05 & $61.5 \%$ & $1.24 \mathrm{E}-04$ & 2.84E-04 \\
\hline $100 \%$ & 2.01E-04 & $42 \%$ & $8.45 \mathrm{E}-05$ & 1.17E-04 & $21.0 \%$ & $6.00 \mathrm{E}-05$ & $63.0 \%$ & $1.27 \mathrm{E}-04$ & 2.86E-04 \\
\hline $100 \%$ & 2.01E-04 & $43 \%$ & 8.65E-05 & $1.15 \mathrm{E}-04$ & $21.5 \%$ & $6.18 \mathrm{E}-05$ & $64.5 \%$ & 1.30E-04 & $2.88 \mathrm{E}-04$ \\
\hline $100 \%$ & 2.01E-04 & $44 \%$ & 8.85E-05 & 1.13E-04 & $22.0 \%$ & 6.37E-05 & $66.0 \%$ & $1.33 \mathrm{E}-04$ & $2.90 \mathrm{E}-04$ \\
\hline $100 \%$ & 2.01E-04 & $45 \%$ & $9.05 \mathrm{E}-05$ & 1.11E-04 & $22.5 \%$ & $6.56 \mathrm{E}-05$ & $67.5 \%$ & $1.36 \mathrm{E}-04$ & 2.92E-04 \\
\hline
\end{tabular}

\subsubsection{Secondary Insert Hydrogen}

Table 5.1.2 shows the results of the test calculations for Equation 2.2.5 for ammonia decomposition up to $45 \%$. Of the 45 calculations, there were 15 where the new calculation gave a different answer to reversing the calculation in the model data with an average difference of $2.03 \times 10^{-20}$, maximum $5.42 \times 10^{-20}$ and minimum $3.39 \times 10^{-21}$.

Equation 2.2.5

$$
n_{H F}=\%_{H F}\left(n_{I}+\frac{2}{3} \times \%_{H F} \times n_{I}\right)
$$

\begin{tabular}{|c|c|c|c|c|c|}
\hline \begin{tabular}{|l|} 
Final Total \\
Molar Flow
\end{tabular} & $\begin{array}{l}\text { Final Molar } \\
\text { Flow } \\
\text { Hydrogen }\end{array}$ & $\begin{array}{l}\text { Final } \\
\text { Percentage } \\
\text { Hydrogen }\end{array}$ & $\begin{array}{l}\text { molar } \\
\text { hydrogen } \\
\text { flow by } \\
\text { reverse model }\end{array}$ & $\begin{array}{l}\text { Molar } \\
\text { hydrogen flow } \\
\text { by Equation } \\
2.2 .5\end{array}$ & $\begin{array}{l}\text { Difference } \\
\text { in molar } \\
\text { flow } \\
\text { calculations }\end{array}$ \\
\hline $\mathbf{n}_{\mathrm{TF}}$ & $\mathbf{n}_{\mathrm{HF}}$ & $\% \%_{\mathrm{HF}}$ & $\begin{array}{l}n_{\mathrm{HF}}= \\
\%_{\mathrm{HF}} *(\mathrm{n}+\mathrm{d})\end{array}$ & $n_{H F}$ & \\
\hline $2.01 \mathrm{E}-04$ & $0.00 \mathrm{E}+00$ & $0.0 \%$ & 0 & & \\
\hline 2.03E-04 & 3.02E-06 & $1.5 \%$ & 3.05E-06 & 3.05E-06 & $0.00 \mathrm{E}+00$ \\
\hline 2.05E-04 & 6.03E-06 & $3.0 \%$ & 6.15E-06 & 6.15E-06 & $0.00 \mathrm{E}+00$ \\
\hline 2.07E-04 & 9.05E-06 & $4.5 \%$ & 9.32E-06 & 9.32E-06 & $0.00 \mathrm{E}+00$ \\
\hline 2.09E-04 & $1.21 \mathrm{E}-05$ & $6.0 \%$ & $1.25 \mathrm{E}-05$ & $1.25 \mathrm{E}-05$ & $0.00 \mathrm{E}+00$ \\
\hline 2.11E-04 & $1.51 \mathrm{E}-05$ & $7.5 \%$ & $1.58 \mathrm{E}-05$ & 1.58E-05 & $0.00 \mathrm{E}+00$ \\
\hline 2.13E-04 & $1.81 \mathrm{E}-05$ & $9.0 \%$ & 1.92E-05 & 1.92E-05 & $0.00 \mathrm{E}+00$ \\
\hline $2.15 \mathrm{E}-04$ & 2.11E-05 & $10.5 \%$ & 2.26E-05 & $2.26 \mathrm{E}-05$ & 3.39E-21 \\
\hline 2.17E-04 & 2.41E-05 & $12.0 \%$ & 2.61E-05 & 2.61E-05 & 3.39E-21 \\
\hline 2.19E-04 & 2.71E-05 & $13.5 \%$ & 2.96E-05 & 2.96E-05 & $0.00 \mathrm{E}+00$ \\
\hline
\end{tabular}


Jonathan Tailby

Table 5.1.2: Test calculations for Equation 2.2.5

\begin{tabular}{|c|c|c|c|c|c|}
\hline $\begin{array}{l}\text { Final Total } \\
\text { Molar Flow }\end{array}$ & $\begin{array}{l}\text { Final Molar } \\
\text { Flow } \\
\text { Hydrogen }\end{array}$ & $\begin{array}{l}\text { Final } \\
\text { Percentage } \\
\text { Hydrogen }\end{array}$ & $\begin{array}{l}\text { molar } \\
\text { hydrogen } \\
\text { flow by } \\
\text { reverse model }\end{array}$ & $\begin{array}{l}\text { Molar } \\
\text { hydrogen flow } \\
\text { by Equation } \\
2.2 .5\end{array}$ & $\begin{array}{l}\text { Difference } \\
\text { in molar } \\
\text { flow } \\
\text { calculations }\end{array}$ \\
\hline $\mathbf{n}_{\mathrm{TF}}$ & $\mathbf{n}_{\mathrm{HF}}$ & $\% \mathrm{HF}$ & $\begin{array}{l}\mathrm{n}_{\mathrm{HF}}= \\
\%_{\mathrm{HF}}{ }^{*}(\mathrm{n}+\mathrm{d})\end{array}$ & $n_{H F}$ & \\
\hline $2.21 \mathrm{E}-04$ & 3.02E-05 & $15.0 \%$ & 3.32E-05 & 3.32E-05 & $0.00 \mathrm{E}+00$ \\
\hline $2.23 \mathrm{E}-04$ & 3.32E-05 & $16.5 \%$ & 3.68E-05 & $3.68 \mathrm{E}-05$ & $0.00 \mathrm{E}+00$ \\
\hline $2.25 \mathrm{E}-04$ & 3.62E-05 & $18.0 \%$ & 4.05E-05 & $4.05 \mathrm{E}-05$ & $6.78 \mathrm{E}-21$ \\
\hline $2.27 \mathrm{E}-04$ & 3.92E-05 & $19.5 \%$ & 4.43E-05 & $4.43 \mathrm{E}-05$ & $6.78 \mathrm{E}-21$ \\
\hline $2.29 \mathrm{E}-04$ & 4.22E-05 & $21.0 \%$ & 4.81E-05 & $4.81 \mathrm{E}-05$ & $6.78 \mathrm{E}-21$ \\
\hline 2.31E-04 & $4.52 \mathrm{E}-05$ & $22.5 \%$ & $5.20 \mathrm{E}-05$ & $5.20 \mathrm{E}-05$ & $0.00 \mathrm{E}+00$ \\
\hline 2.33E-04 & 4.83E-05 & $24.0 \%$ & 5.60E-05 & $5.60 \mathrm{E}-05$ & $0.00 \mathrm{E}+00$ \\
\hline 2.35E-04 & 5.13E-05 & $25.5 \%$ & 6.00E-05 & 6.00E-05 & $6.78 \mathrm{E}-21$ \\
\hline 2.37E-04 & 5.43E-05 & $27.0 \%$ & 6.41E-05 & 6.41E-05 & $1.36 \mathrm{E}-20$ \\
\hline $2.39 \mathrm{E}-04$ & 5.73E-05 & $28.5 \%$ & 6.82E-05 & 6.82E-05 & $1.36 \mathrm{E}-20$ \\
\hline $2.41 \mathrm{E}-04$ & 6.03E-05 & $30.0 \%$ & 7.24E-05 & 7.24E-05 & $0.00 \mathrm{E}+00$ \\
\hline $2.43 \mathrm{E}-04$ & 6.33E-05 & $31.5 \%$ & 7.67E-05 & 7.67E-05 & $0.00 \mathrm{E}+00$ \\
\hline $2.45 \mathrm{E}-04$ & 6.64E-05 & $33.0 \%$ & $8.10 \mathrm{E}-05$ & $8.10 \mathrm{E}-05$ & $0.00 \mathrm{E}+00$ \\
\hline $2.47 \mathrm{E}-04$ & $6.94 \mathrm{E}-05$ & $34.5 \%$ & 8.53E-05 & 8.53E-05 & $0.00 \mathrm{E}+00$ \\
\hline 2.49E-04 & 7.24E-05 & $36.0 \%$ & 8.98E-05 & 8.98E-05 & $0.00 \mathrm{E}+00$ \\
\hline $2.51 \mathrm{E}-04$ & 7.54E-05 & $37.5 \%$ & $9.43 \mathrm{E}-05$ & 9.43E-05 & $0.00 \mathrm{E}+00$ \\
\hline $2.53 \mathrm{E}-04$ & 7.84E-05 & $39.0 \%$ & 9.88E-05 & 9.88E-05 & $0.00 \mathrm{E}+00$ \\
\hline $2.55 \mathrm{E}-04$ & $8.14 \mathrm{E}-05$ & $40.5 \%$ & 1.03E-04 & 1.03E-04 & $0.00 \mathrm{E}+00$ \\
\hline $2.57 \mathrm{E}-04$ & $8.45 \mathrm{E}-05$ & $42.0 \%$ & $1.08 \mathrm{E}-04$ & $1.08 \mathrm{E}-04$ & $0.00 \mathrm{E}+00$ \\
\hline $2.59 \mathrm{E}-04$ & 8.75E-05 & $43.5 \%$ & 1.13E-04 & 1.13E-04 & 2.71E-20 \\
\hline 2.61E-04 & $9.05 \mathrm{E}-05$ & $45.0 \%$ & 1.18E-04 & 1.18E-04 & 2.71E-20 \\
\hline $2.63 \mathrm{E}-04$ & 9.35E-05 & $46.5 \%$ & 1.23E-04 & 1.23E-04 & $0.00 \mathrm{E}+00$ \\
\hline $2.65 \mathrm{E}-04$ & $9.65 \mathrm{E}-05$ & $48.0 \%$ & 1.27E-04 & 1.27E-04 & $0.00 \mathrm{E}+00$ \\
\hline $2.67 \mathrm{E}-04$ & 9.95E-05 & $49.5 \%$ & 1.32E-04 & 1.32E-04 & $0.00 \mathrm{E}+00$ \\
\hline $2.69 \mathrm{E}-04$ & 1.03E-04 & $51.0 \%$ & 1.37E-04 & 1.37E-04 & $0.00 \mathrm{E}+00$ \\
\hline $2.71 \mathrm{E}-04$ & 1.06E-04 & $52.5 \%$ & 1.43E-04 & 1.43E-04 & $0.00 \mathrm{E}+00$ \\
\hline $2.74 \mathrm{E}-04$ & 1.09E-04 & $54.0 \%$ & $1.48 \mathrm{E}-04$ & $1.48 \mathrm{E}-04$ & 2.71E-20 \\
\hline $2.76 \mathrm{E}-04$ & 1.12E-04 & $55.5 \%$ & 1.53E-04 & 1.53E-04 & 2.71E-20 \\
\hline $2.78 \mathrm{E}-04$ & 1.15E-04 & $57.0 \%$ & $1.58 \mathrm{E}-04$ & $1.58 \mathrm{E}-04$ & 2.71E-20 \\
\hline $2.80 \mathrm{E}-04$ & 1.18E-04 & $58.5 \%$ & 1.64E-04 & 1.64E-04 & $5.42 \mathrm{E}-20$ \\
\hline $2.82 \mathrm{E}-04$ & 1.21E-04 & $60.0 \%$ & 1.69E-04 & 1.69E-04 & $0.00 \mathrm{E}+00$ \\
\hline $2.84 \mathrm{E}-04$ & 1.24E-04 & $61.5 \%$ & 1.74E-04 & 1.74E-04 & $0.00 \mathrm{E}+00$ \\
\hline $2.86 \mathrm{E}-04$ & 1.27E-04 & $63.0 \%$ & 1.80E-04 & 1.80E-04 & $0.00 \mathrm{E}+00$ \\
\hline $2.88 \mathrm{E}-04$ & 1.30E-04 & $64.5 \%$ & 1.85E-04 & 1.85E-04 & $0.00 \mathrm{E}+00$ \\
\hline $2.90 \mathrm{E}-04$ & 1.33E-04 & $66.0 \%$ & 1.91E-04 & 1.91E-04 & $0.00 \mathrm{E}+00$ \\
\hline 2.92E-04 & 1.36E-04 & $67.5 \%$ & 1.97E-04 & 1.97E-04 & $5.42 \mathrm{E}-20$ \\
\hline
\end{tabular}




\subsubsection{Secondary Insert Nitrogen}

Table 5.1.3 shows 45 calculations of which test Equation 2.2.4 to calculate the final molar flow of nitrogen. Out of the 45 there were 13 calculations where the new calculation gave a different answer to reversing the calculation in the model data with an average difference of $5.28 \times 10^{-21}$, maximum difference of $1.36 \times 10^{-20}$ and minimum non-zero difference of $8.47 \times 10^{-22}$.

Equation 2.2.4

$n_{N F}=\%_{N F} \times\left(n_{I}+2 \times \%_{N F} \times n_{I}\right)$

Table 5.1.3: Test calculations for Equation 2.2.4.

\begin{tabular}{|c|c|c|c|c|c|}
\hline $\begin{array}{l}\text { Final Total } \\
\text { Molar Flow }\end{array}$ & $\begin{array}{l}\text { Final Molar } \\
\text { Flow } \\
\text { Nitrogen }\end{array}$ & $\begin{array}{l}\text { Final } \\
\text { Percentage } \\
\text { Nitrogen }\end{array}$ & $\begin{array}{l}\text { Molar } \\
\text { Nitrogen flow } \\
\text { by reverse } \\
\text { model }\end{array}$ & \begin{tabular}{|l|} 
Molar \\
Nitrogen flow \\
by Equation \\
2.2 .4
\end{tabular} & $\begin{array}{l}\text { Difference } \\
\text { in molar } \\
\text { flow } \\
\text { calculations }\end{array}$ \\
\hline $\mathbf{n}_{\mathrm{TF}}$ & $\mathbf{n}_{\mathbf{N F}}$ & $\%$ NF & $\begin{array}{l}n_{N F}= \\
\%_{N F}^{*}(n+d)\end{array}$ & $\mathbf{n}_{\mathrm{NF}}$ & \\
\hline 2.01E-04 & $0.00 \mathrm{E}+00$ & $0.0 \%$ & $0.00 \mathrm{E}+00$ & $0.00 \mathrm{E}+00$ & \\
\hline 2.03E-04 & 1.01E-06 & $0.5 \%$ & $1.02 \mathrm{E}-06$ & $1.02 \mathrm{E}-06$ & $0.00 \mathrm{E}+00$ \\
\hline 2.05E-04 & 2.01E-06 & $1.0 \%$ & 2.05E-06 & $2.05 \mathrm{E}-06$ & $0.00 \mathrm{E}+00$ \\
\hline 2.07E-04 & 3.02E-06 & $1.5 \%$ & 3.11E-06 & 3.11E-06 & $0.00 \mathrm{E}+00$ \\
\hline 2.09E-04 & $4.02 \mathrm{E}-06$ & $2.0 \%$ & $4.18 \mathrm{E}-06$ & $4.18 \mathrm{E}-06$ & $0.00 \mathrm{E}+00$ \\
\hline 2.11E-04 & 5.03E-06 & $2.5 \%$ & 5.28E-06 & $5.28 \mathrm{E}-06$ & $0.00 \mathrm{E}+00$ \\
\hline 2.13E-04 & $6.03 \mathrm{E}-06$ & $3.0 \%$ & $6.40 \mathrm{E}-06$ & $6.40 \mathrm{E}-06$ & $0.00 \mathrm{E}+00$ \\
\hline 2.15E-04 & 7.04E-06 & $3.5 \%$ & 7.53E-06 & 7.53E-06 & 8.47E-22 \\
\hline 2.17E-04 & 8.04E-06 & $4.0 \%$ & 8.69E-06 & 8.69E-06 & 1.69E-21 \\
\hline 2.19E-04 & 9.05E-06 & $4.5 \%$ & $9.86 \mathrm{E}-06$ & $9.86 \mathrm{E}-06$ & $0.00 \mathrm{E}+00$ \\
\hline 2.21E-04 & 1.01E-05 & $5.0 \%$ & 1.11E-05 & 1.11E-05 & $0.00 \mathrm{E}+00$ \\
\hline 2.23E-04 & 1.11E-05 & $5.5 \%$ & 1.23E-05 & 1.23E-05 & 1.69E-21 \\
\hline 2.25E-04 & $1.21 \mathrm{E}-05$ & $6.0 \%$ & 1.35E-05 & $1.35 \mathrm{E}-05$ & 1.69E-21 \\
\hline 2.27E-04 & 1.31E-05 & $6.5 \%$ & 1.48E-05 & 1.48E-05 & 1.69E-21 \\
\hline 2.29E-04 & $1.41 \mathrm{E}-05$ & $7.0 \%$ & 1.60E-05 & $1.60 \mathrm{E}-05$ & $0.00 \mathrm{E}+00$ \\
\hline $2.31 \mathrm{E}-04$ & $1.51 \mathrm{E}-05$ & $7.5 \%$ & $1.73 \mathrm{E}-05$ & $1.73 \mathrm{E}-05$ & $0.00 \mathrm{E}+00$ \\
\hline 2.33E-04 & 1.61E-05 & $8.0 \%$ & 1.87E-05 & $1.87 \mathrm{E}-05$ & $0.00 \mathrm{E}+00$ \\
\hline 2.35E-04 & 1.71E-05 & $8.5 \%$ & 2.00E-05 & 2.00E-05 & 3.39E-21 \\
\hline 2.37E-04 & 1.81E-05 & $9.0 \%$ & 2.14E-05 & 2.14E-05 & 3.39E-21 \\
\hline 2.39E-04 & 1.91E-05 & $9.5 \%$ & 2.27E-05 & 2.27E-05 & $0.00 \mathrm{E}+00$ \\
\hline 2.41E-04 & 2.01E-05 & $10.0 \%$ & 2.41E-05 & 2.41E-05 & $0.00 \mathrm{E}+00$ \\
\hline 2.43E-04 & 2.11E-05 & $10.5 \%$ & $2.56 \mathrm{E}-05$ & $2.56 \mathrm{E}-05$ & $0.00 \mathrm{E}+00$ \\
\hline $2.45 \mathrm{E}-04$ & 2.21E-05 & $11.0 \%$ & 2.70E-05 & 2.70E-05 & $0.00 \mathrm{E}+00$ \\
\hline 2.47E-04 & 2.31E-05 & $11.5 \%$ & 2.84E-05 & $2.84 \mathrm{E}-05$ & $0.00 \mathrm{E}+00$ \\
\hline 2.49E-04 & 2.41E-05 & $12.0 \%$ & 2.99E-05 & 2.99E-05 & $0.00 \mathrm{E}+00$ \\
\hline 2.51E-04 & 2.51E-05 & $12.5 \%$ & 3.14E-05 & $3.14 \mathrm{E}-05$ & $0.00 \mathrm{E}+00$ \\
\hline 2.53E-04 & 2.61E-05 & $13.0 \%$ & 3.29E-05 & 3.29E-05 & $0.00 \mathrm{E}+00$ \\
\hline
\end{tabular}




\begin{tabular}{|c|c|c|c|c|c|}
\hline $\begin{array}{l}\text { Final Total } \\
\text { Molar Flow }\end{array}$ & $\begin{array}{l}\text { Final Molar } \\
\text { Flow } \\
\text { Nitrogen }\end{array}$ & $\begin{array}{l}\text { Final } \\
\text { Percentage } \\
\text { Nitrogen }\end{array}$ & $\begin{array}{l}\text { Molar } \\
\text { Nitrogen flow } \\
\text { by reverse } \\
\text { model }\end{array}$ & $\begin{array}{l}\text { Molar } \\
\text { Nitrogen flow } \\
\text { by Equation } \\
\text { 2.2.4 }\end{array}$ & $\begin{array}{l}\text { Difference } \\
\text { in molar } \\
\text { flow } \\
\text { calculations }\end{array}$ \\
\hline $\mathbf{n}_{\mathrm{TF}}$ & $\mathbf{n}_{\mathbf{N F}}$ & $\%$ & $\begin{array}{l}\mathrm{n}_{\mathrm{NF}}= \\
\% \mathrm{oNF}^{*}(\mathrm{n}+\mathrm{d})\end{array}$ & $\mathbf{n}_{\mathrm{NF}}$ & \\
\hline $2.55 \mathrm{E}-04$ & 2.71E-05 & $13.5 \%$ & $3.45 \mathrm{E}-05$ & 3.45E-05 & $0.00 \mathrm{E}+00$ \\
\hline 2.57E-04 & 2.82E-05 & $14.0 \%$ & 3.60E-05 & 3.60E-05 & $0.00 \mathrm{E}+00$ \\
\hline 2.59E-04 & 2.92E-05 & $14.5 \%$ & $3.76 \mathrm{E}-05$ & 3.76E-05 & $6.78 \mathrm{E}-21$ \\
\hline $2.61 \mathrm{E}-04$ & 3.02E-05 & $15.0 \%$ & 3.92E-05 & 3.92E-05 & $6.78 \mathrm{E}-21$ \\
\hline 2.63E-04 & 3.12E-05 & $15.5 \%$ & 4.08E-05 & $4.08 \mathrm{E}-05$ & $0.00 \mathrm{E}+00$ \\
\hline $2.65 \mathrm{E}-04$ & 3.22E-05 & $16.0 \%$ & $4.25 \mathrm{E}-05$ & $4.25 \mathrm{E}-05$ & $0.00 \mathrm{E}+00$ \\
\hline 2.67E-04 & 3.32E-05 & $16.5 \%$ & 4.41E-05 & 4.41E-05 & $0.00 \mathrm{E}+00$ \\
\hline 2.69E-04 & 3.42E-05 & $17.0 \%$ & $4.58 \mathrm{E}-05$ & $4.58 \mathrm{E}-05$ & $0.00 \mathrm{E}+00$ \\
\hline 2.71E-04 & 3.52E-05 & $17.5 \%$ & $4.75 \mathrm{E}-05$ & $4.75 \mathrm{E}-05$ & $0.00 \mathrm{E}+00$ \\
\hline $2.74 \mathrm{E}-04$ & 3.62E-05 & $18.0 \%$ & 4.92E-05 & 4.92E-05 & $6.78 \mathrm{E}-21$ \\
\hline $2.76 \mathrm{E}-04$ & 3.72E-05 & $18.5 \%$ & 5.10E-05 & 5.10E-05 & $0.00 \mathrm{E}+00$ \\
\hline 2.78E-04 & 3.82E-05 & $19.0 \%$ & 5.27E-05 & 5.27E-05 & $1.36 \mathrm{E}-20$ \\
\hline 2.80E-04 & 3.92E-05 & $19.5 \%$ & 5.45E-05 & 5.45E-05 & $6.78 \mathrm{E}-21$ \\
\hline 2.82E-04 & 4.02E-05 & $20.0 \%$ & 5.63E-05 & 5.63E-05 & $0.00 \mathrm{E}+00$ \\
\hline 2.84E-04 & 4.12E-05 & $20.5 \%$ & 5.81E-05 & 5.81E-05 & $0.00 \mathrm{E}+00$ \\
\hline $2.86 \mathrm{E}-04$ & 4.22E-05 & $21.0 \%$ & 6.00E-05 & 6.00E-05 & $0.00 \mathrm{E}+00$ \\
\hline $2.88 \mathrm{E}-04$ & 4.32E-05 & $21.5 \%$ & 6.18E-05 & 6.18E-05 & $0.00 \mathrm{E}+00$ \\
\hline 2.90E-04 & $4.42 \mathrm{E}-05$ & $22.0 \%$ & 6.37E-05 & 6.37E-05 & $0.00 \mathrm{E}+00$ \\
\hline 2.92E-04 & 4.52E-05 & $22.5 \%$ & $6.56 \mathrm{E}-05$ & $6.56 \mathrm{E}-05$ & $1.36 \mathrm{E}-20$ \\
\hline
\end{tabular}

\subsubsection{Secondary Insert Ammonia}

Table 5.1.4 shows 45 test calculations that use Equation 2.2.6 to calculate the final molar flow of ammonia with the secondary inserts. Decomposition values $\geq 90 \%$ and $\leq 45 \%$ are tested. Of the 55 test calculations there were 9 results where the new calculation gave a different answer to reversing the calculation in the model data with an average difference of $5.42 \times 10^{-20}$, maximum of $2.71 \times 10^{-20}$ and minimum nonzero difference $6.02 \times 10^{-21}$.

Equation 2.2.6 $n_{A F}=\%_{A F}\left(n_{I}+\left(\%_{A I}-\%_{A F}\right) \times n_{I}\right)$ 
Table 5.1.4: Test calculations for Equation 2.2.6.

\begin{tabular}{|c|c|c|c|c|c|}
\hline $\begin{array}{l}\text { Final Total } \\
\text { Molar Flow }\end{array}$ & $\begin{array}{l}\text { Final Molar } \\
\text { Flow } \\
\text { Ammonia }\end{array}$ & $\begin{array}{l}\text { Final } \\
\text { Percentage } \\
\text { Ammonia }\end{array}$ & $\begin{array}{l}\text { Molar } \\
\text { Ammonia } \\
\text { flow by } \\
\text { reverse model }\end{array}$ & $\begin{array}{l}\text { Molar } \\
\text { Ammonia } \\
\text { flow by } \\
\text { Equation 2.2.6 }\end{array}$ & $\begin{array}{l}\text { Difference } \\
\text { in molar } \\
\text { flow } \\
\text { calculations }\end{array}$ \\
\hline $\mathrm{n}_{\mathrm{TF}}$ & $\mathrm{n}_{\mathrm{AF}}$ & $\% \mathrm{AF}$ & $\mathrm{n}_{\mathrm{AF}}=\% \mathrm{AF}^{*}(\mathrm{n}+\mathrm{d})$ & $n_{\mathrm{AF}}$ & \\
\hline $2.01 \mathrm{E}-04$ & 2.01E-04 & $0.0 \%$ & $0.00 \mathrm{E}+00$ & $0.00 \mathrm{E}+00$ & \\
\hline $2.03 \mathrm{E}-04$ & 1.99E-04 & $0.5 \%$ & $1.02 \mathrm{E}-06$ & 1.02E-06 & $0.00 \mathrm{E}+00$ \\
\hline $2.05 \mathrm{E}-04$ & 1.97E-04 & $1.0 \%$ & $2.05 \mathrm{E}-06$ & $2.05 \mathrm{E}-06$ & $0.00 \mathrm{E}+00$ \\
\hline 2.07E-04 & $1.95 \mathrm{E}-04$ & $1.5 \%$ & 3.11E-06 & 3.11E-06 & $0.00 \mathrm{E}+00$ \\
\hline $2.09 \mathrm{E}-04$ & $1.93 \mathrm{E}-04$ & $2.0 \%$ & $4.18 \mathrm{E}-06$ & $4.18 \mathrm{E}-06$ & $0.00 \mathrm{E}+00$ \\
\hline $2.11 \mathrm{E}-04$ & $1.91 \mathrm{E}-04$ & $2.5 \%$ & $5.28 \mathrm{E}-06$ & $5.28 \mathrm{E}-06$ & $0.00 \mathrm{E}+00$ \\
\hline 2.13E-04 & 1.89E-04 & $3.0 \%$ & 6.40E-06 & 6.40E-06 & $0.00 \mathrm{E}+00$ \\
\hline $2.15 \mathrm{E}-04$ & 1.87E-04 & $3.5 \%$ & 7.53E-06 & $7.53 \mathrm{E}-06$ & $8.47 \mathrm{E}-22$ \\
\hline $2.17 \mathrm{E}-04$ & $1.85 \mathrm{E}-04$ & $4.0 \%$ & 8.69E-06 & $8.69 \mathrm{E}-06$ & 1.69E-21 \\
\hline 2.19E-04 & 1.83E-04 & $4.5 \%$ & 9.86E-06 & 9.86E-06 & $0.00 \mathrm{E}+00$ \\
\hline $2.21 \mathrm{E}-04$ & 1.81E-04 & $5.0 \%$ & 1.11E-05 & 1.11E-05 & $0.00 \mathrm{E}+00$ \\
\hline $2.23 \mathrm{E}-04$ & 1.79E-04 & $5.5 \%$ & $1.23 \mathrm{E}-05$ & $1.23 \mathrm{E}-05$ & $1.69 \mathrm{E}-21$ \\
\hline $2.25 \mathrm{E}-04$ & 1.77E-04 & $6.0 \%$ & 1.35E-05 & $1.35 \mathrm{E}-05$ & $1.69 \mathrm{E}-21$ \\
\hline $2.27 \mathrm{E}-04$ & $1.75 \mathrm{E}-04$ & $6.5 \%$ & 1.48E-05 & 1.48E-05 & 1.69E-21 \\
\hline $2.29 \mathrm{E}-04$ & 1.73E-04 & $7.0 \%$ & 1.60E-05 & 1.60E-05 & $0.00 \mathrm{E}+00$ \\
\hline 2.31E-04 & $1.71 \mathrm{E}-04$ & $7.5 \%$ & 1.73E-05 & 1.73E-05 & $0.00 \mathrm{E}+00$ \\
\hline $2.33 \mathrm{E}-04$ & 1.69E-04 & $8.0 \%$ & 1.87E-05 & $1.87 \mathrm{E}-05$ & $0.00 \mathrm{E}+00$ \\
\hline 2.35E-04 & $1.67 \mathrm{E}-04$ & $8.5 \%$ & 2.00E-05 & 2.00E-05 & 3.39E-21 \\
\hline $2.37 \mathrm{E}-04$ & 1.65E-04 & $9.0 \%$ & 2.14E-05 & 2.14E-05 & 3.39E-21 \\
\hline 2.39E-04 & 1.63E-04 & $9.5 \%$ & 2.27E-05 & 2.27E-05 & $0.00 \mathrm{E}+00$ \\
\hline $2.41 \mathrm{E}-04$ & 1.61E-04 & $10.0 \%$ & 2.41E-05 & 2.41E-05 & $0.00 \mathrm{E}+00$ \\
\hline 2.43E-04 & 1.59E-04 & $100.00 \%$ & 2.01E-04 & 2.01E-04 & $0.00 \mathrm{E}+00$ \\
\hline $2.45 \mathrm{E}-04$ & 1.57E-04 & $99.00 \%$ & 2.01E-04 & 2.01E-04 & $0.00 \mathrm{E}+00$ \\
\hline 2.47E-04 & $1.55 \mathrm{E}-04$ & $98.00 \%$ & 2.01E-04 & 2.01E-04 & $0.00 \mathrm{E}+00$ \\
\hline 2.49E-04 & 1.53E-04 & $97.00 \%$ & 2.01E-04 & 2.01E-04 & $0.00 \mathrm{E}+00$ \\
\hline $2.51 \mathrm{E}-04$ & $1.51 \mathrm{E}-04$ & $96.00 \%$ & 2.01E-04 & 2.01E-04 & $0.00 \mathrm{E}+00$ \\
\hline 2.53E-04 & 1.49E-04 & $95.00 \%$ & 2.01E-04 & 2.01E-04 & $0.00 \mathrm{E}+00$ \\
\hline 2.55E-04 & 1.47E-04 & $94.00 \%$ & 2.00E-04 & 2.00E-04 & $0.00 \mathrm{E}+00$ \\
\hline $2.57 \mathrm{E}-04$ & $1.45 \mathrm{E}-04$ & $93.00 \%$ & 2.00E-04 & 2.00E-04 & 2.71E-20 \\
\hline $2.59 \mathrm{E}-04$ & 1.43E-04 & $92.00 \%$ & 2.00E-04 & 2.00E-04 & $0.00 \mathrm{E}+00$ \\
\hline 2.61E-04 & 1.41E-04 & $91.00 \%$ & 1.99E-04 & 1.99E-04 & $0.00 \mathrm{E}+00$ \\
\hline $2.63 \mathrm{E}-04$ & 1.39E-04 & $90.00 \%$ & 1.99E-04 & 1.99E-04 & 2.71E-20 \\
\hline 2.65E-04 & 1.37E-04 & $89.00 \%$ & 1.99E-04 & 1.99E-04 & $0.00 \mathrm{E}+00$ \\
\hline 2.67E-04 & 1.35E-04 & $88.00 \%$ & 1.98E-04 & 1.98E-04 & $0.00 \mathrm{E}+00$ \\
\hline $2.69 \mathrm{E}-04$ & 1.33E-04 & $87.00 \%$ & 1.98E-04 & $1.98 \mathrm{E}-04$ & $0.00 \mathrm{E}+00$ \\
\hline 2.71E-04 & 1.31E-04 & $86.00 \%$ & 1.97E-04 & 1.97E-04 & $0.00 \mathrm{E}+00$ \\
\hline $2.74 \mathrm{E}-04$ & 1.29E-04 & $85.00 \%$ & 1.97E-04 & 1.97E-04 & $2.71 \mathrm{E}-20$ \\
\hline $2.76 \mathrm{E}-04$ & 1.27E-04 & $84.00 \%$ & 1.96E-04 & 1.96E-04 & $0.00 \mathrm{E}+00$ \\
\hline 2.78E-04 & 1.25E-04 & $83.00 \%$ & 1.95E-04 & 1.95E-04 & $0.00 \mathrm{E}+00$ \\
\hline
\end{tabular}




\begin{tabular}{|c|c|c|c|c|c|}
\hline \multicolumn{6}{|c|}{ Table 5.1.4: Test calculations for Equation 2.2.6. } \\
\hline $\begin{array}{l}\text { Final Total } \\
\text { Molar Flow }\end{array}$ & $\begin{array}{l}\text { Final Molar } \\
\text { Flow } \\
\text { Ammonia }\end{array}$ & $\begin{array}{l}\text { Final } \\
\text { Percentage } \\
\text { Ammonia }\end{array}$ & $\begin{array}{l}\text { Molar } \\
\text { Ammonia } \\
\text { flow by } \\
\text { reverse model }\end{array}$ & $\begin{array}{l}\text { Molar } \\
\text { Ammonia } \\
\text { flow by } \\
\text { Equation 2.2.6 }\end{array}$ & $\begin{array}{l}\text { Difference } \\
\text { in molar } \\
\text { flow } \\
\text { calculations }\end{array}$ \\
\hline $\mathrm{n}_{\mathrm{TF}}$ & $\mathrm{n}_{\mathrm{AF}}$ & $\% \mathrm{AF}$ & $\mathrm{n}_{\mathrm{AF}}=\% \mathrm{AF}^{*}(\mathrm{n}+\mathrm{d})$ & $n_{\mathrm{AF}}$ & \\
\hline 2.80E-04 & 1.23E-04 & $82.00 \%$ & $1.95 \mathrm{E}-04$ & 1.95E-04 & $2.71 E-20$ \\
\hline $2.82 \mathrm{E}-04$ & $1.21 \mathrm{E}-04$ & $81.00 \%$ & $1.94 \mathrm{E}-04$ & $1.94 \mathrm{E}-04$ & $0.00 \mathrm{E}+00$ \\
\hline $2.84 \mathrm{E}-04$ & 1.19E-04 & $80.00 \%$ & 1.93E-04 & 1.93E-04 & $0.00 \mathrm{E}+00$ \\
\hline $2.86 \mathrm{E}-04$ & 1.17E-04 & $79.00 \%$ & 1.92E-04 & 1.92E-04 & $0.00 \mathrm{E}+00$ \\
\hline $2.88 \mathrm{E}-04$ & $1.15 \mathrm{E}-04$ & $78.00 \%$ & 1.91E-04 & 1.91E-04 & $0.00 \mathrm{E}+00$ \\
\hline 2.90E-04 & 1.13E-04 & $77.00 \%$ & 1.90E-04 & 1.90E-04 & $0.00 \mathrm{E}+00$ \\
\hline 2.92E-04 & 1.11E-04 & $76.00 \%$ & 1.90E-04 & 1.90E-04 & $0.00 \mathrm{E}+00$ \\
\hline $2.51 \mathrm{E}-04$ & 1.51E-04 & $75.00 \%$ & 1.89E-04 & 1.89E-04 & $0.00 \mathrm{E}+00$ \\
\hline $2.53 \mathrm{E}-04$ & 1.49E-04 & $74.00 \%$ & 1.88E-04 & 1.88E-04 & $0.00 \mathrm{E}+00$ \\
\hline $2.55 \mathrm{E}-04$ & 1.47E-04 & $73.00 \%$ & $1.86 \mathrm{E}-04$ & $1.86 \mathrm{E}-04$ & $0.00 \mathrm{E}+00$ \\
\hline 2.57E-04 & 1.45E-04 & $72.00 \%$ & 1.85E-04 & $1.85 \mathrm{E}-04$ & $0.00 \mathrm{E}+00$ \\
\hline 2.59E-04 & 1.43E-04 & $71.00 \%$ & 1.84E-04 & $1.84 \mathrm{E}-04$ & 2.71E-20 \\
\hline 2.61E-04 & $1.41 \mathrm{E}-04$ & $70.00 \%$ & 1.83E-04 & 1.83E-04 & $5.42 \mathrm{E}-20$ \\
\hline $2.63 \mathrm{E}-04$ & 1.39E-04 & $69.00 \%$ & 1.82E-04 & 1.82E-04 & $0.00 \mathrm{E}+00$ \\
\hline $2.65 \mathrm{E}-04$ & 1.37E-04 & $68.00 \%$ & 1.81E-04 & 1.81E-04 & $0.00 \mathrm{E}+00$ \\
\hline 2.67E-04 & 1.35E-04 & $67.00 \%$ & 1.79E-04 & 1.79E-04 & $0.00 \mathrm{E}+00$ \\
\hline 2.69E-04 & 1.33E-04 & $66.00 \%$ & 1.78E-04 & 1.78E-04 & $0.00 \mathrm{E}+00$ \\
\hline 2.71E-04 & 1.31E-04 & $65.00 \%$ & $1.76 \mathrm{E}-04$ & $1.76 \mathrm{E}-04$ & $0.00 \mathrm{E}+00$ \\
\hline $2.74 \mathrm{E}-04$ & 1.29E-04 & $64.00 \%$ & $1.75 \mathrm{E}-04$ & 1.75E-04 & $0.00 \mathrm{E}+00$ \\
\hline $2.76 \mathrm{E}-04$ & 1.27E-04 & $63.00 \%$ & $1.74 \mathrm{E}-04$ & 1.74E-04 & $0.00 \mathrm{E}+00$ \\
\hline $2.78 \mathrm{E}-04$ & $1.25 \mathrm{E}-04$ & $62.00 \%$ & 1.72E-04 & $1.72 \mathrm{E}-04$ & $0.00 \mathrm{E}+00$ \\
\hline 2.80E-04 & $1.23 \mathrm{E}-04$ & $61.00 \%$ & $1.71 \mathrm{E}-04$ & $1.71 \mathrm{E}-04$ & $0.00 \mathrm{E}+00$ \\
\hline 2.82E-04 & 1.21E-04 & $60.00 \%$ & 1.69E-04 & 1.69E-04 & $0.00 \mathrm{E}+00$ \\
\hline 2.84E-04 & 1.19E-04 & $59.00 \%$ & 1.67E-04 & 1.67E-04 & $0.00 \mathrm{E}+00$ \\
\hline $2.86 \mathrm{E}-04$ & 1.17E-04 & $58.00 \%$ & $1.66 \mathrm{E}-04$ & 1.66E-04 & $0.00 \mathrm{E}+00$ \\
\hline 2.88E-04 & 1.15E-04 & $57.00 \%$ & 1.64E-04 & 1.64E-04 & 2.71E-20 \\
\hline 2.90E-04 & 1.13E-04 & $56.00 \%$ & 1.62E-04 & 1.62E-04 & 2.71E-20 \\
\hline 2.92E-04 & 1.11E-04 & $55.00 \%$ & 1.60E-04 & 1.60E-04 & 2.71E-20 \\
\hline
\end{tabular}

\subsubsection{Tertiary Insert Hydrogen Permeation}

The test calculations for the tertiary calculations included some new quantities that were not considered or assumed to be zero in the secondary insert calculations. The most important of these was hydrogen permeation, this affected all of the other quantities by reducing the final total molar flow.

For the test calculations the proportion of hydrogen that permeated through the membrane was not yet known, therefore the calculation was tested with assumed 
values. The test calculation shown here assumes $1 / 13^{\text {th }}$ of the hydrogen produced by decomposition permeated through the membrane and was detected in the sweep gas. Background data for the tertiary calculations that was not needed for the secondary calculations is shown in Table 5.1.5.

\begin{tabular}{|c|c|c|c|c|c|c|}
\hline $\begin{array}{l}\text { Percentage } \\
\text { Decomposition }\end{array}$ & \begin{tabular}{|l} 
Percentage \\
Hydrogen
\end{tabular} & $\begin{array}{l}\text { Percentage } \\
\text { Permeate } \\
\text { Hydrogen }\end{array}$ & \begin{tabular}{|l|} 
Molar Flow \\
of \\
Retentate \\
hydrogen
\end{tabular} & $\begin{array}{l}\text { Molar } \\
\text { Flow of } \\
\text { Permeate } \\
\text { Hydrogen }\end{array}$ & $\begin{array}{l}\text { Total } \\
\text { Molar } \\
\text { Flow }\end{array}$ & \begin{tabular}{|l|} 
Retentate \\
Molar Flow
\end{tabular} \\
\hline$\%_{D}$ & $\%_{\mathrm{H}}$ & $\%_{\text {H Perm }}$ & $\%_{\mathrm{HF}}$ & $\mathrm{n}_{\mathrm{H} \text { Perm }}$ & $\mathrm{n}_{\mathrm{T}}$ & $\begin{array}{l}\mathrm{n}_{\mathrm{T}}-\mathrm{n}_{\mathrm{H}} \\
\text { perm }\end{array}$ \\
\hline $0 \%$ & $0.0 \%$ & $0.00 \%$ & $0.00 \mathrm{E}+00$ & $0.00 \mathrm{E}+00$ & $2.01 \mathrm{E}-04$ & 2.01E-04 \\
\hline $1 \%$ & $1.5 \%$ & $0.12 \%$ & $2.78 \mathrm{E}-06$ & 2.32E-07 & $2.03 \mathrm{E}-04$ & 2.03E-04 \\
\hline $2 \%$ & $3.0 \%$ & $0.23 \%$ & 5.57E-06 & 4.64E-07 & 2.05E-04 & 2.05E-04 \\
\hline $3 \%$ & $4.5 \%$ & $0.35 \%$ & 8.35E-06 & $6.96 \mathrm{E}-07$ & $2.07 \mathrm{E}-04$ & $2.06 \mathrm{E}-04$ \\
\hline $4 \%$ & $6.0 \%$ & $0.46 \%$ & 1.11E-05 & 9.28E-07 & $2.09 \mathrm{E}-04$ & $2.08 \mathrm{E}-04$ \\
\hline $5 \%$ & $7.5 \%$ & $0.58 \%$ & 1.39E-05 & $1.16 \mathrm{E}-06$ & $2.11 \mathrm{E}-04$ & 2.10E-04 \\
\hline $6 \%$ & $9.0 \%$ & $0.69 \%$ & 1.67E-05 & 1.39E-06 & 2.13E-04 & 2.12E-04 \\
\hline $7 \%$ & $10.5 \%$ & $0.81 \%$ & $1.95 \mathrm{E}-05$ & $1.62 \mathrm{E}-06$ & $2.15 \mathrm{E}-04$ & $2.14 \mathrm{E}-04$ \\
\hline $8 \%$ & $12.0 \%$ & $0.92 \%$ & 2.23E-05 & $1.86 \mathrm{E}-06$ & 2.17E-04 & 2.15E-04 \\
\hline $9 \%$ & $13.5 \%$ & $1.04 \%$ & $2.51 \mathrm{E}-05$ & 2.09E-06 & $2.19 \mathrm{E}-04$ & 2.17E-04 \\
\hline $10 \%$ & $15.0 \%$ & $1.15 \%$ & $2.78 \mathrm{E}-05$ & $2.32 \mathrm{E}-06$ & $2.21 \mathrm{E}-04$ & 2.19E-04 \\
\hline $11 \%$ & $16.5 \%$ & $1.27 \%$ & 3.06E-05 & $2.55 \mathrm{E}-06$ & $2.23 \mathrm{E}-04$ & 2.21E-04 \\
\hline $12 \%$ & $18.0 \%$ & $1.38 \%$ & 3.34E-05 & 2.78E-06 & $2.25 \mathrm{E}-04$ & 2.22E-04 \\
\hline $13 \%$ & $19.5 \%$ & $1.50 \%$ & 3.62E-05 & $3.02 \mathrm{E}-06$ & $2.27 \mathrm{E}-04$ & $2.24 \mathrm{E}-04$ \\
\hline $14 \%$ & $21.0 \%$ & $1.62 \%$ & 3.90E-05 & 3.25E-06 & $2.29 \mathrm{E}-04$ & 2.26E-04 \\
\hline $15 \%$ & $22.5 \%$ & $1.73 \%$ & 4.18E-05 & $3.48 \mathrm{E}-06$ & $2.31 \mathrm{E}-04$ & $2.28 \mathrm{E}-04$ \\
\hline $16 \%$ & $24.0 \%$ & $1.85 \%$ & $4.46 \mathrm{E}-05$ & $3.71 \mathrm{E}-06$ & $2.33 \mathrm{E}-04$ & 2.30E-04 \\
\hline $17 \%$ & $25.5 \%$ & $1.96 \%$ & 4.73E-05 & 3.94E-06 & $2.35 \mathrm{E}-04$ & 2.31E-04 \\
\hline $18 \%$ & $27.0 \%$ & $2.08 \%$ & 5.01E-05 & 4.18E-06 & $2.37 \mathrm{E}-04$ & 2.33E-04 \\
\hline $19 \%$ & $28.5 \%$ & $2.19 \%$ & 5.29E-05 & $4.41 \mathrm{E}-06$ & $2.39 \mathrm{E}-04$ & 2.35E-04 \\
\hline $20 \%$ & $30.0 \%$ & $2.31 \%$ & 5.57E-05 & 4.64E-06 & $2.41 \mathrm{E}-04$ & 2.37E-04 \\
\hline $21 \%$ & $31.5 \%$ & $2.42 \%$ & 5.85E-05 & 4.87E-06 & $2.43 \mathrm{E}-04$ & 2.38E-04 \\
\hline $22 \%$ & $33.0 \%$ & $2.54 \%$ & 6.13E-05 & 5.11E-06 & $2.45 \mathrm{E}-04$ & 2.40E-04 \\
\hline $23 \%$ & $34.5 \%$ & $2.65 \%$ & 6.40E-05 & 5.34E-06 & $2.47 \mathrm{E}-04$ & 2.42E-04 \\
\hline $24 \%$ & $36.0 \%$ & $2.77 \%$ & 6.68E-05 & 5.57E-06 & $2.49 \mathrm{E}-04$ & 2.44E-04 \\
\hline $25 \%$ & $37.5 \%$ & $2.88 \%$ & $6.96 \mathrm{E}-05$ & 5.80E-06 & $2.51 \mathrm{E}-04$ & $2.46 \mathrm{E}-04$ \\
\hline $26 \%$ & $39.0 \%$ & $3.00 \%$ & 7.24E-05 & 6.03E-06 & $2.53 \mathrm{E}-04$ & 2.47E-04 \\
\hline $27 \%$ & $40.5 \%$ & $3.12 \%$ & 7.52E-05 & 6.27E-06 & $2.55 \mathrm{E}-04$ & 2.49E-04 \\
\hline $28 \%$ & $42.0 \%$ & $3.23 \%$ & 7.80E-05 & $6.50 \mathrm{E}-06$ & $2.57 \mathrm{E}-04$ & $2.51 \mathrm{E}-04$ \\
\hline $29 \%$ & $43.5 \%$ & $3.35 \%$ & 8.08E-05 & 6.73E-06 & $2.59 \mathrm{E}-04$ & 2.53E-04 \\
\hline $30 \%$ & $45.0 \%$ & $3.46 \%$ & 8.35E-05 & $6.96 \mathrm{E}-06$ & $2.61 \mathrm{E}-04$ & 2.54E-04 \\
\hline $31 \%$ & $46.5 \%$ & $3.58 \%$ & 8.63E-05 & 7.19E-06 & $2.63 \mathrm{E}-04$ & $2.56 \mathrm{E}-04$ \\
\hline $32 \%$ & $48.0 \%$ & $3.69 \%$ & 8.91E-05 & 7.43E-06 & $2.65 \mathrm{E}-04$ & $2.58 \mathrm{E}-04$ \\
\hline
\end{tabular}


Jonathan Tailby

\begin{tabular}{|c|c|c|c|c|c|c|}
\hline $\begin{array}{l}\text { Percentage } \\
\text { Decomposition }\end{array}$ & \begin{tabular}{|l} 
Percentage \\
Hydrogen
\end{tabular} & $\begin{array}{l}\text { Percentage } \\
\text { Permeate } \\
\text { Hydrogen }\end{array}$ & $\begin{array}{l}\text { Molar Flow } \\
\text { of } \\
\text { Retentate } \\
\text { hydrogen }\end{array}$ & $\begin{array}{l}\text { Molar } \\
\text { Flow of } \\
\text { Permeate } \\
\text { Hydrogen }\end{array}$ & $\begin{array}{l}\text { Total } \\
\text { Molar } \\
\text { Flow }\end{array}$ & \begin{tabular}{|l|} 
Retentate \\
Molar Flow
\end{tabular} \\
\hline$\%_{\mathrm{D}}$ & $\% \mathrm{H}$ & $\%_{\text {H Perm }}$ & $\%_{\mathrm{HF}}$ & $\mathrm{n}_{\mathrm{H} \text { Perm }}$ & $\mathrm{n}_{\mathrm{T}}$ & $\begin{array}{l}\mathrm{n}_{\mathrm{T}}-\mathrm{n}_{\mathrm{H}} \\
\text { perm }\end{array}$ \\
\hline $33 \%$ & $49.5 \%$ & $3.81 \%$ & 9.19E-05 & 7.66E-06 & 2.67E-04 & 2.60E-04 \\
\hline $34 \%$ & $51.0 \%$ & $3.92 \%$ & 9.47E-05 & 7.89E-06 & 2.69E-04 & 2.62E-04 \\
\hline $35 \%$ & $52.5 \%$ & $4.04 \%$ & 9.75E-05 & $8.12 \mathrm{E}-06$ & 2.71E-04 & 2.63E-04 \\
\hline $36 \%$ & $54.0 \%$ & $4.15 \%$ & 1.00E-04 & 8.35E-06 & $2.74 \mathrm{E}-04$ & 2.65E-04 \\
\hline $37 \%$ & $55.5 \%$ & $4.27 \%$ & 1.03E-04 & 8.59E-06 & $2.76 \mathrm{E}-04$ & 2.67E-04 \\
\hline $38 \%$ & $57.0 \%$ & $4.38 \%$ & $1.06 \mathrm{E}-04$ & $8.82 \mathrm{E}-06$ & $2.78 \mathrm{E}-04$ & 2.69E-04 \\
\hline $39 \%$ & $58.5 \%$ & $4.50 \%$ & $1.09 \mathrm{E}-04$ & 9.05E-06 & $2.80 \mathrm{E}-04$ & 2.70E-04 \\
\hline $40 \%$ & $60.0 \%$ & $4.62 \%$ & $1.11 \mathrm{E}-04$ & $9.28 \mathrm{E}-06$ & 2.82E-04 & 2.72E-04 \\
\hline $41 \%$ & $61.5 \%$ & $4.73 \%$ & $1.14 \mathrm{E}-04$ & $9.51 \mathrm{E}-06$ & 2.84E-04 & 2.74E-04 \\
\hline $42 \%$ & $63.0 \%$ & $4.85 \%$ & 1.17E-04 & 9.75E-06 & $2.86 \mathrm{E}-04$ & $2.76 \mathrm{E}-04$ \\
\hline $43 \%$ & $64.5 \%$ & $4.96 \%$ & $1.20 \mathrm{E}-04$ & 9.98E-06 & $2.88 \mathrm{E}-04$ & 2.78E-04 \\
\hline $44 \%$ & $66.0 \%$ & $5.08 \%$ & $1.23 \mathrm{E}-04$ & $1.02 \mathrm{E}-05$ & $2.90 \mathrm{E}-04$ & 2.79E-04 \\
\hline $45 \%$ & $67.5 \%$ & $5.19 \%$ & $1.25 \mathrm{E}-04$ & $1.04 \mathrm{E}-05$ & 2.92E-04 & 2.81E-04 \\
\hline $46 \%$ & $69.0 \%$ & $5.31 \%$ & $1.28 \mathrm{E}-04$ & 1.07E-05 & $2.94 \mathrm{E}-04$ & 2.83E-04 \\
\hline $47 \%$ & $70.5 \%$ & $5.42 \%$ & 1.31E-04 & $1.09 \mathrm{E}-05$ & $2.96 \mathrm{E}-04$ & 2.85E-04 \\
\hline $48 \%$ & $72.0 \%$ & $5.54 \%$ & $1.34 \mathrm{E}-04$ & $1.11 \mathrm{E}-05$ & $2.98 \mathrm{E}-04$ & 2.87E-04 \\
\hline $49 \%$ & $73.5 \%$ & $5.65 \%$ & $1.36 \mathrm{E}-04$ & $1.14 \mathrm{E}-05$ & $3.00 \mathrm{E}-04$ & $2.88 \mathrm{E}-04$ \\
\hline $50 \%$ & $75.0 \%$ & $5.77 \%$ & 1.39E-04 & $1.16 \mathrm{E}-05$ & 3.02E-04 & 2.90E-04 \\
\hline $51 \%$ & $76.5 \%$ & $5.88 \%$ & $1.42 \mathrm{E}-04$ & $1.18 \mathrm{E}-05$ & 3.04E-04 & 2.92E-04 \\
\hline $52 \%$ & $78.0 \%$ & $6.00 \%$ & $1.45 \mathrm{E}-04$ & $1.21 \mathrm{E}-05$ & $3.06 \mathrm{E}-04$ & 2.94E-04 \\
\hline $53 \%$ & $79.5 \%$ & $6.12 \%$ & $1.48 \mathrm{E}-04$ & $1.23 \mathrm{E}-05$ & $3.08 \mathrm{E}-04$ & 2.95E-04 \\
\hline $54 \%$ & $81.0 \%$ & $6.23 \%$ & $1.50 \mathrm{E}-04$ & $1.25 \mathrm{E}-05$ & 3.10E-04 & 2.97E-04 \\
\hline $55 \%$ & $82.5 \%$ & $6.35 \%$ & $1.53 \mathrm{E}-04$ & $1.28 \mathrm{E}-05$ & 3.12E-04 & 2.99E-04 \\
\hline $56 \%$ & $84.0 \%$ & $6.46 \%$ & $1.56 \mathrm{E}-04$ & $1.30 \mathrm{E}-05$ & 3.14E-04 & 3.01E-04 \\
\hline $57 \%$ & $85.5 \%$ & $6.58 \%$ & $1.59 \mathrm{E}-04$ & 1.32E-05 & $3.16 \mathrm{E}-04$ & 3.03E-04 \\
\hline $58 \%$ & $87.0 \%$ & $6.69 \%$ & 1.62E-04 & $1.35 \mathrm{E}-05$ & $3.18 \mathrm{E}-04$ & 3.04E-04 \\
\hline $59 \%$ & $88.5 \%$ & $6.81 \%$ & $1.64 \mathrm{E}-04$ & $1.37 \mathrm{E}-05$ & $3.20 \mathrm{E}-04$ & $3.06 \mathrm{E}-04$ \\
\hline $60 \%$ & $90.0 \%$ & $6.92 \%$ & 1.67E-04 & $1.39 \mathrm{E}-05$ & 3.22E-04 & 3.08E-04 \\
\hline $61 \%$ & $91.5 \%$ & $7.04 \%$ & $1.70 \mathrm{E}-04$ & $1.42 \mathrm{E}-05$ & $3.24 \mathrm{E}-04$ & 3.10E-04 \\
\hline $62 \%$ & $93.0 \%$ & $7.15 \%$ & $1.73 \mathrm{E}-04$ & $1.44 \mathrm{E}-05$ & $3.26 \mathrm{E}-04$ & 3.11E-04 \\
\hline $63 \%$ & $94.5 \%$ & $7.27 \%$ & $1.75 \mathrm{E}-04$ & $1.46 \mathrm{E}-05$ & 3.28E-04 & 3.13E-04 \\
\hline $64 \%$ & $96.0 \%$ & $7.38 \%$ & $1.78 \mathrm{E}-04$ & $1.49 \mathrm{E}-05$ & 3.30E-04 & 3.15E-04 \\
\hline $65 \%$ & $97.5 \%$ & $7.50 \%$ & $1.81 \mathrm{E}-04$ & $1.51 \mathrm{E}-05$ & 3.32E-04 & 3.17E-04 \\
\hline $66 \%$ & $99.0 \%$ & $7.62 \%$ & $1.84 \mathrm{E}-04$ & $1.53 \mathrm{E}-05$ & 3.34E-04 & 3.19E-04 \\
\hline $67 \%$ & $100.5 \%$ & $7.73 \%$ & $1.87 \mathrm{E}-04$ & $1.55 \mathrm{E}-05$ & $3.36 \mathrm{E}-04$ & 3.20E-04 \\
\hline $68 \%$ & $102.0 \%$ & $7.85 \%$ & $1.89 \mathrm{E}-04$ & $1.58 \mathrm{E}-05$ & 3.38E-04 & 3.22E-04 \\
\hline $69 \%$ & $103.5 \%$ & $7.96 \%$ & 1.92E-04 & $1.60 \mathrm{E}-05$ & 3.40E-04 & 3.24E-04 \\
\hline $70 \%$ & $105.0 \%$ & $8.08 \%$ & $1.95 \mathrm{E}-04$ & $1.62 \mathrm{E}-05$ & $3.42 \mathrm{E}-04$ & $3.26 \mathrm{E}-04$ \\
\hline $71 \%$ & $106.5 \%$ & $8.19 \%$ & $1.98 \mathrm{E}-04$ & $1.65 \mathrm{E}-05$ & $3.44 \mathrm{E}-04$ & 3.27E-04 \\
\hline
\end{tabular}




\begin{tabular}{|c|c|c|c|c|c|c|}
\hline $\begin{array}{l}\text { Percentage } \\
\text { Decomposition }\end{array}$ & $\begin{array}{l}\text { Percentage } \\
\text { Hydrogen }\end{array}$ & $\begin{array}{l}\text { Percentage } \\
\text { Permeate } \\
\text { Hydrogen }\end{array}$ & $\begin{array}{l}\text { Molar Flow } \\
\text { of } \\
\text { Retentate } \\
\text { hydrogen }\end{array}$ & $\begin{array}{l}\text { Molar } \\
\text { Flow of } \\
\text { Permeate } \\
\text { Hydrogen }\end{array}$ & $\begin{array}{l}\text { Total } \\
\text { Molar } \\
\text { Flow }\end{array}$ & \begin{tabular}{|l} 
Retentate \\
Molar Flow
\end{tabular} \\
\hline$\%_{D}$ & $\%_{\mathrm{H}}$ & $\%_{\text {H Perm }}$ & $\%_{\mathrm{HF}}$ & $\mathrm{n}_{\mathrm{H} \text { Perm }}$ & $\mathrm{n}_{\mathrm{T}}$ & $\begin{array}{l}\mathrm{n}_{\mathrm{T}}-\mathrm{n}_{\mathrm{H}} \\
\text { perm }\end{array}$ \\
\hline $72 \%$ & $108.0 \%$ & $8.31 \%$ & $2.00 \mathrm{E}-04$ & $1.67 \mathrm{E}-05$ & $3.46 \mathrm{E}-04$ & $3.29 \mathrm{E}-04$ \\
\hline $73 \%$ & $109.5 \%$ & $8.42 \%$ & 2.03E-04 & $1.69 \mathrm{E}-05$ & $3.48 \mathrm{E}-04$ & 3.31E-04 \\
\hline $74 \%$ & $111.0 \%$ & $8.54 \%$ & $2.06 \mathrm{E}-04$ & 1.72E-05 & $3.50 \mathrm{E}-04$ & 3.33E-04 \\
\hline $75 \%$ & $112.5 \%$ & $8.65 \%$ & $2.09 \mathrm{E}-04$ & $1.74 \mathrm{E}-05$ & $3.52 \mathrm{E}-04$ & $3.35 \mathrm{E}-04$ \\
\hline $76 \%$ & $114.0 \%$ & $8.77 \%$ & 2.12E-04 & $1.76 \mathrm{E}-05$ & $3.54 \mathrm{E}-04$ & $3.36 \mathrm{E}-04$ \\
\hline $77 \%$ & $115.5 \%$ & $8.88 \%$ & $2.14 \mathrm{E}-04$ & 1.79E-05 & $3.56 \mathrm{E}-04$ & 3.38E-04 \\
\hline $78 \%$ & $117.0 \%$ & $9.00 \%$ & 2.17E-04 & $1.81 \mathrm{E}-05$ & 3.58E-04 & 3.40E-04 \\
\hline $79 \%$ & $118.5 \%$ & $9.12 \%$ & $2.20 \mathrm{E}-04$ & $1.83 \mathrm{E}-05$ & $3.60 \mathrm{E}-04$ & $3.42 \mathrm{E}-04$ \\
\hline $80 \%$ & $120.0 \%$ & $9.23 \%$ & $2.23 \mathrm{E}-04$ & $1.86 \mathrm{E}-05$ & $3.62 \mathrm{E}-04$ & $3.43 \mathrm{E}-04$ \\
\hline $81 \%$ & $121.5 \%$ & $9.35 \%$ & $2.26 \mathrm{E}-04$ & $1.88 \mathrm{E}-05$ & 3.64E-04 & $3.45 \mathrm{E}-04$ \\
\hline $82 \%$ & $123.0 \%$ & $9.46 \%$ & $2.28 \mathrm{E}-04$ & 1.90E-05 & $3.66 \mathrm{E}-04$ & 3.47E-04 \\
\hline $83 \%$ & $124.5 \%$ & $9.58 \%$ & 2.31E-04 & 1.93E-05 & $3.68 \mathrm{E}-04$ & 3.49E-04 \\
\hline $84 \%$ & $126.0 \%$ & $9.69 \%$ & 2.34E-04 & 1.95E-05 & 3.70E-04 & 3.51E-04 \\
\hline $85 \%$ & $127.5 \%$ & $9.81 \%$ & 2.37E-04 & 1.97E-05 & $3.72 \mathrm{E}-04$ & $3.52 \mathrm{E}-04$ \\
\hline $86 \%$ & $129.0 \%$ & $9.92 \%$ & 2.39E-04 & $2.00 \mathrm{E}-05$ & $3.74 \mathrm{E}-04$ & 3.54E-04 \\
\hline $87 \%$ & $130.5 \%$ & $10.04 \%$ & $2.42 \mathrm{E}-04$ & $2.02 \mathrm{E}-05$ & $3.76 \mathrm{E}-04$ & $3.56 \mathrm{E}-04$ \\
\hline $88 \%$ & $132.0 \%$ & $10.15 \%$ & $2.45 \mathrm{E}-04$ & $2.04 \mathrm{E}-05$ & $3.78 \mathrm{E}-04$ & 3.58E-04 \\
\hline $89 \%$ & $133.5 \%$ & $10.27 \%$ & $2.48 \mathrm{E}-04$ & $2.07 \mathrm{E}-05$ & $3.80 \mathrm{E}-04$ & 3.59E-04 \\
\hline $90 \%$ & $135.0 \%$ & $10.38 \%$ & 2.51E-04 & 2.09E-05 & 3.82E-04 & 3.61E-04 \\
\hline $91 \%$ & $136.5 \%$ & $10.50 \%$ & $2.53 \mathrm{E}-04$ & $2.11 \mathrm{E}-05$ & $3.84 \mathrm{E}-04$ & $3.63 \mathrm{E}-04$ \\
\hline $92 \%$ & $138.0 \%$ & $10.62 \%$ & $2.56 \mathrm{E}-04$ & 2.13E-05 & $3.86 \mathrm{E}-04$ & $3.65 \mathrm{E}-04$ \\
\hline $93 \%$ & $139.5 \%$ & $10.73 \%$ & $2.59 \mathrm{E}-04$ & $2.16 \mathrm{E}-05$ & $3.88 \mathrm{E}-04$ & 3.67E-04 \\
\hline $94 \%$ & $141.0 \%$ & $10.85 \%$ & 2.62E-04 & 2.18E-05 & 3.90E-04 & 3.68E-04 \\
\hline $95 \%$ & $142.5 \%$ & $10.96 \%$ & 2.65E-04 & 2.20E-05 & 3.92E-04 & $3.70 \mathrm{E}-04$ \\
\hline $96 \%$ & $144.0 \%$ & $11.08 \%$ & 2.67E-04 & 2.23E-05 & 3.94E-04 & 3.72E-04 \\
\hline $97 \%$ & $145.5 \%$ & $11.19 \%$ & $2.70 \mathrm{E}-04$ & $2.25 \mathrm{E}-05$ & $3.96 \mathrm{E}-04$ & 3.74E-04 \\
\hline $98 \%$ & $147.0 \%$ & $11.31 \%$ & $2.73 \mathrm{E}-04$ & $2.27 \mathrm{E}-05$ & $3.98 \mathrm{E}-04$ & $3.75 \mathrm{E}-04$ \\
\hline $99 \%$ & $148.5 \%$ & $11.42 \%$ & $2.76 \mathrm{E}-04$ & 2.30E-05 & $4.00 \mathrm{E}-04$ & 3.77E-04 \\
\hline $100 \%$ & $150.0 \%$ & $11.54 \%$ & $2.78 \mathrm{E}-04$ & 2.32E-05 & 4.02E-04 & 3.79E-04 \\
\hline
\end{tabular}

Equation 2.2.7 was derived in Section 2.2.12.3.3 for calculating the rate of hydrogen permeation from the percentage of hydrogen measured in the sweep gas. Table 5.1.6 shows the test calculation results for Equation 2.2.7. The permeation rates calculated in Table 5.1.5 have been used as data for these calculations. Of the one hundred test calculations, eight gave a value that was different from reversing the test model, with 
Jonathan Tailby

a maximum difference of $1.69 \times 10^{-21}$ compared to a minimum value of $2.32 \times 10^{-7}$ $\mathrm{mol} \mathrm{min}^{-1}$.

Equation 2.2.7 $n_{\text {HPerm }}=\frac{\%_{H P e r m} \times n_{A r}}{1-\%_{H P e r m}}$

\begin{tabular}{|c|c|c|c|c|c|c|}
\hline $\begin{array}{l}\text { Initial } \\
\text { percentage } \\
\text { Argon }\end{array}$ & $\begin{array}{l}\text { Molar } \\
\text { flow } \\
\text { Argon }\end{array}$ & $\begin{array}{l}\text { Molar } \\
\text { Flow of } \\
\text { Permeate } \\
\text { Hydrogen } \\
\end{array}$ & $\begin{array}{l}\text { Percentage } \\
\text { of Permeate } \\
\text { in Sweep } \\
\text { Gas } \\
\end{array}$ & $\begin{array}{l}\text { Molar } \\
\text { Permeation } \\
\text { by reverse } \\
\text { model } \\
\end{array}$ & $\begin{array}{l}\text { Molar } \\
\text { Permeation } \\
\text { by Equation } \\
2.2 .7\end{array}$ & $\begin{array}{l}\text { Difference } \\
\text { between } \\
\text { calculated } \\
\text { Permeations }\end{array}$ \\
\hline$\%_{\text {Ar I }}$ & $\mathrm{n}_{\mathrm{Ar}}$ & $\mathrm{n}_{\mathrm{H} \text { Perm }}$ & $\%_{\text {Hperm }}$ & $\mathrm{n}_{\text {Hperm }}$ & $\mathrm{n}_{\text {Hperm }}$ & $\mathrm{nH}-\mathrm{C} n \mathrm{H}$ \\
\hline $100 \%$ & $2.01 \mathrm{E}-04$ & $0.00 \mathrm{E}+00$ & $0.00 \%$ & $0.00 \mathrm{E}+00$ & & 0 \\
\hline $100 \%$ & 2.01E-04 & 2.32E-07 & $0.12 \%$ & $2.32 \mathrm{E}-07$ & $2.32 \mathrm{E}-07$ & 0 \\
\hline $100 \%$ & 2.01E-04 & $4.64 \mathrm{E}-07$ & $0.23 \%$ & 4.64E-07 & 4.64E-07 & 0 \\
\hline $100 \%$ & 2.01E-04 & $6.96 \mathrm{E}-07$ & $0.34 \%$ & $6.96 \mathrm{E}-07$ & $6.96 \mathrm{E}-07$ & 0 \\
\hline $100 \%$ & 2.01E-04 & 9.28E-07 & $0.46 \%$ & $9.28 \mathrm{E}-07$ & $9.28 \mathrm{E}-07$ & $1.06 \mathrm{E}-22$ \\
\hline $100 \%$ & 2.01E-04 & $1.16 \mathrm{E}-06$ & $0.57 \%$ & $1.16 \mathrm{E}-06$ & $1.16 \mathrm{E}-06$ & 0 \\
\hline $100 \%$ & 2.01E-04 & $1.39 \mathrm{E}-06$ & $0.69 \%$ & $1.39 \mathrm{E}-06$ & $1.39 \mathrm{E}-06$ & 0 \\
\hline $100 \%$ & 2.01E-04 & 1.62E-06 & $0.80 \%$ & $1.62 \mathrm{E}-06$ & 1.62E-06 & $2.12 \mathrm{E}-22$ \\
\hline $100 \%$ & 2.01E-04 & $1.86 \mathrm{E}-06$ & $0.91 \%$ & $1.86 \mathrm{E}-06$ & $1.86 \mathrm{E}-06$ & 0 \\
\hline $100 \%$ & 2.01E-04 & $2.09 \mathrm{E}-06$ & $1.03 \%$ & 2.09E-06 & $2.09 \mathrm{E}-06$ & 0 \\
\hline $100 \%$ & 2.01E-04 & 2.32E-06 & $1.14 \%$ & $2.32 \mathrm{E}-06$ & $2.32 \mathrm{E}-06$ & 0 \\
\hline $100 \%$ & 2.01E-04 & $2.55 \mathrm{E}-06$ & $1.25 \%$ & $2.55 \mathrm{E}-06$ & $2.55 \mathrm{E}-06$ & 0 \\
\hline $100 \%$ & 2.01E-04 & $2.78 \mathrm{E}-06$ & $1.37 \%$ & $2.78 \mathrm{E}-06$ & $2.78 \mathrm{E}-06$ & 0 \\
\hline $100 \%$ & 2.01E-04 & 3.02E-06 & $1.48 \%$ & $3.02 \mathrm{E}-06$ & 3.02E-06 & 0 \\
\hline $100 \%$ & 2.01E-04 & 3.25E-06 & $1.59 \%$ & $3.25 \mathrm{E}-06$ & $3.25 \mathrm{E}-06$ & 0 \\
\hline $100 \%$ & $2.01 \mathrm{E}-04$ & $3.48 \mathrm{E}-06$ & $1.70 \%$ & $3.48 \mathrm{E}-06$ & $3.48 \mathrm{E}-06$ & 4.24E-22 \\
\hline $100 \%$ & 2.01E-04 & $3.71 \mathrm{E}-06$ & $1.81 \%$ & $3.71 \mathrm{E}-06$ & $3.71 \mathrm{E}-06$ & 0 \\
\hline $100 \%$ & 2.01E-04 & $3.94 \mathrm{E}-06$ & $1.92 \%$ & 3.94E-06 & 3.94E-06 & 0 \\
\hline $100 \%$ & 2.01E-04 & $4.18 \mathrm{E}-06$ & $2.03 \%$ & 4.18E-06 & $4.18 \mathrm{E}-06$ & 0 \\
\hline $100 \%$ & 2.01E-04 & $4.41 \mathrm{E}-06$ & $2.15 \%$ & $4.41 \mathrm{E}-06$ & $4.41 \mathrm{E}-06$ & 0 \\
\hline $100 \%$ & 2.01E-04 & $4.64 \mathrm{E}-06$ & $2.26 \%$ & 4.64E-06 & 4.64E-06 & 0 \\
\hline $100 \%$ & 2.01E-04 & 4.87E-06 & $2.37 \%$ & 4.87E-06 & 4.87E-06 & 0 \\
\hline $100 \%$ & $2.01 \mathrm{E}-04$ & 5.11E-06 & $2.48 \%$ & $5.11 \mathrm{E}-06$ & 5.11E-06 & 0 \\
\hline $100 \%$ & 2.01E-04 & $5.34 \mathrm{E}-06$ & $2.59 \%$ & 5.34E-06 & $5.34 \mathrm{E}-06$ & 0 \\
\hline $100 \%$ & 2.01E-04 & 5.57E-06 & $2.69 \%$ & 5.57E-06 & 5.57E-06 & 0 \\
\hline $100 \%$ & 2.01E-04 & $5.80 \mathrm{E}-06$ & $2.80 \%$ & $5.80 \mathrm{E}-06$ & $5.80 \mathrm{E}-06$ & 0 \\
\hline $100 \%$ & 2.01E-04 & 6.03E-06 & $2.91 \%$ & $6.03 \mathrm{E}-06$ & $6.03 \mathrm{E}-06$ & 0 \\
\hline $100 \%$ & 2.01E-04 & 6.27E-06 & $3.02 \%$ & 6.27E-06 & 6.27E-06 & 0 \\
\hline $100 \%$ & 2.01E-04 & $6.50 \mathrm{E}-06$ & $3.13 \%$ & $6.50 \mathrm{E}-06$ & $6.50 \mathrm{E}-06$ & 0 \\
\hline $100 \%$ & 2.01E-04 & $6.73 \mathrm{E}-06$ & $3.24 \%$ & $6.73 \mathrm{E}-06$ & $6.73 \mathrm{E}-06$ & 0 \\
\hline $100 \%$ & 2.01E-04 & $6.96 \mathrm{E}-06$ & $3.35 \%$ & $6.96 \mathrm{E}-06$ & $6.96 \mathrm{E}-06$ & 8.47E-22 \\
\hline $100 \%$ & 2.01E-04 & 7.19E-06 & $3.45 \%$ & 7.19E-06 & 7.19E-06 & 0 \\
\hline
\end{tabular}




\begin{tabular}{|c|c|c|c|c|c|c|}
\hline $\begin{array}{l}\text { Initial } \\
\text { percentage } \\
\text { Argon }\end{array}$ & $\begin{array}{l}\text { Molar } \\
\text { flow } \\
\text { Argon }\end{array}$ & $\begin{array}{l}\text { Molar } \\
\text { Flow of } \\
\text { Permeate } \\
\text { Hydrogen } \\
\end{array}$ & $\begin{array}{l}\text { Percentage } \\
\text { of Permeate } \\
\text { in Sweep } \\
\text { Gas } \\
\end{array}$ & $\begin{array}{l}\text { Molar } \\
\text { Permeation } \\
\text { by reverse } \\
\text { model } \\
\end{array}$ & $\begin{array}{l}\text { Molar } \\
\text { Permeation } \\
\text { by Equation } \\
2.2 .7\end{array}$ & $\begin{array}{l}\text { Difference } \\
\text { between } \\
\text { calculated } \\
\text { Permeations } \\
\end{array}$ \\
\hline $100 \%$ & 2.01E-04 & 7.43E-06 & $3.56 \%$ & 7.43E-06 & 7.43E-06 & 0 \\
\hline $100 \%$ & 2.01E-04 & 7.66E-06 & $3.67 \%$ & $7.66 \mathrm{E}-06$ & $7.66 \mathrm{E}-06$ & 0 \\
\hline $100 \%$ & 2.01E-04 & 7.89E-06 & $3.77 \%$ & 7.89E-06 & 7.89E-06 & 0 \\
\hline $100 \%$ & 2.01E-04 & 8.12E-06 & $3.88 \%$ & 8.12E-06 & 8.12E-06 & 0 \\
\hline $100 \%$ & 2.01E-04 & 8.35E-06 & $3.99 \%$ & 8.35E-06 & 8.35E-06 & 0 \\
\hline $100 \%$ & 2.01E-04 & 8.59E-06 & $4.09 \%$ & 8.59E-06 & 8.59E-06 & 0 \\
\hline $100 \%$ & 2.01E-04 & 8.82E-06 & $4.20 \%$ & $8.82 \mathrm{E}-06$ & $8.82 \mathrm{E}-06$ & 0 \\
\hline $100 \%$ & 2.01E-04 & 9.05E-06 & $4.31 \%$ & $9.05 \mathrm{E}-06$ & $9.05 \mathrm{E}-06$ & 0 \\
\hline $100 \%$ & 2.01E-04 & 9.28E-06 & $4.41 \%$ & $9.28 \mathrm{E}-06$ & $9.28 \mathrm{E}-06$ & 0 \\
\hline $100 \%$ & $2.01 \mathrm{E}-04$ & 9.51E-06 & $4.52 \%$ & $9.51 \mathrm{E}-06$ & $9.51 \mathrm{E}-06$ & 0 \\
\hline $100 \%$ & 2.01E-04 & $9.75 \mathrm{E}-06$ & $4.62 \%$ & $9.75 \mathrm{E}-06$ & $9.75 \mathrm{E}-06$ & 0 \\
\hline $100 \%$ & $2.01 \mathrm{E}-04$ & 9.98E-06 & $4.73 \%$ & $9.98 \mathrm{E}-06$ & $9.98 \mathrm{E}-06$ & 0 \\
\hline $100 \%$ & 2.01E-04 & 1.02E-05 & $4.83 \%$ & $1.02 \mathrm{E}-05$ & $1.02 \mathrm{E}-05$ & 0 \\
\hline $100 \%$ & 2.01E-04 & $1.04 \mathrm{E}-05$ & $4.94 \%$ & 1.04E-05 & $1.04 \mathrm{E}-05$ & 0 \\
\hline $100 \%$ & 2.01E-04 & $1.07 \mathrm{E}-05$ & $5.04 \%$ & $1.07 \mathrm{E}-05$ & 1.07E-05 & 0 \\
\hline $100 \%$ & 2.01E-04 & 1.09E-05 & $5.14 \%$ & 1.09E-05 & $1.09 \mathrm{E}-05$ & 0 \\
\hline $100 \%$ & 2.01E-04 & 1.11E-05 & $5.25 \%$ & 1.11E-05 & 1.11E-05 & 0 \\
\hline $100 \%$ & 2.01E-04 & $1.14 \mathrm{E}-05$ & $5.35 \%$ & $1.14 \mathrm{E}-05$ & $1.14 \mathrm{E}-05$ & 0 \\
\hline $100 \%$ & 2.01E-04 & $1.16 \mathrm{E}-05$ & $5.45 \%$ & $1.16 \mathrm{E}-05$ & $1.16 \mathrm{E}-05$ & 0 \\
\hline $100 \%$ & 2.01E-04 & $1.18 \mathrm{E}-05$ & $5.56 \%$ & $1.18 \mathrm{E}-05$ & $1.18 \mathrm{E}-05$ & 0 \\
\hline $100 \%$ & $2.01 \mathrm{E}-04$ & $1.21 \mathrm{E}-05$ & $5.66 \%$ & $1.21 \mathrm{E}-05$ & $1.21 \mathrm{E}-05$ & 0 \\
\hline $100 \%$ & $2.01 \mathrm{E}-04$ & $1.23 \mathrm{E}-05$ & $5.76 \%$ & $1.23 \mathrm{E}-05$ & $1.23 \mathrm{E}-05$ & 0 \\
\hline $100 \%$ & 2.01E-04 & $1.25 \mathrm{E}-05$ & $5.87 \%$ & $1.25 \mathrm{E}-05$ & $1.25 \mathrm{E}-05$ & 0 \\
\hline $100 \%$ & 2.01E-04 & $1.28 \mathrm{E}-05$ & $5.97 \%$ & $1.28 \mathrm{E}-05$ & $1.28 \mathrm{E}-05$ & 0 \\
\hline $100 \%$ & 2.01E-04 & 1.30E-05 & $6.07 \%$ & 1.30E-05 & 1.30E-05 & 0 \\
\hline $100 \%$ & 2.01E-04 & 1.32E-05 & $6.17 \%$ & 1.32E-05 & $1.32 \mathrm{E}-05$ & 0 \\
\hline $100 \%$ & 2.01E-04 & $1.35 \mathrm{E}-05$ & $6.27 \%$ & $1.35 \mathrm{E}-05$ & $1.35 \mathrm{E}-05$ & 0 \\
\hline $100 \%$ & 2.01E-04 & 1.37E-05 & $6.37 \%$ & 1.37E-05 & 1.37E-05 & 0 \\
\hline $100 \%$ & 2.01E-04 & 1.39E-05 & $6.47 \%$ & 1.39E-05 & $1.39 \mathrm{E}-05$ & 1.69E-21 \\
\hline $100 \%$ & 2.01E-04 & 1.42E-05 & $6.58 \%$ & $1.42 \mathrm{E}-05$ & $1.42 \mathrm{E}-05$ & 0 \\
\hline $100 \%$ & $2.01 \mathrm{E}-04$ & $1.44 \mathrm{E}-05$ & $6.68 \%$ & $1.44 \mathrm{E}-05$ & $1.44 \mathrm{E}-05$ & 1.69E-21 \\
\hline $100 \%$ & 2.01E-04 & 1.46E-05 & $6.78 \%$ & $1.46 \mathrm{E}-05$ & $1.46 \mathrm{E}-05$ & 0 \\
\hline $100 \%$ & $2.01 \mathrm{E}-04$ & 1.49E-05 & $6.88 \%$ & $1.49 \mathrm{E}-05$ & $1.49 \mathrm{E}-05$ & $1.69 \mathrm{E}-21$ \\
\hline $100 \%$ & $2.01 \mathrm{E}-04$ & 1.51E-05 & $6.98 \%$ & $1.51 \mathrm{E}-05$ & $1.51 \mathrm{E}-05$ & 1.69E-21 \\
\hline $100 \%$ & 2.01E-04 & $1.53 \mathrm{E}-05$ & $7.08 \%$ & $1.53 \mathrm{E}-05$ & $1.53 \mathrm{E}-05$ & 0 \\
\hline $100 \%$ & 2.01E-04 & $1.55 \mathrm{E}-05$ & $7.18 \%$ & $1.55 \mathrm{E}-05$ & $1.55 \mathrm{E}-05$ & 0 \\
\hline $100 \%$ & $2.01 \mathrm{E}-04$ & 1.58E-05 & $7.28 \%$ & $1.58 \mathrm{E}-05$ & $1.58 \mathrm{E}-05$ & 0 \\
\hline $100 \%$ & $2.01 \mathrm{E}-04$ & $1.60 \mathrm{E}-05$ & $7.37 \%$ & $1.60 \mathrm{E}-05$ & $1.60 \mathrm{E}-05$ & 0 \\
\hline $100 \%$ & 2.01E-04 & 1.62E-05 & $7.47 \%$ & $1.62 \mathrm{E}-05$ & 1.62E-05 & 0 \\
\hline
\end{tabular}




\begin{tabular}{|c|c|c|c|c|c|c|}
\hline $\begin{array}{l}\text { Initial } \\
\text { percentage } \\
\text { Argon }\end{array}$ & $\begin{array}{l}\text { Molar } \\
\text { flow } \\
\text { Argon }\end{array}$ & $\begin{array}{l}\text { Molar } \\
\text { Flow of } \\
\text { Permeate } \\
\text { Hydrogen }\end{array}$ & $\begin{array}{l}\text { Percentage } \\
\text { of Permeate } \\
\text { in Sweep } \\
\text { Gas } \\
\end{array}$ & $\begin{array}{l}\text { Molar } \\
\text { Permeation } \\
\text { by reverse } \\
\text { model } \\
\end{array}$ & $\begin{array}{l}\text { Molar } \\
\text { Permeation } \\
\text { by Equation } \\
2.2 .7 \\
\end{array}$ & $\begin{array}{l}\text { Difference } \\
\text { between } \\
\text { calculated } \\
\text { Permeations } \\
\end{array}$ \\
\hline $100 \%$ & 2.01E-04 & $1.65 \mathrm{E}-05$ & $7.57 \%$ & $1.65 \mathrm{E}-05$ & $1.65 \mathrm{E}-05$ & 0 \\
\hline $100 \%$ & 2.01E-04 & 1.67E-05 & $7.67 \%$ & 1.67E-05 & 1.67E-05 & 0 \\
\hline $100 \%$ & 2.01E-04 & $1.69 \mathrm{E}-05$ & $7.77 \%$ & 1.69E-05 & $1.69 \mathrm{E}-05$ & 0 \\
\hline $100 \%$ & 2.01E-04 & $1.72 \mathrm{E}-05$ & $7.87 \%$ & $1.72 \mathrm{E}-05$ & $1.72 \mathrm{E}-05$ & 0 \\
\hline $100 \%$ & 2.01E-04 & $1.74 \mathrm{E}-05$ & $7.96 \%$ & $1.74 \mathrm{E}-05$ & $1.74 \mathrm{E}-05$ & 0 \\
\hline $100 \%$ & $2.01 \mathrm{E}-04$ & $1.76 \mathrm{E}-05$ & $8.06 \%$ & $1.76 \mathrm{E}-05$ & $1.76 \mathrm{E}-05$ & 0 \\
\hline $100 \%$ & 2.01E-04 & $1.79 \mathrm{E}-05$ & $8.16 \%$ & $1.79 \mathrm{E}-05$ & $1.79 \mathrm{E}-05$ & 0 \\
\hline $100 \%$ & 2.01E-04 & 1.81E-05 & $8.26 \%$ & $1.81 \mathrm{E}-05$ & $1.81 \mathrm{E}-05$ & 0 \\
\hline $100 \%$ & $2.01 \mathrm{E}-04$ & $1.83 \mathrm{E}-05$ & $8.35 \%$ & $1.83 \mathrm{E}-05$ & $1.83 \mathrm{E}-05$ & 0 \\
\hline $100 \%$ & 2.01E-04 & $1.86 \mathrm{E}-05$ & $8.45 \%$ & $1.86 \mathrm{E}-05$ & $1.86 \mathrm{E}-05$ & 0 \\
\hline $100 \%$ & 2.01E-04 & $1.88 \mathrm{E}-05$ & $8.55 \%$ & $1.88 \mathrm{E}-05$ & $1.88 \mathrm{E}-05$ & 0 \\
\hline $100 \%$ & 2.01E-04 & $1.90 \mathrm{E}-05$ & $8.64 \%$ & $1.90 \mathrm{E}-05$ & $1.90 \mathrm{E}-05$ & 0 \\
\hline $100 \%$ & 2.01E-04 & $1.93 \mathrm{E}-05$ & $8.74 \%$ & $1.93 \mathrm{E}-05$ & $1.93 \mathrm{E}-05$ & 0 \\
\hline $100 \%$ & 2.01E-04 & $1.95 \mathrm{E}-05$ & $8.84 \%$ & $1.95 \mathrm{E}-05$ & $1.95 \mathrm{E}-05$ & 0 \\
\hline $100 \%$ & $2.01 \mathrm{E}-04$ & 1.97E-05 & $8.93 \%$ & 1.97E-05 & $1.97 \mathrm{E}-05$ & 0 \\
\hline $100 \%$ & 2.01E-04 & $2.00 \mathrm{E}-05$ & $9.03 \%$ & $2.00 \mathrm{E}-05$ & $2.00 \mathrm{E}-05$ & 0 \\
\hline $100 \%$ & 2.01E-04 & 2.02E-05 & $9.12 \%$ & 2.02E-05 & 2.02E-05 & 0 \\
\hline $100 \%$ & $2.01 \mathrm{E}-04$ & $2.04 \mathrm{E}-05$ & $9.22 \%$ & $2.04 \mathrm{E}-05$ & $2.04 \mathrm{E}-05$ & 0 \\
\hline $100 \%$ & 2.01E-04 & 2.07E-05 & $9.31 \%$ & 2.07E-05 & 2.07E-05 & 0 \\
\hline $100 \%$ & 2.01E-04 & $2.09 \mathrm{E}-05$ & $9.41 \%$ & $2.09 \mathrm{E}-05$ & $2.09 \mathrm{E}-05$ & 0 \\
\hline $100 \%$ & $2.01 \mathrm{E}-04$ & $2.11 \mathrm{E}-05$ & $9.50 \%$ & $2.11 \mathrm{E}-05$ & $2.11 \mathrm{E}-05$ & 0 \\
\hline $100 \%$ & 2.01E-04 & 2.13E-05 & $9.60 \%$ & 2.13E-05 & 2.13E-05 & 0 \\
\hline $100 \%$ & 2.01E-04 & $2.16 \mathrm{E}-05$ & $9.69 \%$ & $2.16 \mathrm{E}-05$ & $2.16 \mathrm{E}-05$ & 0 \\
\hline $100 \%$ & $2.01 \mathrm{E}-04$ & $2.18 \mathrm{E}-05$ & $9.78 \%$ & $2.18 \mathrm{E}-05$ & $2.18 \mathrm{E}-05$ & 0 \\
\hline $100 \%$ & 2.01E-04 & 2.20E-05 & $9.88 \%$ & 2.20E-05 & $2.20 \mathrm{E}-05$ & 0 \\
\hline $100 \%$ & 2.01E-04 & $2.23 \mathrm{E}-05$ & $9.97 \%$ & 2.23E-05 & $2.23 \mathrm{E}-05$ & 0 \\
\hline $100 \%$ & $2.01 \mathrm{E}-04$ & $2.25 \mathrm{E}-05$ & $10.07 \%$ & $2.25 \mathrm{E}-05$ & $2.25 \mathrm{E}-05$ & 0 \\
\hline $100 \%$ & 2.01E-04 & 2.27E-05 & $10.16 \%$ & 2.27E-05 & 2.27E-05 & 0 \\
\hline $100 \%$ & $2.01 \mathrm{E}-04$ & $2.30 \mathrm{E}-05$ & $10.25 \%$ & $2.30 \mathrm{E}-05$ & $2.30 \mathrm{E}-05$ & 0 \\
\hline $100 \%$ & 2.01E-04 & 2.32E-05 & $10.34 \%$ & 2.32E-05 & 2.32E-05 & 0 \\
\hline
\end{tabular}

There were 37 calculations in Table 5.1.6 where the new calculation gave a different answer to reversing the calculation in the model data with an average difference of $1.77 \times 10^{-21}$, maximum $3.39 \times 10^{-21}$ and minimum $2.65 \times 10^{-23}$. 


\subsubsection{Tertiary Insert Hydrogen Retention}

Equation 2.2.10 was derived in Section 2.2.12.3.3 for calculating the molar flow of hydrogen in the retentate from the percentage of hydrogen measured in the retentate gas. Table 5.1.7 shows the test calculation results for with the first 40 of the permeation rates calculated in Table 5.1.5.

Equation 2.2.10 $n_{H F}=\frac{\%_{H F} n_{I}-\frac{1}{3} \times n_{H P e r m} \times \%_{H F}}{\left(1-\%_{H F} \times \frac{2}{3}\right)}$

\begin{tabular}{|c|c|c|c|c|c|c|}
\hline $\begin{array}{l}\text { Percentage } \\
\text { Hydrogen }\end{array}$ & $\begin{array}{l}\text { Molar } \\
\text { Flow of } \\
\text { Retentate } \\
\text { hydrogen }\end{array}$ & $\begin{array}{l}\text { Hydrogen } \\
\text { in new } \\
\text { Total }\end{array}$ & $\begin{array}{l}\text { Molar } \\
\text { Permeation } \\
\text { by reverse } \\
\text { model }\end{array}$ & $\begin{array}{l}\text { Retentate } \\
\text { Flow by } \\
\text { reverse } \\
\text { model }\end{array}$ & $\begin{array}{l}\text { Retentate } \\
\text { flow by } \\
\text { Equation } \\
2.2 .10\end{array}$ & $\begin{array}{l}\text { Difference } \\
\text { in two } \\
\text { calculated } \\
\text { flows. }\end{array}$ \\
\hline$\% \%_{\mathrm{H}}$ & $\%_{\mathrm{HF}}$ & $\%_{\mathrm{HF}}$ & $\mathbf{n}_{\text {Hperm }}$ & $\mathbf{n}_{\mathrm{HFa}}$ & $\mathbf{n}_{\mathrm{HFb}}$ & $\mathbf{n}_{\mathrm{HFa}-} \mathbf{n}_{\mathrm{HFb}}$ \\
\hline $0.0 \%$ & $0.00 \mathrm{E}+00$ & $0.00 \%$ & $0.00 \mathrm{E}+00$ & $0.00 \mathrm{E}+00$ & $0.00 \mathrm{E}+00$ & 0 \\
\hline $1.5 \%$ & 2.78E-06 & $1.26 \%$ & 2.32E-07 & 2.78E-06 & $2.78 \mathrm{E}-06$ & 0 \\
\hline $3.0 \%$ & 5.57E-06 & $2.49 \%$ & 4.64E-07 & 5.57E-06 & 5.57E-06 & $8.47 \mathrm{E}-22$ \\
\hline $4.5 \%$ & 8.35E-06 & $3.71 \%$ & $6.96 \mathrm{E}-07$ & 8.35E-06 & 8.35E-06 & 0 \\
\hline $6.0 \%$ & 1.11E-05 & $4.90 \%$ & $9.28 \mathrm{E}-07$ & 1.11E-05 & 1.11E-05 & 0 \\
\hline $7.5 \%$ & 1.39E-05 & $6.08 \%$ & $1.16 \mathrm{E}-06$ & 1.39E-05 & 1.39E-05 & 1.69E-21 \\
\hline $9.0 \%$ & 1.67E-05 & $7.23 \%$ & 1.39E-06 & 1.67E-05 & 1.67E-05 & 0 \\
\hline $10.5 \%$ & $1.95 \mathrm{E}-05$ & $8.37 \%$ & $1.62 \mathrm{E}-06$ & $1.95 \mathrm{E}-05$ & $1.95 \mathrm{E}-05$ & 0 \\
\hline $12.0 \%$ & 2.23E-05 & $9.48 \%$ & $1.86 \mathrm{E}-06$ & 2.23E-05 & 2.23E-05 & 0 \\
\hline $13.5 \%$ & 2.51E-05 & $10.58 \%$ & 2.09E-06 & 2.51E-05 & 2.51E-05 & 3.39E-21 \\
\hline $15.0 \%$ & 2.78E-05 & $11.66 \%$ & 2.32E-06 & 2.78E-05 & 2.78E-05 & $6.78 \mathrm{E}-21$ \\
\hline $16.5 \%$ & 3.06E-05 & $12.72 \%$ & $2.55 \mathrm{E}-06$ & 3.06E-05 & 3.06E-05 & 0 \\
\hline $18.0 \%$ & 3.34E-05 & $13.77 \%$ & $2.78 \mathrm{E}-06$ & 3.34E-05 & 3.34E-05 & 0 \\
\hline $19.5 \%$ & 3.62E-05 & $14.80 \%$ & 3.02E-06 & 3.62E-05 & 3.62E-05 & $6.78 \mathrm{E}-21$ \\
\hline $21.0 \%$ & 3.90E-05 & $15.81 \%$ & $3.25 \mathrm{E}-06$ & 3.90E-05 & 3.90E-05 & $6.78 \mathrm{E}-21$ \\
\hline $22.5 \%$ & $4.18 \mathrm{E}-05$ & $16.81 \%$ & $3.48 \mathrm{E}-06$ & $4.18 \mathrm{E}-05$ & $4.18 \mathrm{E}-05$ & 0 \\
\hline $24.0 \%$ & 4.46E-05 & $17.79 \%$ & $3.71 \mathrm{E}-06$ & 4.46E-05 & 4.46E-05 & 0 \\
\hline $25.5 \%$ & $4.73 \mathrm{E}-05$ & $18.76 \%$ & $3.94 \mathrm{E}-06$ & 4.73E-05 & $4.73 \mathrm{E}-05$ & $6.78 \mathrm{E}-21$ \\
\hline $27.0 \%$ & 5.01E-05 & $19.71 \%$ & 4.18E-06 & 5.01E-05 & 5.01E-05 & $6.78 \mathrm{E}-21$ \\
\hline $28.5 \%$ & 5.29E-05 & $20.65 \%$ & $4.41 \mathrm{E}-06$ & 5.29E-05 & 5.29E-05 & 0 \\
\hline $30.0 \%$ & 5.57E-05 & $21.57 \%$ & 4.64E-06 & 5.57E-05 & 5.57E-05 & $6.78 \mathrm{E}-21$ \\
\hline $31.5 \%$ & 5.85E-05 & $22.48 \%$ & 4.87E-06 & 5.85E-05 & 5.85E-05 & 0 \\
\hline $33.0 \%$ & 6.13E-05 & $23.37 \%$ & 5.11E-06 & 6.13E-05 & 6.13E-05 & $1.36 \mathrm{E}-20$ \\
\hline $34.5 \%$ & 6.40E-05 & $24.26 \%$ & $5.34 \mathrm{E}-06$ & 6.40E-05 & 6.40E-05 & 0 \\
\hline $36.0 \%$ & $6.68 \mathrm{E}-05$ & $25.13 \%$ & 5.57E-06 & 6.68E-05 & 6.68E-05 & 0 \\
\hline $37.5 \%$ & 6.96E-05 & $25.98 \%$ & $5.80 \mathrm{E}-06$ & 6.96E-05 & 6.96E-05 & $2.71 \mathrm{E}-20$ \\
\hline $39.0 \%$ & 7.24E-05 & $26.83 \%$ & 6.03E-06 & 7.24E-05 & 7.24E-05 & 0 \\
\hline $40.5 \%$ & 7.52E-05 & $27.66 \%$ & 6.27E-06 & 7.52E-05 & 7.52E-05 & 0 \\
\hline
\end{tabular}




\begin{tabular}{|c|c|c|c|c|c|c|}
\hline $\begin{array}{l}\text { Percentage } \\
\text { Hydrogen }\end{array}$ & $\begin{array}{l}\text { Molar } \\
\text { Flow of } \\
\text { Retentate } \\
\text { hydrogen }\end{array}$ & $\begin{array}{l}\text { Hydrogen } \\
\text { in new } \\
\text { Total }\end{array}$ & $\begin{array}{l}\text { Molar } \\
\text { Permeation } \\
\text { by reverse } \\
\text { model }\end{array}$ & $\begin{array}{l}\text { Retentate } \\
\text { Flow by } \\
\text { reverse } \\
\text { model }\end{array}$ & $\begin{array}{l}\text { Retentate } \\
\text { flow by } \\
\text { Equation } \\
2.2 .10\end{array}$ & $\begin{array}{l}\text { Difference } \\
\text { in two } \\
\text { calculated } \\
\text { flows. }\end{array}$ \\
\hline$\% \%_{\mathrm{H}}$ & $\% \%_{\mathrm{HF}}$ & $\%_{\mathrm{HF}}$ & $\mathbf{n}_{\text {Hperm }}$ & $\mathbf{n}_{\mathrm{HFa}}$ & $\mathbf{n}_{\mathrm{HFb}}$ & $\mathbf{n}_{\mathrm{HFa}-} \mathbf{n}_{\mathrm{HFb}}$ \\
\hline $42.0 \%$ & 7.80E-05 & $28.48 \%$ & $6.50 \mathrm{E}-06$ & 7.80E-05 & 7.80E-05 & 0 \\
\hline $43.5 \%$ & 8.08E-05 & $29.29 \%$ & $6.73 \mathrm{E}-06$ & $8.08 \mathrm{E}-05$ & $8.08 \mathrm{E}-05$ & 0 \\
\hline $45.0 \%$ & 8.35E-05 & $30.09 \%$ & $6.96 \mathrm{E}-06$ & 8.35E-05 & 8.35E-05 & 0 \\
\hline $46.5 \%$ & 8.63E-05 & $30.88 \%$ & 7.19E-06 & 8.63E-05 & 8.63E-05 & $2.71 \mathrm{E}-20$ \\
\hline $48.0 \%$ & 8.91E-05 & $31.65 \%$ & $7.43 \mathrm{E}-06$ & 8.91E-05 & 8.91E-05 & 0 \\
\hline $49.5 \%$ & 9.19E-05 & $32.42 \%$ & $7.66 \mathrm{E}-06$ & 9.19E-05 & 9.19E-05 & $2.71 \mathrm{E}-20$ \\
\hline $51.0 \%$ & 9.47E-05 & $33.18 \%$ & 7.89E-06 & 9.47E-05 & 9.47E-05 & $1.36 \mathrm{E}-20$ \\
\hline $52.5 \%$ & $9.75 \mathrm{E}-05$ & $33.92 \%$ & 8.12E-06 & 9.75E-05 & 9.75E-05 & $1.36 \mathrm{E}-20$ \\
\hline $54.0 \%$ & 1.00E-04 & $34.66 \%$ & 8.35E-06 & 1.00E-04 & 1.00E-04 & 0 \\
\hline $55.5 \%$ & 1.03E-04 & $35.38 \%$ & 8.59E-06 & 1.03E-04 & 1.03E-04 & 0 \\
\hline $57.0 \%$ & 1.06E-04 & $36.10 \%$ & 8.82E-06 & $1.06 \mathrm{E}-04$ & $1.06 \mathrm{E}-04$ & 4.07E-20 \\
\hline $58.5 \%$ & 1.09E-04 & $36.80 \%$ & 9.05E-06 & 1.09E-04 & 1.09E-04 & 4.07E-20 \\
\hline $60.0 \%$ & 1.11E-04 & $37.50 \%$ & $9.28 \mathrm{E}-06$ & $1.11 \mathrm{E}-04$ & 1.11E-04 & $1.36 \mathrm{E}-20$ \\
\hline
\end{tabular}

18 of the calculations in Table 5.1.7 gave a different answer to reversing the calculation in the model data with an average difference of $1.46 \times 10^{-20}$, maximum $4.07 \times 10^{-20}$ and minimum $8.47 \times 10^{-22}$.

\subsubsection{Tertiary Insert Nitrogen}

Equation 2.2.9 was derived in Section 2.2.12.3.3 for calculating the molar flow of nitrogen in the retentate from the percentage of nitrogen measured in the retentate gas. Table 5.1.8 shows the test calculation results for Equation 2.2.9 with the first 40 of the permeation rates calculated in Table 5.1.5.

Equation 2.2.9 $n_{N F}=\frac{\%_{N F}\left(n_{I}-n_{H P e r m}\right)}{1-2 \times \%_{N F}}$

\begin{tabular}{|c|c|c|c|c|c|c|}
\hline $\begin{array}{l}\text { Percentage } \\
\text { nitrogen }\end{array}$ & $\begin{array}{l}\text { Molar } \\
\text { flow of } \\
\text { retentate } \\
\text { nitrogen }\end{array}$ & $\begin{array}{l}\text { Nitrogen } \\
\text { in new } \\
\text { total }\end{array}$ & $\begin{array}{l}\text { Molar } \\
\text { permeation } \\
\text { by reverse } \\
\text { model }\end{array}$ & $\begin{array}{l}\text { Retentate } \\
\text { flow by } \\
\text { reverse } \\
\text { model }\end{array}$ & $\begin{array}{l}\text { Retentate } \\
\text { flow by } \\
\text { Equation } \\
2.2 .9\end{array}$ & $\begin{array}{l}\text { Difference } \\
\text { in two } \\
\text { calculated } \\
\text { flows. }\end{array}$ \\
\hline$\% \mathrm{~N}$ & $\%$ & $\%_{\mathrm{NF}}$ & $\mathbf{n}_{\text {Hperm }}$ & $\mathbf{n}_{\mathrm{NFa}}$ & $\mathbf{n}_{\mathrm{NFb}}$ & $\mathbf{n}_{\text {NFa- }} \mathbf{n}_{\text {NFb }}$ \\
\hline $0.0 \%$ & $0.00 \mathrm{E}+00$ & $0.00 \%$ & $0.00 \mathrm{E}+00$ & $0.00 \mathrm{E}+00$ & $0.00 \mathrm{E}+00$ & 0 \\
\hline $0.5 \%$ & $1.02 \mathrm{E}-06$ & $0.50 \%$ & $2.32 \mathrm{E}-07$ & $1.01 \mathrm{E}-06$ & $1.01 \mathrm{E}-06$ & 0 \\
\hline
\end{tabular}




\begin{tabular}{|c|c|c|c|c|c|c|}
\hline $\begin{array}{l}\text { Percentage } \\
\text { nitrogen }\end{array}$ & $\begin{array}{l}\text { Molar } \\
\text { flow of } \\
\text { retentate } \\
\text { nitrogen }\end{array}$ & $\begin{array}{l}\text { Nitrogen } \\
\text { in new } \\
\text { total }\end{array}$ & $\begin{array}{l}\text { Molar } \\
\text { permeation } \\
\text { by reverse } \\
\text { model }\end{array}$ & $\begin{array}{l}\text { Retentate } \\
\text { flow by } \\
\text { reverse } \\
\text { model }\end{array}$ & $\begin{array}{l}\text { Retentate } \\
\text { flow by } \\
\text { Equation } \\
2.2 .9\end{array}$ & $\begin{array}{l}\text { Difference } \\
\text { in two } \\
\text { calculated } \\
\text { flows. }\end{array}$ \\
\hline$\% \mathrm{~N}$ & $\%_{\mathrm{NF}}$ & $\% \%_{\mathrm{NF}}$ & $\mathbf{n}_{\text {Hperm }}$ & $\mathbf{n}_{\mathrm{NFa}}$ & $\mathbf{n}_{\mathrm{NFb}}$ & $\mathbf{n}_{\text {NFa- }} \mathbf{n}_{\text {NFb }}$ \\
\hline $1.0 \%$ & $2.05 \mathrm{E}-06$ & $1.00 \%$ & 4.64E-07 & $2.01 \mathrm{E}-06$ & $2.01 \mathrm{E}-06$ & $4.24 \mathrm{E}-22$ \\
\hline $1.5 \%$ & 3.11E-06 & $1.51 \%$ & $6.96 \mathrm{E}-07$ & 3.02E-06 & $3.02 \mathrm{E}-06$ & $4.24 \mathrm{E}-22$ \\
\hline $2.0 \%$ & $4.18 \mathrm{E}-06$ & $2.01 \%$ & $9.28 \mathrm{E}-07$ & $4.02 \mathrm{E}-06$ & $4.02 \mathrm{E}-06$ & 0 \\
\hline $2.5 \%$ & $5.28 \mathrm{E}-06$ & $2.51 \%$ & $1.16 \mathrm{E}-06$ & $5.03 \mathrm{E}-06$ & $5.03 \mathrm{E}-06$ & 0 \\
\hline $3.0 \%$ & 6.40E-06 & $3.02 \%$ & 1.39E-06 & $6.03 \mathrm{E}-06$ & $6.03 \mathrm{E}-06$ & 0 \\
\hline $3.5 \%$ & 7.53E-06 & $3.53 \%$ & 1.62E-06 & 7.04E-06 & 7.04E-06 & 8.47E-22 \\
\hline $4.0 \%$ & 8.69E-06 & $4.03 \%$ & $1.86 \mathrm{E}-06$ & $8.04 \mathrm{E}-06$ & 8.04E-06 & 0 \\
\hline $4.5 \%$ & 9.86E-06 & $4.54 \%$ & 2.09E-06 & $9.05 \mathrm{E}-06$ & 9.05E-06 & -21 \\
\hline $5.0 \%$ & 1.11E-05 & $5.05 \%$ & 2.32E-06 & $1.01 \mathrm{E}-05$ & 1.01E-05 & 0 \\
\hline $5.5 \%$ & 1.23E-05 & $5.56 \%$ & $2.55 \mathrm{E}-06$ & $1.11 \mathrm{E}-05$ & 1.11E-05 & 1.69E-21 \\
\hline $6.0 \%$ & $1.35 \mathrm{E}-05$ & & $2.78 \mathrm{E}-06$ & $1.21 \mathrm{E}-05$ & $1.21 \mathrm{E}-05$ & -21 \\
\hline $6.5 \%$ & $1.48 \mathrm{E}-05$ & $6.59 \%$ & 3.02E-06 & $1.31 \mathrm{E}-05$ & 1.31E-05 & 0 \\
\hline $7.0 \%$ & 1.60E-05 & $7.10 \%$ & $3.25 \mathrm{E}-06$ & $1.41 \mathrm{E}-05$ & 1.41E-05 & $1.69 \mathrm{E}-21$ \\
\hline $7.5 \%$ & 1.73E-05 & & $3.48 \mathrm{E}-06$ & $1.51 \mathrm{E}-05$ & 1.51E-05 & 1.69E-21 \\
\hline $8.0 \%$ & $1.87 \mathrm{E}-05$ & $8.13 \%$ & $3.71 \mathrm{E}-06$ & $1.61 \mathrm{E}-05$ & $1.61 \mathrm{E}-05$ & 0 \\
\hline $8.5 \%$ & 2.00E-05 & $8.64 \%$ & 3.94E-06 & $1.71 \mathrm{E}-05$ & 1.71E-05 & 3.39E-21 \\
\hline $9.0 \%$ & 2.14E-05 & & $4.18 \mathrm{E}-06$ & $1.81 \mathrm{E}-05$ & 1.81E-05 & \\
\hline $9.5 \%$ & 2.27E-05 & $9.68 \%$ & $4.41 \mathrm{E}-06$ & $1.91 \mathrm{E}-05$ & 1.91E-05 & 0 \\
\hline $10.0 \%$ & $2.41 \mathrm{E}-05$ & $10.20 \%$ & $4.64 \mathrm{E}-06$ & $2.01 \mathrm{E}-05$ & $2.01 \mathrm{E}-05$ & 0 \\
\hline $10.5 \%$ & $2.56 \mathrm{E}-05$ & $10.71 \%$ & $4.87 \mathrm{E}-06$ & $2.11 \mathrm{E}-05$ & 2.11E-05 & 0 \\
\hline $11.0 \%$ & 2.70E-05 & $11.23 \%$ & 5.11E-06 & $2.21 \mathrm{E}-05$ & 2.21E-05 & 3.39E-21 \\
\hline $11.5 \%$ & 2.84E-05 & $11.75 \%$ & 5.34E-06 & 2.31E-05 & 2.31E-05 & 3.39E-21 \\
\hline $12.0 \%$ & 2.99E-05 & $12.27 \%$ & 5.57E-06 & $2.41 \mathrm{E}-05$ & $2.41 \mathrm{E}-05$ & 3.39E-21 \\
\hline $12.5 \%$ & $3.14 \mathrm{E}-05$ & $12.80 \%$ & 5.80E-06 & $2.51 \mathrm{E}-05$ & 2.51E-05 & $6.78 \mathrm{E}-21$ \\
\hline $13.0 \%$ & 3.29E-05 & $13.32 \%$ & $6.03 \mathrm{E}-06$ & 2.61E-05 & 2.61E-05 & 3.39E-21 \\
\hline $13.5 \%$ & $3.45 \mathrm{E}-05$ & $13.84 \%$ & $6.27 \mathrm{E}-06$ & $2.71 \mathrm{E}-05$ & 2.71E-05 & 0 \\
\hline $14.0 \%$ & 3.60E-05 & $14.36 \%$ & 6.50E-06 & 2.82E-05 & 2.82E-05 & 3.39E-21 \\
\hline $14.5 \%$ & $3.76 \mathrm{E}-05$ & $14.89 \%$ & $6.73 \mathrm{E}-06$ & 2.92E-05 & 2.92E-05 & $6.78 \mathrm{E}-21$ \\
\hline $15.0 \%$ & 3.92E-05 & $15.41 \%$ & $6.96 \mathrm{E}-06$ & $3.02 \mathrm{E}-05$ & 3.02E-05 & 3.39E-21 \\
\hline $15.5 \%$ & $4.08 \mathrm{E}-05$ & $15.94 \%$ & 7.19E-06 & $3.12 \mathrm{E}-05$ & 3.12E-05 & $6.78 \mathrm{E}-21$ \\
\hline $16.0 \%$ & $4.25 \mathrm{E}-05$ & $16.46 \%$ & 7.43E-06 & $3.22 \mathrm{E}-05$ & 3.22E-05 & 0 \\
\hline $16.5 \%$ & 4.41E-05 & $16.99 \%$ & $7.66 \mathrm{E}-06$ & 3.32E-05 & 3.32E-05 & $6.78 \mathrm{E}-21$ \\
\hline $17.0 \%$ & $4.58 \mathrm{E}-05$ & $17.51 \%$ & 7.89E-06 & $3.42 \mathrm{E}-05$ & 3.42E-05 & 0 \\
\hline $17.5 \%$ & $4.75 \mathrm{E}-05$ & $18.04 \%$ & $8.12 \mathrm{E}-06$ & 3.52E-05 & 3.52E-05 & 1.3 \\
\hline $18.0 \%$ & 4.92E-05 & $18.57 \%$ & $8.35 \mathrm{E}-06$ & 3.62E-05 & 3.62E-05 & 0 \\
\hline $18.5 \%$ & 5.10E-05 & $19.10 \%$ & 8.59E-06 & $3.72 \mathrm{E}-05$ & 3.72E-05 & 0 \\
\hline $19.0 \%$ & 5.27E-05 & $19.62 \%$ & 8.82E-06 & 3.82E-05 & 3.82E-05 & 0 \\
\hline $19.5 \%$ & $5.45 \mathrm{E}-05$ & $20.15 \%$ & $9.05 \mathrm{E}-06$ & 3.92E-05 & 3.92E-05 & $1.36 \mathrm{E}-20$ \\
\hline
\end{tabular}




\begin{tabular}{|c|c|c|c|c|c|c|}
\hline $\begin{array}{l}\text { Percentage } \\
\text { nitrogen }\end{array}$ & $\begin{array}{l}\text { Molar } \\
\text { flow of } \\
\text { retentate } \\
\text { nitrogen }\end{array}$ & $\begin{array}{l}\text { Nitrogen } \\
\text { in new } \\
\text { total }\end{array}$ & $\begin{array}{l}\text { Molar } \\
\text { permeation } \\
\text { by reverse } \\
\text { model }\end{array}$ & $\begin{array}{l}\text { Retentate } \\
\text { flow by } \\
\text { reverse } \\
\text { model } \\
\end{array}$ & $\begin{array}{l}\text { Retentate } \\
\text { flow by } \\
\text { Equation } \\
2.2 .9 \\
\end{array}$ & $\begin{array}{l}\text { Difference } \\
\text { in two } \\
\text { calculated } \\
\text { flows. }\end{array}$ \\
\hline$\%{ }_{\mathrm{N}}$ & $\%_{\mathrm{NF}}$ & $\%{ }_{\mathrm{NF}}$ & $\mathbf{n}_{\text {Hperm }}$ & $\mathbf{n}_{\mathrm{NFa}}$ & $\mathbf{n}_{\mathrm{NFb}}$ & $\mathbf{n}_{\mathrm{NFa}-} \mathbf{n}_{\mathrm{NFb}}$ \\
\hline $20.0 \%$ & 5.63E-05 & $20.68 \%$ & $9.28 \mathrm{E}-06$ & 4.02E-05 & 4.02E-05 & 0 \\
\hline
\end{tabular}

There were 22 calculations in Table 5.1 .8 where the new calculation gave a different answer to reversing the calculation in the model data with an average difference of $4.16 \times 10^{21}$, maximum $1.36 \times 10^{20}$, compared to a minimum calculated value of $1.01 \times 10^{-}$ 6.

\subsubsection{Tertiary Insert Ammonia}

Equation 2.2.11 was derived in Section 2.2.12.3.3 for calculating the molar flow of ammonia in the retentate from the percentage of ammonia measured in the retentate gas. Table 5.1.9 shows the test calculation results for Equation 2.2.11 with the first 40 of the permeation rates calculated in Table 5.1.5.

Equation 2.2.11 $n_{A F}=\frac{\%_{A F}\left(2 n_{I}-n_{H P e r m}\right)}{\left(1+\%_{A F}\right)}$

Table 5.1.9: Test calculations for Equation 2.2.11, calculating retentate ammonia flow from percentage ammonia in retentate.

\begin{tabular}{|c|c|c|c|c|c|c|}
\hline $\begin{array}{l}\text { Percentage } \\
\text { ammonia }\end{array}$ & $\begin{array}{l}\text { Molar } \\
\text { flow of } \\
\text { retentate } \\
\text { ammonia }\end{array}$ & $\begin{array}{l}\text { Ammonia } \\
\text { in new } \\
\text { total }\end{array}$ & $\begin{array}{l}\text { Molar } \\
\text { permeation } \\
\text { by reverse } \\
\text { model }\end{array}$ & $\begin{array}{l}\text { Retentate } \\
\text { flow by } \\
\text { reverse } \\
\text { model }\end{array}$ & $\begin{array}{l}\text { Retentate } \\
\text { flow by } \\
\text { Equation } \\
2.2 .11\end{array}$ & $\begin{array}{l}\text { Difference } \\
\text { in two } \\
\text { calculated } \\
\text { flows. }\end{array}$ \\
\hline$\% \mathrm{~A}$ & $\%$ AF & $\%$ AF & $\mathbf{n}_{\text {Hperm }}$ & $\mathbf{n}_{\mathrm{AFa}}$ & $\mathbf{n}_{\mathrm{AFb}}$ & $\mathbf{n}_{\mathrm{AFa}-} \mathbf{n}_{\mathrm{AFb}}$ \\
\hline $100 \%$ & 2.01E-04 & $100.00 \%$ & $0.00 \mathrm{E}+00$ & 2.01E-04 & 2.01E-04 & 0 \\
\hline $99 \%$ & 1.99E-04 & $98.13 \%$ & 2.32E-07 & 1.99E-04 & 1.99E-04 & $2.71 \mathrm{E}-20$ \\
\hline $98 \%$ & 1.97E-04 & $96.30 \%$ & $4.64 \mathrm{E}-07$ & 1.97E-04 & 1.97E-04 & $2.71 \mathrm{E}-20$ \\
\hline $97 \%$ & 1.95E-04 & $94.49 \%$ & 6.96E-07 & $1.95 \mathrm{E}-04$ & $1.95 \mathrm{E}-04$ & 5.42E-20 \\
\hline $96 \%$ & 1.93E-04 & $92.72 \%$ & $9.28 \mathrm{E}-07$ & 1.93E-04 & 1.93E-04 & 0 \\
\hline $95 \%$ & $1.91 \mathrm{E}-04$ & $90.98 \%$ & 1.16E-06 & $1.91 \mathrm{E}-04$ & 1.91E-04 & 0 \\
\hline $94 \%$ & $1.89 \mathrm{E}-04$ & $89.26 \%$ & 1.39E-06 & $1.89 \mathrm{E}-04$ & 1.89E-04 & $2.71 \mathrm{E}-20$ \\
\hline $93 \%$ & 1.87E-04 & $87.58 \%$ & $1.62 \mathrm{E}-06$ & 1.87E-04 & 1.87E-04 & $2.71 \mathrm{E}-20$ \\
\hline $92 \%$ & $1.85 \mathrm{E}-04$ & $85.92 \%$ & $1.86 \mathrm{E}-06$ & $1.85 \mathrm{E}-04$ & $1.85 \mathrm{E}-04$ & $2.71 \mathrm{E}-20$ \\
\hline $91 \%$ & $1.83 \mathrm{E}-04$ & $84.29 \%$ & 2.09E-06 & $1.83 \mathrm{E}-04$ & $1.83 \mathrm{E}-04$ & 0 \\
\hline $90 \%$ & 1.81E-04 & $82.69 \%$ & 2.32E-06 & $1.81 \mathrm{E}-04$ & 1.81E-04 & 0 \\
\hline $89 \%$ & 1.79E-04 & $81.11 \%$ & $2.55 \mathrm{E}-06$ & 1.79E-04 & 1.79E-04 & 0 \\
\hline $88 \%$ & 1.77E-04 & $79.55 \%$ & $2.78 \mathrm{E}-06$ & $1.77 \mathrm{E}-04$ & 1.77E-04 & 0 \\
\hline $87 \%$ & $1.75 \mathrm{E}-04$ & $78.03 \%$ & $3.02 \mathrm{E}-06$ & $1.75 \mathrm{E}-04$ & $1.75 \mathrm{E}-04$ & 0 \\
\hline $86 \%$ & 1.73E-04 & $76.52 \%$ & $3.25 \mathrm{E}-06$ & 1.73E-04 & 1.73E-04 & 0 \\
\hline
\end{tabular}




\begin{tabular}{|c|c|c|c|c|c|c|}
\hline $\begin{array}{l}\text { Percentage } \\
\text { ammonia }\end{array}$ & $\begin{array}{l}\text { Molar } \\
\text { flow of } \\
\text { retentate } \\
\text { ammonia }\end{array}$ & $\begin{array}{l}\text { Ammonia } \\
\text { in new } \\
\text { total }\end{array}$ & $\begin{array}{l}\text { Molar } \\
\text { permeation } \\
\text { by reverse } \\
\text { model }\end{array}$ & $\begin{array}{l}\text { Retentate } \\
\text { flow by } \\
\text { reverse } \\
\text { model }\end{array}$ & $\begin{array}{l}\text { Retentate } \\
\text { flow by } \\
\text { Equation } \\
2.2 .11\end{array}$ & $\begin{array}{l}\text { Difference } \\
\text { in two } \\
\text { calculated } \\
\text { flows. }\end{array}$ \\
\hline$\% \%_{A}$ & $\% \%_{\mathrm{AF}}$ & $\% \%_{\mathrm{AF}}$ & $\mathbf{n}_{\text {Hperm }}$ & $\mathbf{n}_{\mathrm{AFa}}$ & $\mathbf{n}_{\mathrm{AFb}}$ & $\mathbf{n}_{\mathrm{AFa}-} \mathbf{n}_{\mathrm{AFb}}$ \\
\hline $85 \%$ & $1.71 \mathrm{E}-04$ & $75.04 \%$ & $3.48 \mathrm{E}-06$ & $1.71 \mathrm{E}-04$ & $1.71 \mathrm{E}-04$ & 0 \\
\hline $84 \%$ & 1.69E-04 & $73.58 \%$ & $3.71 \mathrm{E}-06$ & 1.69E-04 & 1.69E-04 & 0 \\
\hline $83 \%$ & 1.67E-04 & $72.15 \%$ & 3.94E-06 & 1.67E-04 & 1.67E-04 & 0 \\
\hline $82 \%$ & 1.65E-04 & $70.74 \%$ & $4.18 \mathrm{E}-06$ & $1.65 \mathrm{E}-04$ & $1.65 \mathrm{E}-04$ & 0 \\
\hline $81 \%$ & 1.63E-04 & $69.34 \%$ & $4.41 \mathrm{E}-06$ & 1.63E-04 & $1.63 \mathrm{E}-04$ & 0 \\
\hline $80 \%$ & 1.61E-04 & $67.97 \%$ & $4.64 \mathrm{E}-06$ & $1.61 \mathrm{E}-04$ & 1.61E-04 & 0 \\
\hline $79 \%$ & 1.59E-04 & $66.62 \%$ & $4.87 \mathrm{E}-06$ & $1.59 \mathrm{E}-04$ & 1.59E-04 & $2.71 \mathrm{E}-20$ \\
\hline $78 \%$ & $1.57 \mathrm{E}-04$ & $65.29 \%$ & 5.11E-06 & 1.57E-04 & 1.57E-04 & $2.71 \mathrm{E}-20$ \\
\hline $77 \%$ & $1.55 \mathrm{E}-04$ & $63.98 \%$ & 5.34E-06 & $1.55 \mathrm{E}-04$ & $1.55 \mathrm{E}-04$ & $2.71 \mathrm{E}-20$ \\
\hline $76 \%$ & $1.53 \mathrm{E}-04$ & $62.69 \%$ & 5.57E-06 & $1.53 \mathrm{E}-04$ & $1.53 \mathrm{E}-04$ & 0 \\
\hline $75 \%$ & $1.51 \mathrm{E}-04$ & $61.42 \%$ & $5.80 \mathrm{E}-06$ & $1.51 \mathrm{E}-04$ & $1.51 \mathrm{E}-04$ & 0 \\
\hline $74 \%$ & 1.49E-04 & $60.16 \%$ & $6.03 \mathrm{E}-06$ & 1.49E-04 & 1.49E-04 & $2.71 \mathrm{E}-20$ \\
\hline $73 \%$ & 1.47E-04 & $58.93 \%$ & $6.27 \mathrm{E}-06$ & 1.47E-04 & $1.47 \mathrm{E}-04$ & 0 \\
\hline $72 \%$ & $1.45 \mathrm{E}-04$ & $57.71 \%$ & $6.50 \mathrm{E}-06$ & $1.45 \mathrm{E}-04$ & $1.45 \mathrm{E}-04$ & $2.71 \mathrm{E}-20$ \\
\hline $71 \%$ & 1.43E-04 & $56.50 \%$ & $6.73 \mathrm{E}-06$ & $1.43 \mathrm{E}-04$ & 1.43E-04 & $2.71 \mathrm{E}-20$ \\
\hline $70 \%$ & $1.41 \mathrm{E}-04$ & $55.32 \%$ & $6.96 \mathrm{E}-06$ & $1.41 \mathrm{E}-04$ & $1.41 \mathrm{E}-04$ & 0 \\
\hline $69 \%$ & 1.39E-04 & $54.15 \%$ & 7.19E-06 & 1.39E-04 & 1.39E-04 & 0 \\
\hline $68 \%$ & 1.37E-04 & $53.00 \%$ & 7.43E-06 & 1.37E-04 & 1.37E-04 & 0 \\
\hline $67 \%$ & $1.35 \mathrm{E}-04$ & $51.86 \%$ & 7.66E-06 & $1.35 \mathrm{E}-04$ & $1.35 \mathrm{E}-04$ & 0 \\
\hline $66 \%$ & 1.33E-04 & $50.74 \%$ & 7.89E-06 & $1.33 \mathrm{E}-04$ & 1.33E-04 & 2.71E-20 \\
\hline $65 \%$ & $1.31 \mathrm{E}-04$ & $49.63 \%$ & 8.12E-06 & $1.31 \mathrm{E}-04$ & 1.31E-04 & 2.71E-20 \\
\hline $64 \%$ & $1.29 \mathrm{E}-04$ & $48.54 \%$ & 8.35E-06 & $1.29 \mathrm{E}-04$ & 1.29E-04 & 0 \\
\hline $63 \%$ & $1.27 \mathrm{E}-04$ & $47.46 \%$ & 8.59E-06 & $1.27 \mathrm{E}-04$ & 1.27E-04 & $2.71 \mathrm{E}-20$ \\
\hline $62 \%$ & $1.25 \mathrm{E}-04$ & $46.40 \%$ & 8.82E-06 & $1.25 \mathrm{E}-04$ & 1.25E-04 & 0 \\
\hline $61 \%$ & $1.23 \mathrm{E}-04$ & $45.35 \%$ & $9.05 \mathrm{E}-06$ & $1.23 \mathrm{E}-04$ & $1.23 \mathrm{E}-04$ & 0 \\
\hline $60 \%$ & $1.21 \mathrm{E}-04$ & $44.32 \%$ & $9.28 \mathrm{E}-06$ & $1.21 \mathrm{E}-04$ & $1.21 \mathrm{E}-04$ & 0 \\
\hline
\end{tabular}

Of the 40 calculations in Table 5.1.9, there were 15 calculations where the new calculation gave a different answer to reversing the calculation in the model data with an average difference of $2.89 \times 10^{-20}$ and maximum $5.42 \times 10^{-20}$ compared to a minimum value of $1.21 \times 10^{-4}$.

\subsubsection{Electronic Gas Pressure Control}

After completion of the ammonia decomposition measurements for the secondary inserts, but before beginning the ammonia decomposition experiments with the tertiary inserts, the carrier gas flow became inconsistent, which caused variation in the 
daily standards. The gas flow should have been controlled by the electronic gas pressure control (EPC) in the GC. The initial response was to manage the carrier gas flow at the regulator which controlled the pressure of argon that entered the GC.

Experiments were performed to locate the cause of the gas flow variation by inserting a pressure gauge at the entrance to the GC column and a bubble flow meter after the $\mathrm{TCD}$, at the exit point of the gas.

A bubble flow meter is a vertical glass column with a $\mathrm{T}$ junction near the bottom. This junction allows the gas flow to enter from the side and meet with a soap bubble film pushed up from the bottom of the column to sit across the column above the $\mathrm{T}$ junction. The gas flow pushes the soap film up the column which is marked to indicate set volumes. By timing how long the bubble takes to travel between marks, the rate of gas flow can be calculated, e.g. when the soap film took $39.5 \mathrm{~s}$ to travel between the start mark and the $10 \mathrm{~mL}$ mark the rate of flow could be calculated to be $15.2 \mathrm{~mL} \mathrm{~min}^{-1}$.

The pressure gauge confirmed that changing the set pressure on the EPC had no effect on the column pressure. The EPC displayed the pressure to be the same as the set pressure, but the pressure gauge remained unchanged. Changing the EPC setting also had no effect on the flow rate through the bubble flow meter. These experiments showed that the pressure of the gas entering the GC had more influence on the carrier gas flow rate than the EPC setting.

The pressure control continued to deteriorate until it took a disproportionately long period of each day to standardise the GC. Two possibilities were considered and remedied. Intermittent EPC activity could have been interfering with the carrier flow. This was addressed by cutting into the gas line from the EPC to the TCD reference gas and connecting the gas input to the TCD line. This completely bypassed the EPC so the EPC could have no effect on the gas flow rate and the carrier gas pressure depended entirely on the regulator which controlled the input pressure of argon. To improve the consistency of the input pressure, the Norgren R07-200-RNKG single stage regulator was replaced with the Victor 8PT 2700 two stage regulator. This kept a more stable pressure, but the operating procedure was altered so that subsequent 
measurements were always standardised with measurements from the same day before being compared with measurements from other days. 


\subsection{Results}

\subsubsection{Complete List of Electrodeposition Samples}

\begin{tabular}{|c|c|c|c|c|c|c|c|}
\hline Code & Base & $\begin{array}{l}\text { Time } \\
\text { (s) }\end{array}$ & Min & $\mathrm{V}(\mathrm{V})$ & Characterisation & $\begin{array}{l}\text { Gas } \\
\text { Reaction }\end{array}$ & Notes \\
\hline 090420 & Brass, & 30 & 0.30 & -0.9 & & & \\
\hline 090420-1 & $\mathrm{Al}$ & & & & & & \\
\hline 090420-2 & $\mathrm{Al}$ & 20 & 0.20 & -0.9 & & & \\
\hline 090421-1 & $\mathrm{Al}$ & 180 & 3.00 & -1.4 & & & \\
\hline 090421-2 & $\mathrm{Al}$ & 300 & 5.00 & -1.4 & & & \\
\hline 090421-3 & $\mathrm{Al}$ & 180 & 3.00 & -1.4 & & & \\
\hline 090422-4 & $\mathrm{Al}$ & 30 & 0.30 & up to -2 & & & \\
\hline 090422-5 & $\mathrm{Al}$ & 30 & 0.30 & -0.9 & & & \\
\hline 090422-6 & $\mathrm{Al}$ & 30 & 0.30 & -1.4 & & & \\
\hline $090422-7$ & $\mathrm{Al}$ & 30 & 0.30 & Increasing & & & \\
\hline $090423 a$ & $\mathrm{Al}$ & 300 & 5.00 & up to -3 & & & bubbling at $2.0 \mathrm{~V}$ \\
\hline
\end{tabular}


Table 5.2.1: Complete list of electrodeposition samples.

$\mathrm{OM}$ in characterisation column stands for optical microscopy. The numbers in the characterisation column are the date. CV in the voltage column indicates cyclic voltametry. Concentration in the notes is the plating solution.

\begin{tabular}{|c|c|c|c|c|c|c|c|}
\hline Code & Base & $\begin{array}{l}\text { Time } \\
\text { (s) }\end{array}$ & Min & $\mathrm{V}(\mathrm{V})$ & Characterisation & $\begin{array}{l}\text { Gas } \\
\text { Reaction }\end{array}$ & Notes \\
\hline $090423 b$ & $\mathrm{Al}$ & 666.7 & 11.07 & up to -2 & & & bubbling at $1.6 \mathrm{~V}$ \\
\hline 090428a & $\mathrm{Al}$ & 10 & 0.10 & -1.6 & SEM 29/04/2009 & & \\
\hline $090428 b$ & $\mathrm{Al}$ & 30 & 0.30 & -1.6 & SEM 29/04/2009 & & \\
\hline 090428c & $\mathrm{Al}$ & 120 & 2.00 & -1.6 & SEM 29/04/2009 & & \\
\hline 090429a & $\mathrm{Al}$ & 10 & 0.10 & -1.6 & SEM 29/04/2009 & & \\
\hline 090429b & $\mathrm{Al}$ & 600 & 10.00 & -1.6 & SEM 29/04/2009 & & \\
\hline 090429c & $\mathrm{Al}$ & 30 & 0.30 & -1.6 & SEM 29/04/2009 & & \\
\hline 090429d & $\mathrm{Al}$ & 120 & 2.00 & -1.6 & SEM 29/04/2009 & & \\
\hline $090504 a$ & $\mathrm{Al}$ & & 0.00 & -1.6 & & & \\
\hline 090504b & $\mathrm{Al}$ & 10 & 0.10 & -1.6 & SEM 11/05/2009 & & $0.01 \mathrm{~mol} \cdot \mathrm{L}^{-1}$ \\
\hline 090504c & $\mathrm{Al}$ & 30 & 0.30 & -1.6 & SEM 11/05/2009 & & $0.01 \mathrm{~mol} \cdot \mathrm{L}^{-1}$ \\
\hline 090504d & $\mathrm{Al}$ & 120 & 2.00 & -1.6 & & & $0.01 \mathrm{~mol} \cdot \mathrm{L}^{-1}$ \\
\hline 090504e & $\mathrm{Al}$ & 10 & 0.10 & -1.6 & SEM 11/05/2009 & & $0.1 \mathrm{~mol} \cdot \mathrm{L}^{-1}$ \\
\hline $090504 \mathrm{f}$ & $\mathrm{Al}$ & 30 & 0.30 & -1.6 & SEM 11/05/2009 & & $0.1 \mathrm{~mol} \cdot \mathrm{L}^{-1}$ \\
\hline
\end{tabular}




\section{Table 5.2.1: Complete list of electrodeposition samples.}

$\mathrm{OM}$ in characterisation column stands for optical microscopy. The numbers in the characterisation column are the date. CV in the voltage column indicates cyclic voltametry. Concentration in the notes is the plating solution.

\begin{tabular}{|c|c|c|c|c|c|c|c|}
\hline Code & Base & $\begin{array}{l}\text { Time } \\
\text { (s) }\end{array}$ & Min & $\mathrm{V}(\mathrm{V})$ & Characterisation & $\begin{array}{l}\text { Gas } \\
\text { Reaction }\end{array}$ & Notes \\
\hline $090504 \mathrm{~g}$ & $\mathrm{Al}$ & 120 & 2.00 & -1.6 & & & $0.1 \mathrm{~mol} \cdot \mathrm{L}^{-1}$ \\
\hline 090504h & $\mathrm{Al}$ & 10 & 0.10 & -1.6 & SEM 11/05/2009 & & $1 \mathrm{~mol} \cdot \mathrm{L}^{-1}$ \\
\hline $090504 \mathrm{i}$ & $\mathrm{Al}$ & & 0.00 & -1.6 & & & failure, no current \\
\hline $090504 j$ & $\mathrm{Al}$ & 30 & 0.30 & -1.6 & & & $1 \mathrm{~mol} \cdot \mathrm{L}^{-1}$, dropped \\
\hline 090504k & $\mathrm{Al}$ & 120 & 2.00 & -1.6 & & & $1 \mathrm{~mol} \cdot \mathrm{L}^{-1}$, dropped \\
\hline $090514 a$ & $\mathrm{Al}$ & 30 & 0.30 & -1.6 & SEM 19/05/2009 & & $0.01 \mathrm{~mol} \cdot \mathrm{L}^{-1}$ \\
\hline 090514b & $\mathrm{Al}$ & 30 & 0.30 & -1.6 & SEM 19/05/2009 & & $0.01 \mathrm{~mol} \cdot \mathrm{L}^{-1}$ \\
\hline 090514c & $\mathrm{Al}$ & 30 & 0.30 & -1.6 & SEM 19/05/2009 & & $1 \mathrm{~mol} \cdot \mathrm{L}^{-1}$ \\
\hline $090514 d$ & $\mathrm{Al}$ & 30 & 0.30 & -0.5 & SEM 19/05/2009 & & $0.1 \mathrm{~mol} \cdot \mathrm{L}^{-1}$ \\
\hline $090514 \mathrm{e}$ & $\mathrm{Al}$ & 30 & 0.30 & -1 & SEM 19/05/2009 & & $0.1 \mathrm{~mol} \cdot \mathrm{L}^{-1}$ \\
\hline $090514 \mathrm{f}$ & $\mathrm{Al}$ & 30 & 0.30 & -1.5 & SEM 19/05/2009 & & $0.1 \mathrm{~mol} \cdot \mathrm{L}^{-1}$ \\
\hline $090514 \mathrm{~g}$ & $\mathrm{Al}$ & 30 & 0.30 & -2 & SEM 19/05/2009 & & $0.1 \mathrm{~mol} \cdot \mathrm{L}^{-1}$ \\
\hline 090514h & $\mathrm{Al}$ & 30 & 0.30 & -2.5 & SEM 19/05/2009 & & $0.1 \mathrm{~mol} \cdot \mathrm{L}^{-1}$ \\
\hline 090515a & $\mathrm{Al}$ & 30 & 0.30 & -1.6 & SEM 19/05/2009 & & $1 \mathrm{~mol} \cdot \mathrm{L}^{-1}$ \\
\hline
\end{tabular}


Table 5.2.1: Complete list of electrodeposition samples.

$\mathrm{OM}$ in characterisation column stands for optical microscopy. The numbers in the characterisation column are the date. CV in the voltage column indicates cyclic voltametry. Concentration in the notes is the plating solution.

\begin{tabular}{|c|c|c|c|c|c|c|c|}
\hline Code & Base & $\begin{array}{l}\text { Time } \\
\text { (s) }\end{array}$ & Min & $\mathrm{V}(\mathrm{V})$ & Characterisation & $\begin{array}{l}\text { Gas } \\
\text { Reaction }\end{array}$ & Notes \\
\hline 090602a & $\mathrm{Al}$ & 30 & 0.30 & -3 & & & $0.1 \mathrm{~mol} \cdot \mathrm{L}^{-1}$ \\
\hline 090602b & $\mathrm{Al}$ & 30 & 0.30 & -2 & & & $0.1 \mathrm{~mol} \cdot \mathrm{L}^{-1}$ \\
\hline 090602c & $\mathrm{Pd}$ & 30 & 0.30 & -1 & SEM 11/06/2009 & & $0.1 \mathrm{~mol} \mathrm{~L}^{-1}$, Calculated Ni thickness $0.03 \mu \mathrm{m}$ \\
\hline 090602d & $\mathrm{Pd}$ & 30 & 0.30 & -1.5 & SEM 11/06/2009 & & $0.1 \mathrm{~mol} \mathrm{~L}^{-1}$, Calculated Ni thickness $0.23 \mu \mathrm{m}$ \\
\hline 090602e & $\mathrm{Pd}$ & 30 & 0.30 & -2 & SEM 11/06/2009 & & $0.1 \mathrm{~mol} \mathrm{~L}^{-1}$, Calculated Ni thickness $0.60 \mu \mathrm{m}$ \\
\hline $090602 \mathrm{f}$ & $\mathrm{Pd}$ & 30 & 0.30 & -2.5 & SEM 11/06/2009 & & $0.1 \mathrm{~mol} \cdot \mathrm{L}^{-1}$, Calculated Ni thickness $0.98 \mu \mathrm{m}$ \\
\hline 090602g & $\mathrm{Pd}$ & 30 & 0.30 & -3 & SEM 11/06/2009 & & $0.1 \mathrm{~mol} \cdot \mathrm{L}^{-1}$, Calculated Ni thickness $1.06 \mu \mathrm{m}$ \\
\hline 090602h & $\mathrm{Pd}$ & 30 & 0.30 & -2 & SEM 11/06/2009 & & $\begin{array}{l}0.01 \mathrm{~mol} \cdot \mathrm{L}^{-1} \text {, Calculated Ni thickness } 0.53 \\
\mu \mathrm{m}\end{array}$ \\
\hline $090602 \mathrm{i}$ & $\mathrm{Pd}$ & 30 & 0.30 & -2 & SEM 11/06/2009 & & $1 \mathrm{~mol} \cdot \mathrm{L}^{-1}$, Calculated $\mathrm{Ni}$ thickness $0.53 \mu \mathrm{m}$ \\
\hline $090624 a$ & Reused Al & 12 & 0.12 & Variable & & & \\
\hline 090624b & Reused Al & 1.5 & 0.02 & Variable & & & 0.9 hold \\
\hline 090624c & Reused Al & 12 & 0.12 & Variable & & & 0.9 hold \\
\hline 090624d & Reused Al & 1.5 & 0.02 & Variable & & & 0.9 hold \\
\hline $090624 \mathrm{e}$ & Reused Al & 20 & 0.20 & Variable & & & 0.9 hold \\
\hline
\end{tabular}




\begin{tabular}{|c|c|c|c|c|c|c|c|}
\hline Code & Base & $\begin{array}{l}\text { Time } \\
\text { (s) }\end{array}$ & Min & $\mathrm{V}(\mathrm{V})$ & Characterisation & $\begin{array}{l}\text { Gas } \\
\text { Reaction }\end{array}$ & Notes \\
\hline $090624 \mathrm{f}$ & Reused Al & 5 & 0.05 & Variable & & & 0.9 hold \\
\hline $090624 \mathrm{~g}$ & Reused Al & 10 & 0.10 & Variable & & & 0.9 hold \\
\hline $090716 a$ & $\mathrm{Pd}$ & 0 & 0.00 & -1.6 & & & $0.1 \mathrm{~mol} \cdot \mathrm{L}^{-1}$ \\
\hline 090716b & $\mathrm{Pd}$ & 1 & 0.01 & -1.6 & & & $0.1 \mathrm{~mol} \cdot \mathrm{L}^{-1}$ \\
\hline 090716c & $\mathrm{Pd}$ & 5 & 0.05 & -1.6 & & & $0.1 \mathrm{~mol} \cdot \mathrm{L}^{-1}$ \\
\hline 090716d & $\mathrm{Pd}$ & 10 & 0.10 & -1.6 & & & $0.1 \mathrm{~mol} \cdot \mathrm{L}^{-1}$ \\
\hline 090716e & $\mathrm{Pd}$ & 0 & 0.00 & -1.6 & & & $0.1 \mathrm{~mol} \cdot \mathrm{L}^{-1}$ \\
\hline $090716 \mathrm{f}$ & $\mathrm{Pd}$ & 1 & 0.01 & -1.6 & & & $0.1 \mathrm{~mol} \mathrm{~L}^{-1}$ \\
\hline $090716 \mathrm{~g}$ & $\mathrm{Pd}$ & 5 & 0.05 & -1.6 & & & $0.1 \mathrm{~mol} \cdot \mathrm{L}^{-1}$ \\
\hline 090716h & $\mathrm{Pd}$ & 10 & 0.10 & -1.6 & & & $0.1 \mathrm{~mol} \mathrm{~L}^{-1}$ \\
\hline 090716i & $\mathrm{Pd}$ & 5 & 0.05 & -0.5 & & & $0.1 \mathrm{~mol} \cdot \mathrm{L}^{-1}$ \\
\hline 090716k & $\mathrm{Pd}$ & 5 & 0.05 & -1 & & & $0.1 \mathrm{~mol} \cdot \mathrm{L}^{-1}$ \\
\hline 0907161 & $\mathrm{Pd}$ & 5 & 0.05 & -1.5 & & & $0.1 \mathrm{~mol} \cdot \mathrm{L}^{-1}$ \\
\hline $090716 \mathrm{~m}$ & $\mathrm{Pd}$ & 5 & 0.05 & -2 & & & $0.1 \mathrm{~mol} \cdot \mathrm{L}^{-1}$ \\
\hline
\end{tabular}


Table 5.2.1: Complete list of electrodeposition samples.

$\mathrm{OM}$ in characterisation column stands for optical microscopy. The numbers in the characterisation column are the date. CV in the voltage column indicates cyclic voltametry. Concentration in the notes is the plating solution.

\begin{tabular}{|c|c|c|c|c|c|c|c|}
\hline Code & Base & $\begin{array}{l}\text { Time } \\
\text { (s) }\end{array}$ & Min & $\mathrm{V}(\mathrm{V})$ & Characterisation & $\begin{array}{l}\text { Gas } \\
\text { Reaction }\end{array}$ & Notes \\
\hline $090803 a$ & $\mathrm{Pd}$ & 5 & 0.05 & -2.5 & SEM 6/08/2009 & & $\begin{array}{l}0.01 \mathrm{~mol} \cdot \mathrm{L}^{-1} \text {, Calculated } \mathrm{Ni} \text { thickness } 0.20 \\
\mu \mathrm{m}\end{array}$ \\
\hline 090803b & $\mathrm{Pd}$ & 5 & 0.05 & -3 & SEM 6/08/2009 & & $\begin{array}{l}0.01 \mathrm{~mol} \cdot \mathrm{L}^{-1} \text {, Calculated } \mathrm{Ni} \text { thickness } 0.21 \\
\mu \mathrm{m}\end{array}$ \\
\hline 090803c & $\mathrm{Pd}$ & 5 & 0.05 & -2.5 & SEM 6/08/2009 & & $0.1 \mathrm{~mol} \cdot \mathrm{L}^{-1}$, Calculated $\mathrm{Ni}$ thickness $0.10 \mu \mathrm{m}$ \\
\hline 090803d & $\mathrm{Pd}$ & 5 & 0.05 & -3 & SEM 6/08/2009 & & $0.1 \mathrm{~mol} \cdot \mathrm{L}^{-1}$, Calculated Ni thickness $0.21 \mu \mathrm{m}$ \\
\hline 090803e & $\mathrm{Pd}$ & 1 & 0.01 & -2.5 & & & $0.1 \mathrm{~mol} \cdot \mathrm{L}^{-1}$, Calculated $\mathrm{Ni}$ thickness $0.03 \mu \mathrm{m}$ \\
\hline $090803 f$ & $\mathrm{Pd}$ & 0 & 0.00 & -2.5 & & & $0.1 \mathrm{~mol} \cdot \mathrm{L}^{-1}$, Calculated $\mathrm{Ni}$ thickness $0.00 \mu \mathrm{m}$ \\
\hline $090817 a$ & $\mathrm{Pd}$ & 10 & 0.10 & -2.5 & SEM 25/08/2009 & & $0.01 \mathrm{~mol} \cdot \mathrm{L}^{-1}$ \\
\hline 090817b & $\mathrm{Pd}$ & 15 & 0.15 & -2.5 & SEM 25/08/2009 & & $0.01 \mathrm{~mol} \cdot \mathrm{L}^{-1}$ \\
\hline 090817c & $\mathrm{Pd}$ & 20 & 0.20 & -2.5 & SEM 25/08/2009 & & $0.01 \mathrm{~mol} \cdot \mathrm{L}^{-1}$ \\
\hline 090817d & $\mathrm{Pd}$ & 25 & 0.25 & -2.5 & SEM 25/08/2009 & $20 / 08 / 2009$ & $0.01 \mathrm{~mol} \cdot \mathrm{L}^{-1}$ \\
\hline 090817e & $\mathrm{Pd}$ & 25 & 0.25 & 0 & & & $0.01 \mathrm{~mol} \cdot \mathrm{L}^{-1}$ \\
\hline 090817f & $\mathrm{Pd}$ & 5 & 0.05 & -2.5 & & & $0.01 \mathrm{~mol} \cdot \mathrm{L}^{-1}$ \\
\hline 090827a & $\mathrm{Pd}$ & 20 & 0.20 & -2.5 & Mass 27/8/09 & MS 27/8/09 & $0.01 \mathrm{~mol} \cdot \mathrm{L}^{-1}$ \\
\hline 090827b & $\mathrm{Pd}$ & 20 & 0.20 & -2.5 & Mass 27/8/09 & MS 8/9/09 & $0.01 \mathrm{~mol} \cdot \mathrm{L}^{-1}$ \\
\hline
\end{tabular}




\begin{tabular}{|c|c|c|c|c|c|c|c|}
\hline Code & Base & $\begin{array}{l}\text { Time } \\
\text { (s) }\end{array}$ & Min & $\mathrm{V}(\mathrm{V})$ & Characterisation & $\begin{array}{l}\text { Gas } \\
\text { Reaction }\end{array}$ & Notes \\
\hline $090915 a$ & $\mathrm{Pd}$ & 20 & 0.20 & -2.5 & Mass 15/9/09 & & $0.01 \mathrm{~mol} \cdot \mathrm{L}^{-1}$ \\
\hline 090915b & $\mathrm{Pd}$ & 20 & 0.20 & -2.5 & Mass 15/9/09 & MS 15/9/09 & $0.01{\mathrm{~mol} \mathrm{~L}^{-1}}^{-1}$ \\
\hline $090915 c$ & $\mathrm{Pd}$ & 20 & 0.20 & -2.5 & Mass 15/9/09 & & $0.1 \mathrm{~mol} \cdot \mathrm{L}^{-1}$ \\
\hline $100127 a$ & $\mathrm{Pd}$, & & & -1 to $1 \mathrm{~V} \mathrm{CV}$ & & & \\
\hline $100127 b$ & $\mathrm{Pd}$, & & & -1 to $1 \mathrm{~V} \mathrm{CV}$ & & & \\
\hline $100127 \mathrm{c}$ & $\mathrm{Pd}$, & & & -2 to $0 \mathrm{~V} \mathrm{CV}$ & XRD 28/1/10 & & \\
\hline $100129 a$ & $\mathrm{Pd}$, & & & 1.0 to $-2.5 \mathrm{~V} \mathrm{CV}$ & & & \\
\hline $100129 b$ & $\mathrm{Pd}$, & 60 & 1.00 & -1 & & & \\
\hline $100129 \mathrm{c}$ & $\mathrm{Pd}$, & 60 & 1.00 & +1 & & & \\
\hline $100129 d$ & $\mathrm{Pd}$, & 600 & 10.00 & -1.6 & & & \\
\hline $100202 a$ & $\mathrm{Pd}$, & $\mathrm{CV}$ & $\mathrm{CV}$ & $2 \times 1$ to $-1+0.5$ to $-2 \mathrm{~V}$ & & & \\
\hline $100202 b$ & $\mathrm{Pd}$, & 3600 & 60.00 & -1 & SEM 15/2/10 & & \\
\hline $100202 c$ & ITO glass & $\mathrm{CV}$ & $\mathrm{CV}$ & +5 to $-1 \mathrm{~V}$ & & & Repeated twice \\
\hline $100210 \mathrm{a}$ & Reused Pd, & 600 & 10.00 & -1 & SEM 15/2/10 & & \\
\hline
\end{tabular}


Table 5.2.1: Complete list of electrodeposition samples.

$\mathrm{OM}$ in characterisation column stands for optical microscopy. The numbers in the characterisation column are the date. CV in the voltage column indicates cyclic voltametry. Concentration in the notes is the plating solution.

\begin{tabular}{|c|c|c|c|c|c|c|c|}
\hline Code & Base & $\begin{array}{l}\text { Time } \\
\text { (s) }\end{array}$ & Min & $\mathrm{V}(\mathrm{V})$ & Characterisation & $\begin{array}{l}\text { Gas } \\
\text { Reaction }\end{array}$ & Notes \\
\hline $100210 b$ & Reused Pd, & 60 & 1.00 & -1 & SEM 15/2/10 & & \\
\hline $100210 \mathrm{c}$ & Reused Pd, & 10 & 0.10 & -1 & SEM 15/2/10 & & \\
\hline $100210 d$ & Reused Pd, & 1 & 0.01 & -1 & & & \\
\hline 100211a & Reused Pd, & 20 & 0.20 & -1 & & & \\
\hline $100211 b$ & Reused Pd, & 5 & 0.05 & -1 & SEM 15/2/10 & & \\
\hline 100211c & Reused Pd, & 20 & 0.20 & -1 & SEM 15/2/10 & & \\
\hline $100211 d$ & Reused Pd, & 2.5 & 0.03 & -1 & SEM 15/2/10 & & \\
\hline $100301 a$ & Cleaned Pd & 1 & 0.01 & -1 & & & \\
\hline $100301 b$ & Cleaned Pd & 5 & 0.05 & -1 & & & \\
\hline 100301c & Cleaned Pd & 20 & 0.20 & -1 & & & \\
\hline 100301e & Cleaned Pd & 60 & 1.00 & -1 & & & \\
\hline $100301 \mathrm{f}$ & Cleaned Pd & 600 & 10.00 & -1 & & & \\
\hline 100301y & Cleaned Pd & 60 & 1.00 & -1 & & & \\
\hline $100301 z$ & Cleaned Pd & 10 & 0.10 & -1 & SEM 3/3/10 & & \\
\hline
\end{tabular}




\section{Table 5.2.1: Complete list of electrodeposition samples.}

$\mathrm{OM}$ in characterisation column stands for optical microscopy. The numbers in the characterisation column are the date. CV in the voltage column indicates cyclic voltametry. Concentration in the notes is the plating solution.

\begin{tabular}{|c|c|c|c|c|c|c|c|}
\hline Code & Base & $\begin{array}{l}\text { Time } \\
\text { (s) }\end{array}$ & Min & $\mathrm{V}(\mathrm{V})$ & Characterisation & $\begin{array}{l}\text { Gas } \\
\text { Reaction }\end{array}$ & Notes \\
\hline $100825 a$ & $\mathrm{Pd}$, & 60 & 1.00 & -1.00 & $\begin{array}{l}\text { XRD 20/9/2016, } \\
\text { SEM 27/9/2016 }\end{array}$ & & \\
\hline $100825 b$ & $\mathrm{Pd}$, & 30 & 0.30 & -1.00 & & & \\
\hline $100825 c$ & $\mathrm{Pd}$, & 30 & 0.30 & -1.00 & $\begin{array}{l}\text { X section SEM } \\
27 / 9 / 2016\end{array}$ & & Epoxy \\
\hline $100825 d$ & $\mathrm{Pd}$, & 30 & 0.30 & -1.00 & $\begin{array}{l}\text { X section SEM } \\
27 / 9 / 2016\end{array}$ & & Epoxy \\
\hline 100825e & $\mathrm{Pd}$, & 30 & 0.30 & -1.00 & & & \\
\hline $100825 f$ & $\mathrm{Pd}$, & 30 & 0.30 & -1.00 & SEM 27/9/2016 & & \\
\hline $100825 \mathrm{~g}$ & $\mathrm{Pd}$, & 30 & 0.30 & -1.00 & SEM 27/9/2016 & & \\
\hline $130822 a$ & SS & 300 & 5.00 & -1.2 & & & \\
\hline $130822 b$ & SS & 560 & 9.20 & -0.9 & SEM 4/09/2013 & & \\
\hline $130828 \mathrm{a}$ & SS & 1680 & 28.00 & -0.9 & & & \\
\hline 130829a & SS & 360 & 6.00 & -0.9 & SEM 4/09/2013 & & \\
\hline $130829 b$ & SS & 360 & 6.00 & -0.9 & & & \\
\hline $130902 a$ & SS & 190 & 3.10 & -0.9 & SEM 4/09/2013 & & \\
\hline
\end{tabular}


Table 5.2.1: Complete list of electrodeposition samples.

$\mathrm{OM}$ in characterisation column stands for optical microscopy. The numbers in the characterisation column are the date. CV in the voltage column indicates cyclic voltametry. Concentration in the notes is the plating solution.

\begin{tabular}{|c|c|c|c|c|c|c|c|}
\hline Code & Base & $\begin{array}{l}\text { Time } \\
\text { (s) }\end{array}$ & Min & $\mathrm{V}(\mathrm{V})$ & Characterisation & $\begin{array}{l}\text { Gas } \\
\text { Reaction }\end{array}$ & Notes \\
\hline $130902 b$ & SS & 120 & 2.00 & -0.9 & SEM 4/09/2013 & & \\
\hline $130902 c$ & SS & 180 & 3.00 & -0.9 & & & \\
\hline $130902 d$ & SS & 180 & 3.00 & -0.9 & SEM 4/09/2013 & & \\
\hline $130903 a$ & SS & 900 & 15.00 & -0.9 & & & \\
\hline $130903 b$ & SS & 180 & 3.00 & -0.9 & & & \\
\hline $130903 c$ & SS & 1010 & 16.50 & -0.9 & SEM 4/09/2013 & & \\
\hline $130903 d$ & SS & 1050 & 17.30 & -0.9 & SEM 4/09/2013 & & \\
\hline 030903e & SS & 1050 & 17.30 & -0.9 & SEM 4/09/2013 & & \\
\hline 130815 & SS & $?$ & $?$ & -1.6 & XRD 6/09/2013 & & \\
\hline $130820 a$ & SS & $<600$ & $<10$ & -0.9 & XRD 6/09/2013 & & \\
\hline 130820b & SS & $<600$ & $<10$ & -0.9 & XRD 6/09/2013 & & \\
\hline $130909 a$ & SS & 200 & 3.20 & -0.9 & & & Acid pretreatment $11.78 \times 12.28 \mathrm{~mm}$ \\
\hline $130909 b$ & SS & 600 & 10.00 & -0.9 & & & Acid pretreatment \\
\hline $130909 c$ & SS, Template & 1230 & 20.30 & -0.9 & & & Acid pretreatment \\
\hline
\end{tabular}




\section{Table 5.2.1: Complete list of electrodeposition samples.}

$\mathrm{OM}$ in characterisation column stands for optical microscopy. The numbers in the characterisation column are the date. CV in the voltage column indicates cyclic voltametry. Concentration in the notes is the plating solution.

\begin{tabular}{|c|c|c|c|c|c|c|c|}
\hline Code & Base & $\begin{array}{l}\text { Time } \\
\text { (s) }\end{array}$ & Min & $\mathrm{V}(\mathrm{V})$ & Characterisation & $\begin{array}{l}\text { Gas } \\
\text { Reaction }\end{array}$ & Notes \\
\hline $130909 d$ & SS & 620 & 10.20 & -0.9 & & & \\
\hline $130909 \mathrm{e}$ & SS, Template & 600 & 10.00 & -0.9 & OM 16/09/2013 & $29 / 07 / 2014$ & 140729 \\
\hline $130924 a$ & SS & 720 & 12.00 & -0.9 & & & \\
\hline $130924 b$ & SS, Template & 3720 & 62.00 & -0.9 & & & $12.26 \times 12.41 \mathrm{~mm}$ \\
\hline $130926 a$ & SS & 600 & 10.00 & -1.2 & & & \\
\hline $130926 b$ & SS, Template & 3000 & 50.00 & -1.2 & SEM 1/10/2013 & & \\
\hline $130927 a$ & SS & 60 & 1.00 & -1.2 & SEM 1/10/2013 & & \\
\hline $130927 b$ & SS & 120 & 2.00 & -1.2 & & & \\
\hline $130927 \mathrm{c}$ & SS, Template, & 420 & 7.00 & -1.2 & SEM 1/10/2013 & & Hold \\
\hline $130927 d$ & SS & 180 & 3.00 & -1.2 & & & \\
\hline 130927e & SS, Template & 960 & 16.00 & -1.2 & SEM 1/10/2013 & & \\
\hline $131004 a$ & SS & 120 & 2.00 & -1.2 & & & \\
\hline $131004 b$ & SS, Template & 120 & 2.00 & -1.2 & & & $11.84 \times 12.98 \mathrm{~mm}$ \\
\hline $131004 \mathrm{c}$ & SS, Template & 120 & 2.00 & -1.2 & & & Hold \\
\hline
\end{tabular}


Table 5.2.1: Complete list of electrodeposition samples.

$\mathrm{OM}$ in characterisation column stands for optical microscopy. The numbers in the characterisation column are the date. CV in the voltage column indicates cyclic voltametry. Concentration in the notes is the plating solution.

\begin{tabular}{|c|c|c|c|c|c|c|c|}
\hline Code & Base & $\begin{array}{l}\text { Time } \\
\text { (s) }\end{array}$ & Min & $\mathrm{V}(\mathrm{V})$ & Characterisation & $\begin{array}{l}\text { Gas } \\
\text { Reaction }\end{array}$ & Notes \\
\hline $131004 d$ & SS, Template & 120 & 2.00 & -1.2 & & & \\
\hline $131004 \mathrm{e}$ & SS, Template & 120 & 2.00 & -1.2 & & & $\begin{array}{l}\text { Hold } \\
11.89 \times 12.91 \mathrm{~mm}\end{array}$ \\
\hline $131009 a$ & SS & 600 & 10.00 & -0.9 & & & $13.30 \times 13.00 \mathrm{~mm}$ \\
\hline $131009 b$ & $\mathrm{Pd}$ & 600 & 10.00 & -0.9 & & & \\
\hline $131009 c$ & $\mathrm{Pd}$ & 600 & 10.00 & -1.2 & & & $12.34 \times 13.20 \mathrm{~mm}$ \\
\hline $131009 d$ & $\mathrm{Pd}$ & 600 & 10.00 & -1.2 & & & \\
\hline $131017 a$ & SS & 600 & 10.00 & -1.2 & & & \\
\hline $131017 b$ & Pd, Template & 600 & 10.00 & -1.2 & OM 17/10/2013 & $31 / 07 / 2014$ & $\begin{array}{l}140731 \\
11.00 \times 12.70 \mathrm{~mm}\end{array}$ \\
\hline $131017 \mathrm{C}$ & Pd, Template & 420 & 7.00 & -1.2 & OM 17/10/2013 & & $12.39 \times 12.67 \mathrm{~mm}$ \\
\hline $140821 a$ & SS, discarded & & & & & & \\
\hline $140821 b$ & SS, Template & 420 & 7.00 & -1.2 & & & Hold \\
\hline 140821c & SS, Template & 600 & 10.00 & -1.2 & & & Hold \\
\hline $140821 d$ & SS, Template & 600 & 10.00 & -1.2 & & & Hold \\
\hline $140822 a$ & SS, discarded & & & & & & \\
\hline
\end{tabular}




\begin{tabular}{|c|c|c|c|c|c|c|c|}
\hline Code & Base & $\begin{array}{l}\text { Time } \\
\text { (s) }\end{array}$ & Min & $\mathrm{V}(\mathrm{V})$ & Characterisation & $\begin{array}{l}\text { Gas } \\
\text { Reaction }\end{array}$ & Notes \\
\hline $140822 b$ & Pd, Template, & 420 & 7.00 & -1.2 & OM 22/08/2014 & & Hold \\
\hline $140822 \mathrm{c}$ & Pd, Template, & 420 & 7.00 & -1.2 & OM 22/08/2014 & & Hold \\
\hline $140901 a$ & SS, discarded & & & & & & \\
\hline $140901 b$ & SS, Template & 420 & 7.00 & -1.2 & OM 1/09/2014 & & Hold \\
\hline 140901c & SS, Template & 420 & 7.00 & -1.2 & OM 1/09/2014 & & Hold \\
\hline $140902 b$ & Pd, Template & 420 & 7.00 & -1.2 & OM 2/09/2014 & & Hold \\
\hline 140904 & SS, Template & 300 & 5.00 & -1.2 & OM 4/09/2014 & & Hold \\
\hline $141114 a$ & SS & 480 & 8.00 & -1.2 & & & Hold \\
\hline $141114 b$ & SS & 240 & 4.00 & -1.2 & & & Hold \\
\hline $141114 c$ & SS & 120 & 2.00 & -1.2 & & & Hold \\
\hline $141114 d$ & SS & 40 & 0.40 & -1.2 & OM 14/11/2014 & & Hold \\
\hline $141114 \mathrm{e}$ & SS & 30 & 0.30 & -1.2 & OM 14/11/2014 & & Hold \\
\hline $141117 a$ & SS & 480 & 8.00 & -1.2 & & & Hold \\
\hline $141117 b$ & SS & 480 & 8.00 & -1.2 & & & Hold \\
\hline
\end{tabular}


Table 5.2.1: Complete list of electrodeposition samples.

$\mathrm{OM}$ in characterisation column stands for optical microscopy. The numbers in the characterisation column are the date. CV in the voltage column indicates cyclic voltametry. Concentration in the notes is the plating solution.

\begin{tabular}{|c|c|c|c|c|c|c|c|}
\hline Code & Base & $\begin{array}{l}\text { Time } \\
\text { (s) }\end{array}$ & Min & $\mathrm{V}(\mathrm{V})$ & Characterisation & $\begin{array}{l}\text { Gas } \\
\text { Reaction }\end{array}$ & Notes \\
\hline $141117 \mathrm{c}$ & SS & 40 & 0.40 & -1.2 & & & Hold \\
\hline $141117 t$ & SS, Tube & 180 & 3.00 & -1.2 & & $\begin{array}{l}18 / 11 / \\
2014- \\
19 / 11 / \\
2014\end{array}$ & Hold \\
\hline $150219 a$ & SS & 190 & 3.10 & -1.2 & & & Autolab , Hold \\
\hline $150219 t$ & $\begin{array}{l}\text { SS, tube, } \\
\text { Template }\end{array}$ & 128 & 2.08 & -1.2 & $\begin{array}{l}\text { SEM 20/02/2015- } \\
26 / 02 / 15\end{array}$ & $\begin{array}{l}23 / 02 / 15- \\
23 / 3 / 15+ \\
23 / 6 / 15- \\
22 / 7 / 15+ \\
10 / 9 / 15- \\
14 / 9 / 15+ \\
5 / 10 / 15- \\
16 / 10 / 15\end{array}$ & Autolab, Hold \\
\hline $\begin{array}{l}\text { 150219B } \\
+\end{array}$ & $\begin{array}{l}\text { SS, tube, } \\
\text { Template, Hold }\end{array}$ & 128 & 2.08 & -1.2 & $\begin{array}{l}\text { SEM 20/02/2015- } \\
26 / 02 / 15\end{array}$ & $\begin{array}{l}31 / 8 / 15 /- \\
9 / 9 / 15+ \\
19 / 10 / 15- \\
2 / 11 / 15+ \\
01 / 12 / 15- \\
9 / 12 / 15\end{array}$ & Autolab \\
\hline $\begin{array}{l}150219 \mathrm{C} \\
+ \\
\end{array}$ & $\begin{array}{l}\text { SS, tube, } \\
\text { Template, Hold }\end{array}$ & 128 & 2.08 & -1.2 & $\begin{array}{l}\text { SEM 20/02/2015- } \\
26 / 02 / 15\end{array}$ & & Autolab \\
\hline $160204 a$ & SS & 60 & 1.00 & -1.2 & & & No ammeter, Hold \\
\hline $160204 b$ & SS, & 240 & 4.00 & -1.2 & & & $\begin{array}{l}\text { Deposited on the back of } 160204 a \text {, } \\
\text { no ammeter, Hold }\end{array}$ \\
\hline
\end{tabular}




\section{Table 5.2.1: Complete list of electrodeposition samples.}

$\mathrm{OM}$ in characterisation column stands for optical microscopy. The numbers in the characterisation column are the date. CV in the voltage column indicates cyclic voltametry. Concentration in the notes is the plating solution.

\begin{tabular}{|c|c|c|c|c|c|c|c|}
\hline Code & Base & $\begin{array}{l}\text { Time } \\
\text { (s) }\end{array}$ & Min & $\mathrm{V}(\mathrm{V})$ & Characterisation & $\begin{array}{l}\text { Gas } \\
\text { Reaction }\end{array}$ & Notes \\
\hline $160204 c$ & SS, & 1200 & 20.00 & V varied & & & No ammeter, Hold \\
\hline $160205 a$ & SS, & & & & & & Failure in ammeter \\
\hline $160205 b$ & SS, & $\begin{array}{l}180+2 \\
40+60 \\
0\end{array}$ & $\begin{array}{l}3+4+ \\
10\end{array}$ & -1.2 & & & Ammeter repaired \\
\hline $160216 a$ & SS & 420 & 7.00 & -1.2 & & & Hold \\
\hline $160216 b$ & SS & 420 & 7.00 & -1.2 & & & Hold \\
\hline $160216 c$ & SS & 420 & 7.00 & -1.2 & & & Hold \\
\hline $160223 a$ & SS & 240 & 4.00 & -1.2 & & & Hold \\
\hline $160223 b$ & SS & 240 & 4.00 & -1.2 & & & Hold \\
\hline $160223 c$ & SS & 240 & 4.00 & -1.2 & & & Hold \\
\hline $160223 d$ & SS & 240 & 4.00 & -1.2 & & & Hold \\
\hline $160226 a$ & SS & 240 & 4.00 & -1.2 & & & Hold \\
\hline $160226 b$ & SS & 240 & 4.00 & -1.2 & & & Hold \\
\hline $160226 c$ & SS & 240 & 4.00 & -1.2 & & & Hold \\
\hline
\end{tabular}


Table 5.2.1: Complete list of electrodeposition samples.

$\mathrm{OM}$ in characterisation column stands for optical microscopy. The numbers in the characterisation column are the date. CV in the voltage column indicates cyclic voltametry. Concentration in the notes is the plating solution.

\begin{tabular}{|c|c|c|c|c|c|c|c|}
\hline Code & Base & $\begin{array}{l}\text { Time } \\
\text { (s) }\end{array}$ & Min & $\mathrm{V}(\mathrm{V})$ & Characterisation & $\begin{array}{l}\text { Gas } \\
\text { Reaction }\end{array}$ & Notes \\
\hline $160226 \mathrm{~d}$ & SS & 240 & 4.00 & -1.2 & & & Hold \\
\hline $160226 \mathrm{e}$ & SS & 240 & 4.00 & -1.2 & & & Hold \\
\hline $160226 f$ & SS, Template & 390 & 6.30 & -1.2 & & & Hold \\
\hline $160226 \mathrm{~g}$ & SS, Template & 300 & 5.00 & -0.2 & & & Hold \\
\hline $160229 a$ & & 240 & 4.00 & -1.2 & & & \\
\hline $160229 b$ & & 240 & 4.00 & -1.2 & & & \\
\hline $160229 c$ & & 240 & 4.00 & -1.2 & & & \\
\hline $160309 a$ & SS & 240 & 4.00 & -1.2 & & & Hold \\
\hline $160309 b$ & $\begin{array}{l}\text { SS, Film } \\
\text { Template, } 1 / 2 \\
\text { Covered }\end{array}$ & 1200 & 20.00 & -1.2 & & & $\begin{array}{l}\text { 1/2 SUEX Template, } 1 / 2 \text { nail } \\
\text { polish, Hold }\end{array}$ \\
\hline $160309 c$ & $\begin{array}{l}\text { SS, Film } \\
\text { Template, } 1 / 2 \\
\text { Covered }\end{array}$ & 240 & 4.00 & -1.2 & & & $\begin{array}{l}\text { 1/2 SUEX Template, } 1 / 2 \text { nail } \\
\text { polish, Hold }\end{array}$ \\
\hline $160318 a$ & SS & 240 & 4.00 & -1.2 & & & Hold \\
\hline $160318 b$ & $\mathrm{Pd}$ & 240 & 4.00 & -1.2 & & & Hold \\
\hline $160318 c$ & PdAg & 240 & 4.00 & -1.2 & & & Hold \\
\hline
\end{tabular}




\begin{tabular}{|c|c|c|c|c|c|c|c|}
\hline Code & Base & $\begin{array}{l}\text { Time } \\
\text { (s) }\end{array}$ & Min & $\mathrm{V}(\mathrm{V})$ & Characterisation & \begin{tabular}{|l|} 
Gas \\
Reaction
\end{tabular} & Notes \\
\hline $160318 d$ & $\mathrm{PdAg}$ & 240 & 4.00 & -1.2 & & & Hold \\
\hline $160323 a$ & SS & 240 & 4.00 & -1.2 & & & Freshly polished electrical contacts, Hold \\
\hline $160323 b$ & SS & 300 & 5.00 & -1.2 & & & Hold \\
\hline $160323 c$ & SS & 240 & 4.00 & -1.2 & & & Hold \\
\hline $160426 a$ & SS & 240 & 4.00 & -1.2 & & & 1/2 nail polish, Hold \\
\hline $160426 b$ & SS, $1 / 2$ covered & 240 & 4.00 & -1.2 & & & 1/2 nail polish, Hold \\
\hline $160426 c$ & SS, $1 / 2$ covered & 240 & 4.00 & -1.2 & & & Hold \\
\hline $160426 \mathrm{~d}$ & SS & 240 & 4.00 & -1.2 & & & Hold \\
\hline $160714 a$ & SS & 180 & 3.00 & -1.2 & & & Hold \\
\hline $160714 b$ & SS & 180 & 3.00 & -1.2 & & & Hold \\
\hline $160714 \mathrm{c}$ & $\begin{array}{l}\text { SS, Film } \\
\text { Template }\end{array}$ & 300 & 5.00 & -1.2 & & & $\begin{array}{l}25 \mu \mathrm{m} \text { SUEX Template, into NMP } \\
\text { 18/7/16, Hold }\end{array}$ \\
\hline $160714 d$ & $\begin{array}{l}\text { SS, Film } \\
\text { Template }\end{array}$ & 300 & 5.00 & -1.2 & & & $\begin{array}{l}25 \mu \mathrm{m} \text { SUEX Template, into NMP } \\
\text { 20/7/16, Hold }\end{array}$ \\
\hline
\end{tabular}

\subsubsection{Complete Spray Coat Mass Data}


Table 5.2.2: Complete list of spray coat mass data.

SS in Base column is stainless steel.

\begin{tabular}{|c|c|c|c|c|c|c|c|c|c|}
\hline Code & Date & Base & Min & Rate & Characterisation & $\begin{array}{l}\text { Starting } \\
\text { mass }\end{array}$ & $\begin{array}{l}\text { Final } \\
\text { mass }\end{array}$ & $\begin{array}{l}\text { Mass } \\
\text { gain }\end{array}$ & Notes \\
\hline $110816 a$ & $16 / 08 / 2011$ & $\mathrm{Pd}$ & 5 & 10 & $\begin{array}{l}\text { OM 16/8/2011, } \\
\text { SEM 24/11/11 }\end{array}$ & 0.207770 & 0.207750 & -0.000020 & \\
\hline $110816 b$ & $16 / 08 / 2011$ & $\mathrm{Pd}$ & 0 & 0 & OM 16/8/2011 & & & & \\
\hline $110830 a$ & $30 / 08 / 2011$ & $\mathrm{Pd}$ & 50 & 10 & $\begin{array}{l}\text { OM 13/10/2011, } \\
\text { SEM 24/11/11 }\end{array}$ & 0.208610 & 0.208840 & 0.000230 & $\begin{array}{l}\text { In the last five minutes, } \\
\text { changed from tidy spray } \\
\text { to messy overdeposit }\end{array}$ \\
\hline $110920 a$ & $9 / 20 / 2011$ & $\mathrm{Pd}$ & 25 & 10 & $\begin{array}{l}\text { OM 27/10/2011 } \\
(10.73)\end{array}$ & 0.226910 & 0.226940 & 0.000030 & \\
\hline $110920 b$ & $9 / 20 / 2011$ & $\mathrm{Pd}$ & 35 & 10 & $\begin{array}{l}\text { OM 27/10/2011, } \\
\text { SEM 24/11/11 }\end{array}$ & 0.244243 & 0.244931 & 0.000688 & \\
\hline
\end{tabular}

Spray coater moved to University

\begin{tabular}{|c|c|c|c|c|c|c|c|c|c|}
\hline $111104 a$ & $4 / 11 / 2011$ & $\mathrm{Pd}$ & & $\begin{array}{l}\text { varied } \\
5-20\end{array}$ & & & & & $\begin{array}{l}\text { Observe constancy of } \\
\text { spray rates. }\end{array}$ \\
\hline $111109 a$ & $9 / 11 / 2011$ & $\mathrm{Pd}$ & 15 & 30 & & 0.191860 & 0.191840 & -0.000020 & \\
\hline $111122 a$ & $22 / 11 / 2011$ & $\mathrm{Al}$ & 10 & 40 & SEM 24/11/11 & 0.258790 & 0.258891 & 0.000101 & \\
\hline $111122 b$ & $22 / 11 / 2011$ & $\mathrm{Al}$ & 12 & 40 & & 0.222530 & 0.222630 & 0.000100 & \\
\hline $111122 c$ & $22 / 11 / 2011$ & $\mathrm{Al}$ & 30 & 30 & & & & & \\
\hline 111123 & $23 / 11 / 2011$ & $\mathrm{Pd}$ & 7.15 & 40 & SEM 24/11/11 & 0.222000 & 0.222110 & 0.000110 & \\
\hline 111206rPda & $6 / 12 / 2011$ & $\begin{array}{l}\text { reused } \\
\mathrm{Pd}\end{array}$ & 10 & 30 & & 0.174751 & 0.174747 & -0.000004 & MassDataSummary.xls \\
\hline $111206 \mathrm{rPdb}$ & $6 / 12 / 2011$ & $\begin{array}{l}\text { reused } \\
\mathrm{Pd}\end{array}$ & 10 & 30 & & 0.172702 & 0.172704 & 0.000002 & MassDataSummary.xls \\
\hline 111206rPdc & $6 / 12 / 2011$ & $\begin{array}{l}\text { reused } \\
\mathrm{Pd}\end{array}$ & 10 & 30 & & 0.192064 & 0.192070 & 0.000005 & MassDataSummary.xls \\
\hline $\begin{array}{l}\text { 111206rPd } \\
\text { d }\end{array}$ & $6 / 12 / 2011$ & $\begin{array}{l}\text { reused } \\
\mathrm{Pd}\end{array}$ & 10 & 30 & & 0.163492 & 0.163505 & 0.000012 & MassDataSummary.xls \\
\hline
\end{tabular}


Table 5.2.2: Complete list of spray coat mass data.

SS in Base column is stainless steel.

\begin{tabular}{|c|c|c|c|c|c|c|c|c|c|}
\hline Code & Date & Base & Min & Rate & Characterisation & $\begin{array}{l}\text { Starting } \\
\text { mass }\end{array}$ & $\begin{array}{l}\text { Final } \\
\text { mass }\end{array}$ & $\begin{array}{l}\text { Mass } \\
\text { gain }\end{array}$ & Notes \\
\hline 111206Pd1 & $6 / 12 / 2011$ & $\mathrm{Pd}$ & 10 & 30 & & 0.204878 & 0.204917 & 0.000039 & MassDataSummary.xls \\
\hline 111206Pd2 & $6 / 12 / 2011$ & $\mathrm{Pd}$ & 10 & 30 & & 0.238524 & 0.238564 & 0.000040 & MassDataSummary.xls \\
\hline $111206 \mathrm{Pd} 3$ & $6 / 12 / 2011$ & $\mathrm{Pd}$ & 10 & 30 & & 0.234274 & 0.234296 & 0.000023 & MassDataSummary.xls \\
\hline $111206 \mathrm{Pd} 4$ & $6 / 12 / 2011$ & $\mathrm{Pd}$ & 10 & 30 & & 0.221885 & 0.221899 & 0.000014 & MassDataSummary.xls \\
\hline 111206Al1 & $6 / 12 / 2011$ & $\mathrm{Al}$ & 10 & 30 & & 0.129059 & 0.129084 & 0.000024 & $\begin{array}{l}\text { Large temperature } \\
\text { variation, } \\
\text { MassDataSummary.xls }\end{array}$ \\
\hline 111206Al2 & $6 / 12 / 2011$ & $\mathrm{Al}$ & 10 & 30 & & 0.195565 & 0.195577 & 0.000012 & $\begin{array}{l}\text { cross wind, } \\
\text { MassDataSummary.xls }\end{array}$ \\
\hline $111206 \mathrm{Al} 3$ & $6 / 12 / 2011$ & $\mathrm{Al}$ & 10 & 30 & & 0.125457 & 0.125469 & 0.000013 & MassDataSummary.xls \\
\hline 111206Al4 & $6 / 12 / 2011$ & $\mathrm{Al}$ & 10 & 30 & & 0.123139 & 0.123174 & 0.000035 & MassDataSummary.xls \\
\hline ss1 & $3 / 03 / 29$ & SS & 10 & 30 & & 0.171306 & 0.171398 & 0.000092 & MassDataSummary.xls \\
\hline ss3 & $3 / 03 / 29$ & SS & 10 & 30 & & 0.182268 & 0.182369 & 0.000101 & MassDataSummary.xls \\
\hline ss6 & $3 / 03 / 29$ & SS & 10 & 30 & & 0.160995 & 0.161232 & 0.000236 & MassDataSummary.xls \\
\hline ss7 & $15 / 05 / 29$ & SS & 10 & 30 & & 0.166287 & 0.166314 & 0.000027 & MassDataSummary.xls \\
\hline ss8 & $15 / 05 / 29$ & SS & 10 & 20 & & 0.187050 & 0.187056 & 0.000006 & MassDataSummary.xls \\
\hline ss9 & $15 / 05 / 29$ & SS & 10 & 20 & & 0.168466 & 0.168488 & 0.000022 & MassDataSummary.xls \\
\hline ss10 & $15 / 05 / 29$ & SS & 10 & 20 & & 0.147149 & 0.147649 & 0.000499 & MassDataSummary.xls \\
\hline ss23A & $23 / 12 / 29$ & SS & 10 & 20 & & 0.114837 & & * & MassDataSummary.xls \\
\hline ss23B & $23 / 12 / 29$ & SS & 10 & 20 & & 0.116176 & 0.116303 & 0.000127 & MassDataSummary.xls \\
\hline ss23C & $23 / 12 / 29$ & SS & 10 & 20 & & 0.114727 & 0.114828 & 0.000101 & MassDataSummary.xls \\
\hline ss23D & $23 / 12 / 29$ & SS & 10 & 20 & & 0.114252 & 0.114301 & 0.000050 & MassDataSummary.xls \\
\hline ss24A & $24 / 12 / 29$ & SS & 10 & 20 & & 0.116658 & 0.116691 & 0.000033 & MassDataSummary.xls \\
\hline ss24B & $24 / 12 / 29$ & SS & 10 & 20 & & 0.116407 & 0.116492 & 0.000085 & MassDataSummary.xls \\
\hline
\end{tabular}


Table 5.2.2: Complete list of spray coat mass data.

SS in Base column is stainless steel.

\begin{tabular}{|l|l|l|l|l|l|l|l|l|l|}
\hline Code & Date & Base & Min & Rate & Characterisation & $\begin{array}{l}\text { Starting } \\
\text { mass }\end{array}$ & $\begin{array}{l}\text { Final } \\
\text { mass }\end{array}$ & $\begin{array}{l}\text { Mass } \\
\text { gain }\end{array}$ & Notes \\
\hline ss24C & $24 / 12 / 29$ & SS & 10 & 20 & & 0.114296 & & $*$ & MassDataSummary.xls \\
\hline
\end{tabular}


Jonathan Tailby

\subsubsection{SEM Image Processing Calculations for Electrodeposition} on Aluminium

Table 5.2.3 shows the quantitative analysis of 1000x magnification SEM images to calculate the average diameter and area of deposits at different deposition voltages. Samples a-c represent a concentration series, each deposited at $-1.6 \mathrm{~V}$, but with the different dilutions of the electroplating solution. Samples d-h represent a voltage series, each deposited with a $0.1 \mathrm{~mol} \cdot \mathrm{L}^{-1} \mathrm{Ni}^{2+}$ electroplating solution but with voltage increased in $0.5 \mathrm{~V}$ steps from -0.5 to $-2.5 \mathrm{~V}$

\begin{tabular}{|c|c|c|c|c|c|c|}
\hline $\begin{array}{l}\text { Table } \\
\text { magr } \\
\text { calcu }\end{array}$ & $\begin{array}{l}\text { 2.3: Quan } \\
\text { cation } S E \\
\text { mean de }\end{array}$ & $\begin{array}{l}\text { tive a } \\
\text { of nicl } \\
\text { sit siz }\end{array}$ & $\begin{array}{l}\text { lysis of ima } \\
\text { electrodep } \\
\text { nd total co }\end{array}$ & $\begin{array}{l}\text { age data } \\
\text { osited o } \\
\text { verage. }\end{array}$ & $\begin{array}{l}1000 x \\
\text { iminiun }\end{array}$ & \\
\hline & & Pix & el Measureı & ments & & \\
\hline Image & $\begin{array}{l}\text { Nickel } \\
\text { Concentration }\end{array}$ & $\begin{array}{l}\text { Number } \\
\text { of } \\
\text { Deposits }\end{array}$ & $\begin{array}{l}\text { Area } \\
\text { average }\end{array}$ & $\begin{array}{l}\text { Area } \\
\text { standard } \\
\text { deviation }\end{array}$ & $\begin{array}{l}\text { Diameter } \\
\text { average }\end{array}$ & $\begin{array}{l}\text { Diameter } \\
\text { standard } \\
\text { deviation }\end{array}$ \\
\hline a5 & $0.01 \mathrm{~mol} \cdot \mathrm{L}^{-1}$ & 29 & 481.38 & 521.64 & 22.22 & 11.11 \\
\hline a7 & $0.01 \mathrm{~mol} \cdot \mathrm{L}^{-1}$ & 17 & 506.65 & 401.61 & 23.28 & 10.47 \\
\hline b10 & $0.1 \mathrm{~mol} \cdot \mathrm{L}^{-1}$ & 16 & 1379.00 & 1720.67 & 35.34 & 23.25 \\
\hline b13 & $0.1 \mathrm{~mol} \cdot \mathrm{L}^{-1}$ & 124 & 599.48 & 799.67 & 23.18 & 15.09 \\
\hline $\mathrm{b} 6$ & $0.1 \mathrm{~mol} \cdot \mathrm{L}^{-1}$ & 19 & 2109.16 & 2444.62 & 42.92 & 29.84 \\
\hline c3 & $1.0 \mathrm{~mol} \cdot \mathrm{L}^{-1}$ & 47 & 472.68 & 695.61 & 21.04 & 12.75 \\
\hline & Voltage & $\begin{array}{l}\text { Number } \\
\text { of } \\
\text { Deposits }\end{array}$ & Area average & $\begin{array}{l}\text { Area } \\
\text { standard } \\
\text { deviation }\end{array}$ & $\begin{array}{l}\text { Diameter } \\
\text { average }\end{array}$ & $\begin{array}{l}\text { Diameter } \\
\text { standard } \\
\text { deviation }\end{array}$ \\
\hline $\mathrm{d} 3$ & $-0.5 \mathrm{~V}$ & 15 & 443.27 & 251.56 & 22.73 & 7.17 \\
\hline d6 & $-0.5 \mathrm{~V}$ & 88 & 949.52 & 1329.35 & 30.55 & 16.7 \\
\hline $\mathrm{e} 7$ & $-1.0 \mathrm{~V}$ & 15 & 622.8 & 370.74 & 26.9 & 8.61 \\
\hline $\mathrm{f6} 6$ & $-1.5 \mathrm{~V}$ & 14 & 1648.64 & 1569.96 & 40.47 & 22.28 \\
\hline g6 & $-2.0 \mathrm{~V}$ & 38 & 1971.18 & 2402.38 & 42.81 & 26.37 \\
\hline h3 & $-2.5 \mathrm{~V}$ & 517 & 377.67 & 641.89 & 18.38 & 11.98 \\
\hline h8 & $-2.5 \mathrm{~V}$ & 134 & 915.62 & 1389.69 & 27.65 & 20.1 \\
\hline
\end{tabular}




\begin{tabular}{|c|c|c|c|c|c|c|c|}
\hline Conve & ersion used: 1 & $148 \frac{\text { Cal }}{\text { pixe }}$ & $\frac{\text { culated } \mathrm{N}}{\mathrm{ls}=10 \mathrm{~m}}$ & $\begin{array}{l}\text { Micron Va } \\
\text { icrons }\end{array}$ & lues & & \\
\hline Image & $\begin{array}{l}\text { Nickel } \\
\text { Concentration }\end{array}$ & $\begin{array}{l}\text { Area } \\
\text { average }\end{array}$ & \begin{tabular}{|l|} 
Area \\
standard \\
deviation
\end{tabular} & $\begin{array}{l}\text { Diameter } \\
\text { average }\end{array}$ & $\begin{array}{l}\text { Diameter } \\
\text { standard } \\
\text { deviation }\end{array}$ & $\begin{array}{l}\text { Total } \\
\text { Area }\end{array}$ & $\begin{array}{l}\text { Percentage } \\
\text { Area }\end{array}$ \\
\hline $\mathrm{a} 5$ & $0.01 \mathrm{~mol} \cdot \mathrm{L}^{-1}$ & 2.20 & 2.38 & 1.50 & 0.75 & 63.73 & $0.64 \%$ \\
\hline a7 & $0.01 \mathrm{~mol} \cdot \mathrm{L}^{-1}$ & 2.31 & 1.83 & 1.57 & 0.71 & 39.32 & $0.39 \%$ \\
\hline b10 & $0.1 \mathrm{~mol} \cdot \mathrm{L}^{-1}$ & 6.30 & 7.86 & 2.39 & 1.57 & 100.73 & $1.01 \%$ \\
\hline b13 & $0.1 \mathrm{~mol} \cdot \mathrm{L}^{-1}$ & 2.74 & 3.65 & 1.57 & 1.02 & 339.37 & $3.39 \%$ \\
\hline $\mathrm{b} 6$ & $0.1 \mathrm{~mol} \cdot \mathrm{L}^{-1}$ & 9.63 & 11.16 & 2.90 & 2.02 & 182.95 & $1.83 \%$ \\
\hline c3 & $1.0 \mathrm{~mol} \cdot \mathrm{L}^{-1}$ & 2.16 & 3.18 & 1.42 & 0.86 & 101.42 & $1.01 \%$ \\
\hline Image & Voltage & $\begin{array}{l}\text { Area } \\
\text { average }\end{array}$ & $\begin{array}{l}\text { Area } \\
\text { standard } \\
\text { deviation }\end{array}$ & $\begin{array}{l}\text { Diameter } \\
\text { average }\end{array}$ & $\begin{array}{l}\text { Diameter } \\
\text { standard } \\
\text { deviation }\end{array}$ & \begin{tabular}{|l|} 
Number \\
of \\
Deposits
\end{tabular} & $\begin{array}{l}\text { Percentage } \\
\text { Area }\end{array}$ \\
\hline d3 & $-0.5 \mathrm{~V}$ & 1.15 & 1.54 & 0.48 & 30.36 & 2.02 & $0.30 \%$ \\
\hline d6 & $-0.5 \mathrm{~V}$ & 6.07 & 2.06 & 1.13 & 381.47 & 4.33 & $3.81 \%$ \\
\hline e7 & $-1.0 \mathrm{~V}$ & 1.69 & 1.82 & 0.58 & 42.65 & 2.84 & $0.43 \%$ \\
\hline f6 & $-1.5 \mathrm{~V}$ & 7.17 & 2.73 & 1.51 & 105.37 & 7.53 & $1.05 \%$ \\
\hline g6 & $-2.0 \mathrm{~V}$ & 10.97 & 2.89 & 1.78 & 341.97 & 9.00 & $3.41 \%$ \\
\hline h3 & $-2.5 \mathrm{~V}$ & 2.93 & 1.24 & 0.81 & 891.41 & 1.72 & $8.90 \%$ \\
\hline h8 & $-2.5 \mathrm{~V}$ & 6.34 & 1.87 & 1.36 & 560.14 & 4.18 & $5.59 \%$ \\
\hline
\end{tabular}

\begin{tabular}{|c|c|c|c|c|c|c|c|c|c|c|}
\hline \multicolumn{7}{|c|}{ Results for Concentration Series } & \multirow[b]{2}{*}{$\mathrm{V}(\mathrm{V})$} & \multirow[b]{2}{*}{ t (s) } & \multirow[b]{2}{*}{$\mathrm{C}\left(\mathrm{mol} \cdot \mathrm{L}^{-1}\right)$} & \multirow[b]{2}{*}{ Base } \\
\hline Sample & $\begin{array}{l}\text { Area } \\
\text { average }\end{array}$ & $\begin{array}{l}\text { Area } \\
\text { standard } \\
\text { deviation }\end{array}$ & $\begin{array}{l}\text { Diamete } \\
\text { average }\end{array}$ & $\begin{array}{l}\text { Diameter } \\
\text { standard } \\
\text { deviation }\end{array}$ & Area & $\begin{array}{l}\text { Percentage } \\
\text { Area }\end{array}$ & & & & \\
\hline $090514 a$ & 5.1287 & 3.039 & 1.8480 & 0.486 & 1599.30 & $0.65 \%$ & -1.6 & 30 & 0.01 & $\mathrm{Al}$ \\
\hline $090514 \mathrm{~b}$ & 9.8952 & 8.555 & 2.4581 & 1.008 & 3594.23 & $1.45 \%$ & -1.6 & 30 & 0.1 & $\mathrm{Al}$ \\
\hline $090514 c$ & 6.8133 & 4.137 & 2.0955 & 0.621 & 738.14 & $0.30 \%$ & -1.6 & 30 & 1 & $\mathrm{Al}$ \\
\hline \multicolumn{7}{|c|}{ Results for Voltage Series } & $\mathrm{V}(\mathrm{V})$ & $t(s)$ & $\mathrm{C}\left(\mathrm{mol} \cdot \mathrm{L}^{-1}\right)$ & Base \\
\hline $090514 d$ & 4.5460 & 2.878 & 1.7370 & 0.473 & $\overline{223.05}$ & $0.09 \%$ & -0.5 & 30 & 0.1 & $\mathrm{Al}$ \\
\hline $090514 \mathrm{e}$ & 4.8950 & 4.051 & 1.7915 & 0.535 & 1081.81 & $0.44 \%$ & -1.0 & 30 & 0.1 & $\mathrm{Al}$ \\
\hline $090514 \mathrm{f}$ & 11.808 & 8.914 & 2.6935 & 1.088 & 1830.24 & $0.74 \%$ & -1.5 & 30 & 0.1 & $\mathrm{Al}$ \\
\hline $090514 \mathrm{~g}$ & 11.915 & 11.40 & 2.6633 & 1.188 & 7327.73 & $2.97 \%$ & -2.0 & 30 & 0.1 & $\mathrm{Al}$ \\
\hline $090514 \mathrm{~h}$ & 10.309 & 17.36 & 2.4334 & 1.199 & 13402.9 & $5.42 \%$ & -2.5 & 30 & 0.1 & $\mathrm{Al}$ \\
\hline
\end{tabular}

Figure 5.2.1 to Figure 5.2.4 shows a comparison series of SEM micrographs from different voltages illustrating this difference in shape. The $-2.0 \mathrm{~V}$ and $-2.5 \mathrm{~V}$ samples show some larger deposits but a greater quantity of small deposits and the large deposits have more irregular shapes with higher surface areas. Altogether the data from the SEM images is consistent with the predictions made from the current-time graphs. Higher voltage produced more nickel as shown in Figure 3.1.8, deposited in smaller deposits, as shown in Figure 3.1.9.

With these promising results, the process was transferred from aluminium to palladium substrates. 
Jonathan Tailby

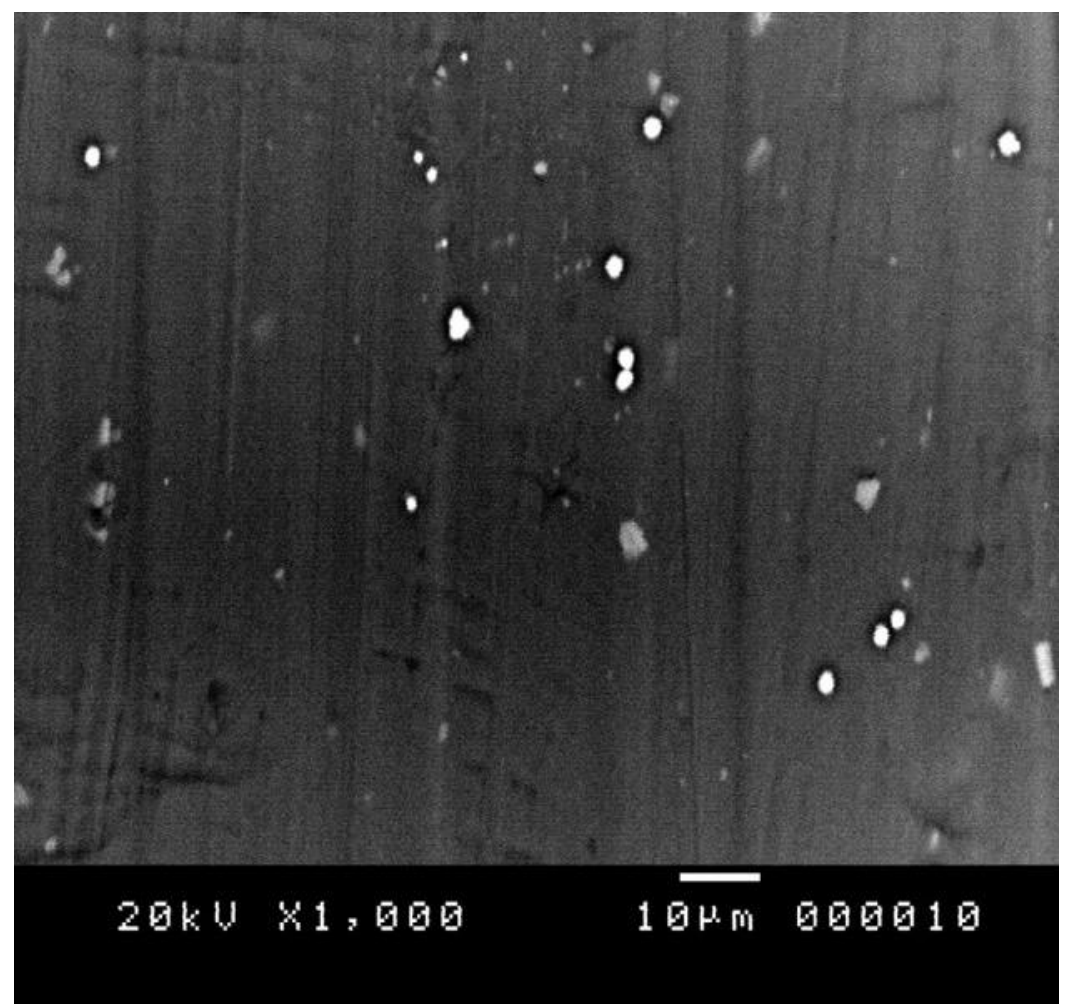

Figure 5.2.1: Electron micrograph of nickel deposited on aluminium at $-1.0 \mathrm{~V}$ for $30 \mathrm{~s}$ in $0.1 \mathrm{~mol} \cdot \mathrm{L}^{-1}$ solution.

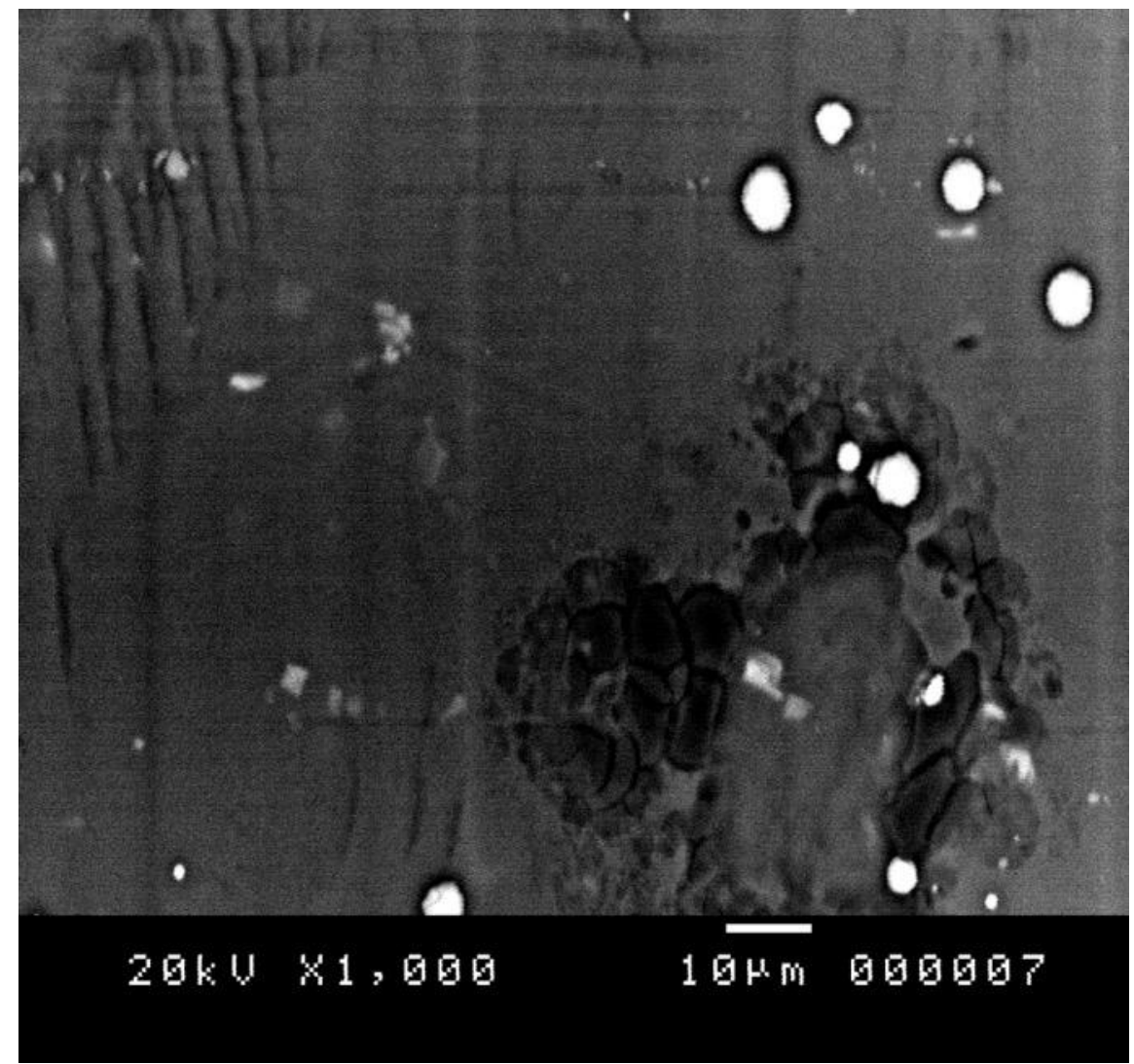

Figure 5.2.2: Electron micrograph of nickel deposited on aluminium at $-1.5 \mathrm{~V}$ for $30 \mathrm{~s}$ in $0.1 \mathrm{~mol} \cdot \mathrm{L}^{-1}$ solution. 
Jonathan Tailby

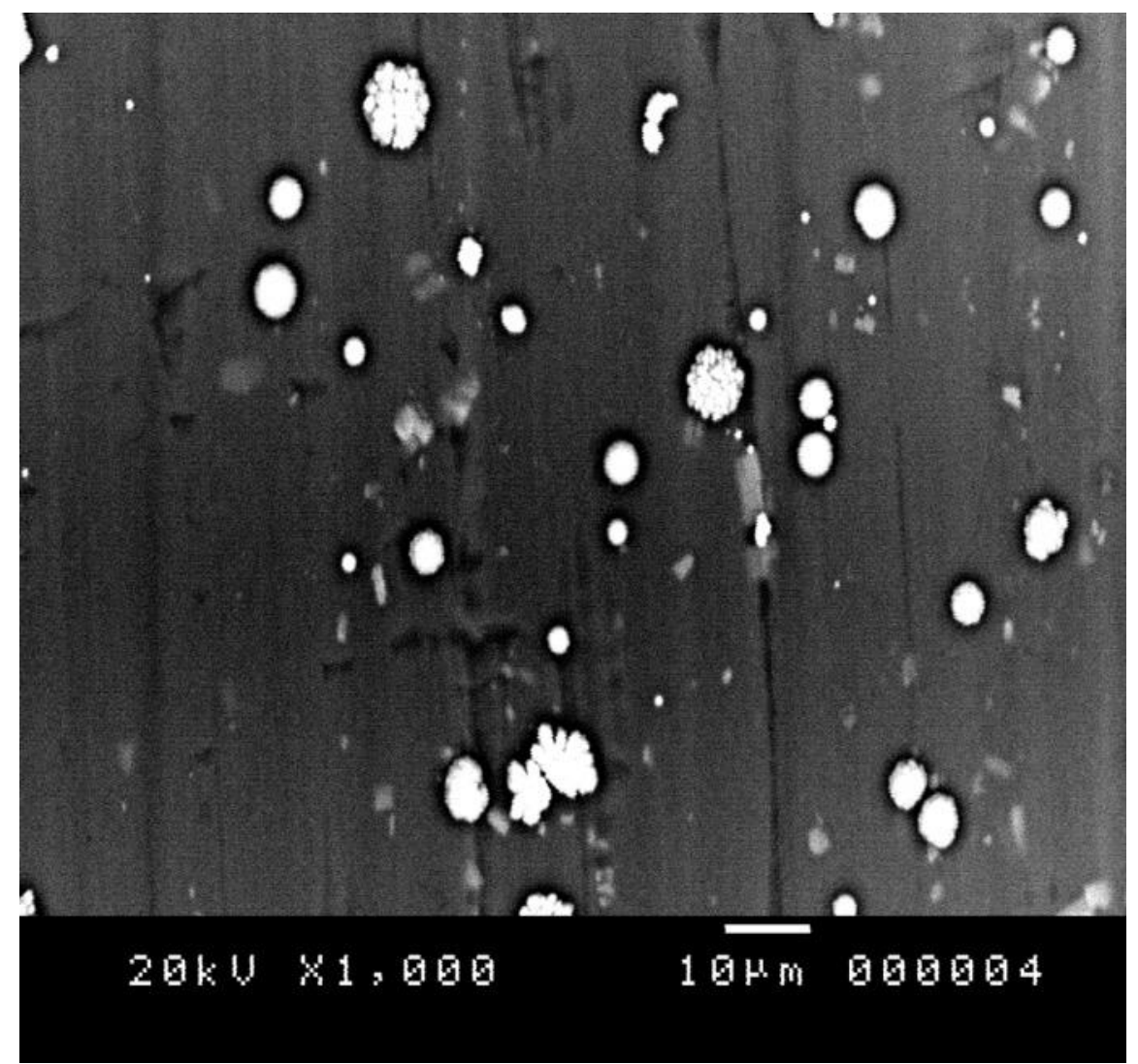

Figure 5.2.3: Electron micrograph of nickel deposited on aluminium at $-2.0 \mathrm{~V}$ for $30 \mathrm{~s}$ in $0.1 \mathrm{~mol} \cdot \mathrm{L}^{-1}$ solution.

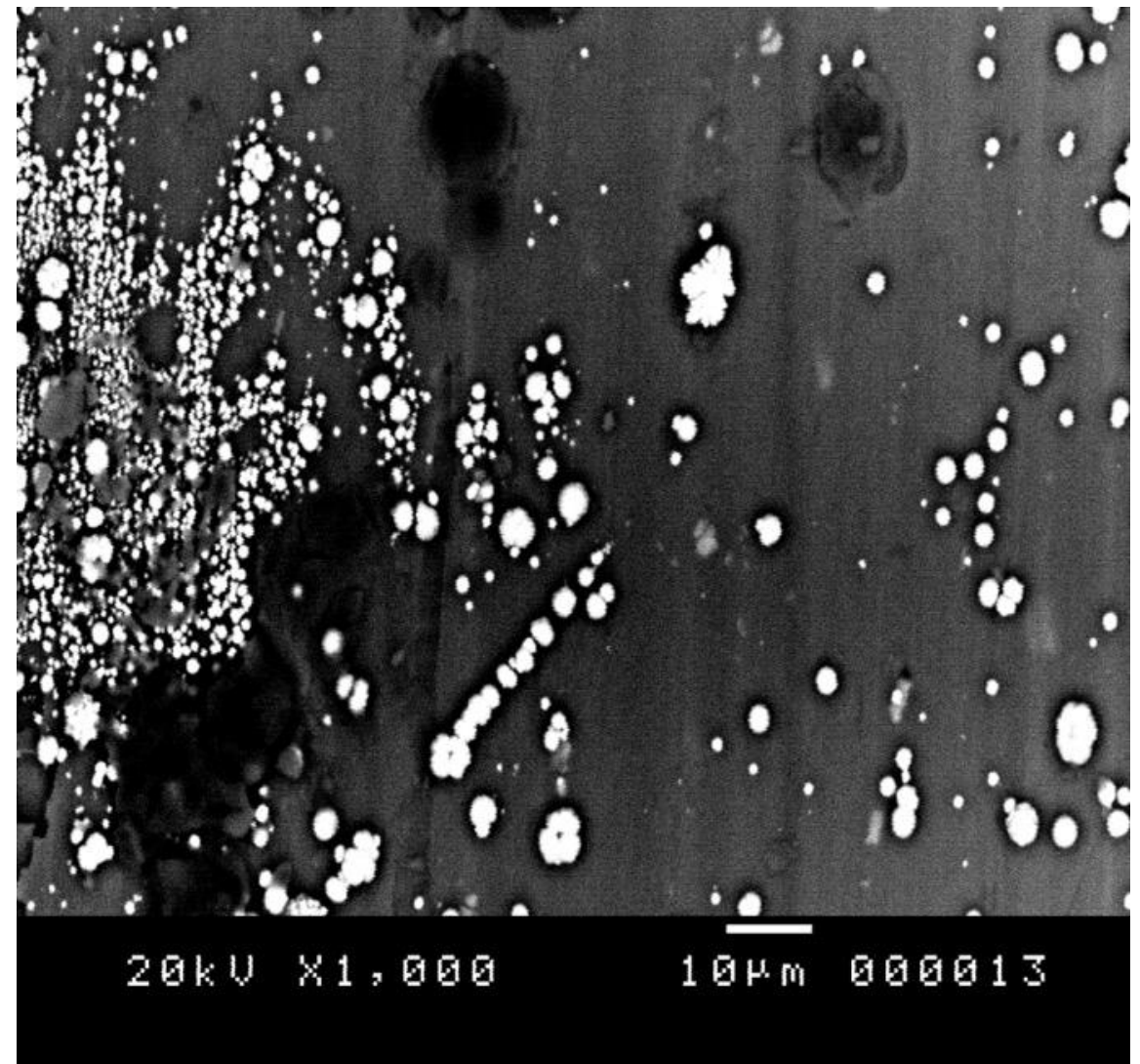

Figure 5.2.4: Electron micrograph of nickel deposited on aluminium at $-2.5 \mathrm{~V}$ for $30 \mathrm{~s}$ in $0.1 \mathrm{~mol} \cdot \mathrm{L}^{-1}$ solution. 
Jonathan Tailby

5.2.4. SEM Image Processing Calculations for Electrodeposition on Palladium

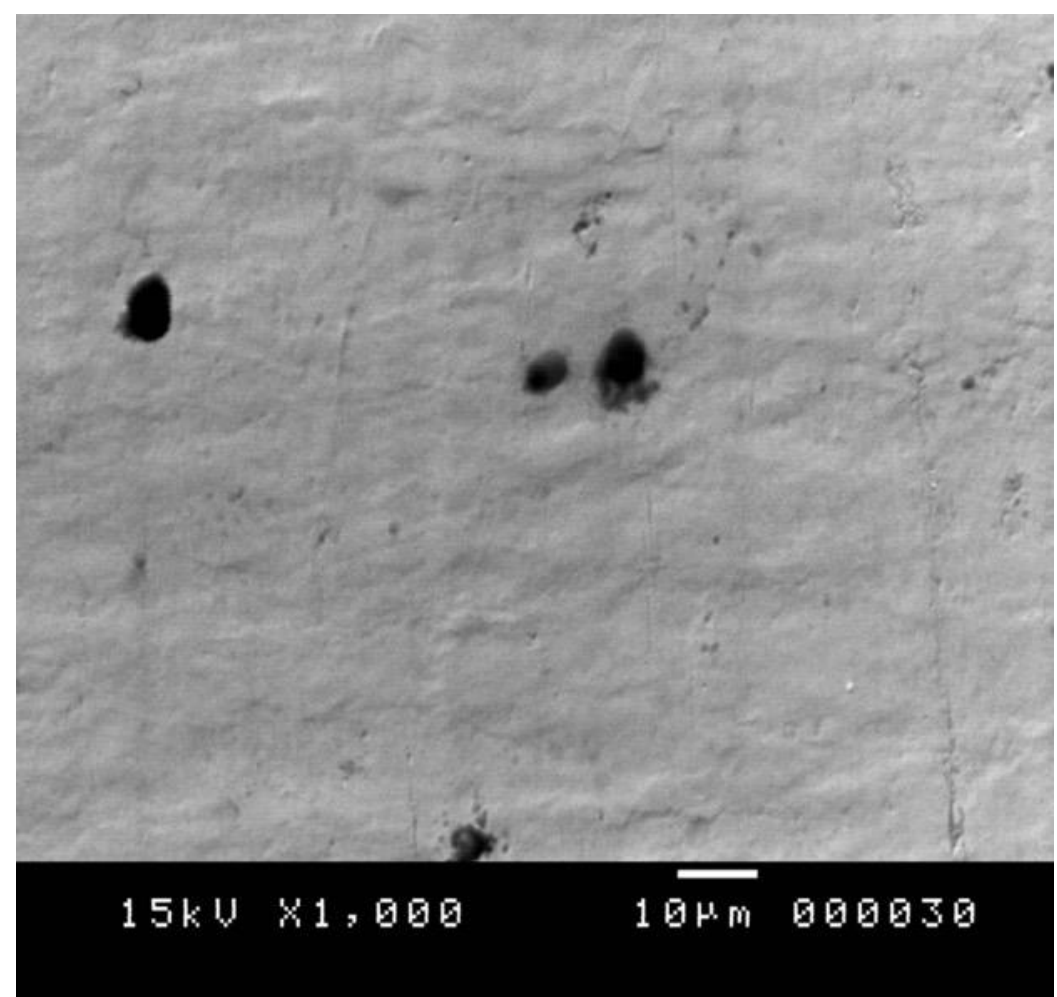

Figure 5.2.5: Electron micrograph of nickel deposited on palladium at $-1.0 \mathrm{~V}$ for $30 \mathrm{~s}$.

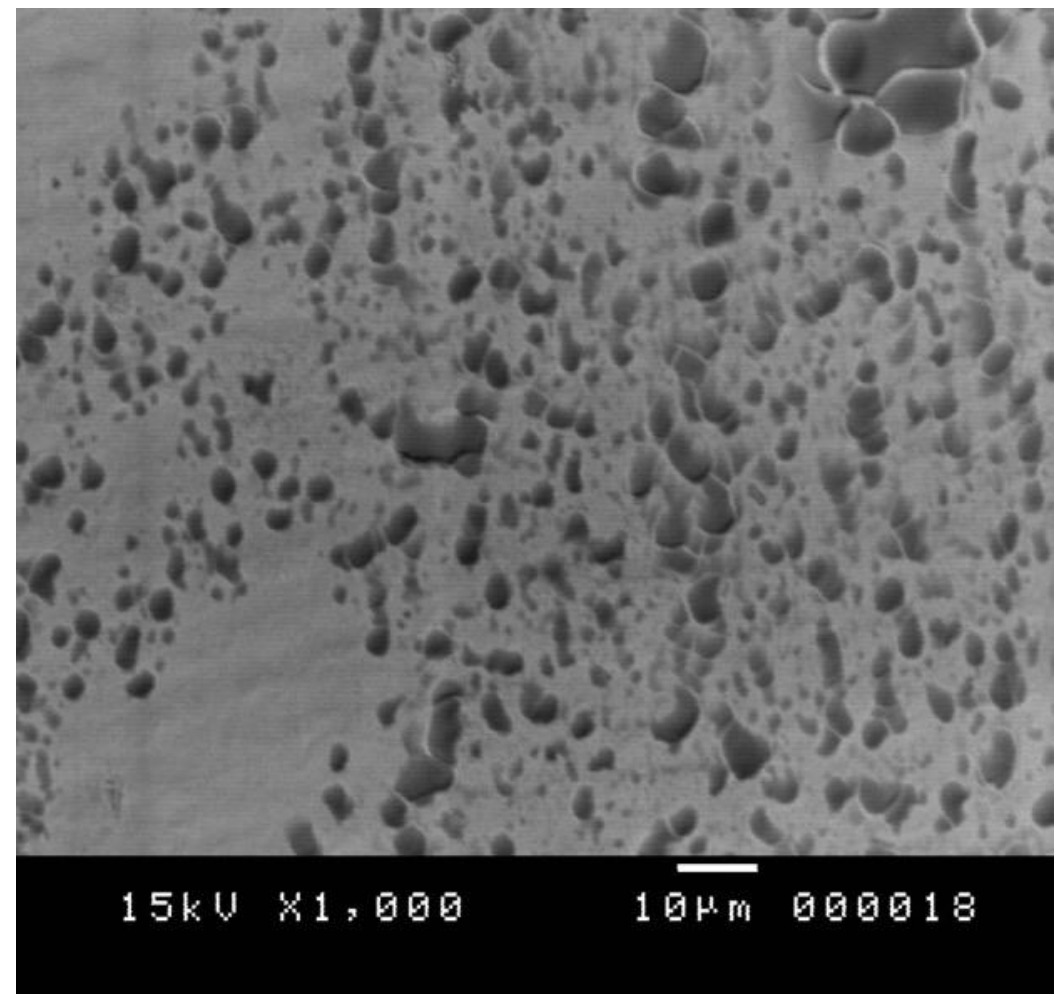

Figure 5.2.6: Electron micrograph of nickel deposited on palladium at $-2.5 \mathrm{~V}$ for $30 \mathrm{~s}$. 


\subsubsection{SEM \& EDS for Spray Coated Samples}

Figure 5.2.7 to Figure 5.2.14 show Electron micrographs and accompanying elemental maps for two more samples of nickel nanoparticles sprayed onto palladium. The SEM images show deposits on the palladium surface. The elemental maps show that these deposits correspond to decreases in the elemental signal for palladium and increases for oxygen and nickel.

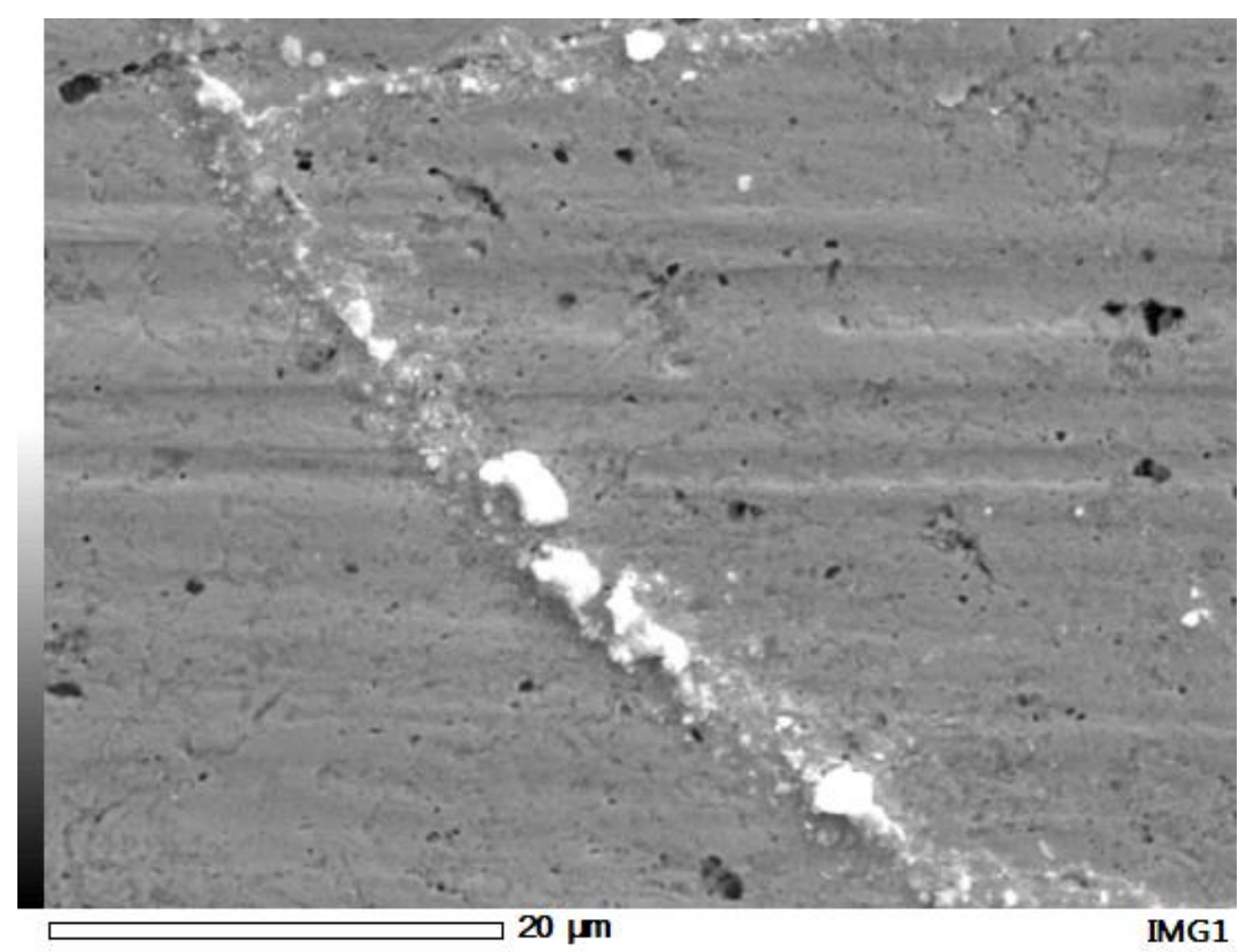

Figure 5.2.7: SEM image of a spray coated palladium sample. 


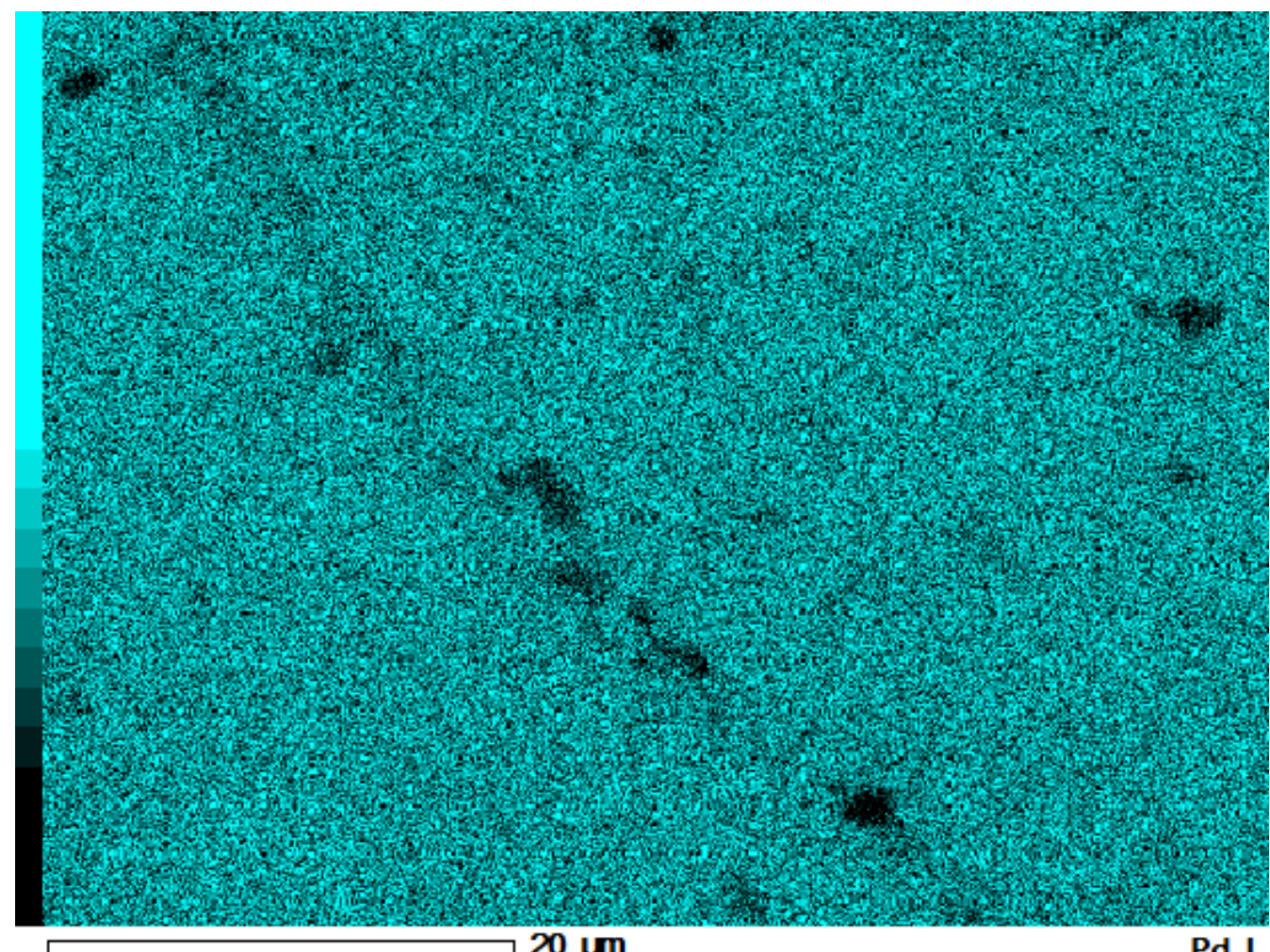

Figure 5.2.8: Elemental map of palladium. Showing decreased palladium in the spray coated areas.

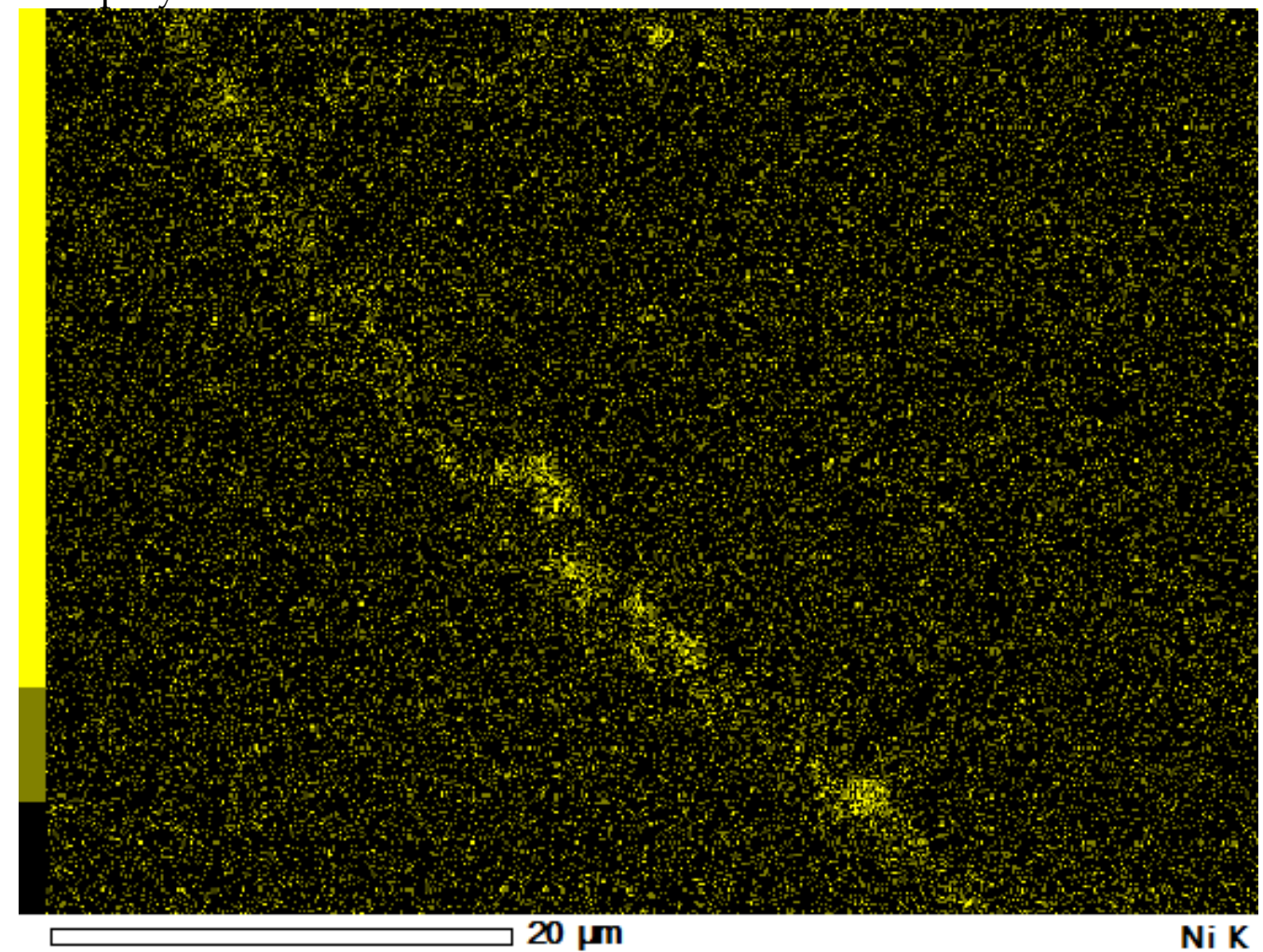

Figure 5.2.9: Elemental map of nickel. Showing increased nickel in the spray coated areas. 


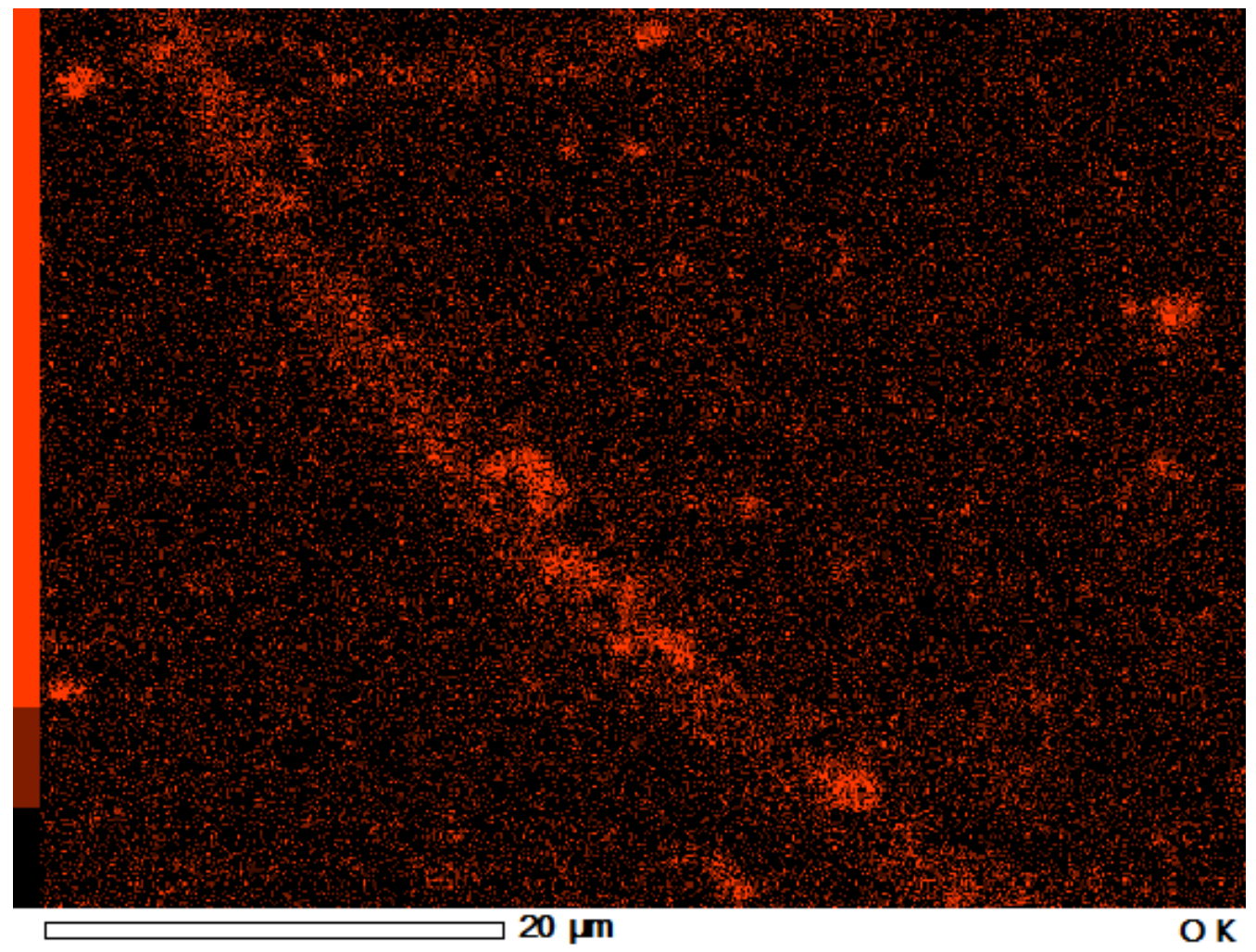

Figure 5.2.10: Elemental map of oxygen. Showing increased oxygen in the spray coated areas and at surface defects.

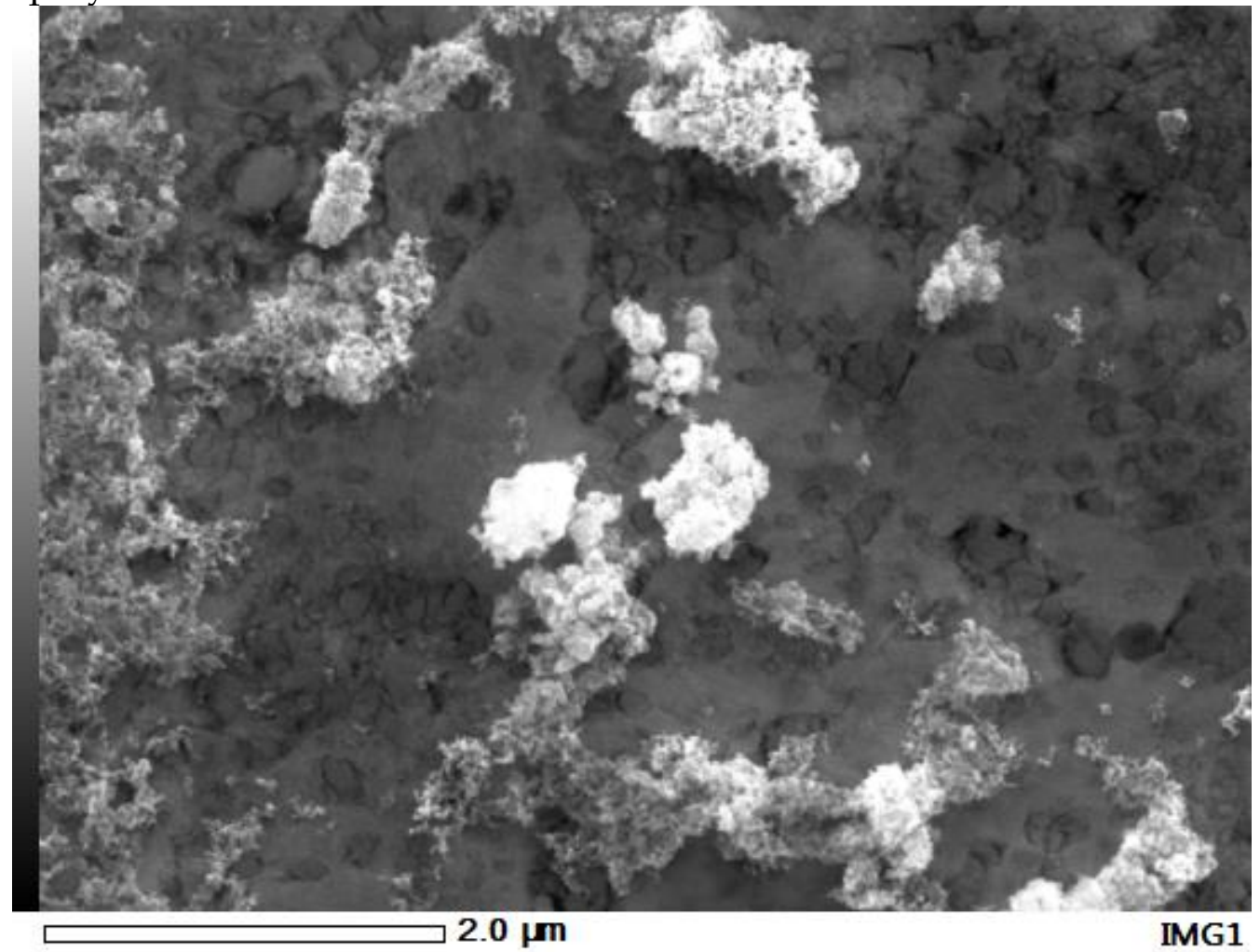

Figure 5.2.11: High magnification SEM image of a spray coated palladium sample. 


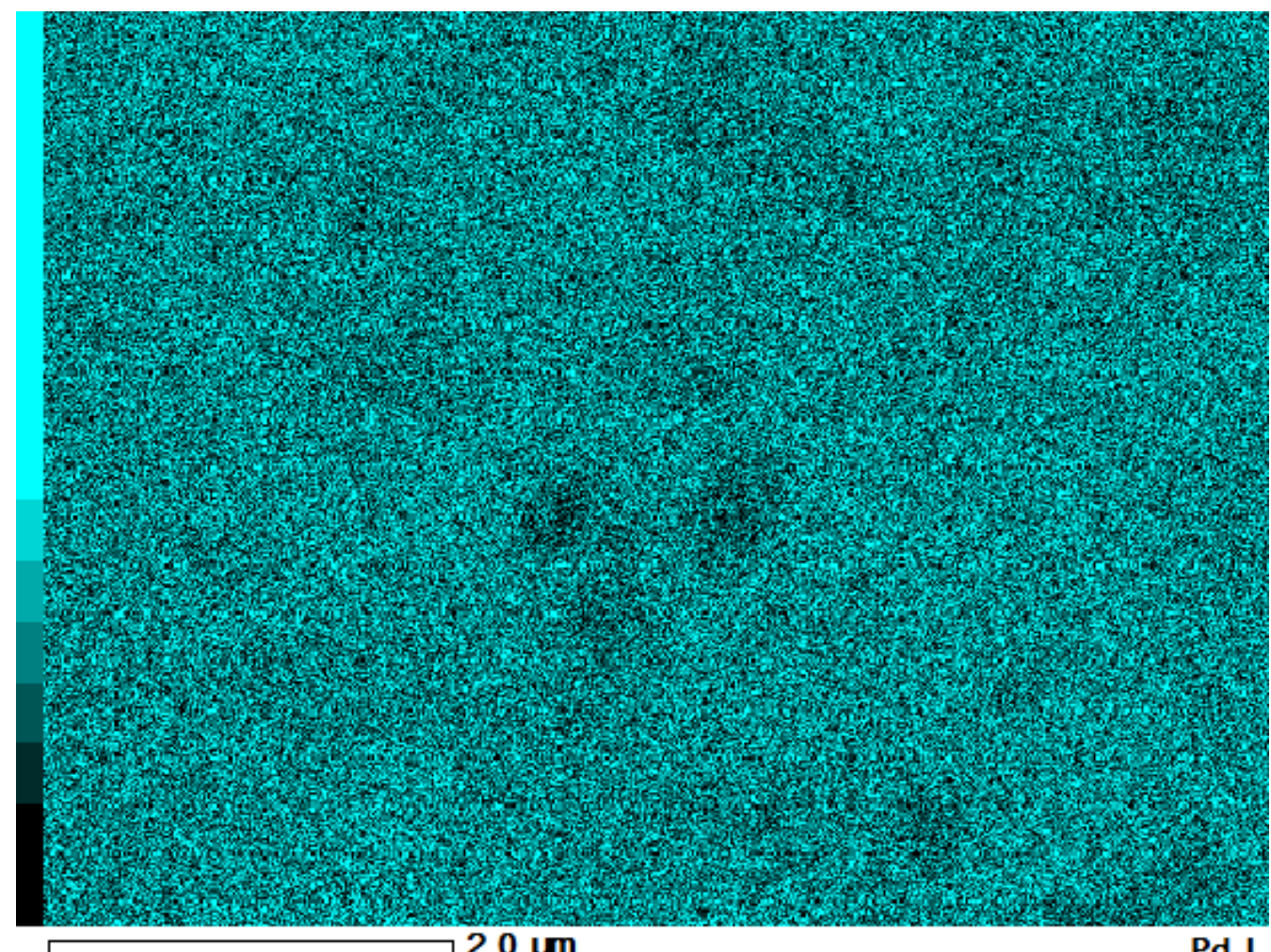

Figure 5.2.12: Palladium elemental map showing decreased palladium only at the largest deposits.

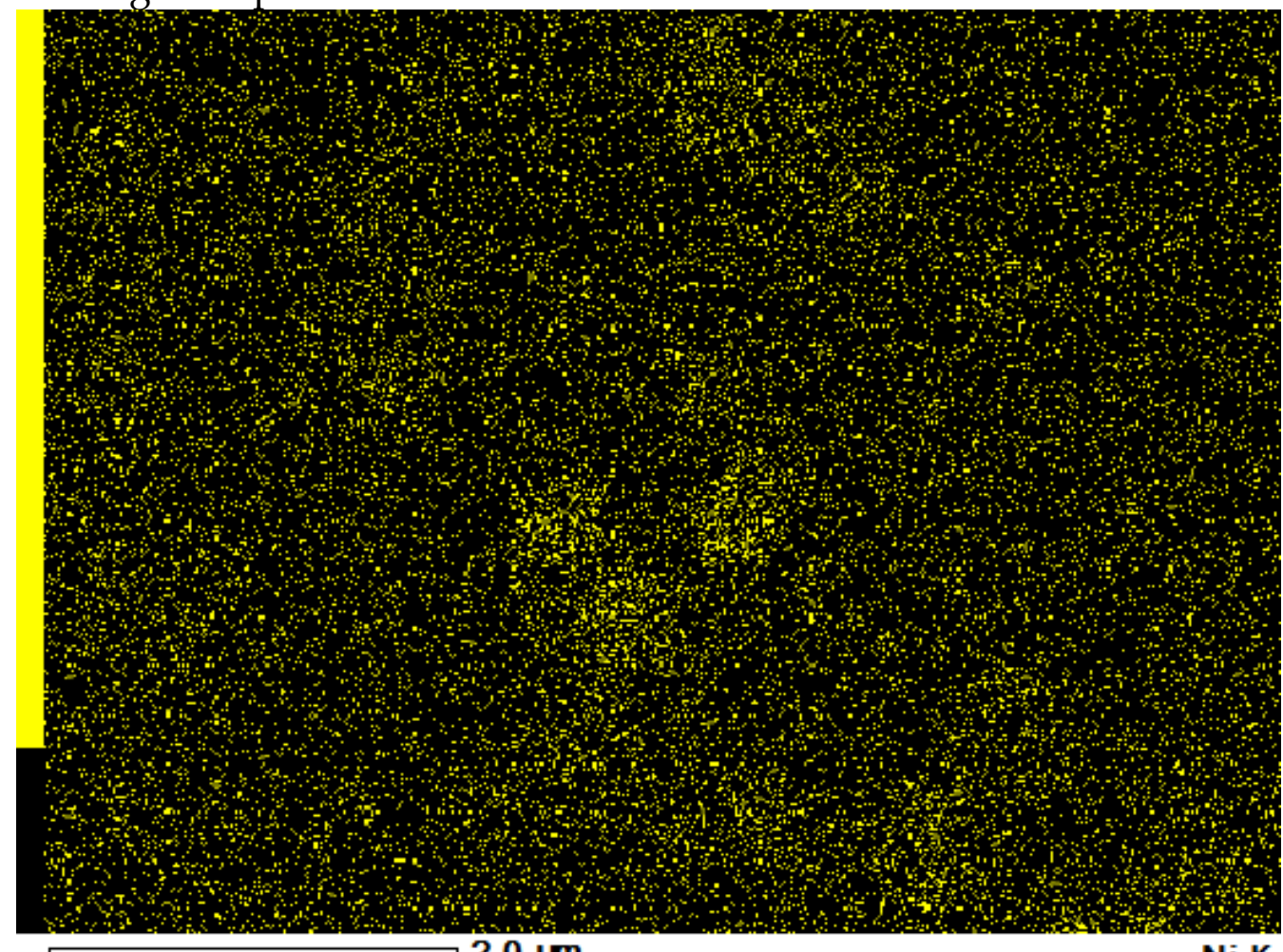

Figure 5.2.13: Elemental map of nickel showing increased nickel only at the largest deposits. 


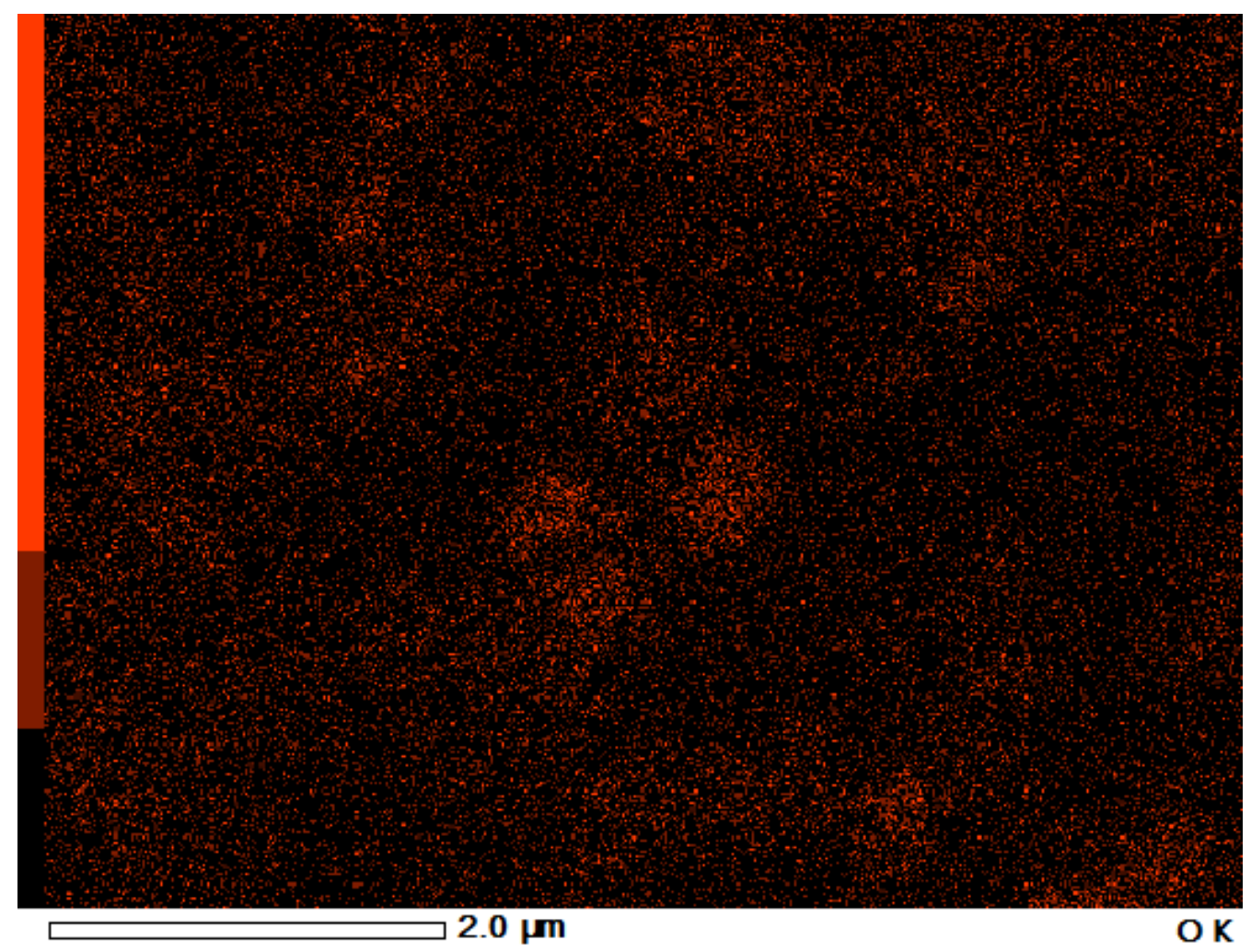

Figure 5.2.14: Elemental map of oxygen showing the greatest increase in oxygen was at the largest deposits.

5.2.6. SEM of Template Electrodeposition on Secondary Insert Tubes

Figure 5.2.15 to Figure 5.2.21 show electron micrographs of templated nickel electrodeposits on steel secondary insert tubes. The variation in these images show that the templated electrodeposition on tubular substrates was not as consistent as the process for flat substrates. 


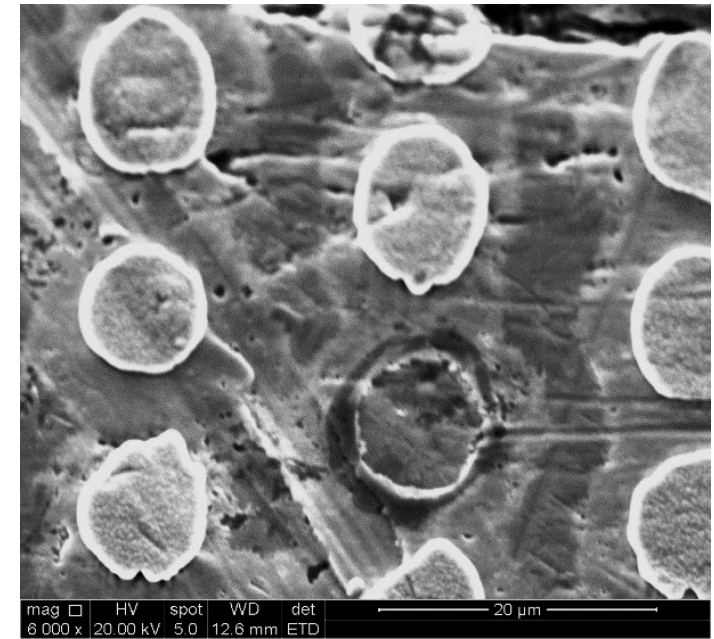

Figure 5.2.15: 6000x magnification secondary electron micrograph of templated electrodeposits on a secondary insert.

This image shows successfully templated electrodeposition.

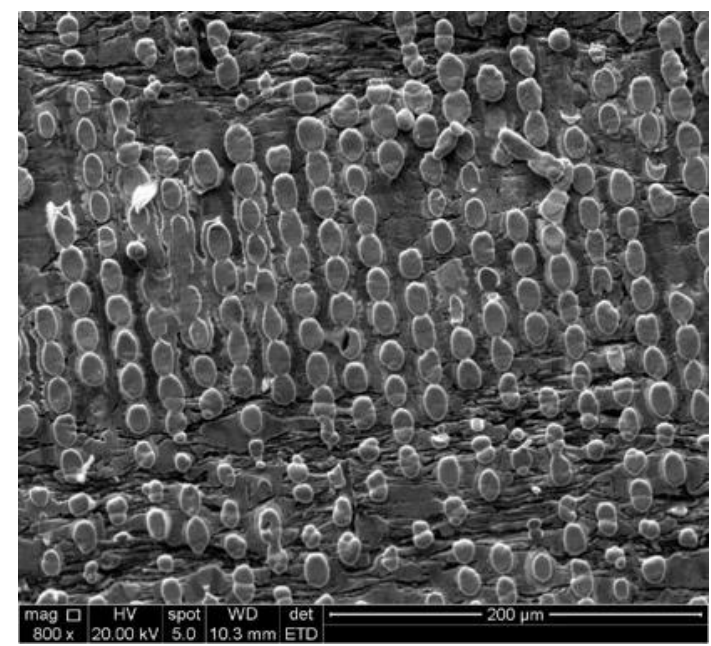

Figure 5.2.17: 800x magnification secondary electron micrograph of templated electrodeposits on a secondary insert.

This image shows elongation of the electrodeposits over most of the field of view.

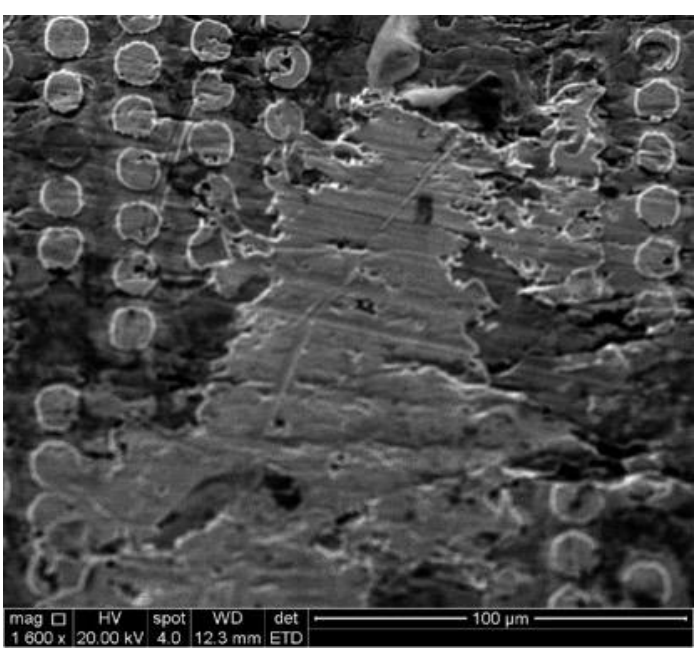

Figure 5.2.16: 1600x magnification secondary electron micrograph of templated electrodeposits on a secondary insert.

This image shows some successfully templated electrodeposits, however, the centre of the image is dominated by a deposit which has not been controlled by the template.

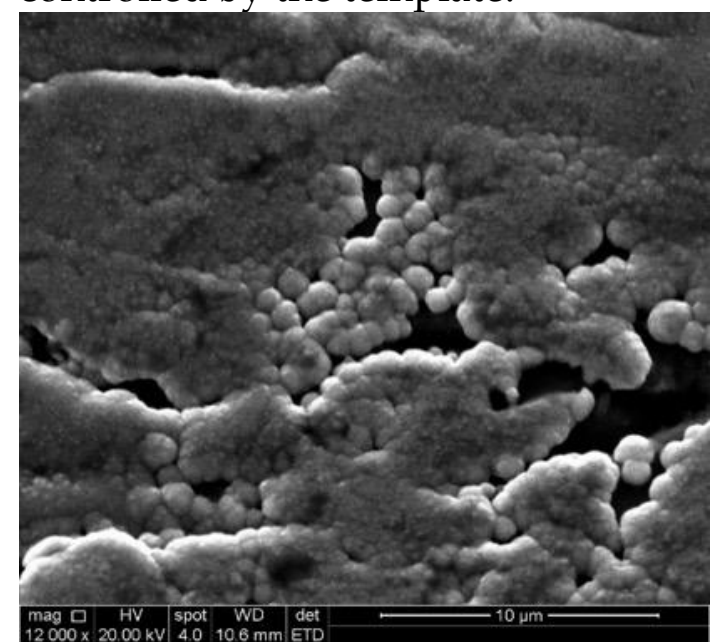

Figure 5.2.18: 1200x magnification secondary electron micrograph of templated electrodeposits on a secondary insert.

This image shows an overdeposited region of the templated electrodeposition.

Figure 5.2.17 to Figure 5.2.19: SEM images of a secondary insert for the gas reactor with templated electrodeposits showing a mix of successfully and unsuccessfully templated regions. 


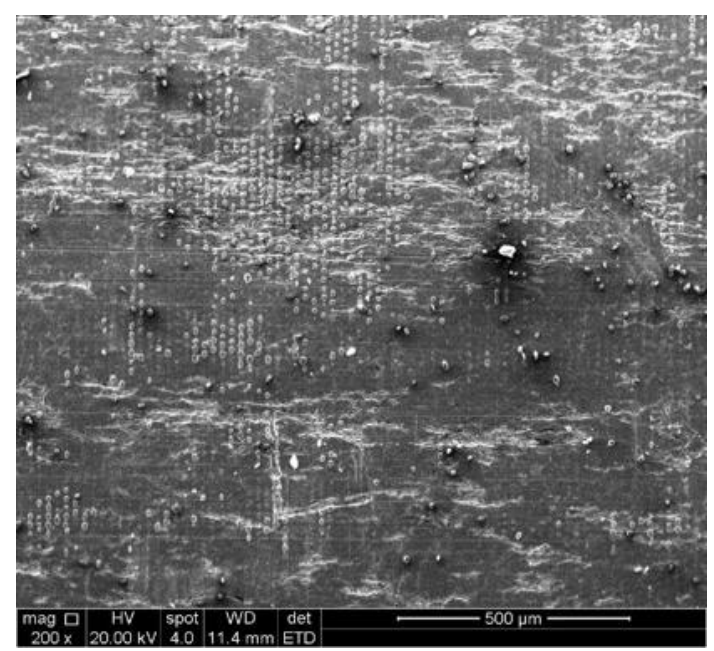

Figure 5.2.19: 200x magnification secondary electron micrograph of an alternate templated electrodeposit on a secondary insert.

This image shows successfully templated electrodeposition over a small portion of the field of view.

Figure 5.2.20 and Figure 5.2.21: SEM images of an alternate secondary insert for the gas reactor with templated electrodeposits showing some successfully templated regions and some unsuccessfully templated regions. Overall the templated electrodeposition was less successful on this insert, this was attributed to a less even template than for the insert shown in Figure 3.1.59 to Figure 3.1.61.

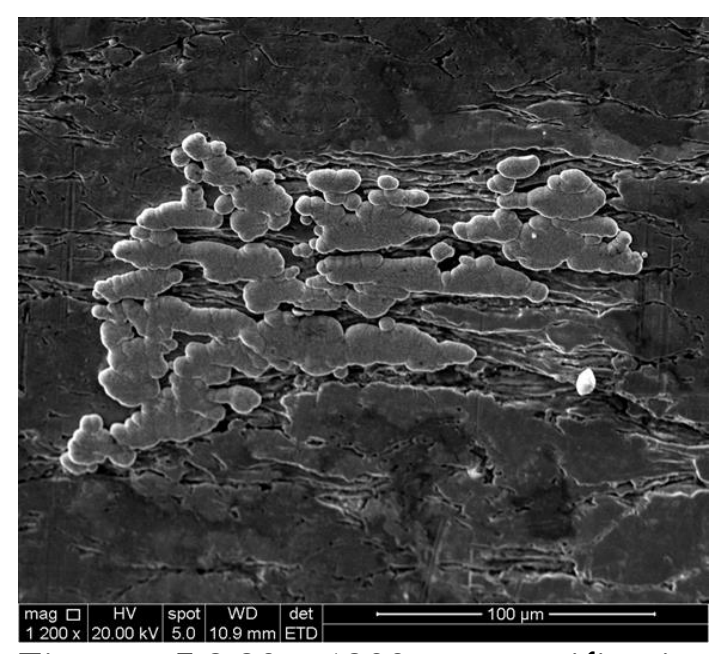

Figure 5.2.20: 1200x magnification secondary electron micrograph of an alternate templated electrodeposit on a secondary insert.

This image shows an overdeposited region with some evidence that it was composed of agglomerated deposits.

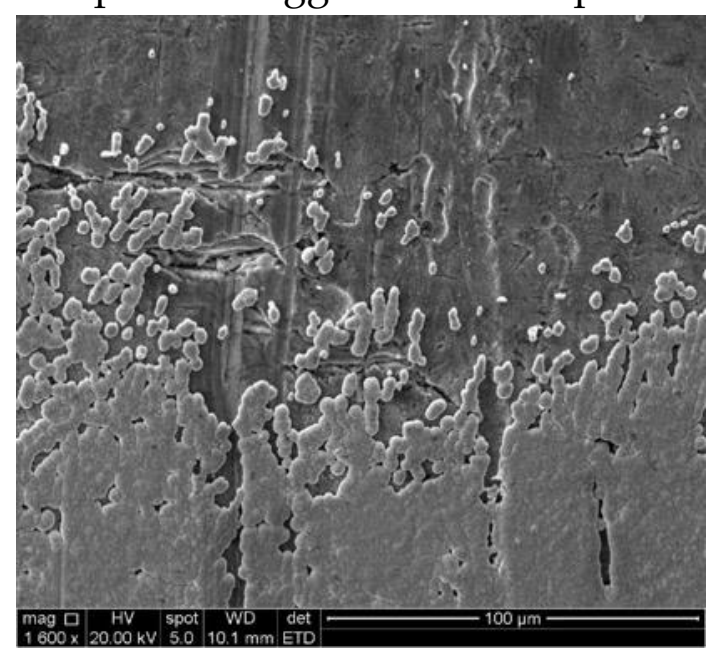

Figure 5.2.21: 1600x magnification secondary electron micrograph of an alternate templated electrodeposit on a secondary insert.

This image shows the end of the deposition region with over deposited template at the bottom of the image giving way to the undeposited region at the top. 
Jonathan Tailby

\subsubsection{Gas Chromatography}

\subsubsection{Catalysts in the Whole Explorer Reactor Volume}

Table 5.2.4 to Table 5.2.8 follow the calculations for determining the outputs and decomposition for experiments that tested different catalyst samples in the full volume of the Explorer reactor at $300-500{ }^{\circ} \mathrm{C}$. The output and decomposition are graphed in Section 3.2.2.2.

The standards used in these calculations are:

5\% Hydrogen $=$ Peak Area 1049.1

95\% Nitrogen $=$ Peak Area 1826.3

100\% Ammonia $=$ Peak Area 5239.4

\begin{tabular}{|c|c|c|c|c|c|c|}
\hline \multicolumn{7}{|c|}{ Hydrogen } \\
\hline Catalyst & 300 & 350 & 400 & 450 & 500 & 550 \\
\hline Blank Steel & & 8.4 & 590.6 & & 2068.0 & \\
\hline $\begin{array}{l}\text { Templated } \\
\text { Steel }\end{array}$ & & 239.2 & 917.8 & 2485.7 & 4591.7 & \\
\hline $\begin{array}{l}\text { Bare } \\
\text { Palladium }\end{array}$ & 50.2 & 187.4 & 730.6 & 2018.0 & 4104.0 & \\
\hline $\begin{array}{l}\text { Templated } \\
\text { Palladium } \\
\end{array}$ & 35.9 & 232.7 & 180.4 & 2215.5 & 4579.8 & 6712.5 \\
\hline \multicolumn{7}{|c|}{ Nitrogen } \\
\hline Catalyst & 300 & 350 & 400 & 450 & 500 & 550 \\
\hline Blank Steel & & 6.1 & 22.7 & & 125.5 & \\
\hline $\begin{array}{l}\text { Templated } \\
\text { Steel }\end{array}$ & & 20.1 & 40.9 & 102.3 & 187.5 & \\
\hline $\begin{array}{l}\text { Bare } \\
\text { Palladium }\end{array}$ & 10.8 & 14.8 & 63.1 & 133.7 & 202.9 & \\
\hline $\begin{array}{l}\text { Templated } \\
\text { Palladium }\end{array}$ & 8.6 & 20.6 & 88.5 & 140.6 & 234.1 & 312.4 \\
\hline \multicolumn{7}{|c|}{ Ammonia } \\
\hline Catalyst & 300 & 350 & 400 & 450 & 500 & 550 \\
\hline Blank Steel & & 5088.5 & 4881.2 & & & \\
\hline $\begin{array}{l}\text { Templated } \\
\text { Steel }\end{array}$ & & 5074.8 & 4829.5 & 4074.8 & 2910.3 & \\
\hline $\begin{array}{l}\text { Bare } \\
\text { Palladium }\end{array}$ & 5154.1 & 5120.5 & 4908.3 & 4323.3 & 319.8 & \\
\hline
\end{tabular}




\begin{tabular}{|l|l|l|l|l|l|l|}
\hline $\begin{array}{l}\text { Templated } \\
\text { Palladium }\end{array}$ & 5239.4 & 5149.2 & 4955.5 & 4389.2 & 3008.3 & 1647.9 \\
\hline
\end{tabular}

\begin{tabular}{|c|c|c|c|c|c|c|}
\hline \multicolumn{7}{|c|}{ Hydrogen } \\
\hline Catalyst & 300 & 350 & 400 & 450 & 500 & 550 \\
\hline Blank Steel & & $0.04 \%$ & $2.82 \%$ & & $9.86 \%$ & \\
\hline $\begin{array}{l}\text { Templated } \\
\text { Steel }\end{array}$ & & $1.14 \%$ & $4.37 \%$ & $11.85 \%$ & $21.88 \%$ & \\
\hline $\begin{array}{l}\text { Bare } \\
\text { Palladium }\end{array}$ & $0.24 \%$ & $0.89 \%$ & $3.48 \%$ & $9.62 \%$ & $19.56 \%$ & \\
\hline $\begin{array}{l}\text { Templated } \\
\text { Palladium }\end{array}$ & $0.17 \%$ & $1.11 \%$ & $0.86 \%$ & $10.56 \%$ & $21.83 \%$ & $31.99 \%$ \\
\hline \multicolumn{7}{|c|}{ Nitrogen } \\
\hline Catalyst & 300 & 350 & 400 & 450 & 500 & 550 \\
\hline Blank Steel & & $0.31 \%$ & $1.18 \%$ & & $6.53 \%$ & \\
\hline $\begin{array}{l}\text { Templated } \\
\text { Steel }\end{array}$ & & $1.05 \%$ & $2.13 \%$ & $5.32 \%$ & $9.75 \%$ & \\
\hline $\begin{array}{l}\text { Bare } \\
\text { Palladium }\end{array}$ & $0.56 \%$ & $0.77 \%$ & $3.28 \%$ & $6.96 \%$ & $10.55 \%$ & \\
\hline $\begin{array}{l}\text { Templated } \\
\text { Palladium }\end{array}$ & $0.45 \%$ & $1.07 \%$ & $4.60 \%$ & $7.32 \%$ & $12.18 \%$ & $16.25 \%$ \\
\hline \multicolumn{7}{|c|}{ Ammonia } \\
\hline Catalyst & 300 & 350 & 400 & 450 & 500 & 550 \\
\hline Blank Steel & & $97.12 \%$ & $93.16 \%$ & & & \\
\hline $\begin{array}{l}\text { Templated } \\
\text { Steel }\end{array}$ & & $96.86 \%$ & $92.18 \%$ & $\begin{array}{l}77.77 \\
\%\end{array}$ & $55.55 \%$ & \\
\hline $\begin{array}{l}\text { Bare } \\
\text { Palladium }\end{array}$ & $98.37 \%$ & $97.73 \%$ & $93.68 \%$ & $\begin{array}{l}82.52 \\
\%\end{array}$ & $6.10 \%$ & \\
\hline $\begin{array}{l}\text { Templated } \\
\text { Palladium }\end{array}$ & $100.00 \%$ & $98.28 \%$ & $94.58 \%$ & $\begin{array}{l}83.77 \\
\%\end{array}$ & $57.42 \%$ & $31.45 \%$ \\
\hline
\end{tabular}


Jonathan Tailby

The Initial gas flow used for these experiments and their calculations was $2.01 \times 10^{-4} \mathrm{~mol} \mathrm{~min}^{-1} \mathrm{NH}_{3}$

Table 5.2.6: Molar output calculated from the percentages using equations developed in Section 2.2.12.3.

Calculated from the percentages in Table 5.2.5 and the initial flow.

Values are in $\mathrm{mol} \mathrm{min}^{-1}$.

\begin{tabular}{|c|c|c|c|c|c|c|}
\hline \multicolumn{7}{|c|}{ Hydrogen } \\
\hline Catalyst & 300 & 350 & 400 & 450 & 500 & 550 \\
\hline Blank Steel & & 8.04E-08 & 5.77E-06 & & 2.11E-05 & \\
\hline $\begin{array}{l}\text { Templated } \\
\text { Steel }\end{array}$ & & 2.31E-06 & 9.05E-06 & 2.57E-05 & 5.04E-05 & \\
\hline \begin{tabular}{|l|} 
Bare \\
Palladium \\
\end{tabular} & 4.82E-07 & $1.81 \mathrm{E}-06$ & 7.17E-06 & 2.06E-05 & $4.45 \mathrm{E}-05$ & \\
\hline \begin{tabular}{|l|} 
Templated \\
Palladium
\end{tabular} & $3.45 \mathrm{E}-07$ & $2.25 \mathrm{E}-06$ & $1.74 \mathrm{E}-06$ & 2.27E-05 & 5.03E-05 & 7.81E-05 \\
\hline \multicolumn{7}{|c|}{ Nitrogen } \\
\hline Catalyst & 300 & 350 & 400 & 450 & 500 & 550 \\
\hline Blank Steel & & 6.37E-07 & 2.43E-06 & $0.00 \mathrm{E}+00$ & 1.48E-05 & \\
\hline \begin{tabular}{|l|}
$\begin{array}{l}\text { Templated } \\
\text { Steel }\end{array}$ \\
\end{tabular} & & $2.15 \mathrm{E}-06$ & 4.46E-06 & $1.18 \mathrm{E}-05$ & 2.34E-05 & \\
\hline \begin{tabular}{|l|} 
Bare \\
Palladium
\end{tabular} & $1.14 \mathrm{E}-06$ & $1.57 \mathrm{E}-06$ & 7.04E-06 & 1.59E-05 & 2.57E-05 & \\
\hline \begin{tabular}{|l|} 
Templated \\
Palladium
\end{tabular} & $9.09 \mathrm{E}-07$ & 2.21E-06 & 1.01E-05 & 1.69E-05 & 3.04E-05 & 4.33E-05 \\
\hline \multicolumn{7}{|c|}{ Ammonia } \\
\hline Catalyst & 300 & 350 & 400 & 450 & 500 & 550 \\
\hline Blank Steel & & 2.01E-04 & 2.00E-04 & & & \\
\hline \begin{tabular}{|l|} 
Templated \\
Steel
\end{tabular} & & $2.01 \mathrm{E}-04$ & 2.00E-04 & 1.91E-04 & 1.61E-04 & \\
\hline \begin{tabular}{|l|} 
Bare \\
Palladium \\
\end{tabular} & 2.01E-04 & 2.01E-04 & 2.00E-04 & 1.95E-04 & 2.38E-05 & \\
\hline \begin{tabular}{|l|} 
Templated \\
Palladium
\end{tabular} & 2.01E-04 & 2.01E-04 & 2.01E-04 & $1.96 \mathrm{E}-04$ & 1.65E-04 & 1.07E-04 \\
\hline
\end{tabular}

Table 5.2.7: Total molar output.

Calculated by the sum of the molar output for each species from . Values are in mol $\mathrm{min}^{-1}$.

Total

Output

\begin{tabular}{|l|l|l|l|l|l|l|}
\hline Catalyst & $\mathbf{3 0 0}$ & $\mathbf{3 5 0}$ & $\mathbf{4 0 0}$ & $\mathbf{4 5 0}$ & $\mathbf{5 0 0}$ & $\mathbf{5 5 0}$ \\
\hline $\begin{array}{l}\text { Blank } \\
\text { Steel }\end{array}$ & & $2.02 \mathrm{E}-04$ & $2.08 \mathrm{E}-04$ & & & \\
\hline $\begin{array}{l}\text { Templated } \\
\text { Steel }\end{array}$ & & $2.05 \mathrm{E}-04$ & $2.13 \mathrm{E}-04$ & $2.29 \mathrm{E}-04$ & $2.35 \mathrm{E}-04$ & \\
\hline $\begin{array}{l}\text { Bare } \\
\text { Palladium }\end{array}$ & $2.03 \mathrm{E}-04$ & $2.04 \mathrm{E}-04$ & $2.15 \mathrm{E}-04$ & $2.31 \mathrm{E}-04$ & $9.40 \mathrm{E}-05$ & \\
\hline $\begin{array}{l}\text { Templated } \\
\text { Palladium }\end{array}$ & $2.02 \mathrm{E}-04$ & $2.06 \mathrm{E}-04$ & $2.12 \mathrm{E}-04$ & $2.35 \mathrm{E}-04$ & $2.45 \mathrm{E}-04$ & $2.28 \mathrm{E}-04$ \\
\hline
\end{tabular}




\begin{tabular}{|c|c|c|c|c|c|c|c|}
\hline \multirow{6}{*}{ Decomposition } & \multicolumn{7}{|c|}{$\begin{array}{l}\text { Table 5.2.8: Rate of ammonia decomposition. } \\
\text { Calculated by the difference between the rate of ammonia input and } \\
\text { the rate of ammonia output. Values are in } \mathrm{mol} \mathrm{min}^{-1} \text {. }\end{array}$} \\
\hline & Catalyst & 300 & 350 & 400 & 450 & 500 & 550 \\
\hline & $\begin{array}{l}\text { Blank } \\
\text { Steel }\end{array}$ & & $1.67 \mathrm{E}-07$ & $9.40 \mathrm{E}-07$ & & & \\
\hline & $\begin{array}{l}\text { Templated } \\
\text { Steel }\end{array}$ & & $1.98 \mathrm{E}-07$ & $1.23 \mathrm{E}-06$ & 9.94E-06 & 3.97E-05 & \\
\hline & $\begin{array}{l}\text { Bare } \\
\text { Palladium }\end{array}$ & 5.32E-08 & $1.03 \mathrm{E}-07$ & 8.03E-07 & $6.15 \mathrm{E}-06$ & 1.77E-04 & \\
\hline & $\begin{array}{l}\text { Templated } \\
\text { Palladium }\end{array}$ & $0.00 \mathrm{E}+00$ & 5.96E-08 & 5.90E-07 & 5.30E-06 & 3.65E-05 & 9.45E-05 \\
\hline
\end{tabular}

\subsubsection{Catalyst squares in the Primary Insert in Explorer Reactor}

Figure 5.2.24 shows the result from ammonia decomposition at $400{ }^{\circ} \mathrm{C}$ with an undeposited stainless steel catalyst sample. Figure 5.2.23 shows a chromatogram for ammonia decomposition at $400{ }^{\circ} \mathrm{C}$ with a stainless steel catalyst sample with templated nickel electrodeposits. The area of the peak from hydrogen increased the most as the temperature increased, but the nitrogen peak also increased. The area of the ammonia peaks decreased as the temperature increased. The peak areas from these chromatograms are summarized in Table 5.2.9 to Table 5.2.13 shows calculations that convert the GC peak areas into gas outputs using the equations from Section 2.2.12.3.2. The rate calculated in Table 5.2.9 to Table 5.2.13 are plotted against temperature in Figure 3.2.7 to Figure 3.2.10

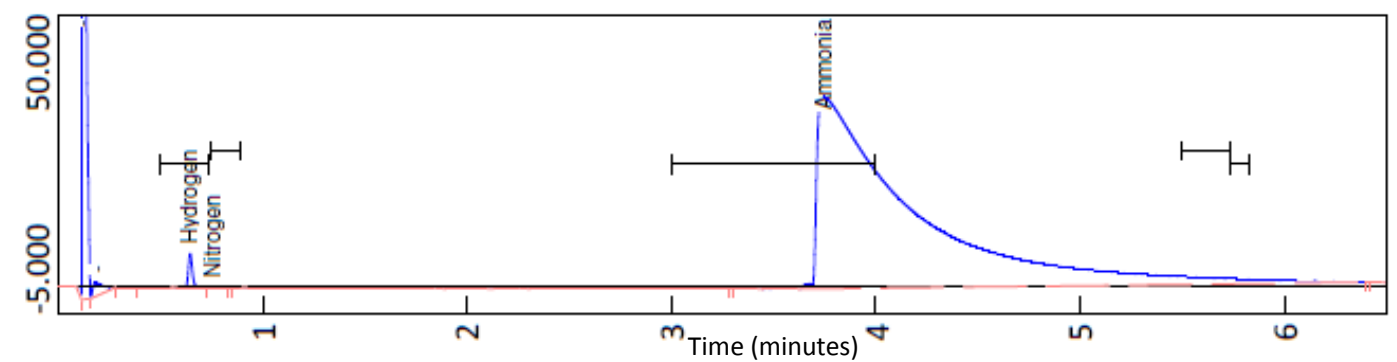

Figure 5.2.22: Decomposition of ammonia with an undeposited stainless steel sample in the primary insert at $400^{\circ} \mathrm{C}$. 


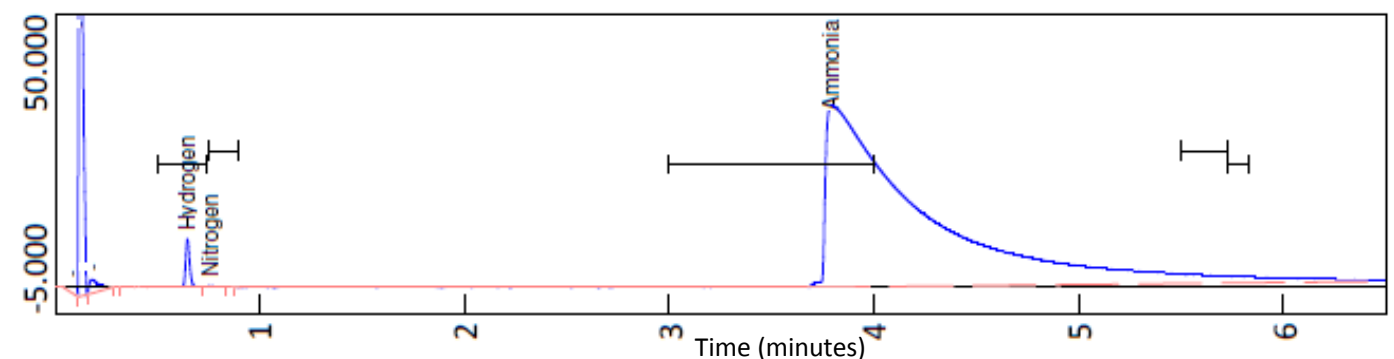

Figure 5.2.23: Decomposition of ammonia in the primary insert at $400{ }^{\circ} \mathrm{C}$ with a stainless steel sample with templated nickel deposits deposited for 2 minutes at $-0.9 \mathrm{~V}$.

Table 5.2.9 to Table 5.2.13 follow the calculation from raw GC measurements to calculated gas output and rate of decomposition.

The standards used in these calculations are:

5\% Hydrogen $=$ Peak Area 1049.1

95\% Nitrogen $=$ Peak Area 1826.3

100\% Ammonia $=$ Peak Area 1710.1

Table 5.2.9: Peak areas recorded by GC after decomposition experiments with three catalysts at $350,400,450$ and $500{ }^{\circ} \mathrm{C}$ in the Explorer Reactor with the primary insert.

\section{Hydrogen}

\begin{tabular}{|l|l|l|l|l|}
\hline & $\mathbf{3 5 0}$ & $\mathbf{4 0 0}$ & $\mathbf{4 5 0}$ & $\mathbf{5 0 0}$ \\
\hline Empty Primary & 2.5 & 9.3 & 84.2 & 291.5 \\
\hline Blank Steel & 0.5 & 8.3 & 46.0 & 213.1 \\
\hline Template Steel & 1.9 & 11.8 & 51.7 & 172.2 \\
\hline
\end{tabular}

Nitrogen

\begin{tabular}{|l|l|l|l|l|}
\hline & $\mathbf{3 5 0}$ & $\mathbf{4 0 0}$ & $\mathbf{4 5 0}$ & $\mathbf{5 0 0}$ \\
\hline Empty Primary & 0.8 & 1.0 & 5.0 & 15.8 \\
\hline Blank Steel & 1.1 & 0.4 & 1.4 & 6.0 \\
\hline Template Steel & 0.2 & 0.5 & 1.7 & 4.7 \\
\hline
\end{tabular}

Ammonia

\begin{tabular}{|l|l|l|l|l|}
\hline & $\mathbf{3 5 0}$ & $\mathbf{4 0 0}$ & $\mathbf{4 5 0}$ & $\mathbf{5 0 0}$ \\
\hline Empty Primary & 1148.2 & 1129.6 & 1080.9 & 1008.1 \\
\hline Blank Steel & 1103.1 & 1140.9 & 1128.6 & 1054.3 \\
\hline Template Steel & 1164.3 & 1132.4 & 1125.8 & 1048.3 \\
\hline
\end{tabular}


Table 5.2.10: Percentage of gases in output for catalysts in the Explorer reactor. Calculated from measurements in Table 5.2.9 and standards.

\begin{tabular}{|c|c|c|c|c|}
\hline \multicolumn{5}{|c|}{ Hydrogen } \\
\hline & 350 & 400 & 450 & 500 \\
\hline Empty Primary & $0.01 \%$ & $0.04 \%$ & $0.40 \%$ & $1.39 \%$ \\
\hline Blank Steel & $0.00 \%$ & $0.04 \%$ & $0.22 \%$ & $1.02 \%$ \\
\hline Template Steel & $0.01 \%$ & $0.06 \%$ & $0.25 \%$ & $0.82 \%$ \\
\hline \multicolumn{5}{|c|}{ Nitrogen } \\
\hline & 350 & 400 & 450 & 500 \\
\hline Empty Primary & $0.04 \%$ & $0.05 \%$ & $0.26 \%$ & $0.82 \%$ \\
\hline Blank Steel & $0.06 \%$ & $0.02 \%$ & $0.07 \%$ & $0.31 \%$ \\
\hline Template Steel & $0.01 \%$ & $0.03 \%$ & $0.09 \%$ & $0.24 \%$ \\
\hline \multicolumn{5}{|c|}{ Ammonia } \\
\hline & 350 & 400 & 450 & 500 \\
\hline Empty Primary & $67.14 \%$ & $66.06 \%$ & $63.21 \%$ & $58.95 \%$ \\
\hline Blank Steel & $64.51 \%$ & $66.72 \%$ & $66.00 \%$ & $61.65 \%$ \\
\hline Template Steel & $68.09 \%$ & $66.22 \%$ & $65.84 \%$ & $61.30 \%$ \\
\hline
\end{tabular}

\begin{tabular}{|c|c|c|c|c|}
\hline \multicolumn{5}{|c|}{$\begin{array}{l}\text { Table 5.2.11: Molar output of each species for catalysts in the } \\
\text { Explorer reactor. } \\
\text { Calculated from molar flow of input and from percentage in } \\
\text { table Table 5.2.10 according to the equations given in Section } \\
\text { 2.2.12.3.2. Values are in } \mathrm{mol} \mathrm{min}^{-1} \text {. }\end{array}$} \\
\hline & \multicolumn{4}{|c|}{ Hydrogen } \\
\hline & 350 & 400 & 450 & 500 \\
\hline Empty Primary & $2.38 \mathrm{E}-08$ & $8.90 \mathrm{E}-08$ & $8.09 \mathrm{E}-07$ & $2.82 \mathrm{E}-06$ \\
\hline Blank Steel & 4.90E-09 & $7.94 \mathrm{E}-08$ & 4.42E-07 & $2.06 \mathrm{E}-06$ \\
\hline \multirow[t]{3}{*}{ Template Steel } & $1.78 \mathrm{E}-08$ & $1.13 \mathrm{E}-07$ & 4.97E-07 & $1.66 \mathrm{E}-06$ \\
\hline & \multicolumn{4}{|c|}{ Nitrogen } \\
\hline & 350 & 400 & 450 & 500 \\
\hline Empty Primary & $8.28 \mathrm{E}-08$ & 1.07E-07 & $5.29 \mathrm{E}-07$ & $1.68 \mathrm{E}-06$ \\
\hline Blank Steel & $1.11 \mathrm{E}-07$ & $4.06 \mathrm{E}-08$ & $1.45 \mathrm{E}-07$ & $6.29 \mathrm{E}-07$ \\
\hline \multirow[t]{3}{*}{ Template Steel } & 1.66E-08 & $5.21 \mathrm{E}-08$ & $1.77 \mathrm{E}-07$ & $4.94 \mathrm{E}-07$ \\
\hline & \multicolumn{4}{|c|}{ Ammonia } \\
\hline & 350 & 400 & 450 & 500 \\
\hline Empty Primary & 1.79E-04 & $1.78 \mathrm{E}-04$ & $1.74 \mathrm{E}-04$ & 1.67E-04 \\
\hline Blank Steel & 1.76E-04 & 1.79E-04 & $1.78 \mathrm{E}-04$ & $1.72 \mathrm{E}-04$ \\
\hline Template Steel & $1.81 \mathrm{E}-04$ & $1.78 \mathrm{E}-04$ & $1.78 \mathrm{E}-04$ & $1.71 \mathrm{E}-04$ \\
\hline
\end{tabular}


Table 5.2.12: Total gas output of the reaction calculated as the sum of the outputs of all gas species from Table 5.2.11

Total output $=$ hydrogen output + nitrogen output + ammonia output.

Values are in $\mathrm{mol} \mathrm{min}^{-1}$.

\begin{tabular}{|l|l|l|l|l|}
\cline { 2 - 5 } \multicolumn{1}{c|}{ Total Output } & $\mathbf{3 5 0}$ & $\mathbf{4 0 0}$ & $\mathbf{4 5 0}$ & $\mathbf{5 0 0}$ \\
\hline Empty Primary & $1.80 \mathrm{E}-04$ & $1.78 \mathrm{E}-04$ & $1.75 \mathrm{E}-04$ & $1.72 \mathrm{E}-04$ \\
\hline Blank Steel & $1.76 \mathrm{E}-04$ & $1.79 \mathrm{E}-04$ & $1.78 \mathrm{E}-04$ & $1.74 \mathrm{E}-04$ \\
\hline Template Steel & $1.81 \mathrm{E}-04$ & $1.78 \mathrm{E}-04$ & $1.78 \mathrm{E}-04$ & $1.73 \mathrm{E}-04$ \\
\hline
\end{tabular}

Table 5.2.13: Rate of ammonia decomposition.

Calculated as the difference between ammonia input and ammonia output.

Values are in $\mathrm{mol} \mathrm{min}^{-1}$.

\begin{tabular}{|l|l|l|l|l|}
\cline { 2 - 5 } \multicolumn{1}{c|}{} & \multicolumn{4}{c|}{ Ammonia Decomposition } \\
\cline { 2 - 5 } \multicolumn{1}{c|}{} & $\mathbf{3 5 0}$ & $\mathbf{4 0 0}$ & $\mathbf{4 5 0}$ & $\mathbf{5 0 0}$ \\
\hline Empty Primary & $2.17 \mathrm{E}-05$ & $2.32 \mathrm{E}-05$ & $2.72 \mathrm{E}-05$ & $3.39 \mathrm{E}-05$ \\
\hline Blank Steel & $2.53 \mathrm{E}-05$ & $2.23 \mathrm{E}-05$ & $2.33 \mathrm{E}-05$ & $2.96 \mathrm{E}-05$ \\
\hline Template Steel & $2.05 \mathrm{E}-05$ & $2.29 \mathrm{E}-05$ & $2.35 \mathrm{E}-05$ & $3.01 \mathrm{E}-05$ \\
\hline
\end{tabular}

\subsubsection{Secondary Insert Decomposition Experiments}

This section contains the chromatograms for ammonia decomposition experiments with the secondary insert catalysts. Table 5.2.14 summarises the average peak areas for these experiments. Table 5.2.15 shows percentages calculated by comparison of the measurements from Table 5.2.14 to the measurements for known standards. The percentages are converted to molar flow, as shown in Table 5.2.16, using the equations from Section 2.2.12.3. Section 5.2.7.3.1 calculates the Activation Energy using the Arrhenius method for all of the rate results from Table 5.2.16.

Figure 5.2.24a-f shows chromatograms for ammonia decomposition with a blank steel secondary insert inside the primary insert of the Explorer reactor. Each chromatogram shows the third and fourth injection cycles from a five injection set. Each injection cycle was 10 minutes long. The injection cycles each had an injection peak at 0.5 minutes; a hydrogen peak at 1.2 minutes; a nitrogen peak at 1.4 minutes; a long-tailed ammonia peak after 5 minutes and a negative peak at the end of the injection cycle when the sample loop switched to its refill setting. 


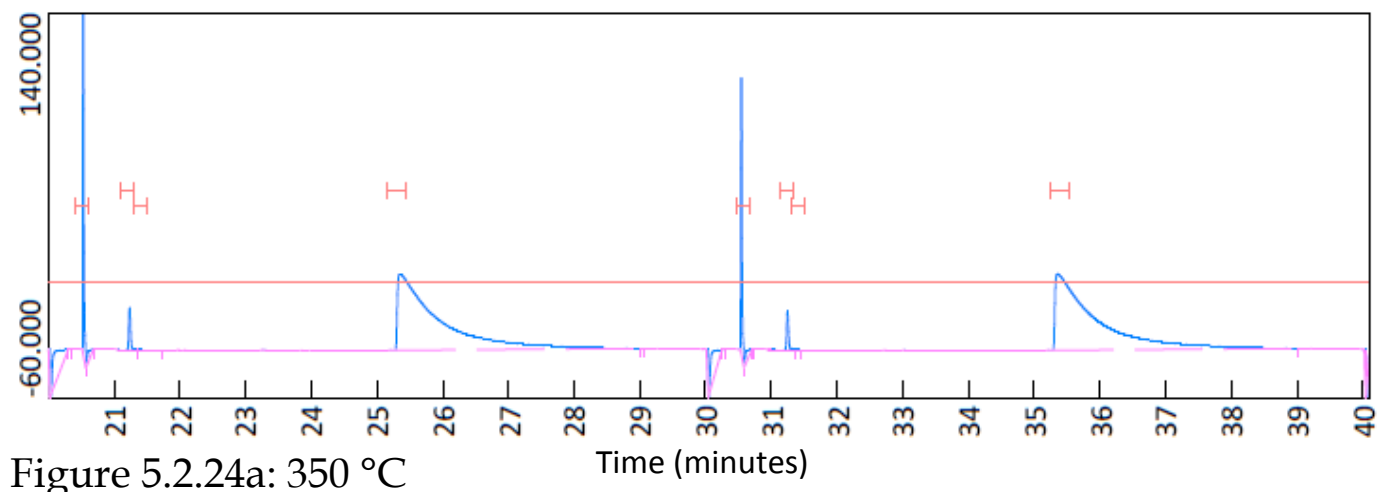

Figure 5.2.24a: $350{ }^{\circ} \mathrm{C}$

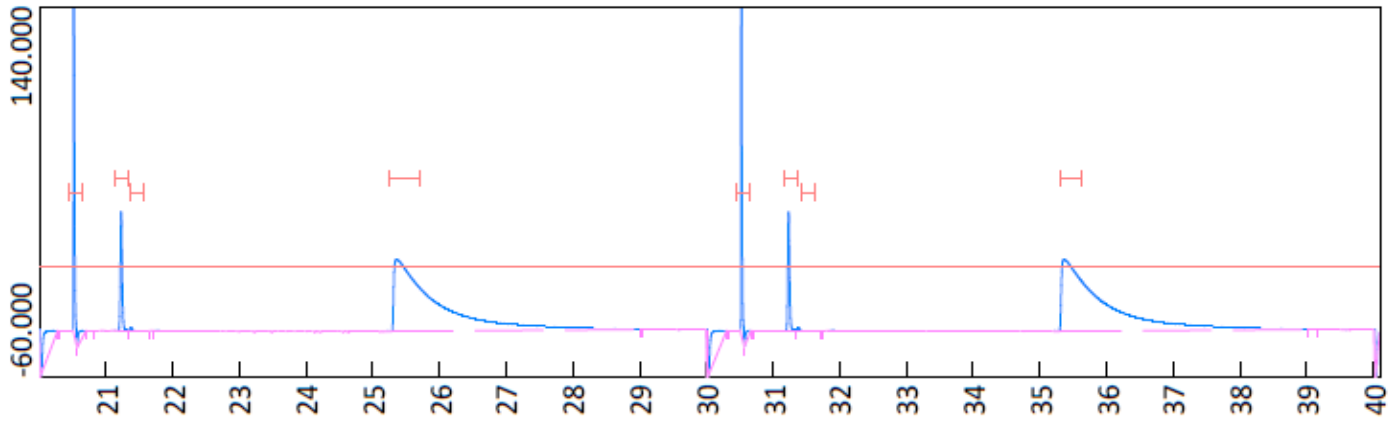

Figure 5.2.24b: $400^{\circ} \mathrm{C}$

Time (minutes)

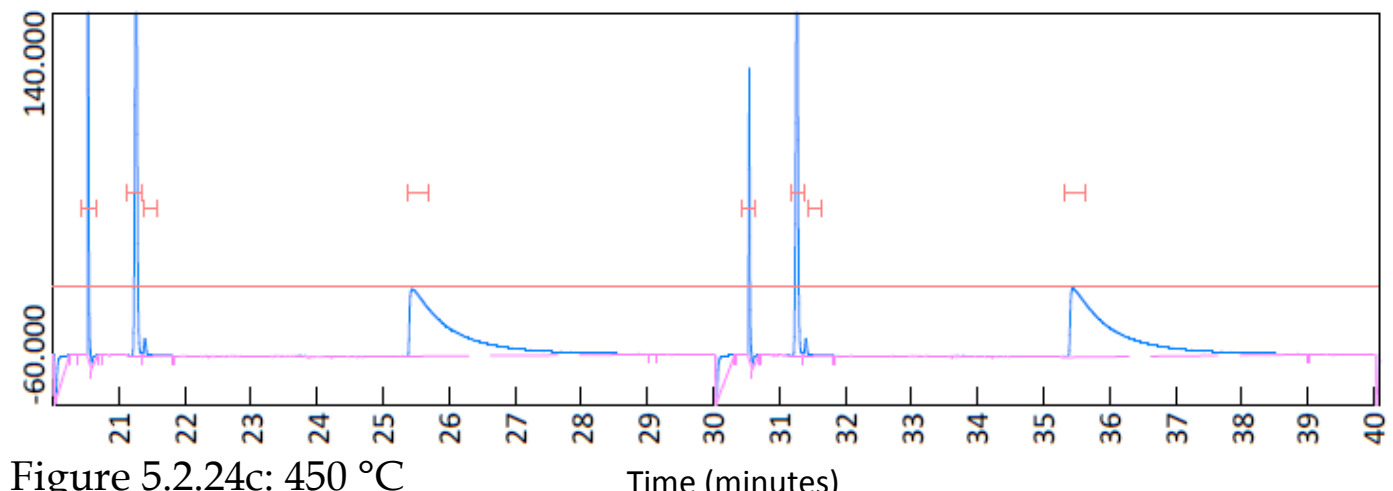

Figure 5.2.24c: $450{ }^{\circ} \mathrm{C}$ Time (minutes)

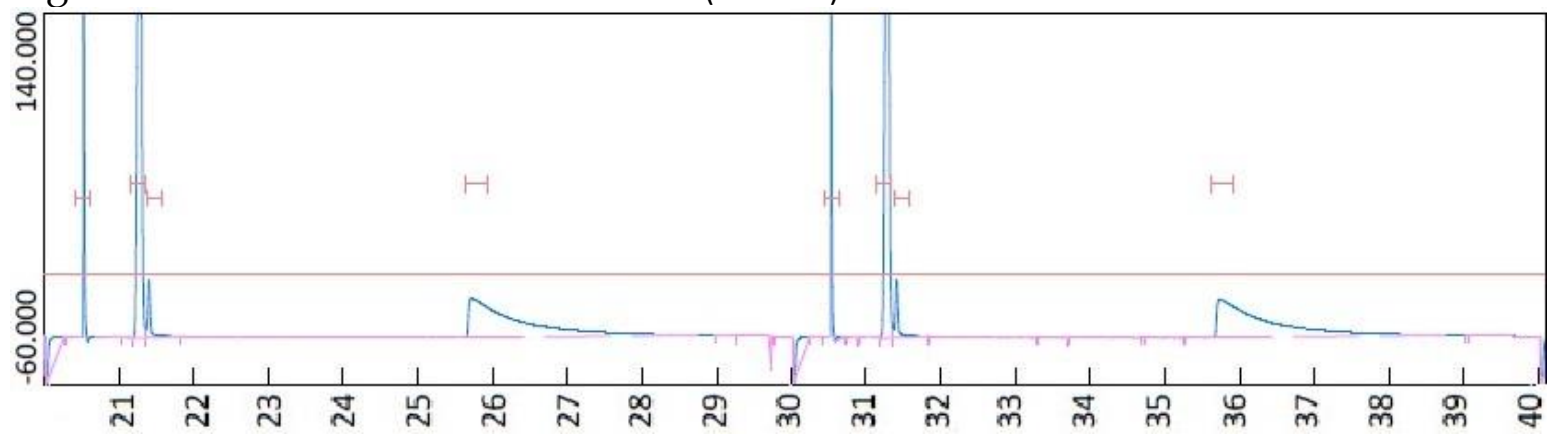

Figure $5.2 .24 \mathrm{~d}: 500^{\circ} \mathrm{C}$

Time (minutes) 


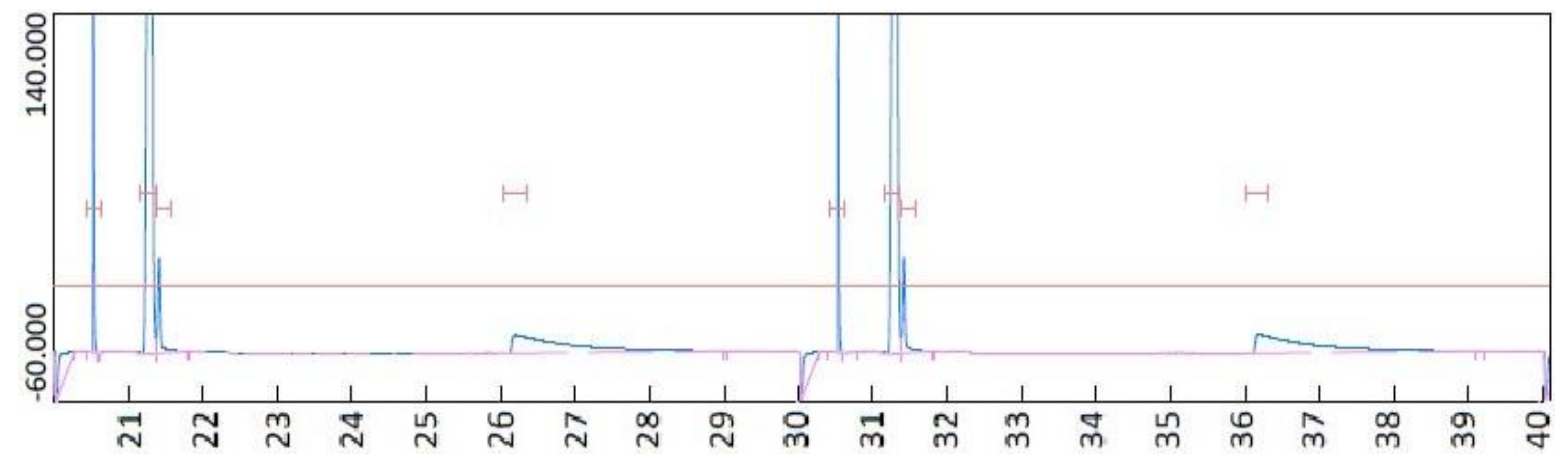

Figure $5.2 .24 \mathrm{e}: 550{ }^{\circ} \mathrm{C}$

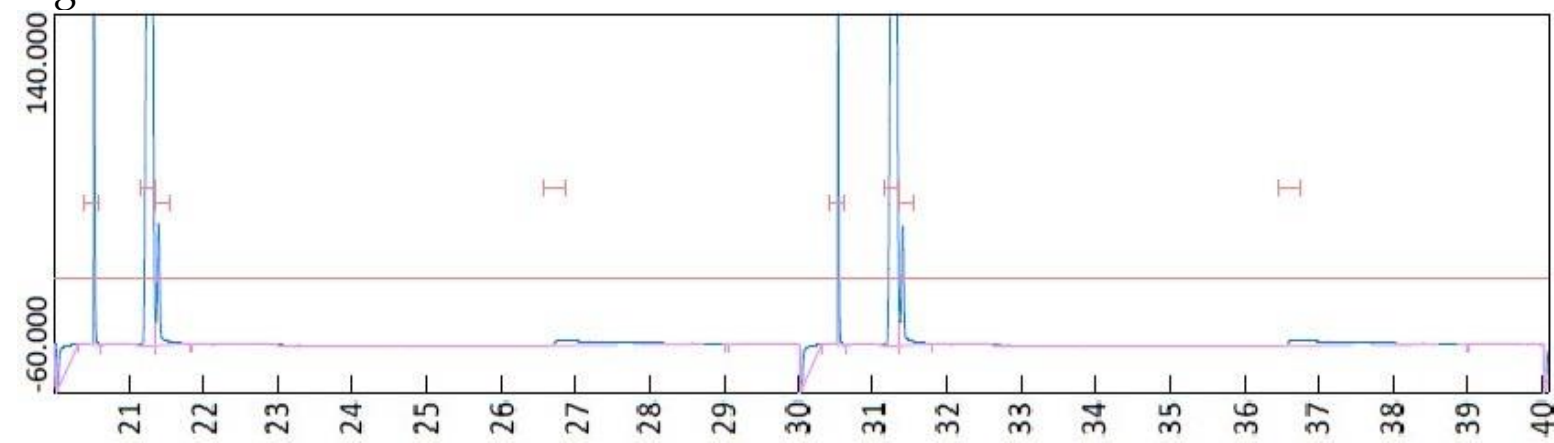

Figure 5.2.24f: $600{ }^{\circ} \mathrm{C}$

Time (minutes)

Figure 5.2.24:TCD chromatograms of ammonia decomposition experiments with an undeposited stainless steel secondary insert.

Table 5.2.14 to Table 5.2.17 follow the calculation of molar output of each gas from ammonia decomposition with the secondary insert catalysts at $300-450{ }^{\circ} \mathrm{C}$. The results for these calculations are graphed in Figure 3.2.11, Figure 3.2.12 and

Hydrogen production vs. Temperature for Secondary Insert Catalysts

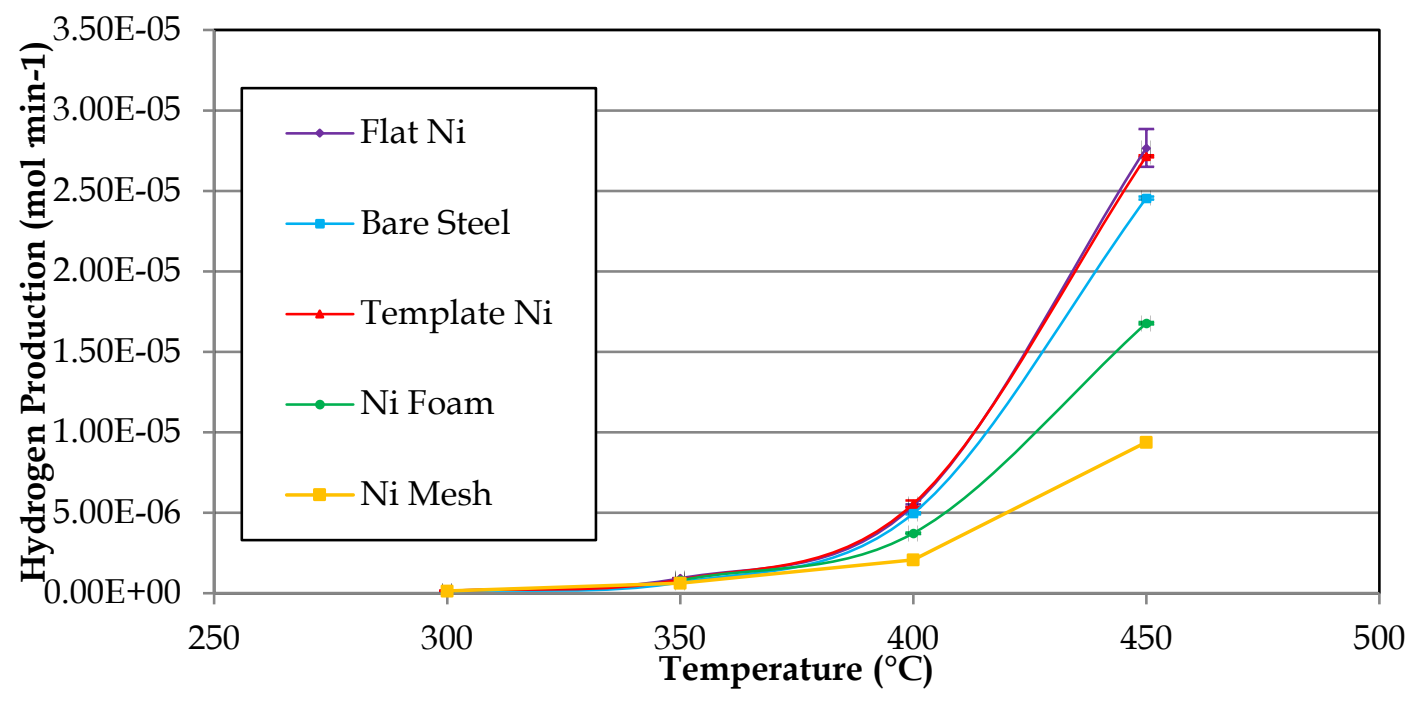

Figure 3.2.13 in section 3.2.2.4. 
The standards used in these calculations are:

5\% Hydrogen $=$ Peak Area 297.925

95\% Nitrogen $=$ Peak Area 1005.6

100\% Ammonia $=$ Peak Area 1710.1

\section{Table 5.2.14: Measured peak areas for the output gases of the ammonia decomposition with secondary insert catalysts at 300-} $450{ }^{\circ} \mathrm{C}$.

\begin{tabular}{|c|c|c|c|c|c|}
\hline \multicolumn{6}{|c|}{ Hydrogen } \\
\hline Catalyst & 300 & 350 & 400 & 450 & $\leftarrow$ Temperature $\left({ }^{\circ} \mathrm{C}\right)$ \\
\hline \multirow[t]{2}{*}{ Flat Ni } & 4.1 & 27.3 & 158.1 & 755.9 & Average \\
\hline & & 0.4 & 2.7 & 34.7 & Standard deviation \\
\hline \multirow[t]{2}{*}{ Bare Steel } & 3.5 & 20.0 & 144.7 & 676.3 & Average \\
\hline & 0.1 & 0.1 & 1.9 & 2.8 & Standard deviation \\
\hline \multirow[t]{2}{*}{ Template Ni } & 4.3 & 24.9 & 161.7 & 742.7 & Average \\
\hline & 0.2 & 1.6 & 6.1 & 1.9 & Standard deviation \\
\hline \multirow[t]{2}{*}{ Ni Foam } & & 23.4 & 109.1 & 472.2 & Average \\
\hline & & 0.5 & 1.4 & 2.2 & Standard deviation \\
\hline \multicolumn{6}{|c|}{ Nitrogen } \\
\hline$\downarrow$ Catalyst & 300 & 350 & 400 & 450 & $\leftarrow$ Temperature $\left({ }^{\circ} \mathrm{C}\right)$ \\
\hline \multirow[t]{2}{*}{ Flat Ni } & 0.4 & 1.2 & 7.0 & 36.2 & Average \\
\hline & 0.1 & 0.1 & 0.1 & 2.9 & Standard deviation \\
\hline \multirow[t]{2}{*}{ Bare Steel } & 0.2 & 0.8 & 6.3 & 34.6 & Average \\
\hline & 0.0 & 0.0 & 0.0 & 0.3 & Standard deviation \\
\hline \multirow[t]{2}{*}{$\begin{array}{l}\text { Template } \\
\mathrm{Ni}\end{array}$} & 0.4 & 1.1 & 7.7 & 36.1 & Average \\
\hline & 0.0 & 0.2 & 0.5 & 0.8 & Standard deviation \\
\hline \multirow[t]{2}{*}{ Ni Foam } & & 0.9 & 4.5 & 22.6 & Average \\
\hline & & 0.1 & 0.3 & 0.1 & Standard deviation \\
\hline \multicolumn{6}{|c|}{ Ammonia } \\
\hline Catalyst & 300 & 350 & 400 & 450 & $\leftarrow$ Temperature $\left({ }^{\circ} \mathrm{C}\right)$ \\
\hline \multirow[t]{2}{*}{ Flat Ni } & 1709.2 & 1712.4 & 1647.2 & 1365.0 & Average \\
\hline & 2.5 & 4.6 & 2.8 & 17.8 & Standard deviation \\
\hline \multirow[t]{2}{*}{ Bare Steel } & 1705.6 & 1703.7 & 1646.6 & 1417.2 & Average \\
\hline & 22.7 & 22.7 & 18.2 & 18.5 & Standard deviation \\
\hline \multirow[t]{2}{*}{$\begin{array}{l}\text { Template } \\
\text { Ni }\end{array}$} & 1674.8 & 1653.3 & 1595.5 & 1364.7 & Average \\
\hline & 9.1 & 4.4 & 9.5 & 2.5 & Standard deviation \\
\hline \multirow[t]{2}{*}{ Ni Foam } & & 1672.4 & 1640.9 & 1505.6 & Average \\
\hline & & 0.9 & 4.5 & 22.6 & Standard deviation \\
\hline
\end{tabular}


Table 5.2.15: Percentages for the gas output of the decomposition reactions with secondary insert catalysts.

Calculated from the peak areas from Table 5.2.14 and the standard measurements.

\begin{tabular}{|c|c|c|c|c|c|}
\hline \multicolumn{6}{|c|}{ Hydrogen } \\
\hline$\downarrow$ Catalyst & 300 & 350 & 400 & 450 & $\leftarrow$ Temperature $\left({ }^{\circ} \mathrm{C}\right)$ \\
\hline \multirow[t]{2}{*}{ Flat Ni } & $0.07 \%$ & $0.46 \%$ & $2.65 \%$ & $12.69 \%$ & Average \\
\hline & $0.00 \%$ & $0.01 \%$ & $0.05 \%$ & $0.58 \%$ & Standard deviation \\
\hline \multirow[t]{2}{*}{ Bare Steel } & $0.06 \%$ & $0.34 \%$ & $2.43 \%$ & $11.35 \%$ & Average \\
\hline & $0.00 \%$ & $0.00 \%$ & $0.03 \%$ & $0.05 \%$ & Standard deviation \\
\hline \multirow[t]{2}{*}{ Template Ni } & $0.07 \%$ & $0.42 \%$ & $2.71 \%$ & $12.46 \%$ & Average \\
\hline & $0.00 \%$ & $0.03 \%$ & $0.10 \%$ & $0.03 \%$ & Standard deviation \\
\hline \multirow[t]{2}{*}{ Ni Foam } & & $0.39 \%$ & $1.83 \%$ & $7.92 \%$ & Average \\
\hline & & $0.01 \%$ & $0.02 \%$ & $0.04 \%$ & Standard deviation \\
\hline \multicolumn{6}{|c|}{ Nitrogen } \\
\hline$\downarrow$ Catalyst & 300 & 350 & 400 & 450 & $\leftarrow$ Temperature $\left({ }^{\circ} \mathrm{C}\right)$ \\
\hline \multirow[t]{2}{*}{ Flat Ni } & $0.04 \%$ & $0.12 \%$ & $0.70 \%$ & $3.60 \%$ & Average \\
\hline & $0.01 \%$ & $0.01 \%$ & $0.01 \%$ & $0.28 \%$ & Standard deviation \\
\hline \multirow[t]{2}{*}{ Bare Steel } & $0.02 \%$ & $0.08 \%$ & $0.62 \%$ & $3.44 \%$ & Average \\
\hline & $0.00 \%$ & $0.00 \%$ & $0.00 \%$ & $0.03 \%$ & Standard deviation \\
\hline \multirow[t]{2}{*}{ Template Ni } & $0.04 \%$ & $0.11 \%$ & $0.77 \%$ & $3.59 \%$ & Average \\
\hline & $0.00 \%$ & $0.02 \%$ & $0.05 \%$ & $0.08 \%$ & Standard deviation \\
\hline \multirow[t]{2}{*}{ Ni Foam } & & $0.09 \%$ & $0.45 \%$ & $2.25 \%$ & Average \\
\hline & & $0.01 \%$ & $0.03 \%$ & $0.01 \%$ & Standard deviation \\
\hline \multicolumn{6}{|c|}{ Ammonia } \\
\hline Catalyst & 300 & 350 & 400 & 450 & $\leftarrow$ Temperature $\left({ }^{\circ} \mathrm{C}\right)$ \\
\hline \multirow[t]{2}{*}{ Flat Ni } & $99.95 \%$ & $100.14 \%$ & $96.32 \%$ & $79.82 \%$ & Average \\
\hline & $0.15 \%$ & $0.27 \%$ & $0.16 \%$ & $1.04 \%$ & Standard deviation \\
\hline \multirow[t]{2}{*}{ Bare Steel } & $99.74 \%$ & $99.63 \%$ & $96.29 \%$ & $82.88 \%$ & Average \\
\hline & $1.33 \%$ & $1.33 \%$ & $1.07 \%$ & $1.08 \%$ & Standard deviation \\
\hline \multirow[t]{2}{*}{ Template Ni } & $97.94 \%$ & $96.68 \%$ & $93.30 \%$ & $79.81 \%$ & Average \\
\hline & $0.53 \%$ & $0.26 \%$ & $0.56 \%$ & $0.15 \%$ & Standard deviation \\
\hline \multirow[t]{2}{*}{ Ni Foam } & & $97.80 \%$ & $95.96 \%$ & $88.04 \%$ & Average \\
\hline & & $0.05 \%$ & $0.26 \%$ & $1.32 \%$ & Standard deviation \\
\hline
\end{tabular}




\begin{tabular}{|c|c|c|c|c|c|}
\hline \multicolumn{6}{|c|}{$\begin{array}{l}\text { Table 5.2.16: Molar ouput of each gas from th } \\
\text { secondary insert catalysts. } \\
\text { Calculated using the initial gas input of } 2.01 \\
\text { percentages from Table } 5.2 .15 \text { and the equati } \\
\text { Values in mol } \mathrm{min}^{-1} \text {. }\end{array}$} \\
\hline \multicolumn{6}{|c|}{ Hydrogen } \\
\hline$\downarrow$ Catalyst & 300 & 350 & 400 & 450 & $\begin{array}{l}\leftarrow \text { Temperature } \\
\left({ }^{\circ} \mathrm{C}\right)\end{array}$ \\
\hline \multirow[t]{2}{*}{ Flat Ni } & $1.37 \mathrm{E}-07$ & $9.26 \mathrm{E}-07$ & $5.43 \mathrm{E}-06$ & 2.77E-05 & Average \\
\hline & 4.53E-09 & $1.30 \mathrm{E}-08$ & 9.11E-08 & 1.17E-06 & Standard deviation \\
\hline \multirow[t]{2}{*}{ Bare Steel } & 1.17E-07 & 6.77E-07 & 4.96E-06 & $2.46 \mathrm{E}-05$ & Average \\
\hline & 3.33E-09 & 3.33E-09 & $6.46 \mathrm{E}-08$ & 9.43E-08 & Standard deviation \\
\hline \multirow[t]{2}{*}{$\begin{array}{l}\text { Template } \\
\mathrm{Ni}\end{array}$} & $1.44 \mathrm{E}-07$ & 8.42E-07 & $5.56 \mathrm{E}-06$ & 2.71E-05 & Average \\
\hline & 8.41E-09 & 5.37E-08 & $2.06 \mathrm{E}-07$ & $6.43 \mathrm{E}-08$ & Standard deviation \\
\hline \multirow[t]{2}{*}{ Ni Foam } & & 7.93E-07 & $3.73 \mathrm{E}-06$ & $1.68 \mathrm{E}-05$ & Average \\
\hline & & $1.74 \mathrm{E}-08$ & 4.72E-08 & 7.49E-08 & Standard deviation \\
\hline \multicolumn{6}{|c|}{ Nitrogen } \\
\hline$\downarrow$ Catalyst & 300 & 350 & 400 & 450 & $\begin{array}{l}\leftarrow \text { Temperature } \\
\left({ }^{\circ} \mathrm{C}\right)\end{array}$ \\
\hline \multirow[t]{2}{*}{ Flat Ni } & 7.09E-08 & $2.46 \mathrm{E}-07$ & $1.42 \mathrm{E}-06$ & 7.75E-06 & Average \\
\hline & 2.28E-08 & 2.27E-08 & 2.73E-08 & $5.76 \mathrm{E}-07$ & Standard deviation \\
\hline \multirow[t]{2}{*}{ Bare Steel } & 3.99E-08 & $1.61 \mathrm{E}-07$ & $1.27 \mathrm{E}-06$ & $7.40 \mathrm{E}-06$ & Average \\
\hline & 4.89E-09 & 4.89E-09 & $6.26 \mathrm{E}-09$ & $6.25 \mathrm{E}-08$ & Standard deviation \\
\hline \multirow[t]{2}{*}{$\begin{array}{l}\text { Template } \\
\mathrm{Ni}\end{array}$} & 7.16E-08 & 2.27E-07 & $1.57 \mathrm{E}-06$ & 7.74E-06 & Average \\
\hline & 4.81E-09 & $4.83 \mathrm{E}-08$ & $9.79 \mathrm{E}-08$ & $1.53 \mathrm{E}-07$ & Standard deviation \\
\hline \multirow[t]{2}{*}{ Ni Foam } & & $1.81 \mathrm{E}-07$ & 9.09E-07 & $4.72 \mathrm{E}-06$ & Average \\
\hline & & $1.06 \mathrm{E}-08$ & 5.77E-08 & $2.82 \mathrm{E}-08$ & Standard deviation \\
\hline \multicolumn{6}{|c|}{ Ammonia } \\
\hline$\downarrow$ Catalyst & 300 & 350 & 400 & 450 & $\leftarrow$ Temperature $\left({ }^{\circ} \mathrm{C}\right)$ \\
\hline \multirow[t]{2}{*}{ Flat Ni } & $2.01 \mathrm{E}-04$ & 2.01E-04 & 2.01E-04 & 1.93E-04 & Average \\
\hline & $5.87 \mathrm{E}-07$ & $1.07 \mathrm{E}-06$ & $6.56 \mathrm{E}-07$ & 4.17E-06 & Standard deviation \\
\hline \multirow[t]{2}{*}{ Bare Steel } & 2.01E-04 & 2.01E-04 & 2.01E-04 & $1.95 \mathrm{E}-04$ & Average \\
\hline & 5.31E-06 & 5.31E-06 & $4.26 \mathrm{E}-06$ & $4.34 \mathrm{E}-06$ & Standard deviation \\
\hline \multirow[t]{2}{*}{$\begin{array}{l}\text { Template } \\
\mathrm{Ni}\end{array}$} & 2.01E-04 & 2.01E-04 & $2.00 \mathrm{E}-04$ & $1.93 \mathrm{E}-04$ & Average \\
\hline & $2.14 \mathrm{E}-06$ & $1.03 \mathrm{E}-06$ & $2.24 \mathrm{E}-06$ & $5.87 \mathrm{E}-07$ & Standard deviation \\
\hline \multirow[t]{2}{*}{ Ni Foam } & & 2.01E-04 & 2.01E-04 & $1.98 \mathrm{E}-04$ & Average \\
\hline & & 2.13E-07 & $1.06 \mathrm{E}-06$ & $5.28 \mathrm{E}-06$ & Standard deviation \\
\hline
\end{tabular}




\begin{tabular}{|c|c|c|c|c|c|}
\hline$\downarrow$ Catalyst & 300 & 350 & 400 & 450 & $\begin{array}{l}\leftarrow \text { Temperature } \\
\left({ }^{\circ} \mathrm{C}\right)\end{array}$ \\
\hline Flat Ni & 2.01E-04 & 2.02E-04 & 2.08E-04 & 2.28E-04 & Average \\
\hline Bare Steel & 2.01E-04 & 2.02E-04 & 2.07E-04 & 2.27E-04 & Average \\
\hline $\begin{array}{l}\text { Template } \\
\mathrm{Ni}\end{array}$ & 2.01E-04 & 2.02E-04 & 2.07E-04 & 2.28E-04 & Average \\
\hline Ni Foam & & $2.02 \mathrm{E}-04$ & $2.05 \mathrm{E}-04$ & $2.20 \mathrm{E}-04$ & Average \\
\hline
\end{tabular}

Figure 5.2.25:a-d show chromatograms for ammonia decomposition on secondary inserts with untemplated nickel electrodeposits at 300 to $450{ }^{\circ} \mathrm{C}$.

The average peak areas for these experiments is included in Table 5.2.14 above. 


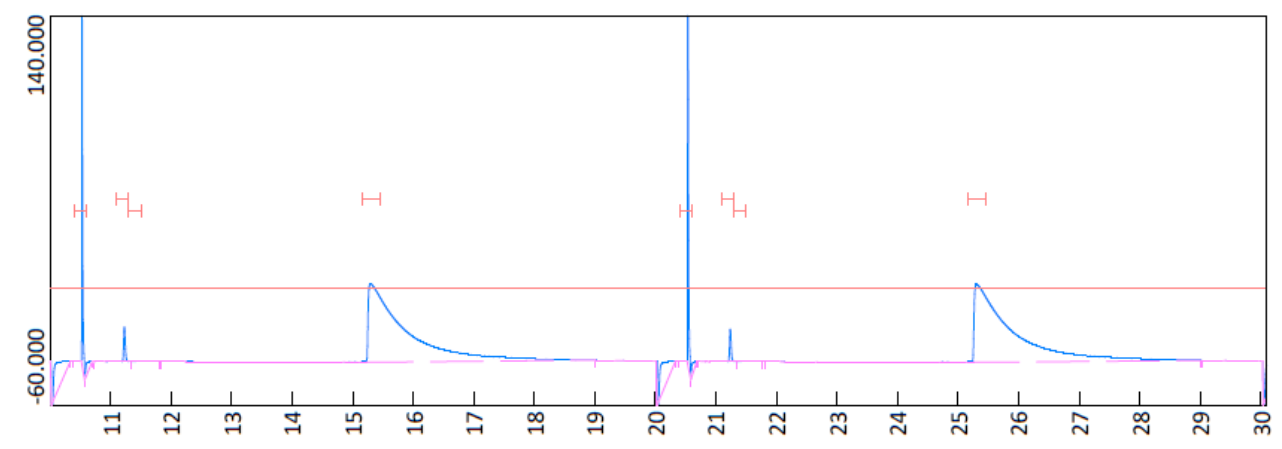

Figure 5.2.25:a: $300^{\circ} \mathrm{C}$

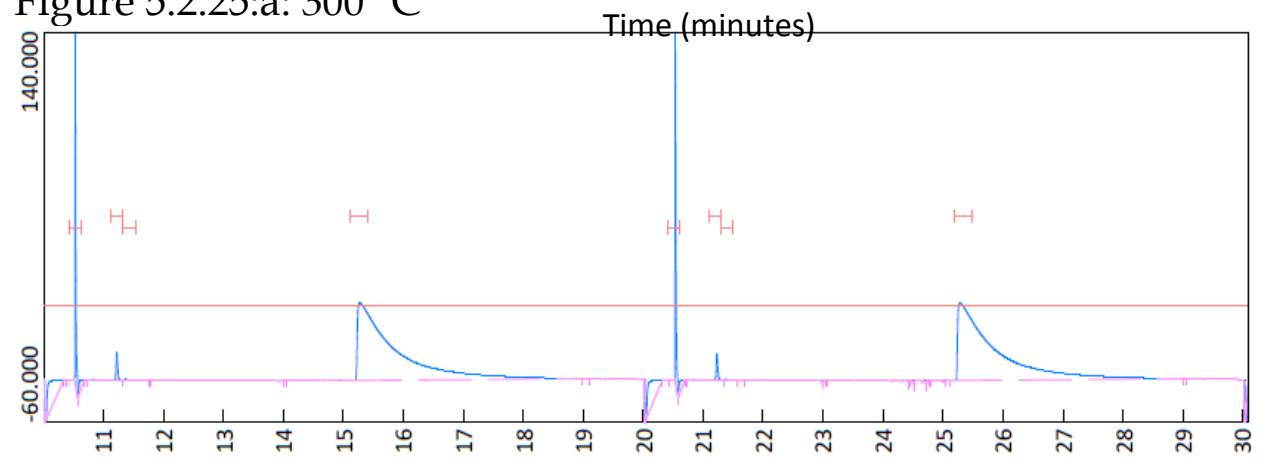

Figure $5.2 .25: \mathrm{b}: 350^{\circ} \mathrm{C}$

Time (minutes)

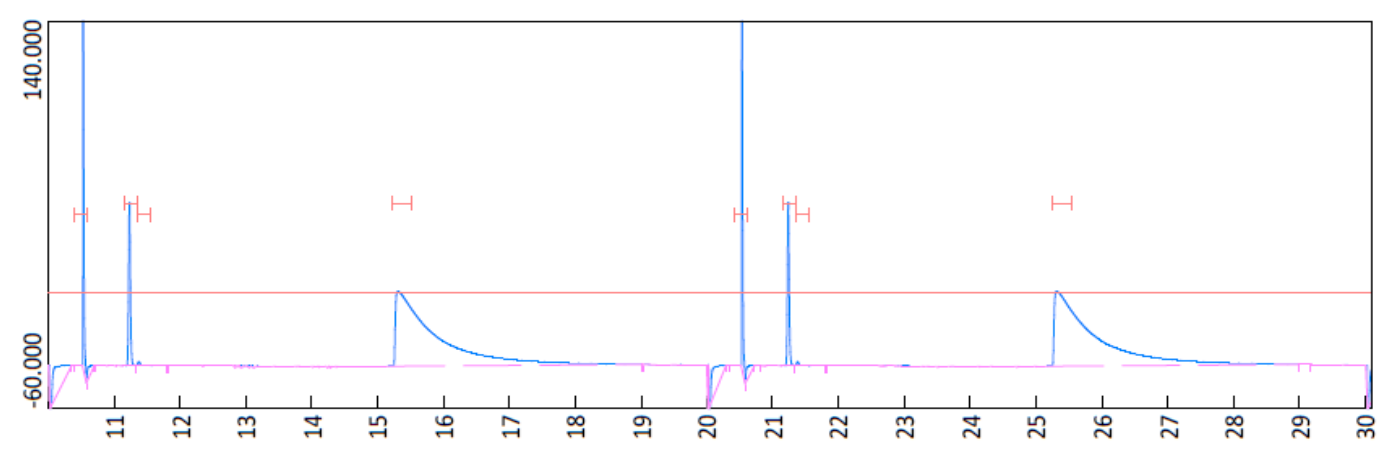

Figure 5.2.25: C: $400^{\circ} \mathrm{C}$.

Time (minutes)

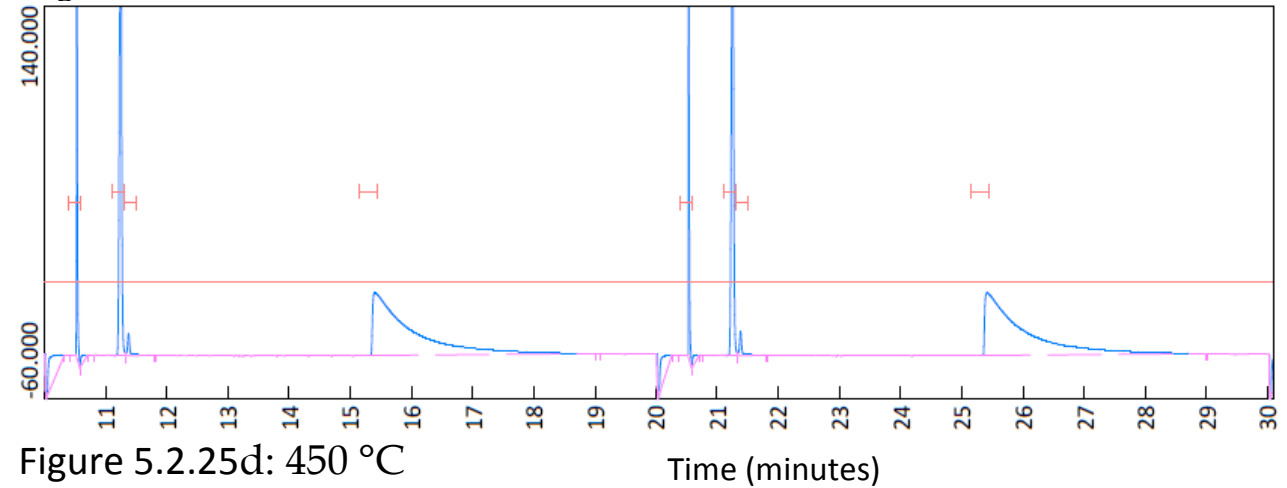

Figure 5.2.25:Typical TCD chromatograms of ammonia decomposition with untemplated nickel deposits on a secondary insert at $300-450{ }^{\circ} \mathrm{C}$.

The chromatograms shown in Figure 5.2.25 have the second and third ten-minute cycles from a set of three injections. From left to right the visible peaks are: 
Jonathan Tailby

injection of sample into the column at 10.5 minutes; hydrogen at 11.2 minutes; nitrogen at 11.4; ammonia at 15.5; and a negative peak at 20.0 from reopening the sample loop to refill; the pattern repeats with injection peak at 20.5; hydrogen peak at 21.2; nitrogen peak at 21.4; ammonia peak at 25.5 and negative peak at 30.0 from reopening the sample loop.

Figure 5.2.26a-d show chromatograms for ammonia decomposition on secondary inserts with templated nickel electrodeposits at 300 to $450{ }^{\circ} \mathrm{C}$. Each chromatogram is the first injection cycle of a three injection set, the injection cycles were each 9 minutes long. The average peak area for these experiments is included in Table 5.2.14 above.

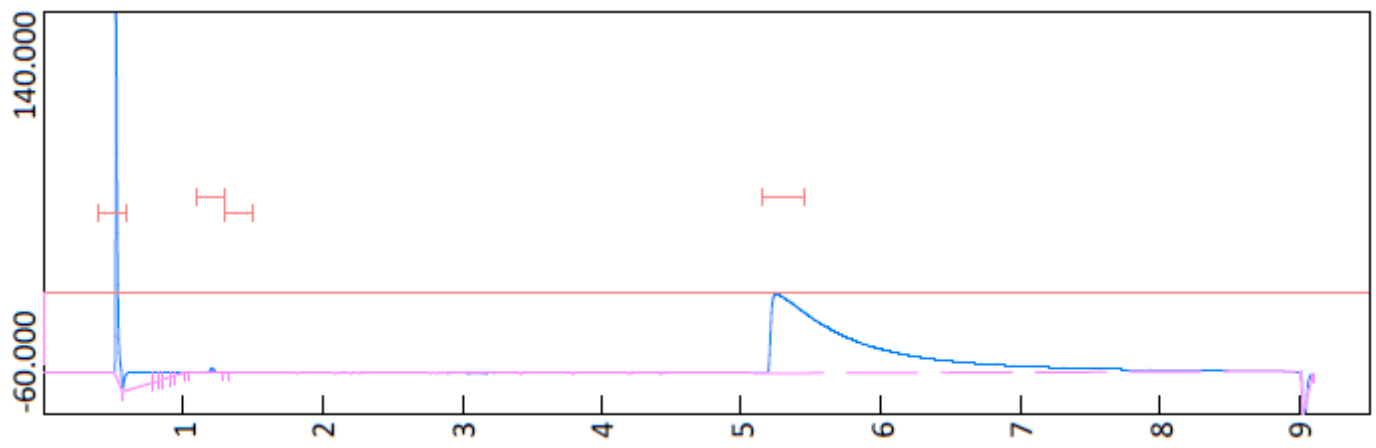

Figure 5.2.26a: $300{ }^{\circ} \mathrm{C}$

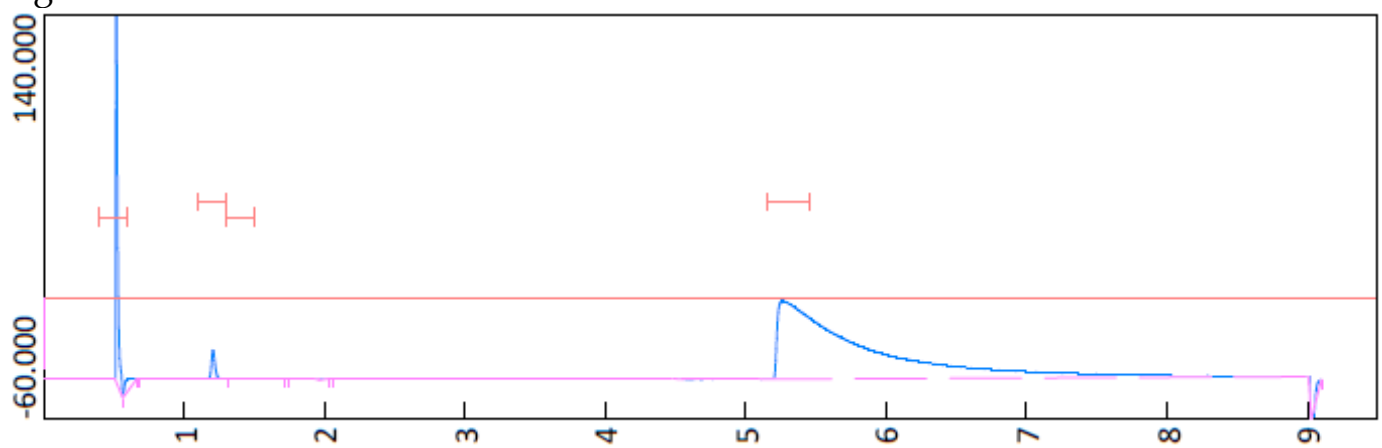

Figure 5.2.26b: $350{ }^{\circ} \mathrm{C}$

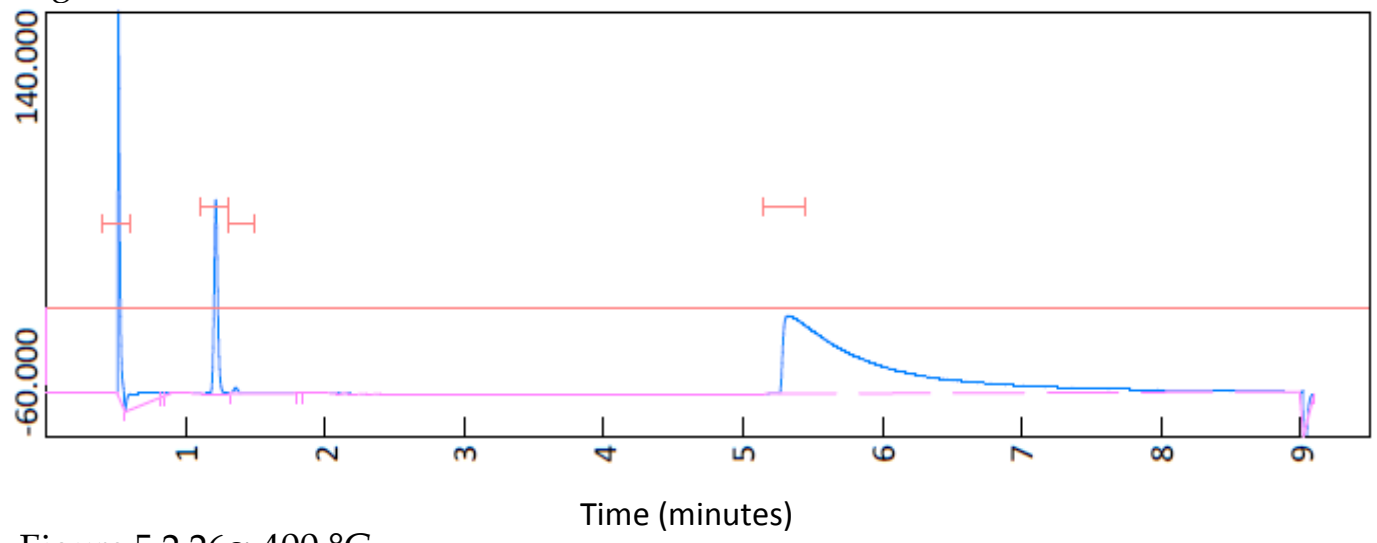

Figure 5.2.26c: $400{ }^{\circ} \mathrm{C}$ 


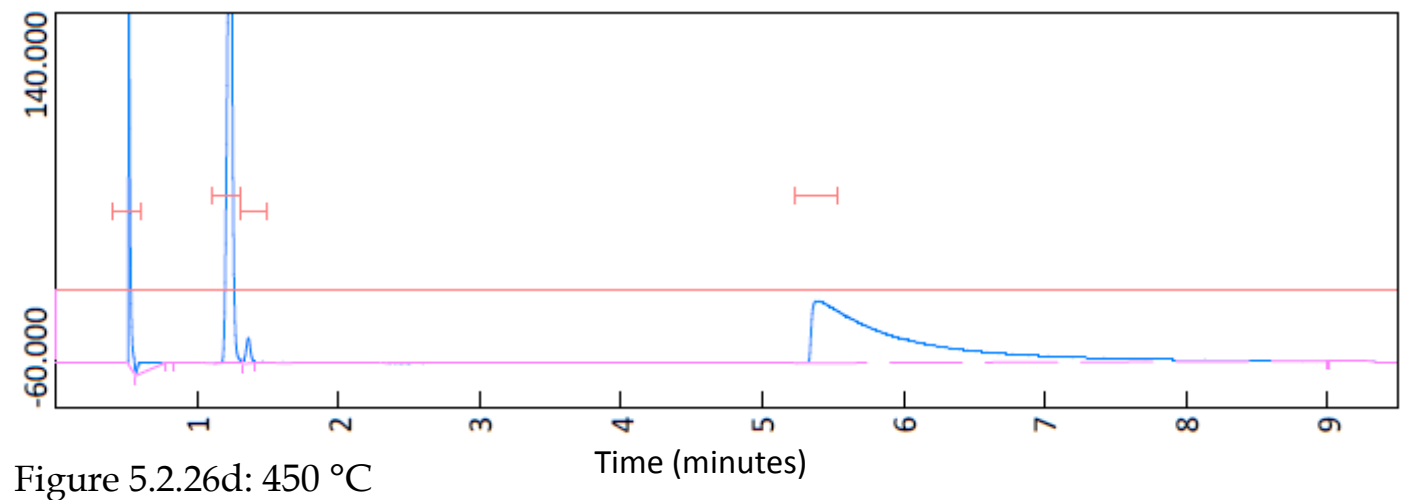

Figure 5.2.26 Representative chromatograms for ammonia decomposition experiments with templated nickel secondary inserts at $300,350,400,450{ }^{\circ} \mathrm{C}$.

Figure 5.2.27 shows typical chromatograms for decomposition experiments with the nickel mesh secondary inserts at 300,350 , and $400^{\circ} \mathrm{C}$. Figure 5.2.27a and b each show the first two injections of a three injection set. Figure 5.2.27c only shows the first injection because the second injection had the negative peaks associated with electrical interference obscuring the peaks. A higher carrier gas pressure has shortened the injection cycle to 7.5 minutes. Figure 5.2.27a and b show two injection cycles of a three injection set, while Figure 5.2.27c only shows the first injection cycle.

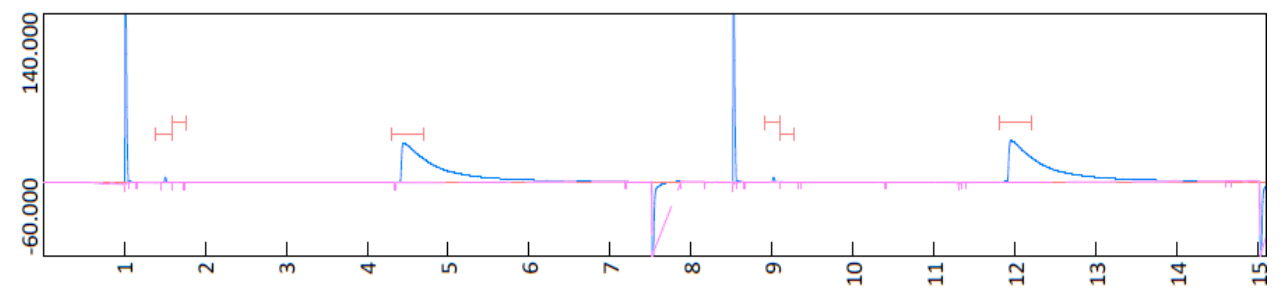

Figure 5.2.27a: $300{ }^{\circ} \mathrm{C}$

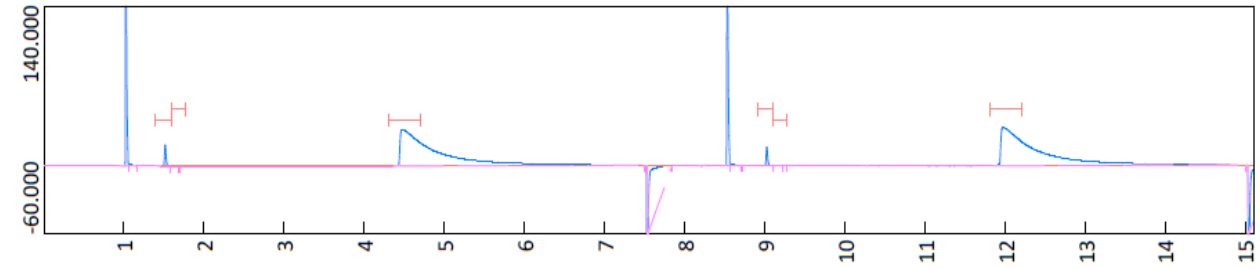

Figure $5.2 .27 \mathrm{~b}: 350{ }^{\circ} \mathrm{C}$

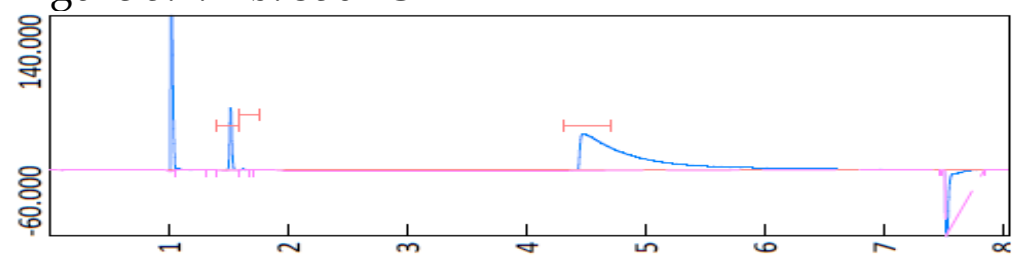

Figure 5.2.27c: $400{ }^{\circ} \mathrm{C}$

Figure 5.2.27: Typical chromatograms for ammonia decomposition with a nickel mesh catalyst. 
Jonathan Tailby

Figure 5.2.28 shows chromatograms for ammonia decomposition on secondary inserts with nickel foam at 350,400 and $450{ }^{\circ} \mathrm{C}$. The average peak area for these experiments is included in Table 5.2.14 above.

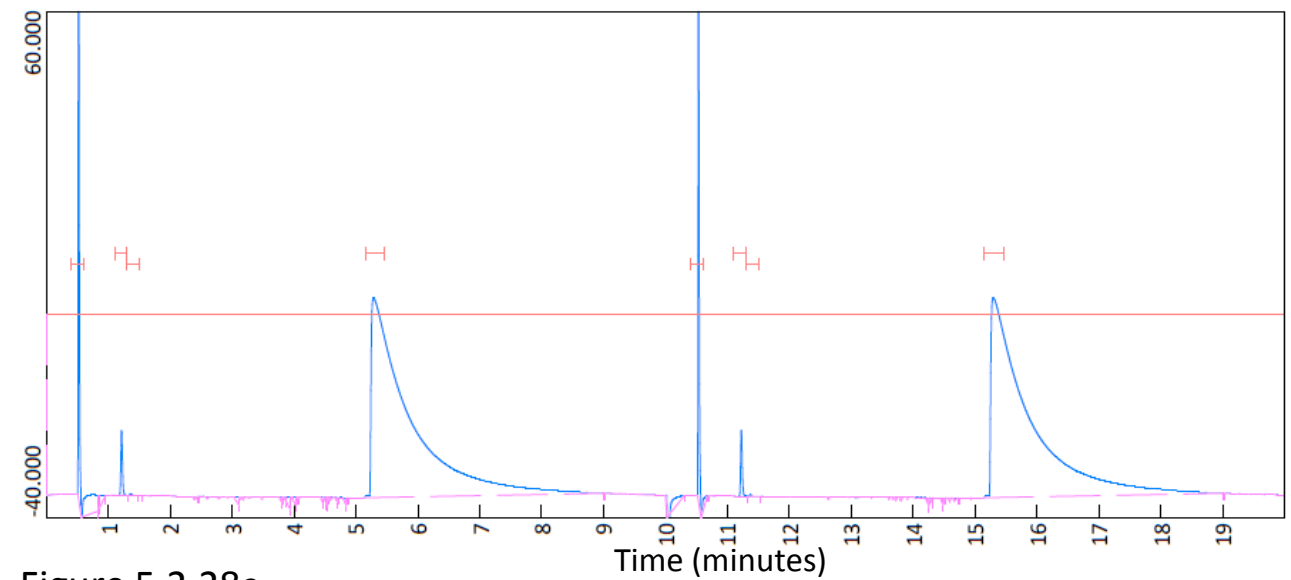

Figure 5.2.28a

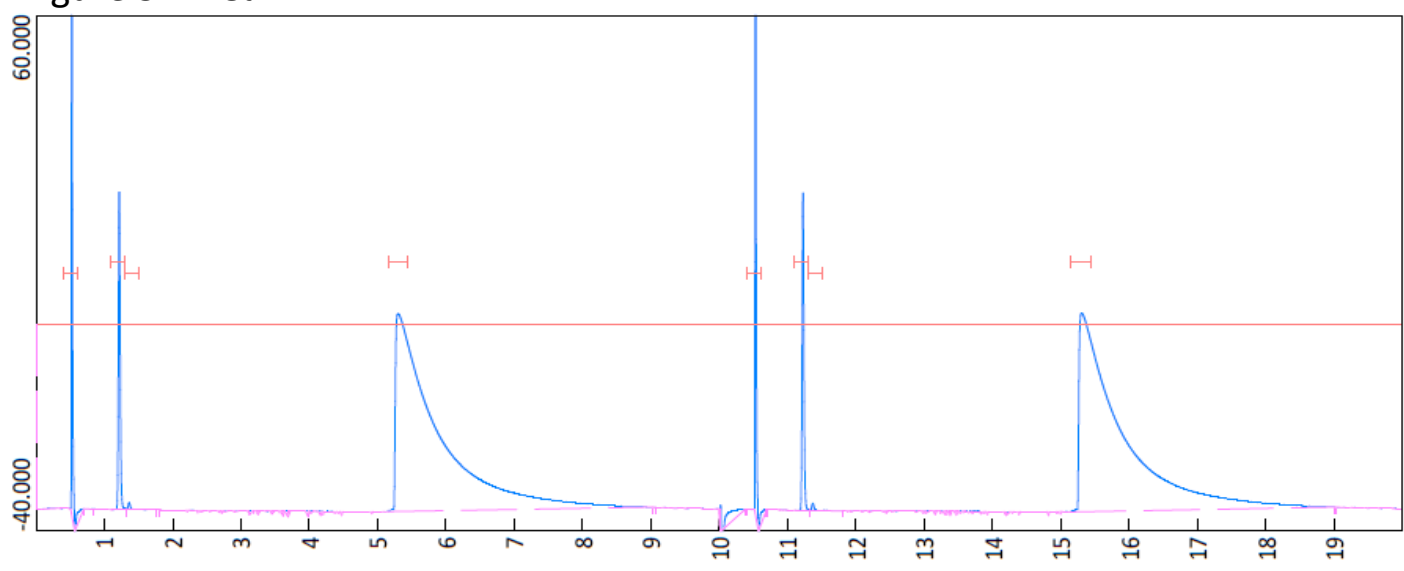

Figure 5.2.28b

Time (minutes)

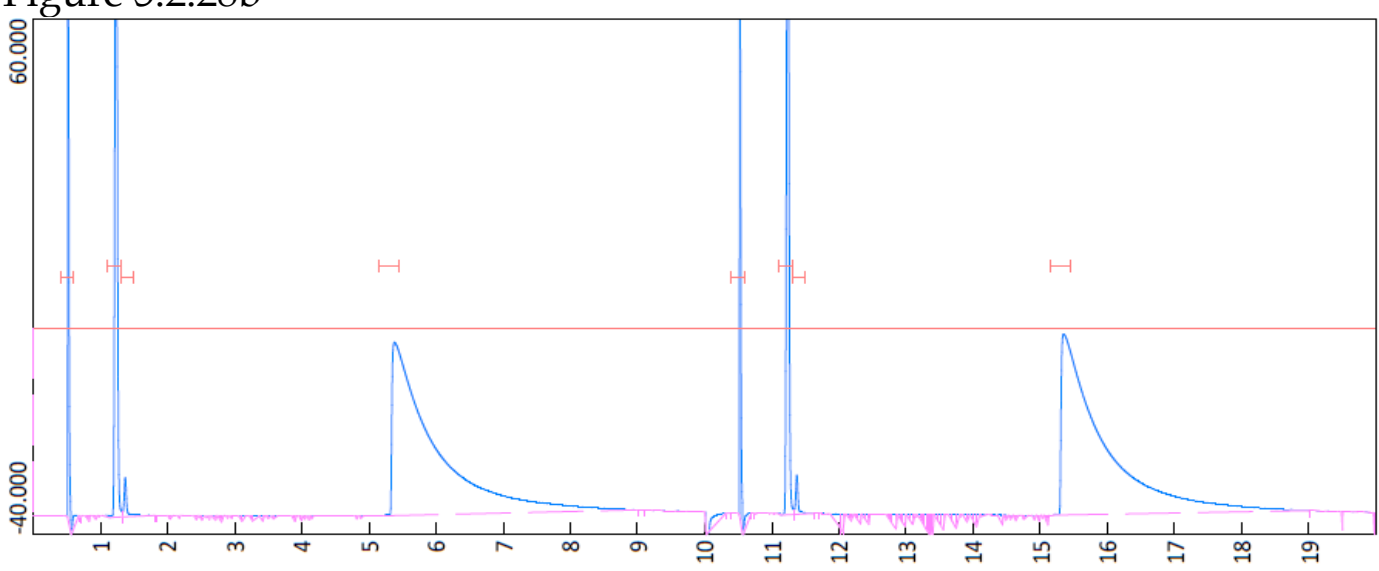

Figure 5.2.28c

Time (minutes)

Figure 5.2.28: Chromatograms for ammonia decomposition with the nickel foam insert showing two injection cycles of 10 minutes each. Each chromatogram represents a different temperature: $\mathrm{a}-350{ }^{\circ} \mathrm{C}, \mathrm{b}-400{ }^{\circ} \mathrm{C}, \mathrm{c}-450{ }^{\circ} \mathrm{C}$. 


\subsection{Activation Energy}

This section covers the application of the Arrhenius method described in Section 2.2.12.4 to the rates determined for the secondary insert catalysts. The three subsections below each calculate the activation energy from the different rate data for the secondary insert catalysts from Table 5.2.16. All of these activation energies are collated in Table 3.2.5 and discussed in section 3.2.2.4.6

\section{Calculation of Activation Energy from the Rate of Ammonia Decomposition}

The activation energy was calculated from the rates of ammonia decomposition for each secondary insert catalyst. Table 5.2.18calculates $1 / \mathrm{T}$ for each temperature and $\ln k$ for each rate. Figure 5.2.29 shows $\ln k$ graphed against $1 / T$ for all of these catalysts.

Table 5.2.18: Calculating Data for Arrhenius plots of Ammonia Rates of secondary inserts.

\begin{tabular}{|l|l|l|l|l|l|}
\hline Temperature $\left({ }^{\circ} \mathrm{C}\right)$ & 300 & 350 & 400 & 450 & \\
\hline Temperature $(\mathrm{K})$ & 573 & 623 & 673 & 723 & \\
\hline T-1 & $1.75 \mathrm{E}-03$ & $1.61 \mathrm{E}-03$ & $1.49 \mathrm{E}-03$ & $1.38 \mathrm{E}-03$ & $\mathrm{~K}-1$ \\
\hline Flat Nickel & $5.41 \mathrm{E}-11$ & $3.73 \mathrm{E}-10$ & $2.72 \mathrm{E}-07$ & $8.19 \mathrm{E}-06$ & $\mathrm{~mol} \mathrm{~min}^{-1}$ \\
\hline $\ln k$ & $-2.36 \mathrm{E}+01$ & $-2.17 \mathrm{E}+01$ & $-1.51 \mathrm{E}+01$ & $-1.17 \mathrm{E}+01$ & \\
\hline Bare Steel & $1.36 \mathrm{E}-09$ & $2.80 \mathrm{E}-09$ & $2.77 \mathrm{E}-07$ & $5.90 \mathrm{E}-06$ & $\mathrm{~mol} \mathrm{~min}^{-1}$ \\
\hline $\ln k$ & $-2.04 \mathrm{E}+01$ & $-1.97 \mathrm{E}+01$ & $-1.51 \mathrm{E}+01$ & $-1.20 \mathrm{E}+01$ & \\
\hline Template Nickel & $8.54 \mathrm{E}-08$ & $2.22 \mathrm{E}-07$ & $9.02 \mathrm{E}-07$ & $8.20 \mathrm{E}-06$ & $\mathrm{~mol} \mathrm{~min}^{-1}$ \\
\hline $\ln k$ & $-1.63 \mathrm{E}+01$ & $-1.53 \mathrm{E}+01$ & $-1.39 \mathrm{E}+01$ & $-1.17 \mathrm{E}+01$ & \\
\hline Nickel Foam & & $9.76 \mathrm{E}-08$ & $3.29 \mathrm{E}-07$ & $6.52 \mathrm{E}-06$ & $\mathrm{~mol} \cdot \mathrm{min}^{-1}$ \\
\hline $\ln k$ & & $-1.61 \mathrm{E}+01$ & $-1.49 \mathrm{E}+01$ & $-1.19 \mathrm{E}+01$ & \\
\hline
\end{tabular}




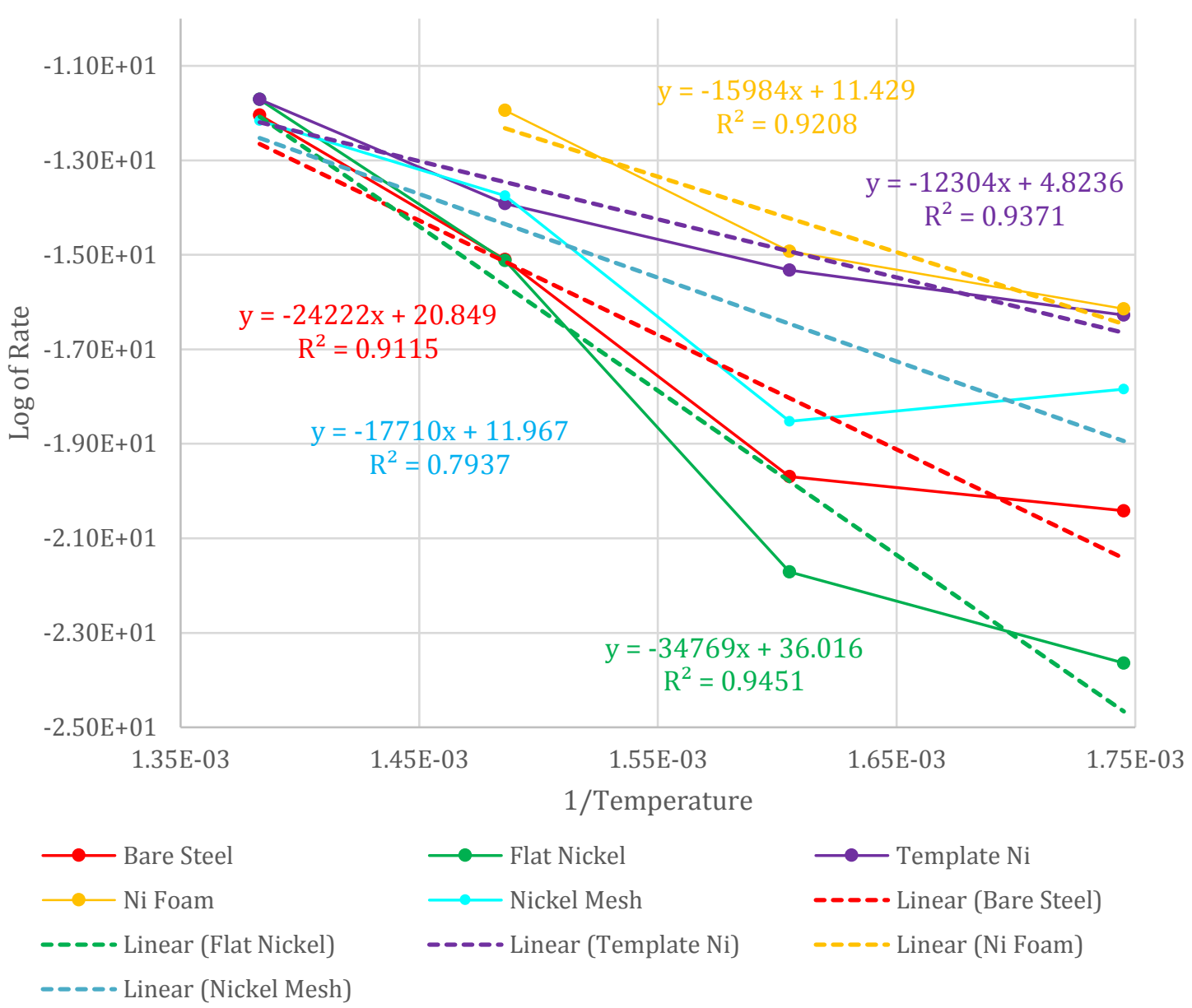

Figure 5.2.29: Arrhenius plot for Ammonia Decomposition with Secondary Insert Catalysts.

Table 5.2.19 uses the equation for the lines from figure $n$ to calculate the activation energy for ammonia decomposition.

Table 5.2.19: Final calculation of Activation Energy for Secondary Insert Catalysts from Rate of Ammonia decomposition.

\begin{tabular}{|l|l|l|l|l|}
\hline Catalyst & Intercept & Slope & $-\mathrm{R} \mathrm{m}$ & $\mathrm{E}_{\mathrm{a}}$ \\
\hline Flat Ni & 36.0 & -34769 & 289069 & 289 \\
\hline Bare Steel & 20.8 & -24222 & 201382 & 201 \\
\hline Template Ni & 4.8 & -12304 & 102295 & 102 \\
\hline Nickel Mesh & 12.0 & -17710 & 147241 & 147 \\
\hline Ni Foam & 11.4 & -15984 & 132891 & 133 \\
\hline & & & $\mathrm{J} \cdot \mathrm{mol}^{-1}$ & $\mathrm{~kJ} \cdot \mathrm{mol}^{-1}$ \\
\hline
\end{tabular}




\section{Calculation of Activation Energy from the Rate of Hydrogen Formation}

The same method was followed to calculate the activation energy, but using the rate of hydrogen formation from the secondary insert catalysts. The data for making the plot is shown in Table 5.2.20. Figure 5.2.30 shows the Arrhenius plot for hydrogen production from the secondary insert catalysts.

Table 5.2.20: Calculating Data for Arrhenius plots of Hydrogen Formation Rates of secondary inserts.

\begin{tabular}{|c|l|l|l|l|l|}
\hline Temperature $\left({ }^{\circ} \mathrm{C}\right)$ & 300 & 350 & 400 & 450 & \\
\hline Temperature $(\mathrm{K})$ & 573 & 623 & 673 & 723 & \\
\hline $\mathrm{T}^{-1}$ & 0.001745 & 0.001605 & 0.001486 & 0.001383 & $\mathrm{~K}-1$ \\
\hline Flat Nickel & $1.37 \mathrm{E}-07$ & $9.26 \mathrm{E}-07$ & $5.43 \mathrm{E}-06$ & $2.77 \mathrm{E}-05$ & $\mathrm{~mol} \mathrm{~min}^{-1}$ \\
\hline $\ln k$ & $-1.58 \mathrm{E}+01$ & $-1.39 \mathrm{E}+01$ & $-1.21 \mathrm{E}+01$ & $-1.05 \mathrm{E}+01$ & \\
\hline Bare Steel & $1.17 \mathrm{E}-07$ & $6.77 \mathrm{E}-07$ & $4.96 \mathrm{E}-06$ & $2.46 \mathrm{E}-05$ & $\mathrm{~mol} \mathrm{~min}^{-1}$ \\
\hline $\ln k$ & $-1.60 \mathrm{E}+01$ & $-1.42 \mathrm{E}+01$ & $-1.22 \mathrm{E}+01$ & $-1.06 \mathrm{E}+01$ & \\
\hline Template Nickel & $1.44 \mathrm{E}-07$ & $8.42 \mathrm{E}-07$ & $5.56 \mathrm{E}-06$ & $2.71 \mathrm{E}-05$ & $\mathrm{~mol} \mathrm{~min}^{-1}$ \\
\hline $\ln k$ & $-1.58 \mathrm{E}+01$ & $-1.40 \mathrm{E}+01$ & $-1.21 \mathrm{E}+01$ & $-1.05 \mathrm{E}+01$ & \\
\hline Nickel Foam & & $7.93 \mathrm{E}-07$ & $3.73 \mathrm{E}-06$ & $1.68 \mathrm{E}-05$ & $\mathrm{~mol} \mathrm{~min}^{-1}$ \\
\hline $\ln k$ & & $-1.40 \mathrm{E}+01$ & $-1.25 \mathrm{E}+01$ & $-1.10 \mathrm{E}+01$ & \\
\hline Nickel Mesh & $1.41 \mathrm{E}-07$ & $6.25 \mathrm{E}-07$ & $2.07 \mathrm{E}-06$ & $9.39 \mathrm{E}-06$ & $\mathrm{~mol} \mathrm{~min}^{-1}$ \\
\hline $\ln k$ & $-1.58 \mathrm{E}+01$ & $-1.43 \mathrm{E}+01$ & $-1.31 \mathrm{E}+01$ & $-1.16 \mathrm{E}+01$ & \\
\hline
\end{tabular}




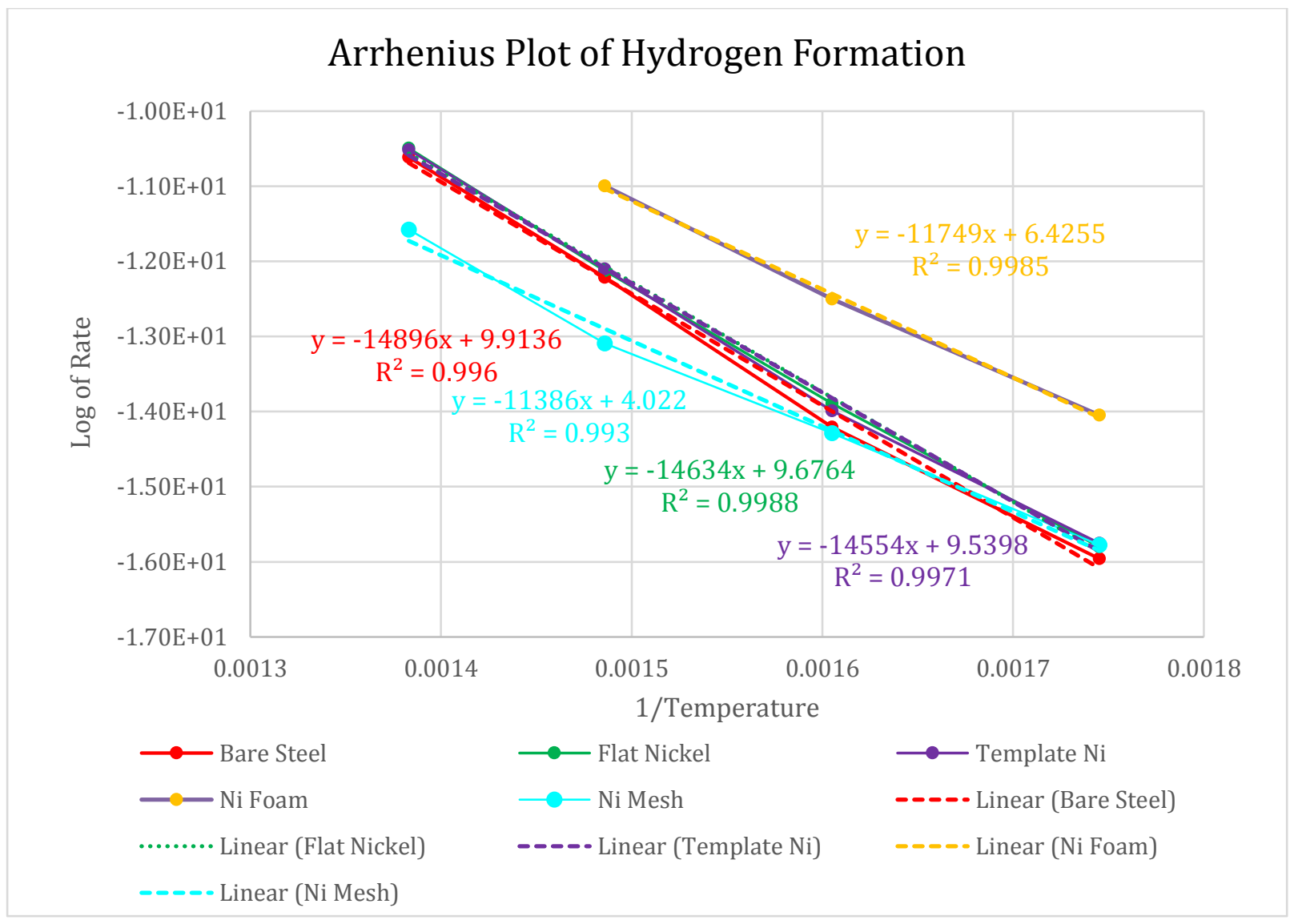

Figure 5.2.30: Arrhenius plot of the Hydrogen Formation data for the Secondary Insert Catalysts.

The gradient of the lines on the Arrhenius plots were used to calculate the activation energy. This data is shown in Table 5.2.21.

Table 5.2.21: Calculating the Activation Energy for the Hydrogen Formation on the Secondary Insert Catalysts.

\begin{tabular}{|l|l|l|l|l|}
\hline Catalyst & Intercept & Slope & $-\mathrm{R} \cdot \mathrm{m}$ & $\mathrm{E}_{\mathrm{a}}$ \\
\hline Flat Ni & 9.6764 & -14634 & 121667 & 121.7 \\
\hline Bare Steel & 9.9195 & -14896 & 123845 & 123.8 \\
\hline Template Ni & 9.5398 & -14554 & 121002 & 121.0 \\
\hline Ni Foam & 6.4255 & -11749 & 97681 & 97.7 \\
\hline Ni Mesh & 4.022 & -11386 & 94663 & 94.7 \\
\hline & & & $\mathrm{J} \cdot \mathrm{mol}^{-1}$ & $\mathrm{~kJ} \cdot \mathrm{mol}^{-1}$ \\
\hline
\end{tabular}

\section{Calculation of Activation Energy from the Rate of Nitrogen Formation}

The process is followed again for calculating activation energy from the rate of nitrogen formation. The data for the Arrhenius plot is prepared in Table 5.2.22, then plotted in Figure 5.2.31. The activation energy is calculated from the gradients of the graphs in Figure 5.2.31 in Table 5.2.23. 
Table 5.2.22: Calculating Data for Arrhenius plots of Nitrogen Formation Rates of secondary inserts

\begin{tabular}{|c|c|c|c|c|c|}
\hline Temperature $\left({ }^{\circ} \mathrm{C}\right)$ & 300 & 350 & 400 & 450 & \\
\hline Temperature (K) & 573 & 623 & 673 & 723 & \\
\hline $\mathrm{T}^{-1}$ & 0.0017452 & 0.0016051 & 0.001486 & 0.001383 & $\mathrm{~K}^{-1}$ \\
\hline Flat Nickel & 7.09E-08 & $2.46 \mathrm{E}-07$ & $1.42 \mathrm{E}-06$ & $7.75 \mathrm{E}-06$ & $\mathrm{~mol} \cdot \mathrm{min}^{-1}$ \\
\hline $\ln k$ & $-1.65 \mathrm{E}+01$ & $-1.52 \mathrm{E}+01$ & $-1.35 \mathrm{E}+01$ & $-1.18 \mathrm{E}+01$ & \\
\hline Bare Steel & 3.99E-08 & $1.61 \mathrm{E}-07$ & $1.27 \mathrm{E}-06$ & $7.40 \mathrm{E}-06$ & $\mathrm{mo}$ \\
\hline $\ln k$ & $-1.70 \mathrm{E}+01$ & $-1.56 \mathrm{E}+01$ & $-1.36 \mathrm{E}+01$ & $-1.18 \mathrm{E}+01$ & \\
\hline Template Nickel & 7.16E-08 & 2.27E-07 & $1.57 \mathrm{E}-06$ & $7.74 \mathrm{E}-06$ & $\mathrm{~mol} \cdot \mathrm{min}^{-1}$ \\
\hline $\ln k$ & $-1.65 E+01$ & $-1.53 E+01$ & $-1.34 \mathrm{E}+01$ & $-1.18 \mathrm{E}+01$ & \\
\hline Nickel Mesh & 4.69E-08 & 2.08E-07 & $6.88 \mathrm{E}-07$ & $3.13 \mathrm{E}-06$ & $\mathrm{~mol} \cdot \mathrm{min}^{-1}$ \\
\hline $\ln k$ & $-1.69 E+01$ & $-1.54 \mathrm{E}+01$ & $-1.42 \mathrm{E}+01$ & $-1.27 \mathrm{E}+01$ & \\
\hline Nickel Foam & & $1.81 \mathrm{E}-07$ & 9.09E-07 & $4.72 \mathrm{E}-06$ & $\mathrm{~mol} \mathrm{~min}^{-1}$ \\
\hline $\ln k$ & & $-1.55 \mathrm{E}+01$ & $-1.39 \mathrm{E}+01$ & $-1.23 E+01$ & \\
\hline
\end{tabular}

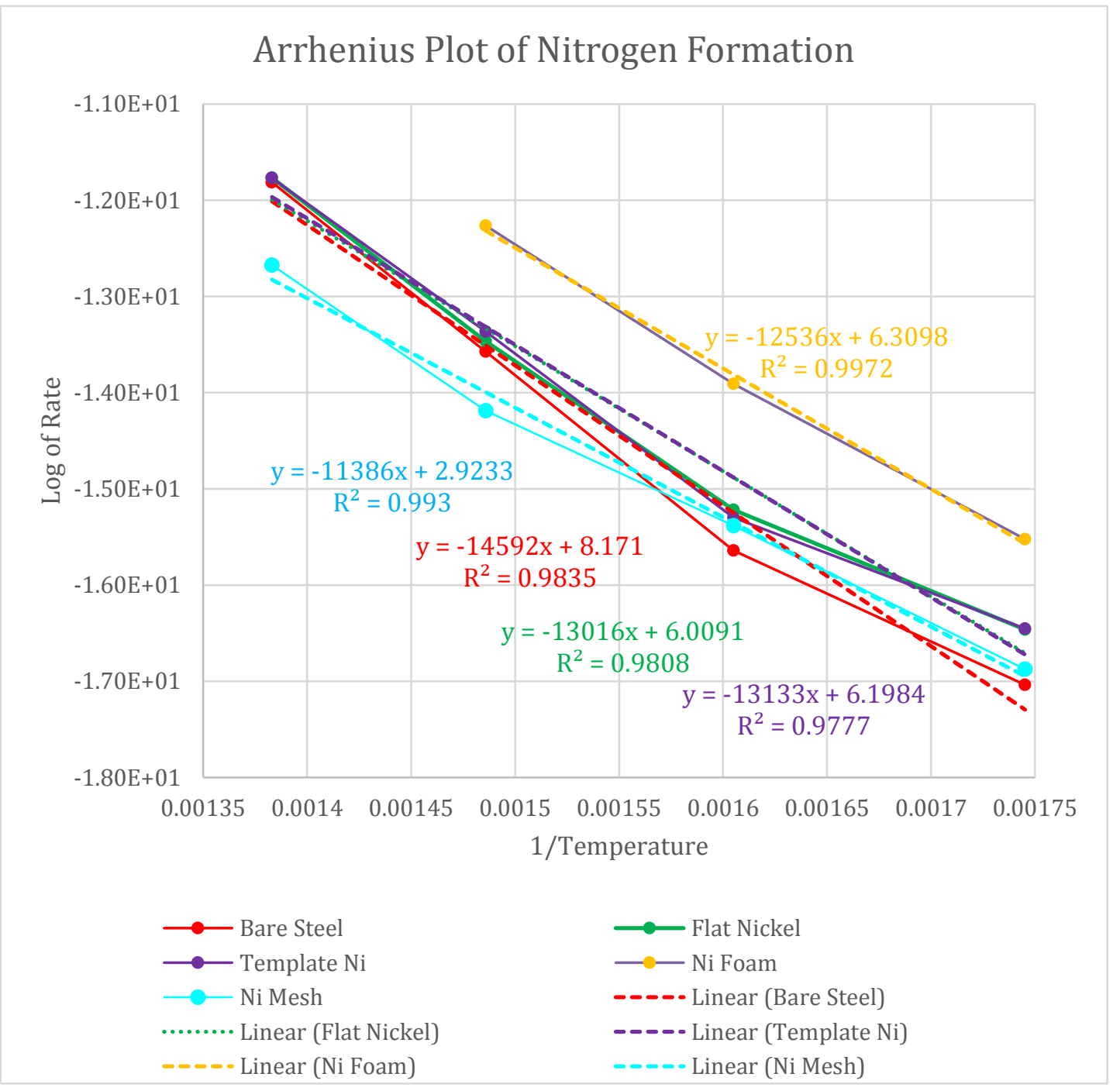

Figure 5.2.31: Arrhenius plot of the Nitrogen Formation data for the Secondary Insert Catalysts. 
Jonathan Tailby

Table 5.2.23: Calculating the Activation Energy for the Nitrogen Formation on the Secondary Insert Catalysts.

\begin{tabular}{|l|l|l|l|l|}
\hline & Constant & Slope & $-\mathrm{R} \mathrm{m}$ & $\mathrm{E}_{\mathrm{a}}$ \\
\hline Flat Ni & 6.0091 & -13016 & 108215 & 108.2 \\
\hline Bare Steel & 8.171 & -14592 & 121318 & 121.3 \\
\hline Template Ni & 6.1984 & -13133 & 109188 & 109.2 \\
\hline Ni Foam & 6.3098 & -12536 & 104224 & 104.2 \\
\hline Ni Mesh & 2.9233 & 11386 & -94663 & -94.7 \\
\hline & & & $\mathrm{J} \cdot \mathrm{mol}^{-1}$ & $\mathrm{~kJ} \cdot \mathrm{mol}^{-1}$ \\
\hline
\end{tabular}

\subsubsection{Tertiary Insert Measurements and Calculations}

Figure 5.2.32a-d show chromatograms for ammonia decomposition with the Nickel-before-Palladium tertiary insert at 300, 350, 400 and $450{ }^{\circ} \mathrm{C}$. The average peak areas for these experiments are collected into Table 3.3.2, Table 3.3.3 and Table 3.3.4. 


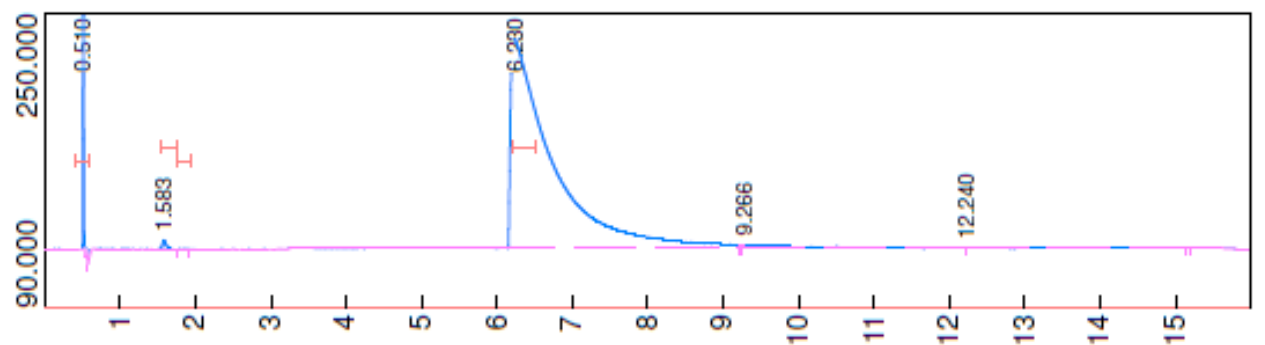

Figure 5.2.32a: $300{ }^{\circ} \mathrm{C}$. Hydrogen: area=18.0. Ammonia area $=4406.7$ to 9.226 minutes

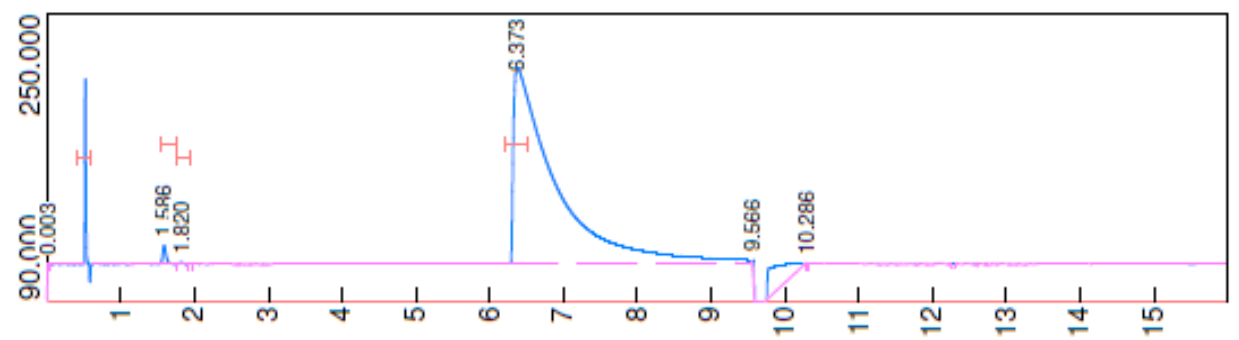

Figure 5.2.32b: $350{ }^{\circ} \mathrm{C}$. Hydrogen area=34.9. Nitrogen area=4.25. Ammonia area $=4355.6$ to 9.566 minutes

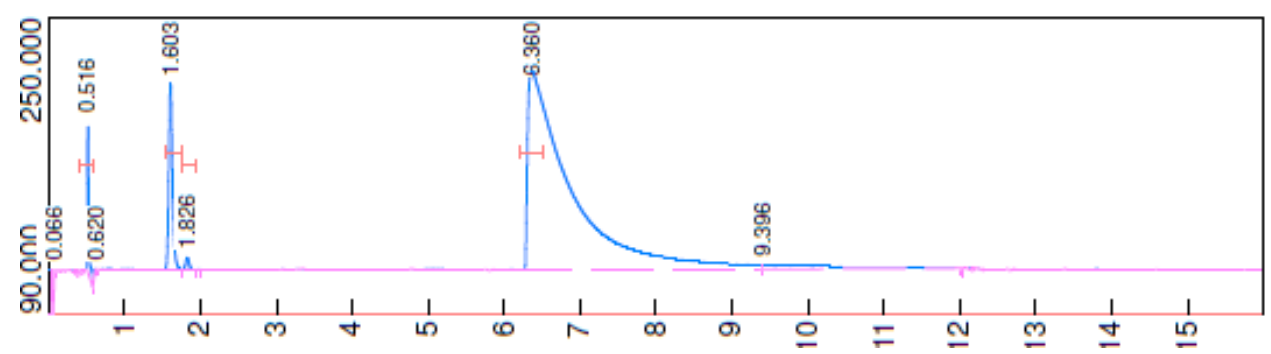

Figure 5.2.32c: $400{ }^{\circ} \mathrm{C}$. Hydrogen: 1.603 minutes, area=336.0. Nitrogen: 1.826 minutes, area=20.7. Ammonia, 6.360 minutes, area=4199.8 to 9.396 minutes

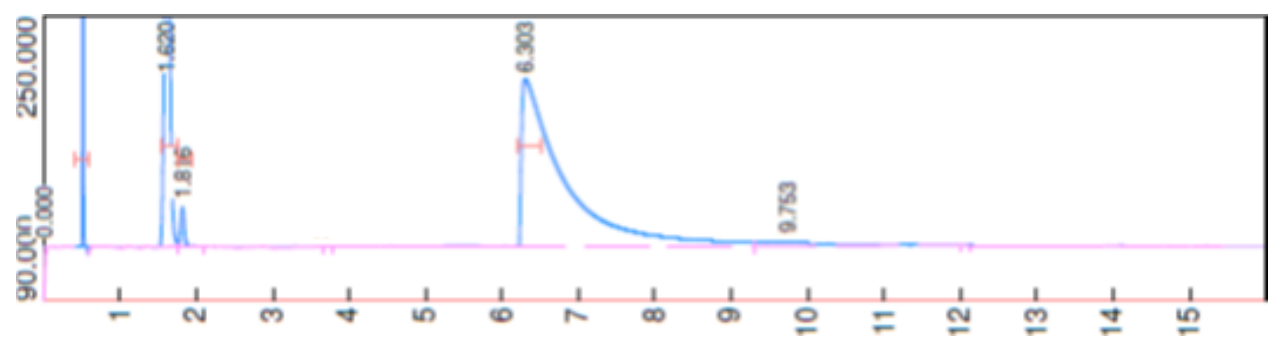

Figure 5.2.32d: $450{ }^{\circ} \mathrm{C}$. Hydrogen area $=1374.8$. Nitrogen area=74.2. Ammonia area $=3600.2$ to 9.753 minutes

Figure 5.2.32: Chromatograms for the retentate gas mixture after ammonia decomposition with the Nickel-before-Palladium tertiary insert.

Figure 5.2.33a-d show chromatograms for the permeate from ammonia decomposition with the Nickel-before-Palladium tertiary insert at 300, 350, 400 and $450^{\circ} \mathrm{C}$. The average peak area for these experiments are collected into Table 3.3.1. 
Jonathan Tailby

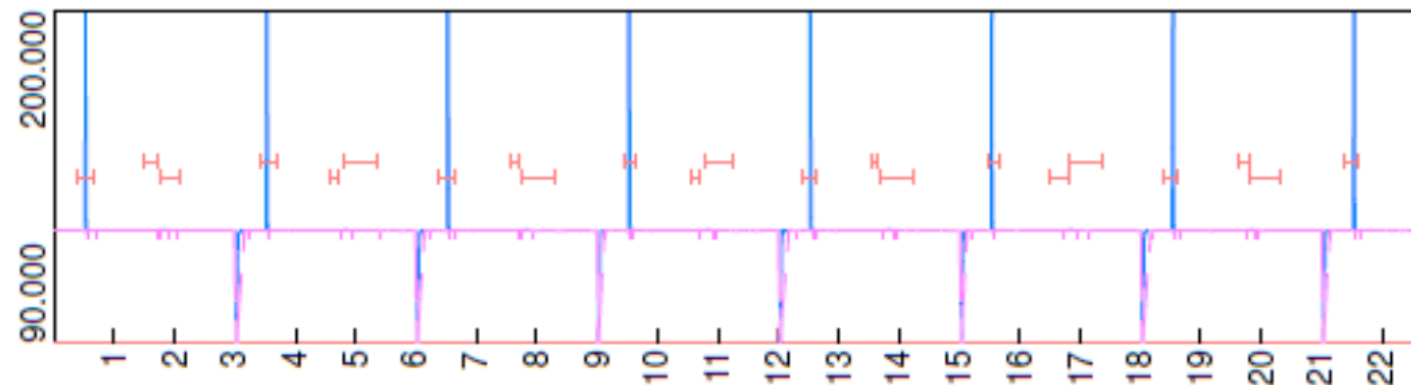

Figure 5.2.33a: $300^{\circ} \mathrm{C}$. Hydrogen: area $=0.69$, Nitrogen: area $=1.79$

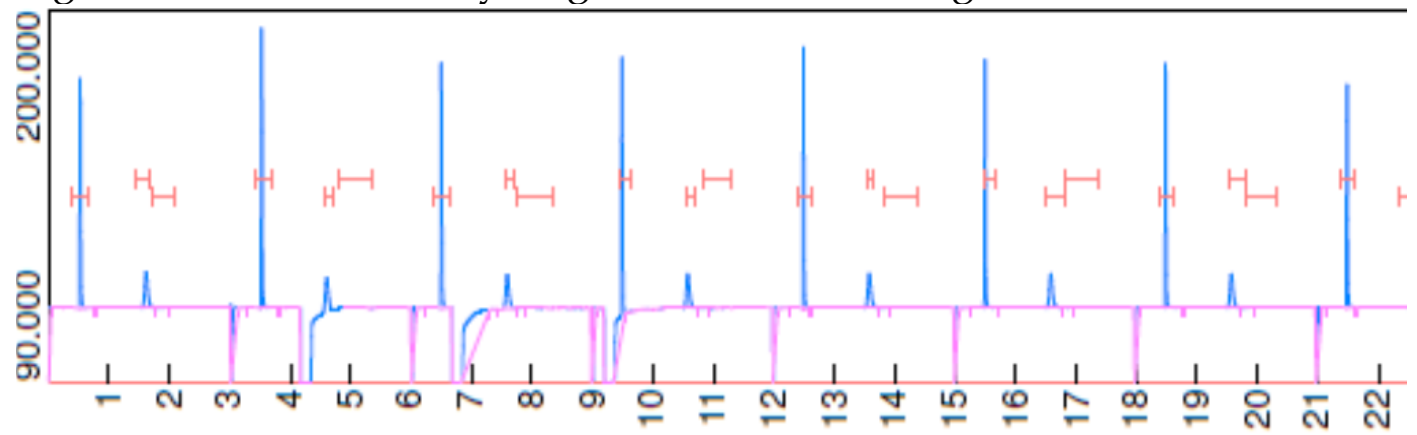

Figure 5.2.33b: $350{ }^{\circ} \mathrm{C}$. Hydrogen: area $=39.2$

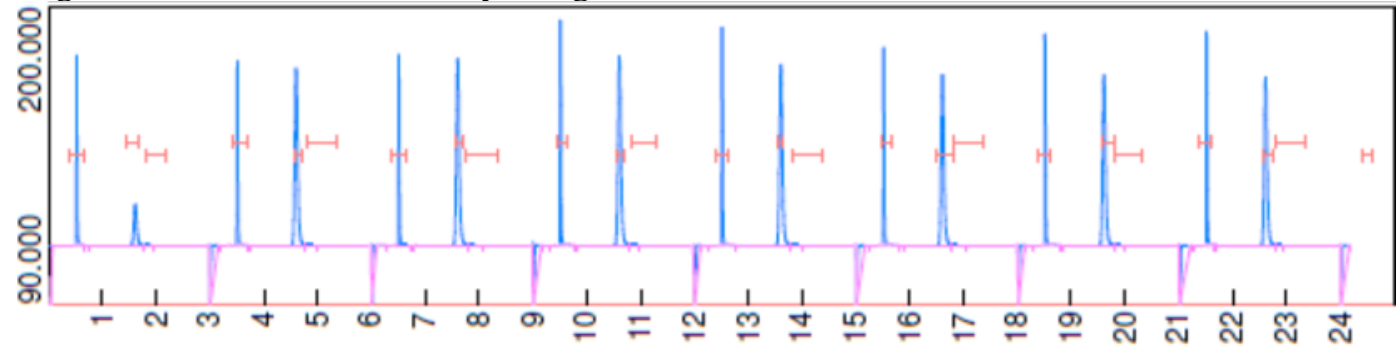

Figure 5.2.33c $400{ }^{\circ} \mathrm{C}$. Hydrogen area $=244.7$

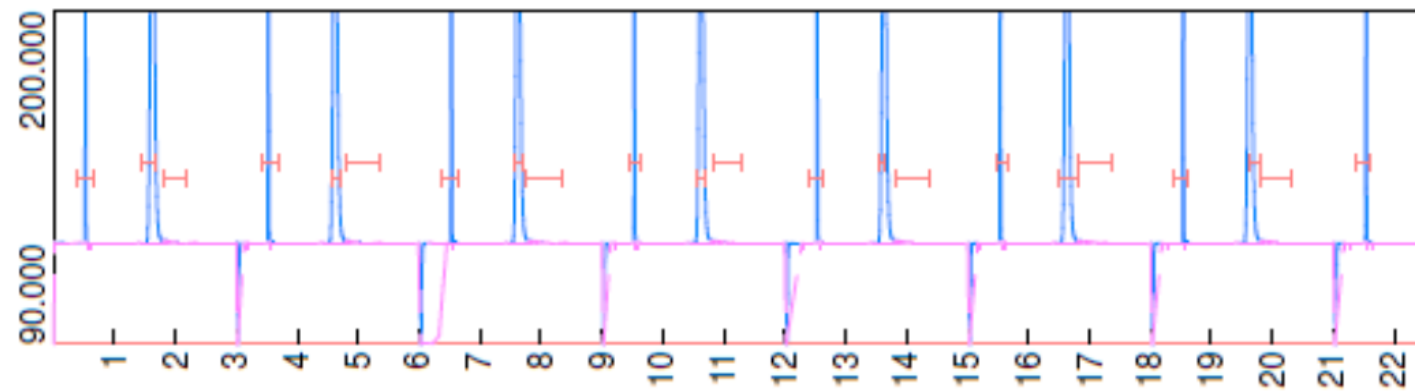

Figure 5.2.33d: $450{ }^{\circ} \mathrm{C}$. Hydrogen: area $=854.0$

Figure 5.2.33: Chromatograms for permeate gas mixture after ammonia decomposition with the Nickel-before-Palladium tertiary insert. 
Figure 5.2.34a-d show chromatograms for ammonia decomposition experiments with the Nickel-on-Palladium-no-Permeation tertiary insert at 300, 350, 400, 450 ${ }^{\circ} \mathrm{C}$.

Ammonia flow of $5 \mathrm{~mL} \mathrm{~min}^{-1}$. The peaks at 0.5 minutes are the injection of the sample into the column. The peaks at 1.6 minutes (1.1 minutes retention time) are hydrogen, 1.8 (1.3) minutes are nitrogen and 6.3 (5.8) minutes are ammonia.

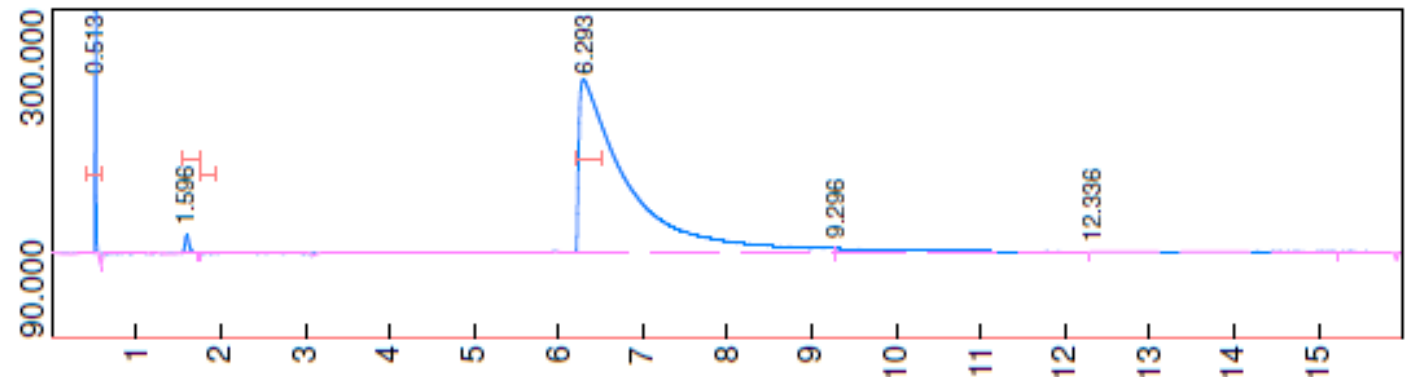

Figure 5.2.34a: $300{ }^{\circ} \mathrm{C}$

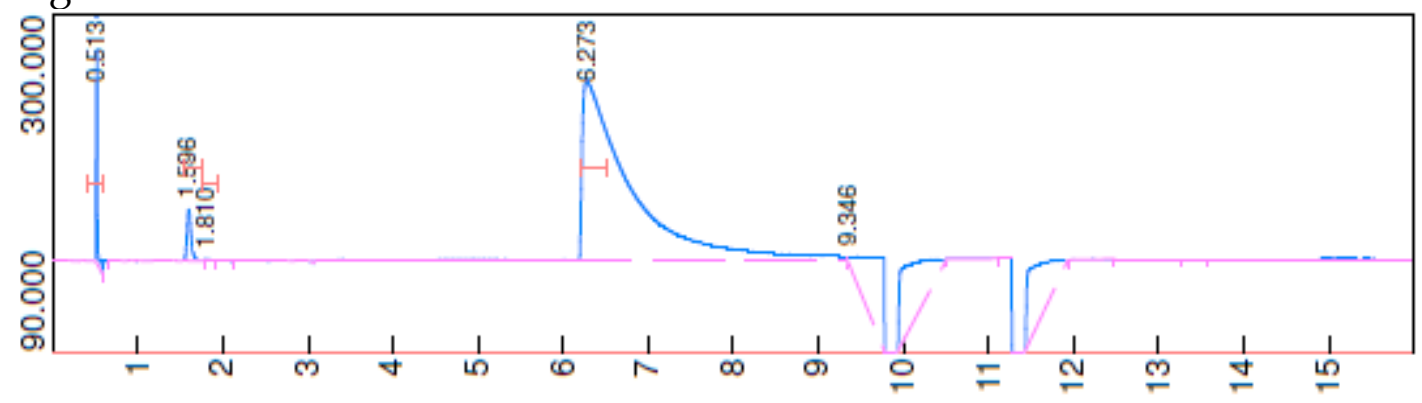

Figure 5.2.34b: $350{ }^{\circ} \mathrm{C}$

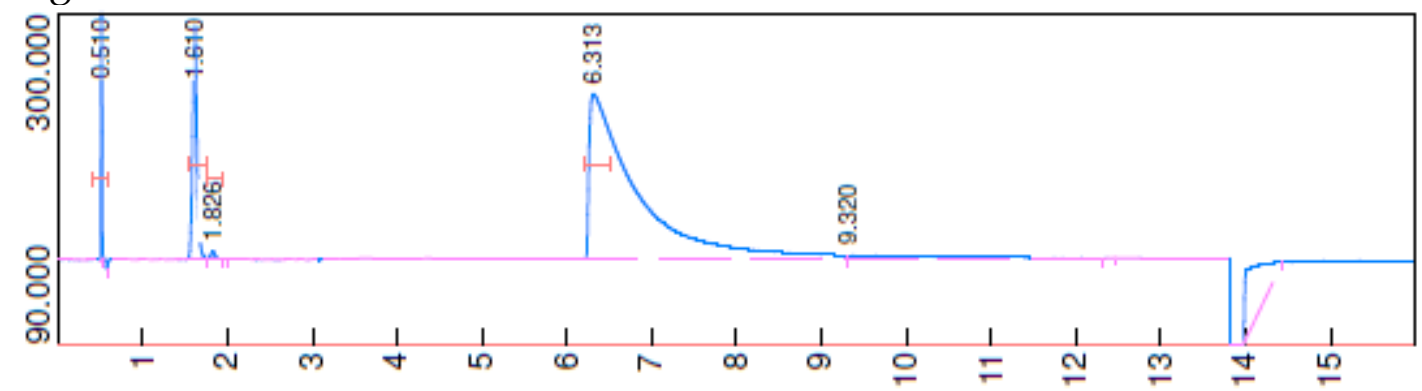

Figure 5.2.34c: $400^{\circ} \mathrm{C}$

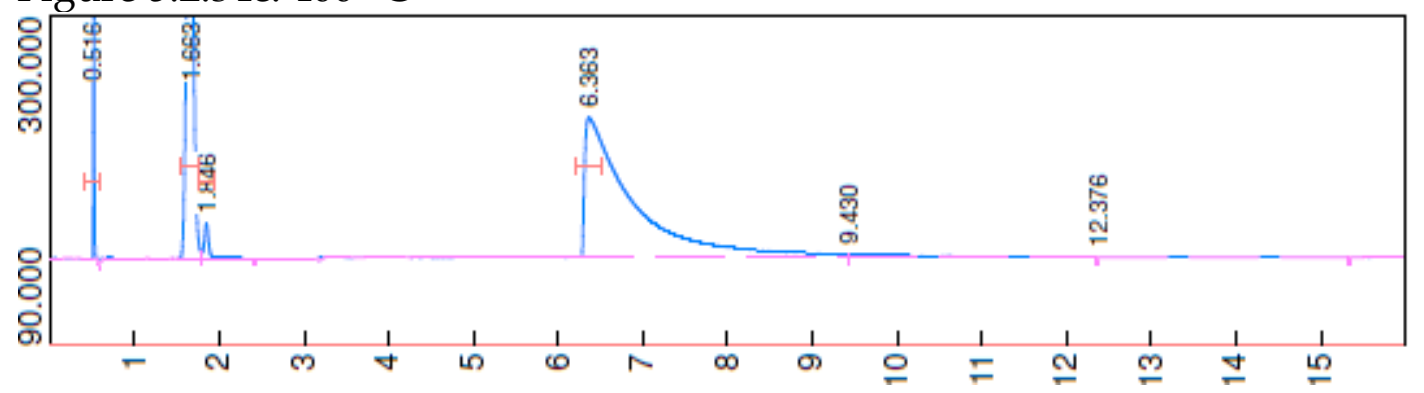

Figure 5.2.34d: $450{ }^{\circ} \mathrm{C}$

Figure 5.2.34: Chromatograms for decomposition of ammonia with the foam catalyst in contact with palladium foil (Nickel-on-Palladium-no-Permeation). 
Jonathan Tailby

Figure 5.2.35a-d show retentate chromatograms for the full temperature range of ammonia decomposition experiments with the Nickel-on-Palladium catalytic spillover membrane.

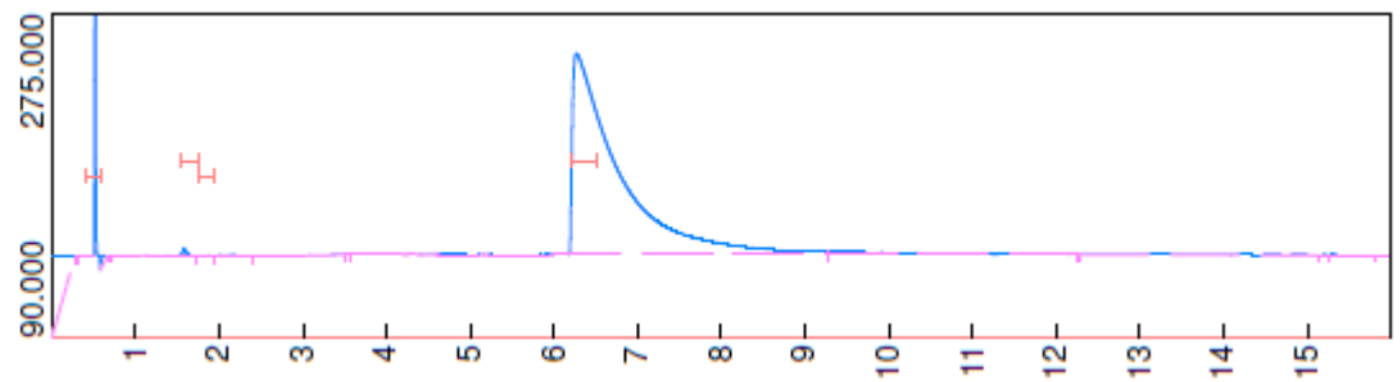

Figure 5.2.35a: $300{ }^{\circ} \mathrm{C}$. Hydrogen: 1.583 minutes, area=12.2. Nitrogen: 1.806 minutes, area $=1.26$. Ammonia, 6.270 minutes, area $=4441.2$ to 9.28 minutes

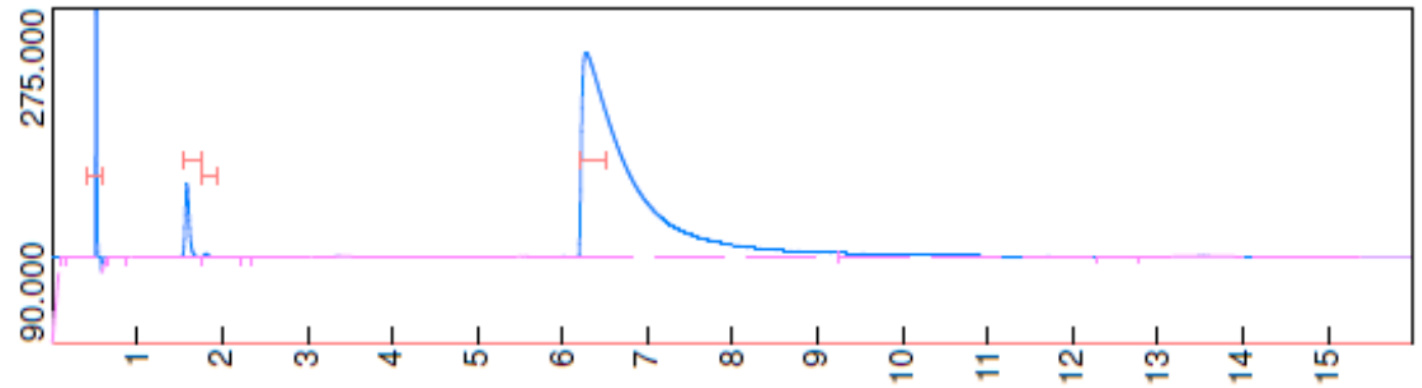

Figure 5.2.35b: $350^{\circ} \mathrm{C}$. Hydrogen: 1.583 minutes, area=131.9. Nitrogen: 1.806 minutes, area $=7.14$. Ammonia, 6.273 minutes, area $=4327.6$ to 9.30 minutes

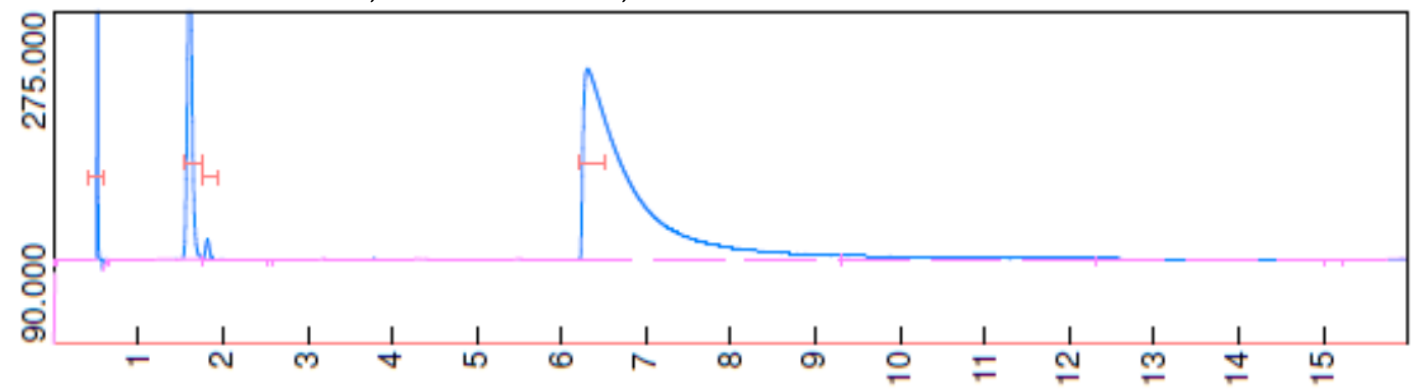

Figure 5.2.35c: $400{ }^{\circ} \mathrm{C}$. Hydrogen: 1.606 minutes, area=735.5. Nitrogen: 1.816 minutes, area $=39.5$. Ammonia, 6.306 minutes, area $=4026.29 .25$ minutes

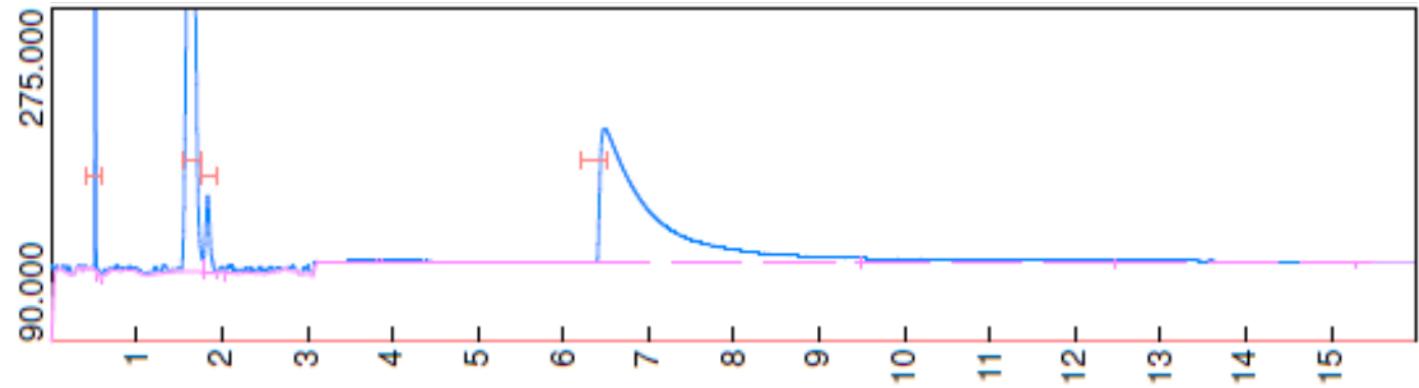

Figure 5.2.35d: $450{ }^{\circ} \mathrm{C}$. Hydrogen: 1.650 minutes, area=2667.9. Nitrogen: 1.833 minutes, area=147.1. Ammonia, 6.483 minutes, area=2900.19.48 minutes Figure 5.2.35: Chromatograms for the retentate of the catalytic spillover membrane when the rate of ammonia flow was retained at $5 \mathrm{~mL} \mathrm{~min}^{-1}$ and the temperature varied from 300 to $450^{\circ} \mathrm{C}$. 
Figure 5.2.36a-d show chromatograms for the permeate from ammonia decomposition at 300 to $450{ }^{\circ} \mathrm{C}$ on the Catalytic Spillover Membrane.

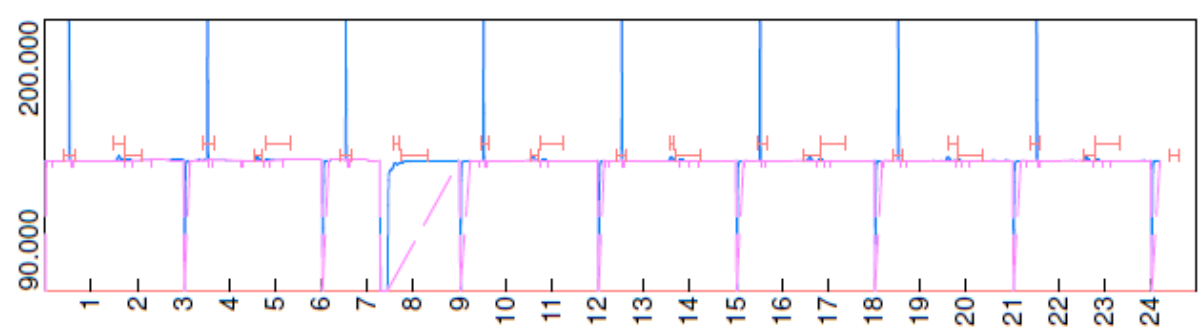

Figure 5.2.36a: $300{ }^{\circ} \mathrm{C}$. Hydrogen area $=6.00$, Nitrogen area $=1.76$

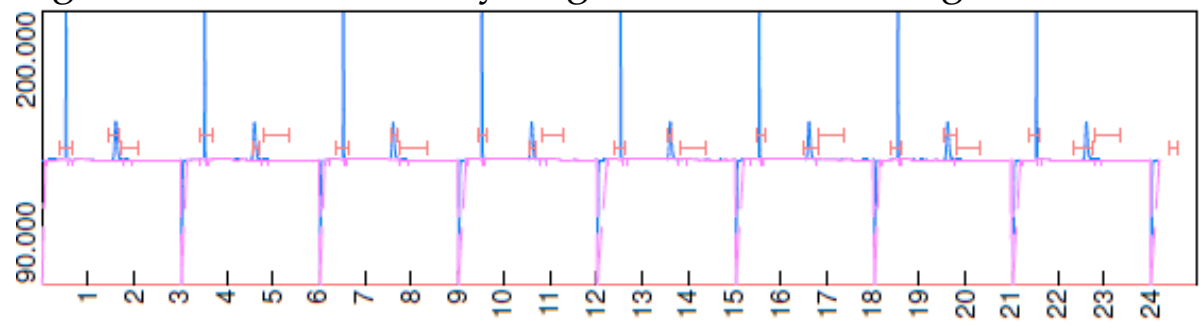

Figure 5.2.36b: $350^{\circ} \mathrm{C}$. Hydrogen area $=56.4$,

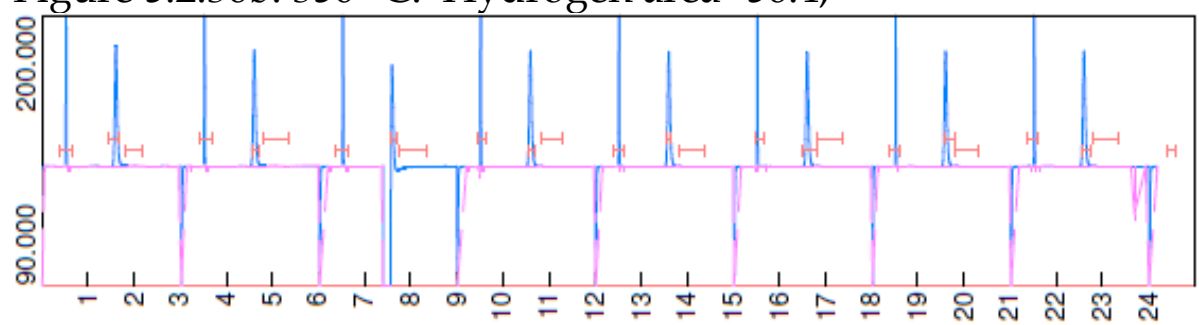

Figure 5.2.36c: $400^{\circ} \mathrm{C}$. Hydrogen area $=181.5$

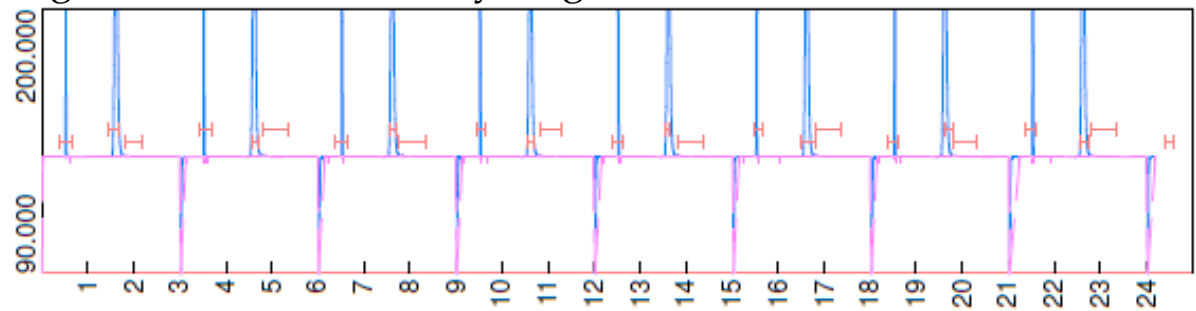

Figure 5.2.36d: $450{ }^{\circ} \mathrm{C}$. Hydrogen area $=660.7 .0$

Figure 5.2.36: Chromatogram for permeate from ammonia decomposition reactions with the Nickel-on-Palladium membrane at $400{ }^{\circ} \mathrm{C}$. 
Jonathan Tailby

Table 5.2.24 summarises the GC measurements and calculated values for the decomposition experiments with the Nickel-on-Palladium-no-permeation at 300 $450^{\circ} \mathrm{C}$. The calculated values are included in graphs in Sections 3.3.2 and 3.3.3.1.1.

\begin{tabular}{|c|c|c|c|c|c|c|}
\hline \multicolumn{7}{|c|}{$\begin{array}{l}\text { Table 5.2.24: Flux calculations for the Nickel-on-Palladium Catalytic } \\
\text { Spillover Membrane using the Richardson's Equation. } \\
\text { Max } E_{a}=48.50 \mathrm{~kJ} \mathrm{~mol}^{-1} \text {, min } E_{a}=12.48 \mathrm{~kJ} \mathrm{~mol}^{-1}, \max \mathrm{Pe} 0=0.38 \times 10-5 \\
\text { mol m s-1 m-2 } \mathrm{kPa}^{-5}\end{array}$} \\
\hline & & & & & & Unit \\
\hline & Temperature: & 300 & 350 & 400 & 450 & ${ }^{\circ} \mathrm{C}$ \\
\hline & & 573 & 623 & 673 & 723 & $\mathrm{~K}$ \\
\hline \multirow[t]{3}{*}{ Permeate } & $3 b \%$ & $0.05 \%$ & $0.54 \%$ & $1.66 \%$ & $6.04 \%$ & \\
\hline & $\mathrm{P}$ & $5.56 \mathrm{E}+01$ & $5.43 \mathrm{E}+02$ & $1.68 \mathrm{E}+03$ & $6.12 \mathrm{E}+03$ & $\mathrm{~Pa}$ \\
\hline & Sqrt $P$ & $7.46 \mathrm{E}+00$ & $2.33 \mathrm{E}+01$ & $4.10 \mathrm{E}+01$ & $7.82 \mathrm{E}+01$ & $\sqrt{\mathrm{Pa}}$ \\
\hline \multirow[t]{6}{*}{ Retentate } & $3 b \%$ & $0.12 \%$ & $1.28 \%$ & $7.01 \%$ & $24.92 \%$ & \\
\hline & $3 a p$ & $1.25 \mathrm{E}+02$ & $1.30 \mathrm{E}+03$ & $7.10 \mathrm{E}+03$ & $2.52 E+04$ & $\mathrm{~Pa}$ \\
\hline & sqrt & $1.12 \mathrm{E}+01$ & $3.60 \mathrm{E}+01$ & $8.43 \mathrm{E}+01$ & $1.59 \mathrm{E}+02$ & $\sqrt{\mathrm{Pa}}$ \\
\hline & & & & & & \\
\hline & ret-perm & $3.74 \mathrm{E}+00$ & $1.27 \mathrm{E}+01$ & $4.33 \mathrm{E}+01$ & $8.07 \mathrm{E}+01$ & $\sqrt{\mathrm{Pa}}$ \\
\hline & $\mathrm{R} \cdot \mathrm{T}$ & $4.76 \mathrm{E}+03$ & $5.18 \mathrm{E}+03$ & $5.60 \mathrm{E}+03$ & $6.01 E+03$ & $\mathrm{~J}_{\mathrm{mol}}{ }^{-1}$ \\
\hline \multirow[t]{2}{*}{$\mathrm{E}_{\mathrm{a}} / \mathrm{RT}$} & $\max E_{a}$ & 1.02E-02 & $9.36 \mathrm{E}-03$ & \begin{tabular}{|l|}
$8.67 \mathrm{E}-03$ \\
\end{tabular} & 8.07E-03 & mol \\
\hline & $\min E_{a}$ & $2.62 \mathrm{E}-03$ & $2.41 \mathrm{E}-03$ & $2.23 \mathrm{E}-03$ & $2.08 \mathrm{E}-03$ & mol \\
\hline \multirow[t]{2}{*}{$-\left(E_{a} / R T\right)$} & $\max E_{a}$ & $-1.02 \mathrm{E}-02$ & $-9.36 \mathrm{E}-03$ & -8.67E-03 & $-8.07 \mathrm{E}-03$ & mol \\
\hline & $\min E_{a}$ & $-2.62 \mathrm{E}-03$ & $-2.41 \mathrm{E}-03$ & $-2.23 \mathrm{E}-03$ & $-2.08 \mathrm{E}-03$ & mol \\
\hline \multirow[t]{2}{*}{$\begin{array}{l}\text { Exp(- } \\
\left.\text { E }_{a} / R T\right)\end{array}$} & $\max E_{a}$ & $9.90 \mathrm{E}-01$ & 9.91E-01 & 9.91E-01 & 9.92E-01 & \\
\hline & $\min E_{a}$ & 9.97E-01 & 9.98E-01 & \begin{tabular}{|l|}
$9.98 \mathrm{E}-01$ \\
\end{tabular} & $9.98 \mathrm{E}-01$ & \\
\hline \multirow{4}{*}{$\begin{array}{l}J_{H_{2}} \\
J_{H_{2}} \\
J_{H_{2}} \\
J_{H_{2}}\end{array}$} & $\max \mathrm{Pe}^{0} \min \mathrm{E}_{\mathrm{a}}$ & $1.41 \mathrm{E}-01$ & 4.78E-01 & $1.63 \mathrm{E}+00$ & $3.04 \mathrm{E}+00$ & $\mathrm{~mol}_{\mathrm{H}} \cdot \mathrm{m}^{-2} \mathrm{~s}^{-1}$ \\
\hline & $\min \mathrm{Pe}^{0} \min \mathrm{E}_{\mathrm{a}}$ & 1.42E-01 & 4.81E-01 & $1.64 \mathrm{E}+00$ & $3.06 \mathrm{E}+00$ & $\mathrm{~mol}_{\mathrm{H}} \cdot \mathrm{m}^{-2} \mathrm{~s}^{-1}$ \\
\hline & $\max \mathrm{Pe}^{0} \max \mathrm{E}_{\mathrm{a}}$ & $3.45 \mathrm{E}+00$ & $1.17 \mathrm{E}+01$ & $4.00 \mathrm{E}+01$ & $7.47 \mathrm{E}+01$ & $\mathrm{~mol}_{\mathrm{H}} \cdot \mathrm{m}^{-2} \mathrm{~s}^{-1}$ \\
\hline & $\max \mathrm{Pe}^{0} \min \mathrm{E}_{\mathrm{a}}$ & $3.48 \mathrm{E}+00$ & $1.18 \mathrm{E}+01$ & $4.03 \mathrm{E}+01$ & $7.51 \mathrm{E}+01$ & $\mathrm{~mol}_{\mathrm{H}} \cdot \mathrm{m}^{-2} \cdot \mathrm{s}^{-1}$ \\
\hline \multirow{2}{*}{$\begin{array}{c}J_{H_{2}} \\
J_{H_{2}}\end{array}$} & $\min$ & $1.41 \mathrm{E}-01$ & 4.78E-01 & $1.63 \mathrm{E}+00$ & $3.04 \mathrm{E}+00$ & $\mathrm{~mol}_{\mathrm{H}} \cdot \mathrm{m}^{-2} \cdot \mathrm{s}^{-1}$ \\
\hline & $\max$ & $3.48 \mathrm{E}+00$ & $1.18 \mathrm{E}+01$ & $4.03 \mathrm{E}+01$ & $7.51 \mathrm{E}+01$ & $\mathrm{~mol}_{\mathrm{H}} \cdot \mathrm{m}^{-2} \mathrm{~s}^{-1}$ \\
\hline
\end{tabular}




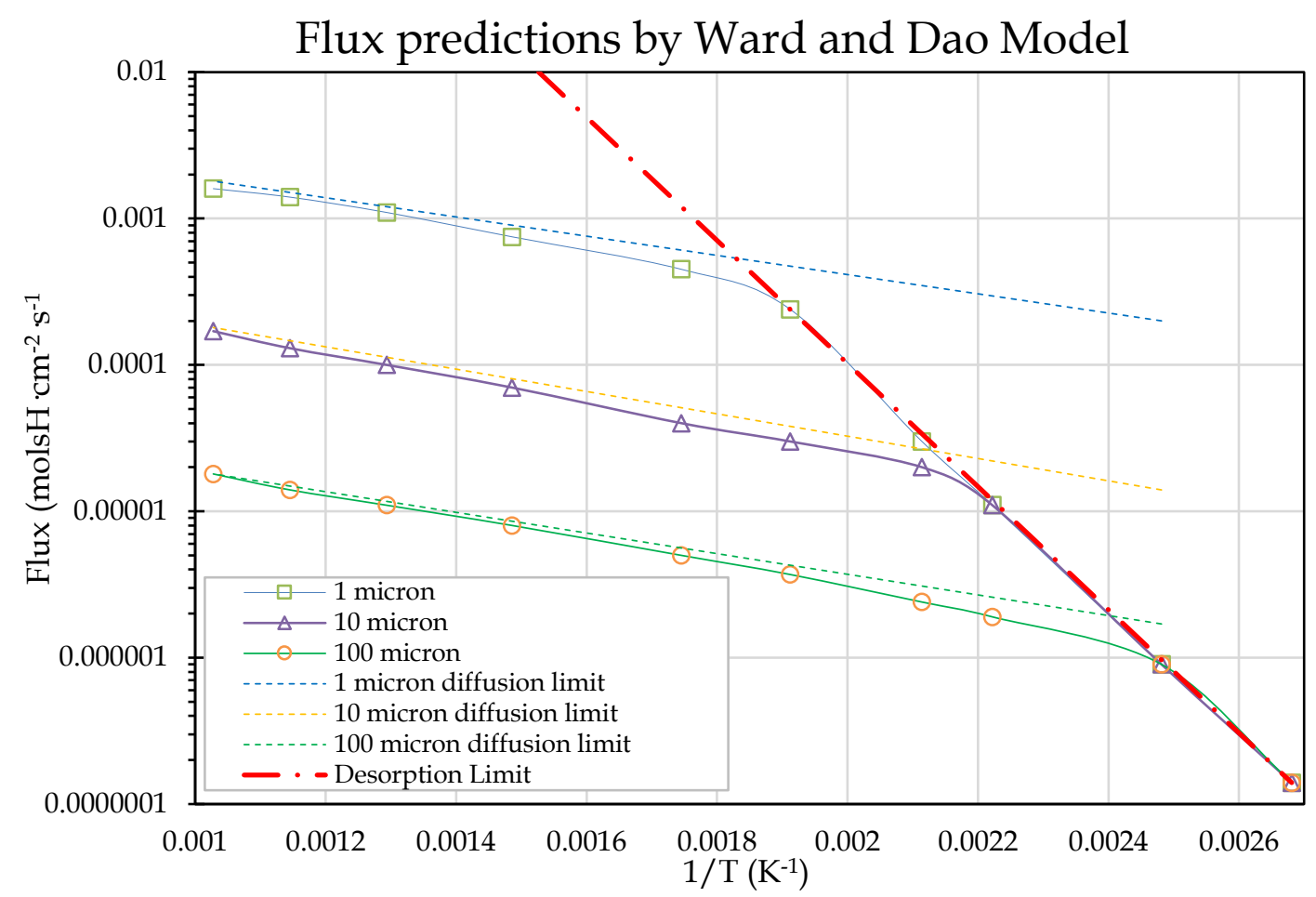

Figure 5.2.37: Reproduction of a Plot of calculations by Ward and Dao that predict hydrogen flux at different temperature for three different thicknesses of palladium [112]. 
Jonathan Tailby

5.2.7.4.1. Arrhenius Activation Energy Calculations for Tertiary Inserts

\section{Calculation of Activation Energy from the Rate of Ammonia Decomposition}

Table 5.2.25 calculates the data for the Arrhenius plots from the rate of ammonia decomposition. Figure 5.2.38 shows the Arrhenius plots and

Table 5.2.26 summarises the final steps of calculating activation energy from the gradients in the Arrhenius plots.

Table 5.2.25: Calculating Data for Arrhenius plots of Ammonia decomposition on Tertiary Insert Nickel Foam Catalysts

\begin{tabular}{|c|c|c|c|c|c|}
\hline Temperature $\left({ }^{\circ} \mathrm{C}\right)$ & 300 & 350 & 400 & 450 & \\
\hline Temperature (K) & 573 & 623 & 673 & 723 & \\
\hline $\mathrm{T}^{-1}$ & 0.001745 & 0.001605 & 0.001486 & 0.001383 & $\mathrm{~K}^{-1}$ \\
\hline Nickel Before & $3.64 \mathrm{E}-07$ & $1.05 \mathrm{E}-06$ & $5.41 E-06$ & $2.44 \mathrm{E}-05$ & $\mathrm{~mol} \cdot \mathrm{min}^{-1}$ \\
\hline $\ln k$ & $-1.48 \mathrm{E}+01$ & $-1.38 \mathrm{E}+01$ & $-1.21 \mathrm{E}+01$ & $-1.06 \mathrm{E}+01$ & \\
\hline Nickel On & $3.02 \mathrm{E}-07$ & $2.64 \mathrm{E}-06$ & $1.06 \mathrm{E}-05$ & 4.18E-05 & $\mathrm{mol} \cdot \mathrm{min}^{-1}$ \\
\hline $\ln k$ & $-1.50 \mathrm{E}+01$ & $-1.28 \mathrm{E}+01$ & $-1.15 \mathrm{E}+01$ & $-1.01 \mathrm{E}+01$ & \\
\hline Nickel On No Perm & $2.51 \mathrm{E}-07$ & 8.82E-08 & 9.35E-07 & 9.34E-06 & $\mathrm{mol} \cdot \mathrm{min}^{-1}$ \\
\hline $\ln k$ & $-1.52 \mathrm{E}+01$ & $-1.62 \mathrm{E}+01$ & $-1.39 \mathrm{E}+01$ & $-1.16 \mathrm{E}+01$ & \\
\hline
\end{tabular}




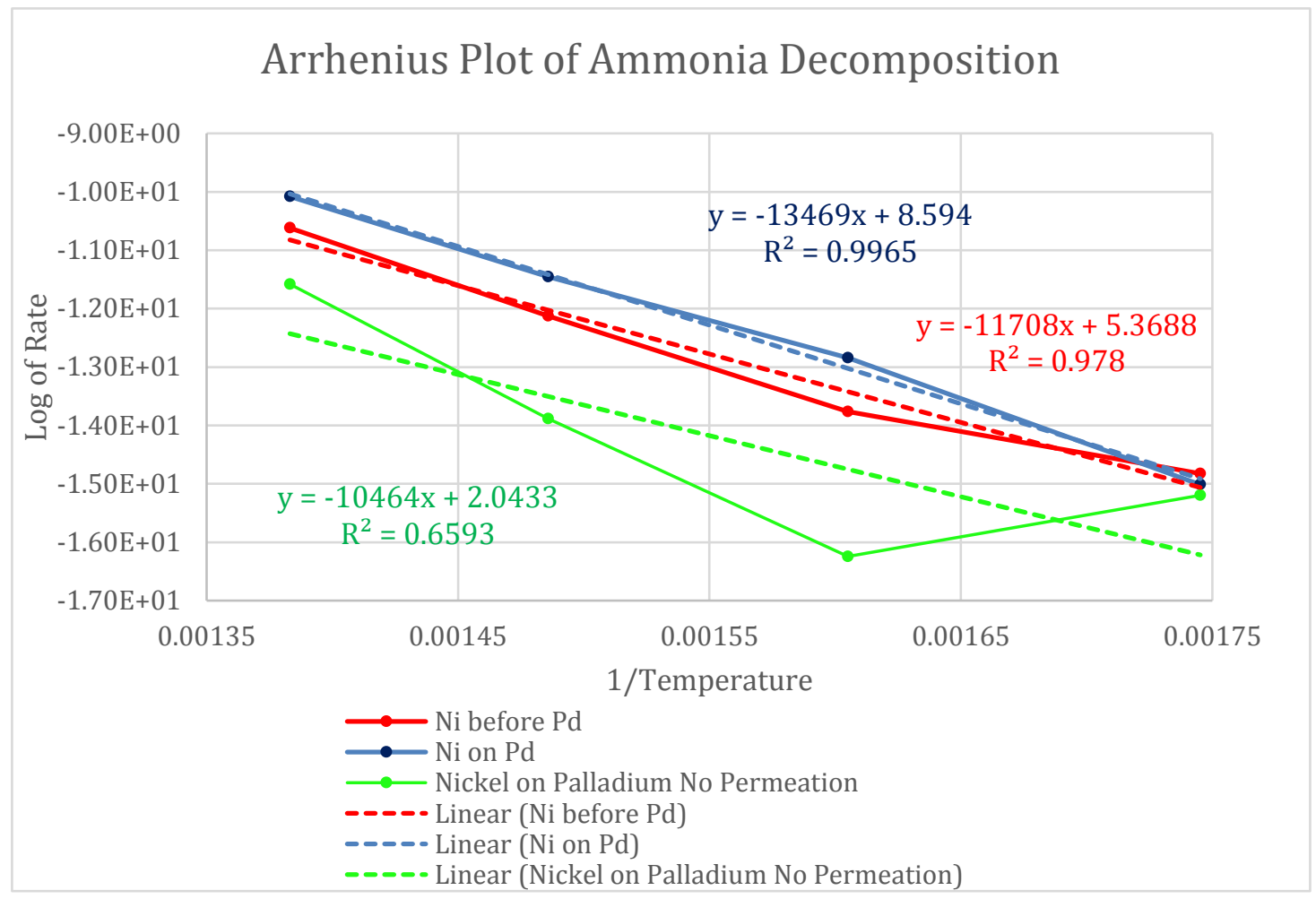

Figure 5.2.38: Arrhenius plot of Ammonia Decomposition on Tertiary Insert Nickel Foam Catalysts.

Table 5.2.26: Calculation of Activation Energy from Arrhenius Plot of Ammonia Decomposition on Tertiary Insert Nickel Foam Catalysts

\begin{tabular}{|l|l|l|l|l|}
\hline & Constant & Slope & $-\mathrm{R} \cdot \mathrm{m}$ & $\mathrm{E}_{\mathrm{a}}$ \\
\hline Nickel Before & 5.3688 & -11708 & 97340 & 97.3 \\
\hline Nickel On & 8.594 & -13469 & 111981 & 112.0 \\
\hline Nickel on no Perm & 2.0433 & -10464 & 86998 & 87.0 \\
\hline & & & $\mathrm{J} \cdot \mathrm{mol}^{-1}$ & $\mathrm{~kJ} \mathrm{~mol}^{-1}$ \\
\hline
\end{tabular}

\section{Calculation of Activation Energy from the Rate of Nitrogen Formation}

The Arrhenius method is repeated for the rate of nitrogen production for each of the tertiary insert nickel foam catalysts. Table 5.2.27 calculates the data for the Arrhenius plots shown in Figure 5.2.39.

Table 5.2.28 shows the Activation energy calculated from the Arrhenius plots in Figure 5.2.39.

Table 5.2.27: Calculating the data for Arrhenius plots of the rates of nitrogen formation on Tertiary inserts nickel foam catalysts.

\begin{tabular}{|l|l|l|l|l|l|}
\hline Temperature $\left({ }^{\circ} \mathrm{C}\right)$ & 300 & 350 & 400 & 450 & \\
\hline Temperature $(\mathrm{K})$ & 573 & 623 & 673 & 723 & \\
\hline $1 / \mathrm{T}$ & 0.001745 & 0.001605 & 0.001486 & 0.001383 & $\mathrm{~K}^{-1}$ \\
\hline
\end{tabular}


Jonathan Tailby

\begin{tabular}{|c|l|l|l|l|l|}
\hline Nickel Before & $1.15 \mathrm{E}-07$ & $7.96 \mathrm{E}-07$ & $4.75 \mathrm{E}-06$ & $1.53 \mathrm{E}-05$ & $\mathrm{~mol} \mathrm{~min}^{-1}$ \\
\hline $\ln k$ & $-1.60 \mathrm{E}+01$ & $-1.40 \mathrm{E}+01$ & $-1.23 \mathrm{E}+01$ & $-1.11 \mathrm{E}+01$ & \\
\hline Nickel On & $2.64 \mathrm{E}-07$ & $1.41 \mathrm{E}-06$ & $8.37 \mathrm{E}-06$ & $3.97 \mathrm{E}-05$ & $\mathrm{~mol} \mathrm{~min}^{-1}$ \\
\hline $\ln k$ & $-1.51 \mathrm{E}+01$ & $-1.35 \mathrm{E}+01$ & $-1.17 \mathrm{E}+01$ & $-1.01 \mathrm{E}+01$ & \\
\hline Nickel On No Perm & $3.21 \mathrm{E}-07$ & $7.95 \mathrm{E}-07$ & $3.59 \mathrm{E}-06$ & $1.88 \mathrm{E}-05$ & $\mathrm{~mol} \mathrm{~min}^{-1}$ \\
\hline $\ln k$ & $-1.50 \mathrm{E}+01$ & $-1.40 \mathrm{E}+01$ & $-1.25 \mathrm{E}+01$ & $-1.09 \mathrm{E}+01$ & \\
\hline
\end{tabular}

\section{Arrhenius Plot of Nitrogen Formation}

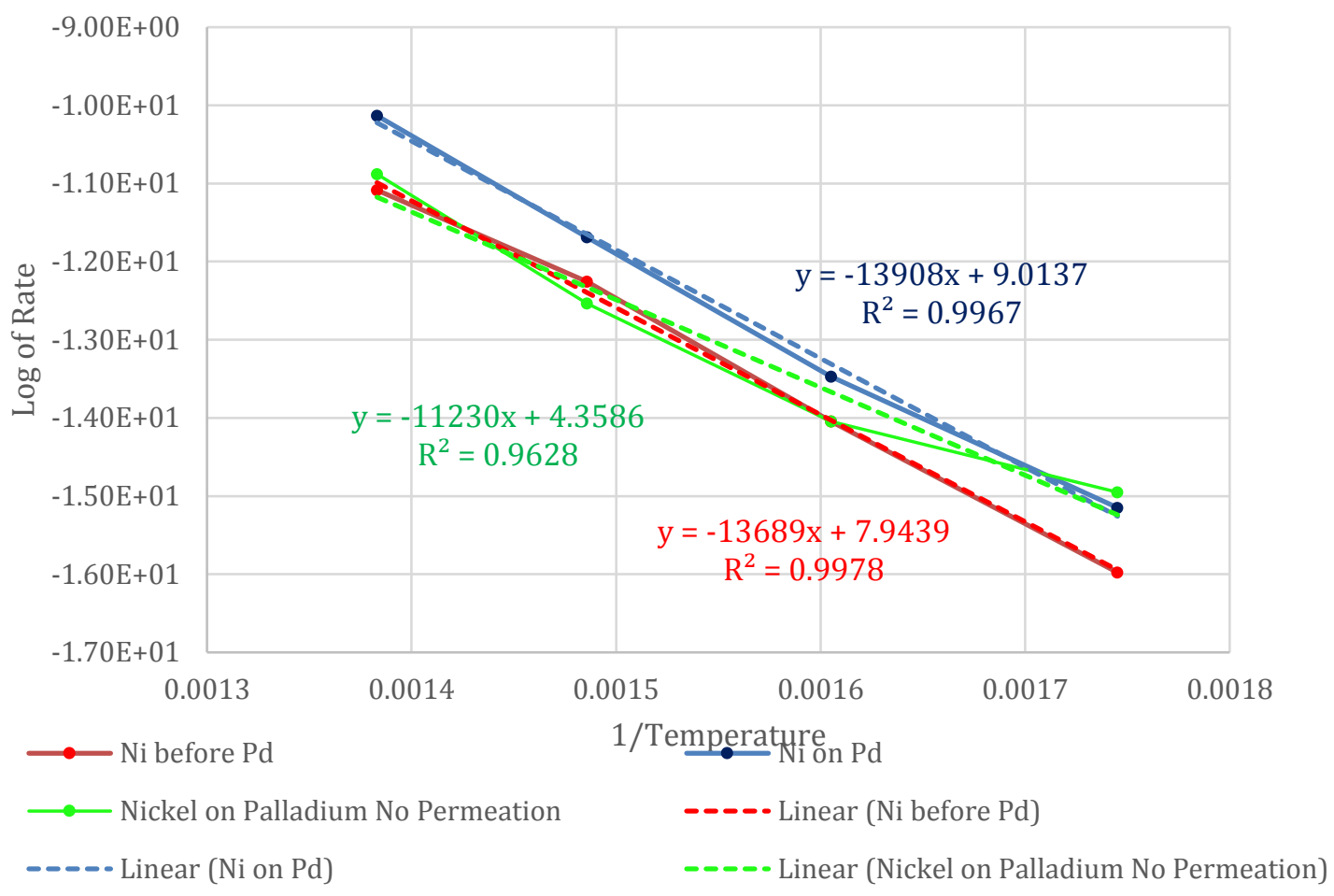

Figure 5.2.39: Arrhenius plots for nitrogen formation on the tertiary insert nickel foam catalysts.

Table 5.2.28: Calculating the activation energy from the Arrhenius plots for nitrogen formation on tertiary insert nickel foam catalysts.

\begin{tabular}{|l|l|l|l|l|}
\hline & Constant & Slope & $-\mathrm{R} \mathrm{m}$ & $\mathrm{E}_{\mathrm{a}}$ \\
\hline Nickel Before & 7.9439 & -13689 & 113810 & 113.8 \\
\hline Nickel On & 9.0137 & -13908 & 115631 & 115.6 \\
\hline Nickel on no Perm & 4.3586 & -11230 & 93366 & 93.4 \\
\hline
\end{tabular}

\section{Calculation of Activation Energy from the Rate of Hydrogen Formation}

The different rates of hydrogen formation for the tertiary catalysts are more difficult to interpret because of the interaction of the formation and the permeation 
reactions. Nevertheless, activation energy was calculated for each hydrogen formation rate and they are included in Table 3.3.7 in Section 3.3.3.1.1.

Table 5.2.29 calculates the data for the Arrhenius plot based on the Retentate hydrogen rate for the tertiary insert catalysts. Figure 5.2.40 is the Arrhenius plot for Retentate Hydrogen for the Tertiary insert catalysts. Table 5.2.30 calculates activation energy from the Arrhenius plot for Retentate Hydrogen on the Tertiary insert catalysts.

Table 5.2.29: Calculating Data for Arrhenius plots of Retentate Hydrogen formation on Tertiary Insert Nickel Foam Catalysts.

\begin{tabular}{|l|l|l|l|l|l|}
\hline & & & & & \\
\hline Temperature $\left({ }^{\circ} \mathrm{C}\right)$ & 300 & 350 & 400 & 450 & \\
\hline Temperature $(\mathrm{K})$ & 573 & 623 & 673 & 723 & \\
\hline $\mathrm{T}^{-1}$ & 0.001745 & 0.001605 & 0.001486 & 0.001383 & $\mathrm{~K}-1$ \\
\hline Nickel Before & $3.40 \mathrm{E}-07$ & $6.64 \mathrm{E}-07$ & $6.61 \mathrm{E}-06$ & $2.37 \mathrm{E}-05$ & $\mathrm{~mol} \mathrm{~min}^{-1}$ \\
\hline $\ln k$ & $-1.49 \mathrm{E}+01$ & $-1.42 \mathrm{E}+01$ & $-1.19 \mathrm{E}+01$ & $-1.06 \mathrm{E}+01$ & \\
\hline Nickel On & $2.49 \mathrm{E}-07$ & $2.59 \mathrm{E}-06$ & $1.46 \mathrm{E}-05$ & $5.77 \mathrm{E}-05$ & $\mathrm{~mol} \mathrm{~min}^{-1}$ \\
\hline $\ln k$ & $-1.52 \mathrm{E}+01$ & $-1.29 \mathrm{E}+01$ & $-1.11 \mathrm{E}+01$ & $-9.76 \mathrm{E}+00$ & \\
\hline Nickel On No Perm & $7.60 \mathrm{E}-07$ & $1.93 \mathrm{E}-06$ & $1.00 \mathrm{E}-05$ & $3.99 \mathrm{E}-05$ & $\mathrm{~mol} \mathrm{~min}^{-1}$ \\
\hline $\ln k$ & $-1.41 \mathrm{E}+01$ & $-1.32 \mathrm{E}+01$ & $-1.15 \mathrm{E}+01$ & $-1.01 \mathrm{E}+01$ & \\
\hline
\end{tabular}




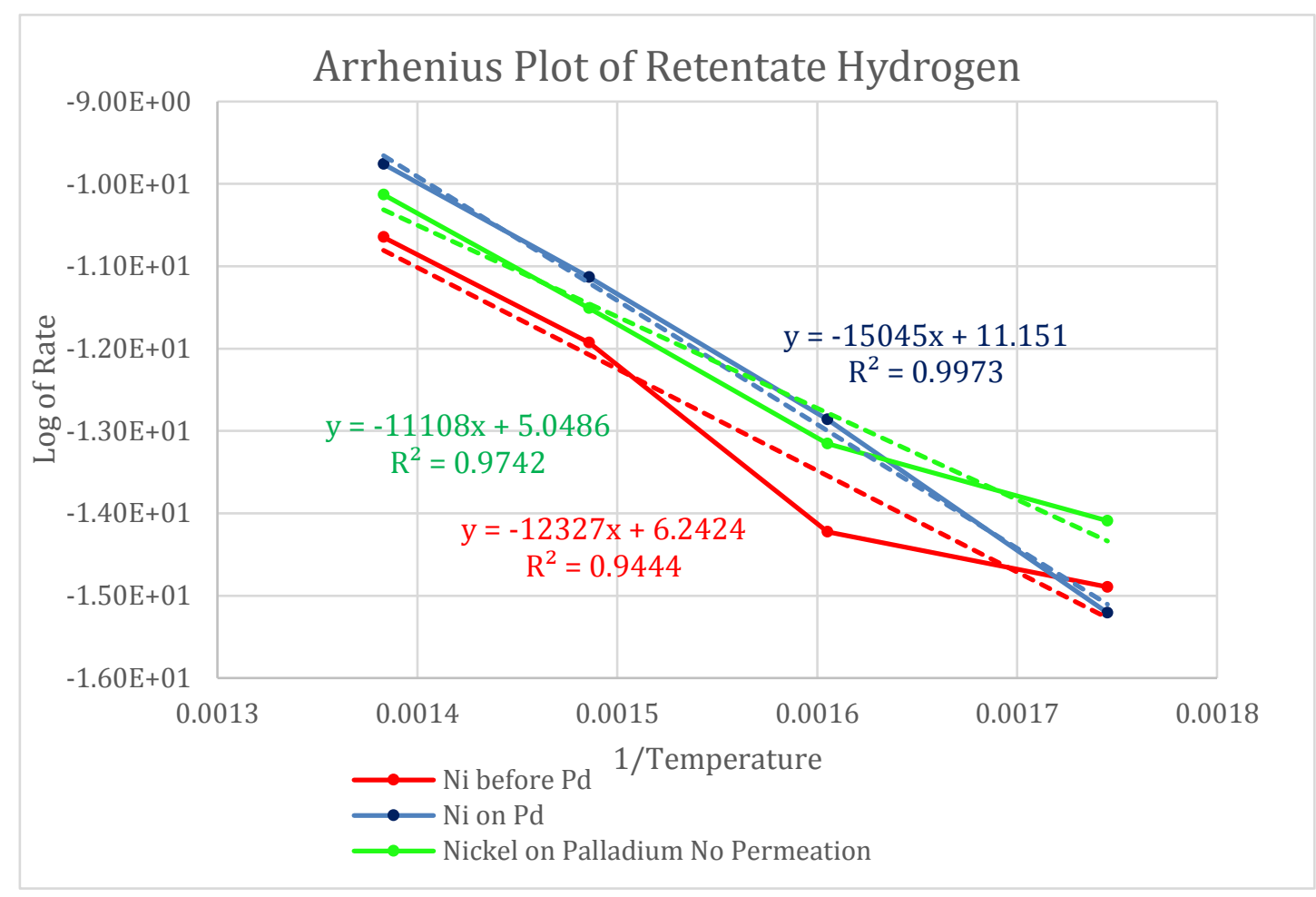

Figure 5.2.40: Arrhenius plots for retentate hydrogen formation on the tertiary insert nickel foam catalysts.

Table 5.2.30: Calculating the activation energy from the Arrhenius plots for retentate hydrogen formation on tertiary insert nickel foam catalysts.

\begin{tabular}{|l|l|l|l|l|}
\hline & Constant & Slope & $-\mathrm{R} \cdot \mathrm{m}$ & $\mathrm{E}_{\mathrm{a}}$ \\
\hline Nickel Before & 6.2424 & -12327 & 102487 & 102.5 \\
\hline Nickel On & 11.151 & -15045 & 125084 & 125.1 \\
\hline Nickel on no Perm & 5.0486 & -11108 & 92352 & 92.4 \\
\hline & & & $\mathrm{J} \mathrm{mol}^{-1}$ & $\mathrm{~kJ} \mathrm{~mol}^{-1}$ \\
\hline
\end{tabular}

Table 5.2.31 calculates the data for the Arrhenius plot based on the permeate hydrogen rate for the tertiary insert catalysts. Figure 5.2.41 shows the Arrhenius plot for permeate hydrogen on Tertiary Insert Catalysts.

Table 5.2.32 calculates the activation energy for permeate hydrogen on the tertiary insert catalysts.

Table 5.2.31: Calculating Data for Arrhenius plots of Permeate Hydrogen formation on Tertiary Insert Nickel Foam Catalysts.

\begin{tabular}{|c|l|l|l|l|l|}
\hline Temperature $\left({ }^{\circ} \mathrm{C}\right)$ & 300 & 350 & 400 & 450 & \\
\hline Temperature $(\mathrm{K})$ & 573 & 623 & 673 & 723 & \\
\hline $\mathrm{T}^{-1}$ & 0.001745 & 0.001605 & 0.001486 & 0.001383 & $\mathrm{~K}-1$ \\
\hline Nickel Before & $1.27 \mathrm{E}-08$ & $7.23 \mathrm{E}-07$ & $4.60 \mathrm{E}-06$ & $1.70 \mathrm{E}-05$ & $\mathrm{~mol} \mathrm{~min}^{-1}$ \\
\hline $\ln k$ & $-1.82 \mathrm{E}+01$ & $-1.41 \mathrm{E}+01$ & $-1.23 \mathrm{E}+01$ & $-1.10 \mathrm{E}+01$ & \\
\hline Nickel On & $1.10 \mathrm{E}-07$ & $1.08 \mathrm{E}-06$ & $3.35 \mathrm{E}-06$ & $1.23 \mathrm{E}-05$ & $\mathrm{~mol} \mathrm{~min}^{-1}$ \\
\hline $\ln k$ & $-1.60 \mathrm{E}+01$ & $-1.37 \mathrm{E}+01$ & $-1.26 \mathrm{E}+01$ & $-1.13 \mathrm{E}+01$ & \\
\hline
\end{tabular}




\begin{tabular}{|c|l|l|l|l|l|}
\hline Nickel On No Perm & $0.00 \mathrm{E}+00$ & $0.00 \mathrm{E}+00$ & $0.00 \mathrm{E}+00$ & $0.00 \mathrm{E}+00$ & $\mathrm{~mol} \mathrm{~min}^{-1}$ \\
\hline $\ln k$ & - & - & - & - & \\
\hline
\end{tabular}

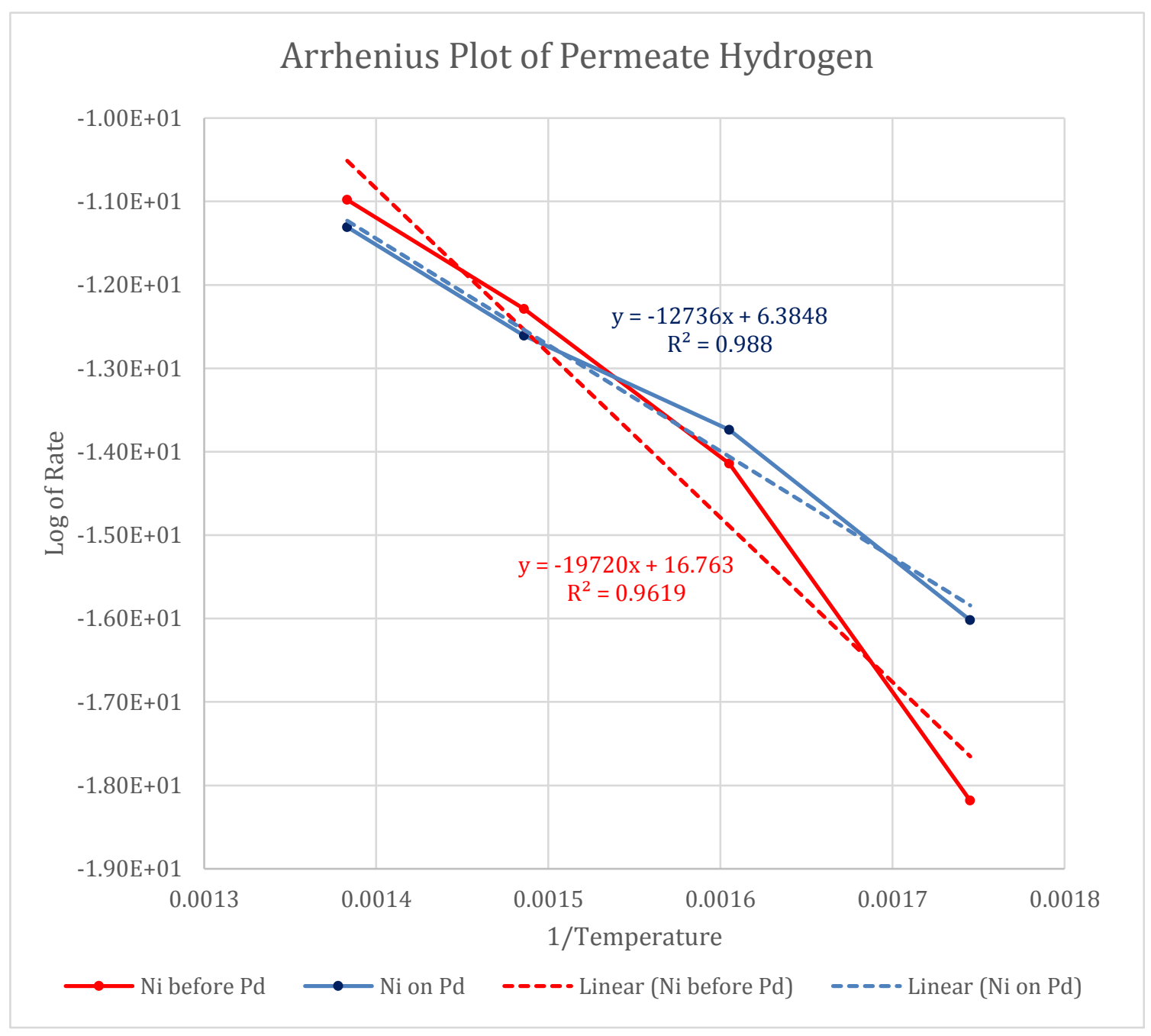

Figure 5.2.41: Arrhenius plots for Permeate hydrogen formation on the tertiary insert nickel foam catalysts.

Table 5.2.32: Calculating the activation energy from the Arrhenius plots for Permeate hydrogen formation on tertiary insert nickel foam catalysts.

\begin{tabular}{|l|l|l|l|l|}
\hline & Constant & Slope & $-\mathrm{R} \cdot \mathrm{m}$ & $\mathrm{E}_{\mathrm{a}}$ \\
\hline Nickel Before & 6.2424 & -12327 & 102487 & 102.5 \\
\hline Nickel On & 11.151 & -15045 & 125084 & 125.1 \\
\hline & & & $\mathrm{J} \mathrm{mol}^{-1}$ & $\mathrm{~kJ} \mathrm{~mol}^{-1}$ \\
\hline
\end{tabular}

Table 5.2.33 calculates the data for the Arrhenius plot based on the total hydrogen rate for the tertiary insert catalysts. Figure 5.2.42 is the Arrhenius plot for Total Hydrogen for the Tertiary insert catalysts. 
Jonathan Tailby

Table 5.2.34 calculates activation energy from the Arrhenius plot for Total Hydrogen on the Tertiary insert catalysts.

Table 5.2.33: Calculating Data for Arrhenius plots of Total Hydrogen formation on Tertiary Insert Nickel Foam Catalysts.

\begin{tabular}{|c|l|l|l|l|l|}
\hline Temperature $\left({ }^{\circ} \mathrm{C}\right)$ & 300 & 350 & 400 & 450 & \\
\hline Temperature $(\mathrm{K})$ & 573 & 623 & 673 & 723 & \\
\hline $\mathrm{T}^{-1}$ & 0.001745 & 0.001605 & 0.001486 & 0.001383 & $\mathrm{~K}-1$ \\
\hline Nickel Before & $3.53 \mathrm{E}-07$ & $1.39 \mathrm{E}-06$ & $1.12 \mathrm{E}-05$ & $4.08 \mathrm{E}-05$ & $\mathrm{~mol} \mathrm{~min}^{-1}$ \\
\hline $\ln k$ & $-1.49 \mathrm{E}+01$ & $-1.35 \mathrm{E}+01$ & $-1.14 \mathrm{E}+01$ & $-1.01 \mathrm{E}+01$ & \\
\hline Nickel On & $3.59 \mathrm{E}-07$ & $3.67 \mathrm{E}-06$ & $1.80 \mathrm{E}-05$ & $7.00 \mathrm{E}-05$ & $\mathrm{~mol} \mathrm{~min}^{-1}$ \\
\hline $\ln k$ & $-1.48 \mathrm{E}+01$ & $-1.25 \mathrm{E}+01$ & $-1.09 \mathrm{E}+01$ & $-9.57 \mathrm{E}+00$ & \\
\hline Nickel On No Perm & $7.60 \mathrm{E}-07$ & $1.93 \mathrm{E}-06$ & $1.00 \mathrm{E}-05$ & $3.99 \mathrm{E}-05$ & $\mathrm{~mol} \mathrm{~min}^{-1}$ \\
\hline $\ln k$ & $-1.41 \mathrm{E}+01$ & $-1.32 \mathrm{E}+01$ & $-1.15 \mathrm{E}+01$ & $-1.01 \mathrm{E}+01$ & \\
\hline
\end{tabular}

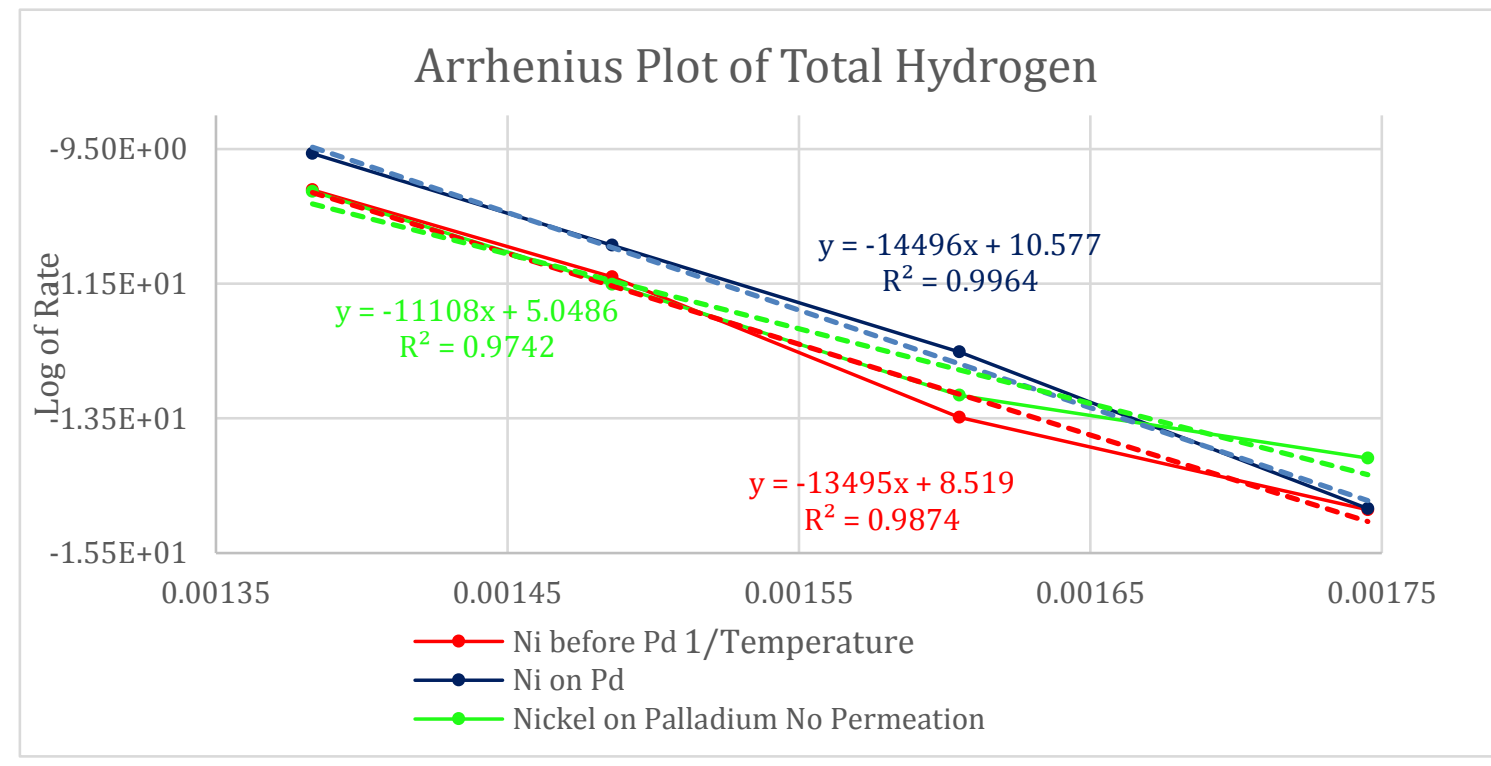

Figure 5.2.42: Arrhenius plots for Total hydrogen formation on the tertiary insert nickel foam catalysts.

Table 5.2.34: Calculating the activation energy from the Arrhenius plots for Total hydrogen formation on tertiary insert nickel foam catalysts.

\begin{tabular}{|l|l|l|l|l|}
\hline & Constant & Slope & $-\mathrm{R} \mathrm{m}$ & $\mathrm{E}_{\mathrm{a}}$ \\
\hline Nickel Before & 8.519 & -13495 & 112197.4 & 112 \\
\hline Nickel On & 10.577 & -14496 & 92351.9 & 92 \\
\hline Nickel on no Perm & 5.0486 & -11108 & 92363.0 & 92 \\
\hline & & & $\mathrm{J} \cdot \mathrm{mol}^{-1}$ & $\mathrm{~kJ} \cdot \mathrm{mol}^{-1}$ \\
\hline
\end{tabular}




\subsection{Flow series}

Figure 5.2.43 shows typical TCD chromatograms for the flow experiments with the Nickel-on-Palladium-no-Permeation insert. Decreasing the rate of ammonia flow was found to increase the proportion of ammonia in the remnant gas, and to increase the proportion of product gases, however the rate of ammonia decomposition increased because more reactant was available.

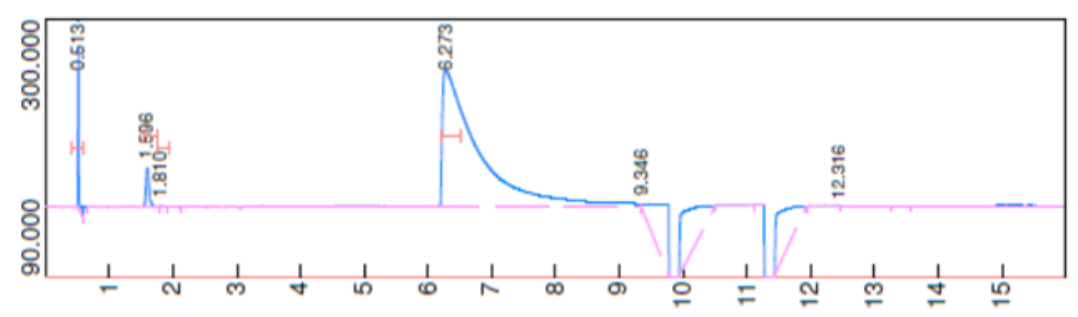

Figure 5.2.43a: $5 \mathrm{~mL} \mathrm{~min}^{-1}$.

Hydrogen area=108.1. Nitrogen area=3.98. Ammonia area=4370.9 to 9.346 minutes

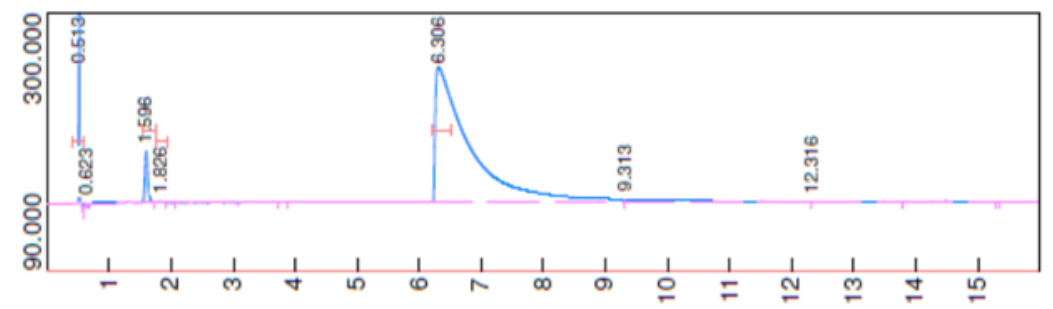

Figure 5.2.43b: $3 \mathrm{~mL} \cdot \mathrm{min}^{-1}$.

Hydrogen area $=139.6$. Nitrogen area $=4.72$. Ammonia area $=4229.3$ to 9.313 minutes

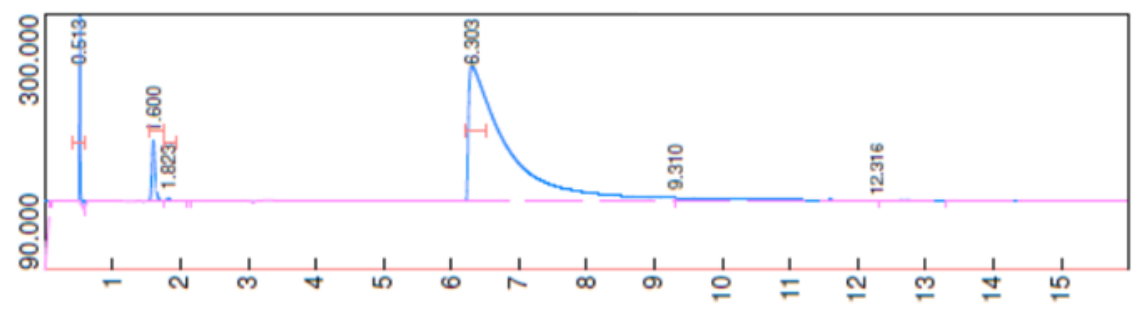

Figure 5.2.43c: $1 \mathrm{~mL} \cdot \mathrm{min}^{-1}$.

Hydrogen area=167.6. Nitrogen area $=10.73$. Ammonia area $=4271.8$ to 9.310 minutes

Figure 5.2.43: Chromatograms for decomposition of ammonia with the foam catalyst in contact with palladium foil (Nickel-on-Palladium-no-Permeation), ammonia flow from 1 to $5 \mathrm{~mL} \cdot \mathrm{min}^{-1}$.

Temperature of $350{ }^{\circ} \mathrm{C}$.

Table 5.2.35 shows the calculation of permeate output for the flow series with the Nickel-before-Palladium insert and the Nickel-on-Palladium insert. 


\begin{tabular}{|c|c|c|c|c|c|c|c|}
\hline Input flow & 6 & 5 & 4 & 3 & 2 & 1 & $\mathrm{~mL} \mathrm{~min}^{-1}$ \\
\hline Input flow & $2.41 \mathrm{E}-04$ & $2.01 \mathrm{E}-04$ & \begin{tabular}{|l|}
$1.61 \mathrm{E}-04$ \\
\end{tabular} & $1.21 \mathrm{E}-04$ & $8.04 \mathrm{E}-05$ & $4.02 \mathrm{E}-05$ & $\mathrm{~mol} \mathrm{~min} \mathrm{~min}^{-1}$ \\
\hline \multicolumn{8}{|l|}{$\begin{array}{l}\text { Nickel-before- } \\
\text { Palladium }\end{array}$} \\
\hline Permeate peak & 6.8 & 9.2 & 11.5 & 12.9 & 15.2 & 17.9 & Peak Area \\
\hline $\begin{array}{l}\text { Hydrogen } \\
\text { standard }\end{array}$ & 538.0 & 538.0 & 538.0 & 538.0 & 538.0 & 538.0 & Peak Area \\
\hline $\begin{array}{l}\text { Permeate } \\
\text { percent }\end{array}$ & $0.063 \%$ & $0.086 \%$ & $0.107 \%$ & $0.120 \%$ & $0.142 \%$ & $0.167 \%$ & $\%$ \\
\hline $\begin{array}{l}\text { Rate of } \\
\text { Permeation }\end{array}$ & $1.52 \mathrm{E}-07$ & $1.73 \mathrm{E}-07$ & $1.72 \mathrm{E}-07$ & $1.45 \mathrm{E}-07$ & $1.14 \mathrm{E}-07$ & $6.72 \mathrm{E}-08$ & mol min $^{-1}$ \\
\hline \multicolumn{8}{|l|}{$\begin{array}{l}\text { Nickel-on- } \\
\text { Palladium }\end{array}$} \\
\hline Permeate peak & & 50.2 & & 58.1 & & 70.6 & Peak Area \\
\hline $\begin{array}{l}\text { Hydrogen } \\
\text { standard }\end{array}$ & & 497.5 & & 497.5 & & 497.5 & Peak Area \\
\hline $\begin{array}{l}\text { Permeate } \\
\text { percent }\end{array}$ & & $0.50 \%$ & & $0.58 \%$ & & $0.71 \%$ & $\%$ \\
\hline $\begin{array}{l}\text { Rate of } \\
\text { Permeation }\end{array}$ & & $1.02 \mathrm{E}-06$ & & 7.09E-07 & & $2.88 \mathrm{E}-07$ & mol min $^{-1}$ \\
\hline
\end{tabular}




\section{References}

1. Abbas, H.F. and W.M.A. Wan Daud, Hydrogen production by methane decomposition: A review. International Journal of Hydrogen Energy, 2010. 35(3): p. 1160-1190.

2. Matsumura, Y. and J. Tong, Methane Steam Reforming in Hydrogen-permeable Membrane Reactor for Pure Hydrogen Production. Topics in Catalysis, 2008: p. 1-10.

3. Iulianelli, A., T. Longo, and A. Basile, Methanol steam reforming reaction in a Pd-Ag membrane reactor for $\mathrm{CO}$-free hydrogen production. International Journal of Hydrogen Energy, 2008. 33(20): p. 5583-5588.

4. Metkemeijer, R. and P. Achard, Comparison of ammonia and methanol applied indirectly in a hydrogen fuel cell. International Journal of Hydrogen Energy, 1994. 19(6): p. 535-542.

5. Dicks, A.L., Hydrogen generation from natural gas for the fuel cell systems of tomorrow. Journal of Power Sources, 1996. 61(1-2): p. 113-124.

6. Schlapbach, L., Technology: Hydrogen-fuelled vehicles. Nature, 2009. 460(7257): p. 809-811.

7. Brown, L.F., A comparative study of fuels for on-board hydrogen production for fuel-cellpowered automobiles. International Journal of Hydrogen Energy, 2001. 26(4): p. 381397.

8. Gallucci, F., et al., Methanol and ethanol steam reforming in membrane reactors: An experimental study. International Journal of Hydrogen Energy, 2007. 32(9): p. 12011210.

9. Umegaki, T., et al., Hydrogen production via steamre forming of ethyl alcohol over palladium/ indium oxide catalyst. Research Letters in Physical Chemistry, 2009. 2009.

10. Alagharu, V., S. Palanki, and K.N. West, Analysis of ammonia decomposition reactor to generate hydrogen for fuel cell applications. Journal of Power Sources, 2010. 195(3): p. 829-833.

11. Chellappa, A.S., C.M. Fischer, and W.J. Thomson, Ammonia decomposition kinetics over Ni-Pt/Al2O3 for PEM fuel cell applications. Applied Catalysis A: General, 2002. 227(1-2): p. 231-240.

12. Chiuta, S., et al., Reactor technology options for distributed hydrogen generation via ammonia decomposition: A review. International Journal of Hydrogen Energy, 2013. 38(35): p. 14968-14991.

13. Stone, C. and A.E. Morrison, From curiosity to "power to change the worldÂA". Solid State Ionics, 2002. 152-153: p. 1-13.

14. Justi, E.W., Seventy years fuel cell research. British Journal of Applied Physics, 1963. 14(12): p. 840-853.

15. Hamrock, S.J. and M.A. Yandrasits, Proton exchange membranes for fuel cell applications. Polymer Reviews, 2006. 46(3): p. 219-244.

16. Wu, J.P., et al., Palladium coated porous anodic alumina membranes for gas reforming processes. Solid State Sciences, 2010. 12(11): p. 1912-1916.

17. Byron Smith, R.J., L. Muruganandam, and S. Murthy Shekhar, CFD analysis of water gas shift membrane reactor. Chemical Engineering Research and Design, 2011. 89(11): p. 2448-2456. 
18. Huang, D.C., et al., Preparation of Ru-Cs catalyst and its application on hydrogen production by ammonia decomposition. International Journal of Hydrogen Energy, 2012.

19. Bell, T.E. and L. Torrente-Murciano, H2 Production via Ammonia Decomposition Using Non-Noble Metal Catalysts: A Review. Topics in Catalysis, 2016. 59(15-16): p. 1438-1457.

20. Hashimoto, K. and N. Toukai, Decomposition of ammonia over a catalyst consisting of rutheniurn metal and cerium oxides supported on Y-form zeolite. Journal of Molecular Catalysis A: Chemical, 2000. 161(1-2): p. 171-178.

21. Papapolymerou, G. and V. Bontozoglou, Decomposition of NH3 on Pd and Ir comparison with Pt and Rh. Journal of Molecular Catalysis A: Chemical, 1997. 120(13): p. 165-171.

22. Jedynak, A., et al., Ammonia decomposition over the carbon-based iron catalyst promoted with potassium. Applied Catalysis A: General, 2002. 237(1-2): p. 223-226.

23. Ohtsuka, Y., et al., Decomposition of ammonia with iron and calcium catalysts supported on coal chars. Fuel, 2004. 83(6): p. 685-692.

24. Zhang, J., H. Xu, and W. Li, High-purity COx-free $\mathrm{H} 2$ generation from NH3 via the ultra permeable and highly selective Pd membranes. Journal of Membrane Science, 2006. 277(1-2): p. 85-93.

25. Rei, M.H., et al., Catalysis-spillover-membrane. III: The effect of hydrogen spillover on the palladium membrane reactor in the steam reforming reactions. Journal of Membrane Science, 2011.

26. Conner Jr, W.C. and J.L. Falconer, Spillover in heterogeneous catalysis. Chemical Reviews, 1995. 95(3): p. 759-788.

27. Prins, R., Hydrogen spillover. Facts and fiction. Chemical Reviews, 2012. 112(5): p. 2714-2738.

28. Rei, M.H., A decade's study and developments of palladium membrane in Taiwan. Journal of the Taiwan Institute of Chemical Engineers, 2009. 40(3): p. 238-245.

29. Energy, U.S.D.o. Multi-Year Research, Development and Demonstration Plan: Planned Program Activities for 2005-2015

2009 April 2009 [cited 201128 July]; Available from: http://www1.eere.energy.gov/hydrogenandfuelcells/mypp/pdfs/storage.pdf.

30. Stetson, N.T., FY 2012 Progress Report For the DoE Hydrogen and Fuel Cells Program S. Satyapal, Editor. 2012, US Department of Energy.

31. Energy, U.S.D.o. Multi-Year Research, Development and Demonstration Plan: Planned Program Activities for 2011-2020

2011 June 2015 [cited 201625 September]; Available from: http://energy.gov/sites/prod/files/2015/05/f22/fcto_myrdd_storage.pdf.

32. Das, D. and T.N. VeziroÇelu, Hydrogen production by biological processes: A survey of literature. International Journal of Hydrogen Energy, 2001. 26(1): p. 13-28.

33. Miltner, A., et al., Renewable hydrogen production: A technical evaluation based on process simulation. Journal of Cleaner Production, 2010. 18(SUPPL. 1).

34. Singh, S., et al., Hydrogen: A sustainable fuel for future of the transport sector. Renewable and Sustainable Energy Reviews, 2015. 51: p. 623-633. 
35. Fujishima, A. and K. Honda, Electrochemical photolysis of water at a semiconductor electrode. Nature, 1972. 238(5358): p. 37-38.

36. Azad, A. and S.J. Kim, A unique approach for high performance photoelectrochemical water splitting: Utilizing coating and doping methods. Materials Research Bulletin, 2016. 84: p. 474-479.

37. Grimes, C.A., et al. Photonic fuels and photovoltaics: Application of self-assembled 1D TiO2 nanotube/wire arrays. in ACS National Meeting Book of Abstracts. 2010.

38. Li, L., et al., Sub-10 nm rutile titanium dioxide nanoparticles for efficient visible-lightdriven photocatalytic hydrogen production. Nature Communications, 2015. 6(5881): p. 10 .

39. May, M.M., et al., Efficient direct solar-to-hydrogen conversion by in situ interface transformation of a tandem structure. Nature Communications, 2015. 6.

40. Li, L. and J.A. Hurley, Ammonia-based hydrogen source for fuel cell applications. International Journal of Hydrogen Energy, 2007. 32(1): p. 6-10.

41. Jena, P., Materials for hydrogen storage: Past, present, and future. Journal of Physical Chemistry Letters, 2011. 2(3): p. 206-211.

42. Principi, G., et al., The problem of solid state hydrogen storage. Energy, 2009. 34(12): p. 2087-2091.

43. Eberle, U., M. Felderhoff, and F. Schüth, Chemical and physical solutions for hydrogen storage. Angewandte Chemie - International Edition, 2009. 48(36): p. 6608-6630.

44. Broom, D.P., et al., Outlook and challenges for hydrogen storage in nanoporous materials. Applied Physics A, 2016. 122(3): p. 1-21.

45. Züttel, A., Materials for hydrogen storage. Materials Today, 2003. 6(9): p. 24-33.

46. Orimo, S.I., et al., Complex hydrides for hydrogen storage. Chemical Reviews, 2007. 107(10): p. 4111-4132.

47. Woolf, H., Light Metal Boronitrides, in School of Chemical and Physical Sciences. 2007, Victoria University: Wellington. p. 165.

48. Yu, X.B., D.M. Grant, and G.S. Walker, A new dehydrogenation mechanism for reversible multicomponent borohydride systems - The role of $\mathrm{Li}-\mathrm{Mg}$ alloys. Chemical Communications, 2006(37): p. 3906-3908.

49. Yamamoto, H., et al., Recyclable hydrogen storage system composed of ammonia and alkali metal hydride. International Journal of Hydrogen Energy, 2009. 34(24): p. 97609764.

50. Klerke, A., et al., Ammonia for hydrogen storage: Challenges and opportunities. Journal of Materials Chemistry, 2008. 18(20): p. 2304-2310.

51. Zamfirescu, C. and I. Dincer, Using ammonia as a sustainable fuel. Journal of Power Sources, 2008. 185(1): p. 459-465.

52. Lan, R., J.T.S. Irvine, and S. Tao, Ammonia and related chemicals as potential indirect hydrogen storage materials. International Journal of Hydrogen Energy, 2012. 37(2): p. 1482-1494.

53. Yin, S.F., et al., A mini-review on ammonia decomposition catalysts for on-site generation of hydrogen for fuel cell applications. Applied Catalysis A: General, 2004. 277(1-2): p. $1-9$. 
54. Christensen, C.H., et al., Towards an ammonia-mediated hydrogen economy? Catalysis Today, 2006. 111(1-2): p. 140-144.

55. Saika, T.a., Nakamura, M.b, Nohara, T.c, Ishimatsu, S.d Study of Hydrogen Supply system with Ammonia Fuel. JSME International Journal, 2006. 49(1).

56. Rarog-Pilecka, W., et al., Carbon-supported cobalt-iron catalysts for ammonia synthesis. Applied Catalysis A: General, 2006. 300(2): p. 181-185.

57. Pfromm, P.H., Towards sustainable agriculture: Fossil-free ammonia. Journal of Renewable and Sustainable Energy, 2017. 9(3).

58. Lan, R., J.T.S. Irvine, and S. Tao, Synthesis of ammonia directly from air and water at ambient temperature and pressure. Scientific Reports, 2013. 3.

59. Kuriyama, S., et al., Catalytic transformation of dinitrogen into ammonia and hydrazine by iron-dinitrogen complexes bearing pincer ligand. Nature Communications, 2016. 7.

60. Bičáková, O. and P. Straka, Production of hydrogen from renewable resources and its effectiveness. International Journal of Hydrogen Energy, 2012. 37(16): p. 1156311578.

61. Rahimpour, M.R., H.R. Mottaghi, and M.M. Barmaki, Hydrogen production from urea wastewater using a combination of urea thermal hydrolyser-desorber loop and a hydrogenpermselective membrane reactor. Fuel Processing Technology.

62. HSC Chemistry. GCD.

63. Rei, B.M.H., G.T. Yeh, and C.W. Pan, Catalysis-spillover-membrane-2 [1] the rate enhancement of methanol steam reforming reaction in a membrane catalytic reactor. Catalysis Today, 2004. 97(2-3 SPEC. ISS.): p. 167-172.

64. Cui, H.Z., et al., Iron-based composite nanostructure catalysts used to produce COx-free hydrogen from ammonia. Science Bulletin, 2016. 61(3): p. 220-226.

65. Podila, S., et al., Hydrogen production by ammonia decomposition using high surface area Mo2N and Co3Mo3N catalysts. Catalysis Science and Technology, 2016. 6(5): p. 14961506.

66. Varisli, D. and T. Rona, COx free hydrogen production from ammonia decomposition over platinum based siliceous materials. International Journal of Chemical Reactor Engineering. 10(1).

67. Simonsen, S.B., et al., Alloyed Ni-Fe nanoparticles as catalysts for NH 3 decomposition. Applied Catalysis A: General, 2012. 447-448: p. 22-31.

68. Yin, S.F., et al., Nano Ru/CNTs: A highly active and stable catalyst for the generation of COx-free hydrogen in ammonia decomposition. Applied Catalysis B: Environmental, 2004. 48(4): p. 237-241.

69. Hill, A.K. and L. Torrente-Murciano, Low temperature H2 production from ammonia using ruthenium-based catalysts: Synergetic effect of promoter and support. Applied Catalysis B: Environmental, 2015. 172-173: p. 129-135.

70. Hill, A.K. and L. Torrente-Murciano, In-situ H2 production via low temperature decomposition of ammonia: Insights into the role of cesium as a promoter. International Journal of Hydrogen Energy, 2014. 39(15): p. 7646-7654.

71. Varisli, D. and N.G. Kaykac, CO $x$ free hydrogen production over cobalt incorporated silicate structured mesoporous catalysts. Applied Catalysis B: Environmental, 2012. 127: p. 389-398. 
72. Li, G., et al., Preparation of a novel bimodal catalytic membrane reactor and its application to ammonia decomposition for CO $x$-free hydrogen production. International Journal of Hydrogen Energy, 2012. 37(17): p. 12105-12113.

73. Li, G., M. Kanezashi, and T. Tsuru, Highly enhanced ammonia decomposition in a bimodal catalytic membrane reactor for COx-free hydrogen production. Catalysis Communications, 2011. 15(1): p. 60-63.

74. Li, G., et al., Ammonia decomposition in catalytic membrane reactors: Simulation and experimental studies. AIChE Journal, 2013. 59(1): p. 168-179.

75. Roduner, E., Understanding catalysis. Chemical Society Reviews, 2014. 43(24): p. 8226-8239.

76. Boisen, A., et al., Why the optimal ammonia synthesis catalyst is not the optimal ammonia decomposition catalyst. Journal of Catalysis, 2005. 230(2): p. 309-312.

77. Lakhapatri, S.L. and M.A. Abraham, Analysis of catalyst deactivation during steam reforming of jet fuel on $\mathrm{Ni}-(\mathrm{PdRh}) / \hat{1}^{3}-\mathrm{Al} 2 \mathrm{O} 3$ catalyst. Applied Catalysis A: General, 2012. 405(1-2): p. 149-159.

78. Choudhary, T.V., C. Sivadinarayana, and D.W. Goodman, Production of COx-free hydrogen for fuel cells via step-wise hydrocarbon reforming and catalytic dehydrogenation of ammonia. Chemical Engineering Journal, 2003. 93(1): p. 69-80.

79. Yin, S.F., et al., Investigation on the catalysis of COx-free hydrogen generation from ammonia. Journal of Catalysis, 2004. 224(2): p. 384-396.

80. Al-Shammeri, K.K. and J.M. Saleh, Adsorption and decomposition of ammonia on metal films of nickel, palladium, tungsten, and aluminum. Journal of Physical Chemistry, 1986. 90(13): p. 2906-2910.

81. Logan, S.R. and C. Kemball, The catalytic decomposition of ammonia on evaporated metal films. Transactions of the Faraday Society, 1960. 56: p. 144-153.

82. Li, g.c.d.L., Chromium Oxide Catalyst for COx free hydrogen generation via catalytic ammonia decomposition. Journal of Molecular Catalysis A: Chemical, 2008.

83. Takahashi, A. and T. Fujitani, Kinetic analysis of decomposition of ammonia over Nickel and Ruthenium catalysts. Journal of Chemical Engineering of Japan, 2016. 49(1): p. 22-28.

84. Ganley, J.C., et al., A priori catalytic activity correlations: The difficult case of hydrogen production from ammonia. Catalysis Letters, 2004. 96(3-4): p. 117-122.

85. Tsai, W. and W.H. Weinberg, Steady-state decomposition of ammonia on the Ru(001) surface. Journal of Physical Chemistry, 1987. 91(20): p. 5302-5307.

86. Rei, M.H., et al., Steam reforming reaction of methanol with a membrane reactor catalysis-spillover-membrane, in Studies in Surface Science and Catalysis. 2002. p. 103108.

87. Vajo, J.J., W. Tsai, and W.H. Weinberg, Mechanistic details of the heterogeneous decomposition of ammonia on platinum. Journal of Physical Chemistry, 1985. 89(15): p. 3243-3251.

88. Bradford, M.C.J., P.E. Fanning, and M.A. Vannice, Kinetics of NH3 decomposition over well dispersed Ru. Journal of Catalysis, 1997. 172(2): p. 479-484.

89. Tamaru, K., A "new" general mechanism of ammonia synthesis and decomposition on transition metals. Accounts of Chemical Research, 1988. 21(2): p. 88-94. 
90. Vilekar, S.A., I. Fishtik, and R. Datta, The peculiar catalytic sequence of the ammonia decomposition reaction and its steady-state kinetics. Chemical Engineering Science, 2012. 71: p. 333-344.

91. Maier, S., et al., Bonding of ammonia and its dehydrogenated fragments on $R u(0001)$. Journal of Physical Chemistry C, 2012. 116(48): p. 25395-25400.

92. Uemiya, S., et al., Steam reforming of methane in a hydrogen-permeable membrane reactor. Applied Catalysis, 1990. 67(1): p. 223-230.

93. Borgognoni, F. and S. Tosti, Pd-Ag multi-membranes module for hydrogen production by methane auto-thermal reforming. International Journal of Hydrogen Energy, 2012. 37(2): p. 1444-1453.

94. Matsuka, M., M. Higashi, and T. Ishihara, Hydrogen production from methane using vanadium-based catalytic membrane reactors. International Journal of Hydrogen Energy, 2013. 38(16): p. 6673-6680.

95. Mei, I.L.S., et al., Thermo-catalytic methane decomposition for hydrogen production: Effect of palladium promoter on Ni-based catalysts. Bulletin of Chemical Reaction Engineering and Catalysis, 2016. 11(2): p. 191-199.

96. Tosti, S., et al., Pressure effect in ethanol steam reforming via dense Pd-based membranes. Journal of Membrane Science, 2011. 377(1-2): p. 65-74.

97. Iulianelli, A., et al., The oncoming energy vector: Hydrogen produced in Pd-composite membrane reactor via bioethanol reforming over $\mathrm{Ni} / \mathrm{CeO}<$ inf $>2</$ inf $>$ catalyst. Catalysis Today, 2015.

98. Gallucci, F., et al., Ethanol steam reforming in a dense Pd-Ag membrane reactor: A modelling work. Comparison with the traditional system. International Journal of Hydrogen Energy, 2008. 33(2): p. 644-651.

99. Basile, A., Hydrogen Production Using Pd-based Membrane Reactors for Fuel Cells. Topics in Catalysis, 2008: p. 1-16.

100. Dincer, I., Green methods for hydrogen production. International Journal of Hydrogen Energy, 2012. 37(2): p. 1954-1971.

101. Garcia-Garcia, F.R., et al., High purity hydrogen production by low temperature catalytic ammonia decomposition in a multifunctional membrane reactor. Catalysis Communications, 2008. 9(3): p. 482-486.

102. Brown, I.W.M., et al., Nanostructured alumina ceramic membranes for hydrogen separation, in A Global Road Map for Ceramic Materials and Technologies: Forecasting the Future of Ceramics, International Ceramic Federation - 2nd International Congress on Ceramics, ICC 2008, Final Programme. 2008: Verona.

103. Gepert, V., et al., Ceramic supported capillary Pd membranes for hydrogen separation: Potential and present limitations. Fuel Cells, 2006. 6(6): p. 472-481.

104. Armor, J.N., Applications of catalytic inorganic membrane reactors to refinery products. Journal of Membrane Science, 1998. 147(2): p. 217-233.

105. Rothenberger, K.S., et al., High pressure hydrogen permeance of porous stainless steel coated with a thin palladium film via electroless plating. Journal of Membrane Science, 2004. 244(1-2): p. 55-68.

106. Gallucci, F., et al., Recent advances on membranes and membrane reactors for hydrogen production. Chemical Engineering Science, 2013. 92: p. 40-66. 
107. Israni, S.H., B.K.R. Nair, and M.P. Harold, Hydrogen generation and purification in a composite Pd hollow fiber membrane reactor: Experiments and modeling. Catalysis Today, 2009. 139(4): p. 299-311.

108. Chen, Y., et al., Hydrogen production capacity of membrane reformer for methane steam reforming near practical working conditions. Journal of Membrane Science, 2008. 322(2): p. 453-459.

109. Abdollahi, M., et al. Palladium membrane reactors: An enabling technology for pure hydrogen production from reformate mixtures. in 11AIChE - 2011 AIChE Annual Meeting, Conference Proceedings. 2011. Minneapolis, MN.

110. Li, A., C.J. Lim, and J.R. Grace, Staged-separation membrane reactor for steam methane reforming. Chemical Engineering Journal, 2008. 138(1-3): p. 452-459.

111. Dunbar, Z.W. and D. Chu, Thin palladium membranes supported on microstructured nickel for purification of reformate gases. Journal of Power Sources, 2012. 217: p. 47-53.

112. Ward, T.L. and T. Dao, Model of hydrogen permeation behavior in palladium membranes. Journal of Membrane Science, 1999. 153(2): p. 211-231.

113. Iulianelli, A., T. Longo, and A. Basile, CO-free hydrogen production by steam reforming of acetic acid carried out in a Pd-Ag membrane reactor: The effect of co-current and counter-current mode. International Journal of Hydrogen Energy, 2008. 33(15): p. 4091-4096.

114. Holladay, J.D., et al., An overview of hydrogen production technologies. Catalysis Today, 2009. 139(4): p. 244-260.

115. Plazaola, A.A., et al., Recent advances in pd-based membranes for membrane reactors. Molecules, 2017. 22(1).

116. Cropper, M.A.J., S. Geiger, and D.M. Jollie, Fuel cells: A survey of current developments. Journal of Power Sources, 2004. 131(1-2): p. 57-61.

117. Halseid, R., P.J.S. Vie, and R. Tunold, Effect of ammonia on the performance of polymer electrolyte membrane fuel cells. Journal of Power Sources, 2006. 154(2): p. 343-350.

118. Shao, G., et al., Study on the initial electrodeposition behavior of Ni-P alloys. Materials Chemistry and Physics, 2005. 90(2-3): p. 327-332.

119. Grujicic, D. and B. Pesic, Electrodeposition of copper: The nucleation mechanisms. Electrochimica Acta, 2002. 47(18): p. 2901-2912.

120. Penner, R., Mesoscopic Particles and Wires by Electrodeposition. Journal of Physical Chemistry, 2002. 106: p. 3339-3353.

121. Adams, R.N., Electrochemistry at solid Electrodes. 1969, Marcel Decker Inc: New York.

122. Sahari, A., et al., Electrochemical study of cobalt nucleation mechanisms on different metallic substrates. Materials Chemistry and Physics, 2008. 108(2-3): p. 345-352.

123. Crousier, J., et al., Influence of substrate on the electrodeposition of nickel-molybdenum alloys. Journal of Applied Electrochemistry, 1992. 22(8): p. 749-755.

124. Zach, M.P. and R.M. Penner, Nanocrystalline nickel nanoparticles. Advanced Materials, 2000. 12(12): p. 878-883.

125. Ebrahimi, F. and Z. Ahmed, The effect of current density on properties of electrodeposited nanocrystalline nickel. Journal of Applied Electrochemistry, 2003. 33(8): p. 733-739. 
Jonathan Tailby

126. Ebrahimi, F., et al., Mechanical properties of nanocrystalline nickel produced by electrodeposition. Nanostructured Materials, 1999. 11(3): p. 343-350.

127. Motoyama, M., et al., Initial stages of electrodeposition of metal nanowires in nanoporous templates. Electrochimica Acta, 2007. 53(1): p. 205-212.

128. Lamer, V.K. and R.H. Dinegar, Theory, production and mechanism of formation of monodispersed hydrosols. Journal of the American Chemical Society, 1950. 72(11): p. 4847-4854.

129. Roland, U., T. Braunschweig, and F. Roessner, On the nature of spilt-over hydrogen. Journal of Molecular Catalysis A: Chemical, 1997. 127(1-3): p. 61-84.

130. Roessner, F. and U. Roland, Hydrogen spillover in bifunctional catalysis. Journal of Molecular Catalysis A: Chemical, 1996. 112(3): p. 401-412.

131. Khoobiar, S., Particle to particle migration of hydrogen atoms on platinum-alumina catalysts from particle to neighboring particles. Journal of Physical Chemistry, 1964. 68(2): p. 411-412.

132. Weigle, J.C. and J. Phillips, Novel Dual-Bed Reactors: Utilization of Hydrogen Spillover in Reactor Design. Langmuir, 2004. 20(4): p. 1189-1193.

133. Baber, A.E., et al., An atomic-scale view of palladium alloys and their ability to dissociate molecular hydrogen. ChemCatChem, 2011. 3(3): p. 607-614.

134. Li, L., et al., Fe-assisted Ru clusters supported on porous and graphitic carbon for ammonia decomposition to $\mathrm{CO}_{x}$ free hydrogen. RSC Advances, 2016. 6(104): p. 102336-102342.

135. Pelka, R., K. Kiełbasa, and W. Arabczyk, Catalytic ammonia decomposition during nanocrystalline iron nitriding at $475^{\circ} \mathrm{C}$ with $\mathrm{NH} 3 / \mathrm{H} 2$ mixtures of different nitriding potentials. Journal of Physical Chemistry C, 2014. 118(12): p. 6178-6185.

136. Šotola, J. and Z. Knor, Field Emission Microscopy Study of Supported Bimetallic Catalysts $\mathrm{Pd}-\mathrm{Mo} / \mathrm{Al}_{2} \mathrm{O}_{3} / \mathrm{W}$ : Nitrogen Spillover. Journal of Catalysis, 1994. 145(2): p. 501-507.

137. Knor, Z., et al., Nitrogen spill-over effect in the palladium-tungsten system. Applied Surface Science, 1986. 25(1-2): p. 107-117.

138. Konda, S.K. and A. Chen, Palladium based nanomaterials for enhanced hydrogen spillover and storage. Materials Today, 2016. 19(2): p. 100-108.

139. Hakamada, M., et al., Hydrogen storage properties of nanoporous palladium fabricated by dealloying. Journal of Physical Chemistry C, 2010. 114(2): p. 868-873.

140. Ross, D.K., Hydrogen storage: The major technological barrier to the development of hydrogen fuel cell cars. Vacuum, 2006. 80(10): p. 1084-1089.

141. Adhikari, S. and S. Fernando, Hydrogen membrane separation techniques. Industrial and Engineering Chemistry Research, 2006. 45(3): p. 875-881.

142. Caravella, A., G. Barbieri, and E. Drioli, Modelling and simulation of hydrogen permeation through supported Pd-alloy membranes with a multicomponent approach. Chemical Engineering Science, 2008. 63(8): p. 2149-2160.

143. David, E. and J. Kopac, Development of palladium/ceramic membranes for hydrogen separation. International Journal of Hydrogen Energy.

144. Yun, S. and S. Ted Oyama, Correlations in palladium membranes for hydrogen separation: A review. Journal of Membrane Science. 375(1-2): p. 28-45. 
145. Hatlevik, O., et al., Palladium and palladium alloy membranes for hydrogen separation and production: History, fabrication strategies, and current performance. Separation and Purification Technology, 2010. 73(1): p. 59-64.

146. Winkler, A., Adsorption, reaction and desorption of hydrogen on modified $\operatorname{Pd}\left(\begin{array}{lll}1 & 1 & 1\end{array}\right)$ surfaces. Applied Surface Science, 2009. 256(4): p. 1114-1119.

147. Dong, W., et al., Hydrogen adsorption on palladium: A comparative theoretical study of different surfaces. Surface Science, 1998. 411(1-2): p. 123-136.

148. Flanagan, T.B. and W.A. Oates, The palladium-hydrogen system. Annual Review of Materials Science, 1991. 21(1): p. 269-304.

149. Simons, J.W. and T.B. Flanagan, Diffusion of hydrogen in the It+-phase of the palladiumhydrogen system. Journal of Physical Chemistry, 1965. 69(10): p. 3581-3587.

150. Jose, D. and B.R. Jagirdar, Nature of hydrogen atom trapped inside palladium lattice. International Journal of Hydrogen Energy, 2010. 35(13): p. 6804-6811.

151. McLeod, L.S., F.L. Degertekin, and A.G. Fedorov, Non-ideal absorption effects on hydrogen permeation through palladium-silver alloy membranes. Journal of Membrane Science, 2009. 339(1-2): p. 109-114.

152. Cizek, J., et al. Hydrogen-induced defects in Pd films. in Physica Status Solidi (C) Current Topics in Solid State Physics. 2009. Kolkata.

153. Dillon, E., et al., Factors influencing the tensile strength, hardness, and ductility of hydrogen-cycled palladium. Materials Science and Engineering A, 2009. 524(1-2): p. 89-97.

154. Basile, A., et al., CO-free hydrogen production by ethanol steam reforming in a Pd-Ag membrane reactor. Fuel Cells, 2008. 8(1): p. 62-68.

155. Basile, A., et al., Hydrogen production by methanol steam reforming carried out in membrane reactor on $\mathrm{Cu} / \mathrm{Zn} / \mathrm{Mg}$-based catalyst. Catalysis Today, 2008. 137(1): p. 1722.

156. Flanagan, T.B., D. Wang, and K.L. Shanahan, Diffusion of H through Pd membranes: Effects of non-ideality. Journal of Membrane Science, 2007. 306(1-2): p. 66-74.

157. Gielens, F.C., et al., High-flux palladium-silver alloy membranes fabricated by microsystem technology. Desalination, 2002. 147(1-3): p. 417-423.

158. McLeod, L.S., F.L. Degertekin, and A.G. Fedorov, Determination of the rate-limiting mechanism for permeation of hydrogen through microfabricated palladium-silver alloy membranes. Journal of Membrane Science, 2009. 341(1-2): p. 225-232.

159. Guazzone, F., E.E. Engwall, and Y.H. Ma, Effects of surface activity, defects and mass transfer on hydrogen permeance and n-value in composite palladium-porous stainless steel membranes. Catalysis Today, 2006. 118(1-2): p. 24-31.

160. Lundin, S.T.B., et al., The role (or lack thereof) of nitrogen or ammonia adsorptioninduced hydrogen flux inhibition on palladium membrane performance. Journal of Membrane Science, 2016. 514: p. 65-72.

161. Wang, W., et al., The effect of co-existing nitrogen on hydrogen permeation through thin $P d$ composite membranes. Separation and Purification Technology, 2007. 54(2): p. 262-271. 
162. Rizzuto, E., P. Palange, and Z. Del Prete, Characterization of an ammonia decomposition process by means of a multifunctional catalytic membrane reactor. International Journal of Hydrogen Energy, 2014. 39(22): p. 11403-11410.

163. Deng, M.J., et al., Electrodeposition behavior of nickel in the water-and air-stable 1-ethyl3-methylimidazolium-dicyanamide room-temperature ionic liquid. Electrochimica Acta, 2008. 53(19): p. 5812-5818.

164. Xu, Y., X.M. Ge, and J.Z. Jiang, Preparation and growth behavior of amorphous $P d<s u b>40</ s u b>N i<s u b>40</ s u b>P<s u b>20</ s u b>$ film by electrodeposition. Journal of Solid State Electrochemistry, 2009. 13(5): p. 713-720.

165. Rasband, W., ImageJ. 2005, National Instutes of Mental Health: Bethesda, Maryland, USA.

166. Majumder, M., et al., Insights into the physics of spray coating of SWNT films. Chemical Engineering Science, 2010. 65(6): p. 2000-2008.

167. Itoh, N., et al., Kinetic enhancement of ammonia decomposition as a chemical hydrogen carrier in palladium membrane reactor. Catalysis Today, 2014. 236(PART A): p. 70-76.

168. Su, Q., et al., Layered double hydroxides derived $\mathrm{Ni}_{x}\left(\mathrm{Mg}_{y} A l_{z} \mathrm{O}_{n}\right)$ catalysts: Enhanced ammonia decomposition by hydrogen spillover effect. Applied Catalysis B: Environmental, 2017. 201: p. 451-460.

169. Yao, Y. and D.W. Goodman, Direct evidence of hydrogen spillover from $\mathrm{Ni}$ to $\mathrm{Cu}$ on $\mathrm{Ni}$ $\mathrm{Cu}$ bimetallic catalysts. Journal of Molecular Catalysis A: Chemical, 2014. 383-384: p. 239-242.

170. Zieliński, M., et al., Hydrogen storage in nickel catalysts supported on activated carbon. International Journal of Hydrogen Energy, 2007. 32(8): p. 1024-1032.

171. Nikolić, V.D., et al., Nickel-based catalysts: Dependence of properties on nickel loading and modification with palladium. Hemijska Industrija, 2016. 70(2): p. 137-142.

172. Leppert, L., R. Kempe, and S. Kümmel, Hydrogen binding energies and electronic structure of Ni-Pd particles: a clue to their special catalytic properties. Physical Chemistry Chemical Physics, 2015. 17(39): p. 26140-26148.

173. Liu, H., et al., Promotion effect of cerium and lanthanum oxides on Ni/SBA-15 catalyst for ammonia decomposition. Catalysis Today, 2008. 131(1-4): p. 444-449.

174. Nishimura, C., et al., V-Ni alloy membranes for hydrogen purification. Journal of Alloys and Compounds, 2002. 330-332: p. 902-906.

175. Waters, Gas Chromatography Column Packing Materials, W. Corporation, Editor. 